\title{
Asthma
}

\section{Pediatric Asthma}

Asthma has been recognized as a disease since the earliest times. In the Corpus Hippocraticum, Hippocrates used the term " $\alpha \sigma \theta \mu \alpha$ " to indicate any form of breathing difficulty manifesting itself by panting. Aretaeus of Cappadocia, a well-known Greek physician (second century A.D.), is credited with providing the first detailed description of an asthma attack [13], and to Celsus it was a disease with wheezing and noisy, violent breathing. In the history of Rome, we find many members of the Julio-Claudian family affected with probable atopic respiratory disorders: Caesar Augustus suffered from bronchoconstriction, seasonal rhinitis as well as a highly pruritic skin disease. Claudius suffered from rhinoconjunctivitis and Britannicus was allergic to horse dander [529]. Maimonides (1136-1204) warned that to neglect treatment of asthma could prove fatal, whereas until the 19th century, European scholars defined it as "nervous asthma," a term that was given to mean a defect of conductivity of the ninth pair of cranial nerves.

If, from a clinical point of view, asthma can be defined with a fair degree of precision, much doubt still surrounds its etiopathogenesis, although the important role played by inflammation has been largely clarified. To this day, asthma still retains the characteristics of frequency and unexpected severity that can negatively affect a child, leading to considerable concern in the family. Asthma begins early in life [305] within the 1 st year of life (Table 5.5) with the developing immune system interacting with environmental influence [305]. Moreover, cases of pediatric asthma are increasing, as can be seen from epidemiological figures (Tables 5.10, 5.12), even though cases may be differently labeled, especially when symptoms are mild or moderate, and subsequently often underdiagnosed [446]. A large body of epidemiological evidence shows that allergic disorders in the pediatric population represent a current major therapeutic and preventive challenge for pediatricians. The surprising upsurge of severe asthma is occurring mainly in infants and children (Figs. 5.1, 5.3, 5.8). Until recently, asthma was defined as a disease fundamentally characterized by a state of bronchial hyperreactivity (BHR). In the last few years, it has been concluded that BHR and bronchoconstriction could be the result of the inflammation primed by Th2 T cells [340].
This change in our understanding has stimulated new lines of research and led to a new therapeutic approach [340]. Progress has derived from information gained by new investigative methods such as the study of bronchoalveolar lavage fluid (BALF) cells, using immunohistochemical and molecular biology techniques, and widespread use of more precise fiberoptic bronchoscopy, especially in children [501]. Asthma is a disorder involving all bronchial structures and depends on a complex interaction between the respiratory tract and inflammatory cells, mediators and adhesion molecules. Release of mediators by metachromatic cells primes both activation and migration of inflammatory cells that cause various degrees of airway obstruction over relatively short periods of time, alterations in the mucociliary system and hyperreactivity of the bronchial smooth muscles. It follows that both inflammatory cells and their products play a key role in provoking an airway inflammation, capable of triggering severe symptoms in predisposed children when specific and aspecific stimuli are present. Pediatric asthma differs from adult asthma by its etiopathogenesis, treatment and prognosis. Studies reporting that the future prospects for treating asthmatic babies and children are optimistic [604] have not taken into account the severity of childhood symptoms [264]. The understanding that events during both fetal and postnatal life may be the major determining factors of chronic asthma is also making progress [589].

The classic concept of asthma is that of a chronic inflammatory disease with a multifactorial pathogenesis, characterized by a state of hyperresponsiveness to various stimuli and a level and intensity that for the most part do not cause disturbances in healthy subjects, which consequently leads to a diffuse airway obstruction, either partially or fully reversible, either spontaneously or following treatment. The confusion still existing in terminology (Chap.5), the reluctance of doctors to use the term "asthma" and of parents to accept it, serve only to delay the diagnosis and treatment. It has long been debated whether two forms of asthma exist: allergic, or extrinsic, and infection-induced, or intrinsic. Some [61] have not confirmed the existence of the latter and others [552] have not found any difference between the IgE values in normal subjects and in subjects with so-called intrinsic asthma. Probably many patients thus classified may be sensitized to unknown 
allergens and/or are not included in the usual allergen battery of tests. For example, patients from Catalonia showed $8.5 \%$ of sensitization to an unusual pollen of the profilin family, perhaps an emerging pollen (Chap.6). This chapter also includes bronchiolitis, extrinsic allergic alveolitis (EAA), and allergic bronchopulmonary aspergillosis $(\mathrm{ABA})$.

\section{Definitions}

Asthma. To date asthma is a severe pediatric disease, affecting a great number of infants during their very first years of life (Table 5.5), which requires early and specific cures. Pediatric asthma should be viewed as a syndrome of lung dysfunction with an imbalance between the forces that maintain airway patency and those forces that operate to narrow or close the pediatric airway [274]. Apart from symptomatic management, pediatric asthma can be cured by anti-IgE, antileukotriene drugs (anti-LT) (see "Leukotriene Modifiers") and SIT (specific immunotherapy) or respiratory desensitization.

Canny and Levison have suggested that any child, in any age group, who has $\geq 3$ episodes of afebrile bronchospasm should be considered as suffering from asthma until the contrary can be proven [79]. This is the definition we and others also prefer [574]. However, the number of asthmatic episodes is not always a helpful guide: $50 \%$ of children suffering $\geq 4$ attacks at age $4-5$ years had no symptoms by the time they reached the age of 10 [471]. Asthma is a chronic lung inflammation, whose characteristics may be summarized as an acute onset of symptoms with bronchoconstriction (clinical data), reversible either spontaneously or with appropriate treatment (pharmacological data) $[465,594]$, accompanied by BHR to diverse stimuli (functional data) and by an inflammation of varying degree, which conditions its persistence, duration and severity (biological data) $[465,739]$. Asthma can be clinically defined as an airway response to different stimuli, with paroxysmal dyspnea, wheezing and coughing, variable in form from mild to severe, or ultimately status asthmaticus [594]. Asthma is characterized by lymphocytes and eosinophils infiltrating in the submucosa. chronic airway inflammation leading to BHR, mucous gland hyperplasia, microvascular leakage, mucus hypersecretion, thickening of the subepithelial collagen layer, epithelial desquamation, mast cell degranulation, airway tissue hyperplasia and hypertrophy, causing variable airflow obstruction $[31,739]$ that requires longterm recovery.

Wheezing. Wheezing is an onomatopoeic word reproducing the sound made by air rushing through narrowed airways in a flow that is no longer laminar but turbulent, which may also be defined as wheezing and/or dyspnea.
BHR. BHR is the particular ease with which the bronchi constrict in response to stimuli [154]: specific stimuli, the allergens, which provoke bronchospasm in a restricted number of subjects defined, therefore, as allergic; aspecific stimuli cause wheezing even in nonallergic children [603] suffering from bronchial disorders of various types [722] and even in healthy children, if the intensity is at least fivefold stronger than in asthmatic children [335]. BHR is therefore dependent on a markedly lowered threshold of the bronchial response to causative factors, which results in exaggerated bronchoconstriction, following which the bronchi constrict too readily and excessively [154]. Bronchoconstriction depends partly on inflammation, partly on smooth muscle spasm, on the mucosal edema and on the modification of the secretions, with formation of viscous plugs that often fill the bronchioles [255]. Some authors, in consideration of the fact that BHR occurs in the presence of nonallergic stimuli [636], propose the synonym "aspecific bronchoreactivity," which, however, ignores the specific forms [179, 481]. In fact, nonallergic stimuli work through specific, though different, mechanisms, so it seems imprecise to apply such terminology. We believe that it seems clearer to define BHR by the agent that provokes it [238].

\section{Prevalence}

Asthma is the most prevalent chronic breathing disease that affects subjects of all pediatric ages, but begins in the first few years. In $85 \%$ of cases, it begins in children by the age of 1 year, $80 \%-85 \%$ by 5 years of age (when statistics begin) and $94 \%-97 \%$ within 10 years. This is confirmed by $10.8 \%$ of new cases in the 2nd decade of life (Table 5.5); therefore, $90 \%$ of cases occur in infancy, considering that sensitization to inhalants is established very early [98] and that cases of sensitization from the 1 st year of life onward occur very frequently. During 1991 , there were approximately 1.6 million visits for pediatric asthma care. Asthma accounted for $16.9 \% \pm 9.0 \%$ of all pediatric emergency department (ED) visits [121]. During 2000, a nationwide survey among 437,873 Chinese children aged $0-14$ found a $1.69 \%$ prevalence in children aged $\geq 3$ years and of $0.23 \%$ in those aged $\leq 3$ years [100]. Among these children are those with $\mathrm{AD}$ (atopic dermatitis) who in $21 \%-79 \%$ of cases, subsequently develop asthma (Table 5.8). The prevalence is also increasing as in our study in 592 children compared to controls (Chap. 5), documenting an $88.8 \%$ increase in severe pediatric asthma. In 411 children ( 220 males and 191 females) ranging in age from 7 to 13 years, we have found asthma in $31.5 \%$ and AR in $25.8 \%$ of them. Respiratory allergy affects children even more than food allergy $(21.7 \%)$. Moreover, pollutants represent an adjuvant factor in the onset of respiratory allergy [521] (Figs. 4.26, 4.27) and we wonder whether similar interactions increase its prevalence. 


\section{Defense Mechanisms in the Airways}

\section{BALT (Bronchus-associated lymphoid issue) and Specific Defenses}

The respiratory apparatus's first and qualitatively most notable line of defense against excessive local immune responses is the defensive barriers, both physical and immunological, that range from safeguarding the tight junctions among epithelial cells to IgA secretion in the bronchial lumen. The respiratory epithelium is subjected to a virtually uninterrupted exposure to environmental antigens present in the 8,000-12,000 1 of air inhaled daily by an average person [520]. The key factor in the maintenance of airway immunological homeostasis is the immune system's ability to discriminate between intrinsically inoffensive antigens and/or those associated with pathogenic microorganisms, and the exclusion of the latter from the respiratory apparatus by the mucociliary system censoring mechanism, before they reach the underlying immune system. The defenses are not always well equipped and penetration of small quantities of allergens can be viewed as a normal occurrence [533].

Parallel to that which has been described in Chap. 9, a common mucosal immune system (MALT) exists, to which BALT is connected, located in the respiratory mucosa [40]. It has been hypothesized that in newborn babies and healthy children BALT is absent, or at least reduced [54] and that it could be an inducible system that develops in the airways of infants and children, especially in concomitance with exposure to antigens and pathogenic agents [193]. Several studies on humans [40] have, however, confirmed the existence of BALT. Anatomically, it consists of a well-developed lymphatic system originating from the NALT (nasal-associated lymphoid tissue) at the airway entrance, and continues with follicles and submucosal lymphoid aggregations distributed along the whole respiratory tract up to the bronchoalveolar junction [40] (Fig. 11.1). The tissue is covered by a layer of flat epithelial cells, with few B lymphocytes, but essentially with $\mathrm{T}$ lymphocytes $\left(\mathrm{CD}^{+}\right.$, $\mathrm{CD}^{+}$or $\mathrm{CD}^{+}$), grouped in clumps of lymphoid and follicles distributed in a seemingly homogeneous manner over the entire epithelial layer in the main bronchi of the pulmonary lobes, principally at the bifurcation with the bronchioles [193]. The most common lymphocytes are intraepithelial (IEL), situated close to the basement membrane (BM), generally not affected by pathological changes typical of epithelial structures and, as with GALT, CD4 T cells dominate over CD8 T cells [193]. Even in the lymphocytes distributed under the $\mathrm{BM}$ of the epithelial layer, $\mathrm{T}$ subsets are more numerous than $\mathrm{B}$ cells [193]. Specific to IEL is CD103, widely expressed on $\mathrm{T}$ cells, of which up to $80 \%$ are CD45RO. As in the intestine CD7 expression is frequent; expression of CD25 is lower [520]. The TcR with $\gamma / \delta$ chains plays a prominent
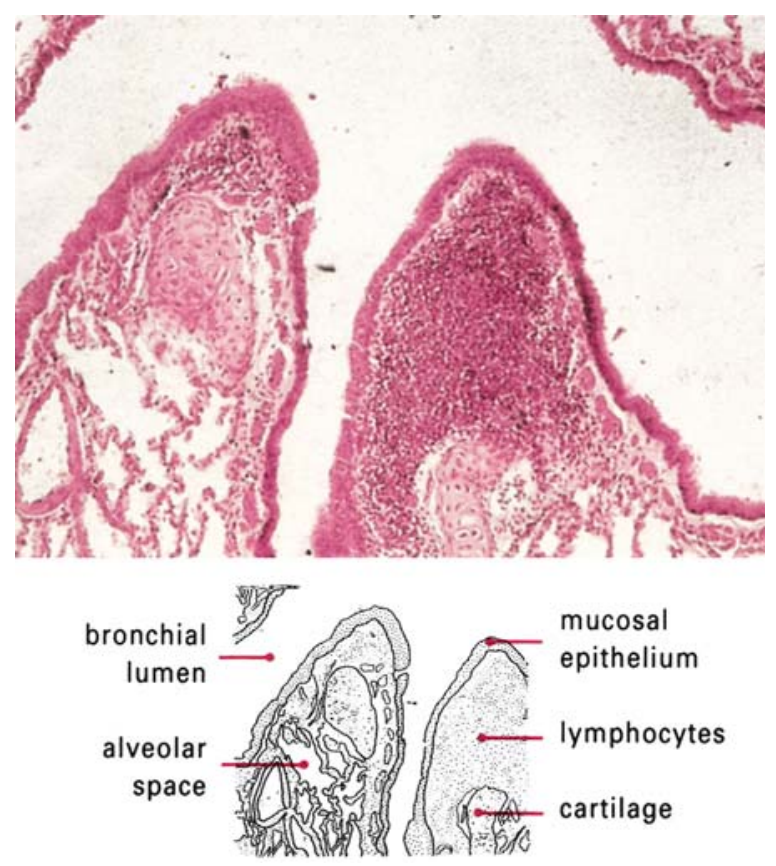

Fig. 11.1. BALT. Section of lung showing a diffuse accumulation of lymphocytes in the bronchial wall

role in the pulmonary first line of defense [250], and there is greater evidence, on the other hand, of TcR $\alpha \beta$, which display a large number of $\mathrm{V} \beta$ genes [520]. Seen in this light, it is important that in asthmatics the $\gamma \delta$ are reduced in the circulation parallel to eosinophil increase, even if the two phenomena seem to be unrelated: nevertheless, low $\gamma \delta$ levels in the airways could be detrimental (see Chap. 1 for further details).

The lymphocytes passing from blood flow to the airways reach BALT, devoid of afferent lymphatics adhering to the HEV (high endothelial postcapillary venules), which in BALT function similarly to the mesenteric lymph nodes where $\mathrm{B}$ and $\mathrm{T}$ cell numbers are similar [40]. BALT follicles contain small and medium lymphocytes but lack capsules and germinal centers characteristic of true lymph nodes [40]; T and B cell rates are similar to those of GALT: $20 \%$ of T and $40 \%-80 \%$ of B cells. This rate is reversed in both lymph nodes and circulation: $70 \%$ of $\mathrm{T}$ and $15 \%-20 \%$ of B cells [533]. Mast cells and macrophages with APC (antigen-presenting cells) activity are present in BALT; therefore antigens can be directly presented to lymphocytes in this location [520]. Additionally BALT might function as a repository for immunoglobulin (Ig)-bearing cells, but because it is poorly provided with plasma cells, further B-cell differentiation should occur outside the BALT; therefore, $\mathrm{B}_{\mathrm{IgA}}$ migrate directly to the lamina propria along the respiratory tract to produce antibodies for the mucosal surface [533], that is in the NALT, which appears to be the principal supplier of B precursors in the upper airways [54]. All Igs are diffused along the airways: plasma 
Fig. 11.2. Schematic representation of immune homeostasis in the

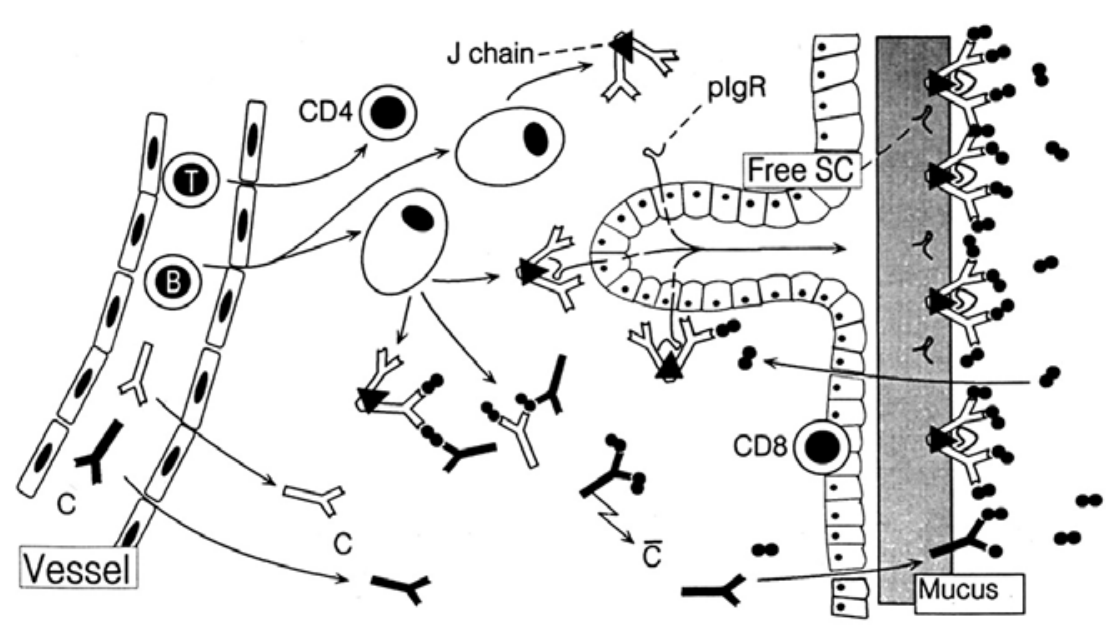

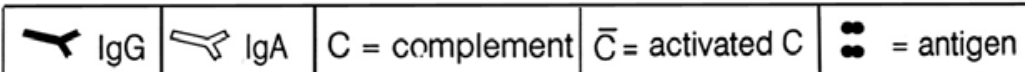
airway mucosa. Mucosal slgA antibodies act as a first-line defense by performing antigen immune exclusion. Antigens circumventing this barrier meet mucosal IgG antibodies. The resulting immune complexes activate complement, and inflammatory mediators most likely formed locally. However, the adverse inflammatory development is usually inhibited by serum $\operatorname{lgA}$ and by locally produced polymeric and monomeric $\lg A$. Antigens may be returned in a noninflammatory way to the vasal lumen by plgRmediated transport mechanisms. The final homeostasis depends on the profile of adhesion molecules expressed by vascular endothelium, normally facilitating preferential extravasation of $B$ and $T$ cells belonging to the MALT (for details see text). plgR Polymeric Ig receptor, slgA secretory $\lg A$

cells of the lamina propria pass into the lumen across the BM and epithelium. In the upper airways, IgA antibodies predominate, with the capacity of neutralizing the RSV (respiratory syncytial virus) Rhinovirus and influenza virus and of agglutinating microorganisms, increasing the mucociliary clearance. In the trachea and bronchial tree secretory $\operatorname{IgA}(\operatorname{sig} \mathrm{A})$ are $10 \%$ of the total proteins; progressing to the alveoli, IgA decrease as low as $5 \%$, while IgG increase up to $10 \%-15 \%$ [533]. Under normal conditions, the respiratory immune system is able to suppress immune responses triggered by allergens circumventing the barrier, in that a successful sIgA defensive system is ready [54]. As seen in Fig. 11.2 [54], sIgA are generated from locally produced polymeric IgA (pIgA), armed with J chain, which is transported by the pIgR (polymeric Ig receptor) to the lumen along with the SC (secretory component) [54]. Consequently, immune tolerance is established after the first contact with non-self agents with a two-phase mechanism related to genetic factors, but, mainly because of environmental factors capable of negatively affecting the immune system, a sensitization with the same mechanisms that we have seen play a part in food allergy (FA) can occur [54].

\section{Nonspecific Defenses}

Nonspecific defenses are essentially represented by bronchial secretions that block and neutralize soluble toxic substances and by the activity of the mucociliary system that propels them upward. Bronchial secretion emitted by the submucosal glands and/or by the goblet cells, and enriched by transudation fluids or exudation of blood origin, is made up of glycoproteins originating in the mucosa, arranged in a type of fibrillar network and of a component, more precisely a fluid, containing anti-infective substances (Ig, lysozyme, lactoferrin) and C3 [648]. Additionally, the bronchial secretion performs a major role by hydrating the inhaled air and maintaining the ionic balance. The mucociliary system, already in place in the first days of life, is made up of ciliated cells, each possessing, on average, 200 cilia, 5-7 $\mu \mathrm{m}$ long and $0.25 \mu \mathrm{m}$ wide. Each cilium is formed structurally of nine pairs of contractile longitudinal microtubules [648], connected two by two by means of nexin links, and arranged in circles around a central pair. All the tubules converge toward the tip of the cilium, forming a hood connected to the ciliary membrane. Two dynein bridges catalyze hydrolysis of ATPase and transduce the energy into a mechanical force in the form of ciliary bending. The cilia act on the mucus blanket shed from the mucosal glands and especially that from goblet cells. At the moment of the active phase of the beat (15/s), the mucus is driven by the ciliary tip toward the oropharynx at increasing speed from the periphery toward the trachea, accelerating from $2-4 \mu \mathrm{m} / \mathrm{min}$. At the alveolar level, the alveolocapillary membrane is covered with surfactant, produced by type II epithelial cells and nonciliated Clara cells, situated on the branching border between bronchial ducts and bronchioles, which synthesize the surfactant A-C proteins. This substance, having surface-active properties, changes the surface-charge properties, making foreign particles less viscid and therefore more easily cleared [648]; in addition, it promotes the intervention of the phagocytes. Ciliary clear- 
ance is substituted in the distal airways by that of macrophages and by cough reflexes [648].

The alveolar macrophages, in the presence of antigens, pollutants, or chronic irritants, pass from a state of quiescence to one of activation and, if the stimulus persists, to one of intense phagocytic activity, with IL (interleukins) production. In particular, inorganic substances are taken up and transported mechanically to the mucociliary system, while organic substances are decayed by cytoplasmic and lysosomal enzymes, and microorganisms are phagocytized and subjected within the phagosomes to the action of free radicals and cationic proteins [330]. Macrophages can also have a deleterious effect on the airways, by releasing enzymes with lytic activity, oxidants and proteases normally neutralized by anti-proteases and by ILs produced by macrophages. Free radicals, ozone $\left(\mathrm{O}_{3}\right)$ and nitrogen dioxide $\left(\mathrm{NO}_{2}\right)$ can, in turn, alter the local protease/antiprotease equilibrium. Lastly, the macrophages have the role of controlling T-mediated activation of the immune system: in subjects with BHR, but without symptoms of inflammation and asthma, macrophage hyperactivity may result in the suppression of local $\mathrm{T}$ cells, while a reduced activity could be fundamental in triggering $\mathrm{T}$ lymphocyte hyperactivation and up-regulating chronic inflammation, which could further exacerbate BHR up to a pattern of full-blown disease [503].

\section{Alterations of the Defense Mechanisms}

- Immaturity. At birth not all the mechanisms contributing to the protection of the airways have reached their full functional potential. The mucociliary system develops very early during intrauterine life and proves to be fully suited to the mechanical purification of the particles inhaled from the very first hours of life. The alveolar macrophages, on the other hand, are late in reaching the airways. This is because they are immature cells endowed with phagocytic activity, but without an oxidizing metabolism or cationic proteins able to ensure efficient bactericidal action. In addition, the factors of acquired immunity have not yet been stimulated and therefore, the appropriate protection is temporarily taken care of by the maternal antibodies.

- Constitutional disease. Diverse genetically transmitted diseases share the same characteristic of appearing in the first years of life with recurrent respiratory infections (RRI), for example, cystic fibrosis (CF), primary ciliary dyskinesia, etc.

- Immunodeficiencies (IDs), which reduce the airway resistance (Raw) to infections, including primary $\operatorname{IgA}$ deficiency, chronic granulomatosis, etc. (Chap. 22).

- Deficiency of $\alpha 1$-antitrypsin. The primary deficiency lies in an insufficiency of the anti-protease defense, corresponding to phenotype $\mathrm{ZZ}$, which favors the development of emphysematous lesions.

\section{Genetic Factors}

Asthma can be considered a multifactorial disease in that different genetic and environmental factors contribute to influencing its phenotypic expression [340]. The pathogenesis is consistently related to genetic factors, family history (FH), and the particular role of atopy, intimately interrelated.

Four sets of data have emerged recently: a clear-cut genetic component of 125 genes related to the causation and progression of asthma symptoms (Table 4.2), $I L_{13}$ overexpression [321], $I L_{12}$ deficient expression [415], and Th1 phenotype downregulation due to reduced expression of T-bet (Th1 transcription factor) [188] while GATA-3 is overexpressed [328], which is implicated in the Th2 development [430].

Several studies on the genetics of asthma confirmed the linkage between asthma and genetic markers on 13 chromosome regions including $5 q$ and $11 q$. Recently, the ADAM33 gene encoded on chromosome 20p13 (Table 4.2) was found to be associated with small-airway remodeling in patients with asthma. ADAM-33 polymorphisms may accelerate the proliferation of smoothmuscle cells and fibroblasts, leading to BHR and subepithelial fibrosis [582]. $\mathrm{IL}_{13}$ is thought to be especially critical in asthma (Table 1.5): normal signal transducer and activator of transcription 6 (STAT6) expression in epithelial cells is both necessary and sufficient to $\mathrm{IL}_{13}$ to induce BHR, eosinophil inflammation, mucus production, lung emphysema, and other central features of asthma in the absence of inflammation. However, mice lacking STAT6 were protected from all pulmonary effects of $\mathrm{IL}_{13}$ [321]. Two new ILs are more destructive. The $\mathrm{IL}_{17}$ family resulted in bronchoalveolar lavage neutrophilia and inflammatory gene expression in the lung. In addition, intranasal administration of $\mathrm{IL}_{25}$ protein resulted in the production of $\mathrm{IL}_{4}$, $\mathrm{IL}_{5}, \mathrm{IL}_{13}$, and eotaxin mRNA in the lung, marked eosinophilia in the BALF and lung tissue, eosinophil chemotaxis and activation mast cell stimulation, epithelial cell hyperplasia, increased mucus secretion, and BHR [269]. In CRH (corticotropin-releasing hormone) deficiency, such as in CRH knockout mice, increased levels of $\mathrm{IL}_{4}, \mathrm{IL}_{5}, \mathrm{IL}_{13}$, IFN- $\gamma$, RANTES and eotaxin in BALF were observed, thus increasing asthma severity [588]. FP in vitro impairs $\mathrm{IL}_{13}$ production by PHA (phytohemagglutinin)-stimulated PBMCs (peripheral blood mononuclear cells) from asthmatic and control subjects [150].

Thus the pathogenesis is associated with ILs, namely $\mathrm{IL}_{12}$ (down) and $\mathrm{IL}_{13}$ (up). Children heterozygous (HET) for $\mathrm{IL}_{12} \mathrm{~B}$ promoter polymorphism (associated with reduced $\mathrm{IL}_{12}$ gene transcription) have a greater risk for progression to severe asthma, irrespective of disease cause, but with no difference between nonatopic and atopic children with asthma [415]. As a consequence, 
everything is ready to up-regulate aberrant Th2 responses in atopic children, probably leading to a class switch to IgE antibody formation.

Outcomes in adult asthma may be determined primarily in early childhood. In an unselected birth cohor, $>25 \%$ children had wheezing that persisted from childhood to adulthood or that relapsed after remission. At age 21, 26.9\% had continuing symptoms of asthma; $14.5 \%$ had persistent wheezing from onset with no remission, and $12.4 \%$ had relapsed after remission. The factors predicting persistence or relapse were sensitization to Der p, BHR, female sex, smoking, and early age at onset [554]. It was previously found that at 25 years $88 \%$ of symptomatic subjects had BHR, a proportion statistically higher in the asthmatics than in the group of controls (12.8\%) [218]. The reason is that a very early presence of eosinophilic inflammation and even remodeling of airway wall occur early in the natural history of pediatric asthma and are present well before asthma would be diagnosed based on clinical symptoms [501]. That the pattern of asthma during childhood predicts outcome is confirmed by the Melbourne study in the original children followed-up at age 42 . Most children with persistent asthma (70\%), frequent asthma (69\%), and infrequent asthma (69\%) had severe asthma into adult life and reduced PFTs [264].

Stimulating findings have opened up an unexpected facet of asthma pathogenesis. Studies suggest that TNF- $\alpha$, a proinflammatory IL that participates in the inflammatory reaction in asthmatic patients, or nearby genes, including those in the HLA region, may contribute to the development of asthma in the Japanese population [449]. Platelet-activating factor (PAF), also implicated in the pathophysiology of inflammation in asthma, is degraded and inactivated by PAF acetylhydrolase: its deficiency is found more frequently in children with atopic asthma [275]. Polymorphism in the activation-induced cytidine deaminase gene might be associated with the pathogenesis of atopic asthma and the regulation of total serum IgE levels in children aged $<3$. The related deficiency leads to a complete defect in class-switching, resulting in a hyper-IgM phenotype and lack of IgG, IgA, and IgE. An area requiring further study is on the role of CC16 (Clara cells), which is secreted in the airway epithelium and plays a key role in inhibiting airway inflammation, but CC16 levels are reduced in patients with current asthma due to increased levels of its $A 38 G$ allele [325]. This allele was associated with increased BHR at age 1 month and increased risk of asthma at age 6 [324]. It might be an intriguing candidate gene determining asthma severity in children with the $\mathrm{CC} 16^{\star} 38 \mathrm{~A}$ phenotype by increasing Th2 IL production in their airways [242].

Even if the existence of a genetic predisposition to asthma has been proved (Table 4.2), the precise mode of hereditary transmission is not yet clear; according to several investigators it could appear to be of the autosomal dominant type. The same model has been attrib- uted to BHR, because of the significant difference between parents of asthmatic children (50\%) and those of healthy children (10\%) and, based on hyperreactivity to histamine, between nonasthmatic atopics, nonasthmatics $(33 \%)$, and the general population (6\%) [179]. In twins, the concordance rate of asthma (about 50\%) is $14.7 \%-19 \%$ in monozygotes (MZ) and $4.8 \%-8.7 \%$ in dizygotic (DZ) twins [174]; $44.5 \%$ of asthmatic children have positive skin prick tests $\left(\mathrm{SPT}^{+}\right)$vs $20.7 \%$ of healthy children [209]; also, total IgE and sIgE (specific) to inhalants are significantly high in asthmatic children, but normal in controls [433], indicating that genetic predisposition is an essential prerequisite, that increased BHR risk is associated with atopy and that severity is correlated to that of atopic manifestations. Although total serum IgE tracks with age, children who are predisposed to persistent wheezing and early sensitization to local aeroallergens already have high levels of IgE at age 6 [305] and 9 months [572].

Martinez et al [383] clearly indicate that a FH of asthma imposes an increased risk for childhood asthma and that elevated serum IgE levels measured during the first year of life are associated with subsequent asthma [383]. Total serum IgE levels were high at age 6 months in a cohort of 150 children at risk for developing asthma as offspring of mothers with asthma; IgE levels were still significantly higher for the asthmatic children at age 6-8 years with a GM (geometric mean) $=38.32$ as compared with nonasthmatic ones $(\mathrm{GM}=12.28)$. IgE levels were higher when the infants were 6 months of age [305]. Within the same family group, some members can have BHR and elevated IgE, others only high levels of $\operatorname{IgE}$ [229]. As mentioned in Chap. 5, the risk factor is significant in relation to positive $\mathrm{FH}(\mathrm{FH}+)$ : the prevalence of asthma has increased from four- to tenfold compared to subjects with negative FH [174]; if it is specifically related to asthma, children have an increased risk of becoming asthmatic within the 2nd year of life [746], with a BHR-atopy association correlated to age and to FH [122]. In our everyday work, we see that almost all asthmatic children have a positive $\mathrm{FH}$, and in those with severe asthma, such as the above-cited 592 children, the rate surpasses $95 \%$. A prospective cohort study has shown that even in the absence of respiratory symptoms $\mathrm{FH}+$ children and those with personal atopy could have impaired PFT (pulmonary function tests) early in life [360]. Asthma in adults is usually associated with an elevated PRIST (paper radio immunosorbent test) and with SPT+ to allergens, and therefore with an IgE-mediated mechanism. The concept that IgE was central in allergic asthma [305] was stressed by data from a population-based study, which showed a close and highly significant relationship between asthma and serum IgE levels [63]. Conversely, the existence of the two forms of asthma has not been confirmed because, once IgE levels have been standardized according to sex and age, the intrinsic forms were brought back to an IgE-mediated mechanism [63]. Even in children $[65,572]$ asthma and 
BHR are associated with elevated concentrations of $\operatorname{IgE}$ and atopic sensitization. Additionally, BHR is already present in newborns and in 2- to 10-week-old infants, before acquired factors can come into play [745], in 6-month-old at-risk babies [553], but also in $8 \%$ of apparently normal babies [746]. In early life, children of atopic parents and those with personal atopy have impaired PFTs even in the absence of respiratory symptoms, but with a significant interaction between history of maternal asthma and the child's atopic status [360]. These findings have up to now been observed only in adults and children with significant respiratory disease. The importance of serum IgE in determining BHR is decisive in the pediatric field [64]. If the association of BHR with the $\mathrm{FEV}_{1} / \mathrm{VC}$ (vital capacity) rate is operative only in asthmatic young people with at least moderate levels of IgE, then IgE influences the given relationship by interacting with BHR. Asthma associated with BHR increase is independent of all other factors considered. Introducing IgE levels and PFT results into this calculation, all other diagnostic and/or clinical parameters play a substantially secondary role [64]. Therefore, in infancy, BHR appears to be associated with atopy, with $\mathrm{FH}+$ of atopy (FHA) and asthma (indicating that it is hereditary) and with wheezing beginning very early or after 2 years of age.

In additional studies on pediatric cohorts, a significant correlation between $\mathrm{BRH}$, asthma and atopy was found in $90 \%-100 \%$ of cases [574] and the degree of atopic sensitization was assessed by SPT+ to several allergens and BHR in asthmatic children [65]. Agreement on a positive correspondence between BHR and atopy is not unanimous [574]: this was confirmed in two longitudinal studies $[4,107]$. Moreover, BHR is significantly more often found in atopic children with wheezing than in nonatopic children [108]. The correlation is absent in other children [108] and in adults followed up from age 7 [289]; infants at risk aged 6.5 months have reacted to the histamine test with BHR independently of wheezing [106]. Atopy can nonetheless be considered the main independent determining factor that contributes to BHR and even acquires a predictive character when accompanied by altered PFTs [574], in that subjects atopic from birth are those affected with more severe BHR in childhood [666]. Certainly, in childhood an airway inflammatory reaction manifests itself early, strictly related to atopy, which underlies on the one hand BHR and on the other - whether directly or not PFT reduction [574]. The even greater relationship between atopy, elevated IgE levels, infant asthma and BHR seems to us especially significant [16]. Another possible pathogenic factor is the finding of IgG subclass deficiency, with or without associated IgA deficiency, in children with chronic and/or severe asthma, especially if accompanied by RRI. Table 11.1 summarizes the intimate links between infant asthma and atopy.
Table 11.1. Pediatric asthma and atopy

Asthma is present in a high rate of parents of asthmatic children

In the parents and relatives, atopic disease and SPT positivity to allergens are increased

Up to $90 \%$ of asthmatic children have one or two atopic parents

If children suffer from atopic asthma, asthma is even more frequent in their family

The risk of asthma is related to the atopy degree

Asthma severity is correlated to severity of atopy

Early onset of atopic asthma is predictive

of subsequent BHR severity

Allergic rhinitis is present in $28 \%-61 \%$

and atopic dermatitis in $50 \%$ of asthmatic children

More than $80 \%$ of asthmatic children

have $\lg \mathrm{E}$ specific to one or more allergens

Even in the absence of specific lg $\mathrm{E}$,

the challenge test with allergens may be

positive in some children

Asthma frequency was the exception in developing countries before the arrival of an industrialized society

$B H R$ bronchial hyperreactivity, SPT skin prick test.

The pathogenic role of allergens as propitiatory of asthmatic attacks is expressed not so much in immediate reactions as in being the cause of long-term inflammation. Instead, symptom worsening over a short period is more easily released by irritants such as $\mathrm{O}_{3}$, physical exertion, variations in $\mathrm{T}$ (temperature), etc. [333]. However, none of these factors has been formally identified as the cause of airway inflammation [499]. Often, intercurrent respiratory infections are able to amplify not only the clinical features, but also the chronic inflammation, because of the increase in the airways of eosinophils and cationic proteins, including MBP (major basic protein) $[158,341,477]$. This is significant, given the positive interaction between Rhinovirus infection and childs response to allergen exposure [341].

Other details regarding the child:

1. BHR can be present in children with $A D$, with greater probability in those displaying an early onset of AD.

2. BHR associated with an increase of total IgE levels can be present in children who have never suffered from asthma.

3. Altered PFT can constitute a factor of asthma risk in the first 3 years of life.

4. The onset of atopic asthma in infancy is liable to continue into adulthood if not diagnosed in time and treated appropriately. 


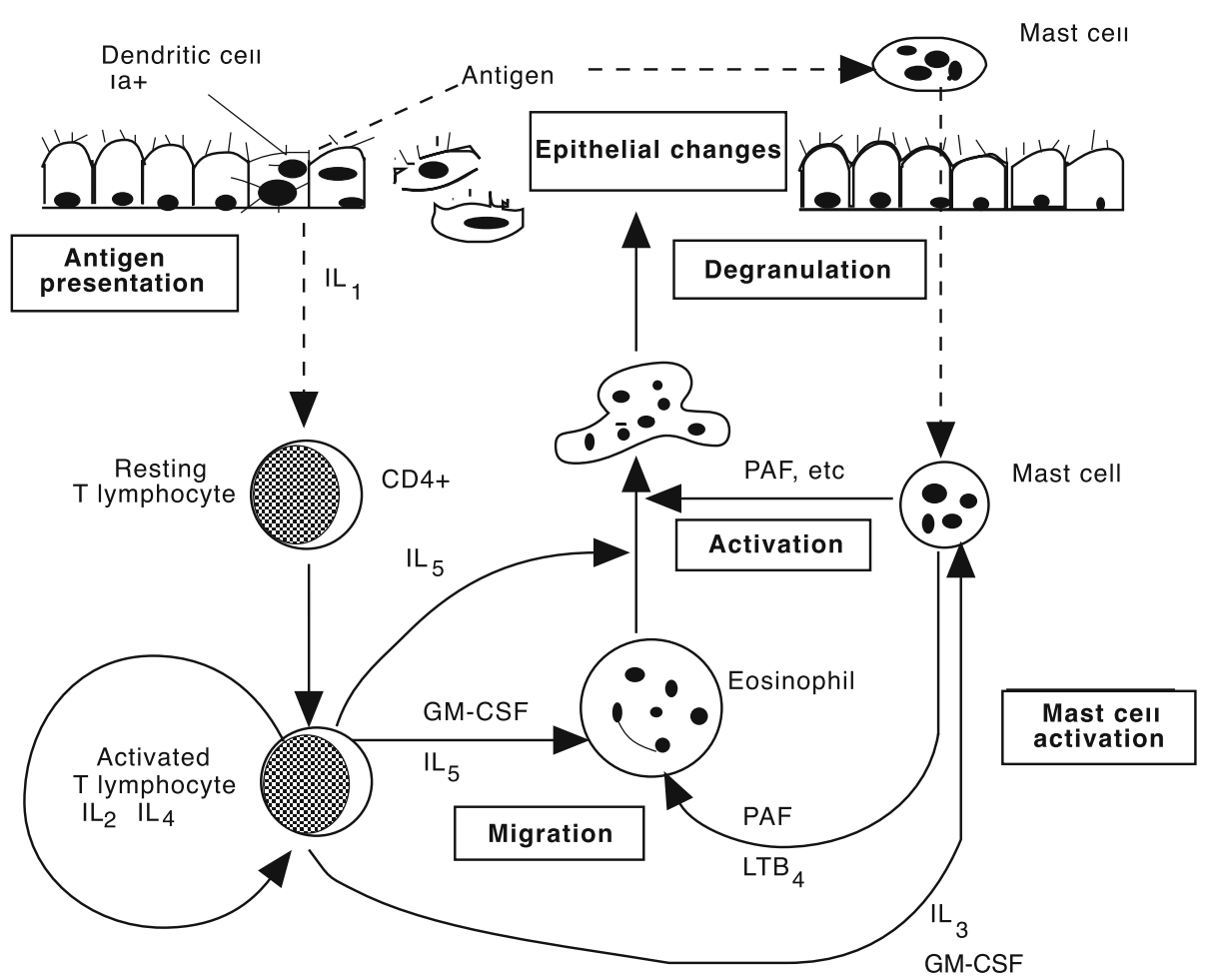

Fig. 11.3.

Subsequent phases of the asthmatic immune inflammation, showing the interactions between $T$ lymphocytes, mast cells and eosinophils, and the consequent changes involving the epithelial cells. (Modified from [194])

\section{Pathogenesis}

\section{Role of Immune Inflammation}

Airway inflammation is a characteristic feature of asthma and contributes significantly to many features of this disease. No doubts remain regarding the pathogenic role of airway inflammation (Fig. 11.3) [194], either in severe, prolonged attacks of asthma or in children with chronic disease. Since 1906 [173], anatomopathological studies of patients who died after an asthmatic attack have highlighted the presence of numerous

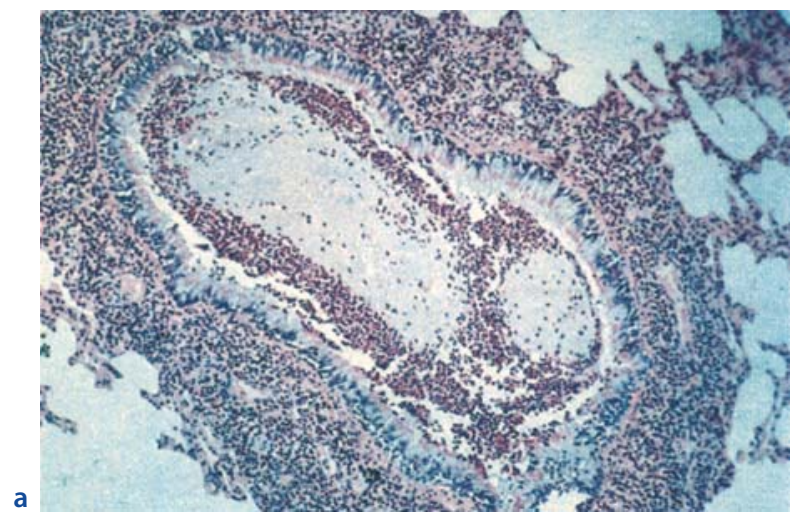

Fig. 11.4. a Histopathology of bronchial mucosa from a subject who died from asthma, showing intense infiltration with eosinophils and mononuclear cells, inflammation and thickening of basement membrane. The lumen of the bronchiole is changes in the bronchial mucosa $[255,516,600]$ (Figs. 11.4, 11.5):

- Clear inflammatory response with subepithelial infiltration of inflammatory cells, eosinophils and activated lymphocytes, with class II antigens, mast cells and macrophages more numerous than in controls.

- Desquamation of the bronchial epithelium with shedding of epithelial cells from basal cells and swollen ciliated cells.

- Hypertrophy and hyperplasia of smooth muscle.

- Edema of the mucosa and the submucosa as a consequence of increased permeability of the microvasculature and plasma leakage.

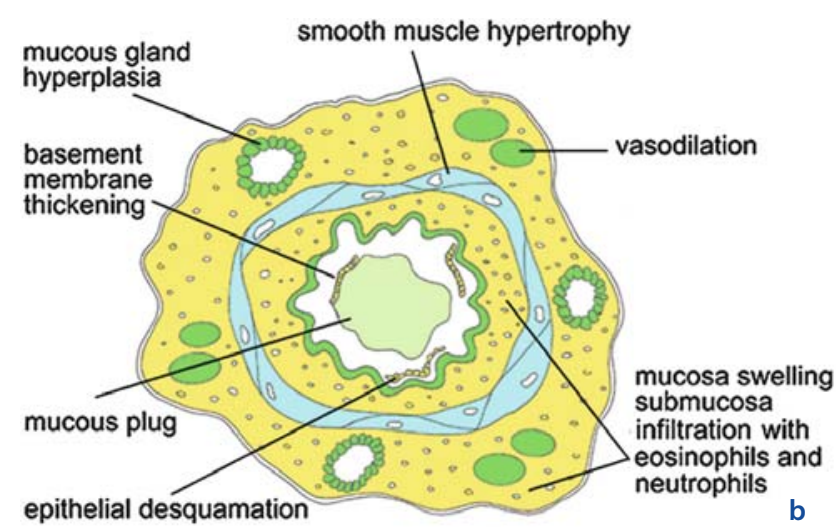

completely occluded with mucus, eosinophils and cellular debris. b Pathological changes in asthma: diagram of cross-section on an airway in severe asthma showing the characteristic hypertrophy of smooth muscles 


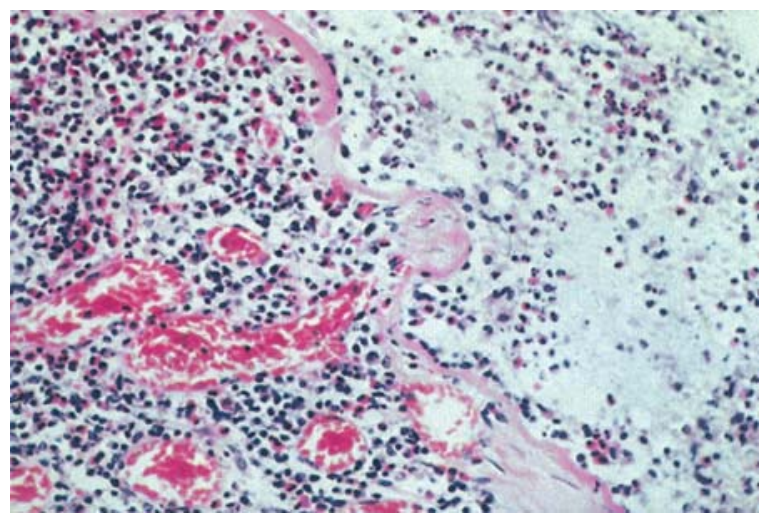

Fig. 11.5. Denudation of the epithelium in an asthma death. Epithelial cells are absent; only the thickened basement membrane remains, with hyperemia and eosinophil infiltration

- Mucus hypersecretion with mucus plugging in the airways (also containing desquamated epithelia, lymphocytes, Charcot-Leyden crystals (CLC) and Curschmann's spirals) up to the segmental bronchi and bronchioles.

- Numerical increase of epithelial goblet cells.

- Mucosal gland hyperplasia.

- Fibrotic pseudo-thickening of the BM by deposits of interstitial collagen under the epithelium.

The end result is a further compromise of the airway lumen [340].

In these studies, however, a greater number of neutrophils and a lesser number of eosinophils may be present, while fibroscopy has made it possible to demonstrate that asthma is prevalently a chronic airway inflammation in which eosinophils and metachromatic cells play a primary role in provoking tissue damage: the bronchial surface is left naked by epithelial desquamation, from which the remodeling processes originate, regenerative of the epithelium with squamous and calyciform metaplasia [53]. If adults with mild forms of asthma show a severe pathological picture, and de-

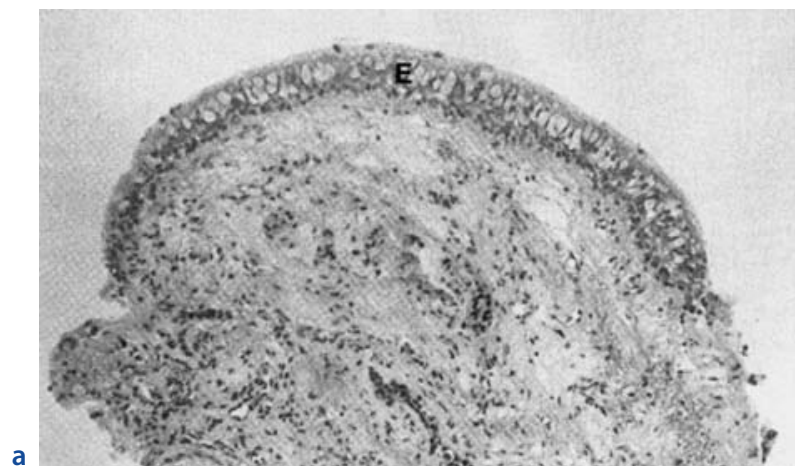

Fig. 11.6. a Bronchial biopsy specimen of a control subject. The well-preserved mucosa is made of normal epithelium $(E)$. Occasional inflammatory cells are observed in connective tissue. b Bronchial biopsy specimen of an asthmatic subject dur- ceased subjects suffering from asthma during life are found to have relatively normal bronchi [287], the damage found in specimens from lung biopsies in children with asthma in remission were similar to pathological specimens of others of the same age who died from status asthmaticus and even of patients who died of causes unrelated to asthma [255]. Figures 11.6 and 11.7 [50] show the differences between the bronchial state of a healthy subject and that of an asthmatic one during the pollen season. It should be emphasized that consistent damage of a chronic airway inflammatory process caused by more than one cell type is observed in subjects with newly diagnosed asthma, having asthma for $<1$ year (Fig. 11.8) [327], even after 2-3 months of asthma duration, showing a greatly decreased number of ciliated cells in the airway epithelium as compared with those in the control subjects [328].

If anatomopathologists underline the inflammatory aspects, clinicians favor that of spontaneously reversible bronchoconstriction, or reversible following specific treatment, whereas physiologists consider the increased BHR as the dominant factor. Even if the concepts of reversibility and of hyperactivity have long attracted a great deal of attention, the idea that asthma should be regarded as a pulmonary inflammation has only recently been given its due attention. Based on recent research, BHR is related to the degree of airway inflammation: as such, both tissue damage and BHR can find a unifying pathogenetic mechanism in the interaction of the different mediators discharged by different cells [255]. It does not, however, seem to be caused by the basic inflammation, since it does not regress with corticosteroid (CS) therapy [94].

Figure 11.9 [499] illustrates the etiology of asthma, BHR and bronchoconstriction. Figure 11.10 [287] shows the relationships between $\mathrm{T}$ and $\mathrm{B}$ lymphocytes and eosinophils in acute and chronic asthma [274]. Figure 11.11 [513] illustrates the physiopathological correlation and Table $11.2[162,538]$ the main differences between immediate asthmatic reactions (IAR) and

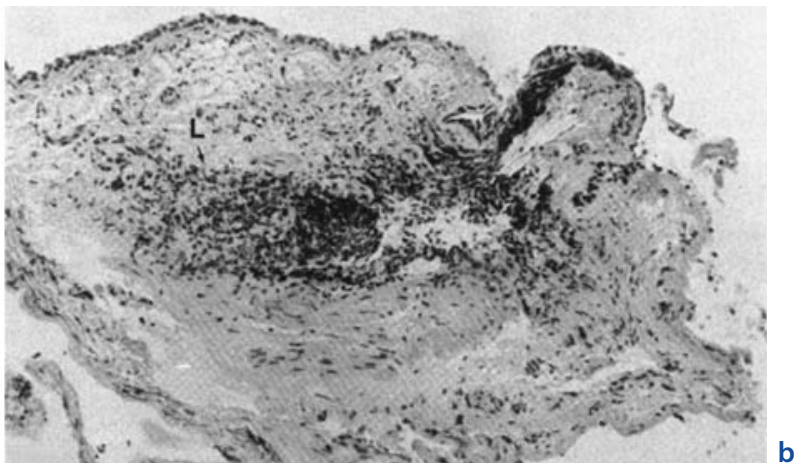

ing seasonal pollen exposure, showing a partial lessening of epithelial cells and inflammatory cells in connective tissue are abundant, mainly lymphocytes $(L) . \times 250$ 


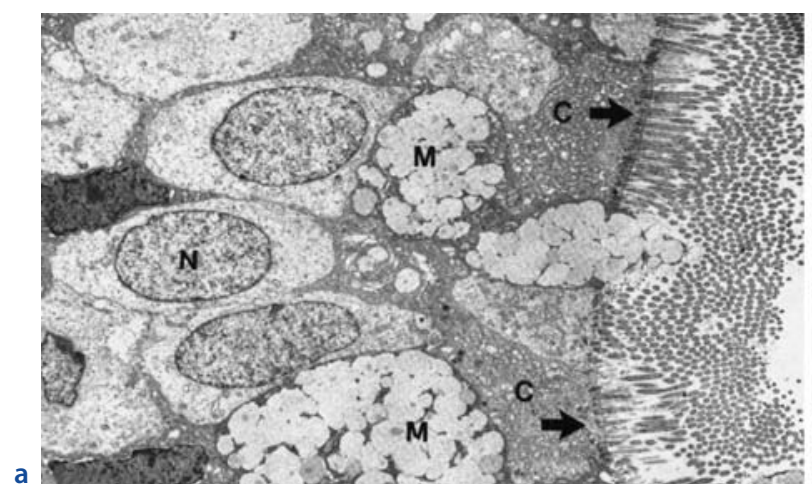

Fig. 11.7. a Electron micrograph of a bronchial biopsy specimen of a control subject, showing ciliated $(C)$ and mucous cells $(M)$, with several cilia and basal corpuscles (arrows). Nuclei $(N)$ were ovoid in shape with vesicular chromatin. $\times 3,400$. b Electron micrograph of an asthmatic subject out of pollen

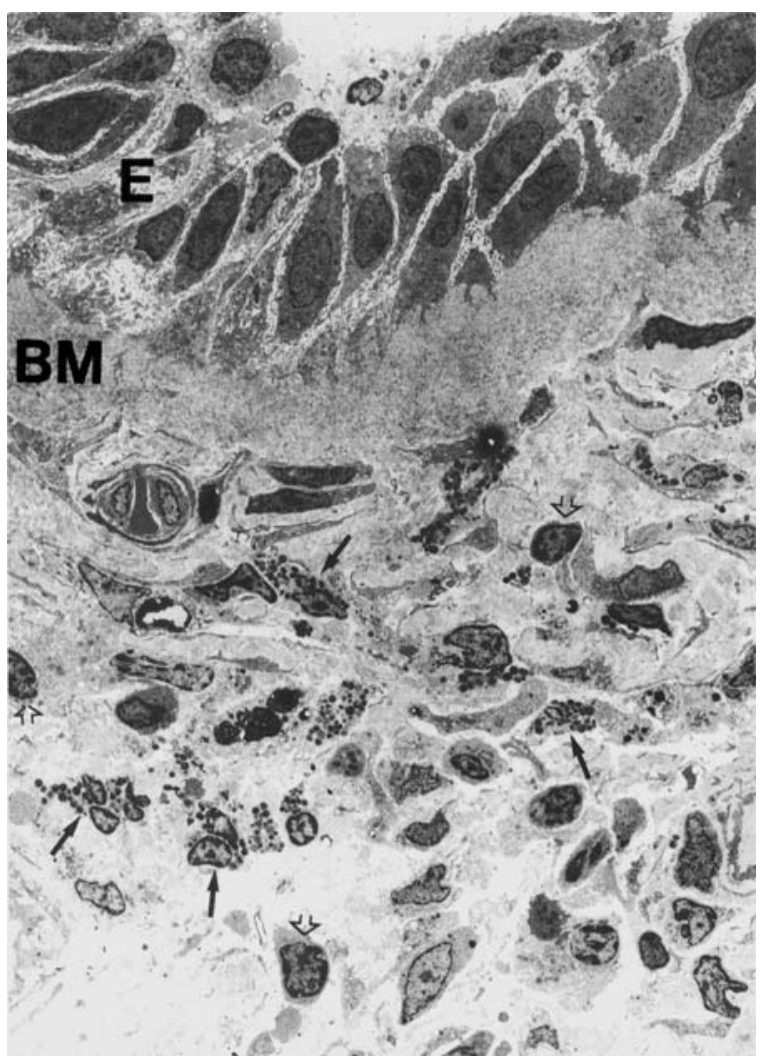

Fig. 11.8. Electron micrograph of a bronchial biopsy specimen of an asthmatic subject with asthma for less than 1 year. The airway epithelium $(E)$ is damaged, and the subepithelial basement membrane is thickened (BM). A strong inflammatory reaction is seen in lamina propria with eosinophils (black arrows) and lymphocytes (open arrows). $\times 2,000$

late asthmatic reactions (LAR), whose characteristics are summarized in Fig. 1.9. The immediate phase is summed up in IgE production in response to allergenic

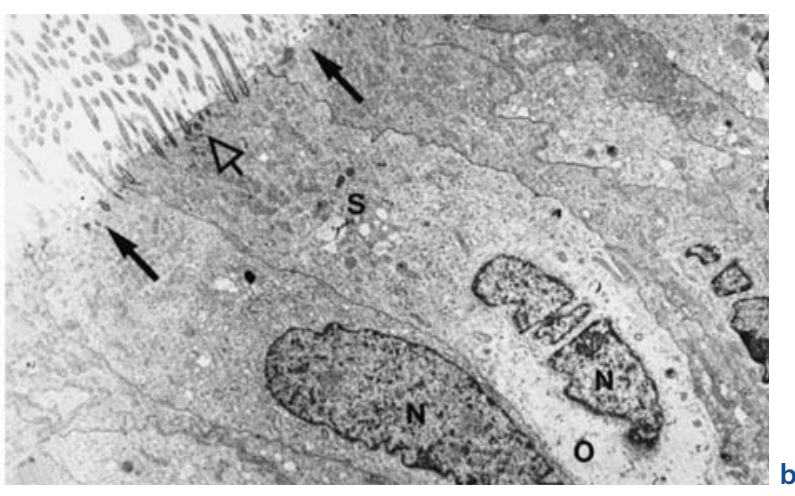

season with an area of ciliated cells. The chromatin is dense with irregularly shaped nuclear membranes. One cell (open arrows) shows a modest number of cilia, but two other cells (black arrows) show almost no cilia. The smooth endoplasmic reticulum $(S)$ is dilated with associated cellular edema. $\times 4,600$

Table 11.2. Differences between immediate and late asthmatic reactions (IAR and LAR)

\begin{tabular}{lll} 
& IAR & LAR \\
Time & Minutes & $4-12 \mathrm{~h}$ \\
\hline Duration & $1-2 \mathrm{~h}$ & $1-3$ days \\
\hline BHR increase & Present & Absent \\
\hline Mechanism & Bronchoconstriction & Inflammation \\
\hline Rate & 35 & 25 \\
\hline \multicolumn{3}{c}{ (associated 40) }
\end{tabular}

Modified from [162, 538].

$B H R$ bronchial hyperreactivity.

stimulus (Fig. 1.40). On the next encounter, in the atopic child, the interaction between allergens and sIgE linked to receptors on effector cell surface initiates a complex chain of biochemical events leading to cellular activation, culminating in mediator release within a few minutes of the allergenic stimulation, and, at the same time, to the appearance of clinical manifestations [207] (Fig. 11.11). Parallel with this, in the airways, $3-12 \mathrm{~h}$ after allergenic exposure, recruitment of eosinophils, neutrophils, basophils and PBMCs, which constitute the primary histopathological infiltrate in status asthmaticus, starts the delayed reaction ultimately resolving in chronicization. A close relationship between basophil number and histamine serum level, and between eosinophil number and MBP concentration [283] is evident. Eosinophil number, $30 \mathrm{~min}$ at most after antigen challenge, reaches its peak after 4-6 h and, in individuals without LAR, after $24 \mathrm{~h}$ [538], so it is assumed that their transition occurs from the mucosa to the secretions. The pathogenesis of this second phase no longer focuses on mast cells, which, nevertheless, participate in releasing ILs and chemotactic factors, but rather on ef- 
Fig. 11.9. Pathogenesis of asthma and bronchial hyperreactivity (BHR) and interactions with bronchial obstruction. 1 The first phase is asymptomatic and several children with serum IgE levels do not experience disturbances, but persistent exposure may cause bronchial inflammation with BHR. 2 The second phase is symptomatic; however, children may have normal lung function. 3 The precipitant factors are the allergens and aspecific stimuli, including passive smoke, exercise, etc. The susceptibility to viral infections is a challenge for pediatricians. (Modified from [499])

Fig. 11.10. The role of mast cells $(M C)$, T lymphocytes, $B$ lymphocytes (B) and eosinophils (Eo) in acute and chronic inflammation in asthma. $L T$ leukotrienes, $P G$ prostaglandins, PAF platelet-activating factor. (Modified from [287])
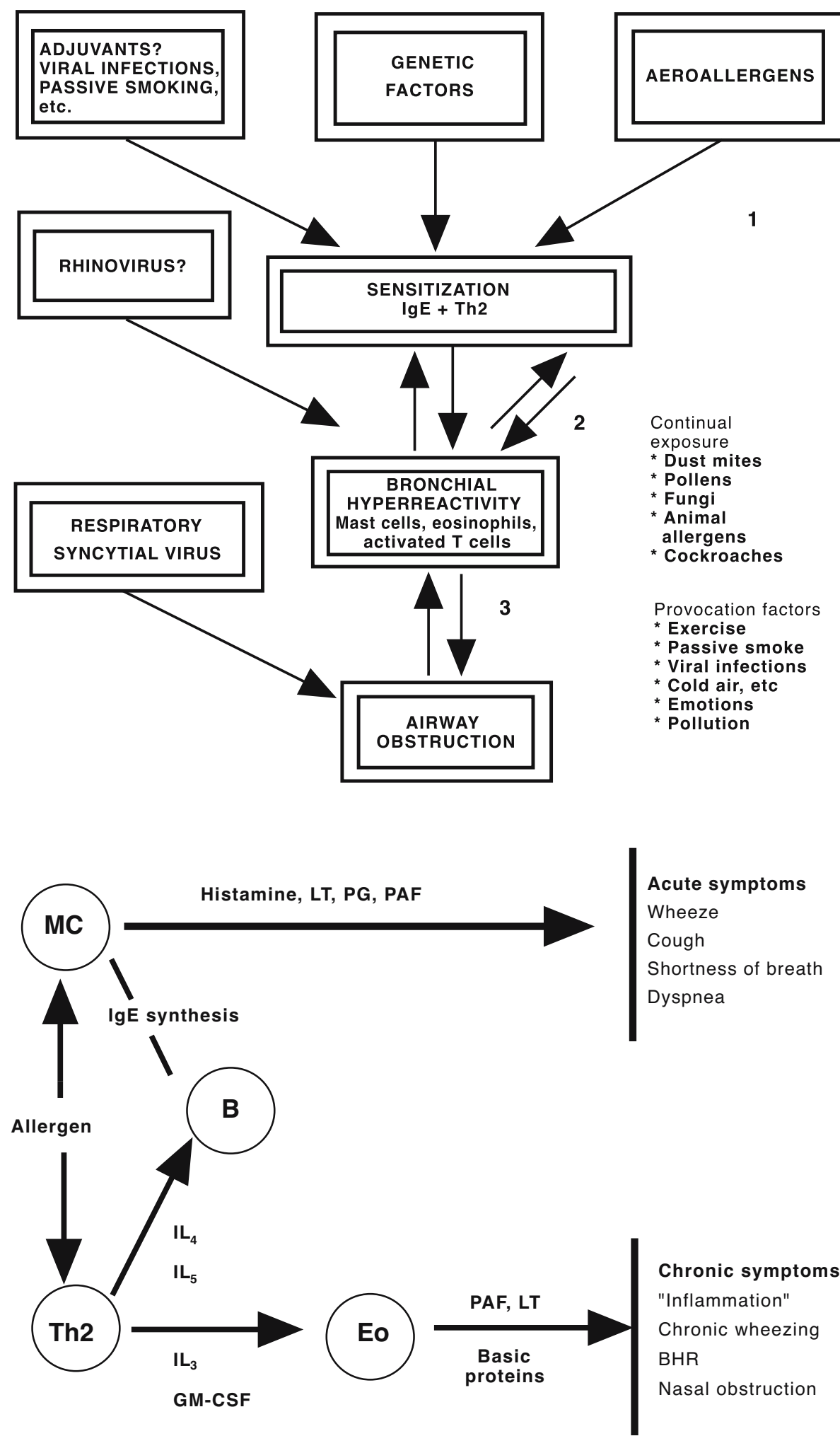

Acute symptoms Wheeze Cough Shortness of breath Dyspnea

Chronic symptoms

"Inflammation"

Chronic wheezing BHR

Nasal obstruction fector cells such as basophils, neutrophils, and, above all, eosinophils, as is demonstrated by cationic protein concentrations [442], ECA (eosinophil chemotactic activity) and NCA (neutrophil chemotactic activity) [240] during a BPT (bronchial provocation test) with aller- gens [240] up to $24 \mathrm{~h}$ later [442]. This role is a prerogative of specific Th2 lymphocytes, ILs, and factors they secrete, that is $\mathrm{LTB}_{4}, \mathrm{PAF}, \mathrm{IL}_{3}-\mathrm{IL}_{6}$ and the chemotactic factor of CD8 T cells [258]. On the other hand, a relative absence of $\mathrm{IL}_{10}$ characterizes the airways of asthmatic 


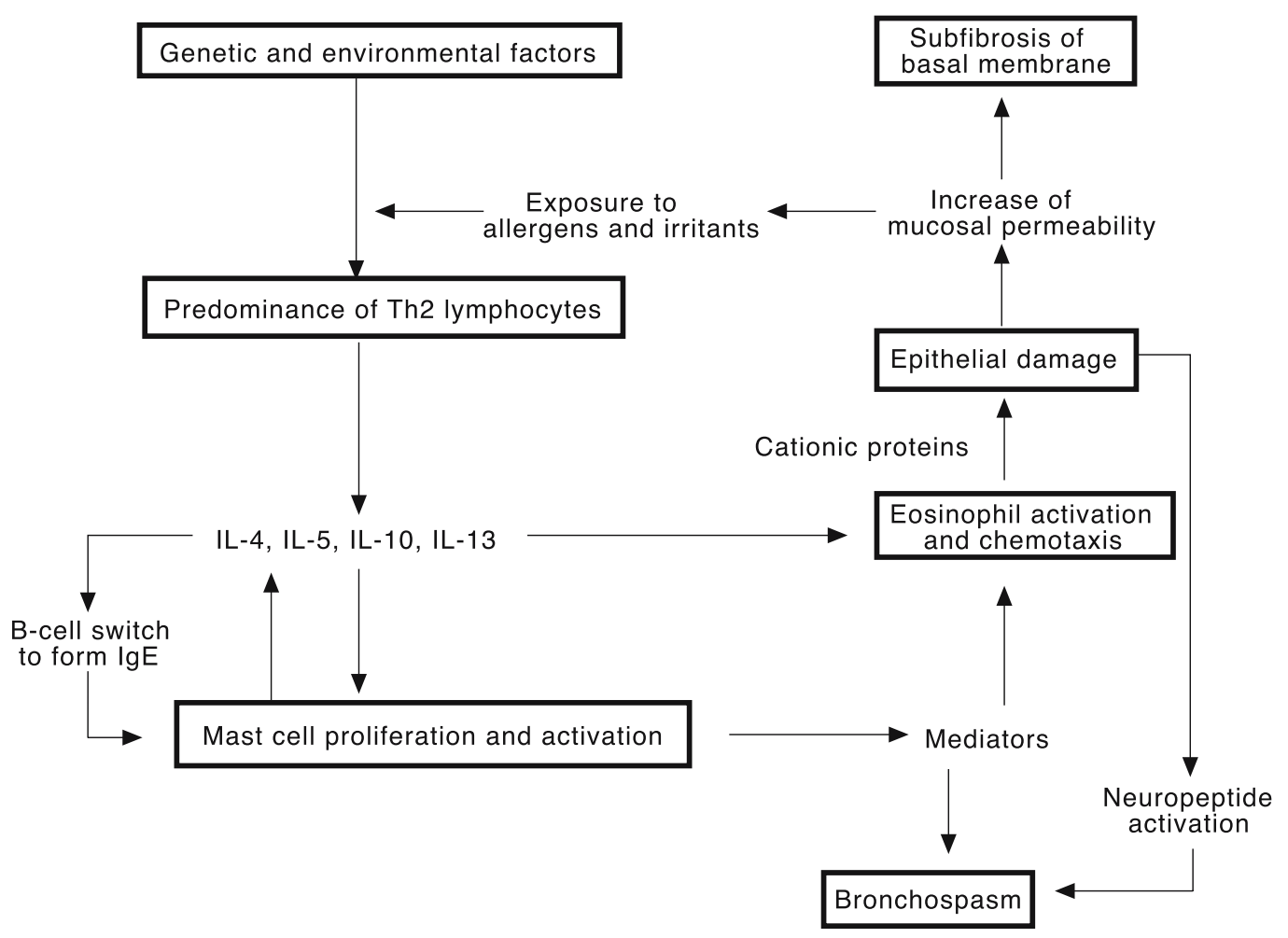

Fig. 11.11. Asthma physiopathology and pathogenesis. (Modified from [513])

sufferers, potentially increasing the severity of allergic inflammation [384]. The role of platelets still remains to be elucidated. On the basis of the latest information available, this reaction appears to be suited for further studies of the physiopathology of asthma and to assess the therapeutic potential of new drugs [162]. Both in asthmatic adults [435] and in children [255], inflammation is usually present. Moreover, a pediatric study has revealed an intimate correlation between activation of both eosinophils and mast cells and BHR [187].

A single pathogenic mechanism does not exist at the basis of the inflammation and consequential BHR; rather, it is likely that the orchestrated action of mediators, their products and cells attracted in this process can play a salient role in provoking BHR [340]. From a histopathological point of view, interesting progress has been made possible by the study of BALF cells via fiberoptic bronchoscopy, which can be performed even in nurslings, using models as small as $1.8-2.3 \mathrm{~mm}$ in diameter [737], and which can also be achieved, in severe cases, using a noninvasive method such as a neonatal catheter [182]. Using these techniques, immune cytochemical research has demonstrated that, in asthmatic children, neither the discriminatory presence of cell types nor their quantitative increase as compared to nonasthmatic atopic controls (Tables 1.40, 1.41) have been found. Studies of BALF cells and lung biopsies have revealed that a great number of $\mathrm{CD}^{+}[15,236]$ are activated by the expression of CD25 and of class II HLA molecules [691]. In atopic asthma sufferers, these T cells have a Th2 phenotype, which is notably relevant in the pathogenesis of allergic disease, especially of the airways, so a great deal of asthma is associated with the activation in the bronchi of $\mathrm{IL}_{3}-\mathrm{IL}_{6}$ and GM-CSF (granulocyte-macrophage colony-stimulating factor), a pattern compatible with Th2 activation [524-526]. $\mathrm{IL}_{13}$ hyperproduction [321] could also bias the differentiation of $\mathrm{T}$ cells toward a Th2 phenotype through its ability to modulate $\mathrm{IL}_{12}$ production from APCs. Whether as a result of exaggerated Th2 cytokine production or as a primary defect, lack of $\mathrm{IL}_{12}$ can clearly influence immune responses toward a skewed Th2 response [676]. Furthermore, alveolar macrophages, bronchial epithelium, eosinophils and mast cells express these and other ILs, whose secretions lead to the pathological findings observed in asthma [15, 236, 691]. Some CXCL and CCL chemokines (Tables 1.54-1.57) act on both basophils and eosinophils; the former could represent the key mediators of immune inflammation. In immune inflammation, it is not easy to draw a balance of the importance of the different cell types or of the mediators involved. It seems more evident that they act together, each armed either with its own weapons or those shared with others, releasing a complex cascade of harmful events [73]. Of no minor importance are the notable alterations experienced by the postcapillary microvasculature: it is known that, in addition to the explicit damaging action carried out by powerful mediators, even vagal nerve stimulation, through neuropeptide release, secreted by neuroendocrine tissues of the airways, including substance 
$\mathrm{P}$ (SP), can contribute to the vasodilation in the microvasculature. In turn, edema contributes to different worsening changes, including the inhibition of mucociliary clearance. In addition, proteins become available that form the substrate for anaphylotoxins of complement derivation and kinins [287].

\section{Role of the Inflammatory Cells}

Different cells are involved in immune inflammation.

\section{T Lymphocytes}

Characteristics [537]:

- The tissues of atopic sufferers show an accumulation of Th2 capable of orchestrating immune inflammation; consequently, Th2 predominate in the BALF of atopic asthmatics.

- Activated Th2 cells are found in blood and airways of asthmatics and their expansion is preferentially allergen-induced.

- Th2 produce proinflammatory ILs and, in particular, $\mathrm{IL}_{3}$ and $\mathrm{IL}_{4}$, with a major role in the synthesis of $\mathrm{IgE}, \mathrm{IL}_{5}$ and GM-CSF, whereas $\mathrm{IL}_{2}$ and IFN- $\gamma$ are absent.

- Th2 are involved in the enrollment of eosinophils, the primary effector cells of inflammation [537].

- During asthmatic attacks, there occurs in children an expansion of allergen-specific Th2 clones, accompanied by very high production of $\mathrm{IL}_{4}$, as demonstrated by an increase in CD23 on B cells and on CD4 ${ }^{+} \mathrm{HLA}^{-\mathrm{DR}^{+}}$[200] (Fig. 11.12) [513].

- In BALF of nonasthmatic children, CD8 T cells are more numerous than CD4 $\mathrm{T}$ cells (Tables 1.40, 1.41), while in atopic pollen-allergic subjects the proportions are more than reversed: CD4 85\% (Th2 48\%, Th0 39.5\% and Th1 $12.5 \%)$, CD8 15\%, allergen-specific $\mathrm{T}$ clones $27 \%$ [141].

Thus an imbalance between Th1 and Th2 lymphocytes primes the Th2 or allergic phenotype, whereas the Th1 phenotype may be underexpressed. A reduced expression of T-bet, in $\mathrm{T}$ cells from airways of asthmatic patients compared with that in $\mathrm{T}$ cells from airways of nonasthmatic patients, suggests that loss of T-bet might be associated with asthma pathogenesis [188].

In a normal lung, $\mathrm{T}$ cells, on the order of $1.5 \times 10^{8}$, are in direct contact with aeroallergens [263]. However, in moderate asthma, their number may not be increased (Fig. 11.13). In the mucosa of lower and upper airways, dendritic cells (DCs) have been found, whose percentage in BALF cells is $1 \%$ [693] and whose number is greatly increased in atopic sufferers [412] and in asthmatic smokers [533]. DCs form a continuous intraepithelial reticulum interdigiting with epithelial cells, similar to the skin process [619]. They do not protrude through the epithelium, being confined by the tight junctions; consequently the allergens that are not removed from

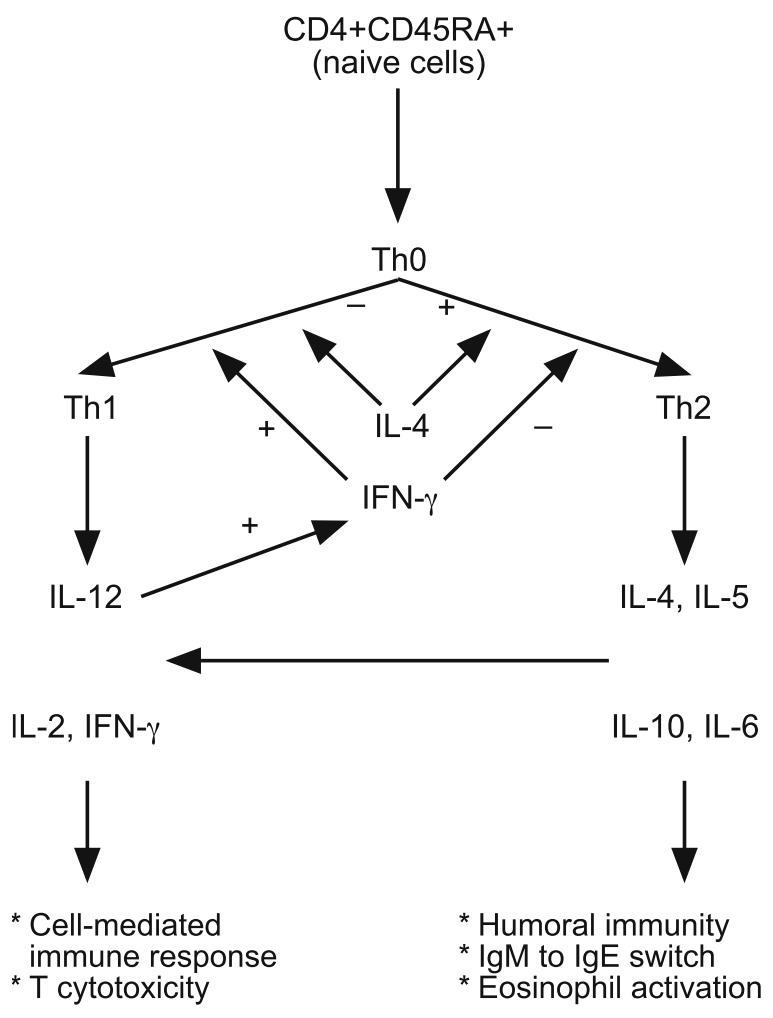

Fig. 11.12. Activation and differentiation of T lymphocytes in asthma pathogenesis. (Modified from [513])

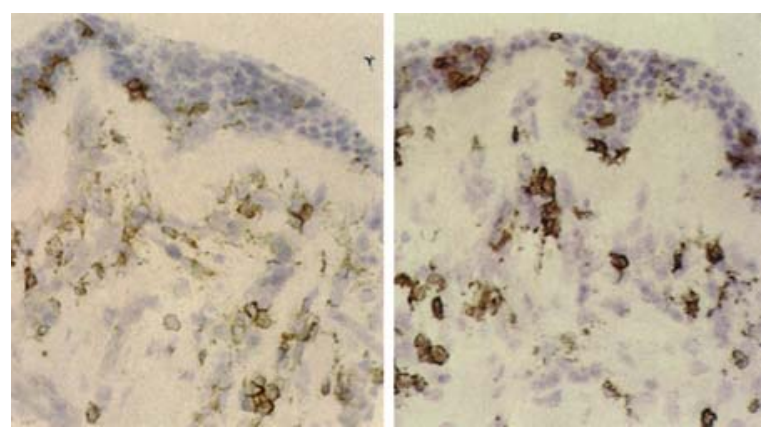

Fig. 11.13. Epithelial and subepithelial T-cell subpopulations (left) and CD4 and CD8 (right) in a bronchial biopsy specimen of an mild asthmatic subject: in mild to moderate asthma $T$ cells are activated, but their total number is unchanged

the mucociliary clearance must cross the mucosal layer and penetrate via the junctions to encounter immunocompetent cells [533]. DCs send the start signal to T cells during the sensitization phase and can also act as synergistic agents of their activation process in the airway walls during IAR. They are equipped with FceRI- $\alpha$ that can facilitate allergen uptake and internalization, enhancing their potential role in the induction and amplification of chronic airway inflammation [660]. Having elevated levels of class II HLA, in vivo they efficiently trap aeroallergens and in vitro are skillful APC for 


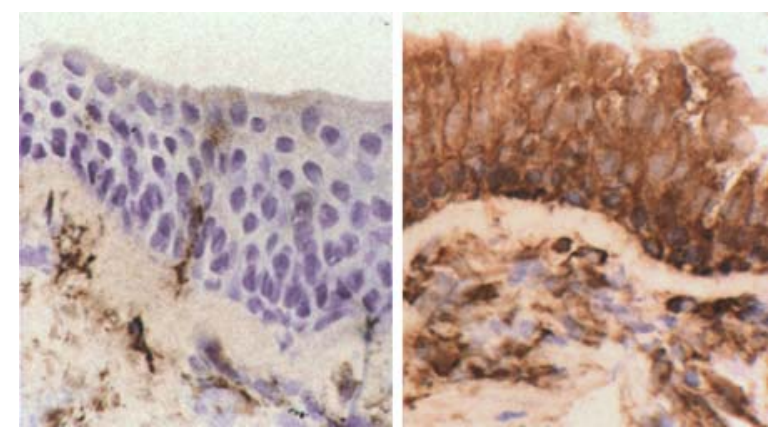

Fig. 11.14. HLA-DR (immunoperoxidase demonstration) in the bronchial epithelium of normal (left) and asthmatic subjects (right)

IELs [263], where they are CD1 $\alpha$-positive only for $30 \%$ [693]. They also are able to transport antigens, by migrating from the respiratory epithelium to the regional lymph nodes, where they undergo a maturing process, acquiring a greater immunostimulating activity [263]. In this way, they provide for antigen processing and presenting to $\mathrm{T}$ lymphocytes, thereby involving HLA-DR molecules, up-regulated in asthmatic subjects (Fig. 11.14), which, by linking to allergenic peptides, form bimolecular complexes easily recognized by TcR-CD3 [258]. Even in the bronchial mucosa in correspondence with lymphatic structures, $M$ cells similar to those found in the intestinal epithelium situated over Peyer's patches have been described. In the airways, they could perform a similar function, transferring antigens from the bronchial lumen to lymphatic structures, even if they do not play a primary role in mucosal immunoregulation [537]. CD8 T cells induced by antigen encounter are in turn activated in the regional lymph nodes that drain the upper respiratory tract [262].

Lymphocytes accumulate in the respiratory tissues during the 1st hour of allergic reaction: in LAR they are attracted by specific receptors located on T cells, as well as on mucosal capillaries and epithelial venules, and activated in sites of immune inflammation, they induce phenotypic alterations in endothelial cells [524]. The predominant clones of allergen-specific $\mathrm{T}$ lymphocytes of atopic asthmatics, which are Th2-like, are able to produce elevated levels of $\mathrm{IL}_{4}$ [535]. Recently, GATA-3 overexpression was found to be implicated in Th2 development in human $\mathrm{T}$ cells and $\mathrm{IL}_{5}$ promoter activity in $\mathrm{CD}^{+} \mathrm{T}$ cells of asthmatic patients was enhanced by GATA-3 [286]. In asthmatic patients, more cells from BALF contain mRNA for $\mathrm{IL}_{3}-\mathrm{IL}_{5}$ and GM-CSF (whose genes are associated in a gene cluster of chromosome 5), and reduced IFN- $\gamma$ levels compared to controls [530]. More precisely, using in situ hybridization, and the immunofluorescence technique on BALF cells from asthmatic subjects, there is an evident imbalance between the reciprocal proportions of $\mathrm{IL}_{4}$ and IFN- $\gamma$ in tissue infiltrates of allergic inflammation [530], with a significantly larger number $(p<0.01-p<0.001)$, in comparison with controls, of cells showing elevated mRNA concentrations for $\mathrm{IL}_{4}$ and $\mathrm{IL}_{3}, \mathrm{CD} 3$-positive (that is $\mathrm{T}$ lymphocytes, predominantly $\mathrm{CD} 45 \mathrm{RO}$ ), and no difference for IFN- $\gamma$ [530]. $\mathrm{IL}_{3}-\mathrm{IL}_{5}$ and GM-CSF are associated with Th2, eosinophil accumulation [531], bronchoconstriction and Aas score [532]. This picture is compatible with the predominant activation of the Th2-like subset, the promotion of an IgE response and generation of pro-inflammatory mediators [524].

In asthmatics, a predominance of Th2 and Th2-like T-cell ILs are reported. T lymphocytes are therefore capable, even supported by the four classes of chemokines, of directly coordinating the accumulation and activation of specific effector cells at the mucosal surface. In particular $\mathrm{IL}_{3}, \mathrm{GM}-\mathrm{CSF}$ and $\mathrm{IL}_{5}$ (with the aid of CD54, $=$ ICAM-1) appear to be very active in attracting and activating eosinophils; GM-CSF and other T-like ILs are active in the differentiation of monocyte-macrophages, $\mathrm{IL}_{3}$ and GM-CSF and metachromatic cells [524]. In this context, the presence of mast cells and basophils assumes great relevance, given that their precursors are able to produce $\mathrm{IL}_{4}$ for Fce receptor cross-linking. $\mathrm{IL}_{4}$ synthesis modulates that of IgE antibodies, by acting directly on B lymphocytes, or by activating Th2-like $\mathrm{CD}^{+}$cells. The latter produce on the one hand $\mathrm{IL}_{4}$ (and therefore $\operatorname{IgE}$ ) and on the other $\mathrm{IL}_{5}$, finally closing the inflammatory cycle [535]. Since $24 \mathrm{~h}$ after BPT with allergens the majority of ILs originate from T cells [531], we may conclude that expanding allergen-specific Th2 lymphocytes directly orchestrates both the development of bronchial inflammation by producing specific IL and the continuous synthesis of IgE, able to elicit decisive influxes on inflammatory cells [525]. Titers of B cells, CD19 and CD23 markers are found to be increased compared to controls [552].

Examination of lymphocyte subsets in asthmatic individuals shows that $\mathrm{CD}^{+}, \mathrm{CD}^{+}, \mathrm{CD}^{+}$and $\mathrm{CD}^{2} 5^{+}$are present in notably greater numbers than in nonasthmatic atopic subjects and in nonatopic controls [15]. The marker $\mathrm{CD} 25$ (receptor of $\mathrm{IL}_{2}, \mathrm{IL}_{2} \mathrm{R}$ ) $[15,119]$ and of HLA-DR and CD49a/CD29 (VLA-1) in greater numbers than in normal controls denotes that such activated cells are able to contribute to the pathogenesis [119]. Soluble $\mathrm{IL}_{2} \mathrm{R}\left(\mathrm{sIL}_{2} \mathrm{R}\right)$ is a marker of their activation, with particularly elevated levels in asthmatic children compared to healthy controls and adults [408]. Even in the pediatric age group, high percentages of both CD4 and CD8 peripheral blood $\mathrm{T}$ cells express activation markers: thus T-cell activation as compared to controls is found [153, $207,252,590]$, with significantly higher differences regarding the increase in absolute numbers of $\mathrm{CD} 4 /$ CD25 $5^{+}$CD4/HLA-DR ${ }^{+}$and CD8/CD25 ${ }^{+}$[207], of T-cell specific markers CD23 and CD25 [252], HLA-DR antigen [590] and PEF-correlated CD25 [153]. Associations between eosinophil activation, $\mathrm{CD} 4^{+}$cell numbers, and a high correlation between CD4-Th2 and eosinophils and severity of asthma [691], are totally confirmed by the evidence in children of CD4-Th2 and CD8 displaying 
CD25 and HLA-DR positively correlated with disease severity $(p=0.03)$ and with a significant increase in total eosinophilia $(p=0.01)$ as compared with the controls [207]. Remarkably, the proportion of CD4 T cells expressing the memory cell marker CD45RO was significantly elevated in both atopic and nonatopic asthmatics compared with controls, whereas the naive marker CD45RA was expressed by a high proportion of CD8 $\mathrm{T}$ cells only in the nonatopic asthmatics compared with controls. Moreover, high rates of these CD4 and CD8 $\mathrm{T}$ cells express mRNA encoding $\mathrm{IL}_{5}$, but only CD4 expressed mRNA encoding $\mathrm{IL}_{4}$ in asthmatic children compared with the controls [208]. The relationship between cells displaying $\mathrm{IL}_{5}$ and activated eosinophils in bronchial biopsies in asthmatics [236, 340] (Fig. 11.10) indicates that high levels of $\mathrm{IL}_{5}$ distinguish between severe asthma [119] and moderate asthma, where they cannot be measured [153], considering that CSs reduce their transcription, together with several others, being capable of inhibiting in atopic asthmatics T-cell activation markers in BALF and in peripheral sites [723] and DC as well [412]. For these reasons, T lymphocytes, with all the cells and factors they stimulate, above all eosinophils, can be considered as the key cells that bring into effect, sustain and perpetuate the immune inflammation, the catalyst element that induces BHR in relation with endogenous and exogenous factors, and triggers the series of events that lead almost inexorably to asthma [287]. This is especially evident when the impossibility of establishing a state of tolerance through Th1 $\mathrm{T}$ cells results in the sensitization to environmental allergens [262], guided by the Th2 in the critical phase of developing the immune system in infants. Tables 1.40 and 1.41 summarize normal data on lymphocytes and other cells in BALF in nonasthmatic children.

\section{Mast Cells}

Characteristics [537]:

- Are present in airways of asthmatics in increased numbers and in various stages of degranulation, but are absent in normal subjects

- Respond to allergens in an IgE-dependent manner with high-affinity IgE receptors (FceRI)

- Have a key role in driving the IgE-mediated reaction

- Directly express CD154 as basophils do, with the immediate implication that synthesis of IgE occurs even in peripheral tissues, other than in germinal centers of lymph nodes

- Produce short-lived mediators and inflammatory ILs Mast cells derive from bone marrow precursors as regulated by the same ILs that are important contributors to the allergen-specific airway inflammation [537]. Their greater concentration is in more peripheral airways, where they localize prevalently in the sub- and intraepithelial layers, and in the bronchial lumen [258]. A striking increase in the number of mast cells was in the bundles of smooth muscle, and in patients with asthma this number was correlated with the hallmark of asthma, BHR [55]. In asthmatics there is no instability of mast cell membrane, which could facilitate degranulation, an event that can be mediated not only by encountering sIgE, but also by sIgG (specific) and anaphylotoxins. Similarly, there is no functional heterogeneity, since mast cells of the human airways belong mainly to the $\mathrm{T}$ type, even if both phenotypes can coexist in the same site with different levels (Table 1.27). Mast cells participate in the immediate phase of allergic reactions: they are found in the BALF of atopic asthmatics taken $15 \mathrm{~min}$ after allergen challenge in the degranulation stage [258], often in proximity to the small vessels and nerve endings and intimately correlated with smooth muscles, with spontaneous release of histamine and eicosanoids [73]. Once the degranulation has started, it is possible that allergen encounter with superficial mast cells provokes an initial mediator release that increases tissue permeability, with consequent enlargement of the intraepithelial tight junction network, thus allowing a greater number of allergens to reach the layers below, where a strong group of mast cells are to be found [283]. The array of primary and secondary mediators further increase the permeability of the epithelial barrier, permitting the entrance of plasma proteins and blood platelets [283]. This is how the true asthma allergy explosion is set off. Consequently histamine, PG, thromboxanes (type $\mathrm{A}_{2}$ ), LTs (leukotrienes), PAF, bradykinins (BKs), granule derivatives and chemotactic factors are crucial for developing an inflammatory stage of immediate reaction [287]. As well as inducing adhesion, diapedesis, directional migration and activation of eosinophils and neutrophils, they also recruit other cells that are fundamental for the establishment and self-maintenance of inflammation, such as monocyte-macrophages, blood platelets, more eosinophils, and neutrophils to the injured site [283]. Mediators participating in the triggering of asthma affect the infiltration of leukocytes, probably aided by afferent vagal stimulations, affecting the bronchospasm as well as mucosal gland secretions, which are unrelated to symptom severity [283]. ILs, whose secretion is associated with histamine release and mast cell proliferation (probably associated with $\mathrm{IL}_{3}$ and $\mathrm{IL}_{4}$ generation), are in turn able to amplify the vasoactive phase of immune inflammation [73]. Mast cells, as well as sharing with basophils the CD154 production with a direct effect on IgE synthesis, express mRNA for $\mathrm{IL}_{4}$ and for $\mathrm{IL}_{5}$, whose release, together with $\mathrm{IL}_{3}$ and $\mathrm{IL}_{6}$, is triggered by an IgE-mediated mechanism [287]. Mast cells, however, appear to be the only cells producing $\mathrm{IL}_{4}$ in the airways of asthmatics: this could indicate their possible intervention in LARs, which, however, has not been fully confirmed. On the other hand, the mediators seem to be correlated to IARs, as demonstrated by the increase in histamine levels in circulation and the increase in histamine and tryptase in BALF of atopic subjects a few minutes after allergen 
stimulation, and by increased urinary methylhistamine in the ensuing hour [283]. Tryptase in BALF is correlated to $\mathrm{PC}_{20}$ (concentration of methacholine inducing a $20 \%$ fall in $\mathrm{FEV}_{1}$ ), but not in serum after TPB with allergens [240], which could suggest that mast cells, along with their mediators, represent the key cells in a child's BHR, given the possibility of a tryptase-induced up-regulation of bronchial smooth muscle tone [187]. Mast cells could also have the function of controlling the immune inflammation by releasing repair mediators such as heparin [467] and $\mathrm{LTB}_{4}$, which is highly chemotactic for both eosinophils and neutrophils [54].

\section{Basophils}

The characteristics of basophils are as follows [376]:

- They produce MBP.

- They express CD154 as do mast cells.

- Anti-IgE modulate their allergen-induced activation [581].

- PAF selectively induces them to release histamine.

- They have an important relationship with the CCL, lesser with CXCL chemokines.

- $\mathrm{IL}_{3}$ induction produces $\mathrm{IL}_{4}$ and activates MCP-1 (monocyte chemotactic protein-1), stimulating their degranulation.

Basophils share with mast cells the IgE-mediated degranulation process, with histamine and $\mathrm{LTC}_{4}$ release that makes them powerful inflammatory cells; however, they do not release $\mathrm{PGD}_{2}$ and are unique since they contain MBP along with eosinophils (Table 1.25). Together with mast cells they are high histamine producers, which release after stimulation by PAF, C5a and antiIgE IgG [581]. Basophils release a greater quantity of histamine in asthmatics than in allergic rhinitis (AR) sufferers [376], even if anti-IgE IgG could down-regulate histamine release [581]. IgE-mediated basophil releasability has a clinical relevance in the pathogenesis of airway reactivity, since they are increased in atopic asthmatics: symptom severity is correlated with basophil responsiveness in vitro [376]. Lymphocytes and other cells promote their maturation and differentiation as well as mediator secretion, favored by various ILs such as $\mathrm{IL}_{1}-\mathrm{IL}_{3}$ and GM-CSF. In particular, $\mathrm{IL}_{3}$ and GM-CSF induce their migration in vitro in picomolar doses and increase their chemokinetic activity and adhesion [742]. Basophils express, together with eosinophils but not with neutrophils, the integrin CD49d/CD29, equal to VLA-4, counter-receptor of VCAM-1, equal to CD106, which allows adhesion to the endothelia [561]. The parallel with eosinophils is strengthened by the community of receptors for $\mathrm{IL}_{5}$ that become active in picomolar doses also on basophils [249]. Recently, it has been established that the basophil role seems to be decisive in $L A R$, coinciding with that carried out in the pathogenesis of AD: $19 \mathrm{~h}$ after the challenge, an increase in mediators released by these cells has been noted [73]. Of equal importance is the link with CCL chemokines and with MCP-1, MCP-2 and RANTES (now CCL2, CCL8, and CCL5, respectively), which stimulate basophil migration (Tables 1.54-1.57), while the CXCL with a function identical to $\mathrm{IL}_{8}$ and CCL5, promote their adhesion, to which several integrins contribute (Tables 1.45-1.49).

\section{Eosinophils (Figs. 11.3, 11.4, 11.8, 11.10, 11.11)}

The characteristics of eosinophils are as follows (see Table 1.24):

- They are markedly increased in number in blood and airways, with statistically significant differences in asthmatic children compared to healthy controls $[254,639]$.

- They are equipped with numerous integrins, CD45, CD59 and HLA-DR among the inducible molecules.

- Dependent on $\mathrm{IL}_{3}, \mathrm{IL}_{5}$ and GM-CSF, as well as on TNF, $\mathrm{PAF}$ and $\mathrm{IL}_{1}$, for development, activation and survival [460] (Table 11.3) [22, 284, 466].

- An increase in the bronchial mucosa of mRNA for $\mathrm{IL}_{5}$ (eosinophilopoietic) [236] associated with GM-CSF (IL para-eosinophilia) [59] indicates its presence and activity $[59,236]$, and also the significant connection with a number of activated $\mathrm{T}$ cells [236].

- They are laden with cationic proteins, which once released may be highly toxic to airway tissues.

- Better than any other cell, they represent the common factor able to correlate IgE hyperproduction and involvement of metachromatic cells, macrophages and of themselves in the immune inflammation [526].

- Some studies, but not all, show the hypodense phenotype and the correlation of ECP (eosinophil cationic protein) levels with clinical manifestations.

Eosinophils also are heterogeneous, as far as density and activity are concerned. Their growth from eosinophil precursors is highly dependent on a set of ILs. $\mathrm{IL}_{5}$ and PAF not only activate eosinophils but transform the normally dense cells into a hypodense phenotype (Fig. $1.35 \mathrm{c}$ ) that responds equally to chemotactic signals $[354,460]$. Numerous studies have evaluated the possible role played by such cells, more cytotoxic [73], with more receptors for IgE and IgG, that produce more $\mathrm{LTC}_{4}$ and more active, from a metabolic point of view, in patients with delayed responses to inhalants [538]. Such data is suggestive, but it is not yet possible to transfer them automatically to the pediatric field since it could more simply be related to immature forms [408], scarcely increased in number $[254,408]$, an effect inhibited by nedocromil sodium [578]. Moreover, the increase in hypodense cells can depend on artifacts resulting from techniques that use dextran or gelatin, or on the significant increase in eosinophilia in asthmatic children, therefore an epiphenomenon [254]. Thus, notwithstanding recurring indications, density appears to be an overly imprecise sign to be useful to recognize a pathogenic role in the pediatric field as an index of cellular activation. 
Table 11.3. Functional correlations between eosinophils and interleukins (IL)

\begin{tabular}{lll} 
Function & ILs & Effects \\
\hline Leukotriene production & $\mathrm{GM}-\mathrm{CSF}, \mathrm{IL}_{3}-\mathrm{IL}_{5}$ & Increase \\
\hline Phagocytosis & $\mathrm{GM}-\mathrm{CSF}$ & Increase \\
\hline Proteoglycan synthesis & $\mathrm{GM}-\mathrm{CSF}, \mathrm{IL}_{3}-\mathrm{IL}_{5}$ & Increase \\
\hline ADCC & $\mathrm{GM}-\mathrm{CSF}, \mathrm{TNF}$ & Increase \\
\cline { 2 - 3 } & $\mathrm{GM}-\mathrm{CSF}, \mathrm{IL}_{3}-\mathrm{IL}_{5}$ & Optimal increase \\
\cline { 2 - 3 } & $\mathrm{TNF}$ & Modest increase \\
\hline Adhesion & $\mathrm{IFM}$ & Delayed increase \\
\cline { 2 - 3 } & $\mathrm{TNF}, \mathrm{IL} \mathrm{L}_{1}$ & CD54 increase \\
\hline Degranulation & $\mathrm{GM}-\mathrm{CSF}, \mathrm{IL}_{1} \beta$ & EAEC increase \\
\hline Survival & $\mathrm{GM}-\mathrm{CSF}, \mathrm{IL}_{3}-\mathrm{IL}_{5}$ & Increase \\
\hline & $\mathrm{IL}$ & Reduction \\
\hline & $\mathrm{IL}$ & Optimal increase \\
\cline { 2 - 3 } & $\mathrm{GM}-\mathrm{CSF}, \mathrm{TNF}_{3}, \mathrm{IFN}$ & Modest increase
\end{tabular}

Data from $[22,284,466]$.

ADCC antibody-dependent cell-mediated cytotoxicity, EAEC eosinophil adhesion to endothelial cells.

Eosinophils have, equal to mast cells, a great number of FceRI and FceRIIb (CD23), which demonstrates their crucial role in atopic IgE-mediated disease. In fact atopy contributes to infantile asthma by the mobilization and activation of such cells [408]. As can be seen from the data in Table 1.24, eosinophils can be primed to express HLA-DR in vivo, implying the presence of HLA class II molecules: therefore they are APCs able to present antigens to $\mathrm{CD} 4 \mathrm{~T}$ cells. The modulating and regulating role carried out at the level of the immediate hypersensitivity reactions is also demonstrated by the increased presence in the IAR sites and by the ability of some of their cellular proteins to inhibit or degrade the mediators of the immune reaction [679]. Especially EPO (eosinophil peroxidase) can degrade $\mathrm{LT}$, and $\mathrm{PGE}_{1}$ combined with $\mathrm{PGE}_{2}$ have been credited with being able to inhibit histamine release [679]. Additionally, eosinophils contain great quantities of VIP (vasoactive intestinal peptide) and produce SP. The mature cells synthesize PAF, which causes an increase in cytotoxicity and superoxide generation [758].

Immunohistochemical studies on BALF cells have shown that eosinophils are in motion 30 min after the antigen encounter $[15,217]$ and that their relative percentage, along with that of ECP in BALF, in the majority of patients, is correlated with the severity of asthma (Fig. 11.15) [51]. In fact they predominate over other cells in BALF, together with MBP, ECP and neurotoxins produced by them [531], data significantly documented in histological specimens of patients who died from asthma [255]. Other studies done to evaluate the differences between asthmatics and healthy controls have

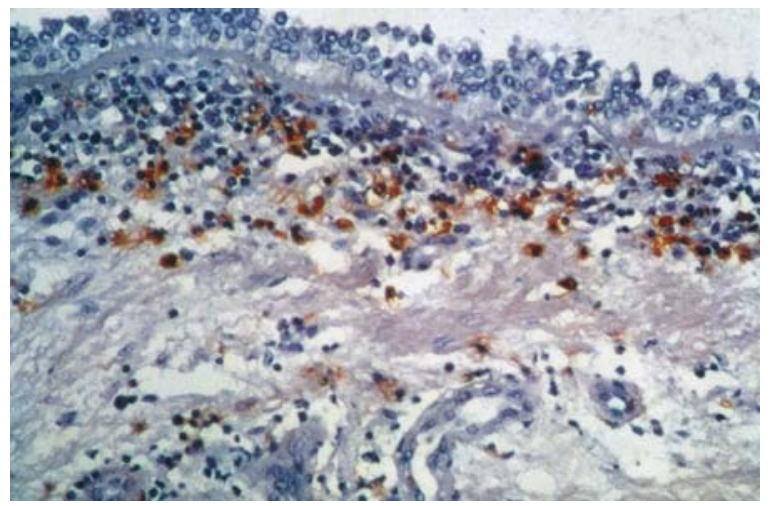

Fig. 11.15. Eosinophilic inflammation in asthma

made it possible to ascertain that in asthmatic subjects, eosinophils appeared in the degranulation stage under the BM and among the basal cells, with a significant parallel between the presence of eosinophils in a degranulation phase and epithelial damage, whereas they were not activated in controls [51]. The asserted verifications of such alterations even in normal subjects can be explained by the potential damage provoked by the biopsy per se and in the preparation of the microscopic specimens [15]. As at the peak of cutaneous reaction, eosinophils are markedly reduced during exacerbations in asthmatic children [605].

Numerous lines of research have given further evidence that activated cells and granule products are implicated in the tissue destruction that accompanies and 


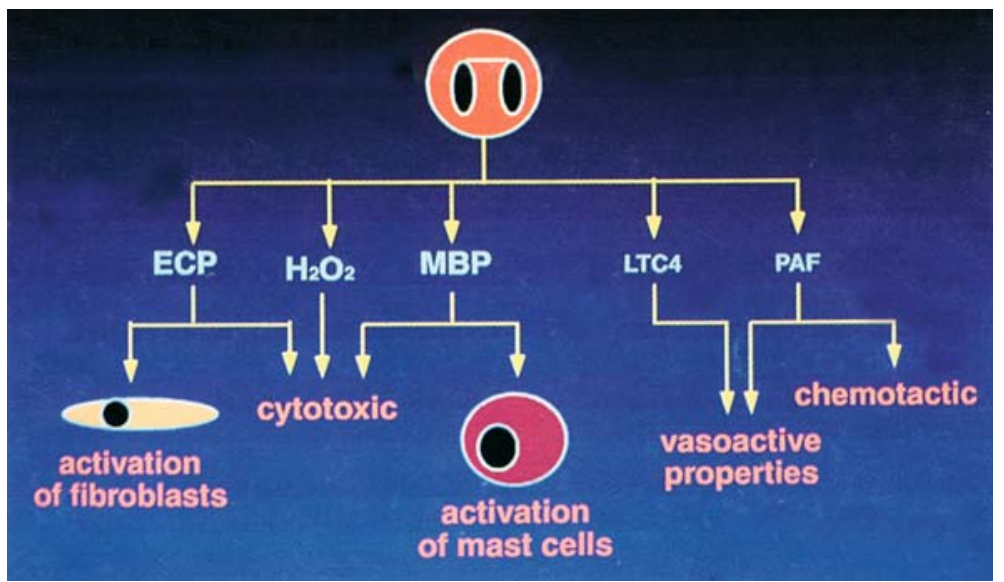

Fig. 11.16. Deleterious role of eosinophils in asthma. ECP eosinophil cationic protein, LTC leukotriene, MBP major basic protein, $P A F$ platelet-activating factor

amplifies immune inflammatory changes (Fig. 11.16) [52]. Moreover, as a result of the change in volume and composition of the secretions of the airways that follows, the mucociliary clearance is compromised (by the parallel effects of $\mathrm{LTC}_{4}$ ). In particular, MBP has a cytotoxic effect on airway epithelium, up to the point of
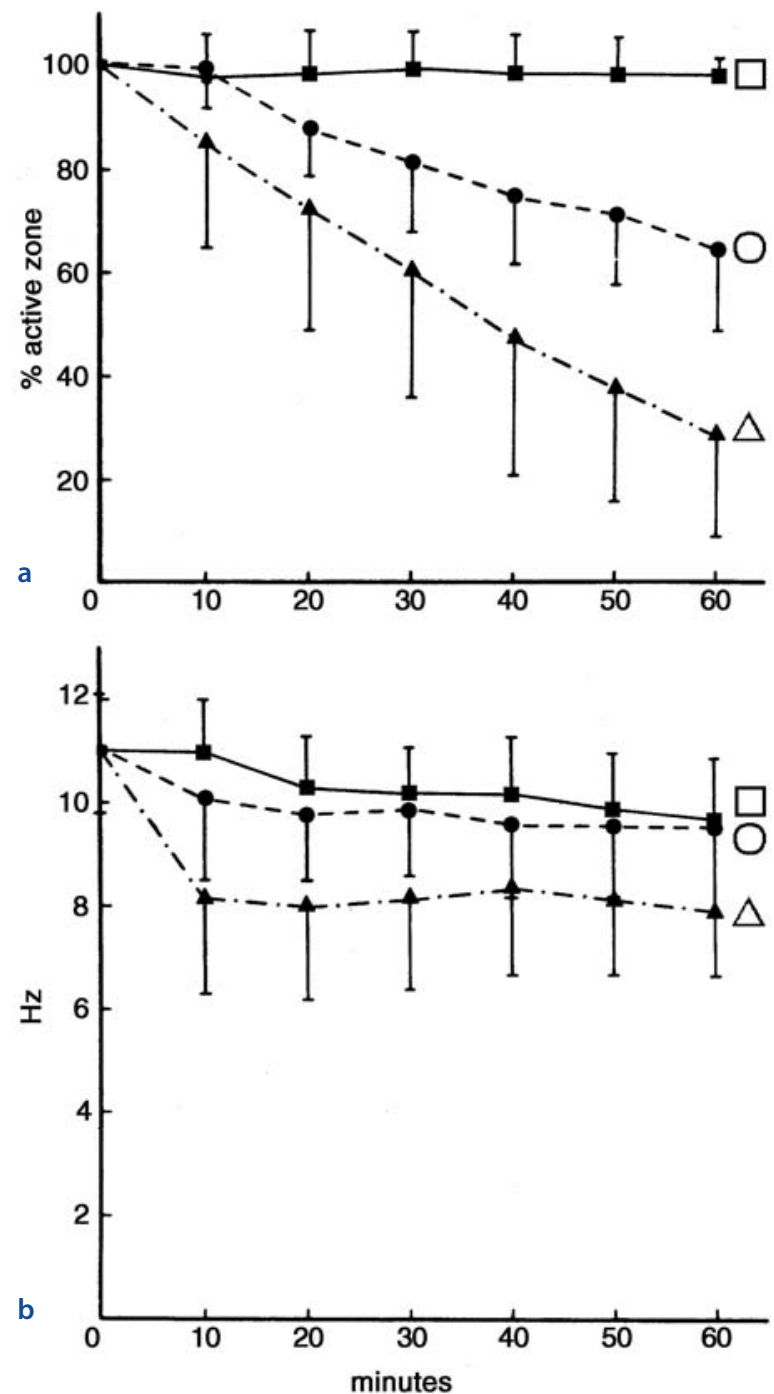

dys-epithelialization [217]. The protein in concentrations of $100 \mu \mathrm{g} / \mathrm{ml}$ provokes in vitro ciliostasis and cell exfoliation within $12-48 \mathrm{~h}$, and, if the dose is increased fivefold, the denudation of the superficial epithelium and of the nerve endings appears, whereas with a level of $700 \mu \mathrm{g} / \mathrm{ml}$, within $30 \mathrm{~min}$, the ciliary beat frequency is inhibited by $20 \%$ and the zone of activity reduced by $40 \%$ (Fig. 11.17) [217]. Experimental research has proven that $\mathrm{EPO}$, in the presence of $\mathrm{H}_{2} \mathrm{O}_{2}$ and halide, tangibly damages the tracheal epithelium [51], while MBP stimulates the tracheal smooth muscles into producing $\mathrm{PGE}_{2}$ and in secreting chlorides [217]; additionally it increases its reactivity to acetylcholine and to histamine, if the epithelium above is normal. If this is not the case, its action is negated [57]. MBP can neutralize the heparin anticoagulant activity [217]. Other highly toxic products derived from the granules play a significant role in determining the epithelial lesions during inflammation, such as ECP, ten times more cytotoxic than MBP, EDN (eosinophil-derived neurotoxin), free $\mathrm{O}_{2}$ radicals, eosinophil-activating factor (EAF) and eosinophil cytotoxicity enhancing factor (ECEF), two factors empowering the eosinophils and their cytotoxicity [679]. An aggravating role is played by complement components, first C5a, which, like PAF, stimulates ECP and EPO release and the production de novo of toxic $\mathrm{O}_{2}$ radicals. Given that components and deposits of the complement components are present in the bronchial secretions and in airway mucosa, it is likely that C5a not only stimulates eosinophils, but also contributes to their activation in tissues close to the inflammatory foci [749].

Fig. 11.17 a,b. MBP effect on ciliary activity (a) and on ciliarybeat frequency (b) in rabbit tracheal explants treated with $1.0 \mathrm{mg} / \mathrm{ml}$ of lysozyme control solution (squares), MBP $100 \mu \mathrm{g} / \mathrm{ml}$ (circles) and $700 \mu \mathrm{g} / \mathrm{ml}$ (triangles). The decline in percent active zone in a, after addition of MBP $0.1 \mathrm{mg} / \mathrm{ml}$ and $0.7 \mathrm{mg} / \mathrm{ml}$ became significantly different from control at 20 and $10 \mathrm{~min}$; in $\mathrm{b}$ the decrease in ciliary-beat frequency is significantly different from the control at 10, 20, $40 \mathrm{~min}$ for MBP $0.1 \mathrm{mg} / \mathrm{ml}$ and from $10 \mathrm{~min}$ onward for MBP $0.7 \mathrm{mg} / \mathrm{ml}$ 


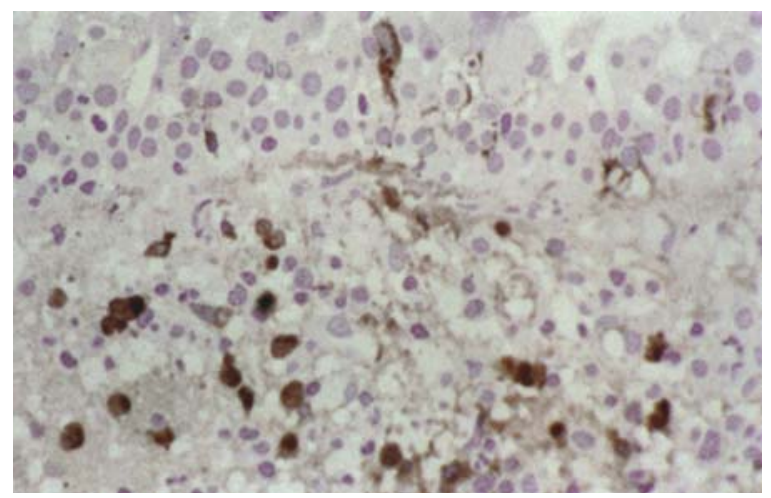

Fig. 11.18. Immunohistochemical demonstration in an asthmatic patient of migrating eosinophils stained for ECP (in brown)

The role exercised by eosinophils in causing inflammation therefore appears dominant, as demonstrated by a significant increase in asthmatic adults in both number and ECP levels (Fig. 11.18) and in the correlation between the two concentrations, with statistically significant differences compared to controls [442, 678]. In this regard, data is controversial in atopic children [240,442, $627,639,756]$, making asthma monitoring problematic given that the rates decrease with treatment [756], but not when asthma is stable [756], or in asymptomatic children [240]. More convincing seems to be the significant increase in $\mathrm{FEV}_{1}$ after treatment, correlated with an ECP decrease, whose levels could be predictive of the results of treatment and of eventual relapses [755]. Such discrepancies are only apparent when one recalls that ECP levels depend on those of active eosinophils when inflammation is present and not on total eosinophilia. $B H R$ is therefore secondary to the epithelial damage caused by eosinophils and their cationic proteins. In fact, at BHR resolution, eosinophils remain unvaried numerically, but with a reduced activation, as proved by the normalization of EPO levels, which is accompanied by the transitory increase in neutrophils and in the levels of MPO (myeloperoxidase) [229].

There exist ample interactions with ILs (Table 11.3) and adhesion molecules (Tables 1.44-1.49 and Fig. 1.59). There are three highly selective phases: expression of CD62E, CD62P and CD62L ligands (Table 1.50); activation of CD11a/CD18, CD11b/CD18, and CD49d/ CD29 (VLA- $4=\alpha_{4} \beta_{1}$ ); and transendothelial transmigration through adhesion molecules of the Ig superfamily (CD106=VCAM-1, CD54 and CD102 = ICAM-1 and -2) (Table 1.4), with a putative mast cell role in binding them to eosinophils through CD62 $\mathrm{P}$ and $\mathrm{IL}_{4}$ (Fig. 11.19) [54]. Like eotaxin- 1 and -2, RANTES and MIP- $1 \alpha$ induce eosinophil chemotaxis during transendothelial transmigration [563]. The adhesion of only eosinophils and basophils to small-vessel endothelium is mediated by the interaction of CD106/CD49d/29, which therefore plays a primary role by recruiting cells to be sent toward

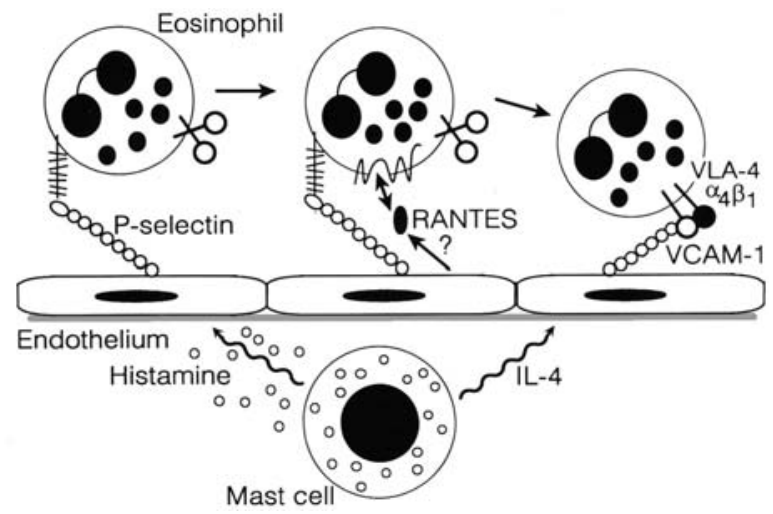

Fig. 11.19. Mast cell putative role on eosinophil transendothelial extravasation. Histamine released by degranulation mediates translocation of preformed CD62P to the endothelial surface and CD62P is involved in eosinophil adhesion. $\mathrm{IL}_{4}$, another mast cell product, induces CD106 expression that binds to $\alpha_{4} \beta_{1}$ on eosinophils
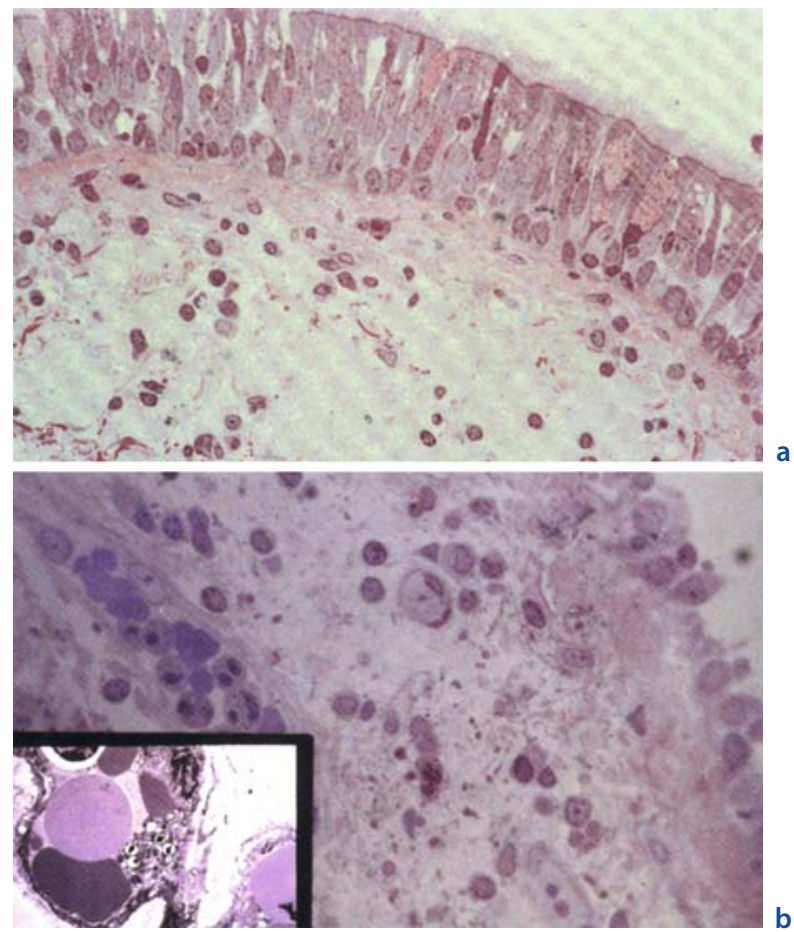

Fig. 11.20. Characteristic aspect of the bronchial mucosa achieved by fiberoptic bronchoscopy from normal (a) and asthmatic individuals (b): the comparison amplifies the extensive inflammatory infiltrate of the asthmatic biopsy and highlights the eosinophil-endothelial interactions (inset)

inflammatory sites in vivo [561]. Let us remember that on a cutaneous level the CD62E selectin facilitates eosinophil and/or neutrophil adhesion, allowing them to migrate through epithelial layers, suggesting that endothelial activation, in the late stage, and due to ILs, covers a primary role in the directional migration of inflammatory cells [258] (Fig. 11.20). The glycoprotein 


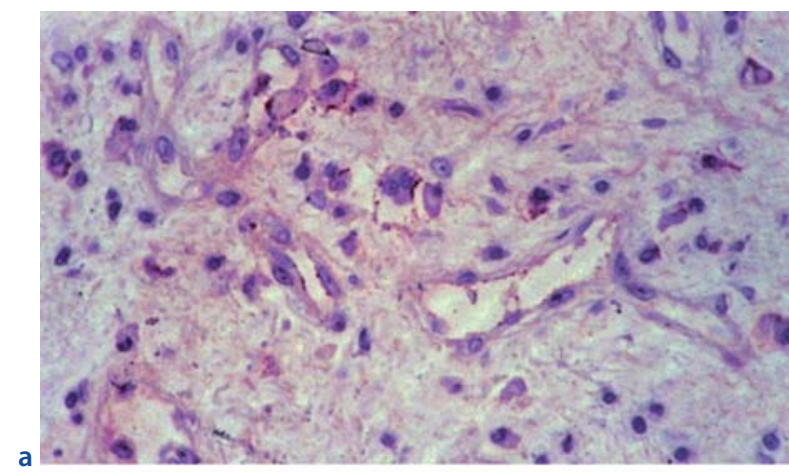

a

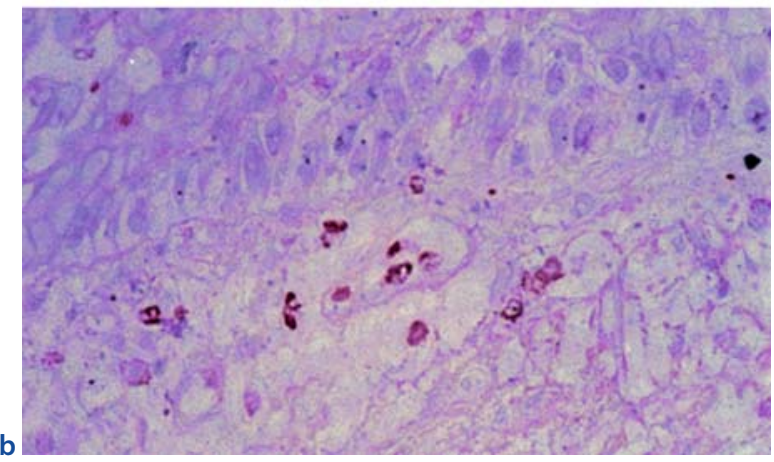

Fig. 11.21. Basal CD54 expression on endothelium of subepithelial blood vessels in a patient with symptomatic asthma (a) and of CD11a/CD18 receptor on T lymphocytes and eosinophils in an asthmatic bronchial biopsy specimen (b)

membrane of the eosinophils interacts with CD62E; thereafter eosinophils are linked via $\beta 1$ and $\beta 2$ integrins to endothelial cell membrane and activated under the influence of $\mathrm{IL}_{3}, \mathrm{IL}_{5}$ and GM-CSF produced by lymphocytes and mast cells [561]. Eosinophils adhere to endothelium in vitro, $\mathrm{IL}_{5}$ induces them to express on membrane CD11a/CD18 CD54, they express their ligand on HEVs, and eosinophils are also bound to CD102, CD62E and CD106, which can contribute to their migration to the inflamed tissue in vivo [233]. They adhere to epithelium activated by $\mathrm{IL}_{1}$ or $\mathrm{IL}_{4} ; \mathrm{IL}_{4}$ can synergize with $\mathrm{IL}_{1}$ or TNF in promoting the eosinophil link and successive transmigration [563]. Linking to CD102, CD62E and CD106 is crucial in encouraging their accumulation in the airways of asthmatics, and is so characteristic as to be able to consider the levels of CD54 and CD106 as activation indexes that are more sensitive than those based on ECP [233].

Activated eosinophils migrate to mucosal sites and through the action of $\mathrm{IL}_{5}$ and PAF progress to the bronchial epithelium [460]. Elegant data indicate that only $\mathrm{IL}_{5}$ is correlated with eosinophil enrollment and degranulation. $\mathrm{IL}_{5}$ promotes the differentiation of mature eosinophils from precursor cells and a current hypothesis suggests that along with GM-CSF and $\mathrm{IL}_{1}$, it can stimulate their degranulation, even in pollen-allergic subjects during the pollen season [87]. $\mathrm{IL}_{5}$ is also able to prolong their survival for $24-48 \mathrm{~h}$, with a lesser role compared to other ILs, and to activate them in successive phases, along with elaboration of lipid mediators and PAF activation [460]. In particular [563], it carries out a chemotactic action on eosinophils. Moreover, since it is a growth factor inducing pro-inflammatory modifications in these cells and acting on B lymphocytes, it increases $\mathrm{IL}_{4}$-induced $\mathrm{IgE}$ synthesis, making it possible to conclude that $\mathrm{IL}_{4}$, after its release from IgEactivated mast cells, can foster eosinophil recruitment and differentiation, both in blood and in the airways. As pointed out previously, the comment that $\mathrm{IL}_{5}$ regulates eosinophil adhesion, an event underlying a fundamental role in their persistence in airway tissue, is relevant [460].

Studies on animals have widened the field of experimental knowledge, indicating that CD54 expression is induced on endothelial and epithelial cells of the airways in the hours following an inflammatory stimulus [81] (Fig. 11.21), that repeated antigen inhalations concretely increase CD54 manifestation on tracheal vascular epithelium and endothelium with a consequent rise in BHR and eosinophils in the airways, and also that anti-CD54 monoclonal antibodies reduce neutrophil adhesion and migration and inhibits (partial) in vitro eosinophil adhesion to endothelial cells [230]. AntiCD54 delivered in vivo lessens or eliminates eosinophil infiltration and aspecific BHR induced by methacholine [230]. These experimental data clearly indicate that CD54 can play a substantial role in BHR pathogenesis [705], while blocking it by a monoclonal antibody can provide a therapeutic approach for the treatment.

\section{Monocyte-Macrophages}

Pluripotent monocyte-macrophage cells are implicated in the pathogenesis of asthma, performing important functions in chronic inflammation, due also to their long life-span (about 180 days). Similarly to mast cells, they are divided into two subpopulations: low-density cells that appear in a state of activation, and high-density cells that are in a state of quiescence.

Immunohistochemical studies on cells taken from BALF have shown evidence that:

- Their numbers are higher in atopic asthmatics than in healthy controls.

- Many cells carry monocyte markers, suggesting that they are of recent derivation from blood monocytes.

- They are provided with receptors for IgE (FceRIIb) and therefore are activated $[330,693]$.

\section{Monocytes}

The characteristics of monocytes are the following [330]:

- They produce ILs stimulated by endothelin-1 (ET-1) and endothelin-4 (ET-4) [129].

- They liberate thermostable factors. 
- They express HLA class II antigens and activation molecules CD11a and CD11b.

Such expression is suppressed by hydrocortisone in sensitive subjects, but not in those who are resistant [330]. They migrate in the alveolar cavities as a consequence of a series of factors related to endothelial microvasculature and chemotaxis phenomena [537]. In the airways, they actively participate in inflammation, secreting numerous ILs with pro-inflammatory activity such as $\mathrm{IL}_{1}$, TNF, M-CSF, chemokines as well as thermostable factors that stimulate eosinophils and neutrophils to release LT and ECEF. Monocytes in asthmatics are activated, as demonstrated by $\mathrm{IL}_{5}$ production in atopics with peripheral eosinophilia; they produce GMCSF in vivo that, on its own or in concert with other ILs, can induce bone marrow progenitors to generate new colonies [330]. Monocytes show a striking ability to produce $\mathrm{O}_{2}{ }^{-}$and, when they are linked by FceRIIb to the receptors, can activate NADPH oxidase, thereby playing a significant role in chronic inflammation by their ability to release toxic products, in part through their linking to CD23-IgE [144]. Co-expression of $\mathrm{CD} 14^{+} / \mathrm{CD} 23^{+}$is significantly increased, while the specific CD14 marker shows no change [552]. ET- 1 and ET-4 are potent stimuli for monocyte production of TNF- $\alpha, \mathrm{IL}_{1 \beta}, \mathrm{IL}_{6}, \mathrm{IL}_{8}$ and CM-CSF [129].

\section{Macrophages}

The characteristics of macrophages are [537]:

- They are increased in number in asthmatic airways and BALF.

- They can produce proinflammatory ILs such as $\mathrm{IL}_{1}$, $\mathrm{IL}_{6}, \mathrm{IL}_{8}, \mathrm{TNF}$, and also PGs, LTs, PAF, $\mathrm{NO}$ (N oxide) and $\mathrm{O}_{2}$ free radicals.

- They express FceRIIb.

It is hypothesized that the majority of active macrophages derive from circulating monocytes [97] (Fig. 11.22). The lung alveolar macrophage is the primary phagocytic cell in the alveolar space [533]. Although their numbers are significantly increased in asthmatics [503], their proliferation in the airways is generally negligible, leading to underestimating their contribution to asthmatic inflammation [330]. On the contrary, they are equipped with HLA class II molecules, becoming, in this association, the predominant cell type in the airways [537], and they are able to process and present antigens to histocompatible CD4 T cells, even though alveolar macrophages are APCs of little effect [287]. After interacting with CD4 $\mathrm{T}$ cells, active macrophages pass to a quiescent stage, which precludes the perpetuation of the inflammation [97]. They synthesize a wide range of mediators: eicosanoids of both metabolic cycles, including $\mathrm{LTC}_{4}, \mathrm{LTD}_{4}$ and $\mathrm{LTE}_{4}, \mathrm{PAF}$, and $\beta$-glucuronidase. Moreover, they produce $\mathrm{O}_{2}$ free radicals, pro-inflammatory ILs of toxic effect on the bronchi, various proteins and peptides, C5a, PDGF (platelet-derived growth factor)

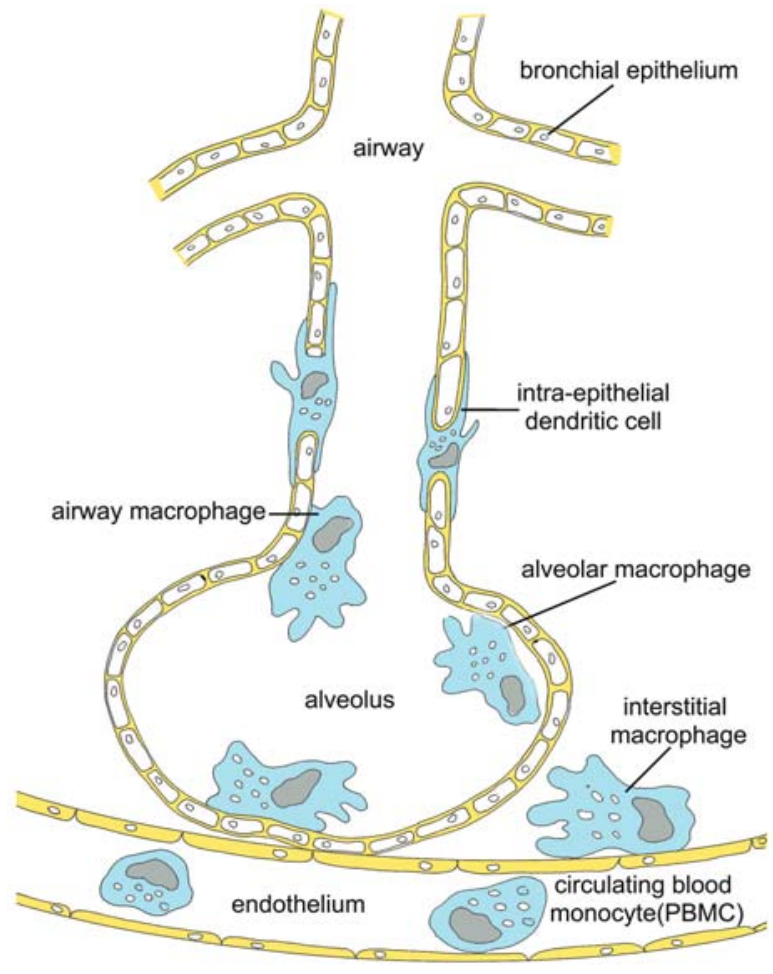

Fig. 11.22. Airway macrophages are significantly increased in asthmatic subjects

with consequent release of other mediators (Fig. 11.22) and, if $\mathrm{Ca}$ is present, HRF, and they also participate in LARs [564]. In contrast to their ability to release factors impairing ciliary function, macrophages also express a potent capacity to synthesize ILs that may up-regulate ciliary beats [648]. In $75 \%$ of cases, macrophages react with GM-CSF [330], which activates them, stimulating their response to IgE via CD23, so IgE occupying the CD23 molecule on macrophages leads to a potential loop for allergens to activate macrophages [537].

As effector cells they are charged with clearance of cellular debris ingestion and killing of microorganisms. They have the ability to migrate to sites of inflammatory reactions where they produce growth factors, bioactive lipids, free $\mathrm{O}_{2}$ radicals, $\mathrm{NO}$ and nitrites [537]. In particular, the marked production of NO [443] may have important effects on vascular and bronchial smooth muscle tone and on bronchial epithelial cells: therefore, the role played by macrophages in airway inflammation [212] is significant. Alveolar cells synthesize elastase and metalloproteins, capable of breaking down macromolecules of the extracellular matrix (ECM), among which elastin is an element in connection with the elastolysis found in asthmatics [53]. As regards the relationship with other cellular types, they contribute to the recruitment and immunological activation of granulocytes, attracted by $\mathrm{LTB}_{4}$ and $\mathrm{IL}_{8} / \mathrm{NAP}-1$ (neutrophil activating peptide-1), of eosinophils by PAF, GM-CSF and $\mathrm{IL}_{5}$, of neutrophils by $\mathrm{IL}_{8} / \mathrm{NAP}-1$ and of mast cells [287]. 


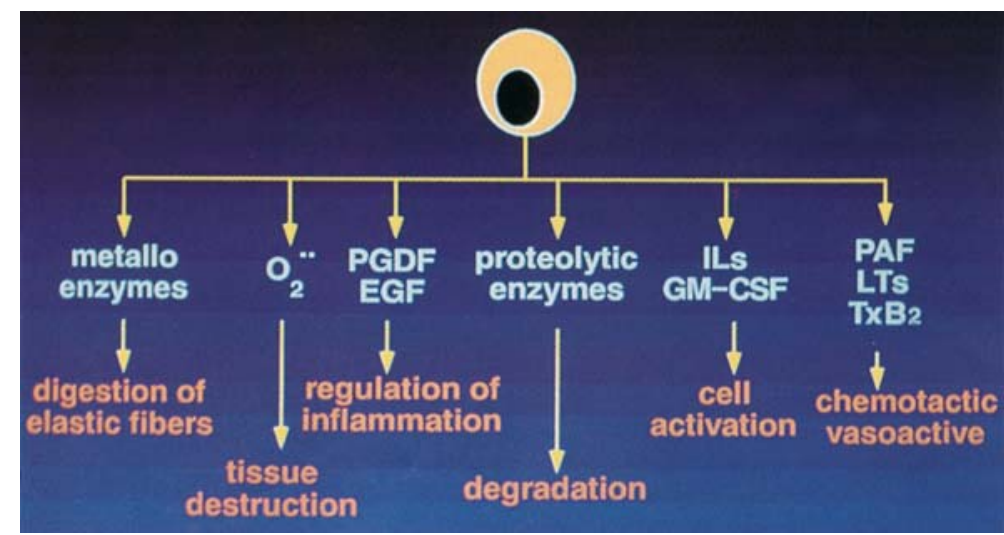

Fig. 11.23. Macrophage role in asthma. EGF epidermal growth factor, GM-CSF granulocyte macrophage-colony stimulating factor, IL interleukins, LTC leukotriene, $P A F$ platelet-activating factor, $P D G F$ platelet-derived growth factor, $T x B$ thromboxane $B$
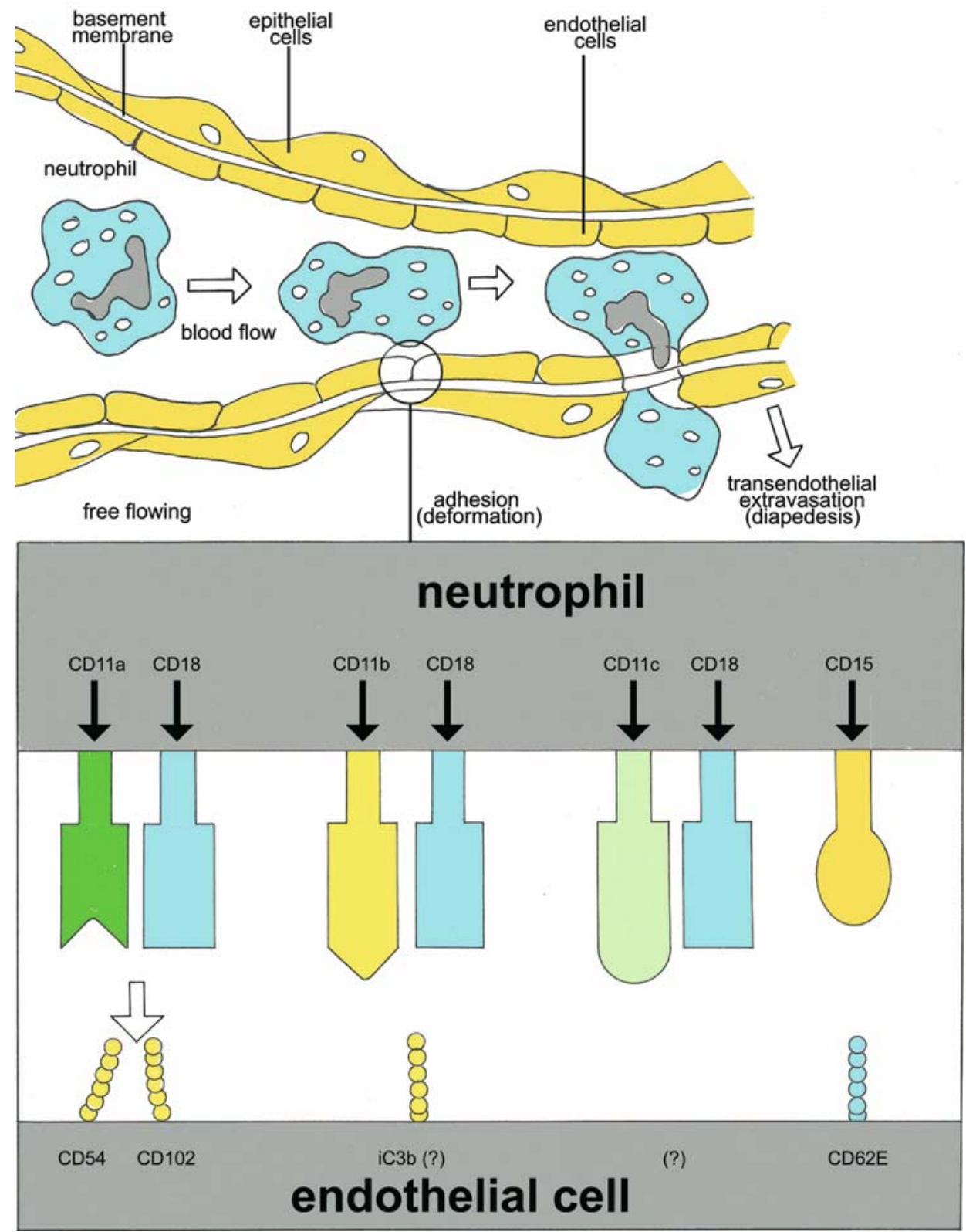

Fig. 11.24. Neutrophil extravasation 
To summarize, macrophages participate in regulating the inflammation by releasing ILs and growth factors, and in the growth and activation of mast cells and eosinophils. All these effects stimulate the release of vasoactive mediators capable of provoking bronchoconstriction and mucus secretion. Recently, it has been reported that macrophages produce CCL chemokines such as MIP-1 and -2, MCP-1-3, and that alveolar cells are activated by MCP-1, with increased levels in asthmatics [330]. It follows that even macrophages could play the role of key cells in immune inflammation [52], as depicted in Fig. 11.23. Moreover, the virtual involvement in the fibrosis process in the reparative stage [53] demonstrates their wide powers. Finally, they constitute the only cell line that does not undergo the positive effects of therapy [212].

\section{Neutrophils}

The characteristics of neutrophils are:

- They can have cytotoxic enzymes and toxic radicals of $\mathrm{O}_{2}$.

- They appear to be more active in LARs.

- They enter into close contact with selectins and integrins.

- They secrete numerous ciliostatic substances.

Neutrophils are active in acute asthma in both IARs and LARs, as shown by the presence in both phases of neutrophil chemotactic factor (NCF). Neutrophils accumulate in the sites of IgE-mediated acute allergic reactions within a time that varies from $30 \mathrm{~min}$ to a few hours. Therefore, these effects are carried out after the initial action, when NAP-1 and NCF come into play, promoting an accumulation of inflammatory cells that prolongs the inflammatory effects of allergen challenge. Their primary role seems to be that undertaken in LAR, depending on the time needed to produce NCF. Neutrophils are found at the center of inflammatory lesions because of two specific cell sites: the cytoplasmic membrane and the intracellular granules, which contain a vast array of digestive enzymes (Table 1.23). Elastase, collagenase and gelatinase, which attack the vasal endothelium of the respiratory apparatus, are particularly cytotoxic. Not all authors are in agreement regarding the attribution of a specific role to neutrophils $[53,600]$, even if $\mathrm{IL}_{17}$ secreted by $\mathrm{T}$ cells could involve them in the airways (Table 1.5).

Recently, however, a better understanding has been gained of the role they play in immune inflammation, which is summed up in the stages described above and which require the recruitment of CD11a/CD18, $\mathrm{CD} 11 \mathrm{~b} / \mathrm{CD} 18, \beta 2$ integrins of neutrophils, which link to their counterparts on endothelial cells, CD54 and CD102 [420] (Fig. 11.24). Furthermore, in vitro studies have shown that the duo NAP- $1 / \mathrm{IL}_{8}$ [266], stimulated by CD62E, directs neutrophils to transmigrate through the endothelium [420], and that SP directs them toward inflammatory sites, joining with VIP and somatostatin in modulating their functions, in concert with various ILs [88]. As underlined in Chap. 1, PAF and SP favor neutrophil adhesion to the walls of vascular endothelium and their successive migration in the interstice of respiratory airways of asthmatics, where they can produce several negative effects on both structure and function [88]. In a mouse model, the link between allergen-induced $\mathrm{T}$ cell activation and neutrophil influx induced early $\mathrm{IL}_{17}$ mRNA expression in inflamed lung tissue, concomitant with a prominent bronchial neutrophil influx [243]. In asthmatic children, the manifestation of CD11b, localized in specific granules, is also increased in the neutrophils, thus carrying out an important role in their migration together with eosinophils: the induction of complement receptors, in particular of CD35, could constitute the first step in the inflammation [37]. The negative effect of neutrophils on ciliated cells caused by proteases such as elastase, by oxidants that impair their motility, as well as by bioactive lipids such as PAF that up-regulate mucociliary clearance $[413,648]$, has now been confirmed.

\section{Epithelial Cells}

The role of epithelial cells is to provide passive support to sensorial receptors, which are opportunely protected by tight junctions and regulate the hydroelectrolytic perireceptor environment, also ensuring, by means of mucus and cilia, the clearance of abnormal substances and performing antimicrobial, antioxidant and antiprotease activities [648]. According to recent information, they also play an immune role as APCs, as they are equipped with FceRII and class II antigens that they can present to CD4 T cells (Fig. 11.13), and express enzyme pathways for cyclooxygenase and lipoxygenase. Oxygenated metabolites have robust effects on smooth muscles, nerves and glands, as well as on inflammatory cells and epithelial cells [52] (Fig. 11.25). Due to specific phospholipases, these cells release eicosanoids, NCF and endopeptidase, able to degrade neuropeptides and fibronectin that mediate cell adhesion together with integrins, and participate in epithelium regeneration [525]. Epithelial cells contribute, therefore, by means of these mechanisms, to the pathogenesis of asthma, promoting both infiltration and local activation of granulocytes and T lymphocytes [374].

The epithelial cells also produce:

- $I L_{1}$, an $\mathrm{IL}_{6}$ synergistic component of $\mathrm{T}$ lymphocyte, $\mathrm{IL}_{8}$ growth and activation [374], $\mathrm{IL}_{10}, \mathrm{IL}_{11}$, and $\mathrm{IL}_{16}$ [189]

- NAP-1/IL $L_{8}$, chemotactic for T cells, neutrophils and above all eosinophils, in contrast with normal subjects [33]

- Growth factors: TNFR-1 (CD120a), endothelins, lipids, NO, PDGF [189]

- Adhesion molecules such as CD54, CD102, CD62E and CD62P, ligands of the counterparts on $\mathrm{T}$ lymphocytes (CD11a, b/CD18, CD49d/CD29) and CD62L 


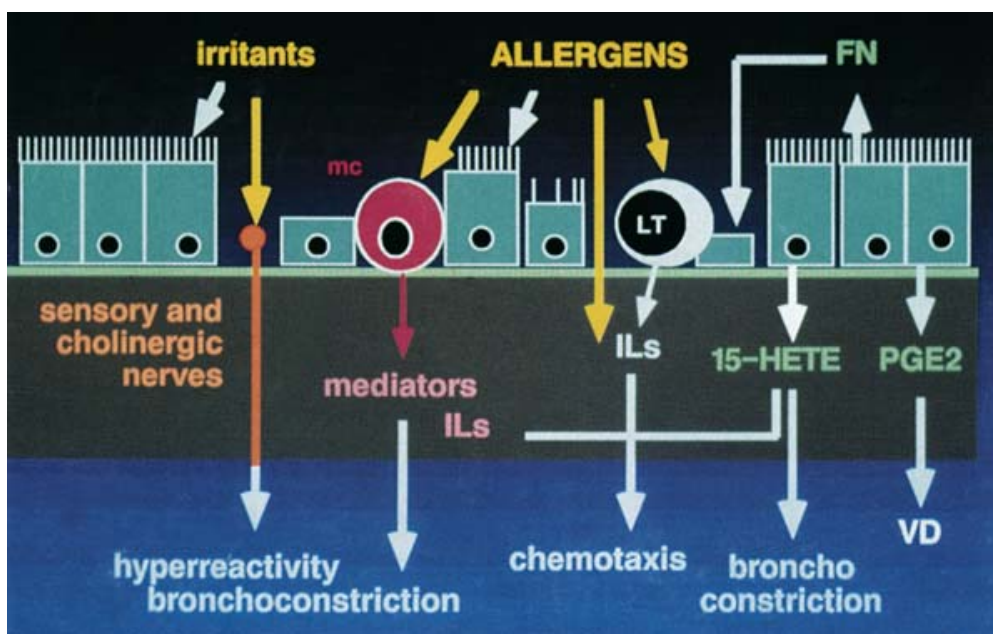

Fig. 11.25. Putative role of epithelium in asthma. $F N$ fibronectin, IL interleukin, LTT-cell, MC mast cell, VD peripheral vasodilation

- Chemokines: MCP-1 and GM-CSF specific for monocytes, MCP-4, GRO- $\alpha$ and GRO- $\gamma$ [189]

The report that $\mathrm{IL}_{1 \beta}$ produced by monocytes induces an increased synthesis of IL brought about by epithelial cells denotes the possible existence of an amplifying network among epithelial cells and monocytes/macrophages.

Recently the effects in the airways of ET-1, provided with a powerful bronchoconstriction action - less so in endothelin-2 and -3, whose synthesis is codified by an equivalent number of genes identified in the human genome - have been defined [431]. Bronchial epithelium of symptomatic patients produces larger quantities of it compared to asymptomatic patients. ET-1 binding sites are present in bronchial and bronchiolar muscles [4]. Additionally, such peptide stimulates the lipoxygenase activity with an increase of LTs, with both chemotactic and constriction effect on smooth muscle [4]: in a pediatric cohort it reflected the degree of effort required by asthma [12]. In confirmation of its autochthonous production, ET-1 levels in BALF are high; the output is regulated by $\mathrm{TNF}-\alpha, \mathrm{IL}_{1}$, lipopolysaccharides (LPs) (endotoxin) [431] and histamine [4]. Consequently, the airway epithelium, like vascular endothelium, plays an important, double role in regulating the muscular tone of the area of interest, releasing both constriction factors, such as ET-1, as well as the epithelium-derived relaxing factor (EDRF) [431], identified with NO [10].

The potentially harmful activity of epithelial cells also comes about through 15-HETE, a biological mediator that stimulates the infiltration of inflammatory cells by inducing the release of mucosal glycoproteins, influencing in leukocytes 5-lipoxygenase activity and stimulating in asthmatics a premature bronchoconstriction in response to inhaled allergens. They are therefore activated cells, which, with their products, can play a deleterious role in the genesis and persistence of bronchial inflammation and BHR (Fig. 11.25), while their changes are the heart of remodeling [491].

\section{Endothelial Cells}

The vascular endothelium, in addition to contributing to the passive barrier and also taking part in airway remodeling [490], seems to be involved in asthma pathogenesis and in inflammatory processes associated with its severe forms. It is considered a true endocrine gland, with a surface made up of about $10^{3}$ cells corresponding, in an adult, to about $1.5 \mathrm{~kg}$ of tissue [112]. Its cells locally regulate the inflammatory cells that, to reach inflammation sites, must cross endothelial walls. This process is articulated in two phases: first adherence and then passage through endothelial cells. The regulatory activity consists of impeding the adhesion and aggregation of blood platelets and other blood cells, maintaining bloodstream regularity by endothelium-released $\mathrm{NO}$, with an action that is both vasodilatory, especially at the arteriolar level, and antiaggregating along with $\mathrm{PGI}_{2}$ [10]. Once activated, endothelial cells induce:

- An increased expression of adhesion molecules

- Production of GM-CSF, $I L_{8}$ and RANTES, potentially reduced by inhalation of beclomethasone dipropionate (BDP)

- Production of chemotactic factors for neutrophils and $\mathrm{T}$ lymphocytes, and of PAF and $\mathrm{IL}_{5}$ [654]

Basophils adhere to endothelia by means of CD49d/ CD29 [561], and TNF- $\alpha$ and $\mathrm{IL}_{1}$ induce CD54 on endothelium (a prerogative also shared by macrophages) and CD62E for leukocyte adhesion. Activated endothelium could be capable of self-protection against TNF- $\alpha$ negative effects, which exposes the barrier to $\mathrm{O}_{2}$ toxicity, thus increasing its permeability [654]. Nor should one forget the complex interactions between subsets of lymphocytes and endothelial tissues in the control of lymphocyte recirculation (Fig. 1.5). Hence, it is not out of the question to also attribute to these cells a participation in the inflammatory process. 
Fig. 11.26. Altered immunological homeostasis in respiratory mucosa. The altered mucosal homeostasis is reflected by distorted B lymphocyte accumulation because of aberrant lymphocyte extravasation, and emigration of mast cells $(M C)$ armed with $\operatorname{lgE}$ and partly primed eosinophils (EOs) is facilitated by cytokines modulating the profile of endothelial adhesion molecules. A second line of defense is set up in the mucosa to perform immune elimination, including local lgG production to limit dissemination of foreign antigens. Due to the proinflammatory IgG properties, a vicious circle may develop with further increase in epithelial antigen penetration, complement hyperactivation, massive phagocyte recruitment and inflammatory mediator release (for details see text)

\section{Mucosal stroma}

Epithelium Lumen

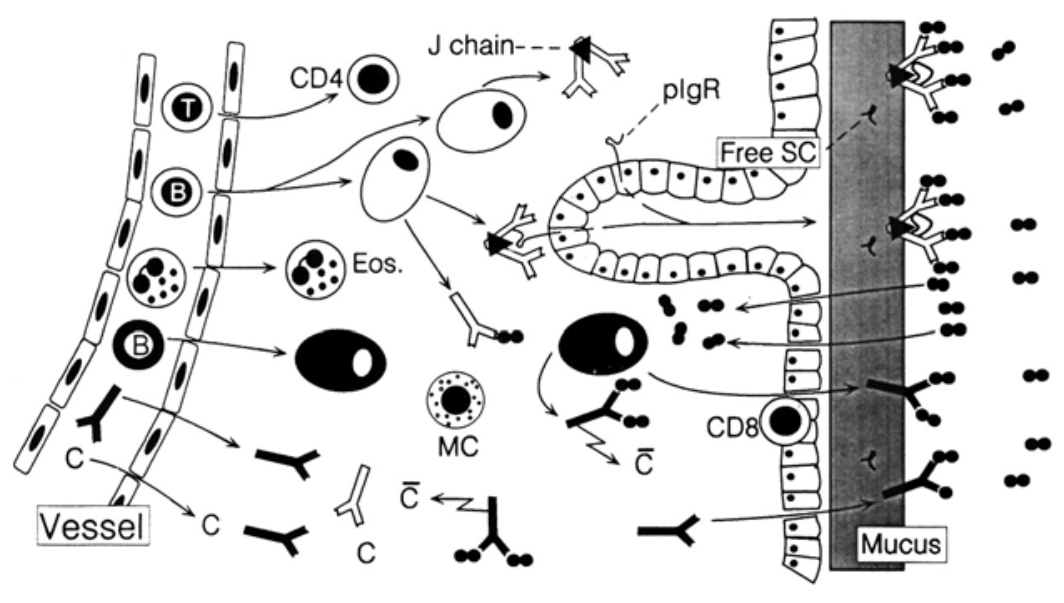

\begin{tabular}{|r|l|l|l|l|}
\hline $\lg G$ & $\sim \lg A$ & $C=$ complement & $\bar{C}=$ activated $C$ & $\boldsymbol{\infty}$ \\
\hline
\end{tabular}

Fig. 11.27. Central role played by lgE and mast cells in development of late-phase allergic reaction. Processed peptide is exposed by APC to T lymphocytes in the context of HLA class II molecules, while intact allergen cross-bridges IgE antibodies on the surface of mast cells. Cytokines generated by CD4 and mast cells enhance preferential $\lg$ E production triggering mast cells, thus polarizing T-cell cytokines to a Th2 pattern (for details see text)

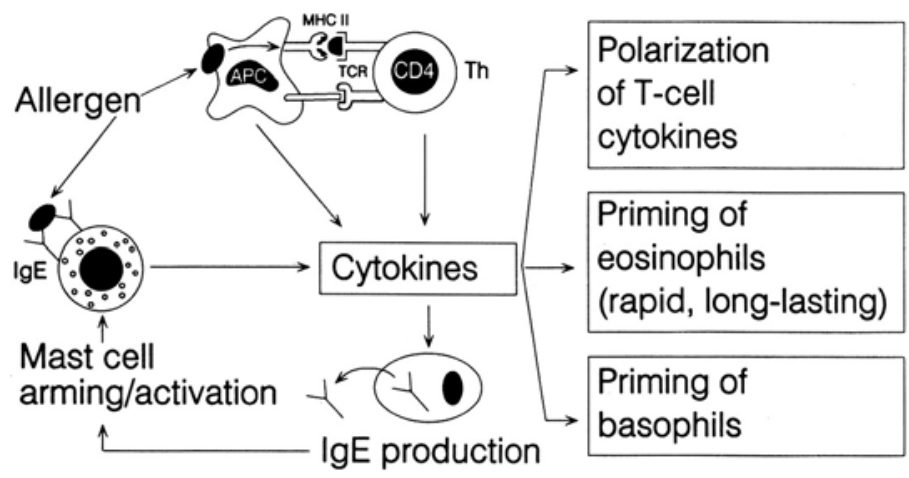

\section{Blood Platelets}

Blood platelets provided with FceRII migrate by diapedesis to inflamed tissues, acting as effector cells in the immune inflammation. An inappropriate activation leads to eosinophil recruitment by the expression, either singularly or in combination, of PF4, PAF, $\mathrm{PGE}_{2}$, and thereby to BHR and asthma. In asthmatics, thrombocytopenia, an increase in associated circulating blood platelets in the microvasculature, of blood platelets in BALF, of PF4 levels and thromboxane $\mathrm{B}_{2}\left(\mathrm{TXB}_{2}\right)$, as well as the prolonged bleeding time and a reduction in the time needed for blood platelet regeneration and survival have been identified. These cells liberate TGF- $\beta$ and PDGF, the former being able to stimulate fibroblast proliferation, the latter smooth muscle hypertrophy. $\beta \mathrm{TG}$ ( $\beta$ thromboglobulin) generates NAP-2 and PF4, chemotactic for neutrophils and monocytes/macrophages, respectively (Table 1.57), suggesting platelet involvement in infiltrating and perpetuating structural alterations which, in the long run, lead to subepithelial fibrosis and smooth muscle proliferation at the basis of BHR [743]. The hypothesis that $\beta \mathrm{TG}$ and PF4 perform the role of plateletactivation markers has not been confirmed [743].

\section{The Role of IgE}

As already noted, the production of IgE is slight in the respiratory mucosa which, instead, is populated by mast cells armed with IgE in the epithelium and connective tissue, which, along with basophils, produce CD154, whereas eosinophils have the CD40-CD154 duo (Chap. 1). In making a comparison with Fig. 11.2, in the mucosa a contrast with the alteration of immune homeostasis can be noted (Fig. 11.26) [54], provoked by an excessive local accumulation of B lymphocytes following a combination of an aberrant extravasation of lymphocytes, an increase in vascular permeability and an excessive exposure to antigens by the local immune system. All this is transformed into a worsening and perpetuation of the inflammation, laying the foundations for a chronic disease of the mucosa [54]. The increased production of IgE and mast cell positive regulation play a role in the outcome of the late reaction which cannot take place without $\mathrm{T}$ cell cooperation (see "Role of the Inflammatory Cells") (Fig. 11.27) [54].

Parallel to the suggestion of interpreting to interpret $\mathrm{AD}$ as a failed attempt by Th2 $\mathrm{T}$ cells to inhibit Th1 $\mathrm{T}$ cells to restore local homeostasis, asthma could be the 
result of a failure of the normal defense of the organism to control IgE-mediated immune responses [263].

\section{Role of the Mediators}

A host of mediators, some preformed such as histamine (Fig. 1.56) and others generated ex novo, participate, directly and indirectly, in inflammation and bronchoconstriction $[162,538]$. Many of those initially released are widespread, far from the discharge site to go toward metabolization or inactivation. Histamine, BK and PAF are at the basis of IAR (Table 11.2); others such as PGs, thromboxanes (TXs) and LTs provoke both IAR and LAR. Other inflammatory factors are $\mathrm{PGD}_{2}$ and $\mathrm{TXA}_{2}$, which produce vasodilation, LTs, which induce mucus secretion and increase vascular permeability, and factors released by neutrophils, basophils and eosinophils, discarded by mast cells after allergen/IgEFceRI interaction and drawn back to the site of immune inflammation by chemoattractant factors (BCF, NCF, ECF, $\mathrm{LTB}_{4}$ ). The peptide LTs such as $\mathrm{LTB}_{4}$, discarded by stimulated neutrophils, monocytes and macrophages, $\mathrm{LTC}_{4}$ by mast cells and eosinophils, are powerful mediators of asthma attacks.

\section{Lipid Mediators}

PAF is a vasoactive mediator released by a range of cells that carries out a bronchoconstriction activity after exposure to a specific allergen. It has chemotactic and activating action on neutrophils and, above all, on eosinophils. Additionally, it increases eosinophil cytotoxicity, provoking the release of granule content; it stimulates $\mathrm{O}_{2}^{--}$generation (less in neutrophils), promoting the release of MBP and ECP cationic proteins and, in blood platelets, the flow of $\mathrm{Ca}$ ions and aggregation [758]. PAF linked to specific receptors can stimulate complex mechanisms of intercellular transduction, by which it induces $\mathrm{G}$ proteins and $\mathrm{C}$ protein kinase activation, and inositol phosphate and intracellular $\mathrm{Ca}$ turnover increase, the result being the secondary activation of phospholipase $\mathrm{A}_{2}$ with subsequent discharge of arachidonate by membrane phospholipids, with subsequent production of a mediator cascade, including TXA [607]. For eosinophils, it is the most potent chemotactic factor which causes, in vivo, their accumulation in the airways, amplifying their activity both in IAR and LAR. Additionally, TXA promotes their adhesion to human vascular endothelium, stimulating eosinophils to synthesize $\mathrm{LTC}_{4}$, an effect amplified by the fact that, when eosinophils come into contact with PAF, they release further quantities [607]. Eosinophils can attract other cells through PAF, with an increase in vascular permeability and bronchoconstriction, to the point of destroying the bronchial epithelium of asthmatic children [558]. Exudation of serum proteins in tissues - also due to a com- bined action with $\mathrm{LTC}_{4}$ - is at the forefront in contributing to the amplification and maintenance of long-term immune inflammation [607]. The selective attractionactivation eosinophil ratio as also documented by the orchestration of cationic proteins, and powerful mediators, makes PAF the leading player in eosinophil inflammation, able to reproduce the entire sequence of typical asthma events. PAF plays a notable regulatory role in pediatric asthma re-exacerbations, as demonstrated by its marked increase during acute asthmatic attacks and by the parallel decrease after SIT [265], and by higher levels in asthmatic children, as compared to asymptomatic children and controls [558].

\section{Eicosanoids}

Eicosanoids are secreted in the airways by mast cells, alveolar macrophages, neutrophils, eosinophils and epithelial cells [362]. Among those derived from mast cell membrane, via the arachidonic acid metabolism, the second class of cysteine derivatives in particular possess a spasmogenic action, while $\mathrm{LTB}_{4}$ carries out a chemotactic action. Lipoxygenase leads to the formation of various PGs, which, together with $\mathrm{TXA}_{2}$, cause bronchoconstriction, milder if induced by $\mathrm{PGE}_{2}$.

$L T s$ are assuming an ever more important role in the pathogenesis of asthma as based on new experimental data (Fig. 1.57). Inhalation of cysteine derivatives provokes an acute bronchoconstriction that is prolonged and stronger than that caused by histamine in asthmatic sufferers, whose upper air tract is particularly sensitive to LT bronchospastic actions [387]. Higher levels in BALF and urine of asthmatics during a severe, acute crisis can always be noted. LTs also exert a sensitizing action on the airways, making them vulnerable and susceptible to BHR to triggering agents such as allergens, intense physical activity or cold air [387]. Additionally, LTs increase vascular permeability, mucus hypersecretion and the production of $\mathrm{IL}_{1}$ by monocytes [362]. $\mathrm{LTE}_{4}$, in particular, recruits eosinophils and neutrophils in the ratio of $10: 1$, able to increase their numbers in only $4 \mathrm{~h}$ [329], while $\mathrm{LTC}_{4}$ reduces the activity of respiratory cilia in vitro [709], a negative effect confirmed by the report that pretreatment with cromones inhibits LTs [578]. $\mathrm{LTB}_{4}$, on the other hand, possesses striking chemotactic activities for neutrophils, eosinophils and monocytes, also regulating the expression of membrane receptors and of IgE-FceRII. Recent data show that concentrations of exhaled LT and 8-isoprostane (a marker of oxidative stress) are increased in atopic asthmatic but not in atopic nonasthmatic children. Exhaled $\mathrm{LTE}_{4}$ concentrations are reduced by $18 \%$ by inhaled CS treatment whereas $\mathrm{LTB}_{4}$ and 8-isoprostane are not reduced [588].

Prostaglandins $\mathrm{PGF}_{2 \mathrm{a}}$ and $\mathrm{PGD}_{2}$ are powerful bronchoconstrictors. PGE produces bronchodilation in healthy subjects and bronchoconstriction in asthmatics. $\mathrm{PGI}_{2}$ has little effect on smooth muscle but, since it 
Table 11.4. Airway effects of the mediators implicated in asthma

\begin{tabular}{|c|c|c|c|c|c|}
\hline Mediators & $\begin{array}{l}\text { Bronchocon- } \\
\text { striction }\end{array}$ & $\begin{array}{l}\text { Airway hypo- } \\
\text { secretion }\end{array}$ & $\begin{array}{l}\text { Permeability } \\
\text { increase }\end{array}$ & Chemotaxis & $\begin{array}{l}\text { Bronchial } \\
\text { hyper-reactivity }\end{array}$ \\
\hline Acetylcholine & + & + & - & - & - \\
\hline Adenosine & + & $?$ & $?$ & $?$ & - \\
\hline Bradykinin & + & + & ++ & - & - \\
\hline Complement fragments & + & + & + & ++ & - \\
\hline Histamine & + & + & + & + & - \\
\hline $\mathrm{LTB}_{4}$ & - & - & \pm & ++ & \pm \\
\hline $\mathrm{LTC}_{4}, \mathrm{D}_{4}, \mathrm{E}_{4}$ & ++ & ++ & ++ & $?$ & \pm \\
\hline NKA & ++ & + & + & - & - \\
\hline PAF & ++ & + & ++ & ++ & ++ \\
\hline $\mathrm{PGD}_{2}, \mathrm{PGF}_{2 \alpha}$ & ++ & + & $?$ & $?$ & + \\
\hline $\mathrm{PGE}_{2}$ & - & - & - & + & - \\
\hline $\mathrm{O}_{2}$ radicals & + & $?$ & + & $?$ & - \\
\hline Serotonin & \pm & $?$ & + & - & - \\
\hline Substance P & +++ & ++ & \pm & - & \\
\hline TXA & ++ & $?$ & - & \pm & $?$ \\
\hline
\end{tabular}

Modified from from [22].

TXA thromboxane A, PAF platelet activating factor, NKA neurokinin A.

Table 11.5. Effects of mediators in the airways

\begin{tabular}{|c|c|}
\hline Effects & Mediators \\
\hline $\mathrm{BHR}$ & ECFs, NCF, HETEs, LTB $_{4}$ \\
\hline Bronchospasm & $\begin{array}{l}\text { Histamine }\left(\mathrm{H}_{1} \text { receptor), } \mathrm{LTC}_{4}, \mathrm{LTD}_{4},\right. \\
\mathrm{LTE}_{4}, \mathrm{PGD}_{2}, \mathrm{PGF}_{2}, \mathrm{TXA}_{2}, \mathrm{BK}, \mathrm{PAF}\end{array}$ \\
\hline $\begin{array}{l}\text { Epithelial } \\
\text { desquamation }\end{array}$ & $\mathrm{H}_{2} \mathrm{O}_{2}, \mathrm{OH}^{-}, \mathrm{O}_{2}^{--}$, proteolytic enzymes \\
\hline Mucosal edema & $\begin{array}{l}\text { Histamine }\left(\mathrm{H}_{1} \text { receptor }\right), \mathrm{LTC}_{4}, \mathrm{LTD}_{4} \\
\mathrm{LTE}_{4}, \mathrm{PGE}, \mathrm{BK}, \mathrm{PAF}\end{array}$ \\
\hline Mucus secretion & $\begin{array}{l}\text { Histamine }\left(\mathrm{H}_{2} \text { receptor }\right), \mathrm{LTC}_{4}, \mathrm{LTD}_{4} \\
\mathrm{LTE}_{4}, \mathrm{PGE} \text {, acetylcholine, } \mathrm{C} 3 \mathrm{a}, \mathrm{C} 5 \mathrm{a}\end{array}$ \\
\hline
\end{tabular}

Modified from [283].

has a striking vasodilatory activity, can cause, in concert with other mediators, edema and therefore BHR [362].

Tables 11.4 and $11.5[22,283]$ summarize the mediator activities and the pathological events for which they are responsible.

\section{Role of Cytokines}

These immunotransmitters, able to induce their own release besides that of other mediators and of regulating the expression of the receptors, have specific effects on immune inflammation acting on both growth and differentiation of the progenitors of hematopoietic cells, which are released at the sites of immune inflammation. ILs (Table 1.5), many of which can be found in BALF, are released $[58,334]$ :

- many that can be found in BALF are released by $[58,334]: \mathrm{IL}_{3}-\mathrm{IL}_{5}, \mathrm{IL}_{9}, \mathrm{IL}_{11}, \mathrm{IL}_{13}, \mathrm{IL}_{17}, \mathrm{IL}_{25}$, and GM-CSF by Th2 lymphocytes and mast cells

- $\mathrm{IL}_{2}, \mathrm{IL}_{9}$ and IFN- $\gamma$ by Th1 lymphocytes

- $\mathrm{IL}_{1}, \mathrm{IL}_{6}$ and TNF- $\alpha$ by macrophages

- GM-CSF, $\mathrm{IL}_{3}, \mathrm{BaDF}, \mathrm{NGF}$ by epithelial cells

- GM-CSF, $\mathrm{IL}_{6}$ by fibroblasts and endothelial cells (see the complete list in Table 1.5, also including $\mathrm{IL}_{16}$ and $\left.\mathrm{IL}_{17}\right)$

Allergic airway disease is associated with skewed Th2 cytokine production, although the underlying cause of this aberrant immune response is not well understood. GM-CSF, $\mathrm{IL}_{3}$ and $\mathrm{IL}_{5}$ prolong eosinophil survival and stimulate their degranulation. GM-CSF stimulates granulocytes to generate LT and PAF, basophils to release histamine and neutrophils and macrophages to secrete more cytokines, among which are found $\mathrm{IL}_{1}$, TNF, G-CSF and M-CSF [58]. All ILs act according to what is summarized in Table 1.5, in concert with the newly released and activated factors (Fig. 1.56), perpetuating the allergic inflammation and forming the molecular basis of its becoming chronic. $\mathrm{IL}_{4}$ plays a highly important role in promoting the isotypic switching of $\mathrm{B}_{\mathrm{IgG}}$ cells into $\mathrm{B}_{\mathrm{IgE}}$ cells and, as a consequence, increases the concentrations of total IgE and sIgE recorded in allergic asthma. $\mathrm{IL}_{6}$ en- 
Intercellular junction

Tight junction

(zonula adherens)

(zonula occludens)

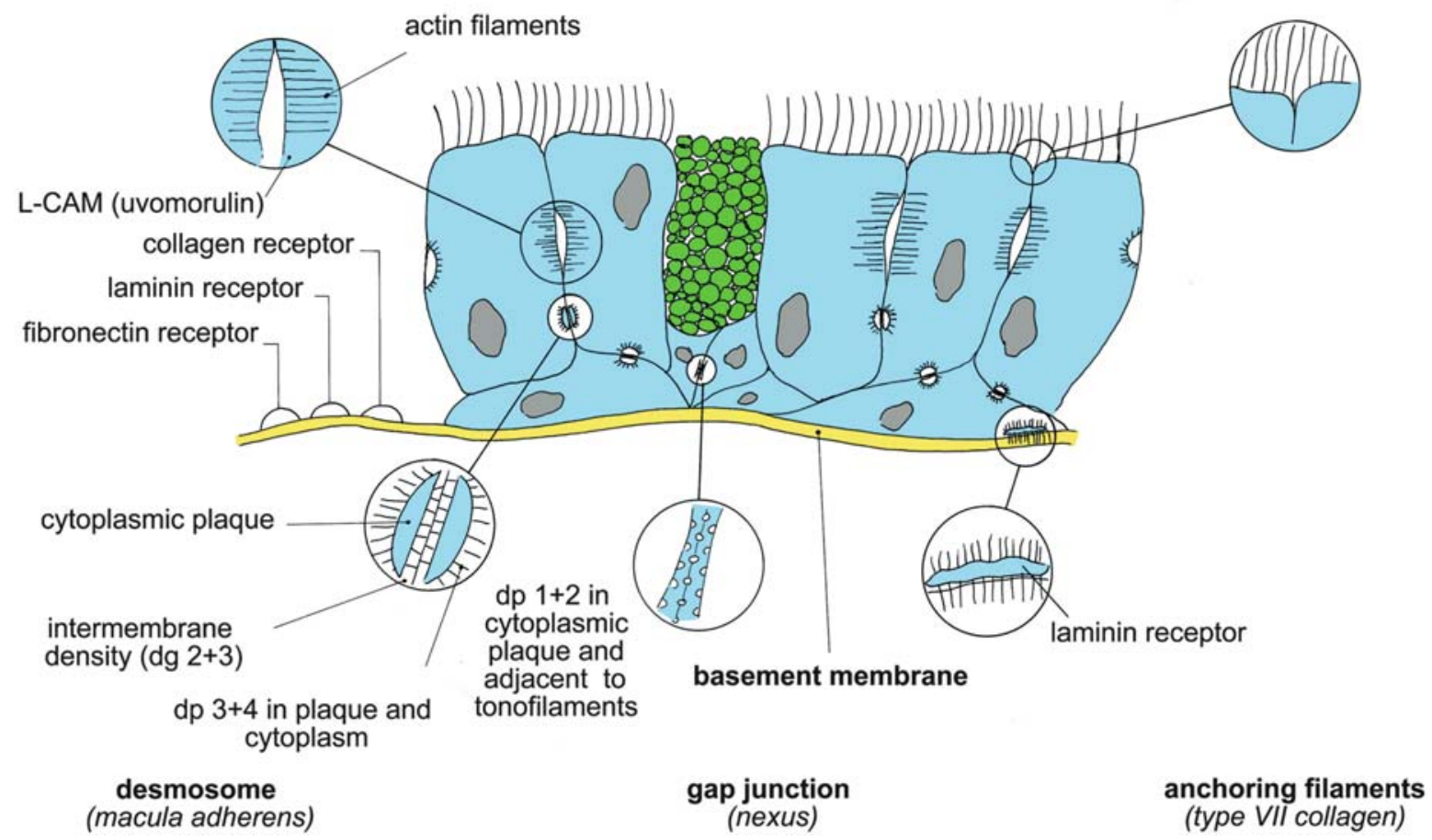

Fig. 11.28. Intercellular adhesion molecules responsible for maintaining bronchial epithelium integrity

hances in $\mathrm{B}$ lymphocytes $\mathrm{IL}_{4}$-induced production of IgE antibodies. All these data reinforce the opinion that ILs perform a leading role in propitiating in the airways the conditions that trigger asthmatic manifestations. Wild et al [721] found that the administration of $\mathrm{IL}_{18}$ increased the production of ragweed-specific $\operatorname{IgE}$ and $\mathrm{IgG}_{1}$ in serum in a mouse model of allergic asthma, effects consistent with the support of a Th2 phenotype. Furthermore, intranasal application of $\mathrm{IL}_{18}$ together with ragweed increased the production of BALF eosinophilia, suggesting the stimulation of an allergic sensitization when coadministered with an allergen [721]. On the contrary, $\mathrm{IL}_{11}$ has a profound inhibitory effect on antigen-induced inflammatory responses in the lung. This inhibitory response is associated with marked diminution in eosinophil recruitment, Th2 cell accumulation, Th2-like T cell IL production, and antigen-induced endothelial cell CD106 expression.[692]. Thus, $\mathrm{IL}_{11}$ is an important mediator of the remodeling response in the asthmatic airway and its elaboration reflects, at least in part, an attempt at healing and repair in this setting [752].

\section{Airway Remodeling}

In all inflammatory diseases the processes of recovery begin early in the course of asthma (premodeling). They start in the first stages of cell denudation and lead either to restitution to a former state (restitutio ad integrum), which does not leave a residue, or leaves changes in connective tissue deposition and to permanently altered airway structure which, in its permanent state, constitutes cicatrization [681]. Epithelial BM collagen deposition and thickening is already apparent in asthmatic children before the age of 3 years, compared to symptom-free children [501]. Normally, healthy epithelium is in contact with cylindrical cells, contact which is characterized by tight junctions in such a way that the adjacent cells oppose an impermeable barrier to the intercellular passage of macromolecules, inhaled pollutants, infectious agents, and other particulate matter. The mucosa generally absorbs small molecules as well as proteins by paracellular means, but when exposed to foreign agents it responds with plasma protein leakage. However, persistent allergic airway inflammation in asthma is accompanied by airway remodeling changes, including hyperplasia of airway mucus glands, myofibroblasts, smooth muscle and vasculature, and the thickening of the airway wall with subepithelial fibrosis [261]. The prospect of developing irreversible airway obstruction should prompt early treatment decisions [189]. $\mathrm{IL}_{11}$ induces an airway remodeling response in the asthmatic airways characterized by tissue fibrosis, deposition of types I and III collagen, and myocyte and myofibroblast hyperplasia [752].

It follows therefore that the remodeling process can also involve other cells such as leukocytes and ECM [491]. 


\section{Fibroblasts}

Their biological activity is regulated by a range of cytokines and growth factors:

- $\mathrm{IL}_{1}$, PDGF and TGF- $\beta$ stimulate their proliferation and collagen synthesis. Furthermore, $\mathrm{IL}_{1}$ can activate $\mathrm{T}$ lymphocytes that respond with TNF.

- GM-CSF induces the formation of CD106, CD54 or CD62E and is able to stimulate the eosinophils either directly or through the induction of $\mathrm{IL}_{5} \mathrm{R}$ (CD125].

- $\mathrm{IL}_{1}, \mathrm{TNF}, \mathrm{TGF}-\alpha$, histamine and heparin regulate mast cell number and function, modulating their content of proteoglycans and enhancing the filling of mast cell granules in the bronchial mucosa.

- $\mathrm{IL}_{8}$ has an important role in directing inflammatory cells in the bronchial mucosa [534].

The fibroblastic activation may be responsible for the deposit of interstitial connective tissue under the epithelial BM [534]. During the initial processes of recovery, fibroblasts proliferate actively, secreting proteoglycans and collagen. These cells, which perform a vital role in the secretion of ECM components, can change phenotype in response to environmental signals and differentiate in myofibroblasts [534]. Macrophages, lymphocytes, mast cells and eosinophils, cells which probably participate in various ways in ECM fibrosis and/or remodeling [53], are always almost present in the granulation tissue. As the recovery process progresses, tenascin, a glycoprotein, qualitatively increases, while fibroblasts and the newly formed vessels diminish [189]. Myofibroblasts, deposits of fibronectin and laminin, and collagen hyperplasia are also found. Even in case of restitution to a former state, the regeneration of the submucosa is always, in part, abnormal [53].

\section{Epithelial Cells}

Epithelial cells release a wide spectrum of molecules participating in airway repair including:

- Fibronectin, growth factors

- Cytokines including $\mathrm{IL}_{9}, \mathrm{IL}_{11}, \mathrm{IL}_{16}$ and $\mathrm{IL}_{18}$

- Chemokines such as GM-CSF and eotaxin

- Adhesion molecules such as CD40 and CD54 [681]

The bronchial epithelium is actively engaged in defense of the airways by secreting mucus and many specific and nonspecific cytoprotective molecules that trap and inactivate inhaled components. If the asthmatic immune inflammation is protracted over time, the adhesion mechanisms are compromised, which should ensure the maintenance of epithelial integrity, represented by desmosomes, hemidesmosomes and tight junctions (zonula occludens) and zonula adherens, which is located immediately below the intercellular joints (Fig. 11.28) [327], as well as adhesion molecules such as $\beta_{1}$-integrins and CD62E [413]. In some cases, a pseudostratified epithelium can be observed with increased goblet cells and vacuolized ciliated cells, often

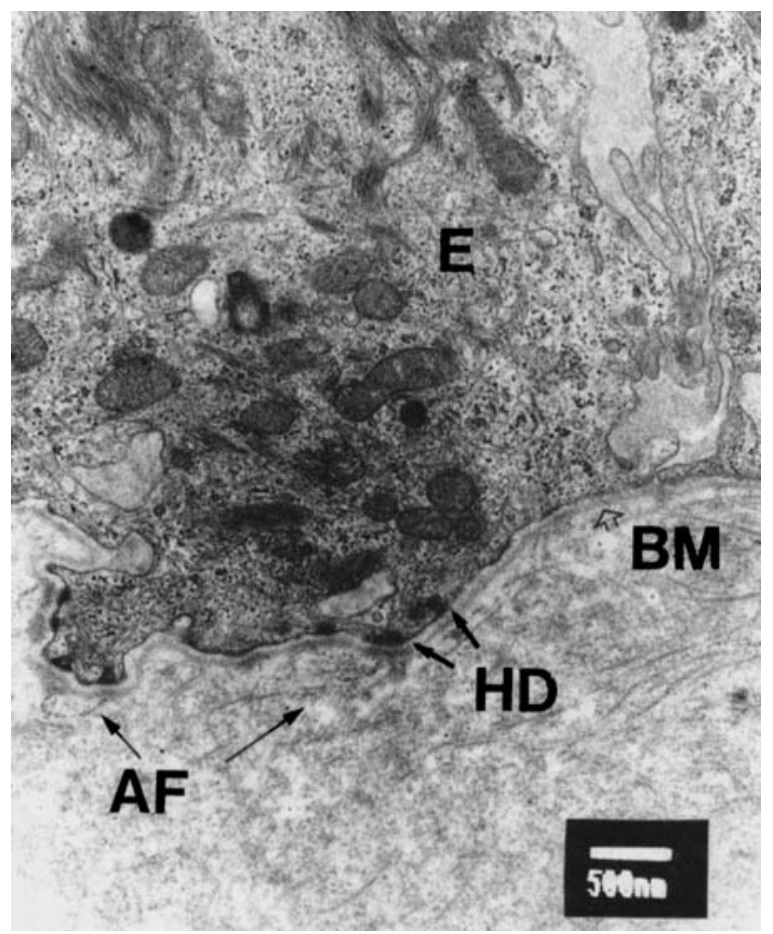

Fig. 11.29. Electron micrograph of thickened subepithelial basement membrane (BM). Basal cells possess hemidesmosomes $(H D)$, by which they adhere to the superficial part of BM. Anchoring filaments $(A F)$ are part of adhesion complex. (E) Airway epithelium

devoid of cilia [51]. This tissue often exfoliates, with separation of mucosal cells, leaving the basal layer exposed but intact, though weakening the connection system between columnar and basal cells. It is clear, therefore, that to induce the effects described, destructive activities must take place, concentrated in both space and time (Figs. 11.30, 11.31) [52, 53]. Epithelial damage could depend not only on the cytotoxic effects of eosinophil-derived basic proteins and oxidants, but also on neutrophil intervention which, in vitro [413] and in vivo [677], have the ability of producing similar effects, as well as the exfoliation and detachment of the epithelium from the BM $[413,677]$. In addition, epithelial injury is mediated by exogenous factors such as air pollutants, viruses and allergens as well as by endogenous factors including the release of proteolytic enzymes from mast cells [260]. It has been suggested that such changes could also originate from an abnormal response of epithelial cells, stimulated by leukocytes [413]. Moreover, $\mathrm{IL}_{13}$ hyperproduction promotes subepithelial fibrosis and thickening of smooth muscle layer [321]. The epithelial response to these stimuli in asthma may be impaired despite up-regulation of CD44 capable of enhancing presentation of EGF (epidermal growth factor) ligands to EGF receptors (EGFR) [260]. 


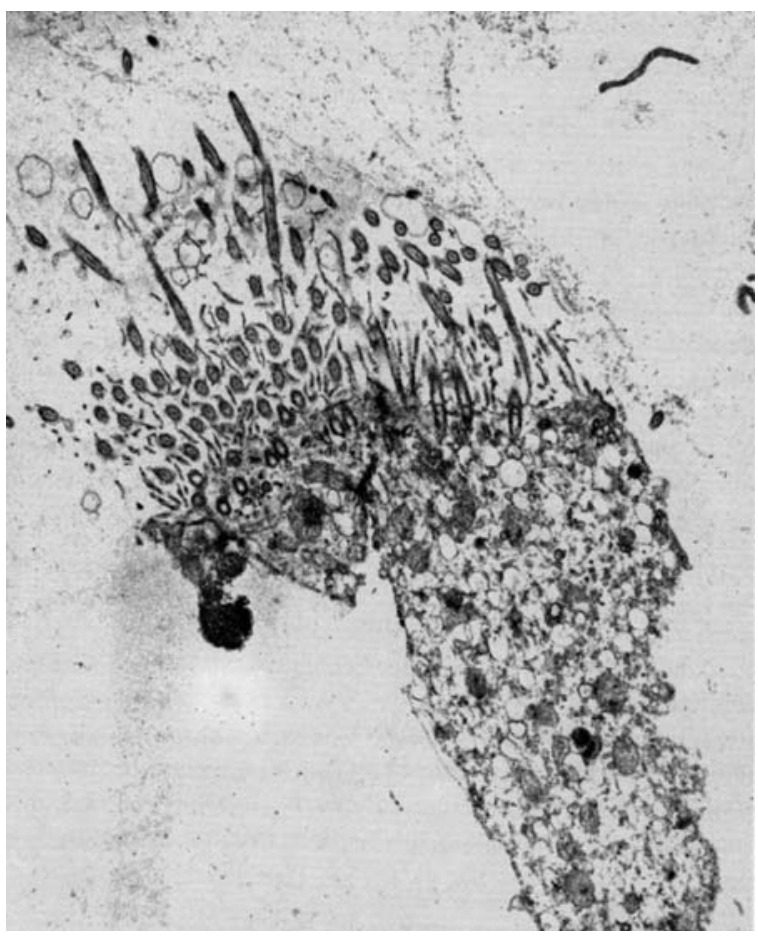

Fig. 11.30. Desquamated epithelial cells undergoing necrosis recovered by BALF from the airways of an asthmatic patient after an exacerbation

However, regenerative activities begin with the reepithelialization of the denuded surface, as demonstrated by various stages of ciliogenesis related to nonciliated superficial epithelium, and by a quantitative increase in goblet cells in the ciliated epithelium. The speed and rapidity with which restitution to a former state begins and continues is surprising. Vascular endothelial cells proliferate, forming granulation tissue, the newly formed vessels have open junctions that permit the release of proteins and erythrocytes, the microvasculature also responds by recruiting neutrophils [490]. It is likely that bronchial epithelial cells originally involved with the lesion are able to initiate these reparatory activities, producing chemotactic factors for intact epithelial cells and for PMNs [600]. Facilitated by an adequate hydraulic pressure, the plasma proteins enter the lumen of the tight junctions of the intact cylindrical cells that circumscribe the denuded area [491]. These cells respond immediately, leading to a supposed intervention of specific factors in vivo: substitute secretory cells, both ciliated and basal cells, undifferentiate, become flattened and migrate beyond the membrane - a process which takes place rapidly in the 1st min following denudation (about $3 \mu \mathrm{m} / \mathrm{min}$ ) [490]. A fibrin and fibronectin gel, also rich in neutrophils, contributes in covering up the stripped areas where it is continually enriched with plasma until the epithelium has been regenerated with the tight junctions [491] (Fig. 11.32). The fibronectin, present in the ECM with a great number of binding sites for the cells and other molecules, appears to participate in epithelium regeneration since it is responsible for cell adhesion, a mediator role that is shared, via different receptors, with collagen and laminin [600]. However, the simple restitution of epithelial cells has the potential of being involved in many aspects of structural alterations, present in human airways, that are at the base of symptoms similar to asthma (Table 11.6) [491].

\section{Extracellular Matrix}

ECM is a complex and dynamic meshwork influencing many cell biological functions such as development, migration, and proliferation. ECM plays an essential supporting structural role, which differs somewhat in the three physiological zones of the lung: the proximal conducting airways and vasculature, the distal gas-exchanging respiratory zone (alveoli), and the intervening transitional zone (respiratory bronchioles). In the conducting airways, the ECM of the rigid cartilage (composed largely of proteoglycans and collagen) and the more flexible interstitial tissue support the adjacent epithelial and smooth muscle cells. ECM allows some mobility to

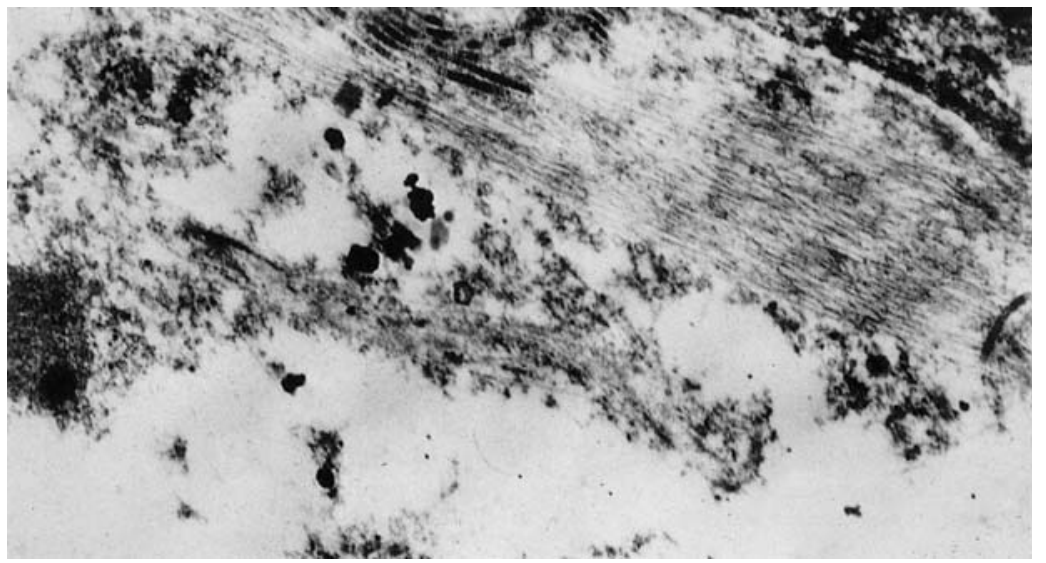

Fig. 11.31. Elastolysis in asthmatic airways: electron microscopic study 
Fig. 11.32. Plasma exuded through barriers in the airway microvasculature and mucosa. Plasma leakage from subepithelial microcirculation multiplies its solutes and expands in volume. It surrounds the basolateral aspects of the epithelial cells; by increasing the hydrostatic pressure the exudate may compress the sides of these cells. At a certain pressure, the tight junctions at the apical pole of epithelial cells would separate due to the increased pressure load. Plasma leakage through the ensuing holes in the venular wall. Tight junctions are reestablished as soon as the interstitial pressure returns to normal levels

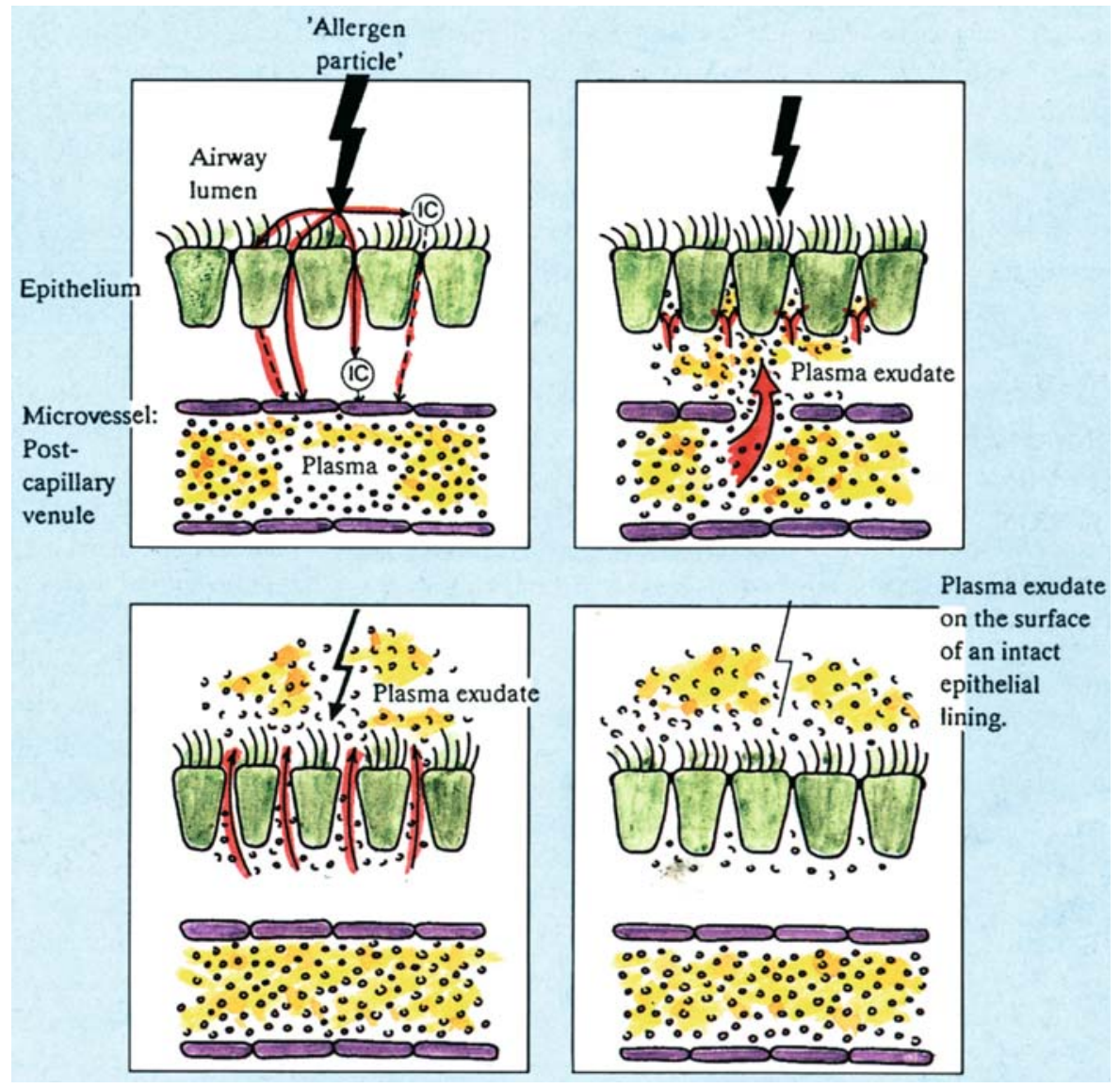

Table 11.6. Asthma-like effects aroused by reparation processes

\begin{tabular}{lll} 
Pathophysiology & Leukocyte activity & Structural alterations \\
Plasma leakage & Eosinophil traffic and activation & Epithelial metaplasia \\
\hline Secretion & Neutrophil accumulation and activation & Adhesion molecules of plasma origin \\
& (Pseudo) thickening of basal membrane \\
& Proliferation of fibroblasts \\
& and/or smooth muscle cells
\end{tabular}

Modified from [491].

regulate airway and vascular diameter and acts as an essential stabilizer for preventing airway collapse during expiration. In the respiratory and transitional zones, the ECM is more dynamic to accommodate the constant fluctuations in alveolar volume that accompany inspiration and expiration. Macromolecules constituting ECM are secreted locally and consist of fibrous proteins (fibronectin and laminin) embedded in a hydrated polysaccharide gel containing several glycosaminoglycans including hyaluronic acid. ECM is a dynamic structure, and an equilibrium between ECM synthesis and degradation components is required to maintain its homeostasis [681].

\section{Basement Membrane}

The BM of surface epithelium is composed of two layers: the basal lamina (referred to as the true BM) and the lamina reticularis. The basal lamina is of normal thickness in asthma. However, thickening of the lamina reticularis is a characteristic feature of the asthmatic bronchus, occurring early in the disease process [681]. Studies of postmortem reports initially led to the conclusion that in asthmatics the BM becomes thicker as a result of edematous collagen deposits, with fibrils of a plexiform appearance. Electron microscopy (EM) and immunohistochemical investigations subsequently made it clear that BM is normal. Airway thickening be- 
neath the BM occurs with collagen deposition and other ECM proteins, including fibronectin and tenascin in the connective tissue layer surrounding the blood vessels, and alveolar interstitium [261]. The degree of collagen deposition into the BM lamina reticularis in children who underwent fiberoptic bronchoscopy is such that airway fibrosis evolves in parallel with eosinophilic inflammation before a clear clinical diagnosis of bronchial asthma could be made [501]. Instead, reticular BM thickness or inflammatory cell number determined in ultrathin sections of endobronchial biopsies were not present in 53 infants aged 3.4-26 months with severe wheeze and/or cough, with reversible airflow obstruction [548]. In healthy subjects, BM, independent of asthma etiology, gravity and duration, shows a pseudothickening [51] caused by a deposit of collagen III, V and I (to a lesser extent) and of fibronectin in the lamina reticularis situated below the BM [534], confirming that at the basis of this process, is the activation of contractile myofibroblasts in the subepithelial site, rather than an epithelial dysfunction [53]. Since BM appears to be the main structure appointed to the regeneration organized by connective tissue, forming a sort of framework for parenchymal cell replication, its structural alterations, in the airways of asthmatics, can result in an ECM deficiency of the submucosa that could be at the basis of airway remodeling.

\section{Elastic Fibers}

Most patients with asthma have an abnormal superficial elastic fiber network with fibers appearing fragmented. The deeper layer of elastic fibers is also abnormal in most patients with asthma: fibers are often patchy, tangled, and thickened. EM studies show that a severe elastolytic process occurs in patients with asthma, and in some patients, disruption of fibers has been observed.

\section{Role of Bronchial Hyperreactivity}

The pathogenesis of asthma (Fig. 11.11) is in large part identified with BHR which, together with epithelial alterations, secondary to immune inflammation, constitutes the causative element in triggering the chain of events that result in an asthmatic attack. On the whole, BHR measurement could represent a useful means of identifying in advance subjects at risk of PFT worsening, well correlated with severe manifestations and treatment implications. Nevertheless, in children a noticeable variation in clinical symptoms is found, which is why, in practice, the usefulness of measuring BHR is somewhat reduced. Furthermore, the uncertainty of a certain correlation of this parameter with the clinical state and its diversity over time renders BHR not very reliable as a diagnostic test for selecting an adequate treatment and for determining a prognosis [108]. Other authors have demonstrated that reduction of activated $\mathrm{T}$ cells and CD25 is correlated with that of BHR [119] but with an improvement of $\mathrm{FEV}_{1}$, the number of eosinophils and probably of $\mathrm{IL}_{5}$ [665] decreases.

With the aim of prospecting the nosological picture, it should be clear that $[110,603,722]$ :

- BHR is not synonymous with asthma, neither is it systematically predictive of an asthmatic state. Rather it is one of the factors that suggest asthma presence, without possessing the characteristics of a diagnostic symptom in se and per se.

- BHR is not, therefore, the only component of the physiopathological mosaic of asthma. The etiopathogenic mechanisms of mucus hypersecretion and of bronchial edema are equally noteworthy factors not to be ignored in the overall picture [238].

- BHR does not lessen with age [344] as previously suggested [414] because a small child is more susceptible to BHR also because of airway caliber reduction [584]. BHR is present even in chronic airway disease such as CF [186].

Virtually all asthmatic children have BHR, but not all children with BHR suffer from asthma $[108,476]$ : more precisely it can be present in apparently healthy children and absent in others who are asthmatic, while nonasthmatic subjects can manifest signs of BHR - for example if faced with a cold air challenge [238]. It is also possible that BHR is present without any ongoing symptoms, which can be ascribed to patients unable to describe correctly the symptoms during initial history [496]. On the other hand, transitory BHR can be present in children with viral infections; therefore exogenous events increase BHR in both healthy and asthmatic subjects [753]. Even exposure to small allergen doses can trigger BHR, without, however, altering airway caliber [722], whereas in asthmatic children the opposite occurs: continuous or repeated exposure to aeroallergens induces a $B H R$ increase, causing an almost unnoticeable progressive increase, persisting for weeks or even months. This gives rise to the premises of a vicious cycle, consequently subsequent exposures, even to nonallergic or banal stimuli easily provoke BHR. Even if inflammatory injuries caused in loco are numerous and severe, when administering histamine parenterally bronchoconstriction is likely to occur [167]; therefore what results is the product of a series of joint causes.

At present, the contribution of environmental factors is considered important in eliciting BHR, as demonstrated by a greater asthma prevalence in southern countries, where SPT + to aeroallergens is less [287]. The pathogenic links between damage provoked by pollutants, mediators, inflammation and BHR are harder to characterize [238]. Strongly suggestive in this regard is that BHR may be possibly generated by $\mathrm{O}_{3}$ with a potent inflammatory action on airways. Injected in test animals, it induces NCA and of $\mathrm{LTB}_{4}$ formation in epithelial tissues, provoking in the respiratory mucosa a PMN in- 
filtration from the microvasculature which, once activated, release $\mathrm{TXA}_{2}$ able to sensitize the smooth muscles or the nerve terminals, thereby causing BHR [287, 317] (Fig. 4.22). $\mathrm{O}_{3}$ induces $\mathrm{BHR}$, be it through oxidizing damage [317], in concentrations of $0.12-0.15 \mathrm{ppm}$ [606], or through indirectly stimulating the tachykinins to induce BHR [315]. As a result of BHR, the threshold of bronchial response to various stimuli that unleash factors with bronchoconstriction effects, is noticeably lowered. Among these, the role of mediators should be considered. As seen in Tables 11.4 and 11.5, those capable of inducing BHR are many: PAF and PG, and, to a lesser extent, TXA and LT, even if it is clear that there is not only one but a combined interaction at work [283]. These factors are therefore capable of causing a clinical response, increasing and/or maintaining BHR, as a result of which vicious cycles are born between clinical response, immune inflammation, triggering factors and symptoms. BHR degree is often correlated with disease severity with important clinical implications, when these are clearly the important correlation in children between BHR to histamine and the increase in mast cell tryptase and of the number of eosinophils/mm of BALF [187]. As opposed to other children, serum tryptase levels did not increase after BPT with allergens [240]. Recent studies suggest that depletion of $\mathrm{IL}_{12}$ [415] and overexpression of $\mathrm{IL}_{13}$ [321] increase susceptibility to development of BHR, even in the absence of inflammation [308].

To better explain the pathogenesis, various theories have been put forward based on the intervention of endogenous and exogenous factors.

\section{Endogenous Factors}

Among the endogenous factors, the following may be enumerated [154]:

1. Autonomic nervous system (ANS) alterations

- Deficit of adrenergic receptors

- Vagal hyperactivity

- Altered neuropeptide release at the sensory terminals

2. Anomalies of smooth bronchial muscles

3. Anomalies in airway epithelium

- Desquamation of the epithelium

- Loss of EDRF

4. Changes in the biochemical homeostasis

5. Effects of NO

\section{ANS Alterations}

Several inflammatory mediators effecting neurotransmitter release by airway nerve endings, or able to act upon ANS receptors, have been identified. Conversely, neuromechanisms could contribute to the inflammatory reaction at the bronchial level. It is therefore possible that some BHR mechanisms are linked to an imbalance of the lower airway nervous control mechanism, entrusted either to the sympathetic (adrenergic system) or parasympathetic system (cholinergic system). These systems are usually in balance: the cholinergic system promotes bronchoconstriction; the adrenergic system, in contrast, modulates bronchial smooth muscle relaxation. At the present time, the ANS airway innervation is considered to be far more complex. Given that sympathetic fibers do not directly innervate smooth muscles, in addition to the usual adrenergic and cholinergic fibers, fibers belonging to a third system called NANC (nonadrenergic and noncholinergic) have been reported, which include both e-NANC (excitatory noncholinergic/nonparasympathomimetic structures) and i-NANC (nonadrenergic/nonsympathomimetic inhibitory structures) [21].

\section{Cholinergic Systems}

The fibers of cholinergic nerves travel along the tenth pair with synapse in the airway parasympathetic ganglia, spreading to smooth muscles and submucosal glands. Stimulation of vagal receptors present in the airways provokes one of the most powerful bronchoconstriction reflexes, besides mucus secretion. In particular, mechanical or pharmacological stimulation of vagal terminals occasion, by reflex action, characteristic asthma symptoms and signs such as coughing and rapid, shallow breathing. Cholinergic innervation of upper airways is most dense, thinning out in the periphery. Animal studies have shown that stimulation produces little effect in the lower airways [21]. Given that in humans the muscarinic $(\mathrm{M})$ receptors are equal to parasympathomimetic, which are diffused even in the lower airways, investigations to ascertain whether in asthma there was an increase in their activity [719] were made. This view point is supported by a study showing that many stimuli having a bronchospastic action, such as $\mathrm{S}$ dioxide $\left(\mathrm{SO}_{2}\right)$, histamine, PG and BK, also affect afferent receptors, therefore inducing reflex bronchoconstriction inhibited by anticholinergics. There could also be an increase in neurotransmitter activity in cholinergic ganglia, either as a result of other neurotransmitter or mediator release, or because postganglion nerve terminals carry out an action favoring acetylcholine release [18]. Given that adrenergic nerves are able to inhibit this production via receptors $\beta$ or $\alpha_{2}$, it is likely that a deficiency in adrenergic responses is reflected in cholinergic tone increase by an increase in muscarinic receptors or others related to them. Asthmatics have an exaggerated bronchospastic response to cholinergic action, but an analogous effect can also be brought about by other spasmogens; therefore an isolated deficit of muscarinic receptors with inhibitor action is unlikely.[21].

The presence in vivo in humans of five muscarinic receptors (only the first three are found in the human lung), each encoded by different genes, has been 
reported. Stimulation of such receptors results in bronchial secretions and in smooth muscle contractions, and correlated $\mathrm{G}$ proteins have also been identified and cloned [318]. Specific studies have clarified the functionally active types [77, 719]:

- $\mathrm{M}_{1}$, located in the parasympathetic ganglia, submucosal glands and in alveolar walls, regulating both vagal tone and mucus secretion. They are inhibited by pirenzepine.

- $\mathrm{M}_{2}$, located on the postganglion nerves in the presynaptic position, with autoreceptor functions, inhibit acetylcholine release and, consequently, the reflex vagal bronchoconstriction actions. They are blocked by gallamine.

- $\mathrm{M}_{3}$, located in the bronchial and bronchiolar smooth muscles in the postsynaptic position and in submucosal glands promote muscular contraction and mucus secretion. They are inhibited by hexahydroxyl-diphenidol. - $\mathrm{M}_{4}$, located in the postganglionic cholinergic nerves, airway smooth muscle and alveolar walls, inhibits acetylcholine release.

- Human skin fibroblasts also express $M_{2}, M_{4}$, and $M_{5}$. In particular, $\mathrm{M}_{1}$, like $\alpha_{1}$ adrenoreceptors, are linked to $\mathrm{Gq}$, and activate phospholipase C (PLC), from which results the turnover of phosphatidylinositol-bisphosphate (PIP2) and $\mathrm{Ca}^{++}$release, whereas $\mathrm{M}_{2}$ are connected to $\mathrm{Gi}$ containing $\mathrm{Gi}_{2}$ to bind to GTP; the stimulation of the receptor inhibits adenylcyclase and modulates the ionic channels for $\mathrm{K}^{+}$and $\mathrm{Ca}^{++}[21]$.

The inhibiting activity is not operative in asthmatics, probably due to a functional deficiency of the $M_{2}$ receptors, which could be expressed by an exaggerated cholinergic activity because of the loss of a normal acetylcholine release retroinhibition, thereby explaining the sometimes dramatic bronchoconstriction action of $\beta$-blockers in asthmatics. In fact, a block of $\beta$-receptors could abrogate the antagonistic actions of cholinergic nerve activation, thus achieving an excessive acetylcholine release, which is not autoregulatory in asthmatics [21]. Therefore, nonselective antagonists of muscarinic receptors, for example, atropine or ipratropium bromide (IB), are able to mediate both bronchodilation $\left(\mathrm{M}_{1}\right.$ and $\mathrm{M}_{3}$ receptors $)$ and bronchoconstriction $\left(\mathrm{M}_{2}\right.$ receptor) [525].

The potential deficits of the parasympathetic cholinergic system affecting BHR are the following:

- Increased cholinergic reflex activity

- Stimulation of the efferent nerve terminals, exposed as a result of inflammation

- Vagal hyperreactivity

- Increased acetylcholine release via ganglionic or postganglionic effects

- Smooth muscle hyperresponsiveness or hypersensitivity to acetylcholine-mediated cholinergic activity

- Possible decrease or dysfunction of protective $\mathrm{M}_{2}$, which turns into exaggerated cholinergic activity

The $\mathrm{M}_{2}$ deficit is confirmed by the simple observation that pilocarpine, their antagonist, blocks $\mathrm{SO}_{2}$-induced bronchoconstriction by limiting the acetylcholine, whereas in asthmatics such a block does not occur. It is significant that influenza virus selectively damages $M_{2}$ receptors rather than $M_{1}$, probably by an effect of neuraminidase action on $\mathrm{M}_{2}$ sialic acid residues, providing a valid mechanism to explain increased BHR in subjects after viral infection [77].

\section{Adrenergic Systems}

The bronchodilator effect of adrenergic stimulation seems to depend on the basal vagal tone. The adrenergic receptors are of the $\alpha$ type, divided into three $\alpha 1$, subdivided in $A, B$ and $C$, four $\alpha 2$ further divided into $A, B, C$ and $D$, and type $\beta$, with $\beta 1, \beta 2$ (present in the lungs with a relationship of about $1: 3$ ) and $\beta 3$ with a more limited diffusion. Also $G$ proteins have been cloned for these re-

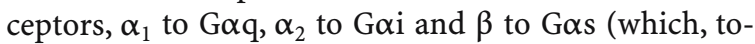
gether with $\mathrm{G}_{\mathrm{i} 2}$ form the four subfamilies of the $\alpha$ subunit) [318]. Under normal conditions, the sympathetic tone (specifically the $\beta$ receptors), represents the main balance unit by antagonizing the vagal tone and diminishing vagus-induced bronchoconstriction. The $\beta$-receptor antagonists have no effect on the cholinergic tone of a healthy subject; on the contrary, they cause bronchospasm in asthmatics with a greater efficacy the more the vagal tone is raised, $\alpha$ stimulation produces mucus secretion and, above all, mast cell mediators are discharged. $\beta 2$ receptors are widely distributed over the bronchial area: they can be found on smooth muscule fibers and in epithelial and glandular cells. In asthmatics, due to a poor reactivity of the adrenergic system, it is likely that the main role in regulating the bronchomotor tone is played by circulating catecholamines. Epinephrine inhibits the bronchoconstriction action of histamine, acting as a true circulating hormone, and could play a protective role with regard to bronchoconstriction agents. In asthmatics and other atopic subjects, a deficit of $\beta$-adrenergic receptors and a parallel $\alpha$-receptor hyperresponsiveness, which could be expressed into blocking the balance of vagal action, has been demonstrated. A supposed genetic deficit of $\beta$-receptors in asth$m a$ has remained an enigma since the beginning [636], even if this anomaly can be hypothesized in a few patients [502]. The postulated interconversion between $\beta$ and $\alpha$ receptors has been negated by studies with monoclonal antibodies on receptor structures [90]. Generally, potential deficits of the adrenergic system can be summarized as follows:

- An increase in $\alpha$ receptors in the airways

- A decrease in $\alpha$-receptor antagonists

- A quantitative and/or qualitative decrease in $\beta$ receptors

- Anomalies in receptor splitting, with $\alpha, M_{1}$ and $M_{3}$ increase causing a reduction in the number of $\beta$ and $M_{2}$ receptors 
Table 11.7. NANC system and potential functions

\begin{tabular}{llll} 
Action & Innervation & Site of action & Neuropeptides \\
Bronchoconstriction & Sensory C-fiber axon & Smooth muscle & NKA/SP \\
& Microganglia & Smooth muscle & SP \\
\hline Bronchodilation & Parasympathetic & Smooth muscle & VIP/PHM \\
& Microganglia & Smooth muscle & VIP \\
\hline Gland secretion & Parasympathetic & Glands & VIP/PHM \\
& Sensory C-fiber axon & Glands, microganglia & SP \\
& Microganglia & Glands & SP+VIP, GRP \\
\hline Vasoconstriction & Sympathetic & Arterioles & NPY \\
& & AV anastomoses & NPY \\
\hline Vasodilation & Parasympathetic & Arterioles & VIP/PHM \\
& & AV anastomoses & VIP/PHM \\
\cline { 2 - 3 } & Sensory C-fiber axon & Arterioles & NKA/SP
\end{tabular}

Modified from [18].

AV Arteriovenous; see Table 11.8 for other abbreviations.

Thus it appears that the postulated $\beta$-receptor deficiency is secondary to asthma, probably as a result of the inflammation. Additionally, if the increased $\alpha$ receptors were of any significance, the $\alpha$ blockers could have a therapeutic effect, which, however, does not happen; consequently these possible anomalies have few clinical implications. Finally, given that treatment with $\beta$-blocking drugs can yield bronchoconstriction in asthmatics (but not in normal subjects), a deficit of this system is excluded [77].

Given the existence of a certain amount of polymorphism related to some $\beta_{1}$ loci, for example at codon 16 (Gly 16), which is more evident in subjects with nocturnal asthma [661], it has been hypothesized that this phenotype predisposes to the nocturnal reduction of the $\beta_{2}$-adrenergic function and therefore to the scant effect of $\beta 2$-adrenergics on the clinical symptoms. It has also been suggested that substituting glutamic acid with Glu 27 (glutamine at codon 27), which has a receptor with reduced suppression, is associated with a bronchoconstriction reduction [234]. In conclusion, G protein studies are justified by a possibly better understanding of the action mechanism of related drugs.

\section{NANC and Neuropeptides}

The functions of NANC, composed of naked nerve fibers that are found free in the submucosa, are summed up in Table 11.7 [18]. NANC was originally described in the gastrointestinal tract. Given that the respiratory system also derives from the cephalic portion of the archenteron, it is logical that NANC and related peptides can be found in both systems; they are, however, present in all organs and can be produced by cells other than CNS cells [21]. It has been suggested that anomalies of the NANC system, in its two components (e-NANC and i-NANC), represent another important component in asthma pathogenesis. Numerous stimuli release neuropeptides $[18,90]$ or tachykinins in the respiratory tract, neurotransmitters that send signals not only to nerve cells, but also to other cells or systems. So-called neurogenic inflammation is based on the contribution of numerous neuropeptides to the pathogenesis of a major part of the anatomopathological lesions thus far reported, thereby determining a series of biological responses during the recurrences of acute asthmatic attacks such as vasodilation, plasma extravasation into postcapillary venules, an increase in vascular permeability, exudation of capillaries in the bronchial lumen and edema development, contraction of bronchial smooth muscle cells, mucus hypersecretion, coughing, activation of inflammatory cells and their adhesion to endothelial cells [426] (Fig. 11.33).

\section{Bronchoconstrictors (e-NANC System)}

As seen in Table 11.8 [18], several tachykinins released by sensitive nerve endings belong to e-NANC: SP, NKA, NKB (neurokinin A and B), CPS (capsaicin), NPY (neuropeptide $\mathrm{Y}$ ) and CGRP (calcitonin gene-related peptide) [21].

$S P$ is released by afferent nerves to sensitive amyelinated terminations known as C-fibers. SP binding sites are diffused throughout the smooth muscles of the whole tracheobronchial tree. Injected intravenously, in humans SP provokes an evident vasodilation, probably 


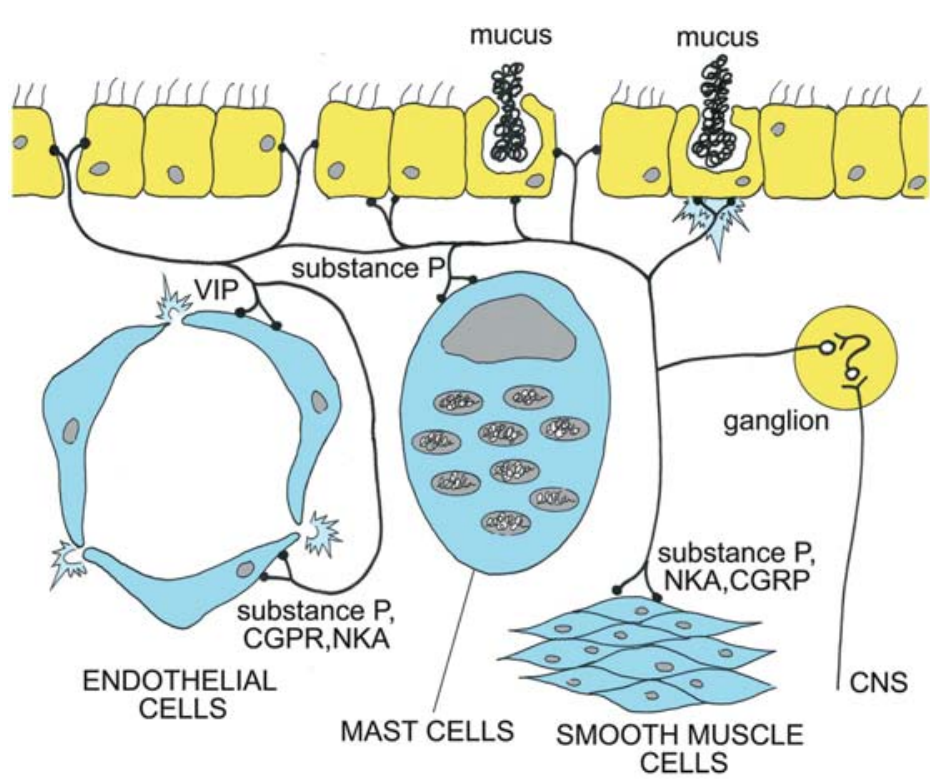

Fig. 11.33. Neurogenic inflammation (left) and VIP localization to the airway nerves and adjacent blood vessel. CGRP calcitonin gene-related peptide, NKA neurokinin A, CNS central nervous system, VIP vasoactive intestinal peptide
Table 11.8. Neuropeptides in human airway mucosa

\begin{tabular}{|c|c|}
\hline \multicolumn{2}{|c|}{$\begin{array}{l}\text { Trigeminal and dorsal root ganglion } \\
\text { cell sensory-motor neurons }\end{array}$} \\
\hline CGRP: & calcitonin gene-related peptide \\
\hline GRP: & gastrin-releasing peptide \\
\hline \multicolumn{2}{|c|}{ Tachykinins } \\
\hline NKA: & neurokinin $\mathrm{A}$ \\
\hline NKB: & neurokinin B \\
\hline SP: & substance $P$ \\
\hline \multicolumn{2}{|c|}{ Postganglionic parasympathetic neurons } \\
\hline ACh: & acetylcholine \\
\hline PHI: & peptide histidine-isoleucine \\
\hline PHM: & peptide histidine-methionine \\
\hline PHV: & peptide histidine-valine \\
\hline VIP: & vasoactive intestinal peptide \\
\hline \multicolumn{2}{|c|}{ Postganglionic sympathetic neurons } \\
\hline NE: & norepinephrine \\
\hline NPY: & neuropeptide Y (tyrosine) \\
\hline \multicolumn{2}{|c|}{ Neurons of undetermined origin } \\
\hline ANP: & atrial natriuretic peptide \\
\hline CALC: & calcitonin \\
\hline DYN: & dynorphin \\
\hline ET-1: & endothelin-1 \\
\hline ET-2: & endothelin-2 \\
\hline ET-3: & endothelin-3 \\
\hline ET-4: & endothelin-4 \\
\hline ENK: & enkephalin \\
\hline GAL: & galanin \\
\hline NT: & neurotensin \\
\hline SOM: & somatostatin \\
\hline
\end{tabular}

Data from $[18,76]$. because of the antidromic activation of local axon reflexes that affect the sensitive C-fibers and also other tachykinins, while PFT alterations result from smooth muscle constriction. SP could, therefore, be the e-NANC neurotransmitter [18]. The presumed mechanism of axon reflexes is that, in response to harmful stimuli in the airway lumen, nerve fibers in the epithelium layer release SP, while other fibers of the same axon, by supplying different targets, release SP by antidromic activation. In this manner, stimulation of epithelial nerve fibers can provoke bronchoconstriction and extravasation of plasma proteins with an axon reflex by SP release near airway smooth muscles and postcapillary bronchial veins [333]. In particular, SP bronchoconstriction activity, at least in vitro, is fulfilled antidromically when reflex cholinergic bronchoconstriction is present, as a result of exposed afferent nerve endings situated under desquamated epithelium, especially those of C-fibers [426]. As well as affecting the muscle, SP also affects bronchial mucus production, stimulating both the submucosal glands to an increased secretion, as well as the myofibrils surrounding glandular ducts to increase their secretion. Furthermore, SP increases mucociliary clearance in the airways [362]. Other evidence suggests that SP induces aspecific degranulation of cutaneous and peritoneal mast cells, but not those of the airways, and causes T-lymphocyte proliferation, neutrophil and alveolar macrophage phagocytosis [90], and enhances neutrophil [88] and eosinophil chemotaxis [90]. Thus, we understand how BHR to SP in asthmatic children is correlated with asthma severity [429].

NKA-induced bronchoconstriction, which in the airways travels in the same fibers containing SP, is dosedependent [126] and stronger than that provoked by SP, which, in turn, is more powerful as a vasodilator and vasopermeabilizer. Therefore, in the present state of our knowledge, NKA and SP exercise a bronchoconstriction 
action (e-NANC) that can clash with i-NANC, which has a bronchodilator effect, as well as with EDRF, with an effect on bronchial smooth muscle equal to NO [10]. Epithelial denudation can involve the loss of these factors, thereby increasing reflex bronchoconstriction - an effect also mediated by tachykinins contained in microganglia [90].

CPS stimulates SP release from sensory terminals and, to the same degree as SP, provokes bronchoconstriction and an increase in vascular permeability. Furthermore, it suppresses not only the inflammatory effects of airway vagal stimulation but also the effects provoked by tobacco smoke and chemical irritants [90].

CGRP is a peptide codified by the same gene as the thyroid C-cells which, in turn, codifies calcitonin. It is located in the sensory nerves where SP is also present. It performs a notable vasodilator action on smooth muscle, strong and persistent, associated with eosinophil infiltration, potently contracting in vitro human airway smooth muscle [96], acting in synergy with immune inflammation mediators, including PAF, BKs and $\mathrm{LTB}_{4}$. It has been credited with a role in blood flow regulation in tracheobronchial small vessels [18] and in APC inhibition [525].

NPY has no direct effect on tracheobronchial smooth muscle contraction, while it stimulates bronchial gland secretion and is a long-acting constrictor of vascular smooth muscles.

The effects of tachykinins are mediated by specific receptors in such a way that each of them activates, by preference, a distinctly separate receptor: NK-1 are activated by SP, and NK-2 by NKA [90].

Possible deficits of the e-NANC system comprise the following mechanisms:

- Enhanced noncholinergic excitatory activity

- Diminished degradation of tachykinins

- Stimulation of the afferent nerve endings in which travel neuropeptides, exposed as a result of inflammation

- Smooth muscle hyperresponsiveness or hypersensitivity to neuropeptide bronchoconstriction activity [96]

\section{Bronchodilators (i-NANC System)}

Human PHM (peptide histidine-methionine), its homologous PHI (peptide histidine-isoleucine), the equivalent in many mammals and VIP, have the same localizations and analogous functions (they are codified by the VIP gene in the same pro-hormone and they have in common $>50 \%$ amino acid structure) [90]. They have a bronchodilator activity, more pronounced with VIP, with an effect 50 times greater than isoproterenol [21]. i-NANC works via parasympathetic nerves containing VIP and/or PHM, these being the only neuropeptides with a bronchodilator action.

VIP, most probably an i-NANC neurotransmitter, is found in parasympathetic ganglia (efferent ways), localized in the cholinergic motor nerve endings in smooth muscle bundles, submucosal glands and bronchial blood vessels. In test animals, VIP has been shown to modulate histamine-induced tracheal smooth muscle contractions, kallikrein, $\mathrm{PGF}_{2 \mathrm{a}}$, $\mathrm{LTB}_{4}$ and NKA, an effect not inhibited by adrenergic or cholinergic receptor activation nor by cyclooxygenase blocking activity [90]. Also characteristic of VIP is an abundant distribution in both upper airways and nasal mucosa, but not in bronchioles, which is why its bronchodilator action is greater in regulating the caliber of large airways, and is practically wholly ineffective in small airways. In consequence, the therapeutic effect in the asthmatic patient is deceptive, either because specific VIP receptors are scarce in this location, as has been said, or because of the effect of peptidases (for example tryptase) capable of degrading VIP which are released by inflammatory cells present in asthmatic airways [333]. It is characteristic that tryptase degrades VIP with bronchodilator effect, but not so SP, thus promoting bronchial reactivity, causing in prospective BHR and bronchospasm [96]. The prevalent conclusion is therefore that i-NANC exercises a modulator activity of cholinergic effect rather than a direct bronchodilator action [96].

Potential alterations of the i-NANC system foresee the following mechanisms:

- Shortage of nonadrenergic neurons with inhibitor activity

- Increased degradation of VIP/PHM

- Reversible blockage of nerve ganglia or nerve endings with nonadrenergic inhibitor activity

- Numerical reduction of VIP/PHM receptors [96]

What, then, is the role of neuropeptides? In a healthy individual at rest, the adrenergic, cholinergic and NANC effects are balanced for a perfect bronchopulmonary homeostasis and there is a balance between the bronchodilator mechanisms ( $\beta$-adrenergic system + VIP/ PHM) and bronchoconstrictors ( $\alpha$-adrenergic/cholinergic + SP/NK/CGRP) [90]. As shown in Table 11.7, NANC displays opposing functions: broncho- and vasoconstriction, and broncho- and vasodilation. Confronting the neuropeptide multiform actions, the host generates numerous defense mechanisms. If, on the one hand, VIP reduction/inactivation could contribute to asthma pathogenesis, on the other, the neutral endopeptidase 24.11 (NEP, CD10) is capable of inactivating neuropeptides in bronchial epithelial cells. The contrasting effects of NKA and SP on the one hand and PHM and VIP on the other have already been mentioned. There could be present anomalies of the intrinsic microganglia, the efferent parasympathetic fibers containing VIP, and the VIP receptor system [18]. On the other hand, the effects of bronchoconstriction NANC hyperactivity could, in some patients, play a negative role, such as a hypersensitivity of sensitive nerves and of axon reflexes, amplifying inflammatory responses by releasing factors stimulating such sensitive endings, or an increased smooth muscle sensitivity to bronchoconstriction tachykinins [426]. Its sensitivity to $\mathrm{O}_{3}$ or to 
lymphocytes macrophages metachromatic

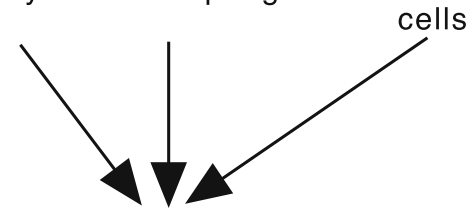

cytokines

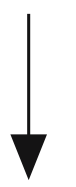

leukocyte priming

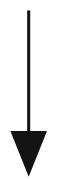

migration into

airway mucosa

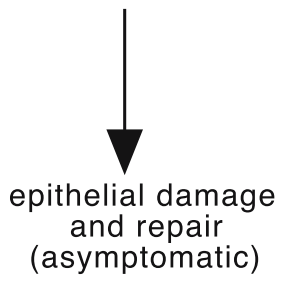

CNS

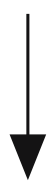

neuropeptides

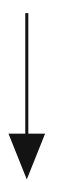

hyperreactive smooth muscle

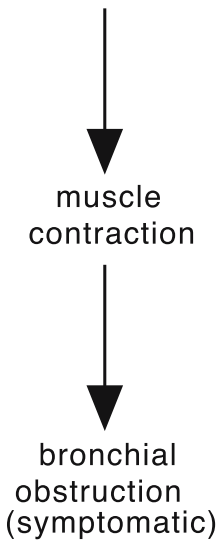

Fig. 11.34. Pathological processes linking inflammatory events to airway hyperreactivity without a causal interrelationship. The autocoids provide the second signal to start both processes, either separately or more frequently conjointly, so that their signal becomes effective. (Modified from [418]) cigarette smoke $[315,337]$ has recently been defined. Furthermore, the afferent sensitive fibers, even terminating normally in the brain, can branch out and, by antidromic stimulation, be induced to release tachykinins and other mediators in peripheral areas via an axon reflex [426]. The final result therefore is that in the asthmatic, unlike in a healthy individual, the balance tends toward bronchoconstriction effects [90]. Neuropeptide action can be linked to that of ILs, with a point of unification represented by the joint action of autacoids, substances physiologically active such as histamine, serotonin, PG, etc. Thus, on the one hand ILs contribute to asymptomatic epithelial damage, while, on the other, neurotransmitters contribute to bronchoconstriction (Fig. 11.34) [418].

\section{Other ANS Dysfunctions}

Another factor can be represented by dysfunctions in the stimuli-response relationship. As is known, Szentivanyi [636] advanced the theory that a $\beta$-receptor anomaly was at the basis of BHR and other related disturbances. However, there is an interesting paradox worth mentioning: $\beta$-adrenergic receptors are quantitatively normal, but cell response to $\beta$-adrenergic stimulation is reduced, as can be noted in atopic patients who have not received treatment. Further, administering $\beta$-adrenergic drugs causes a rapid receptor desensitization [318]: what conclusion can finally be deduced from this? $\beta$-adrenergic receptors are made up of peptides from the cellular membrane which bind either to epinephrine or to norepinephrine. The link to one of these hormones activates Gs - a G-protein - which in turn stimulates adenylyl cyclase to produce cyclic adenosine monophosphate (cAMP), while the Gi inhibit this process. A chain of Gs is deactivated by pertussis toxin. This activity was studied in the murine model in which it reproduces both the immunological anomalies of atopy and hyperreactivity in diverse organs [516]. An experimental model of this type has made possible the definitive demonstration that G-protein deficit is at the basis of signal transduction anomalies [318], or of stimulus-response relationship common to both $A D$ and asthma.

\section{Anomalies of Bronchial Smooth Muscles}

Hyperplasia and hypertrophy of bronchial smooth muscles have been observed, though insufficiently to provide an explanation of BHR pathogenesis, since it is improbable that they are already present in asthmatic children. The reduction of bronchial caliber, especially of bronchioles, which are unique in being surrounded by smooth muscle [255], caused by the joint action of 
mucosal edema, smooth muscle constriction and intraluminal secretion increase, cannot, in itself, constitute the primum movens of the pathology under examination [722]. Mast cells and eosinophils are both able to contribute in a more significant manner - by means of tryptase action on smooth muscle tone [154]. In the asthmatic, smooth muscles could evolve under the effect of certain stimuli from a multiple-unit to a single-unit system. These changes in the contractile properties of smooth muscle in human lungs may be associated with changes in myosin $\mathrm{H}$ chain isoforms, thus contributing to BHR [138].

It has also been suggested that in asthmatics there is a reduction of an epithelium-derived inhibitory factor (EDIF) of the muscle contractility, or of NEP, a factor with a relaxing effect on smooth muscle [675], where it is located. In any event, the result is the loss of the barrier mechanism and therefore of bronchoconstrictor control. A noteworthy impulse has also been given to cAMP study, which that is entrusted with muscle relaxant effects and inhibits mast cell degranulation and consequently histamine discharge, unlike cGMP (cyclic guanosine monophosphate). Normally there is a balance between CAMP and CGMP, which can be broken in either one direction or the other. Maintaining the CAMP level within normal limits is useful to bring adrenergic receptors back to a functional stage in which they can again be activated. Recent research shows that cAMP production induced by adrenergic $\beta$ receptors is not deficient in the PBMCs of asthmatics compared to healthy subjects [155], as was suggested previously. However, PBMCs of patients even with stable asthma do not have the ability to make adenylyl cyclase more powerful [155], so the lack in increase of cAMP concentrations is correlated with a transduction defect. There are two possible pathogenic mechanisms: the existence of a generalized deficit involving bronchial smooth muscles, no longer relaxed under the effect of cAMP metabolism, and therefore the impossibility of potentiating CAMP when facing a bronchoconstriction stimulus, which could result in an equilibrium upset between cAMP and cGMP; or, alternatively, the adenylyl cyclase deficit could reflect negatively on IL production by PBMCs, the tissue inflammation being at the basis of an increased BHR [155]. In vitro, in biopsy specimens of airway smooth muscle of asthmat sufferers, there is a correlation between muscle functions and BHR level, indicating that $\mathrm{BHR}$, which is observed in the presence of a great variety of stimuli, is a demonstration of the noteworthy contribution given to bronchoconstriction by the bronchial smooth muscle spasm. The differences between asthmatics and nonasthmatics have not yet been fully elucidated, nor have the variations induced by different types of stimuli. It is believed that there are other exogenous factors at the basis of this anomaly. Probably, the airway infiltration of inflammatory cells contributes to smooth muscle tone by the action, on a local level, of various mediators such as histamine, PAF and eicosanoids [138].

\section{Anomalies in Airway Epithelium}

The effect on the mucosal epithelium by toxic products of eosinophil origin has been analyzed, together with changes that range from loss of ciliated cells to complete epithelial denudation:

- As previously said, the epithelial barrier permeability is increased by histamine, LTs, BKs, CPS, SP, TNF- $\alpha$, reactive oxygen metabolites, viruses, etc. Consequently, access to the mucosa and submucosa by inhaled molecules such as allergens, microorganisms, dusts, environmental pollutants and inflammatory mediators is made easier. Among those last named, superoxide anion has a particularly harmful effect on the airways with its highly destructive radicals [726]. The epithelial damage exposes intraepithelial mast cells, afferent nerve endings and, in particular, irritating vagal receptors to the combined action of various stimuli. These lie in the upper and lower tract mucosa immediately below the epithelial tight junctions [722], which, though physiologically impermeable, allow a greater absorption of allergenic and irritating substances to take place if altered. In addition, the mucociliary clearance reduces its filtering action of inflammatory substances and airway secretions. - Experimental studies have highlighted the role played by epithelial denudation, which could lead to NEP loss, usually found not only on smooth muscle, but also on target cell membranes such as epithelial, endothelial and alveolar type II cells, submucosal glands, postcapillary venules and nerves, all of which are sites of tachykinin activity [126]. NEP selectivity makes it possible to degrade SP and NKA, blocking their bronchoconstriction activity while the inhibition, especially if associated with $\mathrm{O}_{3}$-induced $\mathrm{BHR}$ [406], potentiates NKA [126] and SP effects [421]. Exfoliation of epithelial cells and other serious cytopathic effects - caused by viruses harmful to cells $[20,72,130]$, chemical substances, pollutants, etc. - points first to a NEP deficiency, thereby preventing both the destruction of bronchoconstriction neuropeptides as well as the synthesis of those with bronchodilator action, also amplifying the inflammatory action of tachykinins and reflex bronchoconstriction [18]. If, as a result of epithelial damage, the sensitive nerve endings of bronchial mucosa remain exposed to the bronchial lumen, having a much lower threshold of stimulation, they are stimulated continually by inflammatory metabolic products [21]. In this way, as a result of antidromic stimulation of these nerve endings, an exaggerated neuropeptide release with a highly rapid and diffuse action on bronchial airway occurs [21]. BK could play a part in allergic asthma since, administered either inhaled or IV, it provokes bronchospasm in asthmatics, but not in healthy subjects. It works indirectly, probably via the stimulation of sensitive nerve terminals, with consequent retrograde release of SP no longer degraded by NEP, NKs and CGRP by an axon reflex $[421,429]$. BK antagonist therapy is based on these premises [18]. Following these phenomena of 
neurogenic inflammation affecting target cells, cholinergic reflex bronchoconstriction, hyperemia, edema and mucus hypersecretion, with an increase in vasal permeability will follow. This axon reflex, amplifying the immune inflammation, could therefore represent an important pathogenic element in provoking asthma and BHR [21].

- Moreover, there is a receptor loss: if they belong to the $\beta$ type, there are no disturbances of note; if they are $\mathrm{H}_{2}$, production of $\mathrm{PGE}_{2}$, which acts by inhibiting, at least in part, the smooth muscle responses to histamine and NKA ceases. The receptor loss by numerous epithelial ILs involves mainly $\mathrm{IL}_{1}$, with a possible amplification of the inflammatory response and consequential jeopardizing of lesion repair [22].

- Other studies have shown the loss of NO production, catalyzed by iNOS (inducible NO synthase) and induced into the epithelium by TNF- $\alpha, \mathrm{IL}_{1 \beta}$ and IFN- $\gamma$, also compromising both vascular muscle regulation and harmful chemical substance clearance [10].

- Another pathogenic hypothesis cites the inhalation of endotoxins present in Gram-negative germs, identified with the somatic antigen, which are located in the cell walls as LPS as a possible culprit. Such toxins, brought into homes by environmental germs, could provoke bronchoconstriction in asthmatics in doses of 20-40 $\mu$ g, and in healthy subjects as well in doses of $200 \mu \mathrm{g}$ [433].

- BHR persistence, also produced by MBP-induced discharge of factors of epithelial origin stimulating muscarinic reactivity [57], could be explained by the thickening of the lamina reticularis below the BM [600]. A crucial point is played by myofibroblasts, capable of producing subepithelial fibrosis, whereas in asthmatics an excess in their numbers below the bronchial epithelium, with a close correlation between the number of myofibroblasts and the collagen layer thickness, has been reported. It is therefore evident that fibrosis jeopardizes the respiratory function and that a network of contractile myofibroblasts underneath the BM markedly contributes to BHR persistence. Moreover, activated eosinophils present in these locations, as well as deposits of ECP, stimulate the synthesis of hyaluronate and proteoglycans in human fibroblasts [600].

\section{Alterations of Biochemical Homeostasis}

The main cause of asthma inflammation, which, apart from smooth muscls spasms, also depends on mucosal edema and on secretion changes, with formation of mucus plugs in the smaller bronchi.

\section{Bronchial Edema}

Bronchial edema is attributable to the increase in capillary permeability with exocytosis of serum proteins in interstitial areas with an array of mediators among which are histamine, BKs, PAF, PGE and LTs, potentiating each other in turn. For example, PAF-produced edema resulting from the harmful action exercised on microvasculature is increased by the coincident action of $\mathrm{LTC}_{4}$ [283]. There are several other edema factors such as cigarette smoke, viral infection, pollutants, neuropeptides and proteases, all capable of altering epithelial integrity and mucosal permeability: thus allergens may penetrate across the junctions that are no longer tight, for their encounter with APCs [619]. Even if the pathophysiological contribution of this process is little known, it has been seen that following an airway effect of local BPT, an extensive edema is formed and that both edema and inflammation increase the thickness of airway walls, thus contributing to bronchospasm in children [619]. Therefore, pharmacological prevention of the increase in vascular permeability requires the inhibition of histamine receptors and of arachidonic acid metabolism through cyclooxygenase and lipoxygenase, with the aim of simultaneously blocking PGs and LTs, respectively. The role of bronchial edema has been the object of few studies and merits a closer examination [283].

\section{Mucus Hypersecretion with Formation of Plugs in the Smaller Bronchi}

The formation of tenacious mucus plugs in the airways is one of the characteristics of asthma [255]. Mucus hypersecretion, a possible consequence of hyperplasia and metaplasia of submucosal glands and goblet cells that cover the respiratory tree, contributes to an exaggerated insufflation and focal atelectasis. The pathogenesis is complex: various mediators and cells of inflammation are able to contribute - along with C-fiber activation also stimulated by BK and inhalants - leading to mucusproducing cell quantitative increase and exocytosis, as well as to myoepithelial cell contraction [362]. The mediators (Tables $11.4,11.5$ ) that are principally responsible are, in order: $\mathrm{LTD}_{4}>\mathrm{LTC}_{4}$ (active in picomolar concentrations) $>$ HETE (nanomolar) $>\mathrm{PGF}_{2 \mathrm{a}}=\mathrm{PGD}_{2}=$ $\mathrm{PGI}_{2}=\mathrm{PGE}_{1}=\mathrm{PGA}_{2}>$ histamine $\mathrm{H}_{2}$ ( $\mu$ molar) [283]. Mucus hypersecretion may be produced by $\mathrm{IL}_{13}$ even in the absence of inflammation [321].

\section{Increase in Permeability}

We summarize the numerous mechanisms that lead to vasodilation and hyperpermeability evidenced so far, with consequent bronchoconstriction amplification. Plasma proteins release from tracheobronchial microvasculature and the forcible fluid passage deriving from it are important inflammation components and, consequently, a basic characteristic of pathogenesis [177]. They occur gradually within 10-20 min after an allergenic BPT in the airways: the stronger the stimulus, the greater the exudation [490] (Fig. 11.32). The continuous 
vascular extravasation can give rise to the production of mediators of inflammation facilitating the intraluminal transmigration of inflammatory proteins. Since the absorption of solutes is directed above all by mediators, the mechanisms that reduce or abolish their activity cause the regression of protein accumulation in the lumen, an effect which can be achieved following treatment with CSs in moderate doses (400 $\mu \mathrm{g} /$ day) [669]. Nonetheless, measurements in controlled asthma have shown little or no increase in airway permeability and no correlation with BHR degree [722].

\section{Surfactant Dysfunctions}

The loss of pulmonary tissue elastic properties can cause bronchoconstriction. Testing immunized animals to BPT with ovalbumin, the resulting protein transudation in the airways inhibits the surfactant, with a consequent Raw increase [356].

\section{Effects of NO}

Until 10 years ago, $\mathrm{NO}$ was known only as one of the components of the harmful gases discharged by vehicles and cigarette smoke, also held to be jointly responsible for acid rain and the $\mathrm{O}_{3}$ hole. Recently $\mathrm{NO}$ equal to EDRF has been localized at the airway epithelium, where it could act as a mediator of bronchodilation under the nervous control to counterbalance the bronchoconstriction caused by mast cell degranulation (or by ET). Moreover, NO, produced in large quantities, provokes an increase in vascular permeability and cytotoxic effects, contributing to epithelial denudation [10]. In this cytotoxic activity, it also mediates $\mathrm{O}_{3}$ effects - a significant fact given the increased incidence/prevalence of asthma [759]. From a pathogenetic point of view, NO deriving from airway epithelium (as well as from macrophages, mast cells and Th1 cells) could play an important role in amplifying and perpetuating the Th2-mediated inflammation (Chap. 4). iNOS can be induced in the epithelium by pro-inflammatory ILs such as TNF- $\alpha$ and $\mathrm{IL}_{1 \beta}$, secreted by macrophages, and IFN- $\gamma$ derived from Th1 cells [759]. It is also feasible that even viral infections induce iNOS production by the epithelium, thus increasing NO secretion during asthmatic attacks [443]. Elevated concentrations of NO thus generated in the airways carry out a suppressive action on Th1 cells and a reductive action on IFN- $\gamma$, resulting in a net proliferation of Th2 cells. CSs inhibit iNOS both directly and by blocking pertinent IL synthesis by macrophages [443]: in fact, in asthmatics treated with oral or inhaled CS (ICS), NO levels are reduced, in contrast to untreated patients [293]. Exhaled NO levels are increased in atopic nonasthmatic children and, all the more, in atopic asthmatic children, but were reduced by $53 \%$ by ICS treatment [587]. Therefore, ICSs inhibit NO production by epithe- lial cells, stimulating the proliferation of Th1 at the expense of Th2 cells [443]. Further improvements in techniques will permit monitoring inflammatory events, by measuring exhaled NO (eNO) levels and providing a noninvasive marker for the early diagnosis (Chap. 6) and a more precisely aimed treatment of pediatric asthma [759].

\section{Main Exogenous Factors [333]}

- Pharmacological stimuli, histamine, methacholine, acetylcholine, hypotonic aerosols [154], all medications may provoke adverse responses (see "Treatment"). In a cross-sectional study on 1,881 children aged 6-7 years, the use of antibiotics during the 1st year of life was significantly associated with wheezing. This increased risk was a prerogative of children genetically predisposed to atopic immune responses [156].

- Physical stimuli: fresh and dry air [206], ultrasonic mist, smog, inert dust, atmospheric pollutants, physical exercise [606].

- Chemical and pollutant stimuli: oxidant, harmful and irritating gases, tobacco smoke [167, 195, 621] (Table 2.26)

- Allergens, especially aeroallergens

- Viral infections [167]

\section{Predisposing Factors}

\section{Anatomical and Physiological Predisposing Factors}

Anatomical and physiological factors, specific to the pediatric age, can predispose to the airway obstruction, as can extrinsic factors more readily predisposing, some of which are even etiological factors.

\section{Strictly Anatomical Dynamic Factors}

Anatomical dynamic factors are represented by an evident reduction, more or less to scale, according to age, of anatomical parameters.

The following [174, 204, 274, 589, 648] show that the child's airways are not to be viewed as a miniature replica of the adult's airways:

- At birth, the lungs weigh $\approx 48 \mathrm{~g}$; at 1 year $130 \mathrm{~g}$; at 12 years $390 \mathrm{~g}$; in the adult $\approx 1,200 \mathrm{~g}$.

- The estimated gas-exchange area at birth is almost 27-fold lower than that of an adult: $2.8 \mathrm{~m}^{2}, 32 \mathrm{~m}^{2}$ at 8 years and $75 \mathrm{~m}^{2}$ in the adult.

- The total pulmonary capacity of a newborn child is $180 \mathrm{ml}$; at the age of 16 it is $5,100 \mathrm{ml}$.

- Bronchi and bronchioles have reduced caliber and length. The diameter increases by $200 \%-300 \%$ from birth to adulthood. 
- The alveoli are reduced in number. The number of alveolar structures increases exponentially from 3032 weeks of intrauterine life until birth, the newborn has only $8 \%-11 \%$ of the alveoli of an adult and with a less differentiated structure, and by the age of 8 the alveoli increase tenfold, and proceeding to adulthood, the airways triple in diameter.

- The cartilaginous tissue, the submucosal glands and the smooth muscle of lower airways complete their development at 8 months; the smooth muscle of proximal airways in adulthood.

- Formed cilia and mucus are found in the airways at 13 weeks of life.

- The thorax is extraordinarily flexible in infancy and it stiffens with age.

Within this anatomical framework, the neonatal epithelium develops structurally and provides important functions in the normal airway development.

\section{Anatomical and Physiological Factors Predisposing Children to Airway Obstruction [584]}

The younger a child, the more evident the anatomical details facilitating the onset of bronchospasm, therefore provoked by trivial causes such as a mucosal edema and/or a catarrhal secretion. The relevant infantile characteristic of the respiratory mechanism in this context is the resistance to air flow. A reduction in bronchioles and/or bronchi caliber translates into a hindrance of air flow so that, in young babies and children, an obstruction, even a limited one, requires great exhaling pressure during exhalation, from which derives a dynamic compression of the peripheral tracts which worsens the obstruction itself. Among the principle causes of this, some of which have been better defined by new methods of investigation (Chap. 6), are the following.

\section{Reduction of the Caliber of the Peripheral Airways}

The relationship between the caliber and upper and lower airways is physiologically reduced as compared to that observed in adults. This condition exposes an infant, often male, to severe bronchoconstriction because the edema, the secretions and the cellular debris cause stenosis more easily in bronchioles of a smaller diameter. We must also consider that Raw varies inversely with the fourth power of the radius of bronchioles. Therefore, it is sufficient that the radius is halved, for example caused by a viral infection, for the Raw to be increased 16 -fold. An increase in caliber occurs only towards 5-6 years of age.

\section{Decreased Lung Elastic Recoil}

The infant chest wall is less rigid, so that the thoracic wall and airways have a greater propensity to deformability. The decreased static elastic recoil properties predispose to an early airway closure even during tidal breathing. On the other hand, greater elasticity means that the airways of babies are more easily susceptible to vibratory movements, which are at the basis of wheezing. The cartilaginous substances of the trachea and segmental bronchi also are less rigid, so that airway collapse is facilitated during expiration. The early airway closure at elevated pulmonary volumes determines an alteration of the ventilation/perfusion rate, potentially complicated by hypoxemia.

\section{Decreased Smooth Muscle in Peripheral Airways}

Decreased smooth muscle in peripheral airways facilitates obstruction especially following an edema, mucous hypersecretion and infiltrations by inflammatory cells. Infants and children are therefore more vulnerable to small airway pathologies. Smooth muscle reduction compared to that of an adult also contributes in many cases to a poorer response to bronchodilators (the younger the baby, the more this is the case).

\section{Mucous Gland Hyperplasia}

Compared to an adult, an increased percentage of mucosal glands in walls of the larger bronchi contributes to intraluminal mucous secretions and in turn to airway obstruction.

\section{Diaphragmatic Disadvantage}

Another anatomical disadvantage from a mechanical point of view is the position of the diaphragm since the angle of insertion on the ribs is horizontal, in contrast to an oblique insertion in adults, which, combined with the compliance of the bronchial wall devoid of cartilaginous support and that of the thorax with low skeletal or muscular support, cause retraction of the rib cage during inhalation, therefore requiring greater effort, whereas in adults the diaphragm tends to elevate the rib cage, thus increasing its diameter.

\section{Functional Insufficiency of the Diaphragm}

Functional insufficiency of the diaphragm occurs because the increased effort cannot be sustained efficiently, caused by relative reduction of muscle fibers. The diaphragm must ensure $>70 \%$ of respiratory excursions, given that a child up to the age of $6-7$ years con- 
tributes only in part to thoracic cage expansion, either because of increased compliance (see "Decreased Lung Elastic Recoil"), or because the movements of lifting and lowering the rib cage are conditioned by their almost horizontal position, and by the almost cylindrical form of the thorax, so that diaphragmatic contraction during inhalation can cause subcostal retraction rather than rib elevation.

\section{Decreased Alveolar Pores and Decreased Collateral Ventilation}

The alveolar openings that permit ventilation between alveoli (pores of Khon) and the bronchoalveolar communications (canals of Lambert) are decreased in number and size in the infant lung, thus impeding a normal collateral ventilation. For this reason, young babies and young children are more easily susceptible to atelectasis distal to obstructed airways.

\section{Role of Pulmonary Volume}

The reduced caliber of peripheral airways can be a risk factor for relapsing wheezing. The functional residual capacity (FRC), airway conductance (Gaw) and $V_{\max }$ were found to be significantly lower in young babies suffering from bronchiolitis before the age of 1 year and related relapses [375].

In conclusion, the fact that the airways of the very young face obstructions more easily and more rapidly than older children is not surprising. Therefore the lung is more vulnerable to inflammation in this early age and this may result in persistent airflow limitation [274]. We have so far outlined some of the principal causes for which the particular predisposition needed to develop not only reversible wheezing in the very first period of life, but also bronchoconstriction and respiratory insufficiency, which is at the basis of the high number of hospital admissions and assisted breathing that is recorded in this age group.

\section{Predisposing or Etiological Factors}

Several factors can modulate the onset and severity of asthmatic clinical symptoms in a child [174]. With advancements in research, knowledge regarding the way in which these factors provoke alterations at the airway has been perfected, even if still far from achieving a univocal pathogenetic mechanism. It is certain, however, that asthmatic children display BHR, an abnormal marked sensitivity to most varied stimuli, which in healthy subjects usually do not induce responses of any particular clinical relevance. In asthmatic children, these and other stimuli can provoke smooth muscle spasms of the lower airways, bronchial edema, dense mucous secretions and respiratory dynamic alterations as well [307].

\section{Aeroallergens}

Sensitization to inhalants is rare in very early infancy, but clearly prevalent from 3 years onward (Fig. 9.39), with high successive sensitization to pollens and to Der p 1 (Fig. 5.22), and an unusual and elevated prevalence of positive SPTs to cat derivatives in children $>9$ years (Fig. 7.17) and of sIgE in 17-month-old infants (Fig. 5.21). Multiple sensitization to inhalants (Fig. 5.20, Tables 5.18, 5.19) may be very prevalent among children suffering from multiple FA (Chap. 9).

\section{Other Atopic Disease}

Of children affected with $\mathrm{AD}, 44 \%-53 \%$ are at risk of developing other atopic disease, namely RA or asthma (Table 5.8 ), and $47 \%$ of these children have asthma with or without BHR (Table 7.10). Additionally, some foods can be responsible for asthma in allergic children, more frequently than in adults (Tables 9.18, 9.19). M cells transport allergens to subepithelium where they facilitate allergen access to lymphoid tissues, then interacting with allergen-specific $\mathrm{T}$ cells or with $\mathrm{B}_{\mathrm{IgE}}$ lymphocytes, finally reaching the airways via blood vessels and causing BHR and asthma [537] even in children $<2$ years of age (Fig. 11.35) [450]. In our division, it is not infrequent that children react positively to food provocation testing manifesting wheezing.

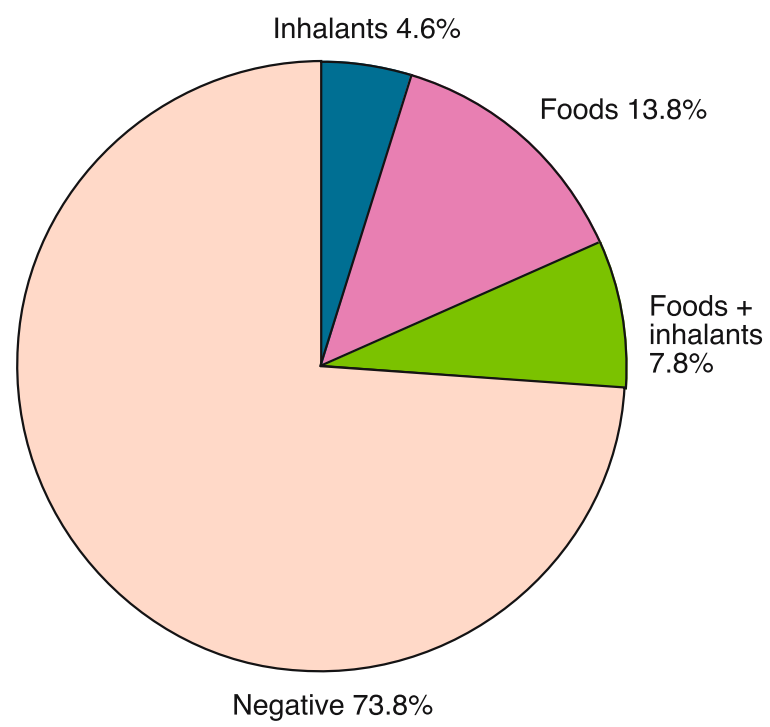

Fig. 11.35. PTC results in $65<2$-years old asthmatic children. (Data from [450]) 


\section{Physical Exercise}

Aretaeus reported cases of exercise-induced asthma (EIA) at the first Olympic games recorded in history [13]. Twenty-four centuries later, approximately 3\%$10 \%$ of athletes taking part in international competitions are affected by asthma. Physical activity regularly leads to a decline in PFT in children and adolescents with asthma. This decline is a consequence of what is known as EIA, a theme of much research in recent years [542]. EIA is found in up to $63 \%$ of asthmatic children (Table 5.11 ), in $30 \%-40 \%$ with RA $[307,584]$, in nearly $3 \%-8 \%$ of schoolchildren and in $13 \%$ of child athletes [542]. The main cause is BHR that typically appears after 3-8 $\mathrm{h}$ of vigorous exercise, so that in diagnosing EIA, the subject is required to run on an equipped treadmill under controlled environmental conditions [39]. After 5-15 min, bronchoconstriction, ranging from moderate to severe and lasting $15-30 \mathrm{~min}$ can occur, followed by a refractory period of $2 \mathrm{~h}$ during which no exertion can provoke EIA. Delayed reactions can occur even $8-12 \mathrm{~h}$ later, and this two-phase reaction is not uncommon [39].

Free-running tests and treadmill tests (Fig. 6.24) induce EIA [542] more easily. Objectively, arduous exercise - for example: running, bicycle riding or crosscountry skiing, but not long-distance skiing [335] - provokes bronchoconstriction in at least $70 \%-80 \%$ of asthmatics, which is neither intense nor prolonged [394]. Running provokes EIA more easily than jogging and this, in turn, more frequently than walking. In evaluating the triggering factors, a variety of issues come into play including cold, dry air, environmental pollutants, a recent allergic exposure or inflammatory mediators. The pathophysiology and cause of EIA remain controversial, but two theories have been offered: 1) the hyperosmolar theory and 2) the airway rewarming theory. Warm air and humidity can accelerate bronchospasm, exacerbated by cold air inhaled during exercise, especially if open-mouth breathing results from increased need for $\mathrm{O}_{2}$ [606]. Hyperpnea induced by physical exertion causes water release via evaporation of mucosal periciliary fluid with consequent local hyperosmolarity [606], which could provoke bronchospasm via direct vagal action, or by induction of mast and other cells to produce mediators, thus activating the successive cascade of immunological events [464]. Other authors, however, place emphasis on heat loss caused by hyperpnea, which could have consequences on subsequent rebreathing, with vasodilation of peribronchial vascular plexus, hyperemia, edema and, finally, bronchoconstriction [66]. Therefore, thermal shock could occur [394]. Since asthmatics have a hyperplastic vascular bed possessing greater permeability, the initial cooling down and subsequent warming up could cause bronchospasm via a true vascular blockage, with the onset of reactive hyperemia and edema involving the bronchial walls [394]. At the moment, however, there is insufficient data to clarify which of the two theories (warming up and hyperosmolarity) is pre-eminent. In studying BALF before and after exercise, no significant differences were found in histamine and tryptase levels. This could make doubtful a possible intervention by the airway mast cells accompanied by liberation of histamine and other mediators [278]. However, Kikawa et al have shown that in children with severe asthma, following physical exercise, $\mathrm{LTE}_{4}$ urinary levels were clearly higher than those of controls, thus concluding that airway reactivity seems to be a salient factor in EIA development [294,295]. The analyses of histaminemia have revealed elevated levels of histamine $10 \mathrm{~min}$ after BPT, returning to normal after $20 \mathrm{~min}$ and significant subsequent reduction of $\mathrm{T}$ lymphocytes 2 and $4 \mathrm{~h}$ after the test [66].

\section{Drugs and Additives}

Certain drugs such as ASA and food additives can induce bronchospasm in asthmatic and nonasthmatic subjects, by means of a nonallergic mechanism probably linked to the interference on mediator regulation (Chap. 10).

\section{Irritant Factors}

Irritant factors [584] like smoke, gases, dusts, vapors and immunotoxic substances, in addition to being harmful to the cells, have a more negative clinical impact on asthmatics compared to the general population, being able to provoke or aggravate asthma in subjects with BHR. Irritants, formaldehyde, fine wood dusts, etc. must be avoided in the home of asthmatic children (Chap. 24). Other factors involve meteorological, hygrometric and/ or barometric variations. Fog, in addition to its specific effect (larger drops deposit in smaller bronchi and trigger bronchoconstriction, smaller drops stratify on bronchial walls increasing mucous fluidity), can also transport harmful dusts and fungi.

\section{Environmental Pollutants}

Tables 4.15-4.17 and Fig. 4.22 illustrate the agents that compromise the immune system in test animals and, prospectively, in human beings. The immunotoxic substances include: exhaust gas, atmospheric dust, industrial smog - notably $\mathrm{SO}_{2}, \mathrm{O}_{3}, \mathrm{NO}, \mathrm{NO}_{2}$ - as well as anion superoxide, from which $\mathrm{H}_{2} \mathrm{O}_{2}, \mathrm{OH}^{-}, \mathrm{O}_{2}$ "singlet" $\left({ }^{1} \mathrm{O}_{2}\right)$ and other $\mathrm{O}_{2}$ radicals are derived, with directly damaging effects on human cells [262]. Small doses induce BHR and high and/or persistent doses induce lasting toxic effects [262]. TNF- $\alpha$ and $\mathrm{IL}_{1 \beta}$ are able to elicit a rapid and temporary increase in ciliary beat frequency following release of NO and, consequently, of iNOS [648]. Experimental data show how in children the in- 
crease of respiratory symptoms and asthma are proportional to that of pollutants [694], even acting in newborn babies (Table 2.26). As yet, no studies have quantified the relationship between atmospheric pollution and BHR [694]. In communities with high $\mathrm{O}_{3}$ concentrations, the RR (relative risk) of developing asthma in children playing three or more sports was 3.3 (95\% CI, 1.9-5.8), compared with children playing no sports; thus, air pollution and outdoor exercise could contribute to pediatric asthma development [390]. Patients exposed to particulate air pollution and gaseous pollutants such as $\mathrm{NO}_{2}$ concentrations can present with an increased prevalence of asthma symptoms and medication use [687]. Children are more at risk both because of the smaller caliber of their airways, and because of the increased RR with a resulting increase in inhaled pollutants per kilogram of body weight [521]; therefore an irritation provoked in an adult may turn into a significant bronchoconstriction in a very young child.

\section{Cigarette Smoke}

Passive smoke could constitute the most important environmental factor in the etiology of early infantile asthma (Tables 4.21-4.23), being involved in 38\%-65\% of cases (Fig. 4.23 and Table 5.11).

\section{Viral Respiratory Infections}

Viral respiratory infections (VRIs) have been related to the onset of recurrent wheezing illness and asthma in infants and are probably the most frequent cause of exacerbations of established disease in children. Studies discussed in Chap. 4 have shown that children with siblings have a lower prevalence of allergies and asthma than do children without siblings, an association attributed to a preventive effect of early infections, according to which early-life infections up-regulate Th1 lymphocytes that may inhibit the expansion of allergen-specific Th2 lymphocytes, thus limiting the development of allergic diseases. The action of respiratory viruses on the airways is modulated by several factors, including atopy, male sex, age, type of virus, possible BHR and development of correlated symptoms [545]. Infection by respiratory viruses can induce in children (even nonatopic children) [72] airway obstruction even though limited to the upper tract, BHR, functional changes in the airways, and PFT changes, which can persist for weeks after recovery (Table 11.9) [20,72, 130].

Infections, especially if contracted in the early stages of life, can damage the mechanisms underlying the mucosal barrier, thereby allowing a massive allergen penetration. As seen in Table 11.9, the necrotizing action that various influenza and parainfluenza viruses can have on the bronchial mucosa has been demonstrated: denuding it of its epithelial covering, they allow a greater penetra- tion of inhaled viral allergens and their absorption [20]. Clinical studies confirm that VRIs markedly aggravate or even directly trigger asthmatic symptoms in a high rate of cases, most especially in very young children [20, 167, 611] (Table 6.1). A re-examination of studies done in pediatric cohorts $(0->17$ years old), with respiratory infections of both upper and lower airways and asthmatic symptoms, found a link with viruses in $25.2 \%$ of general cases and in $35.6 \%$ of asthmatics [69]. In the follow-up of 11- to 17-year-old students with asymptomatic BHR, $45 \%$ developed a symptomatic form and $80 \%$ had suffered from past respiratory infections [753]. More recently, early respiratory infections indicated an increased, rather than a decreased, risk of developing bronchial obstruction during the first 2 years of life and of having asthma at 4 years of age [427]. Two cohort studies indicate that RSV lower respiratory tract infections (LRTIs) during infancy are associated with increased risk of asthma in the following years [585, 597]. Respiratory inflammation (RSV) during the 1st year of life seems to predispose, possibly via $\mathrm{IL}_{13}$-mediated mechanisms, to augmented allergic airway responses [361]. Moreover, both pneumonia and RSV LRTI during the first 3 years of life are associated with an increased risk of asthma or asthma-like symptoms up to 11 years of age $[88,616]$, or up to 5 years in Gambian children [670].

In infants and toddlers, RSV plays an especially important role and can induce respiratory disturbances with variable degrees of severity, culminating in hypoxia [714] and even death [271]. In particular, the course of bronchiolitis in a baby can prove very severe, sufficient to warrant RSV classification as the greatest agent of morbidity and morbility in the 1st year of life [477]. As with other respiratory viruses, RSV causes an increase in $\operatorname{IgE}$ [428], altering their immune regulation, and of eosinophils, which show a greater ability to participate in the immune inflammation [130]. It is highly probable that during infections, virus-specific IgE linked to mast cells react with an RSV antigen, by releasing vasoactive and inflammatory mediators, and start the whole cascade of events leading to BHR already described [72]. The increased airway permeability opens the door to allergens triggering allergic asthma, with an increase in cholinergic responsiveness and a decrease in the $\beta$-adrenergic responsiveness, thereby increasing BHR and airway inflammation.

RSV has an additional specific influence on PMNs producing $\mathrm{O}_{2}^{--}$and $\mathrm{TXB}_{2}$, with a significant chemiluminescence when incubated with RSV, and cytotoxicity for target cells [298]. They are the dominant inflammatory cells in the nasal secretions of babies with RSVinduced bronchiolitis, and in BALF of infants who have undergone ventilation for severe VRI [182]. It appears that PMN-deficient motility (Table 2.10) protects very young babies from RSV. RSV pathogenicity is shown by its ability to activate eosinophils notably producing cationic proteins in greater quantities, and obviously causing persistent BHR and damaging the respiratory 
Table 11.9. Mechanisms of virus-induced wheezing and/or asthma exacerbation

\section{Direct effect on the airway epithelium}

A. Damage to the airway epithelium

Epithelial denudation

Disrupted epithelial integrity

Enhanced allergen penetration

Reduced mucociliary clearance

Loss of neutral endopeptidase, cyclooxygenase

Enhanced contractility to substance $P$

Up-regulation of adhesion molecule expression

Loss of protective mediators, including the endo-

thelium-derived relaxing factor (EDRF)

Altered airway surface fluid osmolarity

Disruption of tight epithelial junctions

Exposure to irritant and cough receptors

B. Effect on vascular endothelium
Increased permeability
Fluid and plasma protein efflux
Production of inflammatory mediators

C. Increased mediator release

Histamine release

Increased arachidonic acid-derived lipoxins

Kinin generation

Enhanced activity of lipid mediators and superoxide

D. Enhanced leukocyte inflammatory function

Activation of basophils, mast cells, macrophages, eosinophils and neutrophils

T-lymphocyte-mediated cytokine release

Activation of cell-mediated immunity

\begin{tabular}{l}
\hline E. Altered neural system function \\
\hline$\beta$-adrenergic blockade \\
Cholinergic stimulation \\
Enhanced neuropeptide release \\
Defective NANC responses \\
\hline
\end{tabular}

F. Antibody-dependent enhancement of inflammation and cytotoxicity

G. Generation of virus-specific IgE antibody

Allergic sensitization

Mediator release

H. Effect on metachromatic cells

Direct activation

Activation via virus-specific $\lg E$ antibodies

Anaphylotoxin activation of complement cascade

Chemokine-mediated activation

IFN- $\gamma$ amplified activation

\section{Effect on lymphocytes}

Stimulation of IFN- $\gamma$ production

Activation of cell-mediated immunity

$\mathrm{CD}^{+}$deficiency

L. Effect on cellular adhesion molecules

Stimulation of interleukin generation

Up-regulation of adhesion molecule expression

Increased eosinophil and neutrophil migration and adhesion

M. Neutrophil activation

Neutrophil respiratory burst up-regulation

\section{Consequences}

1. Mouth breathing

Reduced nasal filtering of inhaled allergens $\rightarrow$ increased penetration to lower airway

Reduced conditioning of inspired air

$\rightarrow$ low temperature/humidity $\rightarrow$ bronchospasm

Reduced lower airway temperature $\rightarrow$ increase of viral replication

2. Increased circulating mediators and/or cytokines

Enhanced bone marrow production and subsequent activation of inflammatory cells

3. Increased serum IgE levels

\section{In conclusion:}

a) Epithelial changes and mucociliary clearance alteration favor a deeper penetration of viral inhaled allergens

b) Increased histamine release and IFN- $\gamma$-induced greater mucosal permeability may prime allergen absorption

c) Respiratory virus produce airway inflammation, and by increasing cholinergic receptor sensitivity promote a vagus-mediated reflex bronchoconstriction

d) Respiratory virus, directly or via metabolites produced by infected cells (including IFN- $\gamma$ ), impair airway $\beta$-adrenergic tone, thus perpetuating the inflammatory changes

e) Respiratory viruses, alter small airway geometry thus leading to bronchiolar wall narrowing and plugging with mucus, a mechanism of virus-induced airway obstruction

f) Virus-specific IgE antibodies trigger mast cells and basophils with subsequent mediator release, which may contribute to airway sensitization

These studies, as a whole, disprove that respiratory infections may be protective toward asthma.

Data from $[20,72,130]$. 
Table 11.10. Potential capacity of Rhinovirus to cause asthma in sensitized subjects

\begin{tabular}{|c|c|c|c|c|c|c|}
\hline \multicolumn{7}{|l|}{ Pathophysiology } \\
\hline $\begin{array}{l}\text { Sublminal exposure } \\
\text { in sensitized subjects }\end{array}$ & $\rightarrow$ & $\begin{array}{l}\text { CD54 induction } \\
\text { on respiratory epithelium } \\
\text { Peristence of inflammation }\end{array}$ & $\begin{array}{l}\rightarrow \\
\rightarrow\end{array}$ & $\begin{array}{l}\text { Increases susceptibility } \\
\text { to Rhinovirus infection } \\
\text { Rhinovirus infection }\end{array}$ & $\rightarrow$ & Asthmatic attack \\
\hline \multicolumn{7}{|l|}{ Clinical manifestations } \\
\hline 1.Clinical latency & & 2. Symptoms & & 3. Bronchospasm & & \\
\hline
\end{tabular}

Modified from [81].

epithelium with subsequent airway exposure of vagal receptors [298]. Studies [201, 298] indicate that $R S V$ triggers eosinophil degranulation by directly activating them [299], a plausible and stimulating link between VRI and asthma exacerbations. Attention has been drawn to the role of alveolar macrophages in RSV infections: once infected, they acquire the ability to replicate RSV, also expressing HLA-DR and immunoregulatory ILs such as $\mathrm{IL}_{1 \beta}$ and TNF- $\alpha$, capable of stimulating phagocyte cytotoxic responses. Consequently macrophages, by regulating local immune responses, perform an antiviral activity conditioning both viral replication and disease severity [401]. Lymphocyte studies have shown that the CD4/CD8 ratio in the lower tract is 22.5:1, and in the upper tract 15:1: thus diminishing the CTL importance, residing, however, in intraparenchymal locations, not intraluminal or bronchiolar [182].

Even influenza and parainfluenza viruses, Rhinovirus, Mycoplasma and Adenovirus often trigger respiratory infections, which can be found in very young babies as copathogenic factors [514], becoming recurrent in older children (Chap. 22). In $70 \%$ of nasal lavages in children $<2$ years with respiratory wheezing, RSV has been identified in $35 \%$ and Rhinovirus in $15 \%$ of cases [161], whereas in the $>2$-year-age group, only $31 \%$ of the cultures proved positive, with Rhinovirus predominant and an almost total lack of RSV [161]. Therefore, in older children, Rhinovirus influenza virus, Mycoplasma pneumoniae, etc. are the most frequent cause of wheezing. In a group of children aged 1-6 years, the viral origin of $45 \%$ out of 115 infection episodes of the airways has been ascertained, underlining, in the following order, the causative role of: Coronavirus, Rhinovirus, influenza virus A, and parainfluenza 1-3 viruses, Adenovirus, and Mycoplasma. The study revealed an RSV absence [400] and a total agreement with the identification process carried out in three other pediatric cohorts [477]. As well as Chlamydia trachomatis active in bronchiolitis, Chlamydia pneumoniae can induce in asthmatic children, especially $<4$ years [428], asthmatic crises whose onset is linked to sIgE production with consequent triggering of symptoms, which can wholly overlap what is known properly asthma. Indeed, the more protracted and pronounced SIgE production, the longer the disease duration [175].
Infection by Rhinovirus is by far the most common cause of wheezing disease in older children [280, 547] (Table 11.10) [81], in that it significantly stimulates mast cell mediator release, contributing to BHR development and possible late reactions [341]. In ten patients with $A R$ and BHR, their responses to BPT to both histamine and allergen (ragweed) were evaluated during an experimental infection from Rhinovirus. Four weeks later, BHR had increased in both tests; specifically, only one patient reacted prior to infection with a late response to the allergen, while $8 / 10$ manifested it during observation, $5 / 7$ persistently (Fisher, 0.0027) [341]. Rhinoviruses are able to increase BHR and promote the appearance of late reactions to the specific allergen. Even if the virus does not usually have a direct effect on the airways, the infection heightens the eosinophil response to challenge [341]. The major risk of causing asthma in children aged $>2$ years was that of the association of Rhinovirus infection with the sensitization to domestic allergens and exposure to passive smoke [161]. In asthmatic children the triggering element of the exacerbations was represented in $66 \%$ of cases by Rhinovirus, compared to $13 \%$ in controls. Furthermore, the relapses and PEF reductions were numerically equal to hospital admission [281]. It is hypothesized that even a subliminal inflammation induces in endothelial cells CD54 expression, CD11a/ CD18 receptor, which plays the role of the linking site for the virus. Linking to CD54, the virus can interfere with CD11a/CD18 and the host's natural defenses, thus inflammatory reactions to viral infection can stimulate Rhinovirus receptors to induce new ones, thereby spreading viral infection [280].

To summarize these reports, a unifying mechanism has been proposed [72], suggesting that virus action has a major implication in triggering asthma. From Table 11.10 , it can be deduced that the epithelial damage and persistent inflammation that follows have an effect on sensitive nerve endings, provoking the onset of bronchoconstriction reflexes. Above all, respiratory viruses, in addition to provoking airway inflammation and prolonging its effects - either directly or through cellular products infected by them (such as IFN- $\gamma$ ) - promote the formation of virus-specific IgE and the release of mediators. 


\section{Emotional Factors}

The influence of emotions on asthmatic attacks has been widely documented; therefore any emotional conflict being experienced by the child must always be taken into consideration (Table 5.11), even if for parents such occurrences are rare (Table 6.1). It is known that in $12 \%-13 \%$ of wheezing increase can be triggered by worries regarding school [307], a forthcoming trip, the birth of a sibling, etc. - all events that carry great emotional weight. Often the child uses the disease as a means of attracting parental attention, or to obtain privileges which might not otherwise be granted, or to divert existing tensions within the family nucleus, thereby avoiding subsequent conflicts. Studies carried out using BPT have confirmed the asthmogenic role of physiological factors: it has been noted that blind placebo (BP) inhalation prior to the test significantly reduced the onset of asthma, while open BPT with placebo, which the children believed to be the specific allergen, provoked an obvious bronchospasm [363].

\section{Crying and Laughter}

Sometimes, a child has bronchospasm following laughter or crying, in the latter case with a frequency of $40 \%$ [339]: this can be explained by the mechanical airway irritations caused by intense and rapid exhalations, as well as by hyperpnea, which, as previously mentioned, plays its part in EIA and can trigger bronchospasm even in healthy subjects. We have observed numerous cases of parents of asthmatic children who literally forbade them to laugh, for fear of setting off an asthmatic attack, and, conversely, other children tried blackmail by threatening to cry [68]. Pediatricians should clarify all these issues both to parents and children, especially explaining that these forms of attack recede spontaneously within a few minutes and should not be viewed as a cause for alarm; but should not be underestimated [68]: a child belonging to a small group of asthmatics under observation over a period of 10 years died following a fit of laughter [339].

\section{Collateral Pathologies}

AR, frequently complicated by sinusitis, can aggravate asthma (Table 11.1). The presence of such conditions should therefore be ascertained in all asthmatic children and, especially, in those affected with chronic asthma who also presented Rx evidence of sinusitis [179].

GER (gastroesophageal reflux) may be a severe disease and is often neglected in the etiopathogenesis of asthma [702]. GER is a pathology more commonly found in younger infants who have not yet learned to sit up and is determined by functional immaturity of the lower esophageal sphincter. GER is caused by the retro- grade movement of gastric contents across this sphincter into the esophagus [702]. The estimated incidence of GER in asthmatic children reaches $50 \%-60 \%$ and can be an aggravating factor of the disease, higher than in the general population [702]. The reflux of acid material provokes bronchospasm, very probably through a vagal reflex. Exposed receptors could be activated with stimulation of the $\mathrm{X}$ afferent fibers situated in the esophageal mucosa. This could provoke a vagus-mediated increase of BHR with amplification of the bronchoconstriction effects of other exogenous stimuli [221]. The activation of this reflex between the esophagus and the airways is also related to the fact that embryologically both derive from the anterior archenteron and that they have a common autonomous innervation (Chap. 9). Based on this pathogenetic mechanism, the cough-producing clinical symptoms that have no other etiologies [170] such as in the child with asthma who is difficult to control, or who shows symptoms $1-2 \mathrm{~h}$ after having gone to bed, etc. [584], can be assessed. In 40 children aged a median of 14 months with persistent respiratory symptoms, a significant decrease was noticed in the number of further GER-related episodes of recurrent bronchopneumonia, reactive airway disease, nocturnal symptoms and in their nutritional status after starting antireflux therapy [277]. An esophageal scintiscanning was used to detect GER in 126 asthmatic children with a mean age of 2.31 years who were refractory to routine antiasthmatic medication [647].

\section{Drugs to Be Used and Routes of Administration}

The treatment of the asthmatic child, summarized in Table 11.11 [26, 360, 476, 508], is determined according to age and according to the severity of the symptoms.

\section{Routes of Administration}

There are four routes of administration:

The parenteral route is recommended for more severe cases, to achieve elevated concentrations of a drug in a short period and to obtain a good bronchodilatory effect. This method does not produce selective effects in that the drug spreads more or less at the same rate to all areas; it is most often used in EDs or in intensive care units (ICU) and, in any event, under medical supervision for asthmatics in severe conditions (in a state of unconsciousness, requiring intubation).

The oral route, having a latency time of $1-2 \mathrm{~h}$ linked to the rate of gastric absorption, has neither rapid nor selective effects, but certainly finds higher compliance. Higher dosages are needed than for the inhalation route to reach effective levels in the bronchial area, possibly with a higher rate of incidence of undesirable effects as a result of $\beta_{2}$ and $\beta_{1}$ receptor stimulation in extrapul- 
Table 11.11. Features of four delivery systems to be used with children

\begin{tabular}{|c|c|c|c|}
\hline MDI & $\begin{array}{l}\text { MDI + spacer } \\
\text { and face mask }\end{array}$ & Dry-powder inhalers & Nebulizers \\
\hline \multicolumn{4}{|l|}{ Requirements } \\
\hline $\begin{array}{l}\text { Shake the canister } \\
\text { thoroughly }\end{array}$ & Rinse the mouth & $\begin{array}{l}\text { Elevated inspiratory flow } \\
\text { after } 6 \text { years of age }\end{array}$ & $\begin{array}{l}\text { Selection and maintenance } \\
\text { of a well-functioning device }\end{array}$ \\
\hline Hold the canister upright & $\begin{array}{l}\text { Shake the canister } \\
+ \text { spacer }\end{array}$ & $\begin{array}{l}\text { Both Turbohaler and Rotohaler } \\
\text { should be held vertically }\end{array}$ & $\begin{array}{l}\text { Preparation of drug solutions } \\
\text { to be nebulized in the correct } \\
\text { proportions }\end{array}$ \\
\hline $\begin{array}{l}\text { Place the inhaler } \\
\text { between the lips }\end{array}$ & $\begin{array}{l}\text { Actuate the MDI } \\
\text { and fill the large } \\
\text { volume spacer }\end{array}$ & Inhale as quickly as possible & $\begin{array}{l}\text { Rinse the mouth after each } \\
\text { inhalation }\end{array}$ \\
\hline Breathe out steadily & $\begin{array}{l}\text { Inhale from the } \\
\text { spacer by five tidal } \\
\text { breaths }\end{array}$ & & $\begin{array}{l}\text { When a close fitting mask is } \\
\text { used, babies should breathe } \\
\text { by their mouth and with no } \\
\text { gap between mask and face }\end{array}$ \\
\hline $\begin{array}{l}\text { Release the dose and } \\
\text { take a slow, deep breath }\end{array}$ & $\begin{array}{l}\text { Synchronize dose } \\
\text { release with inspiration }\end{array}$ & $\begin{array}{l}\text { Gargle/mouth rinse after } \\
\text { steroid administration }\end{array}$ & \\
\hline \multicolumn{4}{|c|}{ Assessment of correct dose (delivered to the lungs) } \\
\hline High (80\%) & Very low $(<5 \%)$ & High $(60 \%)^{a}$ & Very low (5\%) \\
\hline \multicolumn{4}{|c|}{ Advantages (rate of inhaled drug) } \\
\hline Delivery efficiency & Rapid onset of action & Delivery efficiency & Adaptable to doses \\
\hline \multirow[t]{2}{*}{ Rapid onset of action } & Rapid onset of action & $\begin{array}{l}\text { Minimal child coordination } \\
\text { required }\end{array}$ & \\
\hline & $\begin{array}{l}\text { High effectiveness and low } \\
\text { adverse effects }\end{array}$ & $\begin{array}{l}\text { Absence of dispersing } \\
\text { propellants }\end{array}$ & $\begin{array}{l}\text { Utilizable in noncollab- } \\
\text { orating babies }\end{array}$ \\
\hline$(7 \%-14 \%)$ & $(15 \%-25 \%)$ & $(30 \%)^{a}$ & $(10 \%-15 \%)$ \\
\hline \multicolumn{4}{|l|}{ Mean age (years) } \\
\hline 6 or more & $1-5$ & $5-6$ & $1-3$ \\
\hline \multicolumn{4}{|l|}{ Therapeutic issues } \\
\hline None & $\begin{array}{l}\text { In acute and chronic asthm } \\
\text { these are effective, cheape } \\
\text { less time-consuming }\end{array}$ & $\begin{array}{l}\text { Only with medications } \\
\text { with no adverse effects }\end{array}$ & $\begin{array}{l}\text { Severe broncho- } \\
\text { constriction } \\
\text { In absolute absence } \\
\text { of cooperation }\end{array}$ \\
\hline
\end{tabular}

Face mask devices should be applied firmly to the face, especially if a valved spacer is used; with nebulizers the mask should be held as close to the face as practicable without undue disturbance. Any gap reduces the dose dramatically.

Data from $[26,359,476,508]$.

$\mathrm{MDI}$ metered-dose inhaler.

a By Turbohaler.

monary sites. In any event, it is recommended for the very young who are not able to tolerate aerosols and in cases of nonsevere asthma [79].

Inhalation is the preferred route, in that it reaches the tracheobronchial tree immediately and requires lower doses, with a parallel decrease in adverse effects compared to other routes of administration.

The subcutaneous (SC) route is reserved for epinephrine and terbutaline.
To obtain the desired therapeutic effects using inhalatory routes of administration, the following must be considered:

- The size of the inhaled particles

- The appropriateness of the apparatus used

- The child's compliance

- The child's age, strictly linked to the above (Table 11.11), because MDIs (metered-dose inhales) developed for use in adults may be difficult for young children to 
use, because they may have difficulty coordinating actuation with inhalation [28].

Regarding the first point, only particles with a diameter of $1-5 \mu \mathrm{m}$ efficiently reach the lower airways. If they are larger, they will stop in the upper airways and, if smaller, they will reach the alveoli, or be exhaled.

In asthma, there are 4 routes to administer drugs by inhalators:

1. Predosed MDI or pressurized MDI spray can (with spacer)

2. Dry-powder inhaler (DPI)

3. Pneumatic nebulizer with compressor

4. Traditional aerosols

\section{Predosed Pressurized Sprays}

These are predosed spray cans or MDIs that manually release fixed predosed drug doses (puffs). These MDIs, if not used properly, deposit up to $90 \%$ of the drug in the oropharynx with the same effect of a drug administered per os; moreover, steroids may cause dysphonia and predispose the child to oropharyngeal candidiasis [517]. Therefore, the instructions for use should be carefully followed [78]:

- Shake the inhaler.

- Hold it upright and exhale deeply.

- Close the lips around the mouthpiece.

- Press the inhaler while inhaling slowly and as deeply as possible.

- Hold the breath for at least $10 \mathrm{~s}$ (in such way that the drops are deposited on the airways as a result of gravity and not of impact).

- Exhale slowly and wait 1-2 min, if the dose is to be repeated.

MDIs are suitable for children at 10 years (Table 11.11) and 7-8 years of age if properly instructed [273]. The main difficulty arises as a result of poor coordination at the moment of discharge, mostly due to:

- Rapid inhalation

- Blocking the inhalation at the moment when the aerosol discharges its content

- Breathing though the nose

- Premature or delayed breathing (lack of hand-lung coordination) [78]

The major problems can be obviated by keeping the mouthpiece $3-5 \mathrm{~cm}$ away from the lips, an expedient that overcomes the instinctive defense of small children using their tongue to impede any further drug entry to the lower airways: the effect becomes noticeably increased, also allowing for confirmation of its proper use, but it is not always a recommended method for this age group [78]. It should be noted that MDIs contain chlorofluorocarbons (CFCs) 11, 12 and 114, which provoke cough and reduce ventilatory function in one-third of patients [128]. New hydrofluorocarbon inhalers (HFIs) deliver albuterol, BDP, and various other medications.

\section{Spacers or Holding Chambers}

To overcome the difficulty caused by the lack of synchronization between discharge and act of inhaling, spacer devices are available. These are adapters consisting of an expansion chamber placed between the MDI and the patient's mouth, facilitating use in preschool children [273]. It consists of a container in which drug particles are held in suspension, that is held inside the apparatus until the moment of inhalation, discharging the required amount even in situations of noncompliance. Consequently, a minimum deposit in the orophar$y n x$ is assured and a higher percentage of inhaled drug, as well as a decreased incidence of side effects resulting from systemic absorption [25, 220,359]. After the puff, the majority of finer small drops remain suspended inside the spacer for at least $30 \mathrm{~s}$, thereby allowing even the youngest children to undergo treatment. The larger aerosolized particles, on the other hand, are withheld, thus obtaining a more rapid administration of the drug and a more efficient supply and an unquestionably adequate cost-effectiveness ratio [436]. In addition to being helpful to children unable to use MDIs correctly, such adapters become indispensable for those undergoing an ICS treatment, to reduce the pharyngeal drug impact and prevent the side effects listed above [80] (Fig. 11.36).

Another innovation has been the introduction of a valved holding chamber (VHC) at the mouthpiece of large-volume spacers to aid the inhalation by very young babies. The principal MDI models with valve spacers are as follows: with the Aerochamber $(150 \mathrm{ml}$ volume), also available with a mask for infants and young children, the rate of swallowed medication is barely $2 \%-3 \%$ compared to $60 \%$ and $80 \%$, respectively, with the spray or MDI; inhalation is also correct [357], while the rate reaching the airways is $17 \%$ compared to $14 \%$ of an adequate MDI inhalation [357]. Also available are Babyhaler (Fig. 11.37) and Volumatic, more suited for older children, with volumes of $350 \mathrm{ml}$ and $750 \mathrm{ml}$, respectively, Fisonair, Nebuhaler and Turbohaler for DPI budesonide (BUD) (Oxis) Turbuhaler for formoterol and Diskus for salmeterol. In addition, there is the pocket-size spacer-discharger of predosed aerosol (Jet) for BDP (Fig. 11.38), which avoids the synchronization problem mentioned and has an inhalation quota of $30 \%$ and a swallowed one of $10 \%$ [359]; and the breathactuated Autohaler, a dispenser of albuterol. Therefore these last two are useful in acute attacks and for children who have difficulty in using MDIs [128, 485] - not for everyone. Patients aged 5 years have difficulty with the Autohaler since it requires quick inhalation, as with MDIs [128].

For nurslings and very young children, a well-designed mask must be placed on the dispenser (Fig. 11.37), or alternatively, an Aerochamber can be used for nurslings with a closely fitting anatomically designed mask [180], to avoid an empty space between the mask and the face, 


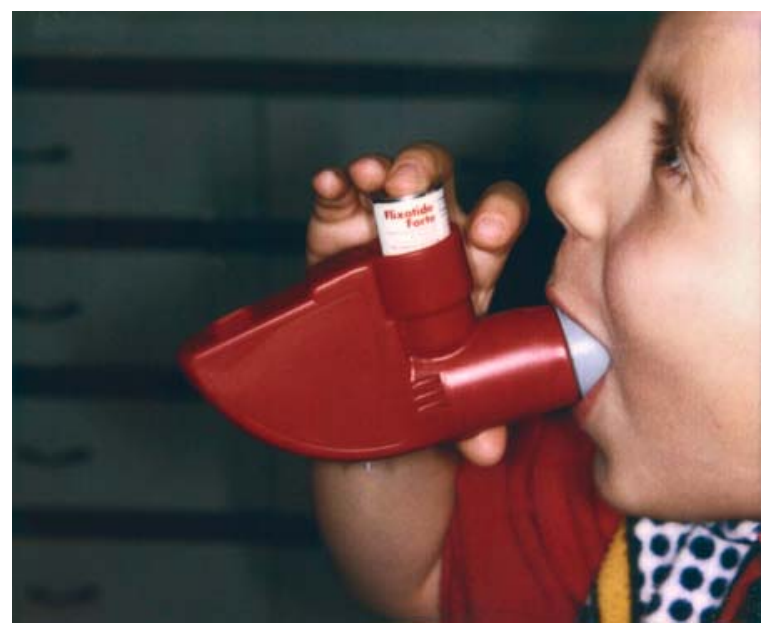

Fig. 11.36. A child about to use a pressurized MDI with incorporated PEF meter

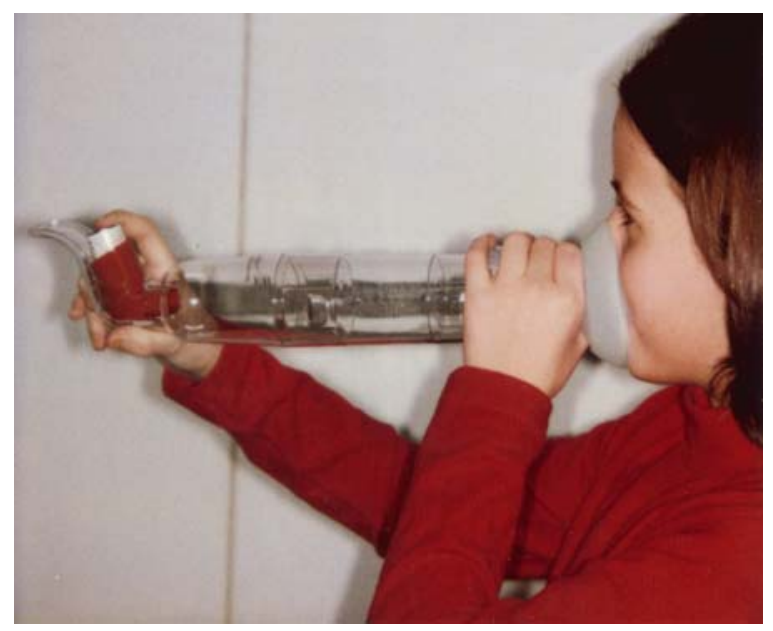

Fig. 11.37. A girl using a valved large-volume spacer with face mask

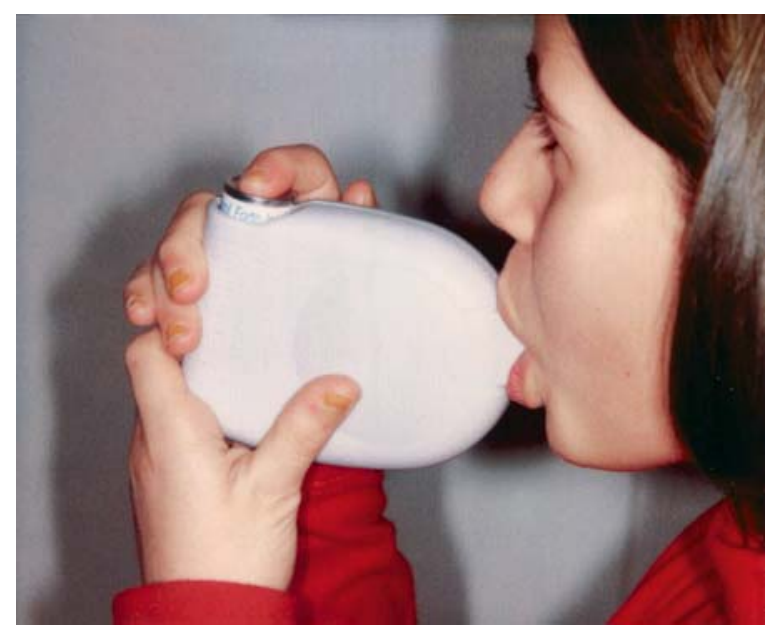

Fig. 11.38. A girl using a pocket MDI with spacer eventually changing the type of mask if necessary. The choice between large or small chambers has been resolved in favor of the latter, which are easier to manage if the child rejects the treatment, and are also less frightening. They release at least the same amount of medication as the larger ones, if not more, at a low tidal volume (TV) rate [180]. If the spacers are not equipped with valves suitable for nurslings, during inhalation the air can be contained within a nonanatomical mask, thus reducing the dosage inhaled [44]. For children unable to tolerate a face mask, a MDI with a polystyrene cup can be used, especially in case of emergency [508]. In conclusion, face mask devices should be applied firmly to the face, especially if a valved spacer is used. With nebulizers the mask should be held as close to the face as practicable without undue disturbance. Any gap reduces the dose dramatically; therefore in very young children high doses of ICS are not effective because a lot of the spray is lost and does not reach the lesser airways, because of the small TV in these babies.

Another characteristic of critical importance is the volume. The right size is a matter of balance. Using a small, low-resistance, two-way chamber with nurslings and children, $38 \%$ of the dose is received, whereas with larger chambers, only $20 \%$ is received [44], obviously depending on details: it is larger, more cumbersome and inconvenient to use.

An alternative to the MDI is the small-volume nebulizer (SVN). Advantages to SVNs include use at any age, or administration while asleep [102]. A recent metaanalysis has found the efficacy of MDI-VHC superior to that of SVNs, particularly in regard to onset of action and reduction of hospitalization [86]. In 123 children $<2$ years of age with moderate-severe exacerbations of wheezing seen in the ED, the response to salbutamol delivered by MDI-VHC and facial mask was faster than to salbutamol delivered by SVN [540].

\section{Dry-Powder Inhalers}

DPIs are ecological since they do not contain CFCs: the micronized powder is contained in capsules, is inhaled by special dosers such as Spinhaler for DSCG (cromolyn); Diskhaler, Rotahaler, and Easyhaler for albuterol, and Turbohaler for BUD and formoterol. They do not require coordination of inhalation techniques since the patient inhales directly from the mouthpiece, the inhalator being breath-actuated. Multi-dose apparatuses, in addition to their convenience, ensure greater compliance, especially from preschool children [232]. Even with this system, difficulties arise. The very young should be taught to rapidly inhale without holding his (her) breath and told not to lean their head backwards [508]. In fact, the micronized particles of the medicine tend to agglutinate in clumps of excessive size. For effective use, children should inhale rapidly with high peaks of inspiratory flow (PIF) of at least 30-60 1/min, to create 


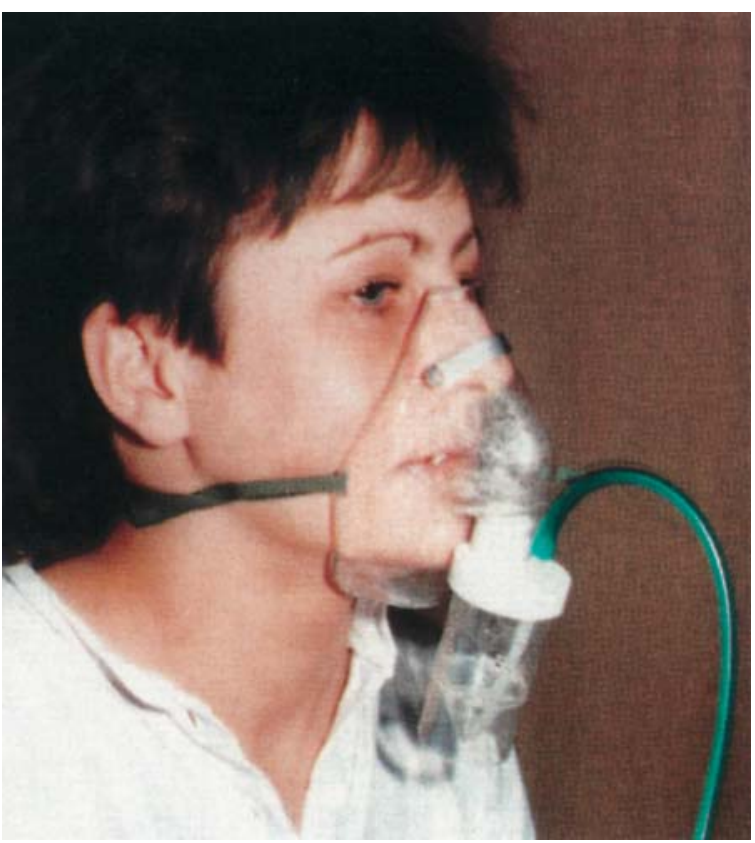

Fig. 11.39. Nebulizer system

sufficient turbulence to break them up: at a lower rate the functionality, for example of the Rotohaler, drops to $10 \%$ [128]. Since large amounts of medication may remain in the mouth, it is advisable to have the child rinse it thoroughly after inhalation [508]. These models are not suited for children suffering from acute asthmatic attacks [44]; they are advisable for use with children aged 5-6 who can use them [44].

\section{Nebulizers}

Jet nebulizers can be used with a mask or directly from the mouthpiece, to avoid the frequent problem of small infants and young children unable to use MDIs, or suffering from acute attacks or persistent bronchoconstriction (Fig. 11.39). The advantages are obvious: even superficial breathing over several minutes can effectively deliver the medication deep into the airways. Compared to traditional MDIs, they produce a larger number of particles in a given time and particles that are also smaller in size $(0.5-5 \mu \mathrm{m}$ instead of $>5 \mu \mathrm{m})$, thereby distributing the drug more quickly. However, if children are not cooperating during the brief time of discharge, this technique will be inadequate. During nebulization, if the mask is distanced even 1 or $2 \mathrm{~cm}$ from the face, the delivered medication is reduced by $85 \%$. Given that even the most tranquil child cannot stay still for more than 5 min, a means of reducing this time frame could be studied [181]. Ultrasonic nebulizers (for example, Fisoneb) ensure rapid nebulization, but are commonly less efficient for drug delivery, especially with suspensions, have a poor cost-benefit ratio and are difficult

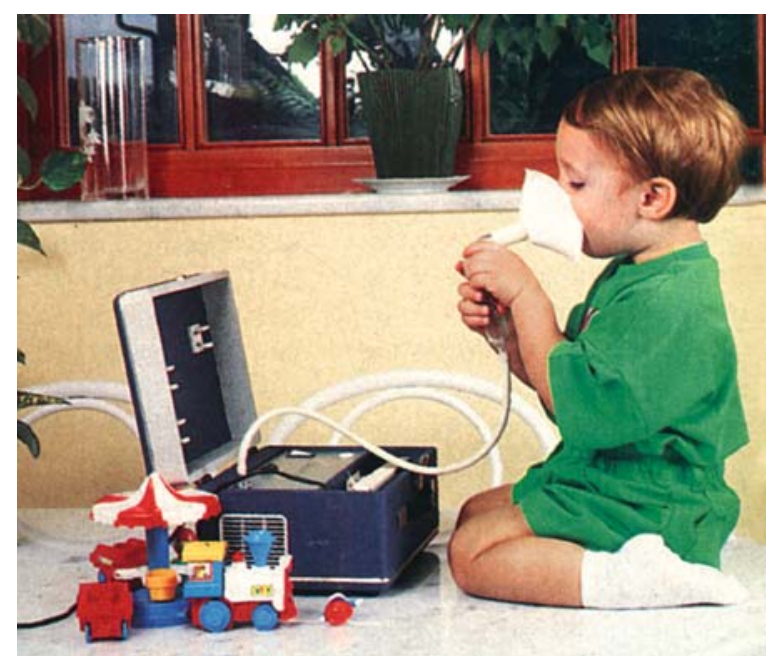

Fig. 11.40. Nebulized $\beta_{2}$-agonist therapy is primary in the management of severe asthma also with toys

to handle [26]. Some nebulizers (for example, Nebula and Soffio) can also be used at home (Fig. 11.40). With nebulizers equipped with electric compressors, the need for good coordination from the moment of discharge and the act of inhalation is eliminated, thereby facilitating a sustained administration [476]. Newer types of hand-held nebulizers are small enough to be safely carried in a briefcase, a purse, or a backpack.

Even with nebulizers some rules should be followed:

- Regular checks of the proper functioning of the machine.

- The drug must be diluted in saline, $4 \mathrm{ml}$.

- Time of nebulization about $10 \mathrm{~min}$.

- Careful rinsing of the oral cavity, especially after using CSs.

- Washing under running water of the flask, mouthpiece and mask after use.

\section{Traditional Aerosols}

The traditional models are suitable for all children who will accept them. Usually, they produce too large particles $(>5 \mu \mathrm{m})$ to reach the airways effectively but, unlike cromolyn and BDP, they are suited to $\beta_{2}$-adrenergics, which are characteristically effective even in low doses, since they are easily absorbed by the oral/nasal mucosa. Even in this case, wheezy infants or toddlers should inhale with an open mouth, otherwise $75 \%$ of the aerosol is blocked, with the nose acting as a filter [549]; nor should the mask be distanced from their mouth [192]. 
Table 11.12. Starting and subsequent drug doses recommended in children $<5$ years in severe conditions (maximum doses and minimum do intervals for domiciliary use in parentheses)

\begin{tabular}{|c|c|c|c|}
\hline Age (years) & Device & Symptomatic & Prevention \\
\hline \multirow[t]{6}{*}{$0-2$} & \multirow{4}{*}{$\begin{array}{l}\text { MDI spacer } \\
\text { and face mask }\end{array}$} & Albuterol $200 \mu \mathrm{g} \mathrm{q} 6$ h (1 mg q 3 h) & Cromolyn 5-10 mg q 6-8 h \\
\hline & & Terbutaline $250 \mu \mathrm{g} \mathrm{q} 6 \mathrm{~h}(2.5 \mathrm{mg} \mathrm{q} 3 \mathrm{~h})$ & BDP $50-200 \mu \mathrm{g}(600 \mu \mathrm{g}) \mathrm{q} 12 \mathrm{~h}$ \\
\hline & & Ipratropium bromide (IB) $1.25 \mu \mathrm{g} \mathrm{q} 6 \mathrm{~h}$ & FP $25-100 \mu \mathrm{g}(250 \mu \mathrm{g}) \mathrm{q} 12 \mathrm{~h}$ \\
\hline & & $(250 \mu \mathrm{g} \mathrm{q} 6 \mathrm{~h})^{\mathrm{b}}$ & BUD $50-200 \mu \mathrm{g}(600 \mu \mathrm{g}) \mathrm{q} 12 \mathrm{~h}$ \\
\hline & \multirow[t]{2}{*}{ Nebulizer } & Albuterol 2.5 mg q 6 h (q 3 h) & Cromolyn $20 \mathrm{mg} \mathrm{q} 6-8 \mathrm{~h}^{\mathrm{c}}$ \\
\hline & & Terbutaline $5 \mathrm{mg} \mathrm{q} 6 \mathrm{~h}(\mathrm{q} 3 \mathrm{~h})$ & BUD up to $800 \mu \mathrm{g} \mathrm{q} 12 \mathrm{~h}$ \\
\hline \multirow[t]{6}{*}{$>2-5$} & \multirow[t]{4}{*}{ MDI+spacer } & Albuterol $200 \mu \mathrm{g} \mathrm{q} 6 \mathrm{~h}(1 \mathrm{mg} \mathrm{q} 3 \mathrm{~h})$ & Cromolyn $10 \mathrm{mg} \mathrm{q} \mathrm{6-8} \mathrm{h}$ \\
\hline & & Terbutaline $500 \mu \mathrm{g} \mathrm{q} 6 \mathrm{~h}(2.5 \mathrm{mg} \mathrm{q} 3 \mathrm{~h})$ & BDP $50-200 \mu \mathrm{g}(600 \mu \mathrm{g}) \mathrm{q} 12 \mathrm{~h}$ \\
\hline & & & FP $25-100 \mu \mathrm{g}(250 \mu \mathrm{g}) \mathrm{q} 12 \mathrm{~h}$ \\
\hline & & & BUD 50-200 $\mu \mathrm{g}(600 \mu \mathrm{g}) \mathrm{q} 12 \mathrm{~h}$ \\
\hline & \multirow[t]{2}{*}{ Nebulizer } & Albuterol 2.5-5 mg q 6 h (q 3 h) & BUD up to $800 \mu \mathrm{g} \mathrm{q} 12 \mathrm{~h}$ \\
\hline & & Terbutaline $5-10 \mathrm{mg} \mathrm{q} 6 \mathrm{~h} \mathrm{(q} 3 \mathrm{~h}$ ) & \\
\hline
\end{tabular}

a Doses of inhaled corticosteroids are recommended for children in such severe condition that they are no longer controlled by nonsteroidal anti-inflammatory drugs. However, higher doses and concentrations may be required for younger children because of the relative inefficiency of delivery devices (see Table 11.13).

b IB should not be administered at intervals less than $6 \mathrm{~h}$ to avoid atropine-like toxicity.

c Cromolyn is more effective if administered at age 9 months by nebulizer [479].

When prescribing steroids, pediatricians should always prescribe the lowest dose required for symptom control; when symptoms remit, steroids may be stepped down and started up again when symptoms return.

Data from $[56,604]$.

$B D P$ beclomethasone dipropionate, $B U D$ budesonide, cromolyn, FP fluticasone propionate.

\section{Age Ranges for Inhalant Therapy}

To summarize $[476,508,699]$ (Tables $11.10,11.12$ ) [56, 604]:

- Children 1-2 years of age

Nebulizers are useful for acute attacks not requiring the child's total cooperation [272]. The more economical MDIs with valve expanders are also recommended. Both are provided with face masks suitable for use even with nurslings and toddlers if the problem of keeping the appliance in position on the face can be overcome, adapting the expansion chamber volume to the specific needs listed in the previous section $[180,181]$. Only $0.3 \%-$ $1.5 \%$ of the nominal dose inhaled by children, even 9-16 months old, reaches the lungs, significantly more if nebulized [549]. A recent trial has established that MDIs with spacers may be as efficacious as nebulizers for the ED treatment of wheezing in children aged $\leq 2$ years [143]. For decades we have used in children aged 6 months and older with wheezing a BDP or flunisolide suspension for nebulization dosed in drops ( $1 \mathrm{drop} / \mathrm{kg}$ ) plus albuterol solution for inhalations (half dose), 3-4 doses/day as needed. The symptoms are improved in $90 \%-95 \%$ of cases.

- Children 3-5 years of age

MDIs with VHC spacer plus face mask $[44,56]$ are preferred; however, many children probably use nebulizers because that is the route they began [78]. New cases should first try MDIs with spacers [56]. Devices have been created that allow the child to breathe while receiving the medication, and placebo spray cans produce excellent preventive training. Breath-actuated MDIs are not recommended for this age group [57].

- Children 6-8 years of age

At this age children spend a great deal of time outdoors, and they need a portable model that is easy to use and efficient. The aerochamber fulfills all these requirements well. The DPI is useful for subjects who have not yet become accustomed to MDIs. Both systems avoid the problems associated with CFCs.

- Children aged $>10$ and adolescents

Young people of this age are able to use MDIs, which are economical and fulfill the requirements needed [476].

The instructions provided with the device are unsuitable for younger children (Table 11.13) [476, 486], and even adults have found difficulty in using the equipment $[103,350]$, to the point that manufacturers were invited to change the mouthpiece [350]. Additionally, there are problems related to the dosage absorbed and to be delivered [112]. In the first instance, a pharmacokinetic analysis revealed that nurslings and toddlers absorb only $0.13 \%-0.33 \%$ of the dose being delivered by MDI or nebulizers, respectively [549], and on average only $14 \%$ of nebulized medication is deposited in the airways, increasing from $9 \%$ to $19 \%$ in older children [357]. Reports have shown nebulizer reactions including tachyphylaxis or increased BHR due to $\beta$-agonist overuse 
Table 11.13. Frequency of the most common problems encountered by 217 children using an inhaler (\%)

\begin{tabular}{|c|c|c|}
\hline Problems & $\begin{array}{l}\text { MDI } \\
\text { inhaler } \\
\text { (no. 132) }\end{array}$ & $\begin{array}{l}\text { Tube } \\
\text { spacer } \\
\text { (no. 85) }\end{array}$ \\
\hline Forgetting to shake the canister & 49 & 34 \\
\hline $\begin{array}{l}\text { Forgetting to exhale } \\
\text { before inhaling }\end{array}$ & 45 & 51 \\
\hline Neck flexed during inhalation & 12 & 14 \\
\hline Coordination problems & 55 & 17 \\
\hline Too rapid inhalation & 67 & 28 \\
\hline Breath holding for $7 \mathrm{~s}$ & 42 & 39 \\
\hline $\begin{array}{l}\text { Stopping inspiration } \\
\text { when firing aerosol }\end{array}$ & 38 & 6 \\
\hline Nasal inspiration & 24 & 32 \\
\hline Submaximal inspiration & 23 & 19 \\
\hline Help from the parents & 5 & 6 \\
\hline \multicolumn{3}{|l|}{$\begin{array}{l}\text { Possible explanations } \\
\text { of the poor compliance: }\end{array}$} \\
\hline \multicolumn{3}{|l|}{ Misunderstanding } \\
\hline \multicolumn{3}{|l|}{ Poor reliance on drug effectiveness } \\
\hline \multicolumn{3}{|l|}{ Fear of adverse effects } \\
\hline \multicolumn{3}{|l|}{ Rebellion } \\
\hline \multicolumn{3}{|c|}{ Coordination problems with administration technique } \\
\hline \multicolumn{3}{|c|}{ Coordination problems with administration timing } \\
\hline
\end{tabular}

Data from $[475,486]$.

or allergic reactions to the medication or the excipient. Recently two asthmatic children experienced a lifethreatening exacerbation of their symptoms after nebulizer use. Cockroaches were detected in the reservoir of the nebulizers used [48].

\section{Dosages for the Very Young}

The point of departure is the anatomical differences found in the very young. Nurslings inhale with a relatively rapid TV and in so doing retain the therapeutically useful particles for a shorter time [436]. Nonetheless it should be noted that TV and PIF are reduced, compared to older babies and preschool children, so that infants $<6$ months inhale the aerosol directly, thereby receiving a greater dosage of the undiluted medication, whereas an older infant or preschool child inhaling with normal TV, but at ambient air, receives a comparatively smaller dose, underscoring the dosage $/ \mathrm{kg}$ problem [112]. Taking into consideration that the reduction in caliber of the lower airway tracts reduces the penetration drug in the peripheral areas [584], and that newborn infants tend to breathe through their nose, in the best hypothe- sis the amount of the drug that is deposited in the airways is only $33 \%-50 \%$ of that inhaled per os [344]. Also, the correlation between the drug concentration in the aerosolized or nebulized solution (Cs) and that per inhaled volume unit of gas (Ci) appears to be inconstant, since for a given $\mathrm{Cs}$ the $\mathrm{Ci}$ can be five times greater in a young infant compared to an older child, in whom the inhaled dosage is independent of weight [112]. Therefore, doses for children aged 6 months to 5 years have to be regulated according to weight in $\mathrm{kg}$, to balance the discrepancies that arise from a dosage based on a single measure for all [112]. If no correction is made, children aged 1-5 might receive a larger dosage than those aged 5-10 [344, 488]. However, based on studies conducted related to the plasmatic concentrations of albuterol, it has been noted that doses calculated on the basis of weight can prove to be less in children aged 6 months to 5 years. Therefore in cases of severe asthma, the dose can be increased - even up to $25 \%-50 \%$ [ 488 ] or more - with no risks, whether it be the result of greater drug clearance in the airways or of an insufficient inhalation coordination between the method of discharge and the technique [488]; and finally - we should add - whether it also be because of the great differences among individuals. One could settle for a fixed dose for all patients, given that the results produced are similar [459]. Further research will ascertain which dosage is the most suitable in early infancy, based also on pharmacokinetic studies of each drug.

\section{Drugs to Be Used}

The drugs to be used are the following:

- Adrenergics

- Anticholinergics

- Theophylline

- Corticosteroids

Table $11.14[124,617,634,635]$ sets forth the action mechanisms whereby drugs that can be used to treat acute asthma work; Table 11.15 [174] those of $\beta_{2}$-adrenergics; Table $11.16[35,56,406,437,552,582]$ specifies the dosage for each method of administration.

\section{Adrenergic Drugs}

Adrenergic or sympathomimetic agonists can be classified, according to their respective actions on the receptors, as follows:

Drugs acting on receptors $\alpha, \beta_{1}$ and $\beta_{2}$ : epinephrine Drugs acting on receptors $\beta_{1}$ and $\beta_{2}$ : isoprenaline, orciprenaline

Drugs with selective action on receptors $\beta_{2}\left(\beta_{2}\right.$-adrenergics, $\beta_{2}$-agonists), further divided into:

- Catecholics: isoprenaline

- Resorcinolic: fenoterol, orciprenaline, reproterol, terbutaline 
Table 11.14. Mechanism of action of asthma medications: comparative effects

\begin{tabular}{|c|c|c|c|c|}
\hline & \multirow[b]{2}{*}{ Bronchodilation } & \multicolumn{2}{|l|}{ Protection } & \multirow[t]{2}{*}{ Improvement ${ }^{b}$} \\
\hline & & Allergens ${ }^{a}$ & Histamine & \\
\hline \multicolumn{5}{|l|}{ Nonbronchodilators } \\
\hline Cromolyn & - & I, L, BHR & - & $++^{c}$ \\
\hline Nedocromil & - & I, L, BHR & - & +++ \\
\hline Inhaled steroids & - & L, BHR & - & +++ \\
\hline Antileukotrienes $^{d}$ & ++ & $\mathrm{I}, \mathrm{L}$ & - & +++ \\
\hline Zileuton & ++ & $\mathrm{I}, \mathrm{L}$ & ND & ND \\
\hline Zafirlukast & \pm & $\mathrm{I}, \mathrm{L}$ & ND & ND \\
\hline Montelukast & ++ & $\mathrm{I}, \mathrm{L}$ & ++ & ND \\
\hline \multicolumn{5}{|l|}{ Bronchodilators } \\
\hline \multicolumn{5}{|l|}{$\beta$-Agonists } \\
\hline Short-actinge & +++ & 1 & +++ & - \\
\hline Salmeterol & +++ & $\mathrm{I}, \mathrm{L}$ & +++ & - \\
\hline Theophylline & ++ & $\mathrm{I}, \mathrm{L}$ & + & + \\
\hline Anticholinergic agents & + & - & ND & ND \\
\hline
\end{tabular}

Data from $[124,617,634,635]$.

ND not done.

a Blocks immediate $(I)$ and/or late-phase $(L)$

reactions and the consequent bronchial hyperreactivity.

b BHR reduction by chronic therapy.

c Full-dose therapy.

d Montelukast, personal data.

e Salbutamol.

with SC epinephrine compared to those given terbutaline inhalation (11\%). In Chap. 20, b/w based doses of inhaled epinephrine are detailed. In case of a severe attack, epinephrine remains the preferred drug [352].

$\beta_{2}$-Adrenergics for predosed MDIs or nebulizers (Fig. 11.40) offer the best results because of rapidity of action, good efficacy and limited adverse effects on the cardiovascular apparatus. Given the recognized benefits of short-acting $\beta_{2}$-adrenergics, attention was directed to extending the duration of effect of $\beta_{2}$-adrenergic agonists. As a result, long-acting $\beta_{2}$-adrenergics with a 12-h duration of action were introduced: salmeterol and formoterol. For an immediate effect, lasting 4-6h, albuterol is recommended: to limit the adverse effects of short-acting $\beta$-agonists, the stereoisomer of albuterol, levalbuterol, compared to racemic albuterol significantly reduced the number of hospitalizations in the ED management of acute asthma in children aged 1-18, but the hospitalization length of stay was not significantly shorter between the two groups [84]. For an effect with 5 min of latency, but with long-term effect $(12 \mathrm{~h})$, salmeterol $25 \mu \mathrm{g}$ is recommended. A dose of $50 \mu \mathrm{g}$ has induced a significant HR increase in the first $5 \mathrm{~min}$ [19] in children of $13.4 \pm 2.5$ years. Formoterol has the same latency and lasts $12 \mathrm{~h}$ [441], while systemic administration of formoterol (via the oral route) displays a short duration of action (4h). Salmeterol is a long-acting $\beta_{2}$-agonist with a long side chain, diffuses quickly from

vials are available. Symptoms of epinephrine overdose (Chap. 20) may be frequent ( $47 \%)$ in children treated 
Table 11.16. Pediatric doses and routes of administration of epinephrine and main $\beta_{2}$-adrenergic (quick-relief) medications

\begin{tabular}{|c|c|c|c|}
\hline Medication & Route & Dosage form & Dose \\
\hline $\begin{array}{l}\text { Adrenaline } \\
\text { (epinephrine) }\end{array}$ & SC & $\begin{array}{l}1 \mathrm{ml} \text { vial of a } \\
\text { 1:1,000 dilution }\end{array}$ & $\begin{array}{l}0.01 \mathrm{ml} / \mathrm{kg} \text { repeated q } 15 \mathrm{~min} \text { for } 3-4 \text { doses }^{\mathrm{a}} \\
\text { as needed. Up to a maximum of } 0.3-0.5 \mathrm{ml} \\
\text { per dose or inhaled (Chap. 20) }\end{array}$ \\
\hline Racemic & $\begin{array}{l}\text { Nebulizer solution } \\
(2.25 \%)\end{array}$ & & $\begin{array}{l}0.1 \mathrm{ml} / \mathrm{kg} \text { diluted to a total of } 2 \mathrm{ml} \text { in } 0.9 \% \mathrm{NaCl} \text {, } \\
\text { or " } 2 \text { years: } 0.3 \mathrm{ml} \text { in } 1.5 \mathrm{ml} \text { of saline } \\
<2 \text { years: } 0.5 \mathrm{ml} \text { in } 2.0 \mathrm{ml} \text { of saline }\end{array}$ \\
\hline \multirow[t]{7}{*}{$\begin{array}{l}\text { Albuterol } \\
\text { (salbutamol) }\end{array}$} & Oral & $\begin{array}{l}\text { Tablets } 2-4 \mathrm{mg} \\
\text { Syrup } \\
(10 \mathrm{ml}=4 \mathrm{mg})\end{array}$ & $\begin{array}{l}\text { Children }<6 \text { years } 0.1-0.2 \mathrm{mg} / \mathrm{kg} \times 3-4 \text { daily } \\
\text { Children } 6-12 \text { years } 2 \mathrm{mg} / \mathrm{kg} \times 3-4 \text { daily } \\
\text { Children }>12 \text { years } 2-4 \mathrm{mg} \times 3-4 \text { daily }\end{array}$ \\
\hline & Rotocap DPI & (200 $\mu \mathrm{g} /$ capsule) & $200 \mu \mathrm{g} \times 3-4$ daily prn or $\mathrm{q} 4-6 \mathrm{~h}$ \\
\hline & \multicolumn{2}{|l|}{ Nebulizer solution $(0.6 \%)$} & $2.6 \mathrm{mg}(0-2$ years) to $5 \mathrm{mg}$ ( $2-5$ years) prn or q $4-6 \mathrm{~h}$ \\
\hline & MDI & (100 $\mu \mathrm{g} /$ puff) & $100-200 \mu g$ (1-2 puffs prn or 3-4 puffs daily) \\
\hline & IM/IV vials & $100 / 500 \mu \mathrm{g}$ & $2-6 \mu \mathrm{g} / \mathrm{kg} \times 3-4$ daily \\
\hline & Levalbuterol & $\begin{array}{l}\text { Unit dose vials of } \\
0.31 \mathrm{mg}, 0.63 \mathrm{mg}, 1.25 \mathrm{mg}\end{array}$ & Pediatric 1.25 mg qid, or q 20 min (max 6 doses) \\
\hline & Racemic & $\begin{array}{l}\text { Solution of } 5 \mathrm{mg} / \mathrm{ml} \\
\text { or unit dose vials }\end{array}$ & Pediatric 2.5 mg qid, or q $20 \min$ ( $\max 6$ doses) \\
\hline \multirow[t]{2}{*}{ Bambuterol } & \multirow[t]{2}{*}{ Oral } & $\begin{array}{l}\text { Solution in 2- to } \\
5 \text {-year-old children }\end{array}$ & $10 \mathrm{mg}$ in the evening \\
\hline & & $\begin{array}{l}\text { Tablets in 6- to } \\
\text { 12-year-old children }\end{array}$ & $10-20 \mathrm{mg}$ in the evening \\
\hline \multirow[t]{2}{*}{ Bitolterol } & MDI solution $0.2 \%$ & (1 puff $=0.5 \mathrm{mg})$ & Children 4-12 years 1-2 puff q 4-6h prn \\
\hline & \multicolumn{2}{|l|}{ Nebulizer solution $(0.1 \%)$} & $1 \mathrm{mg}$ (range $0.5-1.5 \mathrm{mg}$ ) q 4-6 h prn \\
\hline \multirow[t]{3}{*}{ Fenoterol ${ }^{b}$} & Oral & Syrup $0.05 \%(10 \mathrm{ml}=5 \mathrm{mg})$ & $0.1-0.2 \mathrm{mg} / \mathrm{kg} \times 3-4$ daily \\
\hline & \multicolumn{2}{|l|}{ Nebulizer solution $0.1 \%$} & $\begin{array}{l}\text { Children }<6 \text { years } 50 \mu \mathrm{g} / \mathrm{kg} \\
\text { Children }>6 \text { years } 100 \mu \mathrm{g} / \mathrm{kg} \\
\times 1-3 \text { daily }\end{array}$ \\
\hline & MDI & (100 $\mu \mathrm{g} /$ puff) & $100-200 \mu \mathrm{g}$ (1-2 puffs) x 3-4 daily \\
\hline \multirow[t]{2}{*}{ Formoterol } & MDI & (9-12 $\mu \mathrm{g} /$ puff) & Children $>6$ years $1-2$ puffs $\times 2$ daily or $\times 3-4$ daily prn \\
\hline & DPI Turbohaler & (9-12 $\mu \mathrm{g} /$ capsule) & Children $5-12$ years $12-24 \mu \mathrm{g} \times 2$ daily \\
\hline Pirbuterol & \multicolumn{2}{|l|}{ MDI $250 \mu \mathrm{g} /$ dose } & $>12$ years 2 puffs $\times 3-4$ daily or prn \\
\hline \multirow[t]{3}{*}{ Procaterol } & \multirow[t]{2}{*}{ Oral } & Tablets $5 \mu \mathrm{g}$ & $1 \mu \mathrm{g} / \mathrm{kg} \times 2$ daily \\
\hline & & $\begin{array}{l}\text { Syrup } 5 \mu \mathrm{g} / \mathrm{ml} \\
(10 \mathrm{ml}=50 \mu \mathrm{g})\end{array}$ & $1 \mu \mathrm{g} / \mathrm{kg} \times 2$ daily \\
\hline & MDI & (10-25 $\mu \mathrm{g} /$ puff) & $10-25 \mu \mathrm{g}(1-2$ puffs $) \times 2$ daily \\
\hline Salmeterol & MDI & (25 $\mu \mathrm{g} /$ puff) & $25-50 \mu \mathrm{g}(1-2$ puffs $) \times 2$ dailyc \\
\hline \multirow[t]{4}{*}{ Terbutaline } & \multirow[t]{2}{*}{ Oral } & Tablets $2.5-5 \mathrm{mg}$ & $\begin{array}{l}\text { Children }<12 \text { years } 0.05-0.1 \mathrm{mg} / \mathrm{kg}(\max 5 \mathrm{mg}) \mathrm{q} \\
6-8 \mathrm{~h} \text {, } \\
\text { Children } \geq 12 \text { years } 2.5-5 \mathrm{mg} \mathrm{q} 6-8 \mathrm{~h}\end{array}$ \\
\hline & & Solution (0.1\%) & $0.01 \mathrm{ml} / \mathrm{kg}$ up to $0.3 \mathrm{ml} \mathrm{SC}$ \\
\hline & $1 \mathrm{mg} / \mathrm{ml}$ & & q 2-6 h prn \\
\hline & MDI & (250 $\mu \mathrm{g} /$ puff) & $0.25 \mathrm{mg}$ dose (1 puff) q 6-8 h prn \\
\hline
\end{tabular}

Two dosages (10-25 $\mu \mathrm{g} /$ puff) means that two different preparations are available, or that two different doses are suggested. The rate of inhaled drug may be lower than $30 \%$ (Table 11.11). In small children, spacers with a mask are imperative. Epinephrine is suggested for babies with bronchiolitis and children with status asthmaticus. MDI metered-dose inhaler, DPI dry-powder inhaler.

Data from [35, 56, 158, 406, 437, 552, 583, C Kercsmar, pers. comm. Nov 30, 2005] and Aucoin RG. Respiratory pharmacotherapy (Accessed at http://search.yahoo.com/search?fr=fp-pull-web-t\&p; http://picuBOOK.net/1999/04-001(e1).htm).

a Acts within 5-6 s, duration is of $30 \mathrm{~min}$; more data on epinephrine and terbutaline are in Chap. 20.

b Decreased doses have been suggested, see text for details.

c Adult dosage, the 25 - to $50-\mu$ g dose should be the highest in children aged 6-12 years [582]. 
the aqueous phase into cell membranes of the lung with maximal bronchodilation $\approx 1 \mathrm{~h}$ after administration. Formoterol has a medium-sized side chain and diffuses both into the aqueous phase and cell membranes of the lung with an onset of action within minutes. Two recent articles have reported that formoterol provided superior bronchodilator efficacy over $12 \mathrm{~h}$ compared with salmeterol [501], or at least as effective as salmeterol [183]. So once daily oral bambuterol may be an interesting and less expensive alternative to twice-daily inhaled formoterol and salmeterol [158]. The increased efficacy of the first (single) dose compared to regular follow-up treatment should be kept in mind, being equal to a $\mathrm{PD}_{20}$ improvement of 1.7 or 0.7 [680]. Therefore, with the new, long-acting $\beta_{2}$-adrenergics the late reaction is inhibited and adverse effects are reduced. Nevertheless, some undesirable side effects can result, especially with continued and/or high-dose use:

- Muscular effects: muscle tremors (as a result of action on $\beta_{2}$ muscle receptors)

- Metabolic effects: hyperglycemia (for glycogenolysis) with hyperinsulinism, hyperkalemia and therefore hypokalemia as a result of $\mathrm{K}^{+}$deposits at the cellular level

- CNS effects: agitation, insomnia

- Cardiovascular effects (in cases of overdose): tachycardia, extrasystole, hypertension

Possible effects resulting from prolonged usage include:

- Tachyphylaxis (reduction in therapeutic responsiveness)

- Difficult management of the disease: asthma worsening, BHR increase [571] due to tolerance increase [456]

\section{An Assessment of $\beta_{2}$-Adrenergics}

We draw attention to the results of certain studies focusing on $\beta_{2}$-adrenergic use, especially when used over a prolonged period and/or in high dosages [697].

1 . Sears et al [571] obtained better results when $\beta_{2^{-}}$ adrenergic drugs were used only as needed. Control of the disease is reduced in patients who treat asthma with bronchodilators continually, for a long time and regularly, patients becoming dependent, and thus inhaling ever higher doses: in this way desensitization of $\beta$-adrenergic receptors occurs, that is repeated administration reduces the bronchodilator effect.

2. According to Page [467], this is probably caused by a disrupted equilibrium between inflammatory and antiinflammatory factors, as a result of reduced anti-inflammatory release, caused by the powerful antidegranulation accomplished by $\beta_{2}$-adrenergics on mast cells. In time, this leads to the deactivation of natural defense mechanisms, allowing the inhaled allergens to penetrate in the bronchial tree more deeply [467], with loss of the action inhibiting BM thickening and smooth muscle hypertrophy, deleterious aspects for asthmatics [516] likely leading to an irreversible chronicity [467].
3. According to Spitzer et al [608], these drugs aggravate asthma, rendering the airways more susceptible to stimuli. In effect, though reducing the acute episode, they promote delayed bronchial inflammation, which is at the basis of chronic asthma.

4. Burrows and Lebowitz [62] agree with these findings and advise against regular, high dosages of $\beta_{2}$-adrenergics.

5. Prospective studies in children have shown that prolonged usage of $\beta_{2}$-adrenergics does not reduce BHR [571].

6. In patients treated with terbutaline, a rebound type of BHR can occur when treatment is stopped: conversely, in children 1.5-11 years admitted to an ICU, the same drug was shown to be safe and effective [288].

7. It should be noted that for $\beta_{2}$-adrenergics, dosages that double the usual ones, even if only for 7 days, amplify airway reactivity even in the late reaction [110]. Chronic use of high doses results in hypokalemia, because ATP stimulation causes $\mathrm{K}^{+}$passage within the skeletal muscles, an effect that is strengthened by hypercapnia, hypoxia and hypokalemia, characteristics of acute asthma-status asthmaticus [310, 697]; even theophylline can provoke hypokalemia, and together with CSs, hyperkaluria [310].

8. Few and contradictory results have been obtained with these drugs in bronchiolitis: epinephrine was more efficacious than salbutamol [401]. Probably, the negative results depend on having measured the Raw index of seriously ill infants with widespread edema and mucous secretions, thereby reducing the dose of the aerosolized drug [112]. Moreover, the total resistance of upper airways can be reduced by $50 \%$ at this age, making measurements unreliable [206]. This can also be related to a reduced response to $\beta$-adrenergic stimuli effected by respiratory viruses and therefore to that of $\beta$-adrenergic drugs [73].

9. In conclusion, there is no clear benefit of using $\beta_{2^{-}}$ adrenergics in the management of recurrent wheeze in the first 2 years of life, although there is conflicting evidence [140].

\section{Anticholinergic Drugs}

These drugs stimulate the muscarinic receptors with reduction in intracellular cGMP by competitively inhibiting $\alpha$-adenylcyclase [77]. The bronchodilator effect works on medium- or large-caliber bronchi; it is less rapid (15 min after administration, maximum effect after $30 \mathrm{~min}$ to $2 \mathrm{~h}$ ), but longer-lasting than short-acting $\beta_{2}$-adrenergics (exhausted after 3-5 h). They should be used in the intercritical periods, or in association with inhaled $\beta 2$-adrenergics, which allows the dose of the latter to be reduced. This also reduces the risk of side effects as a consequence of the therapeutic margin of these drugs [79]. 
IB has an anticholinergic activity that is similar to that of atropine, but also a reduced systemic absorption. IB is most often used with other drugs, providing good bronchodilator action and few side effects (mucosa dryness) [371, 663] when utilizing a DPI - preferred by young children rather than an MDI [663] - or a spray with spacer [371].

\section{Theophylline Drugs}

Theophylline drugs are enjoying a renaoissance mainly because of their rediscovered anti-inflammatory properties [274]. Theophylline is a methylxanthine similar to theobromine and caffeine. The mechanisms of its action $[124,635,740]$ and the anti-inflammatory effects $[101$, $114,461,658]$ are summarized in Tables 11.17 and 11.18 $[101,114,124,461,635,658,736]$, from which the induction of apoptosis in eosinophils clearly emerges (Fig. 11.41). Bronchodilation is caused by PDE inhibition, which causes an increase in cAMP levels. It also displays considerable anti-inflammatory properties (Table 11.14) and promotes the production of anti-inflammatory $\mathrm{IL}, \mathrm{IL}_{10}$, deficient in asthmatic airways, depressing instead that of IFN- $\gamma$ [513]. Significantly, in young asthmatics, abatement of IgE levels occurs with slow-release theophylline, but not with cromolyn or BPD [658].

In examining the pharmacological aspects, it is useful to review a few basic facts $[404,706]$ :

- The therapeutic range of theophylline is between 10 and $20 \mu \mathrm{g} / \mathrm{ml}(56-111 \mu \mathrm{mol} / \mathrm{l})$, even though it is known that bronchodilation can take place with therapeutic levels of $5-15 \mu \mathrm{g} / \mathrm{ml}$. Below the minimum level the effects are few and side effects - even severe can occur when the theophylline level exceeds 15$20 \mu \mathrm{g} / \mathrm{ml}$. Thus the therapeutic range is extremely close to the toxic range.

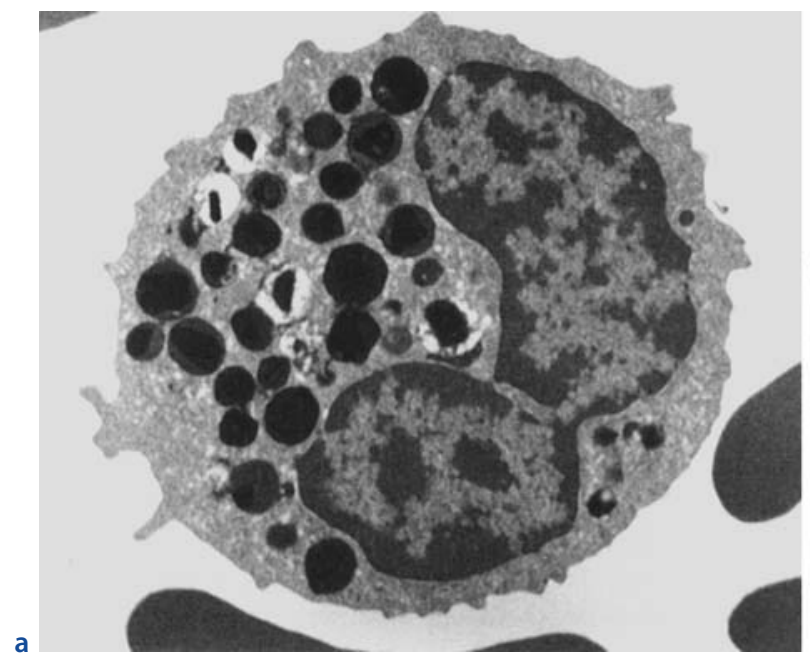

Fig. 11.41 a,b. Morphological changes of eosinophils by adding theophylline to culture medium with $\mathrm{IL}_{5}$. a The eosinophils, without additions, are "normal" in shape. b By
Table 11.17. Anti-inflammatory action of theophylline

\begin{tabular}{l} 
Increases mucociliary transport \\
\hline Increases diaphragmatic contractility \\
\hline Decreases muscle work \\
\hline Bronchodilation \\
\hline Inhibits phosphodiesterase \\
\hline Suppresses histamine release by mast cells \\
and basophils \\
\hline Inhibits vascular permeability \\
\hline Inhibits PG and LT \\
\hline Increases cAMP intracellular concentrations \\
Inhibits immediate and delayed asthmatic reactions, \\
a change not observed in any other group \\
Inhibits airway responsiveness
\end{tabular}

Data from $[124,635,735]$.

Table 11.18. Immunomodulatory effects of theophylline

Inhibits neutrophil and mononuclear cell chemotaxis

Suppresses $\lg \mathrm{M}$, IgG and $\lg \mathrm{A}$ synthesis

Reduces serum IgE levels in 4- to 6-year-old asthmatics

Inhibits TNF- $\alpha$ and $\mathrm{IL}_{1 \beta}$ expression and release

Reduces CD3, CD4, CD8, CD15, and CD25 cell counts

Enhances trafficking of peripheral blood-activated T cells

Inhibits eosinophil influx after allergen challenge and cationic protein production

Enhances eosinophil apoptosis

Data from $[104,114,658]$.

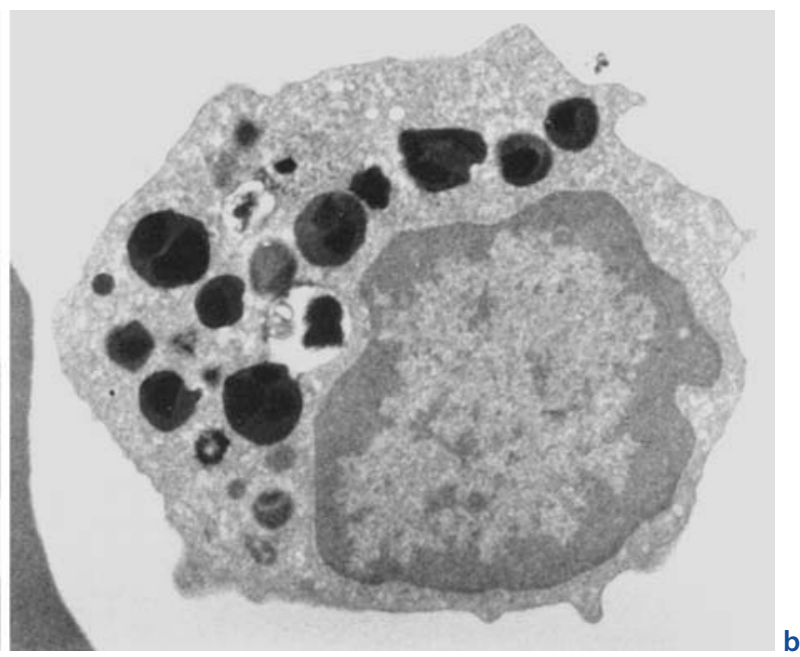

adding theophylline the cells show apoptotic changes including aggregated chromatin, smooth cell surface and cytoplasmic vacuoles 
- Theophylline metabolism results in $85 \%$ of theophylline being metabolized by the enzymatic system of hepatic cytochrome P450, so all factors or drugs that interfere with this system can alter its metabolism (Table 11.19) [244], increasing or reducing theophylline clearance, with reduction or, respectively, increase of its concentrations [706].

- Theophylline clearance (distribution volume $\times$ unchanging level of elimination) varies according to age [404]:

- From birth to 7-9 months, it is reduced.

- Between 7-9 months and 9 years of age it increases $(1.44 \mathrm{ml} / \mathrm{min} / \mathrm{kg})$; therefore, proportionately higher doses are required.

- From 9 to 16 years the clearance is reduced again $(9-12$ years $=1.26 ; 12-16$ years $=0.9 \mathrm{ml} / \mathrm{min} / \mathrm{kg})$.

Such levels are appropriate for good theophylline distribution and function. Other factors that must be borne in mind are [244]:

- Individual variations in the rate of absorption.

- Differences of half-life in children are half that of adults $(\approx 3.5 \mathrm{~h}$ instead of $7 \mathrm{~h})$.

- If the patient is obese, the correct dosage must be prescribed according to ideal standard weight, and not to the actual weight.

Theophylline absorption also depends on circadian rhythms. The indispensable interval of time to reach peak concentration of the medication is significantly shorter (and absorption increased) when oral doses are taken in the morning, compared to evening effects. Analogously, clearance is influenced: the elimination rate is greater with morning doses compared to evening doses in children treated with slow-release or IV medications, so a standard dosage with equal doses at regular intervals will provoke an increase in nocturnal serum levels compared to daytime levels, to the advantage of nocturnal asthma sufferers [32]. All this is of great importance in establishing dosages which must be personalized whenever constant monitoring for an entire $24 \mathrm{~h}$ period become necessary. Similarly, for the study of theophylline pharmacokinetics, the hours at which both doses and blood samples were taken must be recorded [244].

\section{Indications}

- Children with acute asthma who can benefit from theophylline, but reducing the respective doses when associated with $\beta_{2}$-adrenergic.

- Mild or moderate forms of acute attacks, if there has been no response to the $\beta_{2}$-adrenergics. Eventually it can be associated with them, again reducing the respective doses.

- Moderate to severe forms of chronic asthma resistant to other medications [707].

The scientific rationale behind theophylline lies in its anti-inflammatory quality, not shared by other medica-
Table 11.19. Factors and drugs affecting theophylline serum concentrations

Increased concentrations
(decreased clearance)

\section{(\%)}

\begin{tabular}{|c|c|}
\hline \multicolumn{2}{|l|}{ Physiological alterations } \\
\hline Bronchopulmonary dysplasia & $\begin{array}{l}\text { Variable, } \\
\text { notable }\end{array}$ \\
\hline Caffeine (tea, cacao, Coca-cola) & $?$ \\
\hline Cardiac and/or hepatic insufficiency & $\begin{array}{l}\text { Variable, } \\
\text { notable }\end{array}$ \\
\hline Febrile illness & $?$ \\
\hline High-carbohydrate and hypoproteic diets & $?$ \\
\hline Hypothyroidism & 50 \\
\hline Influenza A vaccine & $?$ \\
\hline Obesity & $?$ \\
\hline Premature and newborn babies & $?$ \\
\hline Prolonged fever (>24 h) & 50 \\
\hline Undernourishment & $?$ \\
\hline \multicolumn{2}{|l|}{ Drugs } \\
\hline Allopurinol (high doses) & 25 \\
\hline Cimetidine & 50 \\
\hline Ciprofloxacin & 30 \\
\hline Erythromycin & 25 \\
\hline Methotrexate & 20 \\
\hline Oral contraceptives & 30 \\
\hline Propanolol & 20 \\
\hline Troleandomycin & 50 \\
\hline Low dose & 25 \\
\hline
\end{tabular}

Decreased concentrations $\quad$ (\%) (increased clearance)

\begin{tabular}{ll} 
Physiological alterations & $?$ \\
\hline Cystic fibrosis & 20 \\
\hline Hyperthyroidism & $?$ \\
\hline Ingestion of meat cooked on coal-stoves & $?$ \\
\hline Low-carbohydrate and hyperproteic diets & $? 0$ \\
\hline Tobacco smoking & $?$ \\
\hline Young age (1-16 years) & $?$ \\
\hline Drugs & 60 \\
\hline Barbiturates & 20 \\
\hline Carbamazepine & 75 \\
\hline Isoproterenol (IV) & 80 \\
\hline Phenytoin &
\end{tabular}

Data from [244].

? Unknown rate. 
tions, which we have always taken into consideration. Logically it holds an undisputed and, to this day, unique place ensuring the compliance of young and very young children who are not used to taking inhalation drugs. According to Szefler [634], the clinical effect of chronic therapy with theophylline, cromones, and anti-LT on symptom scores, PEFR, and acute exacerbations (morning, or evening) is comparable.

\section{Dosages and Methods of Administration}

1. IV in severe asthma attacks to achieve a more rapid therapeutic effect.

2. Per os the absorption is slower; depending on the nature of the case the following can be used:

- Fast-acting theophylline.

- Long-acting theophylline, which, having a greater serum half-life, is preferable for long-term treatment. Also, it is suitable for the very young [77].

Dosages advised for fast-acting theophylline are as follows, four doses every $6 \mathrm{~h}[244,707]$ :

1. Initial dosage for children 6 months old: about $10 \mathrm{mg} /$ $\mathrm{kg} /$ day up to a maximum of $300 \mathrm{mg} /$ day. After 3 days, if the dose is tolerated:

- First increment: about $13 \mathrm{mg} / \mathrm{kg} /$ day up to a maximum of $450 \mathrm{mg} /$ day. After a further 3 days, if the dose is tolerated:

- Second increment: about $16 \mathrm{mg} / \mathrm{kg} /$ day up to a maximum of $600 \mathrm{mg} /$ day.

In view of the rapid metabolism that the drug undergoes in the child, an early and periodic monitoring of theophylline to verify that the levels are not subtherapeutic $(5.6 \mu \mathrm{g} / \mathrm{ml})$ is indispensable. We suggest measuring theophylline levels after at least 3 days to coincide with the administration of the maximum dose tolerated, 4 or $8 \mathrm{~h}$ after the morning dose, depending on the medication being used. Once theophylline levels have been monitored, the dosage should be adjusted based on serum concentrations in $\mu \mathrm{g} / \mathrm{ml}$ [244]:

$<7.5$ Increase dose about $25 \%$ (check serum theophylline levels for guidance in further dose adjustment).

$<7.6-10$ If tolerated increase dose about $25 \%$.

10-15 If tolerated maintain dose (recheck serum theophylline levels at 6- to 12 -month intervals).

15.1-19.9 Consider a 10\% dose reduction (to provide greater margin of safety.

20-25 Skip next dose and resume treatment based on the last increase tolerated.

25

Skip next two doses and resume the initial dosage, or that of the last increase.

Whenever side effects occur, dosage for all children should be reduced to the minimum dose previously tolerated.
2. Dosages for infants $<1$ year of age [244]:

- Initial dosage in $\mathrm{mg} / \mathrm{kg} /$ day: (0.2) (age in weeks) +5.0; this equation results in levels of theophylline between 5 and $10 \mathrm{mg} / \mathrm{l}$ [244]. Serum theophylline concentrations should be monitored within 6-12 h after start of treatment [256].

- Subsequent dosages should be based on serum theophylline levels measured at least 3 days after start of treatment.

The average dosage of long-acting theophylline is $7-10 \mathrm{mg} / \mathrm{kg}$ every $12 \mathrm{~h}$ [83]. This formula, having a greater half-life, is recommended for treating nocturnal asthma, among other reasons because of its protracted bronchodilator effect. Additionally, capsules that can be emptied and mixed with foods minimize the child's opposition [83]. For cases using long-acting theophylline, a noninvasive, reliable, and convenient method for measuring theophylline levels in saliva has been proposed [640]. Three different controls are recommended, to characterize around-the-clock theophylline profiles:

- Good symptom control, no side effects: measurement $4 \mathrm{~h}$ after morning dose, every 3-6 months

- Nonoptimal control: as above with the addition of a further measurement just before evening doses

- Poor or no clinical response: $24 \mathrm{~h}$ profile, even at home [640]

In conclusion, the use of theophylline has but one disadvantage: for safe treatment we recommend measuring theophylline levels to reach and maintain levels encompassed within the therapeutic range [706]. This is also valid to avoid undesired side effects [244].

Notwithstanding what has been said, theophylline is completely safe. A study of 35,000 patients who filled 222,000 prescriptions for 9 years registered only one case of seizures in a child (without specifying the cause), equal to $0.003 \%$ of cases and to $0.00045 \%$ of the prescriptions [404].

\section{Theophylline Assessment}

From time to time, North American and North European authors have underlined the negative effects of theophylline with the result that experts tend to relegate this drug to a secondary role among the forms of treatment $[272,435,504]$, thereby contributing to an unjustified decline in its use $[536,635]$. In particular, three recent studies $[89,148,622]$ excluded its use in hospital treatments of children aged 2-18 years with moderate to severe asthma and, specifically, in association with MDIdelivered albuterol and IV-administered methylprednisolone, even though no undesired side effects were found to justify the exclusion [622]. Nevertheless, the studies did not include patients in severe conditions, a limitation that prevents the evaluation of theophylline ability to preclude the necessity of ICU admission and/or assisted ventilation. Furthermore, in our opinion, the above-mentioned associations have not been 


\section{hydrocortisone}<smiles>CC12CCC(=O)C=C1CCC1C2C(O)CC2(C)C1CC[C@H]2O</smiles>

prednisolone<smiles>CC12C=CC(=O)C=C1CCC1C2C(O)CC2(C)C(=O)CCC12</smiles>

dexamethasone<smiles>C[C@H]1CC2C3CCC4=CC(=O)C=CC4(C)C3(F)C(O)CC2(C)C1C(=O)CO</smiles>

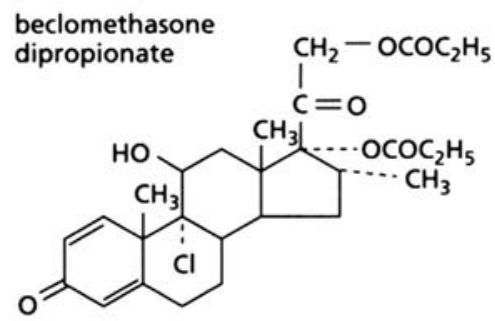<smiles>CCOCCO[C@H]1CC2C3CCC4=CC(=O)C=CC4(C)C3C(O)CC2C1(C(=O)CO)C(C)(C)C</smiles>

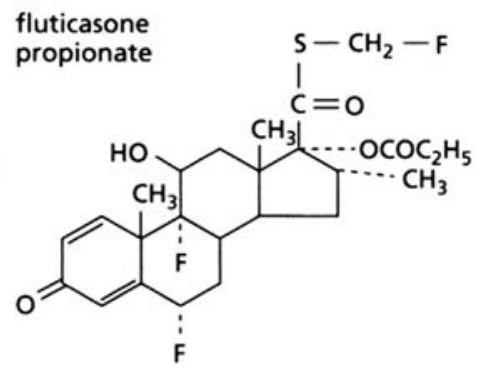

Fig. 11.42. Structural formulas of common corticosteroids.

properly evaluated since children had already reached maximum bronchodilation with "liberal and elevated" doses of nebulized albuterol [89]. Even in children or teenagers with nonsevere asthma treated with albuterol and IV-administered methylprednisolone, DiGiulio et al [148] did not find clinical differences between theophylline and placebo, although at the cost of possible type II errors. Although concern was expressed about the dangers of improper use of theophylline [89], no related adverse effects were reported in either study: nausea was the most toxic symptom encountered [622]. On the other hand, several studies have shown theophylline effectiveness (ref in 615). In patients admitted to ED and treated with theophylline, discharges were more frequent than admittance to hospital (6\%), which differs from patients treated with metaproterenol and cortisone $(21 \%)$, and with an overall cost fivefold lower [740]. Mechanical reasons, especially in life-threatening forms of infantile asthma, can impede $\beta_{2}$-adrenergic passage. This is theophylline's strong point: administered IV in these forms it produces an almost immediate clinically detectable, objective bronchodilation [664]. In 94 children aged 1-17 in severe status asthmaticus admitted to the PICU theophylline safely hastened the recovery of these children who were also receiving albuterol, IB, and CS therapies $[49,515]$.

On a therapeutic level, other side effects were noted [706] such as learning and behavioral problems. Re-examined by various researchers in ten studies, they were unable to definitively verify this correlation, partly due to methodological errors [123], or because the former were completely absent, whereas the rest normalized, or improved, within 2 weeks [613]. No school-related differences were found between asthmatics (whether theophylline-treated or not) and controls [353]. Parents were unable to decide to which treatment (theophylline or placebo) to attribute the improvement in memory and attention [34]. Other researchers, not finding any side effect, suggest that the potential vulnerability only concerns children already suffering from similar problems [562]. In a systematic review of 12 pediatric studies, no behavioral or cognitive adverse effects of theophylline were identified [597a].

\section{Corticosteroids}

The CS action mechanism (Tables 11.20 [23, 249, 284], 11.21 [120], $11.22[23,203,284,633]$ and 11.23 [56, 117, 437, 581], Fig. 11.42 [105]) is performed on adenylyl cyclase by increasing cAMP levels, and on the inflammation, reducing the edema and inhibiting histamine release [703]. In particular, cAMP level maintenance within a normal range is useful for bringing the adrenergic receptors back to a functional state in which they can again become activated [703]. CSs inhibit the metachromatic cell mediators and basophil releasability, and reduce circulating eosinophil number and presumably also those in the tissues, but not of macrophages [212]. These effects are due to IL inhibition that activates these cells and stimulates them into reaching inflammatory sites [570]. In asthmatic children ( $80 \%$ atopic), serum concentrations of $\mathrm{IL}_{5}, \mathrm{CD} 25$, and ECP remained significantly higher than controls, even after treatment with oral CSs. Thus, $\mathrm{T}$ cell-mediated inflammation may persist in childhood asthma despite apparent clinical remission associated with conventional treatment [172]. The most powerful action of endogenous CSs is directed against inflammation, markedly exacerbated if the adrenal gland is removed [570]. In this process, the main activity is aimed at suppressing the transcription of genes involved in inflammation, and, to this end, their re- 


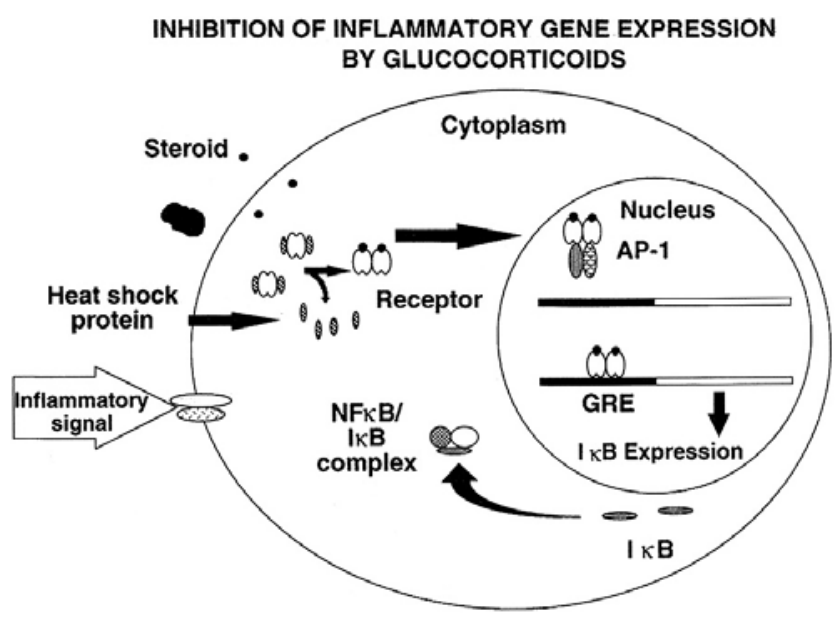

Fig. 11.43. Inhibition of inflammatory gene expression by CSs. $A P-1$ apolipoprotein $1,1 \kappa B$ inhibitor of NF- $k B, N F-\kappa B$ nuclear factor $\mathrm{kB}$

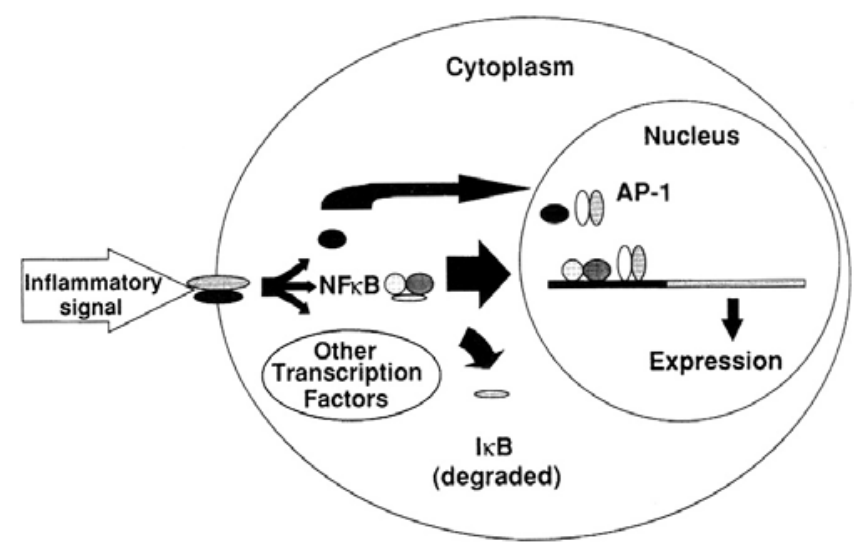

Fig. 11.44. Activation of inflammatory gene expression. $A P-1$ apolipoprotein $1, / \kappa B$ inhibitor of NF- $\mathrm{KB}$

ceptors interact with AP-1 (activator protein-1) and NF$\mathrm{\kappa B}$ (nuclear factor of chain $\kappa$ of Bs) (Fig. 11.43) [568] or, diversely, render more potent the inhibitor of the chain $\kappa$ of $B$, type $\alpha(\kappa B-\alpha)$, inhibitor of NF- $\kappa B$ (Fig. 11.44) [568]. However, the bronchial smooth cells lack the CCAAT/enhancer binding protein $\alpha(\mathrm{C} / \mathrm{EBP} \alpha)$ required to form a complex with the CS receptor, therefore CSs cannot contrast the ADAM33 accelerated proliferation of bronchial smooth-muscle cells and the resulting BHR [539]. From a clinical point of view, the latency time is lengthened $(6-12 \mathrm{~h})$ compared to other drugs [683], and a maximum bronchoprotecting effect can be attained after many months of continuous treatment [570]. Consequently, although CSs are an effective treatment on ameliorating asthma symptoms and represent the most effective anti-inflammatory therapy of asthma, this therapy is symptomatic since the disease flares when treatment is stopped [517, 645, 671, 680]. If ICSs are discontinued, the asthmatic children return to
Table 11.20. Molecular mechanisms of corticosteroid (CS) action in asthma

\section{A. Specific effects of CSs on transcription of genes relevant to asthma}

Increased transcription
a. Lipocortin-1
b. $\beta_{2}$-Adrenoreceptor
c. Endonuclease

Inhibition of transcription
a. Leukocyte protease
b. Cytokines

Decreased transcription
a. Cytokines $\left(\mathrm{IL}_{1}-\mathrm{IL}_{6}, \mathrm{IL}_{8}, \mathrm{IL}_{11}-\mathrm{IL}_{13}, \mathrm{IL}_{16}, \mathrm{TNF}-\alpha, \mathrm{GM}-\mathrm{CSF}\right)$
b. Chemokines (eotaxin, MIP-1 $\alpha$, RANTES, SCF)
c. iNOS
d. Inducible cyclooxygenase
e. Inducible $\mathrm{PLA}_{2}$
f. ET-1
g. CD54
h. $\mathrm{N \kappa}_{1}$ receptors

\section{B. Effect on IgE synthesis}

a. Reduction

b. Probable enhancement ${ }^{a}$

C. Effects on mast cells, basophils and eosinophils

a. Reduction in mast cell number

b. Inhibition of mast cell proliferation

c. Inhibition of mediator release

d. Inhibition of histamine synthesis

e. Inhibition of eicosanoid release

\section{Effects on lymphocytes}

E. Reduction in lymphocytes with activation markers (CD3, HLA-DR)

\section{F. Reduction in cellular traffic and functions}

G. Effect on specific processes

a. Inhibition of late reactions

b. Reduction in vascular permeability

c. Inhibition of mucosal secretion

d. Induction of vasoconstriction

\section{H. Potential relevant mechanisms}
a. Anti-inflammatory action
b. Increase in number and sensitivity of $\beta$-adrenergic receptors
c. Adenylate cyclase activation
d. Increased CAMP
e. Eosinophilopenia, basophilopenia, monocytopenia

The IL-13 inhibition by steroids may, at least in part, account for their therapeutic effects [150].

See Tables 7.17 and 7.18 for side effects of CSs employed topically in atopic dermatitis.

Data from $[23,249,284]$.

a See text. 
Table 11.21. Adverse effects of inhaled CSs in 163 children (\%)

\begin{tabular}{ll} 
1. Hypertension & 88 \\
\hline 2. Cushingoid features & 66 \\
\hline 3. HPA-axis suppression & 56 \\
\hline 4. Myopathy & 50 \\
\hline 5. Osteopenia & 46 \\
\hline 6. Growth suppression & 39 \\
\hline 7. Obesity & 30 \\
\hline 8. Hypercholesterolemia & 30 \\
\hline 9. Posterior subcapsular cataracts & 14
\end{tabular}

Osteopenia was strongly associated with growth suppression (odds ratio, 5.6). Note: $50 \%$ of children required chronically administered oral CS therapy.

Data from [120].

their baseline PFT and indices of airway inflammation after 2 weeks [593]. CSs do not relieve symptoms promptly and children may not improve within a few days [517]. So CS efficacy is significant in late asthmatic responses, chronic asthma and, more generally, in suppression of chronic inflammation [570]. This effective anti-inflammatory action on mast cells and eosinophils occurs in the epithelium (each 2.5-fold) and in submucosa (from 2- to 10-fold, respectively) and, on clinical grounds, in BHR improvement [258] (Table 11.14). Basically, there are no definitive effects, since the underlying inflammation is still active in childhood asthma despite apparent clinical remission even after years on ICSs [476]. Thus, any beneficial CS effect on BHR is not due to the prevention or resolution of remodeling of the airway wall [645]. The reason is that CSs have marked effects in inhibiting T-cell activation, but these effects are not reflected in changes of overall cell numbers in the circulation. So there is neither a discernible effect on the underlying mechanisms of inflammation [208], nor on methacholine responsiveness [645]. In addition, during the period of alveolar development, CS administration may result in decreased lung-cell mass and in the presence of too few abnormally large alveoli [727]. Also, for this reason it may be prudent to avoid CS use in the very young, because they appear not to be very effective, as is also demonstrated by their lack of impact on PFT [620]. $\mathrm{FP}$, mometasone furoate (MF) and, to a lesser extent, BDP are thought to be second-generation ICSs in that they display both increased anti-inflammatory potency and systemic bioavailability [434].

\section{An Assessment of Corticosteroids}

In addition to dysphonia and oral candidiasis, several undesirable effects of inhaled CS (including osteoporosis) occurred in 163 children aged $14.4 \pm 2.1$ years with
Table 11.22. Mechanisms possibly explaining a poor response to CSs in asthma

1. Additional respiratory disorders (cystic fibrosis, vocal cord dysfunction, etc.)

2. Overwhelming allergen exposure

3. Irreversible airway hyperreactivity

4. Poor compliance (children, parents, etc.)

5. Psychological problems

6. Inadequate dose for severity of asthma

7. Pharmacokinetic motives
a. Rapid metabolism
b. Poor distribution at the site of action
c. Poor/partial absorption from oral administration

8. Immunological resistance

\section{A. Monocytes}

a. Unable to decrease MCR and CRI expression

b. Unable to decrease PHA-induced proliferation

c. Unable to decrease cytokine production (TNF)

d. Quantitative and functional defect of intracytoplasmic receptors

\section{B. Lymphocytes}

a. Unable to decrease PHA-induced proliferation

b. Unable to decrease cytokine production $\left(\mathrm{IL}_{2}\right.$, IFN- $\gamma$ )

c. Unable to inhibit activation (CD25, HLA-DR)

\section{Abnormal receptor or postreceptor} down-regulation

The use of high doses of inhaled $\beta_{2}$-agonists in acute asthma exacerbations may result in resistance to high-dose intravenous CSs in the treatment of these exacerbations [23].

Data from [23, 203, 284, 633].

CAMP cyclic adenosine monophosphate, CRE complement receptor increase, HPA hypothalamic-pituitary-adrenal, $M C R$ monocyte complement receptor.

severe asthma receiving high-dose inhaled CS therapy $(1675 \pm 94 \mu \mathrm{g} / \mathrm{d}), 50 \%$ of whom required chronic oral CS therapy [120] (Tables 11.20-11.22) [21, 120, 203, 249, 284, 633]. Pharmacokinetics studies suggest that the fraction deposited in the airways is absorbed in an active form in variable concentrations, and passes, in turn, into the circulation. In fact $80 \%$ of the amount deposited in the oropharynx is absorbed by the intestine, passing through the liver where most of it is metabolized. Part of it reaches the systemic circulation where it joins with the amount originated in the airways. These two rates are aggregated, but are continually reduced as a result of recirculation and hepatic deactivation, as is evident from Fig. 11.45 [703]. Nonetheless, there is a different bioavailability (the drug rate bypassing the hepatic filter) and a rapid CS metabolic clearance CS reaching the circulation $[22,359]$. Substandial differences can 


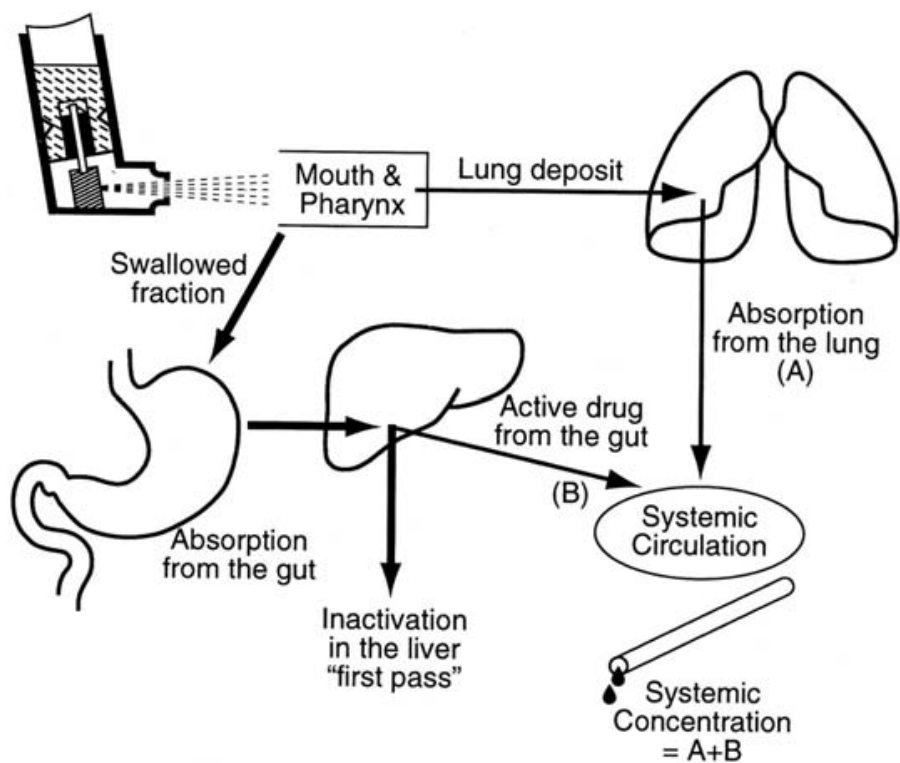

Fig. 11.45. The fate of inhaled CS. The amount of an inhaled CS that reaches the systemic circulation is the sum of pulmonary and orally bioavailable fractions. The fraction deposited in the oral cavity will be swallowed and systemic availability will be determined by absorption from the gastrointestinal tract and the degree of liver inactivation. The systemic concentration will be reduced by continuous recirculation and liver inactivation

Table 11.23. Usual dosage for corticosteroids in pediatric asthma

\begin{tabular}{|c|c|c|c|}
\hline Medication & Route & Dosage form & Dose \\
\hline \multirow[t]{2}{*}{$\begin{array}{l}\text { Beclomethasone } \\
\text { Dipropionate }\end{array}$} & Aerosol & Metered-dose inhaler & $\begin{array}{l}42 \mu \mathrm{g} \text { inhalation } \\
2-4 \text { puffs } \times 2-4 \text { daily }\end{array}$ \\
\hline & Inhalation & Dry-powder inhaler & $\begin{array}{l}100 \mu \mathrm{g} \\
4 \text { inhalations daily }\end{array}$ \\
\hline Betamethasone & Oral & $\begin{array}{l}\text { Tablets, } 0.6 \mathrm{mg} \\
\text { Solution, } 0.6 \mathrm{mg} / \mathrm{ml}\end{array}$ & $0.1-0.2 \mathrm{mg} / \mathrm{kg}$ daily \\
\hline Budesonide $^{a}$ & Aerosol & Metered-dose inhaler & $\begin{array}{l}200 \mu \mathrm{g} \text { inhalation } \\
1 \text { inhalation } \times 2 \text { daily }\end{array}$ \\
\hline \multirow[t]{2}{*}{ Deflazacort } & Oral & Tablets, 6-30 mg & $1.2-2.4 \mathrm{mg} / \mathrm{kg}$ daily \\
\hline & Oral & Drops, 1 drop/mg & $1-2 \mathrm{mg} / \mathrm{kg}$ daily \\
\hline Dexamethasone & Oral & Tablets & $\begin{array}{l}0.1-0.2 \mathrm{mg} / \mathrm{kg} \text { daily } \\
0.5-0.75 \mathrm{mg}\end{array}$ \\
\hline Flunisolide & Aerosol & $\begin{array}{l}\text { Solution } 0.1 \%(1 \mathrm{mg} / \mathrm{ml}) \\
\text { Metered-dose inhaler }\end{array}$ & $\begin{array}{l}0.5-1 \mathrm{mg} \times 2 \text { daily } \\
250 \mu \mathrm{g} \times 2-3 \text { puffs daily }\end{array}$ \\
\hline Fluticasone propionate & Aerosol & $\begin{array}{l}\text { Metered-dose inhaler } \\
\text { DPI 30, 100, } 250 \mu \mathrm{g} / \text { dose }\end{array}$ & $\begin{array}{l}44 \mu \mathrm{g} \times 2-4 \text { Puffs } \\
50 \mu \mathrm{g} 2-4 \text { inhalations }\end{array}$ \\
\hline Methylprednisolone & Oral & 2-, 4-, 8-, 16-, 32-mg tablets & $0.25-2 \mathrm{mg} / \mathrm{kg}$ daily as needed \\
\hline Mometasone furoate & Inhalation & Dry-powder inhaler & $440 \mu \mathrm{g}$ once-daily \\
\hline Prednisone & Oral & $1-, 2.5-, 10-, 20-, 25-\mathrm{mg}$ tablets & $1-2 \mathrm{mg} / \mathrm{kg}$ daily \\
\hline Prednisolone & Oral & 2.5- to 5-mg tablets & $0.5-2 \mathrm{mg} / \mathrm{kg}$ daily \\
\hline Triamcinolone acetonide & Aerosol & Metered-dose inhaler & $\begin{array}{l}100 \mu \mathrm{g} \text { inhalation } \\
4-8 \text { puffs daily }\end{array}$ \\
\hline
\end{tabular}

Data from $[56,117,437,495,581]$.

a by Turbohaler.

also derive from the metabolism of single molecules. BDP is metabolized in monopropionate in various tissues, including the airways, but it is not clear which rates are absorbed or metabolized in humans. FP (fluticasone propionate) and BUD, among the commercialized molecules, appear to be equipped with the lowest oral bioavailability as a result of a very high liver metabolism of first passage, so that $99 \%$ of FP and $90 \%$ of BUD are 
deactivated by the liver, and only a limited amount, equal to $1 \%$ and $10 \%$ [23], reaches the circulation. Side effects are few with preparations active by inhalation and with reduced systemic absorption, which, undoubtedly, have resulted in therapeutic progress - among these preparations are BDP, BUD, deflazacort, flunisolide [26], even if giving rise to controversy [668]. FP, because of its more favorable risk-benefit ratio, is preferable to BDP for the long-term treatment of children with asthma, especially if moderate doses are required [135]. It should, however, be clarified that with inhaled doses of $400 \mu \mathrm{g} /$ day and using a valve spacer, the systemic absorption is reduced to zero, given that all of the drug ingested remains in the spacer, thereby eliminating any difference in the bioavailability of different medications [359]. Both BPD used with the Volumatic or the Jet as well as BUD taken with Nebuhaler have a lesser systemic activity compared to the same dose inhaled with an MDI or a DPI, even if rinsing the mouth after use reduces or abates ingestion, as previously mentioned [26]. Additionally, drop formulations (deflazacort is prescribed in doses of $1-2 \mathrm{mg}=1-2 \mathrm{drops} / \mathrm{kg}$ ) favor personalized prescriptions and ensure compliance in younger children.

\section{Suppression of the Hypothalamic- Pituitary-Adrenal (HPA) Axis}

Other adverse phenomena, such as HPA axis suppression, growth retardation $[120,285]$, and are, in our opinion, provoked by prolonged treatment and by administering high dosages, chiefley orally [120], which can lead to a potential axis inhibition, with reduced ACTH secretion due to negative feedback mechanisms and the adrenal gland inability to respond to adrenocorticotropic stimulation, giving way to severe risks in patients prone to respiratory arrest during severe acute attacks [517]. A complete functional restoration may require up to 1 year, whereas treatment limited to 1 week is usually devoid of negative consequences: $8-10$ days after stopping treatment, HPA function returns to normal [560]. In adults, a possible axis deficiency can be revealed only through metapyrone or insulin tolerance tests, or through a CRH test [560]. Recent data suggest that $\mathrm{CRH}$ and its CS regulation may be essential for the control of airway inflammation and the maintenance of lung homeostasis in asthma [588]. Several studies have not found such anomalies $[487,684]$, or a small but significant degree of growth impairment in children receiving only $400 \mu \mathrm{g} /$ day of BDP [157]. Regarding the dosage factor, important undesired systemic effects of chronically and systemically administered medications (Tables 11.20-11.22) are found in children 7-10 years old with severe asthma, treated with doses of FP increased from $800-1500 \mu \mathrm{g}$ up to $2,250 \mu \mathrm{g} / \mathrm{day}$, leading to an increased risk of systemic side effects such as undetectable cortisol levels [650]. Acute adrenal crises have been reported by several studies, all in children treated with inhaled FP [630, 650-652, 736, 757] (see "Linear
Growth"). Similarly, 22 children aged $3.3-10$ years (with an incidence of $3.1 \%$ of total cases) treated with inhaled FP (91\%), with doses ranging from 500 to $2,000 \mu \mathrm{g}$ /day presented with acute adrenal crisis, acute hypoglycemia, and with decreased levels of consciousness, coma, or coma and convulsions. The remaining six children presented mainly with lassitude, weakness, nausea, and dizziness [652]. Severe growth retardation and adrenal suppression were described in children taking FP doses $>1,000 \mu \mathrm{g} /$ day [715], and acute adrenal crisis associated with inhaled FP in three children presenting with hypoglycemic coma and convulsions [651]. A probable explanation for these findings is that FP taken twice a day accumulates in the blood, which has a half-life of 7-8 h and a distribution 4 to 5 -fold greater than other drugs, leading to an increased risk of systemic side effects such as growth retardation and adrenal insufficiency [61]. HPA was also reported in 4 children aged $4.2-4.8$ years receiving FP $500 \mu \mathrm{g} / \mathrm{day}$, in 1 child aged 7.2 treated with FP 1,000 $\mu \mathrm{g} /$ day, in 3 children aged 4.8-6.1 treated with BUD $400 \mu \mathrm{g} /$ day and a 9-year-old treated with BDP $600 \mu \mathrm{g} /$ daily [475]. Adrenal suppression was found in 9-18 children aged $7-17$ receiving FP $477 \mu \mathrm{g} / \mathrm{m}^{2} /$ day for 5-16 weeks [368]. Given to asthmatic children in high dosages, flunisolide has provoked no negative effects on the axis, nor on glycolipid metabolism [215, 494]. These results have been confirmed with BUD, administered for 3-5 years in doses of $200 \mu \mathrm{g} /$ day [685], with measurement of 24-h plasma and urine cortisol [684, 685], 24-h urinary cortisol alone [157, 487]. Yet, other studies found no HPA deficiency, nor growth deficiency in children [541] or in schoolchildren undergoing treatment with BUD [646]. In asthmatic children under treatment for 3 months with inhaled BDP in doses of $400 \mu \mathrm{g} /$ day, a $63 \%$ decrease of plasma cortisol after evening doses and a $29 \%$ decrease after morning doses [637] was observed. Such data were subsequently confirmed by reductions of serum cortisol [493], urinary cortisol [505], or both [439], after treatment with BDP and BUD in doses of $400 \mu \mathrm{g}$ [445], or with FP in doses of $250 \mu \mathrm{g}$ [757], and with an MDI without a spacer [220]. Among the possible causes could be the use of MDI spray cans without spacers, given that they increase oropharyngeal deposits [220].

\section{Bone Density}

Another question being debated is whether inhaled medications, properly used, have a negative effect on bone density, an effect which, however, is independent of HPA-axis suppression, possibly related to the inhibition of insulin-like growth factors [517]. Using the equivalent of $8 \mu \mathrm{g} / \mathrm{kg}$ of BDP [649], a child should be considered at risk of HPA-axis suppression or of delayed growth [198]. The effects on the skeletal system have been evaluated by dosing two markers correlated with osteosynthesis and growth rate, osteocalcin and the carboxy terminal propeptide of type I procollagen (PCIP), showing a growth decrease after 1 and 5 months of 
treatment with BUD, both in full doses of $800 \mu \mathrm{g} / \mathrm{m}^{2} /$ day and half doses, with no variations in growth measured by conventional parameters [603]. The combined examination of PCIP and amino terminal propeptide of type III procollagen (PCIIINP) has shown the suppression of both bone turnover and collagen after twice-weekly treatment with DPI BUD and BDP $(800 \mu \mathrm{g} /$ day $)$, more evident in the latter case [42]. The effects on PCIIINP [734] and on osteocalcin were subsequently confirmed by other studies $[655,733]$. Bone density was also measured using a densitometer and an absorptiometer, without noting any significant differences between asthmatic children and controls. Vice-versa, osteocalcin was shown to be reduced in the densitometer compared to the absorptiometer, a result that can be ascribed to the disease and not to BDP, according to the authors [312]. Some maintain that osteocalcin is not a marker sensitive to bone turnover, in that asthma per se can decrease it tangibly, making it of little use in evaluating the effects [312]. Others have measured bone density without finding any significant difference $[17,300]$.

\section{Linear Growth}

Concern has been raised that the use of ICSs in children may be associated with growth suppression. Recent evidence presents conflicting results regarding reduced $[157,593,649,690]$ and normal growth $[444,684,718]$, including a wide and well-documented study [8] that meta-analyzed 21 pediatric studies, based on statistical analysis and excluding the studies employing different methods of evaluation [729]. A recent meta-analysis [583] has concluded that moderate doses of the inhaled steroid BDP in children with mild to moderate asthma has been shown to significantly affect linear growth. Also, for the effect of moderate doses of inhaled fluticasone, a statistically significant difference was revealed. However, two recent long-term studies have been reassuring $[6,645]$. The growth of a cohort of 142 children with mild to moderate asthma was followed until they reached adult height; the subjects received a mean dose of BUD of $412 \mu \mathrm{g} /$ day for a mean of 9.2 years. The adult height of this cohort on long-term inhaled BUD therapy was $0.3 \mathrm{~cm}$ greater than expected. Its sole weakness was a high rate (72\%) of drop-outs in the control group [6], as in other conventionally treated children [568]. In the Childhood Asthma Management Program (CAMP) study, a randomized trial for more than 5 years in $>1,000$ children with mild to moderate asthma, neither inhaled BUD nor nedocromil was better than placebo in terms of PFT, but BUD provided substantial clinical improvement as compared with nedocromil or placebo [645]. Significantly, both studies reported a transient reduction in growth velocity only in the 1st year of treatment $[6,645]$. In longer clinical trials, despite a strict selection process, compliance problems can be noted. Problems increase with duration of participation, increasing child age, and the presence of less family cohesion or attention problems in children [625]. Height may diminish with an increase in dose and return to normal when treatment is reduced or stopped [646]. The attitude of inhaled CS overdosing in children was recently clearly documented. In 8,913 children, the CS inhaled at daily dose ranges equivalent to BDP were $350-2400 \mu \mathrm{g}$, $265-3400 \mu \mathrm{g}$, and $300-4800 \mu \mathrm{g}$ for the $0-4,5-11$, and 12 to 16-year-olds [171]. We believe that many results not associated with HPA-axis suppression may depend on the use of safe doses of 100-200 $\mu \mathrm{g} /$ day, which should proportionally be reduced as compared to adult doses, better in $\mu \mathrm{g} / \mathrm{kg}$, as has previously been pointed out. Although inhaled FP was found to reduce morning serum cortisol concentrations by $17 \%-43 \%$, even at low doses of $176 \mu \mathrm{g} /$ day [169] or of $200 \mu \mathrm{g} /$ day; prepubescent children treated with FP $100 \mu \mathrm{g}$ and $200 \mu \mathrm{g}$ daily for 1 year showed no statistically significant differences in growth rates, similarly to placebo-treated control subjects [7]. FP DPI 100-200 $\mu$ g administered daily to 437 children (4-11 years old) with persistent asthma for 12 weeks in a randomized, DB, parallel-group, multicenter trial did not affect 24-h urinary free-cortisol excretion, and improved PFT in children even as young as 4 and 5 years old [484]. Often, good results are obtained in children with low doses [205, 438, 487, 684, 729], and even in adults, doses can be reduced with the same results $[231,285]$. A 12 -month $100-\mu$ g daily treatment of FP in 625 children aged $1-3$ years with mild to moderate recurrent wheeze resulted in improved symptom control with no effect on growth rate, and a serum and urinary cortisol concentration suppression of $10 \%$ and $14 \%$, respectively [45]. A recent study has set up a milestone in the treatment of pediatric asthma, having demonstrated that repeated bursts of oral CS at $1-2 \mathrm{mg} / \mathrm{kg} /$ day (maximum $=50 \mathrm{mg}$ ) for a 5 -day period are not only effective, but above all are safe in children, since they are not associated with any lasting perturbation in bone metabolism, bone mineralization or adrenal function [160]. In addition, low cortisol levels normalized after discontinuing inhaled FP [757]. Others have noted that growth decline is reduced after the first 6 weeks or at most within 18 weeks [159]. A 6-month treatment with inhaled BUD and FP did not induce body fat accumulation in 21 of 26 asthmatic children; however, in five children aged from 4.3 to 5.3 years, the treatment was associated with growth velocity below the third percentile [550]. The magnitude of these changes in linear growth has varied between other studies using different ICS preparations, indicating on the one hand that either study design or specific steroid preparation/dose may be important considerations [434], and on the other the lack of reliable data on compliance may seriously confound the study of long-term growth effects of ICSs [732].

$\mathrm{DB}$, randomized placebo-controlled tests have also been carried out [729-731, 733], employing knemometry (from $\kappa \nu \eta \mu \eta$, leg), which ensures reproducible findings (Figs. 11.46-11.48) [365], to evaluate ICS effects on lower limb linear growth, which always proved to be re- 


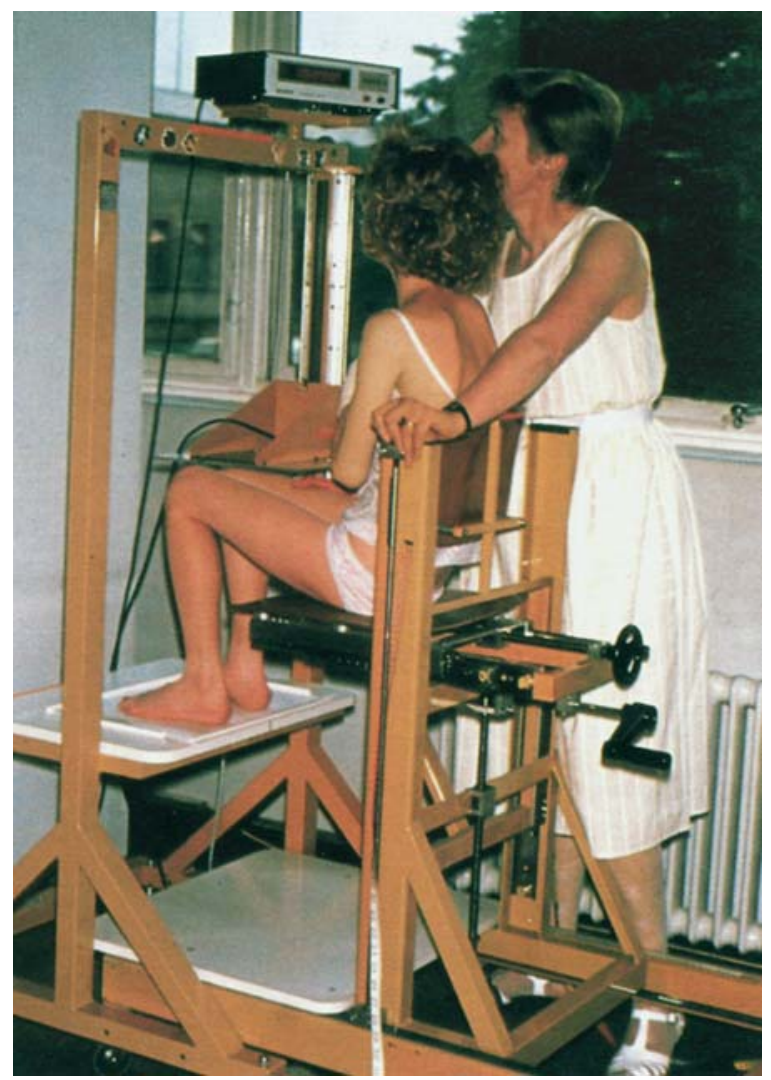

Fig. 11.46. A knemometer. The child is positioned so that the knee is positioned at a right angle and the feet are comfortably placed. At the first visit a template is made by drawing around the child's feet, so that at the subsequent visit positioning is identical.

Fig. 11.48.

Changes in total length on alternate day steroid dose

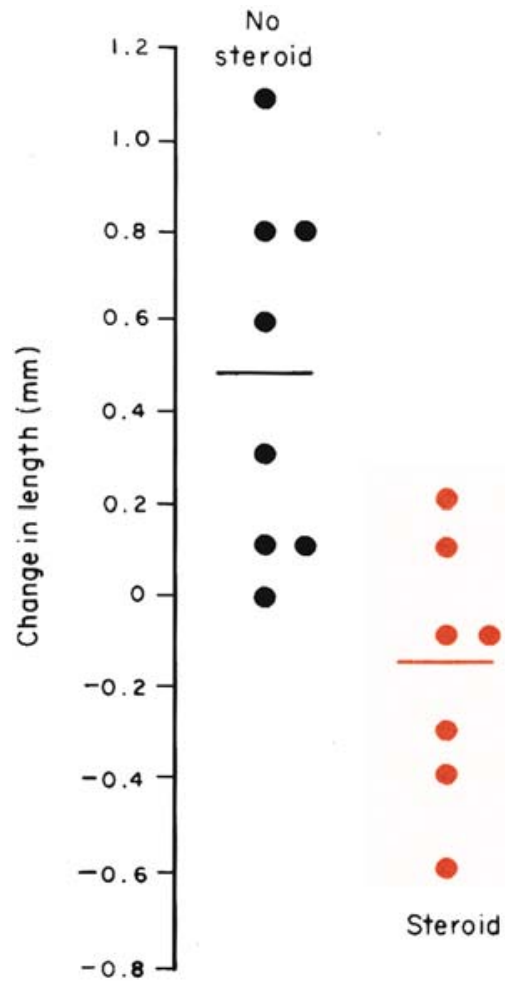

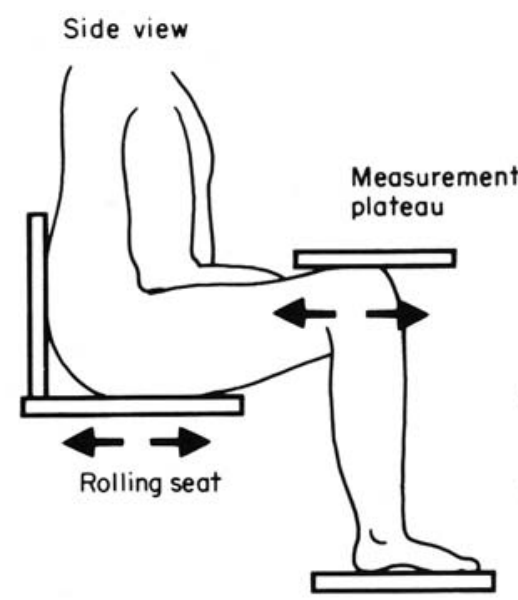

Front view
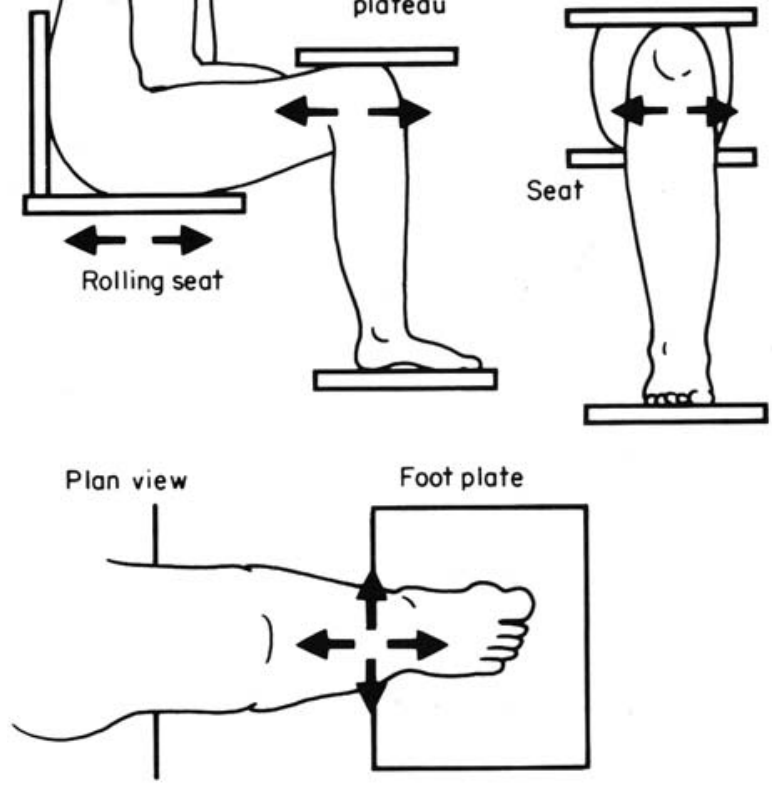

Fig. 11.47. Diagram showing knemometer and manipulation of the lower leg. The lower leg is moved around in order to gain maximum reading of the digital ruler. Alternatively, knemometry may be measured by a hand-held knemometer, provided with an electronic caliber, which measures the length of the lower leg from above the knee to below the heel. Measurements are taken with the child in a supine position with a $90^{\circ}$ flexion in hip, knee, and ankle joints. A fixed cap is held against the knee and the other, adjustable cap is placed under the heel. Both caps are parallel, and the distance is ongoing measured electronically with a resolution of $10 \mu \mathrm{m}$. An investigator stabilizes the knee cap with one hand, while slowly compressing the heel cap up to a predefined pressure of $80 \mathrm{~g}$ with the other hand, as determined by an interposed spring. At this pressure, a microswitch is activated and the reading is recorded on a computer

duced, though not with FP [731]. The results on PCIIINP of the same group [734], completed by measuring cortisol and insulin-like growth factor levels [735], and additional data have confirmed these studies [365]. In children aged 0-3 years, within a 3 -week period the increases in the lower-leg length during placebo, BUD 400, and FP 400 treatments $(200 \mu \mathrm{g}$ inhaled twice daily) were 85 , 45 , and $34 \mu \mathrm{m} / \mathrm{d}$, respectively [11]. Nevertheless, some aspects of the assessment of safety are unique to younger children, including the rapid growth velocity and their different metabolism. The rapid growth in the first 2-3 years of life may make the child more vulnerable to the adverse effects of drugs and/or disease. More seriously, the safety findings in adults and schoolchildren cannot be extrapolated uncritically to younger children [11]. We deem that the concern about potential dysfunc- 
tions is valid in cases of oral and/or high doses as an intercurrent necessity [347]. A meta-analysis suggests that moderate doses of inhaled BDP in four studies and 450 children and FP in one study and 183 children with mild to moderate asthma cause a decrease in linear growth velocity of $1.51 \mathrm{~cm} /$ year and $0.43 \mathrm{~cm} /$ year, respectively [583]. However, the increase in height of 277 children aged 4-11 years with chronic asthma was greater with FP than with BDP, 5.01 vs $4.10 \mathrm{~cm} /$ year, respectively [151]. These findings should not limit ICS administration in children with moderate/severe asthma $[28,282]$. A possible choice is flunisolide, which is freer of adverse effects $[215,228,494]$, provides better penetration and a more homogeneous distribution in the distal airways, also parenterally administered if asthma is more severe [706]. In moderate to severe asthma, the benefits may outweigh the risks associated with continuous use [703]. Various immune parameters are normal in long-term ICS-treated children [347], but eosinophils are reduced only in patients taking monodoses [497]. In conclusion, short-term and/or alternate-day treatments are safer [438], with a return to normal basal parameters in a short time, as already detailed [285,757]. To further reduce potential damage, preparations should be chosen with a longer duration of action and to begin treatment between 3:00 and 5:00 PM (monodose), as there are no differences with qid therapy, nor does it influence 24-h plasma or urinary cortisol levels [497]. It will be sufficient to monitor the effects of treatment in individual patients, while in other cases the risk-benefit analysis is strongly in favor of preventive drugs and of theophylline [285]. However, refer to the comparison of SIT/steroids in Chap. 13. We deem, however, that periodically measuring the growth of asthmatic children on long-term treatment with ICS is a useful practice [728]. An FDA mandate (November 9, 1998) requires labels on inhaled and intranasal CS warning of a potential reduction in linear growth in children demonstrates this concern (accessed from: http://www.fda.gov/cder/news/ cs-label.htm).

Table 11.21 enumerates CS side effects and the consequent measures required, and indicates a few possible causes of treatment failure with these drugs. No posterior subcapsular cataracts have been observed in children treated with ICS [592], which were seen, however, in $11.3 \%$ of 274 children [718], and in $15 \%-21 \%$ of 163 children [120], most probably related to chronic oral treatment. Synthetic CS prime IgE synthesis in vitro $[304,452]$ and the increase in serum IgE levels after 7 days of steroid treatment - viewed as being caused by steroid immunomodulator effects on $\mathrm{T}$ lymphocytes which is also associated with an $\mathrm{FEV}_{1}$ reduction [754]. Such effects were not seen ex vivo even after 1 week of steroid treatment [304]. It is not excluded, however, that with larger doses and/or long-term treatments, significant increases in IgE can be seen [318]; such reports require further evaluations; however, in this respect ICS may not be suitable in children. An in vitro study has demonstrated that hydrocortisone enhances total and allergen-specific IgE production by PBMCs from atopic subjects in vitro. The induction of Der p-specific IgE synthesis in subjects with high serum allergen-specific IgE levels was even greater than that of $\mathrm{IL}_{4}$. This is the first report to show that hydrocortisone enhances Der p-specific IgE production from circulating B cells in atopic asthma [101]. The immediate in vivo interpretation of these findings would be that CSs promote allergy, which in theory would mean that the most efficacious treatment for allergic diseases is detrimental regarding disease progress. However, the underlying mechanism by which CSs enhance IgE production in vivo is unknown [101]. High dosage can cause an increase in anxiety, depression, memory reduction, and psychological vulnerability, with a potentially negative impact on asthma severity and on the child's own ability to cope with the disease and its treatment. If, on the other hand, CSs are used in brief cycles, school performance, memory and behavior remain unchanged [432]. In conclusion, CS-induced adverse effects in 163 children with severe asthma are inadmissibly high. Given the frequency with which they occur, evaluation and close monitoring of potential adverse effects is clearly warranted, even in those patients who are not CS-dependent but require high-dose ICS and frequent rescue oral CS therapy [120].

\section{Comparison of the Various Forms of Drugs}

Some data will be useful in making a more prudent choice regarding medications to be used, especially in cases of severe asthma (Table 11.14) (in parentheses the variable effects) [284]:

- Muscle relaxant: $\beta_{2}$-adrenergics, theophylline

- Antiedematous: $\beta_{2}$-adrenergics, theophylline

- Anti-mucus secretion: CS, $\left(\beta_{2}\right.$-adrenergics, theophylline)

- Prevention of late reaction: CS

- Inhibition of mast cell degranulation $\left(\beta_{2}\right.$-adrenergics, theophylline)

- Attenuation or resolution of hyperreactivity: CS

$\beta_{2}$-Adrenergics, compared to other drugs, are characterized by:

- Greater selectivity

- Quick relief (greater effect on early asthmatic response)

- Very brief latency time by inhalation (a few minutes)

- Lower incidence of side effects

Duration of effectiveness: catecholics 2-4 h; resorcinol and salagen 4-6h; clenbuterol and procaterol $>6 \mathrm{~h}$; fenoterol and salmeterol $12 \mathrm{~h}$, and oral bambuterol $24 \mathrm{~h}$ [158].

Anticholinergics, compared to $\beta_{2}$-adrenergics, induce a lesser degree of bronchodilation, are more delayed but more prolonged in duration (compared to most $\beta_{2}$ adrenergics. IB is to be preferred because of the lesser 
impact of side effects and protracted duration of its action: 4-5 h. Nevertheless, other controlled studies are needed before they can be used effectively and safely in maintenance therapy.

Theophylline drugs, compared to $\beta_{2}$-adrenergics, have a more gradual action with more frequent and more significant side effects. Long-acting theophylline [83] has proved useful in young children who do not readily accept inhaled procedures, in children with asthma or wheezing in the morning as the only symptom, in cases of chronic asthma to avoid waking the child during the night, and in adolescents with severe symptoms before adding CS to their maintenance treatment [706], or to reduce doses when possible [566].

CSs compared to $\beta_{2}$-adrenergics and theophylline drugs have a greater latency time and are the preferred choice for use in severe crises for short periods. In prolonged treatments, the wide spectrum of side effects makes it preferable to use hydroxylase compounds in acute phases because of their short plasma half-life and the faster bioavailability of their active principle [633]. Preparations that interfere less with the metabolic processes are preferred for chronic forms [633].

$\beta_{2}$-Adrenergics can be combined - depending on the case - with anticholinergics, theophylline and CS:

- In the first case a functional synergy is obtained without causing an amalgamation of the side effects of the two drugs considered individually.

- With the combination of $\beta_{2}$-adrenergics and theophylline, an increased bronchodilation in cases of severe acute asthma and a better control of the symptoms in the several forms with a chronic trend could be achieved, but there are few recent studies that could confirm the usefulness of this association, when carried out on a regular basis, whereas if doses and the frequency of administration of inhaled $\beta_{2}$-adrenergics are reduced, the association with theophylline is effective on bronchodilation.

- CSs together with $\beta_{2}$-adrenergics enhance their therapeutic efficiency by acting on cAMP; cAMP levels are maintained within normal limits, with the above-mentioned effects, also improving oxygenation [545]. Furthermore, $\beta_{2}$-adrenergics with long-term half-life provide better control of asthma when symptoms persist, notwithstanding treatment with CS, and considering that this association can be useful in covering the CS latency period [359].

\section{Assessment of Guidelines and Pediatric Compliance}

One major problem is the treatment guidelines, an issue frequently quoted: written guidelines are often difficult to read. In the US, the mean grade levels obtained for the leading guidelines ranged from 4.9 to 9.2 [191]. Only $2.1 \pm 1.0 \%$ of the directors from 376 sampled EDs reported the use of written protocols or guidelines [121].
These guidelines are likely adopted by $5.7 \%-7.9 \%$ of readers of NIH guidelines [121]. Even within private practice, patients' race and ethnicity are associated with clinician nonadherence to national guidelines [463]. In a recent study of children who presented to an ED, 71\% of children (mean age $>8$ years) did not have a written plan. Only $7 \%$ of those with a plan consulted it at the onset of wheezing and $4 \%$ consulted it before going to the ED. Also, $48 \%$ did not use a holding chamber with their MDIs and $66 \%$ did not use their PEF meters. Regarding exacerbation response, $71 \%$ did not have a written action plan, and $89 \%$ did not maintain a symptom diary [557]. In 1114 children aged 6 (mean) evidencebased guidelines for pediatric asthma demonstrated an improvement of asthma management plans, but failed to influence patient outcome [385]. Moreover, US guidelines are structured "for children 5 years of age and older" [435-437], while European consensus guidelines [698-700] consider children aged $0-5$ years as well. Guidelines do not master factors associated with lower adherence to the medical regimen in young children, such as medication taste, and the congestion of too many medications with multiple dosing intervals [27]. Children with many risk factors experienced 0.80 more days of wheezing and 1 more day of activity restriction as a result of asthma compared with children with few risks for nonadherence [27]. For children younger than 5 years, expert recommendations are based on extrapolations of studies in older children [434]. The issues related to minorities in the populations are pertinent: black and Latino children had worse asthma status and less use of preventive asthma medications than white children within the same managed Medicaid populations [351].

The parents may not always be aware of the severity of the disease: in one trial, $36 \%$ of parents stated that they would administer a $\beta_{2}$-agonist, but $57 \%$ would go to the ED without giving a $\beta_{2}$-agonist first. Also, fewer than $5 \%$ would call the physician and use a PFM [696]. In a separate sample, the figures were $13 \%$ and $1 \%$, respectively [338]. One-third or fewer parents followed other NHLBI recommended steps, including using a PFM, calling or going to see the doctor, or going to the ED [557].

\section{Bronchiolitis}

\section{Definition}

Bronchiolitis is an acute respiratory disease that affects infants, generally between 2 and 10 months of age [499] and involves chiefly the small arteries, is characterized by an acute onset of wheezing, expiratory dyspnea, tachypnea, fever, with or without diffuse fine crepitations of rhonchi and rales on auscultation, and with radiological appearance of emphysema [69]. This definition prevails in Europe and Australia, and by American authors all first-time wheezing associated with a respi- 
ratory tract infection in infants is included [674] as wheezing-associated respiratory infection (WARI). It probably represents the first episode of asthma disease. Episodes of lower airway illness labeled bronchiolitis in the 2 nd year of life is more likely to be asthma [747].

\section{Prevalence}

Bronchiolitis is highest in infants (50\%-60\% of cases), but can also be seen in the 2 nd year of life, even if with a much lower prevalence. It is more frequent in winter months and usually affects males, as does asthma. Since 1980 , the rate of hospitalization for children with bronchiolitis has increased from $5.4 \%$ to $16.4 \%$, whereas mortality rates for the disease have remained constant [372]. In the Netherlands, the national number of bronchiolitis hospitalizations significantly increased from 1991 to 1999 in children aged 0-4 years [674].

\section{Predisposing Factors}

The host's characteristics stand out in the clinical manifestation of the disease [226, 271,391,417, 512,611,711]: - Age ( $<1$ year) holds first place, given the reduced caliber of nursling bronchioles. Our studies indicate that the onset age $<6$ months is even more significant (Tables 11.24, 11.25) [69].

- Genetic predisposition.

- Patient's immune state.

- Prematurity.

- Lungs more or less immature (additional effects from smoking).

- Male sex.

- Bottle feeding.

- Presence of cardiopathies, pneumopathies, polymalformative syndromes.

- Poor socioeconomic status and/or living conditions.

- Exposure to allergens and/or pollutants.

- Passive smoke, particularly maternal.

Breast feeding plays a key role, since maternal milk contains anti-RSV $\operatorname{sg} A$. Breast feeding for at least 1 month and, negatively, parental smoke equal to $>20$ cigarettes are highly significant compared to controls [391]. Assay of cotinine levels emphasizes a significant relationship between admissions to hospital and mother's and parents's secondhand smoke $(p<0.0005)$, compared to a similar group not affected by bronchiolitis ( $p=0.0181$ ) [519]. In the cohort of 240 infants, $29 \%$ had a smoking mother [747]. The impact of daycare attendance on the incidence of infantile LRTI amounts to a doubled increase in the risk of such infections. In a cohort of children prospectively studied in the first 3-6 months of life by a polyfactorial analysis, the factors increasing the risk of a care setting different from the child home have been defined. They include repeated transfers from one place to the other, twice-daily changes of the environment (both heralding potential infections), worries, probably lesser care by persons replacing the mother, and higher prevalence of infections (8.6\%) [257]. Compared to the age of 4 months to 3 years, the presence of $>3$ children of the same age, and/or that of smoking persons in daycare settings (personal experience) exposes the child to the risk of acquiring respiratory infections, but not at home, even with $>$ three siblings, and persons caring for the child or sharing the bedroom, etc. [257].

\section{Etiology}

In $87 \%$ of cases, bronchiolitis is caused by respiratory viruses such as RSV (see Chap. 4) and the remaining rate to parainfluenza 3 and 1 viruses, adenovirus, etc. About $25 \%-50 \%$ of young babies show seroconversion for RSV or parainfluenza virus in the 1st year of life, though only a certain number of babies manifest signs of disease. RSV provokes, in most subjects, nonsevere or imperceptible clinical features, while in infants it can cause bronchiolitis with all ensuing symptoms, showing its greater impact on young infants. With passing time, the number and severity of the episodes decrease [323]. Regarding the frequency of reinfections, it has been reported that a second infection recurs in $75 \%$ of cases and a third in $65 \%$ of cases, thus being the major cause of lower airway disease in infancy. As with all infections sustained by respiratory viruses, bronchiolitis can occur in epidemic forms, more severe in some years and less so in others, and more than once in the same child during the 1st year of life [546].

Genetic factors such as FHA [271] (71\%, [499]) or allergic sensitization [586] and family predisposition to asthma $(43 \%,[499])$ are crucial elements that condition the onset of bronchiolitis, as well as the persistence of bronchoconstriction. It is important to emphasize that one atopic or asthmatic parent is a highly predictive factor relating to the insurgence of asthma in the child [271] (Tables $11.24,11.25$ ). In a prospective study of 47 infants, it is interesting that among children with RSV, 6/11 (54.5\%) children with FHA developed asthma compared with 5/36 (13.8\%) matched controls without FHA. In these children at age $7 \frac{1}{2}$ asthma was found in

Table 11.24. Family history and asthma development in 70 children with bronchiolitis (Follow-up, 6 years)

\begin{tabular}{llll} 
& $\begin{array}{l}\text { No. of cases } \\
70\end{array}$ & Family history \\
\cline { 3 - 4 } & & + & - \\
\hline Asthma + & 28 & 16 & 12 \\
\hline Asthma - & 42 & 4 & 38
\end{tabular}

Fisher $=0.0000$.

Data from [69]. 
Table 11.25. Onset age of the first episode of bronchiolitis and asthma development in 70 children with bronchiolitis (follow-up, 6 years)

\begin{tabular}{llll} 
& $\begin{array}{l}\text { No. of cases } \\
70\end{array}$ & \multicolumn{2}{l}{ Age at the first episode } \\
\cline { 3 - 4 } & 28 & 16 & 12 \\
\hline Asthmanths + & 28 months & 62 \\
\hline Asthma - & 42 & 10 & 32 \\
\hline
\end{tabular}

Fisher $=0.0050$.

Data from [69].

$11 / 47(23 \%)$ of the RSV group and in $2 / 93(2 \%)$ of the controls, and in 7/47 (14.9\%) of the children with FHA and $1 / 93(1 \%)$ of the matched controls without FHA $(\mathrm{RR}=13.88)$. A positive test of $\mathrm{IgE}$ to inhalants was found in $14 / 44(32 \%)$ of the RSV group and in $12 / 87$ $(14 \%)$ of the controls $(\mathrm{RR}=2.31)$ [586]. Moreover, a positive test for IgE was found in 14/44 (31.8\%) RSV children and in 8/92 (8.7\%) control children [587]. In a cohort of 2402 - to 10 -week-old infants, $61 \%$ had a FHA and $40 \%$ a history of asthma [747].

\section{Pathogenesis}

If we consider the infant's still immature immune system, already Th2-controlled at birth, it is evident how, with the decrease of passively acquired antibodies and with the addition of the aggravating factor of low IFN- $\gamma$ generation and progressive decrease in anti-RSV sIgA in maternal milk, the infection can progress. The functional characteristics of young infants are dominated by an insufficient development of bronchial cartilages, smooth muscles and the number of alveoli, and by reduced elastic recoil [512]. RSV has a certain tropism for small airway epithelial cells, whereas, for example, the parainfluenza virus prefers the subglottic epithelial cells [125]. The viruses have immunosuppressive and cytopathic effects on monocytes, macrophages and T lymphocytes, subsequently interfering on the normal process of macrophage-induced lymphocyte activation [503], as previously seen (Table 11.9). It can be assumed that in a selected number of children, either affected previously, or at the time of primary RSV infection, there is an alteration of T-cell regulatory mechanisms [523]. Therefore, bronchiolitis can be regarded as an immune system disorganization related to host immaturity, in which T-cell hyperactivity is unraveled, in parallel with persistent and elevated IgE-mediated responses, which, via increased histamine and other mediator release, can, in future, predispose to asthma and BHR [611]. Infants affected by bronchiolitis have a markedly lower number of $\mathrm{CD} 8^{+}$lymphocytes (inversely proportional to IgE maximum titers) than infants suffering from other types of RSV infection [715] during convalescence. Similarly, CD8 T cells are reduced during bronchiolitis [523] and in infants manifesting $>3$ episodes of wheezing during their 1st year of life [546].

From the fundamental studies of Welliver et al [714716], the presence of anti-RSV sIgE in the nasopharyngeal secretions of children after an episode of bronchiolitis or of pneumonia with wheezing provoked by RSV has been noted, but not in controls also affected by RSV-induced respiratory infections and without bronchoconstriction. Also known is the longer persistence of anti-RSV sIgE in the study babies compared to controls (Table 11.26) [714]. At the same time, an elevated histaminemia and a clear correlation of anti-RSV sIgE titers with hypoxia as the objective measure of disease severity has been displayed, results that are totally overlapping those following infection by parainfluenza viruses [716]. Furthermore, the higher the levels of anti-RSV IgE in children suffering from bronchiolitis, the more easily the wheezing relapses ( $70 \%$ compared to $20 \%$ of those whose levels are not measurable) [715]. IgE produced by RSV therefore has immediate implications on atopic asthma, resulting in the IgE-mast cells linking and mediator and IL release. A subsequent direct mast cell intervention could be inferred from tryptase levels present in BALF of $91.8 \%$ of infants, but not so elevated as compared to the levels found in controls, to imply a confirmation [184].

Table 11.26. Mean RSV-IgE responses in nasopharyngeal secretions according to the illness group and related to respiratory symptoms

\begin{tabular}{|c|c|c|}
\hline \multirow[b]{2}{*}{ Symptoms } & \multicolumn{2}{|c|}{ No. of children with positive RSV lgE titers/number of babies tested } \\
\hline & Acute & Convalescent \\
\hline I. Upper-respiratory-tract disease only & $0 / 9$ & $0 / 4$ \\
\hline II. Pneumonia without wheezing & $0 / 9$ & $1 / 7$ \\
\hline III. Pneumonia with wheezing & $3 / 10$ & $6 / 10$ \\
\hline IV. Bronchiolitis & $21 / 43$ & $17 / 25$ \\
\hline
\end{tabular}

Statistically significant differences between groups III and IV and groups I and II (combined) and between groups III and IV (combined) and groups I and II (combined).

Data from [715]. 
By an aspecific phagocytosis of RSV by the cells or an interaction with a receptor on eosinophils, RSV prime the eosinophils to start the chain of $\mathrm{O}_{2}^{-}$generation and activation and to release mediators in greater quantities, confirming the hypothesis that some inflammatory signs seen in the airways of these children are eosinophil-induced [298]. Such RSV-induced effects on inflammatory cells in bronchiolitis may be much more pathogenic than was formerly believed, as demonstrated by their relationship, which has come to light in recent years, with eosinophils, whose activation appears pathogenically to be more important than that of neutrophils/ macrophages and is characteristic of bronchiolitis but not of other respiratory disease [298]. Significantly higher ECP levels have been reported in infants with RSV-induced bronchiolitis than in controls suffering from URTIs and LRTIs that were also RSV-induced. High ECP levels in the nasopharyngeal secretions are predictive of the development of bronchiolitis at the time of RSV infection, and in parallel of clinical severity [201]. Especially in males, ECP levels are associated with the disease and its severity more than with peripheral eosinophilia, presenting as markers of disease progression [202]. The same difference was observed in infants with other RSV infections, but in females rather than males [751]. ECP was not predictive of asthma development in 6/34 children 2 years old after hospital admission at the age of 3 months [585].

There is, however, a correlation between $\mathrm{LTC}_{4}$ and RSV-specific IgE levels in the nasopharyngeal mucosa $[686,714]$ of $67 \%$ of children with RSV bronchiolitis, vs $33 \%$ of controls with URTIs, also caused by RSV $(p<0.001)$, who, moreover, showed $\mathrm{LTC}_{4}$ concentrations fivefold lower than the study children $(p<0.02)$ [686]. $\mathrm{PaO}_{2}$ titers were lower in children with detectable $\mathrm{LTB}_{4}$ than in those with undetectable $\mathrm{LTB}_{4}$, and $\mathrm{LTB}_{4}$ titers were inversely correlated with initial $\mathrm{PaO}_{2}$,values which suggests a connection with disease severity [203]. Therefore, the damage produced by RSV at the bronchial epithelium, associated with toxic effects of basic proteins, and edema and bronchospasm triggered by LTs, could induce an airway obstruction, followed by a postinfection persistent BHR [323]. Other than BHR, bronchiolitis is dominated by activation of cellular immunity with production of Th2-like T cell ILs, clearly indicating an immune response to RSV and predictive of the development of asthma. An increased rate of $\mathrm{CD} 4^{+}$, $\mathrm{CD} 25^{+}$, and $\mathrm{CD} 23^{+}$lymphocytes was found in infants at 5 months compared with the time of bronchiolitis and with healthy subjects of the same age. Moreover, the $\mathrm{CD}^{+}$increase is not associated with CD8 increase, which remains low, whereas $\mathrm{IL}_{4}$ increased in both groups. Eosinophils also increase significantly and are related to the number of days of wheezing - therefore, a classic Th2 response [523] manifested by RSV-induced $\mathrm{IL}_{13}$ production [670]. It is unclear whether genetic factors condition the phenotypic expression of RSV-in- duced bronchiolitis and the development of asthma, or RSV predisposes infants to asthma.

Even more pathogenetic is soluble CD14 (sCD14), a predictor of subsequent wheezing in children aged 2-14 months with RSV-induced brochiolitis, although not influenced by FHA+, sex, or breast-feeding [601].

Anatomopathological study shows that RSV replication in the airways has cytolytic effects on the epithelium. Significant changes in airway morphology are seen in animals with acute viral respiratory infection: the airways become edematous and infiltrated with inflammatory cells. The lower airways show marked bronchial narrowing and collateral ventilation and elastic recoil reduction. The reduction in caliber of distal airways makes the small bronchi and bronchioles obstructed by cellular debris from virus-specific epithelial necrosis, increased mucus secretion, intraluminal secretions of relatively dense and viscid exudate, bronchiolar inflammatory infiltrates and edema of both submucosa and adventitia. The lesions are aggravated by replacement of necrotic cells by cuboid nonciliated cells, thus impairing mucus clearance, which condenses in obstructive and potentially occlusive plugs [611].

From a functional viewpoint bronchospasm can be absent. Vice versa, alterations of the respiratory mechanism are present, denoted by a $50 \%$ increase in FRC compared to normal, reduced pulmonary compliance, increased resistance to air passage, especially during exhalation, since during exhalation - especially if forced the airway caliber is reduced as a result of bronchiolar obstruction. The work of breathing is aggravated by air trapping with pulmonary hyperdistention or atelectasis, which are at the basis of hypoxemia - warning signs of ventilation-perfusion imbalance [271].

\section{Clinical Presentation}

Bronchiolitis is an acute disease with a sudden onset and a steady worsening in the first $24 \mathrm{~h}$. It presents a severe clinical situation in young infants caused by respiratory changes, with prolonged exhalation, cough, sustained resting polypnea ( $R R \geq 70-80 / \mathrm{min})$, and obstructive type dyspnea, sometimes cyanosis and/or lethargy [474]. Moreover, symptoms can include coughing (100\%), nasal congestion (94\%), wheezing ( $89 \%)$, difficulty breathing $(87 \%)$, poor feeding $(70 \%)$, poor sleeping $(69 \%)$, irritability $(67 \%)$, fever $(59 \%)$, vomiting (51\%), and choking (41\%) [499]. If the disease worsens further, RR decreases (Appendix 11.1) [302, 544] and signs of hypoxia and difficult breathing occur $22 \%$ to $46 \%$ of children so affected are admitted to hospital, especially if RR is $>70$ [499] or are admitted to the PICU at a median age of 1.7-2.27 months [506] and requiring mechanical ventilation in $31.4 \%-34.1 \%$ of cases. Tachypnea makes breast-feeding difficult, not leaving a sufficient interval of time for sucking and swallowing. 
The child can become dehydrated as a result of an increased perspiratio insensibilis and of shock. Fever if present, in most cases does not rise beyond $39^{\circ} \mathrm{C}$ [270].

Objectively, nasal flaring, use of accessory respiratory muscles due to intercostal retractions can be noted. During auscultation, a prolonged expiratory phase can be heard, together with expiratory wheezing and fine diffused rales or rhonchi. The rales indicate an obstruction of the main bronchi, and the rhonchi an alveolar hypoventilation. High-pitched expiratory wheezes in all lung fields may be audible. Hypoxemia and hypercapnia deriving from the alteration of the ventilation-perfusion balance and of $\mathrm{pH}$ and $\mathrm{PaCO}_{2}$ are present. Aspecific symptoms can also be noted in young infants such as lethargy and irritability or other neurological symptoms of hypoxemia. Apnea and/or cyanosis can be the only symptom $[512,594]$.

\section{Diagnosis}

Diagnosis is above all clinical. $\mathrm{SaO}_{2}$ monitoring is determinant to ascertain the degree of airway obstruction, and in cases of severe respiratory distress it should be integrated with the acid-base balance test; arterial samples may verify $\mathrm{PaCO}_{2}$ levels. Pulsus paradoxus is a clinical correlate of cardiopulmonary interaction during asthma and correlates with the severity of the asthma attack. The degree of pulsus paradoxus can be measured directly with a hand-operated BP cuff or estimated from pulse oximetry [372, 717], a noninvasive method for detecting severe cases and for measuring $\mathrm{O}_{2}$ saturation $\left(\mathrm{SaO}_{2}\right)$, but not always reliable [512] because it can be affected by the child's movement and by peripheral vasoconstriction, which occurs in the more severe cases [509]. This pulse may be related to RR in young children [372], but it cannot always be utilized because of HR increase; nor is there a clear correlation between peripheral pulse increase and asthma aggravation [738]. If it is $>20$ Torr, a moderate to severe obstruction is present [174]. In a survey of 519 physicians, the decision to transfer children to an appropriate emergency setting was significantly influenced by the $2 \%$ difference between $\mathrm{SaO}_{2}$ values of $94 \%$ and $92 \%$. This finding may help to explain the increased numbers of admissions for bronchiolitis since the popularization of pulse oximetry [372]. However, in $67 \%$ of cases oximetry is done in pediatric ED as part of the initial procedure and to document improvements after treatment [121]. Full blood counts are normal [125] in $80 \%$ of children [512]. Chest $\mathrm{X}$-rays show typical airway emphysema resulting from air trapping, chest hyperinflation, diaphragmatic flattening and accentuation of the bronchial network; multiple areas of atelectasis can mimic areas of thickening. No correlation between these findings and severity of bronchiolitis is found [512].

Cytological examinations of nasopharyngeal aspirates and titers of specific antibodies may facilitate the etiological agent identification. Confirmation is obtained by using the indirect immunofluorescence technique and by ELISA. In any event, these are roundabout methods and can take more than 10 days to carry out [125]. Even if in 3\%-10\% of cases Chlamydia trachomatis and Mycoplasma pneumoniae [514] are isolated, both illness severity and prognosis are parallel to cases in which only RSV has been found.

Differential diagnosis is summarized in Tables 11.27 and 11.28 [594]. It is made difficult in babies by the frequent analogies between the clinical picture, the objectivity and the X-ray results.

Bronchiolitis has characteristics other than those of asthma [125]:

- It manifests itself in the cold seasons.

- It occurs in epidemic waves.

- Episodes are frequently preceded by rhinorrhea and fever.

- It is particularly common in daycare settings.

- In the same family other members suffer from influenza.

- It is primarily a disease affecting babies in the 1 st year of life, which is why diagnosis can be limited [747], whereas asthma normally occurs after this age (Figs. 4.14 and 5.3).

However, pediatricians generally do not need to differentiate virus and nonvirus-induced severe wheezing, since treatment of airway obstruction is unaffected, apart from an epidemiological interest in etiology [594].

\section{Treatment}

Treatment is symptomatic. If breathing difficulties are severe, there is need to humidify the air and to hydrate the baby adequately (not $>1,500 \mathrm{ml} / \mathrm{m}^{2}$, correcting the acidosis), also to choose the diet and to control hydrosaline balance: in young infants rehydration is cardinal even though cases of severe dehydration are rare [26, 222]. Electrolyte concentrations should be carefully monitored in all infants with acute bronchiolitis [512]. If cyanosis is present and $\mathrm{SaO}_{2}$ is $\geq 95 \%$ in room air, warm and humidified $\mathrm{O}_{2}$ can be delivered into a tent or headbox, adjusting $\mathrm{O}_{2}$ saturation. If necessary, give a dose of epinephrine 1:1,000 (Table 11.16), with a vasoconstriction action and, therefore, an antiedematous action, which can, if required, be repeated after 15-20 min, since it is rapidly metabolized. Inhaled epinephrine is discussed in Chap. 20. Racemic epinephrine [551] (not registered everywhere) has proved most effective, as shown by concrete improvement and few or no side effects. The effect of the racemic form derives from its action on $\alpha$-adrenergic receptors, capable of reducing microvascular leakage by causing constriction of precapillary bronchial arterioles and hence bronchial mucosal edema [551]. The drug is administered through a nebulizer in doses of $0.1 \mathrm{ml} / \mathrm{kg}$ [551]. In severe cases, assisted breathing is needed [271]. Racemic epinephrine proves 
Table 11.27. Pediatric differential diagnosis of bronchiolitis based on clinical features

\begin{tabular}{|c|c|c|}
\hline \multirow[t]{2}{*}{ Symptoms/signs } & \multicolumn{2}{|l|}{ Diseases associated with wheezing } \\
\hline & In infants & In older children \\
\hline Association with positional changes & GER, anomalies of great vessels & GER \\
\hline Failure to thrive & $\begin{array}{l}\text { Cystic fibrosis, tracheoesophageal fistula, } \\
\text { bronchopulmonary dysplasia }\end{array}$ & $\begin{array}{l}\text { Cystic fibrosis, chronic hyper- } \\
\text { sensitivity pneumonitis, } \\
\alpha_{1} \text { antitrypsin deficiency, } \\
\text { bronchiectasis }\end{array}$ \\
\hline Association with feeding & Tracheoesophageal fistula, GER & GER \\
\hline Environmental factors & Allergic asthma & $\begin{array}{l}\text { Allergic asthma, acute } \\
\text { hypersensitivity pneumonia }\end{array}$ \\
\hline Sudden onset & Allergic asthma, croup & $\begin{array}{l}\text { Allergic asthma, foreign body } \\
\text { aspiration, croup, acute } \\
\text { hypersensitivity pneumonia }\end{array}$ \\
\hline Fever & Bronchiolitis, pneumonia & $\begin{array}{l}\text { Acute hypersensitivity } \\
\text { pneumonia, croup }\end{array}$ \\
\hline Rhinorrhea & Bronchiolitis, pneumonia & Allergic asthma, croup \\
\hline Concomitant stridor & $\begin{array}{l}\text { Tracheal or bronchial stenosis, } \\
\text { anomalies of great vessels, croup }\end{array}$ & Foreign body aspiration, croup \\
\hline Finger clubbing & & $\begin{array}{l}\text { Cystic fibrosis, bronchiectasis } \\
\text { chronic lung disease (CLD), } \\
\text { allergic asthma }\end{array}$ \\
\hline
\end{tabular}

Table 11.28. Pediatric differential diagnosis of bronchiolitis and asthma

\begin{tabular}{lll} 
Parameters & Asthma & Bronchiolitis \\
\hline Positive family history & Frequent & May be frequent (Table 11.22) \\
\hline Etiology & Allergens, viruses, etc. & RSV \\
\hline Age at onset & $50 \%$ by 2 years of age & $<2$ years, frequently at $<1$ year \\
\hline Recurrent wheezing & $80 \%$ by 5 years of age & \\
\hline Onset of wheezing & Characteristic & $70 \%$ ( $\leq 2$ episodes) \\
\hline Association with allergic disease & If allergic asthma, allergic rhinitis, & $30 \% \rightarrow$ asthma ( $\leq 3$ episodes) \\
\hline Concomitant symptoms of RRI & atopic dermatitis & Insidious \\
\hline Chest auscultation & High-pitched expiratory rales & Yes \\
\hline PRIST & Acute if allergic or exercise-induced & Fine, sibilant rales, coarse inspiratory \\
\hline Nasal eosinophilia & May be elevated & and expiratory wheezes \\
\hline Response to bronchodilators & Characteristic & Usually normal \\
\hline
\end{tabular}

Updated from [594].

GER gastroesophageal reflux, $R R I$ recurrent respiratory infections, $R S V$ respiratory syncytial virus.

to be better, as has been confirmed in the US [551] where a shorter length of hospitalization of children compared to a group treated with albuterol has been documented
[399]. If the mean percent $\mathrm{SaO}_{2}$ at 60 min was significantly higher in the epinephrine group [399], either the acidity of the solution ( $\mathrm{pH} 3.2$ ), or the content of 
metabisulfites, present in levo-epinephrine preparations as a preservative [543], or in epinephrine acid tartrate as a vehicle [689] or both, might have been responsible [543]. These causes may explain why no improvement was shown in infants with acute bronchiolitis when compared with placebo $[2,226,689]$.

Four recent randomized, DB studies have evaluated nebulized epinephrine in the treatment of infants with bronchiolitis. The delivered types of epinephrine were L-epinephrine [2], racemic epinephrine [237], epinephrine diluted in normal saline solution [373], or epinephrine acid tartrate, $1 \%$ [689]. Nebulizations were administered using a nebulizer and face mask, and $\mathrm{O}_{2}$ was given as needed as above. No significant overall differences were found between the groups [689] or between treated and placebo groups $[2,237]$. A decrease in symptoms and length of hospitalization was reported in 54 infants [373]. When epinephrine diluted in normal saline was nebulized to these infants with viral bronchiolitis, the in-hospital stay was reduced by $25 \%$, from 4 days in the $0.9 \%$ saline solution group (group 1) to 3 days in the $3 \%$ saline solution group (group 2) [373]. This outcome could bear an important economic and clinical impact worldwide; in the US, $>10^{5}$ children are hospitalized annually at a cost of US $\$ 300$ million. Decreasing this burden by almost $25 \%$ could theoretically save nearly US $\$ 75$ million annually in the US alone [373]. A Cochrane review concludes that there is insufficient evidence to support the use of epinephrine for the treatment of bronchiolitis among inpatients aged $\leq 2$ years except a significant change in clinical score at $60 \mathrm{~min}$ post-treatment [239]. Usually, severely ill, hospitalized infants are aged a few days $[2,470]$ or 3-6 months [69, $237,372,373,470,474,499]$. We analyze the differences in epinephrine preparations in Chapter 20.

As has been discussed, the use of bronchodilators is controversial, since usually they increase the state of agitation of infants in whom bronchiolar smooth muscles are barely developed before the 18th month, even if muscular tissue appears at the 23rd week of intrauterine life and by the 25th week is uniformly distributed at all levels of the bronchial tree [632]. As stated, bronchospasm is not a main component of bronchiolitis, and in acutely ill infants the production of mucosal edema and increased mucus secretion may impair medication effects [206]. In some cases, $\beta_{2}$-receptors in healthy infants of 5.6 months [206] have been found, even in reduced numbers, and the efficacy of nebulized albuterol has been reported in infants 1-24 months old [565]. A causative factor, as noted, is the $\beta$-adrenergic receptors' desensitization caused by RSV. The safety of $\beta_{2}$-adrenergics for use in infants of 7.5 months [36, $370,371]$ has been ascertained, whereas in infants $<1-6$ months $[199,250] \beta_{2}$-adrenergics induced a significant $\mathrm{HR}$ increase and a $\mathrm{SaO}_{2}$ reduction. Even if tachycardia can be viewed as an index of effectiveness [199], we believe that $\beta_{2}$-adrenergic may be useful in older children who can no longer be diagnosed as having bronchiolitis. This could account for the controversies summarized elsewhere [323]. As an alternative, consideration should also be given to forced nasal respiration of the infant and to the greater aerosol residual in the oropharynx compared to an adult [549]. In the survey from 519 physicians, most respondents recommended use of bronchodilators (96\%), and few recommended steroids $(8 \%)$, or antibiotics (2\%). Inhaled albuterol was the most common drug specified (84\%). Inhaled epinephrine $(57 \%)$ followed by inhaled albuterol $(35 \%)$ were the most commonly second recommended treatment [372]. Epinephrine was given to $58 \%$ of 237 infants in pediatric EDs in Canada [499]. In 149 infants hospitalized with bronchiolitis, there were no group differences in the effectiveness of therapy of either nebulized epinephrine or albuterol every $1-6 \mathrm{~h}$. This is probably because by the time infants present to medical care, the amount of virus-induced necrosis may already be substantial [474].

Much debated is CS use in $5 \%$ of cases [499]. The rationale for their use is in direct relation to the anti-inflammatory power carried out on the bronchiolar walls and the restoration of $\beta_{2}$-receptors; but the small airways of these infants are, for the most part, unresponsive [711]. Furthermore, since they retain fluids in the tissues, CSs should be used with caution [594] in view of the inappropriate secretion of ADH (antidiuretic hormone), potentially present in these babies along with hyperreninemia [222]. Five percent of infants received CSs while in the hospital and $4 \%$ on discharge [499]. Antibiotics have no therapeutic value in a viral disease; unless a bacterial association is suspected (in $2 \%$ of cases), or confirmed by clinical or laboratory evidence, and severe, clinical signs, such as persistent febrile condition and/or an increase in the indexes of inflammation [512]. However, there are concerns about risks of adverse reactions, and the effect of antibiotics on bacterial resistance (Chap. 19).

Good results have been achieved with ribavirin, a synthetic nucleotide with virostatic properties on many viruses including RSV, interfering with the expression of mRNA of RSV itself, the effects of which on clinical parameters are noticeable $24-48 \mathrm{~h}$ after therapy [599]. The drug is administered by a small-particle aerosol generator and is delivered to a head box, face mask or $\mathrm{O}_{2}$ tent in a solution containing $20 \mathrm{mg} / \mathrm{ml}$ of water, for $12-18 \mathrm{~h}$ consecutively per day for 3-7 days, depending on the disease progression [599]. Given the potential for environmental contamination during treatment, it is preferable to administer it in high doses by means of an $\mathrm{O}_{2}$ hood at $6 \mathrm{~g} / \mathrm{dl}$ water for $2 \mathrm{~h}$ instead of $18 \mathrm{~h}$, achieving the same effects [176]. After the initial positive results, reservations were expressed, due also to the unfavorable cost-benefit ratio [712], its limited strength and the drug's possible toxicity and environmental contamination [176], which is why it is recommended as an optional choice [235]. 


\section{Prevention}

Immunoglobulins with high titers of anti-RSV antibodies [227], IV administered (RSV-IVIg), in a prospective blinded randomized, multicenter study over 3 years, with high (750) or low $(150 \mathrm{mg} / \mathrm{kg})$ monthly infusions can be used for prevention. Compared to controls, highdose RSV-IVIg reduced the incidence of LRTI, RSV-associated hospitalizations, ICU days, and ribavirin doses ( $p=0.01-0.05)$ with $3.3 \%$ adverse reactions. The protection was extended to the follow-up with a clear reduction in severe consequences, even among subjects who were at greater risk [227]. In premature babies, the efficacy is the same, compared to controls, with a reduction in the number of days spent in hospital or ICU associated with RSV infection [226]. Even though offering a valid defense, it is not preventive in $100 \%$ of cases; nor has it lowered the mortality rate [9]. It is administered IV $(750 \mathrm{mg} / \mathrm{kg} / 2 \mathrm{~h}$, once a month, during the RSV season: November to March-April): it causes overhydration, requires the same monthly controls and has an elevated cost, as does ribavirin [235]. In 1996, the FDA approved the use of RSV-IVIg as a prevention against RSV infections, excluding, however, children with congenital cardiopathy. Other DB, randomized studies are further testing the advantages and limitations of RSV-IVIg [9].

Another molecule, palivizumab, a monoclonal antibody directed against RSV, is now marketed for preventing respiratory tract infection by RSV in infants. The results of six trials suggest that the optimal dose is $15 \mathrm{mg} / \mathrm{kg}$ palivizumab by monthly injection throughout the seasonal epidemic period. The AAP has restricted its use to infants with CLD and congenital heart disease (CHD) [9]. Two recent studies [225, 515] have shown that the hospitalization rate for RSV bronchiolitis decreased significantly ( $46.2 \%$ vs 11.8 and $3.8 \%$; p $<0.01$ ) in premature infants with a gestational age $\leq 32$ weeks and with CLD [225]. The emerging problem is that $83 \%$ of the children needing PICU admission for mechanical ventilation for the RSV bronchiolitis treatment from 2000 to 2002 born at term did not have CLD and were not candidates for RSV prophylaxis according to the current recommendations $[9,515]$. Certainly RSV prophylaxis would increase the net cost of care if palivizumab were administered to the population of infants with bronchiolitis.

\section{Prognosis}

Generally speaking the prognosis is favorable. The mortality rate in hospitalized infants is $1 \%$, which rises to $3.5 \%$ in premature infants and babies born with heart diseases, chronic respiratory disease, primary and secondary IDs, etc. [323].

Long-term prognosis can be complicated by asthma. Many studies have examined why children with genetic predisposition to atopy develop bronchiolitis more fre- quently or, afterwards, an asthmatic condition, especially if with IgE elevated levels. The influence of predisposing and confounding factors is well known: passive smoke - especially maternal - environmental pollutants, etc. Nevertheless, RSV infection remains the main factor that facilitates the insurgence of asthma. In this context, the report that more than one-third of children who have asthma during childhood have suffered from RSV-induced bronchiolitis (Tables 11.24, 11.25) seems particularly relevant. Consequently, PRIST is useful for identifying children with persistent wheezing who will continue to suffer from asthma [69], in many of whom the IgE increase is followed by sIgE development [70]. Several studies have indicated the predictive nature of total IgE [300] and/or of CBIgE [368]; others underline SPT effectiveness as a screening method (Chap. 6).

What is the role of atopy? Several authors have expressed controversial opinions [69, 70, 184, 383, 391, 453]. Often the studies have proved inconclusive, some linking the unfavorable prognosis to atopy and maternal passive smoke [391]. Others do not confirm FHA+ with atopy, emphasizing instead the association with parental smoke and sibling presence [400]. The results of Rylander et al [545] appear to be very eloquent: reexamining 79 children 4 years after their admission to hospital, they observed in only 22 of those with recurring wheezing a statistically relevant association with $\mathrm{FH}$ and an equally significant PEF and $\mathrm{MEF}_{25}$ reduction. In 83 children followed-up to the age of $8[313,314,320]$, the risk of atopic children developing asthma or BHR during their school age was significantly related to recurring wheezing that appeared in the 1st year of life and to a premature IgE increase [314] compared to controls. At age 19 wheezing in early childhood was a significant predictor of asthma, and also seemed to predict PFT abnormalities in early adulthood. Thus, although the outcome of children with early wheezing is good at school age they may become symptomatic again as adults [495]. In other entrants, at the age of 4 years, asthma was present in $62.5 \%$ of ex-bronchiolitis sufferers vs $6.3 \%$ of controls and in $88.5 \%$ of children with high IgE titers compared to $32 \%$ of those with normal IgE levels [555]. In children of atopics, at the age of 11 atopy and wheezing were closely related [609].

Many different lines of research agree that RSV-induced bronchiolitis is responsible for a subsequent asthmatic syndrome, persistent for many years after the primary infection. In particular, it is hypothesized that anti-RSV IgE production can constitute a marker for the predisposition to develop specific response to VRI of early and late infancy, able to trigger recurrent asthmatic episodes [453] (Table 11.29) [4, 117, 161, 178, 214, 241, $246,302,381,375,423,447,454,470,495,587,616,637$, $644,713,726,745]$. Studies fail to clarify the pathogenesis of this higher prevalence of asthma in these subjects $[585,587]$. It can be speculated either that the atopic risk preceded and/or provoked RSV-induced bronchiolitis and wheezing episodes, or that, at the origin, a reduced 
Table 11.29. Bronchiolitis, IgE antibodies, atopy and asthma development: a meta-analysis

\section{Relationships between bronchiolitis and IgE antibodies}

Viral infections do not provoke an IgE antibody expansion and a higher atopic risk in children aged $<2$ years, frequent at 2-4 years and more frequent after 4 years [161]

Development of specific lgE in $44 \%$ of asthmatic children and in $17 \%$ of nonasthmatic children up to 2 years [587]

Significant association between FHA/asthma and RSV infection at 3 years, with similar differences in specific lgE titers between study children and controls [587]

Atopy in $67 \%$ of ex-bronchiolitics (aged 19) and in $50 \%$ of controls [495]

Children who were current wheezers at ages 7-8 had detectable RSV-specific lgE during their initial episodes but no relation to alteration of spirometry tests at age 7-8 years [712].

\section{Relationships between bronchiolitis and asthma/recurrent wheezing}

Recurrent viral infections unrelated to BHR at 1 year [644], 3-6 [4,725],7-8 [724], or at 13 years [616]

Aspecific BHR at methacholine inhalation challenge (MIC) in $80 \%$ of children of 12 years hospitalized in the 1 st year of life due to severe bronchiolitis, vs $15 \%$ of controls [178]

BHR to MIC was present in $48 \%$ of ex-bronchiolitics and in $32 \%$ of controls [495]

Recurrent bronchiolitis not followed by asthma in $85 \%$ of cases within the 10th year of life (despite numerous episodes experienced during the 1 st year of life), or significantly predictive of spirometry value reduction, also in RAST-negative children, moreover in $45.2 \%$ of cases methacholine challenge was correlated with dust mite-positive RAST [246]

Among 6-year-old children who were hospitalized with RSV bronchiolitis as infants, there was a $\approx 3$-fold increase in wheezing [423]; at 9-10 years of age, $33 \%$ of these children with RSV bronchiolitis in infancy required bronchodilator therapy, compared with $3 \%$ of the control group [447]

Infants with reduced conductance have a fourfold greater (in males tenfold) virus-induced risk of wheezing within the 1st year of life; in females the risk is 16-fold greater in case of reduced FRC before the illness [382]

Elevated Raw and reduced FRC are predisposing factors for long-term wheezing respiratory illness in infants [381]

Children with wheezing in the 1st year of life and at least one episode in 3 years of follow-up, experience at birth, compared to controls, a $22 \%-25 \%$ PFT reduction [382]

In children with chronic cough and previous LRTI, the intercellular spaces are 17-fold increased with notable edema and the inflammatory cells increased sevenfold ( $91 \%$ of lymphocytes, $9 \%$ of mast cells and eosinophils),

whereas ciliated cells are reduced threefold [241]

In prematurely born babies with gestational age of 29 weeks, a high Raw is significantly associated with respiratory manifestations at 2 and 3 years of life [214]

Prematurely born babies with birth weight of $0.5-1.5 \mathrm{~kg}$ who suffered from $>1$ wheezing illness

in the first 2 years of life develop asthma at 5 years in $18.1 \%$ and at 8 years in $21.3 \%$ of cases vs $10 \%$ of controls [302]

BHR is observed at birth in healthy neonates [744] or at 8 months, 3.6 months after having suffered from bronchiolitis [644]

\section{In conclusion:}

A high proportion of infants hospitalized with bronchiolitis go on to develop asthma-like symptoms [712]; such infants are more likely to have reduced PFT at 1 month of age [637], thus raising the chance that bronchiolitis may identify the infants with poorly developed airways and therefore at an increased risk of developing asthma-like symptoms in response to respiratory infections

Viral bronchiolitis in infancy enhances the risk of asthma and recurrent wheezing in later wheezing by increasing the likelihood of Th2 sensitization to subsequent respiratory infections and to aeroallergens [470]

$25 \%-56 \%$ of bronchiolitic subjects are eventually diagnosed with asthma, more commonly with a personal or family history of asthma [117]

PFT abnormalities may persist in asthmatic children up to 17 years of age and recur in the adult age [454]

Decreased indices of small airway obstruction (PEFR, FEV $, \mathrm{FEF}_{25-75}, \mathrm{FEV}_{1} / \mathrm{FVC}$ ) and increased Raw have been found in children following bronchiolitis [117]

$B H R$ bronchial hyperreactivity, FRC functional residual capacity, PFT pulmonary function testing.

PFT is already present in the first weeks of life [747]. In fact, these alterations, years after an episode of bronchiolitis, can reflect pre-existing damage in the pulmonary system and/or in airway's mechanical properties, already present at birth $[381,638,747]$. A meta-analysis
[453] did not find a causative connection between the initial type of infection and subsequent respiratory changes [611]; therefore bronchiolitis during infancy might not cause a greater morbility for respiratory disease in older children [747], or the long-term prognosis 
is open to all types of complications. It is likely that a percentage of children will suffer from asthma $(15 \%-63 \%)$ [130, 320, 417, 453, 555], especially with $\mathrm{FH}+$ and/or high titers of total and/or specific IgE and $\mathrm{PC}_{20}$-histamine $>2 \mathrm{mg} / \mathrm{dl}, \mathrm{FEF}_{25-75}$ over $70 \%$, and $\mathrm{FEV}_{1} / \mathrm{FVC}$ over $70 \%$ ) of predicted status asthmaticus [453], or independent of FH [523, 616], but with Th2 producers of $\mathrm{IL}_{4}$, virtual absence of CD8 T cells and almost undetectable IFN- $\gamma$ [523]. In 7- to 8-year-old exsufferers of severe bronchiolitis, RSV infection primes memory $\mathrm{T}$ cells that make strong IFN- $\gamma$ responses. However, $\mathrm{IL}_{4}$-producing $\mathrm{T}$ cells responding to $\mathrm{RSV}$ and cat antigens are significantly more frequent in ex-bronchiolitics, thus increasing the risk of allergic sensitization by providing a local $\mathrm{IL}_{4}$-rich environment in which aeroallergens are first encountered [470].

The other important question remaining to be clarified is that together with children in whom wheezing associated with atopy and BHR (development that is a prelude to asthma) began after the age of 2 [423], there are others with early onset of wheezing ( $<2$ years), which ceases, more or less without consequences, at about 10-11 years of age [726], in relation with reduced airway development in this period, and with the particular vulnerability to VRIs [725]. Similarly, the age which should be taken as a reference has proved to be a relative factor [723]. To this end, let us stress that $49 \%$ of children labeled as asthmatic at the age of 5 years were asymptomatic at 10 years [471]. A more valid criterion is allergen sensitization, which is not only linked to the diagnosis of asthma, but is also predictive of asthma in children with wheezing [142]. The differential diagnosis between the two forms is summarized in Table 11.30 $[383,723]$, and that of asthma and bronchiolitis in Tables 11.27 and 11.28.

For the prevalence of wheezing, see Tables 5.10 and 5.12. Wjst et al show that it is on the increase from $10.9 \%$ to $17.6 \%$ in the 1 - to 3 -year age group, then decreasing from $16.4 \%$ to $9.7 \%$ from 4 to 9 years [726], while others report figures of $60 \%$ [638] or $50 \%$ [383]. In this study, the children examined at birth and re-examined at the age of 3 or 6 based on the time of onset and pattern of wheezing symptoms were divided into three wheezing phenotypes:

- $19.9 \%$ with transient wheezing only in the first 3 years of life

- $15 \%$ with late-onset wheezing, not present in the first 3 years, (but with symptoms beginning between 3 and 6 years)

- $13.7 \%$ with persistent wheezing in the first 6 years of life.

Children with transient wheezing showed significantly altered PFTs, to a greater degree than the other groups, and also with a smoking mother (only the 2nd and 3rd group had asthmatic mothers), IgE titers significantly high (higher in the 3rd group), SPT+ and normal PFTs at 1 year of age and altered PFTs at age 6: this is the minority with a predisposition to asthma [383]. Persis-
Table 11.30. Differential diagnosis between wheezing and wheezing/asthma

\begin{tabular}{lll} 
Characteristics & Wheezing & $\begin{array}{l}\text { Wheezing/ } \\
\text { asthma }\end{array}$ \\
\hline Aspect & Episodic & $\begin{array}{l}\text { Alternating/ } \\
\text { episodic } \\
\text { symptoms }\end{array}$ \\
\hline Age & $0.5-3$ years & $>3$ years \\
\hline Etiology & $\begin{array}{l}\text { Viral, passive } \\
\text { smoking mostly } \\
\text { maternal }\end{array}$ & $\begin{array}{l}\text { Atopy } \\
\text { (+ viral } \\
\text { infections) }\end{array}$ \\
\hline IgE & \pm & ++ \\
\hline BHR & Normal & Increased \\
\hline Prognosis & $\begin{array}{l}\text { Transient, } \\
\text { up to school age }\end{array}$ & Persistent \\
\hline
\end{tabular}

Data from $[383,723]$.

tent wheezing is more commonly seen in children with asthmatic parents who have significant LRTIs with RSV [616]. The three groups can be further reduced to two: one with a viral infection and one with an atopic disease, probably aggravated by the infection [547]. Measuring specific Raw (sRaw), 200 children who wheezed at least once during first 3 years of life had significantly higher sRaw than the 303 who had never wheezed in the first 3 years of life, who had significantly higher sRaw if they were atopic or were at high genetic risk of atopy. These high-risk children, even if they had never wheezed, had a higher sRaw than children at medium and low risk. Atopic children had significantly higher sRaw than those who were not atopic, but nonatopic children at high risk had higher sRaw than those at medium risk [351]. Therefore, higher sRaw predominates in atopic or high-risk children independently of the number of wheezing episodes. Persistent symptoms after 3 years of age are associated with the concrete risk of developing asthma [152]. At the age of 11,17 of 21 children still had wheezing and 12 of 21 had BHR [609].

\section{Asthma}

When asthmatic children encounter the allergen, an acute asthmatic episode is triggered that can evolve in three stages. The first begins $20-30 \mathrm{~min}$ after allergen exposure and essentially consists of a bronchospasm, a clinical consequence of metachromatic cell degranulation; the airway caliber generally returns to normal within $2 \mathrm{~h}$. The second stage reaches its maximum intensity about $6 \mathrm{~h}$ after the provoking event, coincident with the eosinophil inflammatory response in the airways. The third stage, which can last up to 3 weeks, is sustained by the intense inflammatory response induced by mediator release and progressive recruiting of 


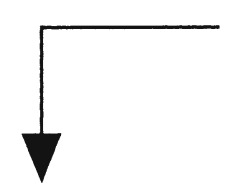

\section{Inflammation}

BHR

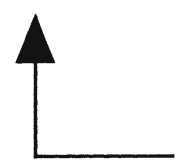

Bronchoconstriction of variable degree

Fig. 11.49. Inflammation contributes to clinical manifestations. (Modified from [454])

Table 11.31. Incidence of prodromal signs of bronchial obstruction (\%)

\begin{tabular}{lcc} 
Prodromal signs & $<6$ Years & $>6$ Years \\
\hline Cough & 89 & 86 \\
\hline Rhinitis $^{\mathrm{a}}$ & 55 & 71 \\
\hline Sleep disorders & 50 & 48 \\
\hline Asthenia & 28 & 37 \\
\hline Nervousness, irritability & 39 & 33 \\
\hline Orbital darkening $^{\prime}$ & 44 & 30 \\
\hline Loss of appetite $^{\text {Fever }}{ }^{\mathrm{a}}$ & 44 & 24 \\
\hline Pruritus & 28 & 26 \\
\hline Abdominal pain & 0 & 15 \\
\hline Headache & 17 & 8 \\
\hline Additional symptoms & 11 & 3 \\
\hline
\end{tabular}

Data from [25].

a Early symptoms.

inflammatory cells: in this stage the airways react acutely to contact irritating substances. All these events lead to the noted effects of inflammation and bronchoconstriction, which, as shown in Fig. 11.49 [454], are expressed by clinical features.

What is defined as asthma is therefore structured on two levels: the asthmatic attack and the intercritical period. The first is the final link in a chain of events and reactions whose immediate causes do not represent the core of the issue. It could be better to analyze the predisposing factors carefully, the latent anomalies, the causes predominating at the beginning of the process and the pathogenic mechanisms at the basis of the re-exacerbations. Clinical features do not present clearly defined aspects and are extremely variable from an almost normal state to one of extreme severity. The onset can be gradual and often the diagnosis is not clear. The early recognition of premonitory signs (Table 11.31) [25] before wheezing is perceptible can be finalized by an often resolutive immediate therapy. The hallmark is wheezing during expiration provoked by air rushing through larger but narrowed airways in sufficient force to generate air vibration, heard as a whistling sound associated with breathing. Additional symptoms are a feeling of a tight chest and a hacking, recurrent cough, especially at night, which can also be the only symptom. After the age of 1 year, the bronchospastic component predominates in acute attacks, which are rapidly reversible. The hypersecretion component dominates in chronic asthma and in more prolonged, severe attacks that are less responsive to therapy with bronchodilators (status asthmaticus). In addition to respiratory dyspnea, children often complain of abdominal pain, or headache, or a general sense of feeling unwell as the first subjective signs. Abdominal pain, particularly in younger children, is secondary to the use of abdominal muscles and of diaphragm. The dry, irritable, nonproductive cough of the first stages is often accompanied by a feeling of anxiety and is found together with tachypnea and tachycardia. The child is almost recumbent in bed, has difficulty in walking and talking, generally adopts particular postures to facilitate breathing, for example, sitting in a rigid position or leaning forward, to better use the auxiliary respiratory muscles. Wheezing may be a late symptom. It results from air being exchanged through partially obstructed airways and occurs in the larger airways where airflow is turbulent. The small airways do not produce wheezing since the airflow is laminar rather than turbulent. Consequently, marked small airway obstruction may be unrecognized on auscultation [481]. This fact, objectively important, does not always reveal the obstruction and precisely defines its evolutive stages. These limitations can be overcome by spirometry.

\section{Classification}

Infantile asthma is classified as acute and chronic; however, rigidly following structured frameworks can lead to imprecise evaluations. Table 11.32 [481] illustrates the assessment of varying factors that trigger the clinical features and the differential prevalence according to age. Classifications of asthma based on clinical presentation (Tables 11.33-11.35) [1, 435-437, 494, 592] are also useful in defining the several phases of treatment [437].

Aas staging [1] is often requested for the purpose of publication in international journals. Table 11.36 [186, 279] presents a summary of the causes of persistent cough. Figure $11.50[694,695]$ presents an indicative diagram on the diagnosis of asthma, which, in most cases, can be made on the basis of history, objective examination and instrumental proofs [102]. 
Clinical history

Physical examination

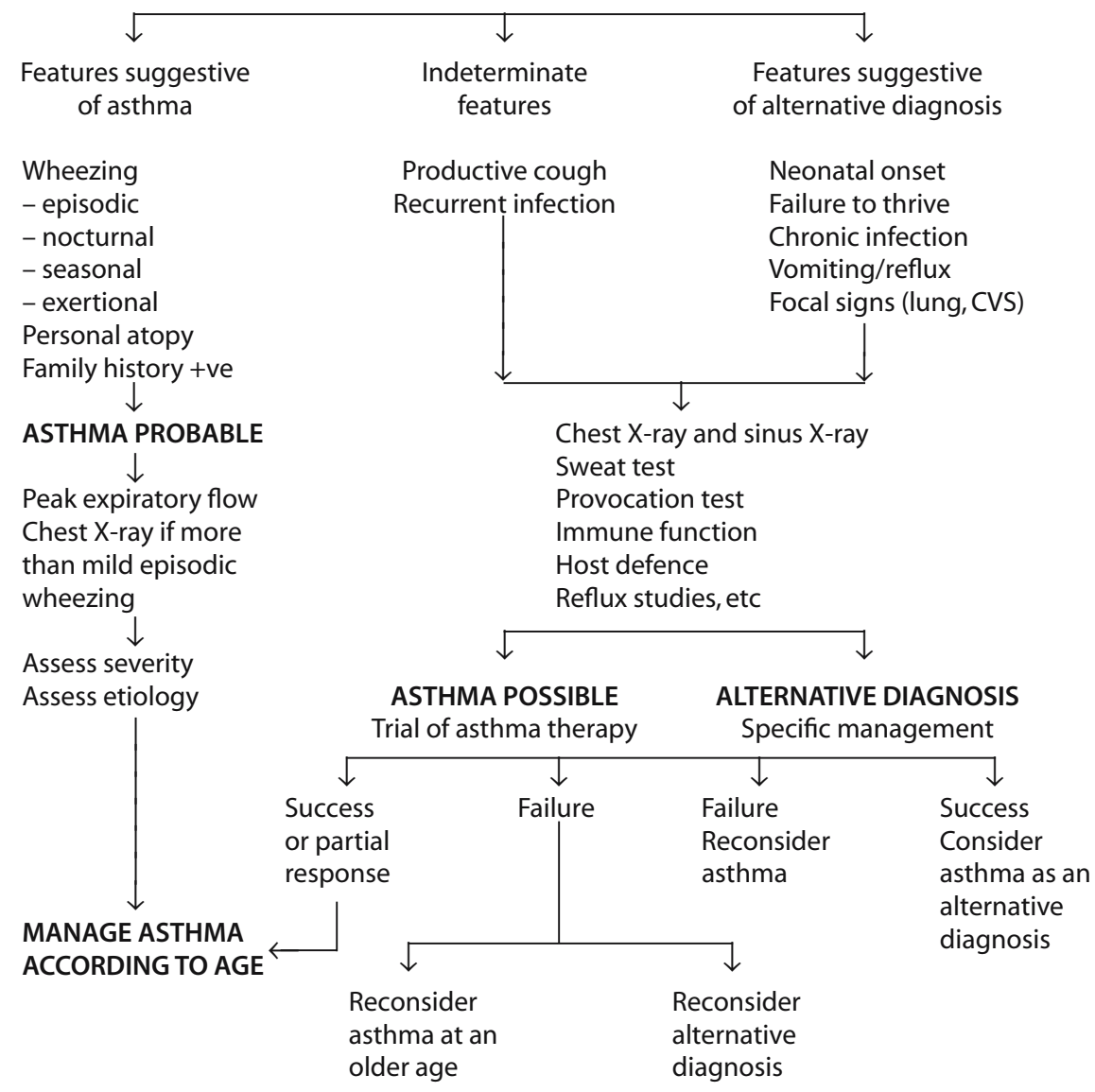

Fig. 11.50. Algorithm for the diagnosis of asthma in children unable to perform lung function tests. CVS cardiovascular system. (Modified from $[698,699]$ )

Table 11.32. Assessment of the parameters underlying the clinical manifestations and the higher age-related incidence

\begin{tabular}{|c|c|c|c|c|}
\hline \multirow[t]{2}{*}{ Parameters } & \multicolumn{3}{|l|}{ Age } & \multirow[t]{2}{*}{ Relative weight (\%) } \\
\hline & $<2$ years & $2-5$ years & $5-12$ years & \\
\hline Foods & ++ & + & \pm & \\
\hline Exercise & + & ++ & +++ & 70 \\
\hline Emotional factors & \pm & \pm & ++ & \\
\hline Irritant factors & + & ++ & +++ & 100 \\
\hline Environmental inhalants & + \pm & +++ & +++ & \\
\hline Viral infections & ++++ & +++ & + \pm & 90 \\
\hline Pollens & + & ++ & +++ & \\
\hline Allergens on the whole & & & & 80 \\
\hline
\end{tabular}

Modified from [481]. 
Table 11.33. Stepwise approach for managing infantile asthma based on symptom frequency

\begin{tabular}{ll} 
Type & Clinical manifestations \\
Episodic & $\begin{array}{l}75 \% \text { of cases; an exacerbation every } 4-6 \text { weeks with infrequent symptoms }(<5 \text { symptomatic days } \\
\text { every month); long periods of well-being, moderate wheezing after prolonged effort; } \\
\text { no urgent therapy, normal PFT between exacerbations }\end{array}$ \\
\hline Frequent & $\begin{array}{l}20 \% \text { of cases; recurrent symptoms ( }<\text { once a week), asymptomatic periods interrupted } \\
\text { by more frequent attacks and wheezing after modest efforts; urgent therapy: from never to often; } \\
\text { rare hospitalization; PFT variability } 20 \%-30 \%\end{array}$ \\
\hline Chronic & $\begin{array}{l}4 \% \text { of cases; frequent symptoms with no periods of well-being (symptoms }>5 \text { days/month, } \\
\text { over }>3 \text { months and } 50 \% \text { of days of each } 1 \text { st month of disease); wheezing after mild effort; } \\
\text { urgent therapy: rarely/less than once/month, variable PFT }\end{array}$ \\
\hline Seasonal & $\begin{array}{l}\text { Symptom development similar to the chronic type, but restricted to seasonal periods due } \\
\text { to exposure to inhalant allergens }\end{array}$
\end{tabular}

Data from [434-437, 494, 592].

\section{Clinical Presentation}

Recognition of the different patterns is useful to evaluate the symptoms based on age (Table 11.32), frequency and severity of the symptoms (Tables 11.33-11.35), and risk factors (Table 11.37) [310, 336, 481, 485, 623]. The procedure will distinguish between noncomplicated/ nonsevere cases or a severe asthmatic attack reflecting status asthmaticus.

\section{Noncomplicated/Nonsevere Cases}

\section{History}

The guidelines indicated in Chap. 6 are followed, examining the content of Table $11.38[164,584]$. If it is ascertained that the child has already manifested recurring episodes of cough and wheezing, likely linked to EIA, diagnosis is almost achieved. Also, it is possible that the child under observation belongs to a group of young patients with chronic and nonproductive nocturnal cough, or with laborious breathing after running, etc., but never wheezes (Table 11.36).

\section{Physical Findings}

The physical examination includes thoracic examination as well as verifying:

- The general condition of the subject

- The state of nutrition, including weight and height

- The possible presence of effort-induced dyspnea

Diffuse hyperphonesis, unequal reduction of breathing sounds according to the conditions, wheezing, expiratory whistling, prolonged expiration, and rales of varying loudness (sometimes only rales can be heard) reflecting airflow limitation are ascertained; liver and spleen are often palpable as a result of diaphragm depression, following marked lung hyperinflation. Wheez- ing is the tip of the iceberg as most of the iceberg is not noticeable, airway obstruction begins well before the tip is evident, and wheezing is heard, therefore its presence can be measured by spirometry before clinical objectivity reveals wheezy breathing [481]. When symptoms occur gradually, it is surprising how children are able to get used to the reduced respiratory function, considering it a normal condition and even expressing a mild sense of well-being so that a $<50 \%$ fall in PEF, and the related reduction of dynamic parameters can be underestimated for a long time. The symptoms that should arouse suspicion in these children are asthenia, a lessened ability to concentrate and persistent sinusitis.

\section{Diagnosis}

\section{Laboratory Examinations}

- Eosinophilia is often higher than $250-400$ cells $/ \mathrm{mm}^{3}$. The count can be repeated in the expectoration. In children this could be unrelated to ECP levels; however, $27 \%$ of 92 children aged $<2$ years had serum ECP $\geq 8 \mu \mathrm{g} / \mathrm{l}$, $76 \%$ of this group developed physician-diagnosed wheezing, and $48 \%$ had hospital admissions for wheezing.

- PRIST is often elevated. SPTs and RAST can be carried out to spot allergic asthma.

- Spirometry can provide useful information with collaborating children [429].

- If necessary, PFTs and/or BPTs (Chap. 6) can exclude other causes of wheezing (Tables 6.2, 6.3, 6.11). Even if BPT has proved useful in excluding the diagnosis of asthma, especially in adolescents, BPT with histamine is not a substitute for traditional medical diagnostic ability; children aged 2 years may be able to perform BPT (Fig. 6.25b).

- Other research has proved futile or misleading. For example, leukocytosis $>15,000 / \mathrm{mm}^{3}$ may be related to child age, the effect of stress and of adrenergic drugs [41]. 
Table 11.34. Stepwise approach for managing infantile asthma based on symptom severity

\begin{tabular}{|c|c|c|c|c|}
\hline Classification & $\begin{array}{l}\text { Mild } \\
\text { intermittent }\end{array}$ & $\begin{array}{l}\text { Mild } \\
\text { persistent }\end{array}$ & $\begin{array}{l}\text { Moderate } \\
\text { persistent }\end{array}$ & $\begin{array}{l}\text { Severe } \\
\text { persistent }\end{array}$ \\
\hline \multicolumn{5}{|l|}{ Clinical manifestations } \\
\hline Symptom frequency & $\begin{array}{l}\text { Intermittent } \\
\leq 2 \text { times a week }\end{array}$ & $\begin{array}{l}\text { Intermittent } \\
>2 \text { times/week, } \\
\text { but <once a day }\end{array}$ & $\begin{array}{l}\text { Daily } \\
\text { Daily symptoms }\end{array}$ & $\begin{array}{l}\text { Continual } \\
\text { Frequent severe } \\
\text { symptoms }\end{array}$ \\
\hline Exacerbations & $\begin{array}{l}\text { From a few hours } \\
\text { to a few days }\end{array}$ & $\begin{array}{l}\text { May affect normal } \\
\text { activity/sleeping }\end{array}$ & $\begin{array}{l}\text { Affects normal } \\
\text { activity/sleeping } \\
\geq 2 \text { times a week }\end{array}$ & Frequent \\
\hline $\begin{array}{l}\text { Between exacerbations } \\
\text { cough/wheeze }\end{array}$ & Asymptomatic & Asymptomatic & Often cough/wheeze & Continual \\
\hline Nocturnal asthma & $\leq 2$ times a month & $\geq 2$ times a month & $\geq$ Once a week & Frequent \\
\hline Exercise tolerance & Not reduced & $\begin{array}{l}\text { Reduced by vigorous } \\
\text { effort }\end{array}$ & Reduced & Very reduced \\
\hline School attendance & Regular & Regular & May be affected & $\begin{array}{l}\text { Frequent } \\
\text { absences }\end{array}$ \\
\hline \multicolumn{5}{|l|}{ Lung function } \\
\hline $\mathrm{FEV}_{1}$ or PEF $\%$ of predicted & $\geq 80 \%$ & $\geq 80 \%$ & $>60-<80 \%$ & $\geq 60 \%$ \\
\hline Daily variability & $<20 \%$ & $20 \%-30 \%$ & $>30 \%$ & $>30 \%$ \\
\hline \multicolumn{5}{|l|}{ Spirometry } \\
\hline Signs of obstruction & Absent & Absent or minimal & Modest & Severe \\
\hline Response to bronchodilators & Normal & $\begin{array}{l}>15 \% \text {, normal values } \\
\text { or modest increase }\end{array}$ & $\begin{array}{l}\text { Incomplete } \\
\text { normalization }\end{array}$ & $\begin{array}{l}\text { Incomplete/ } \\
\text { absent } \\
\text { normalization }\end{array}$ \\
\hline BPT to methacholine $\left(\mathrm{PC}_{20}\right)$ & & $>20 \mathrm{mg} / \mathrm{ml}$ & $2-20 \mathrm{mg} / \mathrm{ml}$ & $<2 \mathrm{mg} / \mathrm{ml}$ \\
\hline
\end{tabular}

Data from $[435,437,494,592]$.

Table 11.35. Grading of clinical severity

\begin{tabular}{|c|c|}
\hline Grading & Clinical criteria \\
\hline 1 & $\begin{array}{l}<5 \text { episodes per year with }>7 \text { days duration of symptoms and functional restriction each time, } \\
\text { and long symptom-free intervals with apparently normal PFT }\end{array}$ \\
\hline 2 & $\begin{array}{l}\text { 5-10 episodes per year with }>7 \text { days duration of symptoms and functional restriction each time, } \\
\text { and long symptom-free intervals with apparently normal PFTa }\end{array}$ \\
\hline 3 & $\begin{array}{l}\text { More than } 10 \text { episodes per year with }<7 \text { days duration of symptoms and functional restriction } \\
\text { each time, and long symptom-free intervals with apparently normal PFT, or more prolonged } \\
\text { periods (totaling } 12 \text { weeks or more per year) with symptomatic bronchial obstruction } \\
\text { or apparently impaired PFTa }\end{array}$ \\
\hline 4 & $\begin{array}{l}\text { More than } 5 \text { episodes per year with prolonged obstruction (totalling } \geq 6 \text { months per year) } \\
\text { following most episodes, or chronic symptomatic obstruction with restriction of function. } \\
\text { Bronchial asthma in need of institutional treatment and/or continuous use of corticosteroid } \\
\text { medication (any route) to classify for grade III or better }\end{array}$ \\
\hline 5 & $\begin{array}{l}\text { Chronic, incapacitating asthma with severe, acute exacerbations despite continuous medication } \\
\text { following appropriate and safe dosage regimen }\end{array}$ \\
\hline
\end{tabular}

The subgroups are formed according to those in Table 11.32 (episodic, frequent, chronic) or Table 11.33 (mild intermittent, mild persistent, moderate persistent, severe persistent) and to $\mathrm{FEV}_{1}$ values between exacerbations.

Modified from [1].

PFT Pulmonary function testing.

a If children have symptoms and signs of more prolonged bronchial obstruction (including subclinical obstruction) the next higher grade is given; simple exercise-induced asthma is not taken into account provided that recovery is complete with rest and/or a single dose of bronchodilator. 
Table 11.36. Differential diagnosis of persistent cough

\begin{tabular}{|c|c|}
\hline System & Causes \\
\hline Central nervous system & Psychogenic \\
\hline \multirow[t]{4}{*}{ Upper airways } & Irritation: foreign bodies, cigarette smoke, dust \\
\hline & $\begin{array}{l}\text { Inflammation: typical asthma, tracheitis, bronchitis, pertussis, recurrent infections } \\
\text { by virus and Chlamydia and Mycoplasma }\end{array}$ \\
\hline & Tumors: benign, malignant \\
\hline & Extrinsic compression: lymphadenopathy, tumors \\
\hline \multirow[t]{3}{*}{ Pulmonary parenchyma } & Inflammation: bronchiolitis, alveolitis, pneumonia \\
\hline & Vascular: pulmonary emboli, cardiac insufficiency \\
\hline & Respiratory disorders: cystic fibrosis, measles, bronchiectasis, bronchomalacia, tuberculosis \\
\hline \multirow[t]{5}{*}{ Extrapulmonary } & Stimulation of vagal auricular branches \\
\hline & Pleural, diaphragmatic or pericardial irritation \\
\hline & Sinusitis \\
\hline & Esophageal disease: tracheal fistula, foreign body aspiration, gastroesophageal reflux \\
\hline & Humoral immune deficiencies \\
\hline \multicolumn{2}{|l|}{ Others to be specified } \\
\hline Medications & $\beta$-Agonists, inhibitor of angiotensin converting enzyme \\
\hline
\end{tabular}

Data from $[186,279]$.

Table 11.37. Risk factors for status asthmaticus

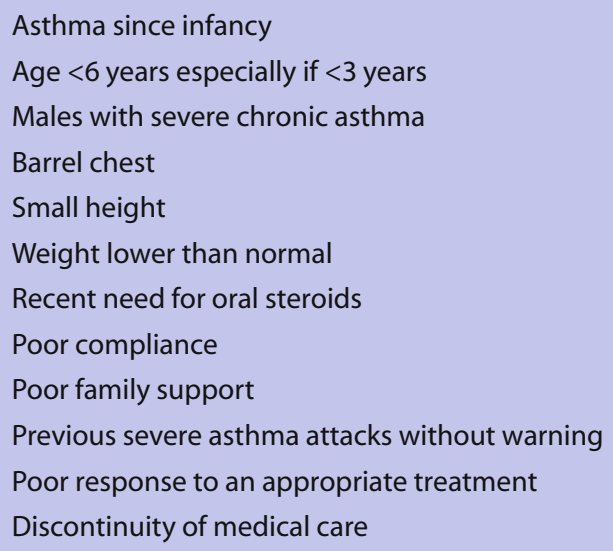

\section{Subjects at high risk}

Younger children:
a. Diagnostic doubts
b. Develop respiratory failure more rapidly

\section{Older children:}
a. Weaning of oral steroids after hospitalization
b. Hospitalization for asthma in past year
c. History of prior severe attacks
d. Poor compliance
e. Psychosocial problems

Children and adolescents

a. Prior admissions to pediatric emergency department

b. $\geq 2$ admissions in past year

c. $\geq 3$ admissions in past months

Data from $[310,336,481,485,623]$.
- Chest X-ray and ECG are not necessary in cases of uncomplicated asthma.

Chest X-ray shows hyperinflated lungs, depressed and not very mobile diaphragm, increased thoracic anterior-posterior diameter, peribronchial interstitial infiltrates, and sometimes parenchymal opacities often leading to atelectasis are found.

In all cases of recurring bronchoconstriction especially with increased susceptibility to infections (Chap. 22), it could be necessary to evaluate immunological parameters such as quantitative serum (Table 1.15) and secretory Igs, and lymphocytes and subpopulations levels (Tables 1.34-1.41, with BALF data). Determination of IgG subclasses is superfluous in the asthmatic child, as there are no differences compared to healthy subjects, with the exception of a few specific cases [468].

If a differential diagnosis with other conditions that should be differentiated from asthma is indicated, specific tests can be asked for such as PFT, BPT, as above, bronchoscopy, diagnostic imagining, bronchography, CT (computerized tomography), scintigraphy, etc., functional and cytological analysis of ciliary structures, and the sweat test.

\section{Differential Diagnosis}

Differential diagnosis includes the most common clinical conditions that mimic asthma at various age levels: Table 11.39 [481] shows the relative prevalences. Table $11.40[186,481,584]$ shows the most common precipitants of asthmatic symptoms and Table 11.36 those of persistent cough. A brief description of the condi- 
tions that most frequently present difficulties $[186,469]$ follows, also based on Appendix 11.2 [186, 469]:

- Aspiration of a foreign body: $40 \%$ of cases occur in children $\approx 2$ years old; if not recognized and treated promptly, it can cause disease and even death.

Table 11.38. History of asthma in infants and children

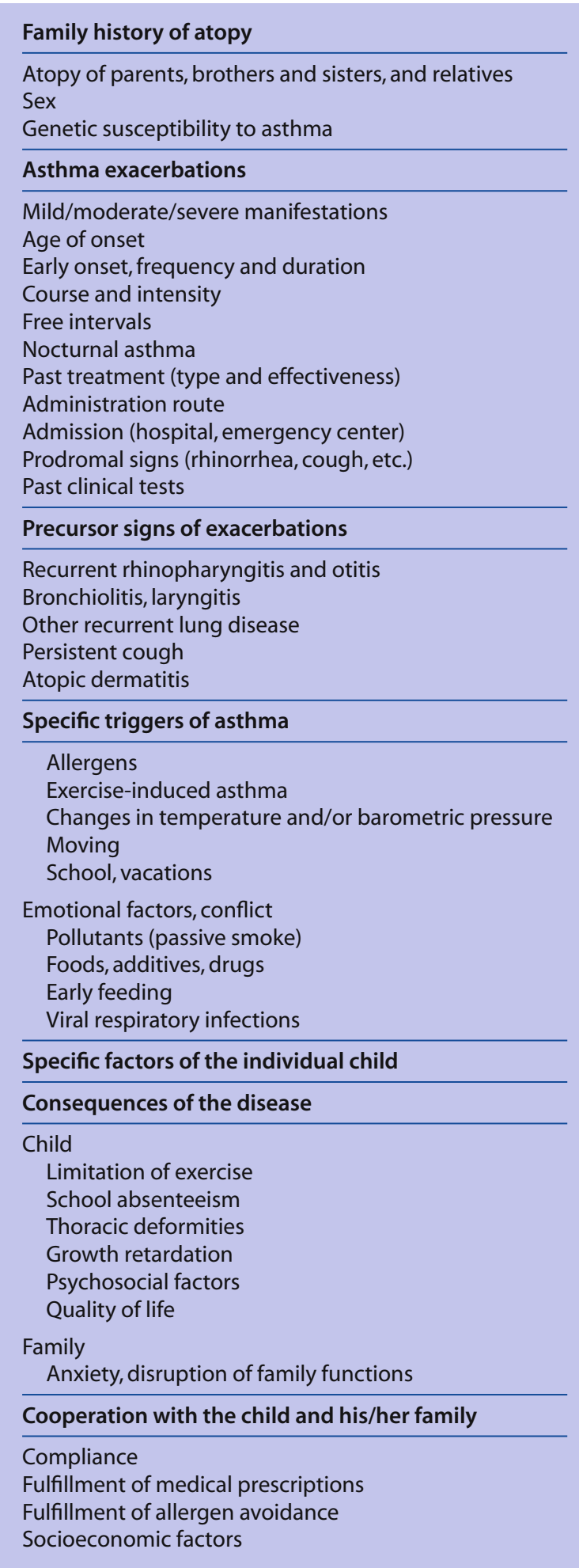

Data from [164, 584].
Usually it begins abruptly in otherwise normal subjects. Limited wheezing reduced to a single hemithorax is characteristic; it can be confused with asthma. Nevertheless, it does not respond equally promptly to bronchodilators, potentially implying that inappropriate treatments were instituted. Smaller bodies may induce progressive symptoms. Chest X-rays are not always discriminatory, since many foreign bodies are radiopaque. - Vocal cord dysfunction is a functional disturbance that mimics asthma with paroxysmal attacks and severe dyspnea and is unresponsive to any treatment. Sounds can be heard on auscultation during inspiration and expiration that is otherwise normal. Instrumental analysis gives consistently negative results.

- Hyperventilation syndrome and panic attacks can coexist with the basic disease. The patient complains of being breathless. There are asthmatic symptoms that contrast with negative objective findings, as do most other tests.

- Bronchiolitis: see Tables 11.27, 11.28, 11.30 and 11.32.

- Pertussis can lead to a mistaken diagnosis that should be avoided through a lymphocyte count and nasopharyngeal cultures.

- Cystic fibrosis: there can be initial asthma-like symptoms and BHR. The sweat test is a decisive means of providing a definitive answer, also necessary, especially considering the marked frequency in Caucasians [469].

- Bronchiectasis: wheezing and BHR are found. CT enables differentiation from asthma.

- Ciliary dyskinesia is suspected when a chronic obstructive respiratory disease is seen, accompanied by rhinosinusitis, otitis and X-rays showing emphysema [469].

\section{Severe Asthmatic Attack - Status Asthmaticus}

\section{Definition}

Status asthmaticus is an attack that lasts more than 1 or 2 days, in whom conventional forms of therapy have failed, and may require admittance to hospital, with the child often in progressive respiratory failure. It is a medical emergency in which the child with acute asthma fails to improve following appropriate aggressive treatment in an ED or outpatient setting [717]. However, children 3-5 years old are significantly more likely to have an ED visit (OR - odds ratio 1.6; $95 \% \mathrm{CI}, 1.3-2.0$; $p<0.0001$ ) or a hospitalization (OR 2.9; $95 \%$ CI, 2.0, 4.3; $p<0.0001$ ) than older children [5].

Risk factors of status asthmaticus are summarized in Table 11.36. The risk of hospitalization is very high with on household smoking (Table 4.25). The risk is greater in children aged $<2-4$ years as a result of the above-mentioned physiological particularities. Bronchial smooth muscles are particularly reduced in children 
Table 11.39. Relative incidence of most common clinical patterns entering the differential diagnosis of asthma in different age groups

\begin{tabular}{|c|c|c|c|}
\hline Disorder & Infants & Schoolchildren & Adolescents \\
\hline Acute laryngotracheobronchitis & ++ & ++ & \\
\hline Aspiration bronchopneumopathy & +++ & \pm & \pm \\
\hline Bronchiectasis & + & + & + \\
\hline Bronchiolitis & +++ & + & \\
\hline Chronic viral infections & +++ & ++ & \\
\hline Congenital anomalies & +++ & + & \\
\hline Cystic fibrosis & +++ & + & \pm \\
\hline Epiglottic laryngitis & +++ & ++ & \\
\hline Foreign body & ++ & +++ & \pm \\
\hline Hyperventilation syndrome & & + & ++ \\
\hline Hypoglottic laryngitis & ++ & + & \\
\hline Laryngotracheobronchomalacia & ++ & \pm & \\
\hline Mitral valve prolapse & & & + \\
\hline Pertussis & +++ & + & \\
\hline
\end{tabular}

Modified from [481].

Table 11.40. Relative incidence of most common precipitants of wheezing in different age groups

\begin{tabular}{llll} 
Disorder & Infants & Schoolchildren & Adolescents \\
Aspirin & $?$ & $?$ & $?$ \\
\hline Exercise & + & ++ & +++ \\
\hline Food allergens & ++ & + & $?$ \\
\hline Inhalant allergens (perennial) & + & +++ & +++ \\
\hline Inhalant allergens (seasonal) & $?$ & ++ & +++ \\
\hline Irritants (ozone, cigarette smoking) & + & ++ & +++ \\
\hline Viral infections & ++++ & +++ & ++
\end{tabular}

Data from $[186,481,584]$.

aged $<3$ years, in whom the obstruction is more a result of edema than of bronchospasm. On the other hand, mucosal glands are numerous, with a consequent increase in the Reid index (mucosal glands/thoracic wall). Furthermore, the relative scarcity of diaphragmatic fibers, which contributes to a reduced muscle resistance to the work required of them, the increased pulmonary peripheral resistance and reduced alveolar surface should not be forgotten. Pulmonary mechanics and volumes are markedly altered in status asthmaticus. Caused by severe lower airway airflow limitation, premature airway closure leads to increases in closing capacity and FRC. Inspiratory muscle activity persists throughout expiration, attempting to counteract expiratory airway closure by increasing the forces holding the airway open. Hyperinflation results [717]. Nonhomo- geneous distribution of areas of premature airway closure and obstruction causes ventilation/perfusion mismatching and hypoxemia results. Increased work of breathing under hypoxic conditions and some degree of dehydration combine to cause accumulation of inorganic acids. This acidosis is initially offset by respiratory alkalosis, but once respiratory failure ensues, a rapid and often profound decrease in $\mathrm{pH}$ will occur [717]. The combination of these factors explains the increased severity of asthma, the higher incidence of hospitalization, and the relatively scant response to bronchodilators in the pediatric population aged $<5$ years, and particularly $<3$ years. 
History

It is of major importance to ascertain [509]:

- Chronology of the episode underway

- Apparent cause

- Severity of the symptoms

- Oral solid and fluid intake in the last $12 \mathrm{~h}$

- The performance - or the contrary - of normal activities

- Type and duration of sleep

- Moods

- Name, dosage, and administration time of any medication ingested during the last $24 \mathrm{~h}$

- Effect of the treatment

- Outcome of the preceding episodes

- Possible admittance to hospital or ICU

- Family ability to carry out necessary therapy

\section{Objective Examination}

Objective examination (Table 11.41) [273, 435-437, 485, $623]$ requires that on inspection, the general conditions and the presence or lack of be observed:

- Possible signs of risk affecting the psyche (anxiety, agitation, apathy, drowsiness)

- Inhalatory retractions and use of the accessory muscle

- Dehydration

- Polypnea

- Forced posture

- Cyanosis

- Sweating

- Tremors

- Breathlessness

After the evaluation of the general conditions, hydration, decubitus, etc., and of thoracic objectivity, several other parameters can be considered to make a diagnosis as quickly as possible and initiate treatment. Table 11.41 provides a general assessment; therefore it is not necessary for all parameters under consideration to be present. However, the greatest risk in respiratory failure dur-

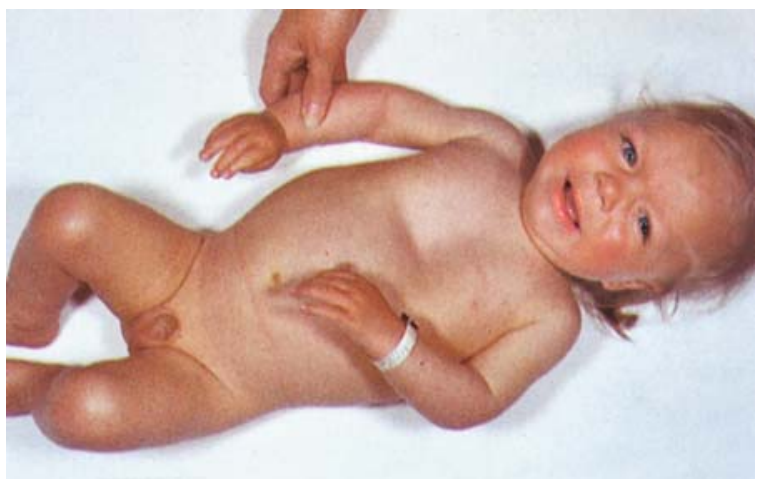

Fig. 11.51. A 13-month-old baby with acute asthma: note the marked indrawing of lower sternum ing episodes of severe asthma regards young children (Table 11.36), in whom PEFR measurement is not always easy; therefore an evaluation of these parameters can permit a strict control of the child's condition [436].

Vigilance is the participation in the environment and can help in evaluating the child's fatigue.

Dyspnea is the parameter most noted by parents and doctors and can be helpful in evaluating the level of airway obstruction. It can be evaluated semiquantitatively by asking the child to repeat a phrase or count to ten within a single breath. The condition improves if the length of the phrase or the numbers counted increase.

The use of auxiliary muscles is an indication of bronchoconstriction. Sternocleidomastoid use is linked to PEFR or $\mathrm{FEV}_{1}<50 \%$ of predicted value. Flaring of nasal wings (Fig. 11.51) is a visible sign of dyspnea and shows the involvement of auxiliary muscles in respiration. Diaphragmatic depressions can be noted and inspiratory retractions, especially intercostal [481] (Figs. 11.51, 11.52).

Status asthmaticus is an ingravescent asthma, resistant to therapy, that progresses to a state of emergency [310]. The symptoms given in Table 11.41 can assist in its diagnosis. It eventually becomes hypercapnic, caused by $\mathrm{CO}_{2}$ accumulation with ensuing development of respiratory acidosis.

On auscultation, prolonged expiration, wheezing, due to obstruction worsening, is audible during both inspiration and expiration, while respiratory sounds are re-

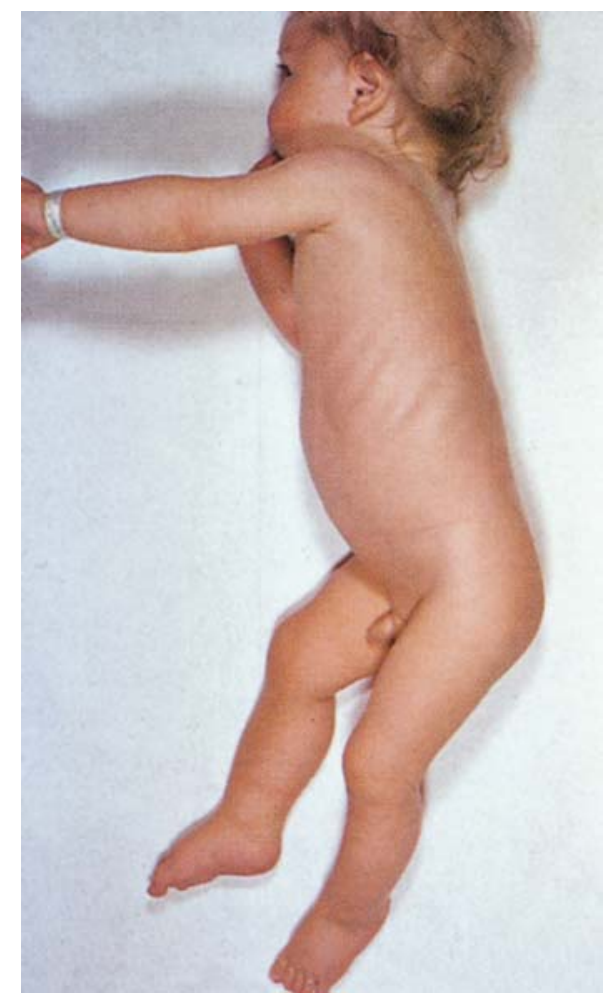

Fig. 11.52. A 1-year-old baby during an acute asthma attack: note the marked indrawing of intercostal spaces 
Table 11.41. General assessment of a severe asthmatic attack in children

\begin{tabular}{|c|c|c|c|c|}
\hline Symptoms & Mild & Moderate & Severe & Resp. arrest imminent \\
\hline Dyspnea & Absent/mild & Moderate & Severe & \\
\hline Older child & Walks, plays & Walks, speaks & Resting, poor speaking & \\
\hline Infant & Softer, shorter cry & Difficulty feeding & Stops feeding/suckling & \\
\hline Decubitus & Can be down & Prefers sitting & Sits upright & \\
\hline Talks in: & Normal sentences & Short phrases & Words or single letters & \\
\hline Alertness & May be agitated & Usually agitated & Always agitated & Drowsy or confused \\
\hline Color & Normal/reduced & Pallor & Cyanosis \pm & \\
\hline Accessory muscles & $\begin{array}{l}\text { Absent/mild } \\
\text { retractions }\end{array}$ & $\begin{array}{l}\text { Modest retractions, } \\
\text { use of sternocleido- } \\
\text { mastoid muscles }\end{array}$ & $\begin{array}{l}\text { Marked retractions, } \\
\text { nasal flaring during } \\
\text { inspiration }\end{array}$ & Paradoxical breathing \\
\hline Wheeze & $\begin{array}{l}\text { Moderate, } \\
\text { end-expiratory }\end{array}$ & $\begin{array}{l}\text { Loud, expiratory } \\
\text { inspiratory }\end{array}$ & $\begin{array}{l}\text { Reduced air } \\
\text { penetration }\end{array} \longrightarrow$ & Silent chest \\
\hline \multicolumn{5}{|l|}{ RR by age [485] } \\
\hline$<3$ months & $<60 /$ min & $60-70 / \mathrm{min}$ & $>70 /$ min & \\
\hline 3-12 months & $<50 /$ min & $50-60 / \mathrm{min}$ & $>60 / \mathrm{min}$ & \\
\hline $1-6$ years & $<40 / \min$ & $40-50 / \mathrm{min}$ & $>50 / \min$ & \\
\hline$>6$ years & $<30 /$ min & $30-40 / \mathrm{min}$ & $>40 /$ min & \\
\hline \multicolumn{5}{|l|}{ HR by age [485] } \\
\hline$<1$ year & $<150$ & $150-170$ & $>170$ & \\
\hline $1-2$ years & $<120$ & $120-140$ & $>140$ & \\
\hline$>2$ years & $<110$ & $110-130$ & $>130$ & Bradycardia \\
\hline Pulsus paradoxus & $\begin{array}{l}\text { Absent } \\
<10 \mathrm{mmHg}\end{array}$ & $\begin{array}{l}\text { Present } \pm \\
10-20 \mathrm{mmHg}\end{array}$ & $\begin{array}{l}\text { Often present } \\
20-40 \mathrm{mmHg}\end{array}$ & Absent ${ }^{b}$ \\
\hline $\begin{array}{l}\text { PEFR (\% of predicted } \\
\text { or personal best) } \\
\text { (pretreatment) } \\
\text { see Figs. } 6.26 \text { to } 6.28 \text { ) }\end{array}$ & $>80 \%$ & $50 \%-80 \%$ & $<50 \%$, life-threatening & $<33 \%$ \\
\hline $\mathrm{SaO}_{2}$ & $>95 \%$ & $91 \%-95 \%$ & $<91 \%$ & \\
\hline $\mathrm{PaCO}_{2}$ & $<35 \mathrm{mmHg}$ & $40 \mathrm{mmHg}$ & $\begin{array}{l}>40 \mathrm{mmHg} \\
\text { possible cyanosis }\end{array}$ & \\
\hline $\mathrm{PaO}_{2}$ (room air) & $90-100 \mathrm{mmHg}$ & $60-90 \mathrm{mmHg}$ & $\begin{array}{l}<60 \mathrm{mmHg} \text {, possible } \\
\text { respiratory insufficiency }\end{array}$ & \\
\hline
\end{tabular}

Children exhibiting moderate symptoms should be considered for admission. Normal age-related RR values are in Appendix 11.1

Data from $[273,435-437,485,623]$.

$\mathrm{SaO}_{2} \mathrm{O}_{2}$ saturation, $\mathrm{PaO}_{2}$ partial pressure of $\mathrm{O}_{2}$ in arterial blood, $\mathrm{PaCO}_{2}$ partial pressure of $\mathrm{CO}_{2}$ in arterial blood, $R R$ respiratory rate.

a It is more reliable when $>20 \mathrm{mmHg}$ (see text).

b Suggests respiratory muscle fatigue.

duced. In children with signs of respiratory distress, wheezing is absent because of airway obstruction.

The severity can also be measured by means of a respiratory score (Table 11.42) [174, 476], especially for children aged $<6$ who have little experience with PFM, or children in significant respiratory distress, in whom it is difficult to obtain an accurate PFM measurement [102].

\section{Laboratory Evaluation}

Blood gas analysis: measurement of $\mathrm{PaCO}_{2}$ is the parameter most indicative of severity [485].

Appendices 6.6-6.12 indicate PEF values according to sex and age. No response to a $\beta_{2}$-adrenergic, whether evaluated clinically or instrumentally, is a sign of severe obstruction related to the degree of asthma severity. 
Table 11.42. Pediatric clinical score to estimate the severity of an acute exacerbation of asthma: clinical score

\begin{tabular}{|c|c|c|c|c|}
\hline & 0 & 1 & 2 & 3 \\
\hline Wheezing & Absent & Expiratory & $\begin{array}{l}\text { Expiratory } \\
\text { and inspiratory }\end{array}$ & $\begin{array}{l}\text { Silent chest (presence } \\
\text { of severe obstruction) }\end{array}$ \\
\hline RR increase & Normal & Normal to $30 \%$ & $30 \%-50 \%$ & Over $50 \%$ a \\
\hline $\mathrm{HR}$ & $<120-140$ & $\approx$ & $\approx$ & $>120-140^{a}$ \\
\hline Accessory muscle use & Normal & $\begin{array}{l}\text { Mild retractions } \\
\text { (negligible) }\end{array}$ & $\begin{array}{l}\text { Moderate } \\
\text { (intercostal } \\
\text { retractions) }\end{array}$ & $\begin{array}{l}\text { Severe (marked tracheosternal } \\
\text { and intercostal retractions) }\end{array}$ \\
\hline Muscle retractions & - & + & ++ & +++ \\
\hline Orthopnea & Normal & & Inconstant & Constant \\
\hline Activity & Normal & $\begin{array}{l}\text { Limitations on } \\
\text { vigorous exercise }\end{array}$ & $\begin{array}{l}\text { Very reduced, } \\
\text { nightly disturbance, } \\
\text { anxiety }\end{array}$ & $\begin{array}{l}\text { Stops feeding, stops sleeping, } \\
\text { agitation and/or prostration }\end{array}$ \\
\hline Duration & $<1 \mathrm{~h}$ & $1-4 h$ & $4-8 h$ & $>8 \mathrm{~h}$ \\
\hline
\end{tabular}

Severity: mild exacerbation $<7$ points, mean exacerbation 7-12, severe exacerbation $>12$.

Data from $[174,476]$.

a With other parameters reaching a score value of $2-3$.

Table 11.43. Pediatric clinical score to estimate the severity of an acute exacerbation of asthma: respiratory score

\begin{tabular}{llll} 
& 0 & 1 & 2 \\
$\mathrm{PaCO}_{2}$ & $<36$ & 40 & $>40$ \\
\hline $\mathrm{PaO}_{2}(\mathrm{mmHg})$ & $90-100$ & $60-90$ (room air) & $<60\left(\mathrm{in} 40 \% \mathrm{O}_{2}\right)$ \\
\hline $\mathrm{SaO}_{2}$ & $>95 \%$ & $91 \%-95 \%$ & $<91 \%$ \\
\hline Cyanosis & Normal & Room air & In $40 \% \mathrm{O}_{2}$ \\
\hline Pulsus paradoxus $(\mathrm{mmHg})$ & $<10$ & $10-20$ & $20-40$ \\
\hline Accessory muscle use & Normal & Moderate & Marked \\
\hline Auscultation (wheeze) & End-expiratory & Inspiratory and expiratory & Loud or absent \\
\hline Alertness & Normal & Normal/decreased & Decreased
\end{tabular}

To each parameter is assigned a score from 0 to 3 . Summing up the single scores, the severity of asthma exacerbations can be estimated according to the following scale: $0-4$, no immediate danger; 5-6, impending respiratory insufficiency; 7 or more =, ongoing respiratory insufficiency.

Data from [174].

$\mathrm{SaO}_{2} \mathrm{O}_{2}$ saturation, $\mathrm{PaO}_{2}$ partial pressure of $\mathrm{O}_{2}$ in arterial blood, $\mathrm{PaCO}_{2}$ partial pressure of $\mathrm{CO}_{2}$ in arterial blood.

The respiratory score (Table 11.43) evaluates the need for hospitalization and monitoring the outcome of clinical symptoms in a hospital setting.

\section{Particularities of Small Children}

- Inspiratory retractions at rest and nasal flaring (in infants rhythmic head flexion during inhalation and extension during expiration).

- HR and RR: RR can vary from 20\%-30\%, depending on whether the infant is awake or asleep. It is advisable to measure it also during sleep.
- $\mathrm{SaO}_{2}$ : toddlers tend to develop hypoxemia earlier than adults [435].

\section{Treatment}

\section{Treatment of Acute Asthma Attack}

An acute attack or asthma exacerbation always requires the greatest medical care [559]. The aims of treatment are summarized in Table $11.44[79,604]$. On the basis of the flow chart in Fig. $11.53[174,435,436]$ and the scores in Tables $11.42,11.43$, it is possible to establish the most 
Table 11.44. Targets of antiasthmatic treatment in children Maximum clinical improvement by minimal use
of medications

Reduction of both frequency and severity of acute attacks

Efforts must be made to reduce visits to emergency wards and hospital admissions

Maximum improvement of lung function tests

Patient education to asthma and its treatment

Normal night rest

No symptoms at awakening

No missed day from school

Participation in physical, sport and social activities with no restrictions

Improve their quality of life

Minimize potential adverse effects of asthma medications

Normal growth

Data from $[79,604]$.

appropriate therapeutic approach, but there are others that are equally valid. Given the variability of the clinical picture, however, they cannot constitute a rigid guide, but should be interpreted on the basis of clinical evidence [268] in which history and progression of clinical symptoms are important [485]. To evaluate the severity of an acute asthma attack in the child, follow
Table 11.41. Immediate management in the hospital receiving room can reduce hospital admissions for acute asthma, allowing more children to be safely managed in the community [115]. As a consequence, $>70 \%$ of asthma hospitalizations could be cared for in alternative settings with supplemental $\mathrm{O}_{2}$, nebulized medication treatments, and close nursing observation [392]. Depending on symptom relevance (mild, moderate or severe), various drugs or combinations of drugs in sequence can be used. Diagnostic tests, if convenient, with the aid of a respiratory score, can evaluate the necessity for recovery: a score of $0-4$ is reassuring, in that an immediate danger of respiratory failure can be excluded; it must, however, be monitored at regular intervals. With scores $>5$, admittance to hospital could be unavoidable [174]. Because of RR variability, it is recommended that it be measured as described below, as well as pulsus paradoxus: if the score is $>20 \%$, a serious airway obstruction is present.

If the child is old enough or can collaborate, the reduction in pulmonary volumes and flows can be measured (spirometry, PEFR) [437]. These findings, if possible, should be obtained at once, then at 30- to 60-min intervals, which must be reduced to $15-30 \mathrm{~min}$ if the child does not respond to treatment [268]. If spirometry cannot be carried out, even because of the severity of the child's conditions, ascertain $\mathrm{PaO}_{2}$ (Table 6.25) and $\mathrm{PaCO}_{2}$ by measuring arterial blood gas level.

Figure 11.54 [140, 174, 435-437, 559, 699] indicates various treatment plans, either at home or in an ED. Therapeutic interventions should be integrated, according to the case, with good hydration, correction of acidosis, and $\mathrm{O}_{2}$ therapy when necessary (see below).

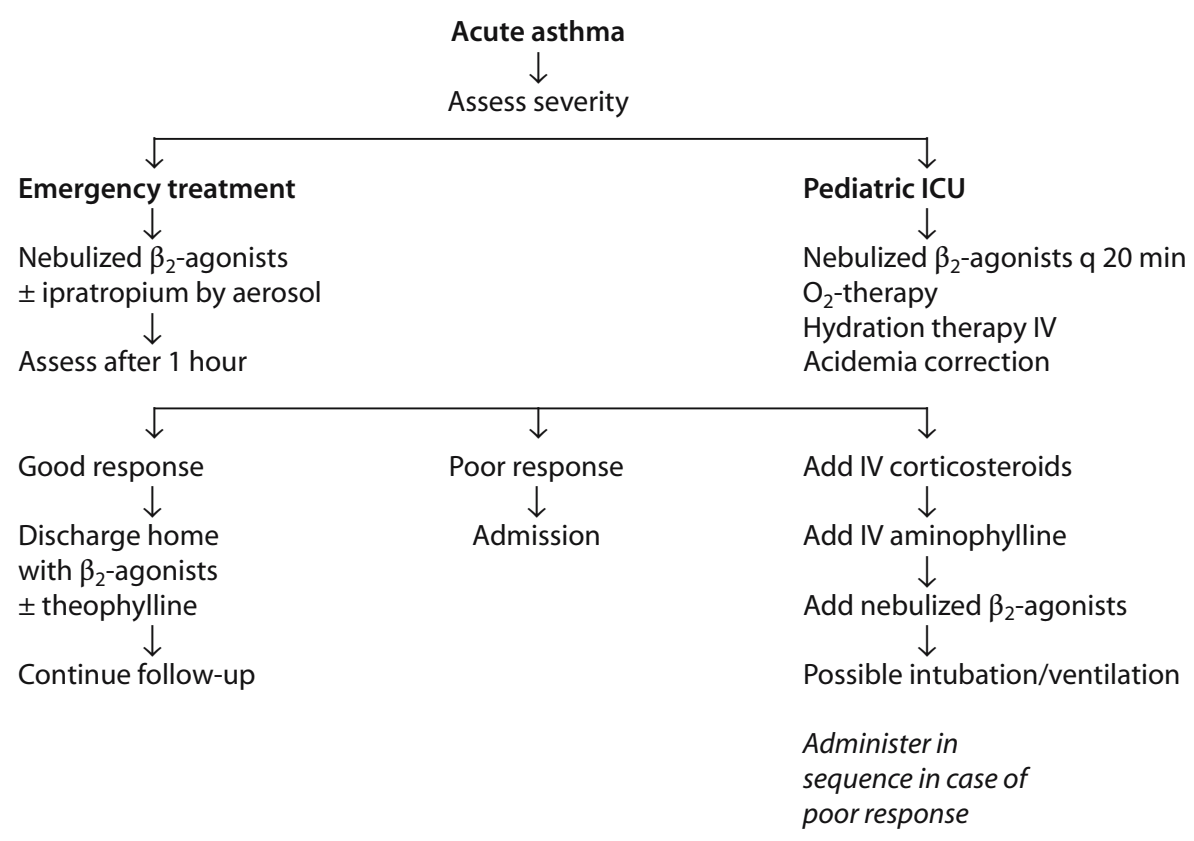

Fig. 11.53. Flow chart for severe pediatric asthma management in a pediatric emergency department or pediatric intensive care unit. (Data from $[174,434,436])$ 
We repeat that in early infancy rehydration is cardinal, since dehydration can easily occur as a result of RR increase and the reduced supply of nutrients and water: a $5 \%$ dehydration should be corrected when necessary.

Children experiencing an asthmatic attack, even if not severe, become hypoxemic more easily than older children [605] as a result of perfusion-ventilation [554]. The degree is related to respiratory obstruction and, above all, to $\mathrm{PaO}_{2}$ and $\mathrm{FEV}_{1}[267,605]$.

$\mathrm{PaCO}_{2}$ increase is an index of severity. The respiratory silence associated with hypoxemia $\left(\mathrm{PaO}_{2}<50\right.$ torr $)$ and/or hypercapnia $\left(\mathrm{PaCO}_{2}>45-50\right.$ torr $)$, or $\mathrm{SaO}_{2}$ $<91 \%$, is also an index of severe respiratory insufficiency, which makes recovery in an ICU urgent [406].

The AAP recommends, as the first treatment in a child whose condition may be defined as severe, the use of SC epinephrine in standard dosages [509]. Moreover, the $\alpha$-stimulating action makes epinephrine the drug of choice [485]. If a moderate attack occurs, which can be treated at home, the first treatment to be done at once should be with epinephrine, which has specific indications in cases of severe asthma, particularly suitable for use with children who, by definition, do not suffer from cardiovascular disturbances, apart from effects due to overdose. For the inhaled racemic form see above and Table 11.16; otherwise it can be substituted with inhaled $\beta_{2}$-adrenergics, for example albuterol: unit dose 100$200 \mu \mathrm{g}$ with dosed MDI, or else $150 \mu \mathrm{g} / \mathrm{kg}$ diluted in $3 \mathrm{ml}$ of saline to be nebulized in $10 \mathrm{~min}$ by facemask or spacer + VHC, measuring periodically the above parameters. All $\beta_{2}$-adrenergics are equivalent, but because of its wide international use, albuterol remains the drug used as a reference point [406].

If, after the use of epinephrine $/ \beta_{2}$-adrenergics, an efficacious clinical response is obtained, a home maintenance treatment can be suggested, without disregarding subsequent treatment plans (Fig. 11.54). If no obvious improvement is observed, IB can be added to nebulized solution (150-250 $\mathrm{\mu g}$ according to the age, to be repeated every $6 \mathrm{~h}$ ). ICS can be added to the treatment [406]. Interestingly, in children with acute asthma a single inhaled dose of BUD decreases ENO and is correlated to a PEFR increase [659], and two doses of dexamethasone may provide similar efficacy with improved compliance and fewer side effects than five doses of prednisone [510]. In two studies in ED children aged $<5[149,556]$, the treatments compared with prednisone were nebulized dexamethasone $1.5 \mathrm{mg} / \mathrm{kg}$ and nebulized BUS $800 \mu \mathrm{g}$ three doses at 30 -min intervals, there were no significant differences in admission rates, with a nonsignificant trend favoring ICS (OR 0.49; 95\% CI, 0.22-1.07); thus oral CS appear to be at least as effective as ICS in children with exacerbations of asthma. Another ED trial specifically including 100 children aged $>5$ with the most severe asthma on presentation $\left(\mathrm{FEV}_{1}\right.$ $<60 \%$ predicted) found prednisone better than $2 \mathrm{mg} \mathrm{FP}$ by MDI and spacer at $4 \mathrm{~h}\left(\mathrm{FEV}_{1} 45 \%\right.$ predicted at trial entry, admission rate $31 \%$ on FP vs $10 \%$ on prednisone)
[567]. Using much higher doses, the bulk of evidence suggests that ICS may be as effective as oral CS in all but the most severe episodes. One trial compared nebulized BUD ( $2 \mathrm{mg} / 8 \mathrm{~h}$ ) with two doses of prednisolone $2 \mathrm{mg} / \mathrm{kg}$ on admission and at $24 \mathrm{~h}$ in 46 children admitted to hospital with severe asthma. Life-threatening episodes were excluded. Outcomes were comparable at $24 \mathrm{~h}$ and at 3- and 24-day follow-up [386].

Predictors of hospitalization in children with acute asthma are five variables: previous ICU admission, baseline $\mathrm{SaO}_{2} \leq 92 \%, 4$-h $\mathrm{SaO}_{2} 92 \%$ or less, 4 - h clinical asthma score $6 / 9$ or higher, and hourly albuterol dosing intervals, associated with long vs short therapy, all with high odds ratio (OR). Probability of long therapy was $91.5 \%-99 \%$ for $\leq 3$ predictors, but only $40.6 \%-61.8 \%$ for individual variables [291].

Otherwise, follow what is indicated in the treatment of status asthmaticus, or take the child immediately to a pediatric $\mathrm{ED}$, especially if there is no response to the $\beta_{2}$-adrenergic administered by MDI of parenterally [41]. Persisting with treatment at home in a child with little response to the bronchodilators leads only to a worsening of the episode, which becomes even more refractory to drugs [267].

\section{Treatment of Status Asthmaticus}

Ingravescent asthma unresponsive to treatment is a lifethreatening condition usually requiring admittance to ICU. Table $11.45[434,435,485]$ indicates the dosages of the drugs to be used in an acute severe attack of status asthmaticus. The clinical characteristics, to be evaluated together with the clinical asthma score in Tables 11.42 and 11.43, are summarized in Table 11.46 [56, 336, 476, 485]. Monitoring of clinical parameters is integrated with laboratory parameters only under a regimen of hospitalization and observation. In emergencies, it is better to treat the child rather than waste time in consulting textbooks or prescribing tests [485]. It should be underlined that because of potential hypoxemia, $\mathrm{SaO}_{2}$ (which must always be $>93 \%$ ) should be measured regularly. Nevertheless $\mathrm{SaO}_{2}$ decrease is often an early sign of moderate or severe obstruction. If it is found to be $<91 \%$, it is an indication that hospitalization is required [663]. Therapy is summed up in the following points [267, 268, 435, 485, 564, 683, 699, 717] (Fig. 11.54, Table 11.45).

An urgent strategy in a child with status asthmaticus is a controlled ventilation. Over 11 years, $11.3 \%$ of 290 PICU admissions for status asthmaticus required ventilation: 13 children (aged 2-18) presented with rapid respiratory failure en route, or within $30 \mathrm{~min}$ of arriving to the ED [366]. Pressure-controlled ventilation (PCV) in 40 children with severe respiratory failure was an effective strategy. Four hours after starting PCV, median $\mathrm{pH}$ increased to $7.31(6.98-7.45, p<.005)$, and $\mathrm{Pco}_{2}$ decreased to 41 torr $(21-121$ torr, $p<.005)$, which were 
below 7.21 (range, 6.65-7.39) and 65 torr (29-264 torr), respectively. For children with respiratory acidosis $\left(\mathrm{PCO}_{2}\right)$ ( $>45$ torr) within $1 \mathrm{~h}$ of starting PCV, the median length of time until $\mathrm{PCO}_{2}$ decreased to $<45$ torr was $5 \mathrm{~h}(1-51 \mathrm{~h}) . \mathrm{SaO}_{2}$ was maintained $>95 \%$ in all patients. Median duration of PCV was $29 \mathrm{~h}$ (4-107 h), PICU stay was $56 \mathrm{~h}(17-183 \mathrm{~h})$, and hospitalization was 5 days (2-20 days). Therefore, PCV represents a therapeutic option in the management of such children [554]. Children with rapid respiratory failure had greater improve- ments in ventilation (significantly shorter) and oxygenation than those with progressive respiratory failure [366].

Status asthmaticus must be treated with IV therapy. Many children are dehydrated due to excessive insensible loss from respiratory effort, hyperdiuresis, and vomiting. Initial therapy must include intravascular volume repletion with normal saline, correction of electrolyte imbalance, and fluid and electrolyte maintenance.

\section{A) HOME TREATMENT}

\begin{tabular}{|c|}
\hline $\begin{array}{l}\text { Assess severity } \\
\text { Measure PEFR value, respiratory rate (RR), heart rate (HR), } \\
\text { note use of accessory muscles, alertness, color }\end{array}$ \\
\hline$\downarrow$ \\
\hline $\begin{array}{l}\text { Initial treatment } \\
\text { Albuterol (Fig. } 11.45 \text { ) up to } 3 \text { administrations } \\
\text { - by nebulizer } 0.1 \mathrm{mg} / \mathrm{kg} / \text { dose or } \\
\text { - MDI/spacer } 2-4 \text { puffs q } 20 \text { minutes up to one hour if needed } \\
\text { or } \\
\text { Theophylline, with no prior dose, } 6.5 \mathrm{mg} / \mathrm{kg}\end{array}$ \\
\hline
\end{tabular}

Evaluate after 1 hour

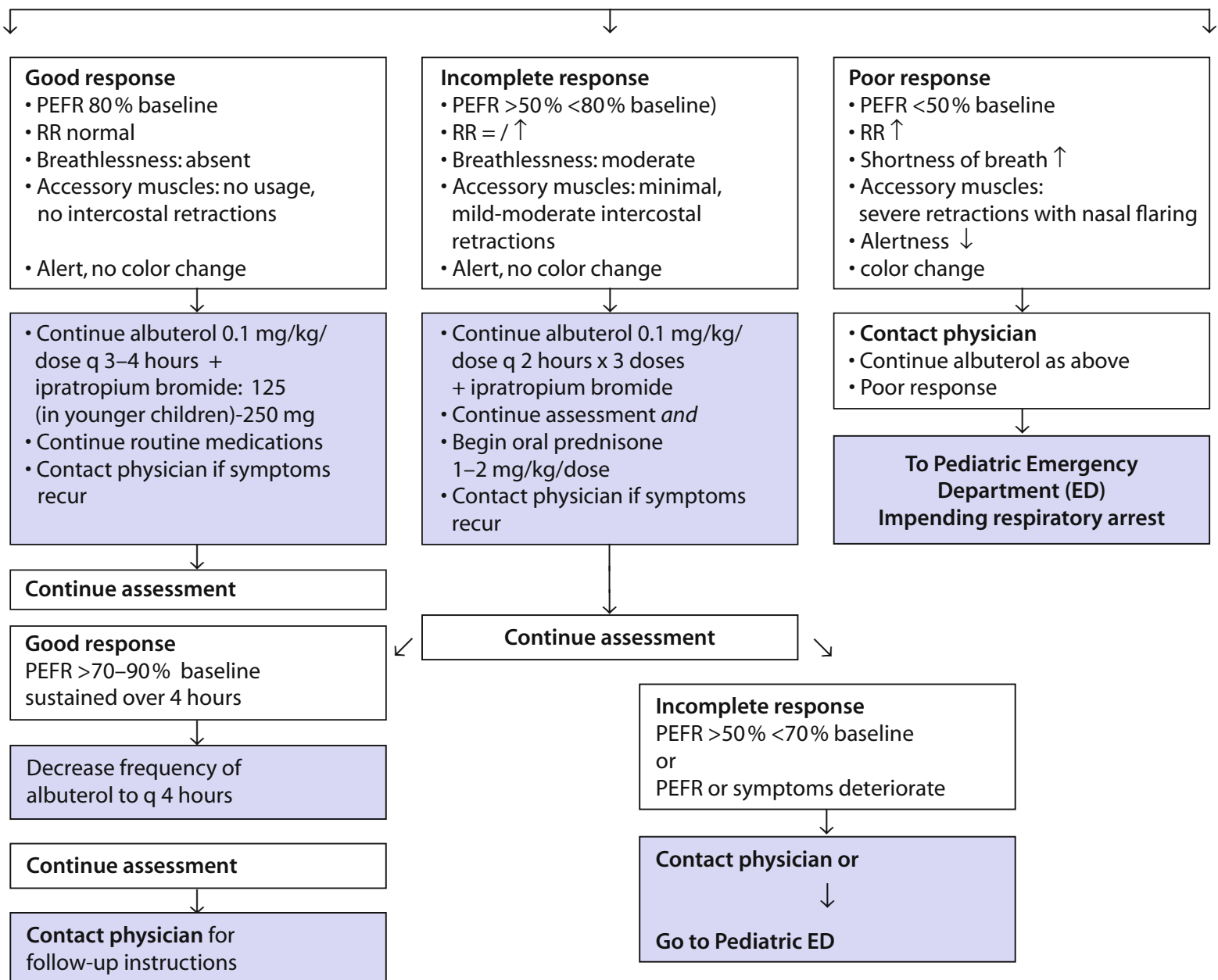

Fig. 11.54. Flow chart for the treatment of pediatric acute asthma exacerbations. A Home treatment. 


\section{B) PEDIATRIC ED}

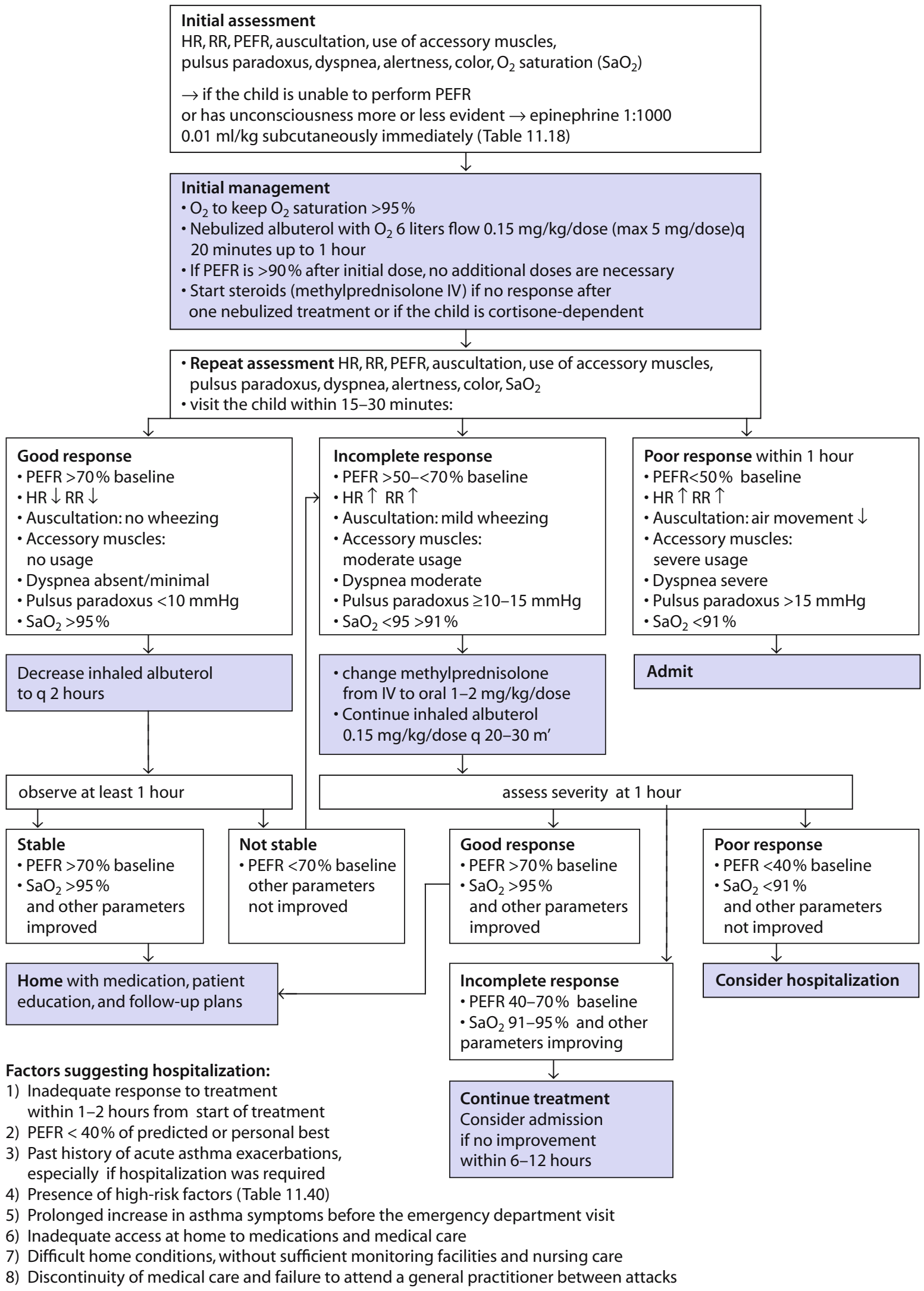

Fig. 11.54 (continued). Flow chart for the treatment of pediatric acute asthma exacerbations. B Treatment in the ED. 


\section{C) HOSPITAL MANAGEMENT}

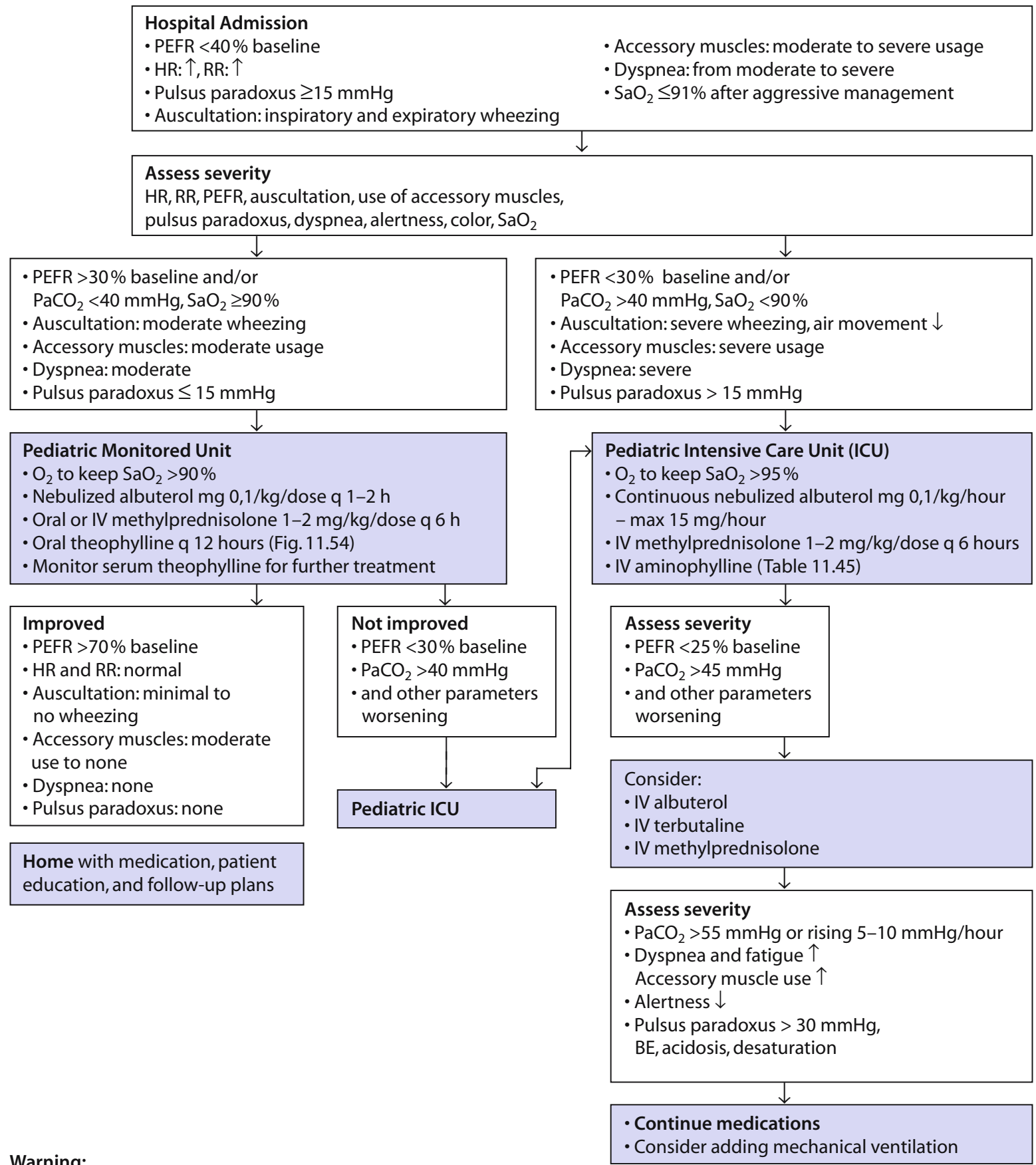

In any moment the child presents:

- excessive fatigue /weakening

- confusion or drowsiness

- PEFR is $<50 \%$ and/or hypercapnia and hypoxia persist or worsen admit the child to ICU

Note: in most cases holding chambers could be substituted for nebulizers to deliver $\beta_{2}$-agonists in acute asthma in the ED [140].

Data from $[174,435-437,559,699]$

Fig. 11.54 (continued). Flow chart for the treatment of pediatric acute asthma exacerbations. C Hospital Management. 
Table 11.45. Medication and dosages for pediatric status asthmaticus

\begin{tabular}{|c|c|c|c|}
\hline Medication & Preparation & \multicolumn{2}{|l|}{ Doses } \\
\hline \multicolumn{4}{|l|}{ A. Inhaled $\beta_{2}$-agonists } \\
\hline \multicolumn{4}{|l|}{ Albuterol } \\
\hline Metered-dose inhaler & $90 \mu \mathrm{g} /$ puff & \multicolumn{2}{|c|}{$4-8$ puffs q $20 \mathrm{~min} \times 3$, then $4-8$ puffs q $1-4 \mathrm{~h}$} \\
\hline \multirow[t]{3}{*}{ Nebulized solution } & $0.5 \%(5 \mathrm{mg} / \mathrm{ml})$ & \multicolumn{2}{|c|}{$\begin{array}{l}0.10-0.15 \mathrm{mg} / \mathrm{kg} \text { (minimum dose } 2.5 \mathrm{mg} \text {, maximum } 5 \mathrm{mg} \text { ) } \\
\text { in } 2-\text { to } 2.5-\mathrm{ml} \text { saline q } 20 \mathrm{~min} \times 3\end{array}$} \\
\hline & & \multicolumn{2}{|c|}{ Or $0.15-0.3 \mathrm{mg} / \mathrm{kg} \mathrm{q} 1-4 \mathrm{~h}$} \\
\hline & & \multicolumn{2}{|c|}{ Or $10-20 \mathrm{mg} / \mathrm{h}$ in saline nebulized continuously } \\
\hline \multicolumn{4}{|l|}{ Terbutaline } \\
\hline Metered-dose inhaler & $200 \mu \mathrm{g} / \mathrm{puff}$ & \multicolumn{2}{|c|}{2 puffs q 5 min up to a total of 12 puffs } \\
\hline \multicolumn{4}{|l|}{ B. Systemic $\beta_{2}$-agonists } \\
\hline Epinephrine & $1: 1,000(1 \mathrm{mg} / \mathrm{ml})$ & \multicolumn{2}{|l|}{ Tables $11.16,11.17$} \\
\hline \multirow[t]{2}{*}{ Terbutaline } & $\begin{array}{l}\text { Solution (0.1\%) } \\
1 \mathrm{mg} / \mathrm{ml}\end{array}$ & \multicolumn{2}{|c|}{$\begin{array}{l}0.01 \mathrm{ml} / \mathrm{kg} \text { up to } 0.3 \mathrm{ml} \mathrm{SC} \\
\mathrm{q} 2-6 \mathrm{~h} \mathrm{prn}\end{array}$} \\
\hline & & \multicolumn{2}{|c|}{$\begin{array}{l}\text { Or IV: load } 10 \mu \mathrm{g} / \mathrm{kg} \text { over } 10 \mathrm{~min} \text { maintenance dose: } 0.4 \mu \mathrm{g} / \mathrm{kg} / \mathrm{min} \text {; } \\
\text { increase by } 0.1-0.2 \mu \mathrm{g} / \mathrm{kg} / \mathrm{min} \text { every } 15-20 \mathrm{~min}\end{array}$} \\
\hline \multicolumn{4}{|l|}{ C. Inhaled anticholinergic } \\
\hline \multicolumn{4}{|l|}{ Ipratropium bromide } \\
\hline Nebulized solution & $500 \mu \mathrm{g} / \mathrm{ml}$ & \multicolumn{2}{|c|}{$\begin{array}{l}<40 \mathrm{~kg}: 250 \mu \mathrm{g}(0.5 \mathrm{ml}) / \mathrm{dose}=40 \mathrm{~kg}: 500 \mu \mathrm{g}(1.0 \mathrm{ml}) / \text { dose } \\
\mathrm{q} 20 \mathrm{~min} \times 3 \text { doses, then q } 2-4 \mathrm{~h}\end{array}$} \\
\hline \multicolumn{4}{|l|}{ D. Methylxanthines } \\
\hline \multirow[t]{12}{*}{ Theophylline } & & \multirow{2}{*}{\multicolumn{2}{|c|}{$\begin{array}{l}\text { IV: initial dose (load): in } 20-30 \mathrm{ml} \text { saline in } 20-30 \mathrm{~min} \\
\text { If theophylline serum levels are known :1 } \mathrm{mg} / \mathrm{kg} / \mathrm{IV} \text { for each } \\
\text { increase of } 2 \mu \mathrm{g} / \mathrm{ml} \text { of theophylline levels }\end{array}$}} \\
\hline & & & \\
\hline & & \multicolumn{2}{|c|}{ If theophylline levels are unknown: } \\
\hline & & \multicolumn{2}{|c|}{$\begin{array}{l}\text { If the child in the past } 24 \mathrm{~h} \text { has not taken oral theophylline, } \\
5 \mathrm{mg} / \mathrm{kg} \text { of aminophylline IV }\end{array}$} \\
\hline & & \multicolumn{2}{|c|}{ If the child has taken oral theophylline, $3 \mathrm{mg} / \mathrm{kg}$ of aminophylline IV } \\
\hline & & \multicolumn{2}{|c|}{$\begin{array}{l}\text { Maintenance IV doses (5-6 doses up to clinical improvement }= \\
\text { stable concentration of } 10-12 \mathrm{mg} / \mathrm{ml})^{\mathrm{a}} \text { or: }\end{array}$} \\
\hline & & $1-6$ months & $0.5 \mathrm{mg} / \mathrm{kg} / \mathrm{h}^{\mathrm{b}}$ \\
\hline & & 6 months - 1 year & $0.7 \mathrm{mg} / \mathrm{kg} / \mathrm{h}$ \\
\hline & & $1-9$ years & $1.0 \mathrm{mg} / \mathrm{kg} / \mathrm{h}$ \\
\hline & & $9-12$ years & $0.8 \mathrm{mg} / \mathrm{kg} / \mathrm{h}$ \\
\hline & & $12-16$ years & $0.7 \mathrm{mg} / \mathrm{kg} / \mathrm{h}$ \\
\hline & & 16 years & $0.6 \mathrm{mg} / \mathrm{kg} / \mathrm{h}$ \\
\hline \multicolumn{4}{|l|}{ E. Corticosteroids } \\
\hline Outpatient children & $\begin{array}{l}\text { Prednisone, } \\
\text { prednisolone, } \\
\text { methylprednisolone }\end{array}$ & \multicolumn{2}{|c|}{$1-2 \mathrm{mg} / \mathrm{kg} /$ day (single or divided doses) } \\
\hline \multirow[t]{2}{*}{ Hospitalized children } & Prednisolone & \multicolumn{2}{|c|}{$\begin{array}{l}\text { Loading dose } 1-2 \mathrm{mg} / \mathrm{kg} / \text { dose }(\max 60 \mu \mathrm{g} \text { ), then } 2 \mathrm{mg} / \mathrm{kg} \text { per } 24 \mathrm{~h} \\
\text { divided into two doses }\end{array}$} \\
\hline & Methylprednisolone & \multicolumn{2}{|c|}{$\begin{array}{l}\text { Loading dose: } 1-2 \mathrm{mg} / \mathrm{kg} / \text { dose } \mathrm{q} 6 \mathrm{~h} \text { per } 24 \mathrm{~h} \text {, then } 1-2 \mathrm{mg} / \mathrm{kg} / \text { day } \\
\text { in divided doses q } 8-12 \mathrm{~h}\end{array}$} \\
\hline
\end{tabular}

See Figs. 11.50 and 11.53 .

Data from $[434,435,485]$.

a Theophylline is indicated in children who have responded to theophylline or who are taking theophylline, have low levels, and are not improving.

b Monitor theophylline serum levels (see text). 
- Rehydration: in general not $>1-1.5$-fold the normal daily needs, monitoring fluid and electrolyte balance because of potential hypersecretion of $\mathrm{ADH}$ and osmolality [336]. Do not exceed the dosage because the high negative pleural pressures during inhalation encourage fluid accumulation in interstitial spaces and therefore the insurgence of pulmonary edema [174]. Provide adequate $\mathrm{K}^{+}$chloride (25-40 mEq/l) also because $\beta_{2}$-adrenergics may produce hypokalemia, and fluid supplementation, $300-400 \mathrm{ml} / \mathrm{m}^{2}$ body surface in the first hour, with $24 \mathrm{~h}$ continuation of $200-300 \mathrm{ml} / \mathrm{m}^{2}$.

- $\mathrm{O}_{2}$ treatment is $\mathrm{O}_{2}$ humidified to $30 \%-40 \%$ via a face mask or nasal cannula or via a partial or nonrebreather mask at a flow rate of $4-6 \mathrm{l} / \mathrm{min}$ not longer than 30-60 min, to keep $\mathrm{PaO}_{2}>85$ torr. It must not be excessive to avoid increased ventilation.

- Inhaled $\beta_{2}$-agonist with albuterol at $0.15 \mathrm{mg} / \mathrm{kg}$ (maximum, $5 \mathrm{mg}$ ) per dose should be started every 15-20 min for at least $1 \mathrm{~h}$, even if some improvement occurs after the first inhalation; inhaled albuterol should be continued at the above doses at $30 \mathrm{~min}$ intervals over an additional $1-2 \mathrm{~h}$, then at hourly intervals for another $2-3 \mathrm{~h}$, and at decreasing intervals, by nebulization with mask in the very young and with MDI-spacer in older children.

- If children fail to respond within $2 \mathrm{~h}$ to $\beta_{2}$-adrenergics, start IB via MDI in the doses indicated; it could be added to IV $\beta_{2}$-adrenergics.

- Continuous infusions of aminophylline in children who do not respond satisfactorily to $\beta_{2}$-adrenergics only. A reasonable starting point is a bolus of $3-6 \mathrm{mg} / \mathrm{kg}$ over $10-20 \mathrm{~min}$, followed by $1 \mathrm{mg} / \mathrm{kg} / \mathrm{h}$ depending on whether the theophylline level is known or not or the child is following an oral therapy (Table 11.45). Measure the theophylline level as soon as possible; it must be within 10 and $20 \mu \mathrm{g} / \mathrm{ml}$, considering that $1 \mathrm{mg} / \mathrm{kg}$ of aminophylline increases by $2 \mu \mathrm{g} / \mathrm{l}$ the serum concentration [707].

- IV CSc. It has been shown the best results by administering a single IV dose: hydrocortisone $4-6 \mathrm{mg} / \mathrm{kg} /$ dose or methylprednisolone $1-2 \mathrm{mg} / \mathrm{kg} /$ dose, both every $6 \mathrm{~h}$. Continue with inhaled doses. If ICSs are not available the oral type can be taken, but it requires $6 \mathrm{~h}$ to reach peak levels. It is recommended that the first dose be administered as soon as bronchoconstriction worsens [683].

- Measure $p H$ and base excess (BE) to correct acidosis with $\mathrm{NaHCO}_{3}=\mathrm{mEq}$ required to be calculated with the formula: $\mathrm{BE} \times 0.3 \times \mathrm{kg}$ in weight.

- The use of sedatives to reduce the state of agitation should be employed with great caution because they depress the respiratory centers [272] (see "Death by Asthma").

If monitoring of the above parameters shows a continuous and significant clinical improvement for at least 4 consecutive h, drug doses can be gradually reduced, but monitoring the drugs necessary in case intensive use be made of $\beta_{2}$-adrenergics. Some children can worsen
Table 11.46. Immediate treatment of status asthmaticus

\section{Severe symptoms}

Dyspnea associated with severe functional limitation ongoing from $>8 \mathrm{~h}$

Response to $\beta_{2}$-agonists poor or of short duration requiring frequent administration $<2-3 \mathrm{~h}$ ).

Child stops sleeping, must sit upright.

Agitation or confusion.

Too breathless to speak: children talk in single words.

Visible accessory muscle retractions.

Pulsus paradoxus: if $>20 \%$ a severe bronchoconstriction is impending.

PEFR (if available) $<50 \%$ of best

Life-threatening features requiring immediate

treatment without carrying out labor test

Cyanosis, sweating

Paradoxical thoracoabdominal movement

No expiratory rales (silent chest)

Fatigue/exhaustion/drowsiness

Agitation, or reduced level of consciousness

Bradycardia and/or severe RR alteration ( $<50 \%)$

Young children develop respiratory failure more readily than can be assessed

$\mathrm{PaO}_{2} \leq 91 \%$

PEFR (if available) $<33 \%$ of best (in collaborating

children $>6$ year)

The presence of any of these life-threatening features should alert the doctor.

Data from $[56,336,476,485]$.

Table 11.47. Indications for hospitalization

1. Deteriorating alertness

2. Suprasternal retractions

3. Retractions of sternocleidomastoid and diaphragmatic muscles

4. Pulsus paradoxus $>20 \%$

5. PEFR $<50 \%$ of best

6. $\mathrm{SaO}_{2}<91 \%$

7. $\mathrm{PaCO}_{2}>40 \mathrm{~mm} \mathrm{Hg}$

8. $\mathrm{PaO}_{2}<60 \mathrm{~mm} \mathrm{Hg}$

9. Poor response to treatment in the $1 \mathrm{st} h$

10. Fatigue or exhaustion

11. Insufficient care at home

12. Poor access to medical care

Data from $[56,485,509]$. 
Table 11.48. Routine emergency treatment of childhood asthma: United States

\begin{tabular}{|c|c|c|}
\hline \multirow[t]{2}{*}{ Intervention } & \multicolumn{2}{|c|}{$\begin{array}{l}\text { Emergency interventions } \\
\text { in } 118 \text { and } 137 \text { pediatric departments (\%) }\end{array}$} \\
\hline & 1988 & 1994 \\
\hline Use of clinical scoring system & 20 & 19 \\
\hline Use of pulmonary function testing & 56 & 73 \\
\hline Chest Rx during the first episode of wheezing & 59 & 50 \\
\hline Oxygen given to all wheezing children & 33 & 40 \\
\hline \multicolumn{3}{|l|}{ First medication given } \\
\hline$\beta_{2}$-Agonists by injection & 72 & 1 \\
\hline$\beta_{2}$-Agonists by inhalation & 17 & 136 \\
\hline Either (no preference) & 11 & 0 \\
\hline \multicolumn{3}{|l|}{ Injected agonist of first choice } \\
\hline Epinephrine & 81 & 63 \\
\hline Terbutaline & 17 & 19 \\
\hline Albuterol & 2 & 2 \\
\hline None & 3 & 12 \\
\hline \multicolumn{3}{|l|}{ Inhaled agonist of first choice } \\
\hline Albuterol & 47 & 95 \\
\hline Metaproterenol & 39 & 3 \\
\hline Isoetharine & 12 & 1 \\
\hline Terbutaline & 9 & 3 \\
\hline Isoproterenol & 2 & 0 \\
\hline \multicolumn{3}{|l|}{ Maximum number of doses (injected and inhaled) } \\
\hline 1 & 1 & 0 \\
\hline 2 & 5 & 0 \\
\hline 3 & 58 & 40 \\
\hline 4 & 16 & 18 \\
\hline 5 or more & 20 & 39 \\
\hline \multicolumn{3}{|l|}{ Corticosteroids usually employed } \\
\hline Early & 21 & 82 \\
\hline At time of disposition & 47 & 11 \\
\hline Variable & 29 & 7 \\
\hline Not at all & 3 & 0 \\
\hline \multicolumn{3}{|l|}{ Inhaled anticholinergic agents } \\
\hline Frequent & 2 & 12 \\
\hline Rarely & 50 & 63 \\
\hline Never & 48 & 23 \\
\hline Variable & 0 & 2 \\
\hline
\end{tabular}

Number exceeds 100 since some hospitals utilized more than one agent.

Data from $[332,536]$. 
Table 11.49. Routine emergency treatment of childhood asthma: Canada

\begin{tabular}{lcl} 
Drug (\%) & ICU & $\begin{array}{l}\text { At home } \\
\text { (oral/inhaled) }\end{array}$ \\
\hline Nebulized $\beta_{2}$-agonists & 100 & 99 \\
\hline IV steroids & 94 & 42 \\
\hline IV albuterol & 38 & \\
\hline Nebulized & 10 & \\
ipratropium bromide & & \\
\hline IV isoproterenol & 10 & 25 \\
\hline Cromolyn & &
\end{tabular}

Data from [615].

however, with progressive respiratory failure. Therefore, dependent on their condition, they should be transferred to the ICU. Table 11.47 [56, 485, 509] lists data evaluating the relevance and urgency of hospitalization. The first item on the list of decisive factors is if the equipment for blood gas level tests or drugs necessary to continue treatment are unavailable.

We recommend that $\mathrm{O}_{2}$ be administered as needed in a hospital environment and, during treatment and that $\mathrm{HR}, \mathrm{RR}, \mathrm{PA}$, blood gas level tests and/or $\mathrm{SaO}_{2}$ and electrolyte levels be monitored [406]. In life-threatening cases, to avoid the need of assisted ventilation [47], terbutaline and albuterol have been injected IV. The first by a bolus of $10 \mu \mathrm{g} / \mathrm{kg}$ administered over $30 \mathrm{~min}$ and repeated after a further $30 \mathrm{~min}$, followed by maintenance

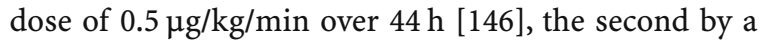
bolus of $10 \mu \mathrm{g} / \mathrm{kg}$ over $10 \mathrm{~min}$, followed by a gradually increased maintenance dose up to an average of $1.7 \mu \mathrm{g} / \mathrm{kg} / \mathrm{min}$ over $36 \mathrm{~h}$ [47]. Kelly et al administered terbutaline to children 4.5-14 years old, nebulized continuously in doses of $0.4 \mathrm{mg} / \mathrm{kg}$ for an average of $9.4 \mathrm{~h}$, for up to $24 \mathrm{~h}$ [288]. Current guidelines [434-436] suggest starting with an albuterol dose of $0.15 \mathrm{mg} / \mathrm{kg}$ (minimum of $2.5 \mathrm{mg}$, maximum of $5 \mathrm{mg}$ ) per nebulizer treatment for all age groups. For infants $<6$ months, half the dose (1.25) can be used if $2.5 \mathrm{mg}$ provoke unacceptable tachycardia. During the first hour of wheezing, 3-4 albuterol nebulizer treatments are given every 15-20 min. If there is an inadequate response to treatment, the child is placed on continuous nebulization. Others have shown that doses of $0.15 \mathrm{mg} / \mathrm{kg}$ of albuterol repeated every 20-30 $\mathrm{min}$ are better than doses of $0.05 \mathrm{mg} / \mathrm{kg}$ and both have no side effects, and that children (under careful surveillance) respond more promptly to higher doses [564]. High doses of albuterol, especially in very young infants, are devoid of side effects and can be justified by above-mentioned studies [488]. Therefore, aggressive doses of albuterol (every $30 \mathrm{~min}$ ) are more effective than those administered every $1-4 \mathrm{~h}$ [116]. To achieve positive results, higher doses of albuterol, which are needed because the decreased airway caliber de- creases the aerosol penetrance, and the quantity of drug reaching the airways decreases as the child pattern of respiration is altered [607], provided that $\beta_{2}$-adrenergics are nebulized with an appropriate face mask, thus ensuring a more rapid improvement, which either makes it unnecessary to send children to an ICU, or shortens the period of hospitalization [683].

A note to explain the rational behind the use of theophylline in severe, life-threatening cases, above all in children [615] who, for anatomical reasons, experience bronchoconstriction more than bronchial obstruction. Inhaled $\beta_{2}$-adrenergics may not reach the inflamed bronchus because of severe bronchospasm, not because of bronchus failure to dilate. On the contrary, the theophylline reaches it systemically, performing anti-inflammatory effects (Tables 11.17, 11.18) and producing a diffuse bronchodilation that reaches the bronchi obstructed by edema and mucus [393]. At this point, $\beta_{2}$-adrenergics, inhaled or nebulized, can take advantage of the opening and reach the terminal airways to the point of inducing a therapeutic effect. Table $11.48[332,536]$ compares the use made in 1988 of various drugs in 118 pediatric EDs in the US, compared to data 6 years later [332], to evaluate the effect of 1991 guidelines [435]. It should be noted that the great use made in the US of epinephrine and the marked increase in albuterol (first choice in $95 \%$ of cases) and CS use, whose route of administration is not specified. In a survey of 376 directors of ED settings, $80 \%$ reported the use of inhaled $\beta$-agonists as the initial treatment. Only $44.7 \% \pm 2.9 \%$ reported the use of steroids if there was a poor response to the initial treatment [121]. Also, in 125 children nebulized $\beta_{2}$-adrenergics in ICU and at home were used in $100 \%$ of the cases (Table 11.49) [615]. A subsequent survey of 348 ED directors confirmed the high preference for inhaled $\beta_{2}$-adrenergics ( $\left.96.5 \%\right)$, significantly more public $(24.6 \%)$ and community hospitals $(17.1 \%)$ than pediatric EDs (3.5\%) reported the use of SC epinephrine as the first medication, compared to steroids $(18.1 \%)$ as a routine part of the treatment [121]. However, North American authors have shown that epinephrine is comparable to inhaled $\beta_{2}$-adrenergics for its rapidity of action, effectiveness and duration of effect [537]. Also notable is how much easier and more practical it is to use epinephrine in the doctor's office, at the child's home or in a busy ED [536, 750], especially if auto-injectable (Chap. 20). It has been noted that if a visit to the $\mathrm{ED}$ is followed by treatment with albuterol every $30 \mathrm{~min}$ for $4 \mathrm{~h}$, and by giving prescriptions to continue treatment at home, after $4 \mathrm{~h} 43 \%$ of children are released, rising to $61 \%$ if a dose of oral prednisolone is added [116]. Also in asthmatics with acute forms and admitted to ED and hospitalized, relapses are reduced by $50 \%$ and nocturnal disturbance by $75 \%$, if referral to an asthma specialist is facilitated and treatment continued thereafter, compared to patients treated by a family doctor [750].

The effectiveness of treatment in subjects with status asthmaticus is such that on the 3rd day of hospital treat- 
ment there is a significant reduction - correlated with amelioration of bronchoconstriction - of $\mathrm{T}, \mathrm{IL}_{2} \mathrm{R}, \mathrm{HLA}$ DR cell levels, present in higher concentrations than in normal controls [119]. An intubated child with status asthmaticus was treated with two doses of intratracheal recombinant human DNase (rhDNase) therapy, a mucolytic agent used to relieve peripheral airway obstruction. The child was extubated $26 \mathrm{~h}$ after receiving the rhDNase treatment with no adverse effects [163]. A dramatic sustained improvement followed this treatment in a 3-year-old boy with acute life-threatening asthma in whom $48 \mathrm{~h}$ of aggressive therapy had failed [473].

\section{Comprehensive Post-Attack Care}

If symptoms evolve towards a concrete improvement, it is suggested that children remain under observation for $12-24 \mathrm{~h}$, monitoring both symptoms and parameters, weaning the child to a drug regimen that includes bronchodilators and steroids, either inhaled or orally, then discharging the child, prescribing therapy for 3-5 days and ensuring that the child is able to follow the inhalatory technique correctly, with a follow-up with the family pediatrician about 1 week after discharge [272, 406, 437], which was positive in $59.8 \%-74 . \%$ of cases [121]. Drugs prescribed at discharge were bronchodilators $(95.3 \%)$, CSs increasing with age group, and theophylline (21\%-34\%) [121]. Having achieved remission, the therapeutic strategy continues the drug regimen for at least 3-4 weeks, during which the family pediatrician will check the clinical progress by means of twice daily measurements of PEFR and sending the child to a center for infantile respiratory physiopathology for necessary PFT follow-up [164]. The month-long interval can be usefully spent establishing how the problems related to environmental and pharmaceutical prophylaxis will be managed, and in informing both the child and family how to handle the situation [434], also to work on a plan of action for frequent asthma, which is dealt with in the next section. We underline that in children aged $<5$, the necessary compliance for PEFR measurement is missing, while the child is at greater risk of a symptom worsening. Therefore attention paid to the clinical parameters (Tables 11.31, 11.34, 11.38 and 11.41) can prove equally valid for defining a post-attack strategy [436]. Given that BHR and/or PFT changes tend to persist for an indeterminate time in the form of chronic coughing, nocturnal asthma, or as a result of physical activity [359], therapy must overcome all of these, before a possible treatment discontinuation can be considered (though not in $<2$ months), also and above all to ensure the normal quality of life [164] recently ensured by omalizumab [342] and formoterol [183] treatment. The family and older children should understand that if asthma is not dealt with decisively and controlled effectively it will eventually become a disease that will render the sufferer an invalid in adulthood [218].

\section{Treatment of Episodic, Frequent, Chronic and Other Forms of Asthma}

Treatment of asthma must be personalized according to the frequency and severity of symptoms (Table 11.32). The main points for consideration are [571, 700]:

- Deciding which drugs to use, whether continuously or prn (if required)

- Determining the most suitable means of administering treatment

- Using an MDI suited to the child's age, if a drug should be inhaled

- Making sure that the medicine is working

- Regularly verifying the effectiveness of treatment

- Discussing with children and their family the general lines of treatment

- Listening to the problems that asthma or its management provoke in children and their family

- Keeping a clinical diary in which daily variations are noted

Below, in order of severity are listed the different types of asthma:

1. Episodic asthma

2. Mild intermittent asthma

3. Chronic asthma (mild, moderate, severe) (Figs. 11.55 to 11.58 )

4. Administration of drugs at various age levels

5. Specific forms (asthma associated with VRI, EIA, nocturnal asthma, cough variant asthma)

6. Unresponsive asthma

7. Collateral pathologies

8. Complications

Differentiating among these types of asthma is essentially didactic, to better understand the various therapeutic particularities. The possibility of finding the therapy suitable for the control of the symptoms will enable asthmatic children to experience a quality of life that as much as possible resembles that of their healthy companions. From a therapeutic point of view, many treatment alternatives exist, proposed by several authors of proven experience, all of which are usually effective. The problems most frequently met with are represented by asthmatic attacks, by severe forms and by the day-by-day or maintenance treatment. We will try to give precedence to the aspects correlated to symptom severity based on the child's age. Evaluating these factors separately, even if in practice a certain overlapping between frequent, more demanding forms and moderate, chronic forms is possible, it seems to us useful for ensuring greater clarity.

\section{Episodic Asthma}

This is a mild and infrequent form; $\approx 75 \%$ of asthmatic children experience mild episodes of coughing and wheezing every 2 months on average (in relation to season and stimuli), then remaining asymptomatic for 


\section{MILD INTERMITTENT}

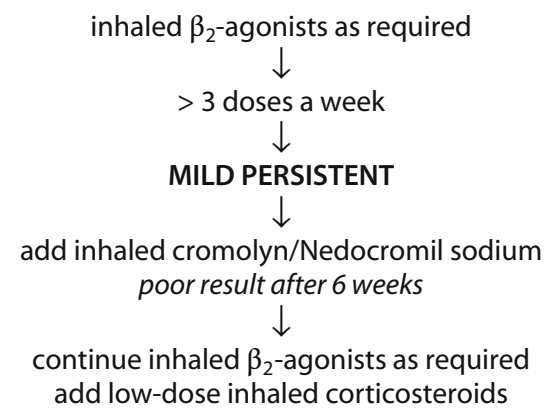

in younger children by nebulizer or spacer and facemask

poor result

$\downarrow$

MODERATE PERSISTENT

$\downarrow$

continue low-dose inhaled corticosteroids

add theophylline

$\downarrow$

poor result

$\downarrow$

SEVERE PERSISTENT

\begin{tabular}{ccc}
\hline$\downarrow$ & $\downarrow$ & $\downarrow$ \\
$\begin{array}{c}\text { Consider } \\
\text { long acting }\end{array}$ & Consider & $\begin{array}{c}\downarrow \\
\text { slow release } \\
\beta_{2} \text {-agonists }\end{array}$ \\
\hline
\end{tabular}

$\downarrow$

poor result

$\downarrow$

continue inhaled corticosteroids

$\downarrow$

add oral corticosteroids

* see Fig. 11.56. Data from [435-437, 699, 700].

Fig. 11.55. Therapeutic algorithm for pediatric mild intermittent asthma: a stepwise approach (step 1)

relatively long periods. They are therefore able to play, attend school and take part in sporting activities without any problems, and usually their sleep is not interrupted by coughing. Prophylactic treatment should be sufficient in most cases. Measurements of PEF in $75 \%$ of these asthmatics is usually of no use [700].

\section{Mild Intermittent Asthma (Step 1)}

No daily medication is usually required. It is characterized by frequent or infrequent attacks, but of moderate severity, use of bronchodilators prn, persistent airway obstruction not correlated with clinical symptoms, reduction of physical activity, night awakenings by asthma 1-2 nights a month, routine reduction in school attendance. A course of treatment is illustrated in Fig. 11.55 [434-437, 699, 700]. Recommendations for treatment of infants and young children with moderate or severe cases of asthma are based on extrapolations from studies in older children and adults [434]. As can be seen, the first choice of drugs is always for inhaled or nebulized $\beta_{2}$-adrenergics. If results are good, step-down treatment with cromones (see "Prevention"); if insufficient, consider stepping in with ICSs and anti-LT or theophylline [434], associating flunisolide with albuterol inhalation [228]. However, albuterol bronchoprotection lasts $<4 \mathrm{~h}$, formoterol DPI at least $8 \mathrm{~h}$ in 2-to 5-year-old asthmatic children [441] and bambuterol (once daily dose) [158] (see Tables 11.12, 11.16 and 11.23 for doses). This therapy is recommended in children with persistent cough or asthma. In moderately severe cases, one or two doses should be sufficient. Furthermore, BUD, DPI or BPD by spacers + VHCs as indicated can be used as a good alternative. In children with almost continuous relapses or suffering from chronic asthma and steroid-dependence who are undergoing systematic treatments, we advise ICS once the acute phase of the crisis has been overcome. Given that steroid maximal effects are usually delayed $6 \mathrm{~h}$, it is necessary that children be under control with antileukotrienes, active on immediate and late reactions. 


\section{Chronic Asthma}

As we have already stated, asthma is an inflammatory process that, beyond the immediate episodes, continues in chronic form as a result of the persistence of inflammatory cells. Usually, chronic asthma is related to children affected with asthma in the first few years of life, but the disease assumes its maximal severity after the age of 5 years. At recruitment the children with severe asthma totaled $8(11 \%)$, but at age 42 the people with severe asthma totaled $33(47 \%)$ with reduced lung function [264]. Generally, the symptoms appear early without an allergic nature being promptly identified, nor proper treatment started. Often, a careful study leads to the discovery of a variety of allergic diseases, for example AR, frequently complicated by sinusitis. It is therefore necessary to ascertain its presence, especially in children affected with chronic asthma. Usually frequent symptoms without perceptible periods of well-being are observed, given that bronchoconstriction and effort-induced dyspnea are constantly present, in practice children learn to live with asthma. This form, whose prevalence is $4 \%-5 \%$, often induces invalidating symptoms, which can be summarized as follows: persistent bronchoconstriction, almost daily symptoms, frequent night-time coughing and limited physical activity [476]. In 37 randomly recruited children with chronic asthma compared to 37 controls with episodic asthma, we noted significant early onset of symptoms, delayed diagnosis, and poorer spirometry results, in addition to a positive personal or FHA, as always a potent determinant of atopy. Especially significant was the greater number of children sensitized to multiple aeroallergens. A strong influence of environmental factors on the development of severe asthma is demonstrated by the significant prevalence of maternal smoke during pregnancy, parental smoke, damp houses, and viral infections. Among the drugs available, we have four possible options: cromones, $\beta_{2}$-adrenergics (even with a long halflife), ICSs, and theophylline in drops or the long-acting type from 2-3 years of age onwards.

Chronic asthma is subdivided into three types, according to whether symptoms are mildly persistent, moderately persistent or severely persistent, each subclass being present in 60,30 and $<10 \%$ of cases, respectively [476]. The intermittent form [436], which could be defined as the transitory form between acute and chronic asthma, is considered to belong to the mild forms, to be treated only as needed [437], with four stages: mild intermittent, mild persistent, moderate persistent and severe [437]. It should be noted that NIH guidelines (Expert Panel Report 2) tabulate treatment as follows: firstline therapy may begin first with ICSs (low dose), then with cromolyn, or nedocromil as preferred therapy, or alternatively with sustained-release theophylline, or an anti-LT [437], or GINA (global initiative for asthma) guidelines introduce a daily base treatment with CSs, adding cromones only for mild forms and using $\beta_{2^{-}}$ adrenergics as symptomatic (rescue) drugs [436]. The Expert Panel Report 2002 believes that a diagnostic trial of inhaled bronchodilators and anti-inflammatory medications may be helpful; however, infants consistently requiring symptomatic treatment more than twice a week should be given daily long-term-control therapy, and ICSs should be the preferred treatment [434]. These guidelines have not been confirmed by three longitudinal studies [210, 465, 575], and the Melbourne 37-year longitudinal study in asthmatic children followed up to adulthood show no differences between those who did and those who did not take steroids [210], nor has the proposal to introduce CS use from the onset of the infantile asthma been validated, not even in the mild forms [465]. The PFT impairment in the group with persistent asthma was greater in those with persistent BHR and in those treated with ICSs [575]. Cromones and/or theophylline are important for reducing CS doses $[437,566]$. Nor are GINA guidelines productive, since a retrospective study of 175 children followed on average for 8.4 years concluded that, contrary to what the guidelines suggested, starting treatment with cromones and not with CSs improves the outcome, while in the mild-moderate forms positive effects are obtained with bronchodilators [311]. Without doubt, further prospective studies are needed. For our part, we have seen the positive results of early treatment with cromones, in agreement with the 3rd Consensus for infantile asthma [700]. One of the tasks of a pediatrician is to strive for a better compliance and quality of life for asthmatic children. There are often reasons for noncompliance to long-term medications when these are numerous, including in adolescence the fear of CS side-effects, a poor understanding of treatment, and a wish to be like one's peers [158]. To our knowledge, one proposition consists of simplifying the treatment program by reducing the frequency of drug administration from twice daily, as usually recommended, to once daily. However, if efficacy is preserved, can prophylactic asthma treatment be prescribed once daily for each drug and for each child, whatever the age, the device and the asthma severity [158]?

\section{Mild Persistent Asthma (Step 2)}

Normally, PFT basic anomalies are absent in mild persistent asthma (Fig. 11.56) [435-437, 699, 700]. There are asymptomatic periods between exacerbations, symptoms often follow physical activity, exposure to environmental allergens or respiratory infections, with a decrease in $\mathrm{FEV}_{1}$ of $20 \%$ or less [198]. PEF offers adequate indications, even if in children of 3-5 years the results may be not significant. Symptoms must be carefully checked: coughing, wheezing, difficulty in daily activities or physical exercise, nocturnal disturbance, even if intermittent [589]. Treatment is begun at the first signs of the illness or if PEF measurement is underway, when 
CLINICAL MANIFESTATIONS

see Table 11.33

$\begin{array}{cc}\downarrow & \downarrow \\ \text { asymptomatic } & \text { symptomatic } \\ \text { PEFR } \geq 80 \% \text { of predicted values } & \text { variability }>20 \%\end{array}$

\begin{tabular}{ll}
\hline & MANAGEMENT \\
& \\
pretreatment as required & \\
2 puffs of $\beta_{2}$-agonist or cromolyn & $\beta_{2}$-agonists (see Table 11.15) \\
before a physical activity, & $<5$ years nebulized or spacer + VHC and facemask \\
or exposure to allergen, etc & $>5$ years inhaled \\
& 2 puffs q 4-6 hours as required or once daily \\
& or depending on severity of symptoms \\
& in alternative sustained release theophylline \\
& or inhaled cromones, or anti-LT*
\end{tabular}

\[ \]
\[ \]
positive
RESULT
- control of symptoms
- normal activity
- absence of nocturnal cough
- PEF stabilization
- normal lung function tests

* anti-LT oral administration is preferred by children: montelukast for children aged $\geq 2$, zafirlukast for children aged $\geq 7$, zileuton for children aged $\geq 12$.

Data from $[435-437,699,700]$.

Fig. 11.56. Therapeutic algorithm for pediatric mild persistent asthma: a stepwise approach (step 2)

it decreases by $10 \%-20 \%$ [273]. During symptomatic periods, children should be treated with nebulized $\beta_{2}$-adrenergics (Table 11.16), every $4-6 \mathrm{~h}$ prn for the duration of the episode, until PEF is stabilized or there is a clear and constant improvement [566]. In children $<5$ years of age, PEF measurements can be substituted by clinical parameters, such as coughing and dyspnea, while in children $>5$ years of age, starting a continuous PEF monitoring period at home to evaluate the severity of asthma is suggested [699]. The choice of drugs is less difficult, since response to $\beta_{2}$-agonists is often variable in young infants, provided they are used correctly [26]. Inhaled $\beta_{2}$-adrenergics can be used prn if control of symptoms can be coupled with a normal level of activity, absence of nocturnal coughing and PEF stabilization, [697]. If, on the other hand, the condition progressively worsens and $\beta_{2}$-agonists are taken more than twice a week, reaching the point of daily intake dosages or 3-4 times/day, long-acting theophylline or cromones can be introduced. In children $\geq 2$ years we suggest that anti-LT can be considered when inhaled medication delivery is suboptimal because of poor technique or adherence, or BUD which fared well at a dose of $0.25,0.5$, or $1 \mathrm{mg}$ once daily in 359 children aged 6 month to 8 years [290]. If control is missing or incomplete, Fig. 11.57 will be useful to reach the most appropriate decisions.

\section{Moderate Persistent Asthma (Step 3)}

Moderate persistent asthma (Fig. 11.57) [435-437, 699, 700 ] is characterized by two or more weekly acute asthma exacerbations with a $60 \%-80 \%$ PEF decrease and with PFT values up to $20 \%$ below existing readings or personal best [198]. For treatment, cromones are considered, which, in addition to their anti-inflammatory action, have the advantage of not being absorbed throughout the system, which renders them preferable to theophylline, remembering that for some cases a long-term prophylactic treatment might be required. If cromones have no effect within $4-8$ weeks, the use of ICSs, which have a good anti-inflammatory action, can be started. We have treated with inhaled BUD (200 $\mu \mathrm{g} /$ day) 74 children aged 3.5-5.9 years (mean 4.2 years) and affected by moderate to severe asthma, despite conventional therapy, compared to 71 controls of similar age range and treated with antihistamines. The primary outcome measure was the change in asthma severity, as measured by the mean asthma score during the last 2 weeks of a baseline period and the last 2 weeks of each treatment. The mean difference in asthma score between BUD and antihistamines was: -7.5 ; $95 \% \mathrm{CI}$, -11.70 to $-3.29(p<0.0001)$. Spirometric data demonstrated a significant improvement. ICSs are suitable for 
CLINICAL MANIFESTATIONS

- daily symptoms

- exacerbations $>2$ times a week

and affect activity level

- visits to pediatric emergency wards

progressively more severe

Lung function tests

PEF $>60$ to $<80 \%$ of predicted values

variability $20-30 \%$ if symptomatic

specialist visit

\begin{tabular}{|c|c|}
\hline MANAGEMENT & \\
\hline$\sqrt{ }$ & $\downarrow$ \\
\hline inhaled $\beta_{2}$-agonists & Alternatives \\
\hline from as required to $3-4$ doses/day & long-acting inhaled $\beta_{2}$-agonists \\
\hline and anti-inflammatory & from as required to $3-4$ doses/day or \\
\hline cromolyn 2 puffs $\times 2-4$ doses/day & long-acting $\beta_{2}$-agonists syrup or tablets \\
\hline in younger children & $\begin{array}{l}\text { oral sustained release theophylline } \\
\text { up to } 5-15 \mu \mathrm{g} / \mathrm{ml} \text { concentration }\end{array}$ \\
\hline
\end{tabular}

$\downarrow$

persisting symptoms

> 3-4 doses/day are inhaled

add low-medium dose ICSs

in younger children by nebulizer or spacer $+\mathrm{VHC}$ and facemask or anti-LT medications *

positive
- control of symptoms
- normal lung function tests
- normal activity level
- rare nighttime symptoms
- reduced PEF variability
- rare exacerbations
- no visits to PICU
* for anti-LT medications see Fig. 11.56.
Data from [435-437,699, 700].

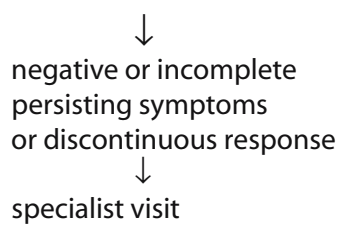

Fig. 11.58

Fig. 11.57. Therapeutic algorithm for pediatric moderate persistent asthma: a stepwise approach (step 3)

children $>5$ years who take cromones, but who also need $\beta_{2}$-adrenergics 3-4 times a day, or who have nocturnal asthma [476]. Recently, with once daily morning treatment, monometasone furoate DPI $440 \mu \mathrm{g}$ significantly changed $\mathrm{FEV}_{1}$ compared with BUD DPI $440 \mu \mathrm{g}$ [117]. $\beta_{2}$-adrenergics, especially those with a long half-life and limited to 1-2 doses a day, are useful together with IB [566]. Long-acting theophylline has the advantage of oral administration. Regularly monitoring its serum concentrations and recording any adverse symptom [198], it proves to be very effective in this form of asth$\mathrm{ma}$, as in mild forms [649]. With regular use of these drugs, $\beta_{2}$-adrenergics can be stopped, or retained as part of the treatment only as needed [435]. When prescribing long-term treatment, parents should be informed of the latency time of the drugs being used.

\section{Severe Persistent Asthma (Step 4)}

Severe asthma increases the risk of the airways progressing into a persistent and unresponsive airflow narrowing [274]. Children with severe asthma (Fig. 11.58) [435-437, 699, 700], despite appropriate treatment, often display PEF variations $>30 \%-50 \%$ and PFT $<60 \%$ of predicted or personal best, which can worsen during most severe exacerbations [198]. For daily therapy, bronchodilators are prescribed, namely long-acting theophylline and $\beta_{2}$-adrenergics prn, up to 3-4 times a day, in association with CSs [559]. In children 16 months and older [136], ICSs are suitable for anti-inflammatory effects, recurring to oral preparations if problems of compliance or financial difficulties arise. Infants use the more economical formulations per os $[435,476]$ more readily and willingly. Steroids with short half-life are 


\section{CLINICAL MANIFESTATIONS}

- continual symptoms

- frequent exacerbations

- limited physical activity

- frequent nighttime symptoms

- visits to pediatric emergency

department and hospitalizations

\section{EVALUATION OF LUNG FUNCTION}

PEF $<60 \%$ of predicted values

Variability of $20-30 \%$

with a routine treatment

\section{DAILY MANAGEMENT}

short-acting inhaled $\beta_{2}$-agonists

from as required to 3-4 doses/day *

$<5$ years by nebulizer or MDI + facemask

$>5$ years inhaled:

2 puffs $x$ 4-6 doses/day

in case of need with spacer + VHC

and

antiinflammatory

- low-dose ICSs

2-4 puffs x 2-4 doses/day

- long-acting inhaled $\beta_{2}$-agonist;

adding or not in case of need

- cromolyn 2 puffs x 2-4 doses/day

to minimize ICS dose

adding or not in case of need

- sustained release theophylline

(especially for nocturnal asthma)

up to a concentration of $5-15 \mu \mathrm{g} / \mathrm{ml}$

to minimize ICS dose
PEF variability $>20-30 \%$

during severe exacerbations

$\downarrow$

Consider oral corticosteroids

$-<5$ years $5-10 \mathrm{mg}$ tapered

to the lowest alternate morning

regimen that provides control

of symptoms and PEF

- > 5 years the lowest AM schedule

is tapered over several days to a week

depending on symptoms and PEF

\section{RESULTS}

- PFT improvement

- reduced PEF variability

- almost normal activity level

- rare nocturnal symptoms

- reduced incidence of relapses

- little need for relievers

- little need for ICSs

- little need for visits to PICUs

- normal growth and development

* if control is not achieved with 3-4 doses/day consider step up or review the therapy plan: eg adding anti-LT medications: see Fig. 11.56.

Data from $[435-437,699,700]$.

Fig. 11.58. Therapeutic algorithm for pediatric severe persistent asthma: a stepwise approach (step 4)

preferred, such as flunisolide $=\mathrm{h} 1.6 \pm 0.35$ and $\mathrm{BUD}=\mathrm{h}$ 2.8 1.1 , compared to the $15 \mathrm{~h}$ of BDP [633], tapered to the lowest effective single regimen (which provides the same results as that of dividing the administration into four doses) [497] taken on alternate days in the early afternoon $(3: 00-5: 30 \mathrm{PM})[378,497]$ to wean to the least undesirable effects, chief among which is that of inhibiting the HPA axis $[198,633]$. The ICS dose should be gradually stepped down to the lowest possible doses of medication required to maintain asthma control and perhaps discontinued if a child remains asymptomatic for more than 1-2 months [198, 290, 418, 566, 634]. By following this guide, danger of the baby or child encountering any sort of problem can be avoided; most of the symptomatic benefit obtainable from ICSs occurs by reducing doses of BUD to 100-200 $\mu \mathrm{g} /$ day, with little effect from dose increments [487]. Such lines have been confirmed in a 4-month pediatric trial, which evaluated many varied parameters: BDP achieved the best clinical results, but associated with albuterol and theophylline proved to be most effective in reducing asthma attacks, and in 10 out of 16 parameters that evaluated the most frequent adverse reactions, it registered the smallest rate (albuterol + theophylline the greatest), including the critical points such as asthma attacks and symptoms [396].

All CS-treated children should receive specific medical advice regarding calcium intake and vitamin $D$ supplementation. Review treatment every 1-6 months; a gradual stepping down in treatment may be possible and is needed to identify the minimum therapy required to maintain control. If control is not maintained, consider stepping up after reviewing patient medication technique, adherence, and environmental control (M.Plan Asthma Expert Panel).

In conclusion, being the cause of acute and chronic symptoms, infantile chronic asthma should be viewed a multiform pathology, whose therapeutic strategy should be based on multiple grounds, that is treating other allergies and eliminating food, environmental and infectious triggering factors, as well as on SIT, which recorded excellent results in asthmatic and rhinitissuffering children (Table 13.2). The pharmacological choices summarized in Table $11.50[335,698,699]$ can be recommended, according to age and symptoms. Table 11.51 focuses on the difficulties that can be encountered in the treatment of the very young [472]. 
Table 11.50. Stepwise and age-related approach for managing children with chronic asthma symptoms

\begin{tabular}{|c|c|c|}
\hline Type of chronic asthma & Younger children ${ }^{a}$ & Older children \\
\hline Mild persistent & $\begin{array}{l}\text { Cromolyn/nedocromil sodium } \\
\text { or inhaled } \beta_{2} \text {-agonists with MDI } \\
\text { and face mask }\end{array}$ & $\begin{array}{l}\text { Inhaled } \beta_{2} \text {-agonists as required } \\
\text { for symptoms or cromolyn/ } \\
\text { nedocromil sodium }\end{array}$ \\
\hline \multirow[t]{3}{*}{ Moderate persistent } & Cromolyn/nedocromil sodium & Cromolyn/nedocromil sodium \\
\hline & Inhaled $\beta_{2}$-agonists & Low-dose inhaled corticosteroids \\
\hline & & Long-acting theophylline \\
\hline \multirow[t]{3}{*}{ Severe persistent } & Inhaled $\beta_{2}$-agonists up to 3 times a day & Medium-dose inhaled corticosteroids \\
\hline & Oral/inhaled corticosteroids & Inhaled $\beta_{2}$-agonists up to 3 times a day \\
\hline & & Antileukotriene medications and/or cromones \\
\hline
\end{tabular}

Data from $[335,698,699]$.

a Inhaled medications by nebulizer or spacer and face mask.

Table 11.51. Issues in the treatment of asthma in the very young

Recurring wheeze and cough have a typical onset with viral respiratory infections, often without positivity of family history

The diagnosis relies almost wholly on clinical symptoms that may be variable, without the objectivity of pulmonary function tests

Treating young babies with inhaled therapy presents unique challenges due to inappropriate devices prescribed for age and capacity of the child, or inadequate training given to enable the child to use spacing/holding chambers effectively

There are very few controlled studies on asthma therapy and they are often related to older children

The response to bronchodilators is variable, at the first place remains epinephrine in case of need

The younger the child is, the more conditions there are that may masquerade as asthma

Modified from [472].

\section{Administration of Drugs at Various Age Levels $[485,566,698-700]$}

- In babies and infants up to 1 year of age (Fig. 11.59), it is unnecessary to treat mild and infrequent symptoms, especially if there is no interference with daily life, night-time sleep and behavior. Often the infant whistles as a result of the greater elasticity of the bronchial tree or for intercurrent VRIs, thus rendering CS use difficult [620]. If the child is well and growing normally, only follow-ups are necessary. If symptoms intensify, even if infants show little therapeutic response to $\beta_{2}$-adrenergics, treatment with spacers/nebulizer and face mask is called for, with recourse to cromones or to theophylline if the desired effect is not achieved. If the condition de- teriorates, intervention with IB and/or CS by nebulizers and/or with epinephrine, and $\mathrm{O}_{2}$ therapy in emergencies is suggested.

- In children 1-3 years of age (Fig. 11.60), inhaled drugs must be administered by nebulizer and face mask. If symptoms are mild, satisfactory results can be obtained with $\beta_{2}$-adrenergic and/or theophylline. If attacks should become of moderate intensity, with persistent wheezing and coughing, the use of associated cromolyn/nedocromil sodium is advised, making use of CSs in the case of a further deterioration of the symptoms, always by nebulizer and face mask and orally in the minimum effective dose, on alternating days in single-doses.

- In children 3-5 years of age (Fig. 11.61) and with mild asthma, $\beta_{2}$-adrenergics may be useful. If coughing and wheezing persist and inhaled bronchodilators fail to elicit a significant improvement, prophylactic treatment with cromolyn/nedocromil sodium or, alternatively, theophylline is called for. The combined use of $\beta_{2^{-}}$ adrenergics and MDI + spacer CSs should be reserved for when symptoms become acute again. A further worsening will require a CS treatment in the forms indicated, such as inhaled BUD at a total dose of $800 \mu \mathrm{g}$ daily, which significantly improved symptom scores, asthma exacerbation rates, PFTs, and BHR in asthmatic children aged 2-5 years [440]. Anti-LT medications should be introduced as symptoms step up.

- In the age range from 5 to 18 years (Fig. 11.62), the differences separating cases of mild and medium/moderate asthma should be observed, alternating or combining the drugs and modifying the routes of administration. As can be seen in the figure, if asthma is severe and persistent, recourse to CS use is necessary. Asthma improves only in $41.3 \%-47.5 \%$ of cases (Table 5.15 ) (the earlier the age of onset, the greater the risk of relapses) [575], so in most adolescents who are not SIT-treated, delivery of medical care may also be challenging [476]. Anti-LT medications should be introduced as symptoms step up. 
Fig. 11.59. Algorithm for management of asthma in children aged 0-1 years: a stepwise approach. Inhaled medications are delivered by $\mathrm{pMDI} /$ with spacer + VHC with face mask or nebulizer. Face mask devices should be close fitting, especially if a valved spacer is used, with nebulizers the mask should be held as close fitting as practicable without undue disturbance. Any gap reduces the dose dramatically. (Data from [485, 566, 698-700])
MILD INTERMITTENT

(no evident symptoms)

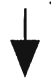

No daily medication required

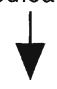

MILD PERSISTENT

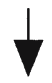

inhaled cromolyn or nedocromil by nebulizer or facemask and spacer

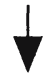

inhaled short-acting $\beta 2$-adrenergics by nebulizer or facemask and spacer

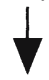

replace or combine with ipratropium bromide as required
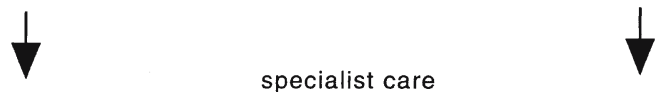

specialist care
MILD PERSISTENT

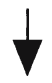

inhaled $\beta 2$-agonists bid-qid as required

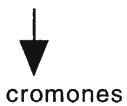

by nebulizer or facemask and spacer

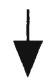

low-dose inhaled corticosteroid
MODERATE PERSISTENT with nebulizer or facemask and spacer

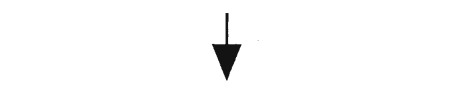

consider cromones or theophylline to minimize steroid dose +low-dose inhaled corticosteroids

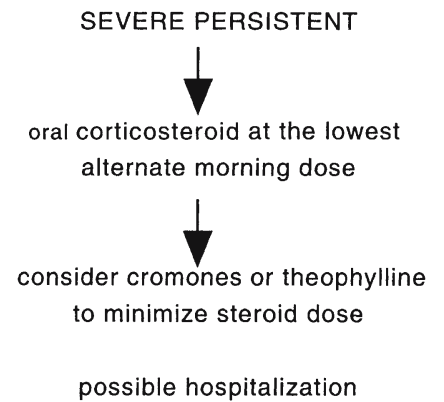

In conclusion, the necessity for a targeted use of the diverse drugs should be recognized in relation to the characteristics of age-related clinical symptoms. Cromones are effective even in the very young, and advantage can be taken of their preventive abilities and of their use in combination with albuterol + flunisolide, all inhaled. Anti LT-medications should be introduced as symptoms step up in alternative with SIT. A three-part respiratory diary should be kept, divided into symptoms (asthma, EIA, malaise, cephalea, abdominal symptoms, insomnia, nervousness, etc.), prevention and therapy, noting in addition PEFR and school absences. Symptoms are classified on a graded scale as follows: 1 intermittent, 2 mild, 3 moderate, 4 severe.

\section{Specific Forms of Asthma}

\section{Asthma Associated with Viral Respiratory Infections}

URTIs make patients vulnerable to asthma development or recurrence and potentially to the establishment of chronic forms. The considerable impact on the asthmatic child who experiences irritations more frequently, often more coincident with VRIs than with the numerical increase of mites in the air or in mattresses, should not be forgotten. For this reason, as virus trigger relapses, CS preventive administration caused a significant decrease in the number of wheezing days, attacks, ED visits, and hospitalizations [60]. 
MILD INTERMITTENT

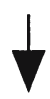

cromones by nebulizer or facemask with spacer and/or theophylline

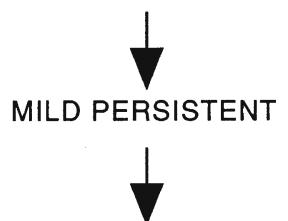

add inhaled short-acting $\beta 2$-agonists by nebulizer or facemask with spacer
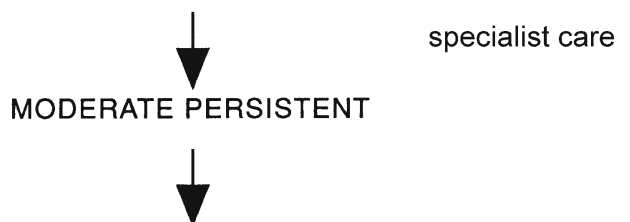

inhaled long-acting $\beta 2$-agonists as needed by nebulizer or facemask with spacer

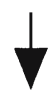

add inhaled corticosteroids by nebulizer or facemask with spacer

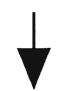

replace with oral corticosteroids at the lowest alternate morning dose

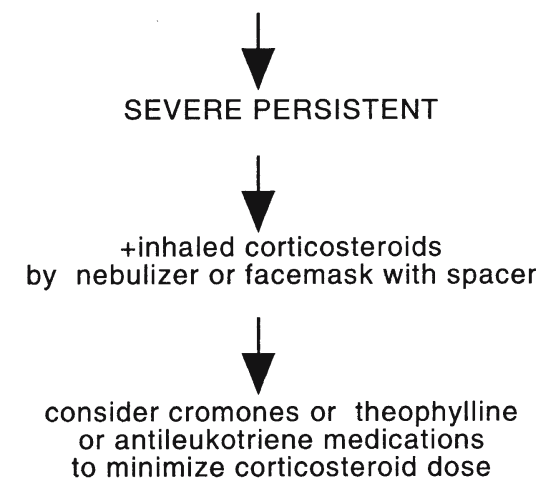

evaluate hospitalization
Fig. 11.60. Algorithm for management of asthma in children aged 1-3 years: a stepwise approach. Inhaled medications are delivered by $\mathrm{pMDI} /$ with spacer + VHC with face mask or nebulizer. Face mask devices should be close fitting to the face, especially if a valved spacer is used; with nebulizers the mask should be held as close fitting as practicable without undue disturbance. Any gap reduces the dose dramatically. (Data from ([485, 595, 698700])

\section{Exercise-Induced Asthma}

The fact that parents often make no mention of their children's EIA seems more closely related to the objective difficulties of not being able to recognize EIA than to its absence; but its occurrence in $63 \%$ of 273 subjects is relevant, the greater part of whom asthma had not been diagnosed [446]. Whereas EIA diagnosis should be made with urgency, given that it can be prevented, thus allowing the child to enjoy a normal social life. Children depend on taking part in sporting activities for correct mental, social and physical development. Parents, often easily troubled by the parallel between exercise and the onset of an attack, often constrain their children to limit, or even stop, these activities, forcing them to adopt a sedentary and solitary life, with negative repercussions. It is therefore indispensable that parents be instructed on the significance of and how EIA can occur, since EIA worsens the prognosis of asthma [214]. The cornerstone on which diagnosis relies is history. Typical EIA appears in asthmatic children, but it may also become clinically manifest with exercise in children experiencing a subclinical degree of obstruction [214]. EIA diagnosis was possible in 88 children of $12.4 \pm 3.2$ years by employing a treadmill test in which the speed was gradually increased, with positive results in $73 \%$; 35 of 36 boys complained of pain and dyspnea, but not of wheezing, entirely reduced after albuterol inhalation [720]. Significantly, without these tests, $64 \%$ of those affected [542] and, often, also $40 \%$ of children complaining of thoracic pains [720] remain undiagnosed. Correct identification therefore is essential. Sporting activity must represent in an asthmatic infant or child - as it does in a healthy one - an appreciable part of life, and the child should be made aware of his or her virtual competitiveness even with healthy subjects [214]. EIA can be effectively pre- 
Fig. 11.61. Algorithm for management of asthma in children aged 3-5 years: a stepwise approach. Inhaled medications are delivered by $\mathrm{pMDI} /$ with spacer + VHC with face mask or nebulizer. Face mask devices should be close fitting to the face, especially if a valved spacer is used; with nebulizers the mask should be held as close fitting as practicable without undue disturbance. Any gap reduces the dose dramatically. (Data from [566, 698-700])
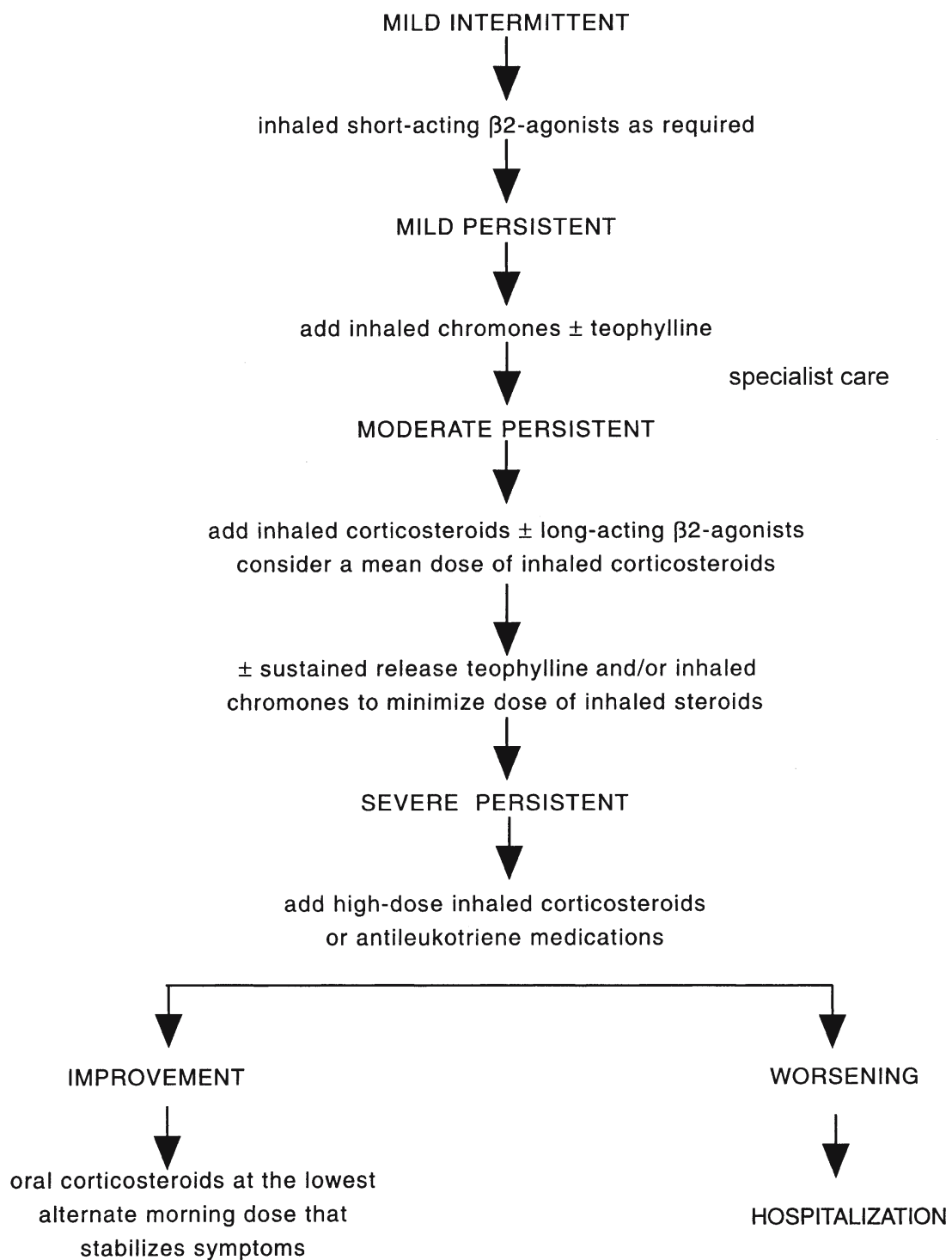

vented both by warming up, as well as by inhalations of $\beta_{2}$-adrenergics about $5 \mathrm{~min}$, or cromones about 15-20 min before beginning the activity [476]. This has proved effective in $70 \%$ of cases [39]. Studies in children have yielded significant results: after administration of cromolyn/nedocromil sodium, 10 or $4 \mathrm{mg}$, respectively, with MDI [113, 134, 451], there was a significant lowering of $\mathrm{FEV}_{1}$ compared to basic tests [134, 451]. Cromolyn remained effective for up to $4 \mathrm{~h}$ and procaterol up to $8 \mathrm{~h}$ after the test, though with reduced effect [ 419 , 450 ]. $\beta_{2}$-adrenergics are preferred due to the better clinical results obtained as well as to their duration of action. Despite recognized shortcomings in the treatment of moderate to severe asthma, cromones are preferred for limited or zero incidence of adverse effects [603]. In more resistant cases, $\beta_{2}$-adrenergics can be associated with cromones: both offer equal protection to children after only $30 \mathrm{~min}$. Formoterol in doses of 9-12 $\mu \mathrm{g}$ has an action lasting $6.5 \mathrm{~h}$ in reducing $50 \%$ of symptoms, while albuterol in doses of $200 \mu \mathrm{g}$ lasts for $1.5 \mathrm{~h}$. Using halved doses, albuterol was shown to be equal to formoterol [247]; therefore effectiveness is linked to a dose of $100 \mu \mathrm{g} /$ puff. Salmeterol has a lasting action of $12 \mathrm{~h} \mathrm{[343]}$ and bambuterol even more [159]. Long-acting theophylline administered to 12-year-old asthmatics $2 \mathrm{~h}$ before the challenge prevented both IAR and LAR $(8 \mathrm{~h})$, thereby covering an ample time period [270]. Among CSs, BUD is indicated in modest doses; $53 \%$ of the maximal effect is reported in children with doses of $200 \mu \mathrm{g} /$ day, and $83 \%$ with doses of $400 \mu \mathrm{g} /$ day, registering significant differences with $100 \mu \mathrm{g} /$ day [487], and parallel results with doses of $400 \mu \mathrm{g} /$ day [216]. Often, children forget preventative medical measures or are reluctant to be seen while they are taking the medication. In such cases, oral bambuterol [158], salmeterol [343], formoterol [441] and theophylline [270], anti-LTs [482], 
MIILD INTERMITTENT

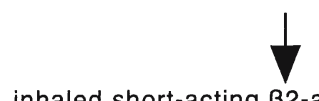

inhaled short-acting $\beta 2$-agonists as required

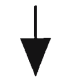

MILD PERSISTENT

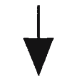

+ cromones

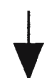

consider inhaled corticosteroids $+\beta 2$-agonists

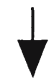

MODERATE PERSISTENT

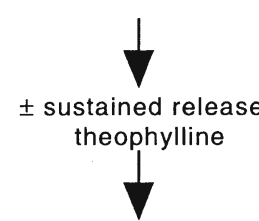

SEVERE PERSISTENT

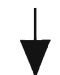

increase inhaled corticosteroid from standard to full dose

or introduce antileukotriene medications

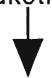

+ inhaled long-acting $\beta 2$-agonists and/or cromones and/or theophylline

to minimize inhaled corticosteroids

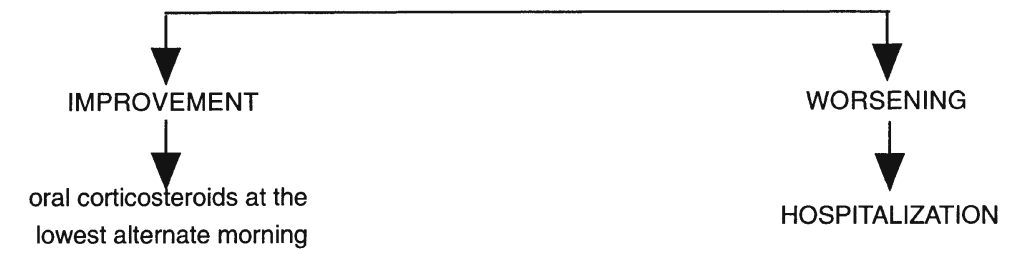

Fig. 11.62. Algorithm for management of asthma in children aged 5-18 years: a stepwise approach. (Data from $[566,698-$ 700])

dose that stabilizes symptoms

levo-cetirizine, and oral bambuterol [158] are useful. As an alternative, the following measures are suggested:

- Before beginning whatever form of activity, choose a warm, humid environment: free running in cold and dry ambient air causes much greater bronchoconstriction than swimming [214].

- Nasal respiration is preferred because, as noted, it humidifies the inhaled air and reduces the cooling effect on the airways.

- Do a gradual warming up exercise almost to the point of provoking a bronchospasm, thus delaying an asthmatic attack about 2-3 h.

- Alternatively, wear a mask with a filter that retains heat and humidity and that has proved to be effective [618].

Usually, the pharmacological pretreatment is followed by a warm-up period of $10 \mathrm{~min}$ requiring light effort. This is followed by intermittent training, which alternates more demanding exercises with lighter ones; and finally a training period (for 6-8 weeks, 2- to 3-fold a week for 45-60 $\mathrm{min}$ ) with a submaximal work-load in such a way that HR does not go beyond 160-170 beats/ min in prepuberal children and 180 in the younger ones [214].

Another frequently encountered problem is the child doing sports. Commonly, asthmatic children seem to be unwilling to take part in sports, but this presumed inaction toward sports in general is often caused by the limitations imposed by the EIA of which they suffer [617]. As for athletes in all branches from sport, including those taking part in athletic competitions, EIA is not a contraindication for children. Not all children are natural sportsmen, but all children should have a chance to share sporting with companions. Usually limitations imposed by asthma on daily childhood activities are not recognized until the child begins to participate, and then gains interest in physical activity [617]. In addition to pretreatment, children should avoid activities in 
places with excessive air pollution and/or high concentrations of allergens; if all conditions are optimal, warmup exercises can begin [419]. Swimming, ball games, relay races and dancing are examples of useful activities in the training and rehabilitation of children and adolescents with EIA. We suggest that asthmatic children and adolescents be helped to participate with others in the most suitable sports (in asthmogenic ascending order): swimming, water polo, canoeing, long-distance skiing, volleyball, free dance, speed walking, baseball, basketball, etc. When treatment does not control the problem, then further diagnostic evaluation should be done to rule out conditions other than EIA, and all children with cough, shortness of breath, or history of recurrent bronchitis should be followed to make sure that the correct diagnosis is made and to make sure that treatment is effective [482].

\section{Nocturnal Asthma}

Cough is the distinctive characteristic of asthma and nocturnal symptoms may be so dominant and bothersome as to disrupt the sleep of both the child and family. Much has been written on the condition, but a significant trend was found with more nights awakened in the past 4 weeks and with reduction in the quantity and quality of sleep and daytime sequelae of nocturnal asthma, including missing school, educational problems, and parents missing work or other usual activities [147]. Nocturnal cough generally reflects PEFR diurnal variations. Usually, examining at what time the episodes occurred, it can be seen that they do not always coincide with PEFR minimum level. Cough is more frequent about $2 \mathrm{~h}$ after the child goes to bed, and then again before awakening in the morning, and not between 3:00 and 5:00 AM when PEFR is lowest, reaching its nadir around 4:00 AM [32]. BALF studies in adults with nocturnal asthma have shown an extraordinary correlation: at 4:00 AM, BALF was found to contain a significantly greater number of lymphocytes, eosinophils, neutrophils and epithelial cell than at 4:00 PM [379]. This data shows that BHR is greatest at 4:00 AM compared to the corresponding afternoon hour, in correlation with $\mathrm{FEV}_{1}$ and $\mathrm{PC}_{20}$ equivalent variations [380]. Such are the effects of circadian rhythms, linked to the hypothalamic clock, which is linked in turn to the solar clock, with night and day alternation, which is why night-shift workers register the best test scores during nocturnal hours [32]. Another potential factor is the nocturnal body cooling independent of ambient $\mathrm{T}$, correlated with clinical evidence that small reductions of body $\mathrm{T}$ provoke cold-induced bronchoconstriction [378]. Relative to allergen influx on circadian rhythms, it has been noted that exposing mild asthmatics without nocturnal asthma to an allergenic burden, they all responded with IAR both in the daytime and night-time, but also showed LAR after the evening test [364]. The connection existing between nocturnal asthma and circadian reductions in histamine concentrations is cited as evidence of a so-called permissive effect on mast cell histamine release, a mechanism that could explain the asthmatic attacks recurring over several consecutive nights following a single exposure to provoking allergens [32].

The relationship between nocturnal asthma severity and PFT during the daytime is still the subject of study, in that as yet no conclusive results are available. A controlled study in children has confirmed that nocturnal asthma is not dependent on an increase in bronchoconstriction [665]. Concerning treatment, the drugs used for daytime treatments, especially inhaled bronchodilators, do not last long enough to cover the night-time as well [364]. In these patients, a dose of long-acting theophylline at the time of sleeping is generally effective [83, 760]. On the basis of previous analysis on theophylline and circadian rhythms, a treatment with equal doses at regular intervals will be able to increase theophylline serum levels at night compared to daytime levels [760]. The persistence of nocturnal symptoms may indicate the necessity of including an anti-inflammatory treatment [379]. This option generally seems to be unnecessary since at precisely 4:00 AM the drug blocks the flow of inflammatory cells in BALF, an effect mediated by $\mathrm{LTB}_{4}$ [316]. If theophylline, and inhaled $\beta_{2}$-adrenergics and steroids taken as late as possible do not control night-time symptoms, it will be necessary to resort to the usual therapy for day-time asthma. In children, slow-release terbutoline is more effective than inhaled $\beta_{2}$-agonists in preventing nocturnal asthma [398], while ICSs are more effective and less harmful if taken between 3:00 and 5:30 PM, as noted above. A pre-eminent position might be held by formoterol [183], salmeterol, bambuterol [159], and long-acting antihistamines may ensure overall night-time symptom reduction, which of course do not offer the anti-inflammatory potency of theophylline [760].

Differential Diagnosis. Nocturnal asthma might identify those children at risk for severe exacerbations caused by lability of airway function, total IgE levels, changes in clinical symptoms and need for albuterol, which are more likely to predict nocturnal awakening than a decrease in PEF [626].

Maximum environmental prevention is essential against dust mites and pet epidermal derivatives, particularly in children allergic to dog or cat when there is a large amount of dog or cat allergen in the environment also rich in Der p 1 [626]. The bulk of evidence stresses the time the child spends at home; the largest part of which is sleeping, commonly spent for the most part in this environment (see Chap.24). The influence of environmental factors also relies on the threshold of bronchial reactivity in hyperreactive subjects that is further reduced at night, not related to airway obstructions, but to fluctuations of circadian rhythms [380]. In some cases, the possible association with GER, often occurring during night-time because of the lack of opposition 
by gravitational forces and, by means of a reflexive obstructive mechanism stimulated by gastric juices, could also be critical [221]. Furthermore, GER could increase bronchoconstriction by activating a vagal reflex (see "Collateral Pathologies"), but the links with nocturnal asthma are not yet clear [398], also because of a lack of precise correlation between esophagus acidity and altered respiratory function [170].

\section{Cough Variant Asthma}

A general summary of the many causes of persistent coughing is given in Table 11.36. This is considered to be a mild form of asthma that is frequently unrecognized, resulting in inadequate treatment. Persistent coughing is a form of asthma that is not always well defined, which, according to recent data, is commonly found in all age groups as a variant of clinical asthma and presents symptoms that frequently result in asthma escaping detection and correct classification. At least onethird of asthmatics suffer from chronic cough [741] and among 10,063 asthmatic children, 785 (7.8\%) had cough variant asthma [100]. Several groups of children with and without wheezing [306, 472, 741] and aged $<18$ months [279] were kept under observation. With the meta-analysis of the data collected from these studies $[151,306,455,472,653]$, we have ascertained that the risk of developing asthma is statistically very significant ( $p=0.0001)$ and we agree with those authors who have defined it as hidden asthma.

Regarding the pathogenesis, the cough depends on the following [151, 306, 455, 472, 653]:

1. Upper airway obstruction where cough receptors are more numerous

2. Respiratory difficulty due to peripheral airway obstruction where receptors are scarce

3. Use of anti-cough medications, potentially in relation to cough receptor hyperresponsiveness

4. Possibly a higher wheezing threshold, apparently with no difference in BHR.

In children with classic asthma as well as in those with the variant form, a marked $\mathrm{FEV}_{1}$ reduction has been noted, as though there was an increased bronchoconstriction.

Symptoms may be summed up in a few points [741]: symptoms reminiscent of a mild form of asthma with chronic, persistent, nonproductive cough that causes interference with sleep, vomiting, and interrupted school attendance, exacerbated by airborne viral infections, physical exercise and inhaled cold air [279].

For diagnostic purposes, the algorithm of Fig. 11.63 $[279,472]$ can be followed. Moreover, chest objectivity is scarce; PFT and BPT can be normal and the only diagnostic confirmation is the positive response to bronchodilators [741]. However, since the prevalence of risk factors (atopy, FHA, and allergy) are similar to classic asthma, it is not easy to diagnose those children who, subsequently, have wheezing $[472,741]$. Among the 785 children, only $1 / 3$ had a correct diagnosis [100]. Above all, differential diagnosis is needed (Table 11.36), and atopy may distinguish groups of coughers from groups of wheezers [395]. The natural history is highly variable: $9 \%-75 \%$ of children (on average $40 \%-50 \%$ ) develop full-blown asthma over 6-96 months [279], while a number of cases not easily quantified (about 50\%) evolves toward a disappearance of clinical symptoms. In two groups of children aged 5.7 (mean) [653] or 7-15 years [306] to $54 \%$ [653] to $55 \%$ [306] developed classic asthma. Interestingly, asthma-positive children developed cough variant asthma at a young age [653]. For example, GER may be the cause of chronic cough and BHR [170]. In children with clinical wheezing the methacholine $\mathrm{PD}_{20}$ test significantly decreased as these children developed wheezing [306]. On the other hand, $83 \%$ of a group who were SPT+ to inhalants and asthma-like night cough and worsened by exercise, was found to have improved at follow-up 2 years later, while $25 \%$ developed recurrent wheezing [348]. In $93 \%$ of cases, these children enjoyed the benefits of antiasthmatic therapy $[151,279]$. To achieve satisfactory control of the disease, it is also necessary to eliminate the triggering mechanisms.

\section{Unresponsive Asthma}

Some infants, children or adolescents continue to suffer from asthma that is either greater or lesser in severity, despite the apparently appropriate treatment they are undergoing. This could be caused by various factors (Table 11.52) [476]: inappropriate doses of medication, poor child compliance, and inability to regularly follow the treatment. A more uncommon cause is a variety of atypical outdoor antigens to be considered in all children with nonresolving chest disease or unresponsive asthma [137]. It could also be the result of objective elements related to asthma severity requiring the continuous use of steroids, especially if elevated doses are needed [633], as well as GER [179]. From a pathogenic point of view, this could be a result of anomalies of the CS receptors (CR) (Table 11.22), whether for an IL-induced reduced linking ability [23] due to a receptor irreversible reduction or because different $\mathrm{T}$ subpopulations are active, which, in resistant subjects, are more activated as they have an increase in CD25 and HLA-DR [131] or because of different CR links to DNA [23].

A cohort of 103 entrants aged 9-17 suffering for years from poorly controlled severe asthma and admitted several times, were hospitalized on average for 75 days to rationalize their treatment [624]. After a year, follow-up visits, admittance to hospital as well as days requiring hospitalization, visits to EDs or doctors' offices for acute asthma were found to be significantly reduced, with an obvious amelioration in $82 \%$ of the hospitalized chil- 

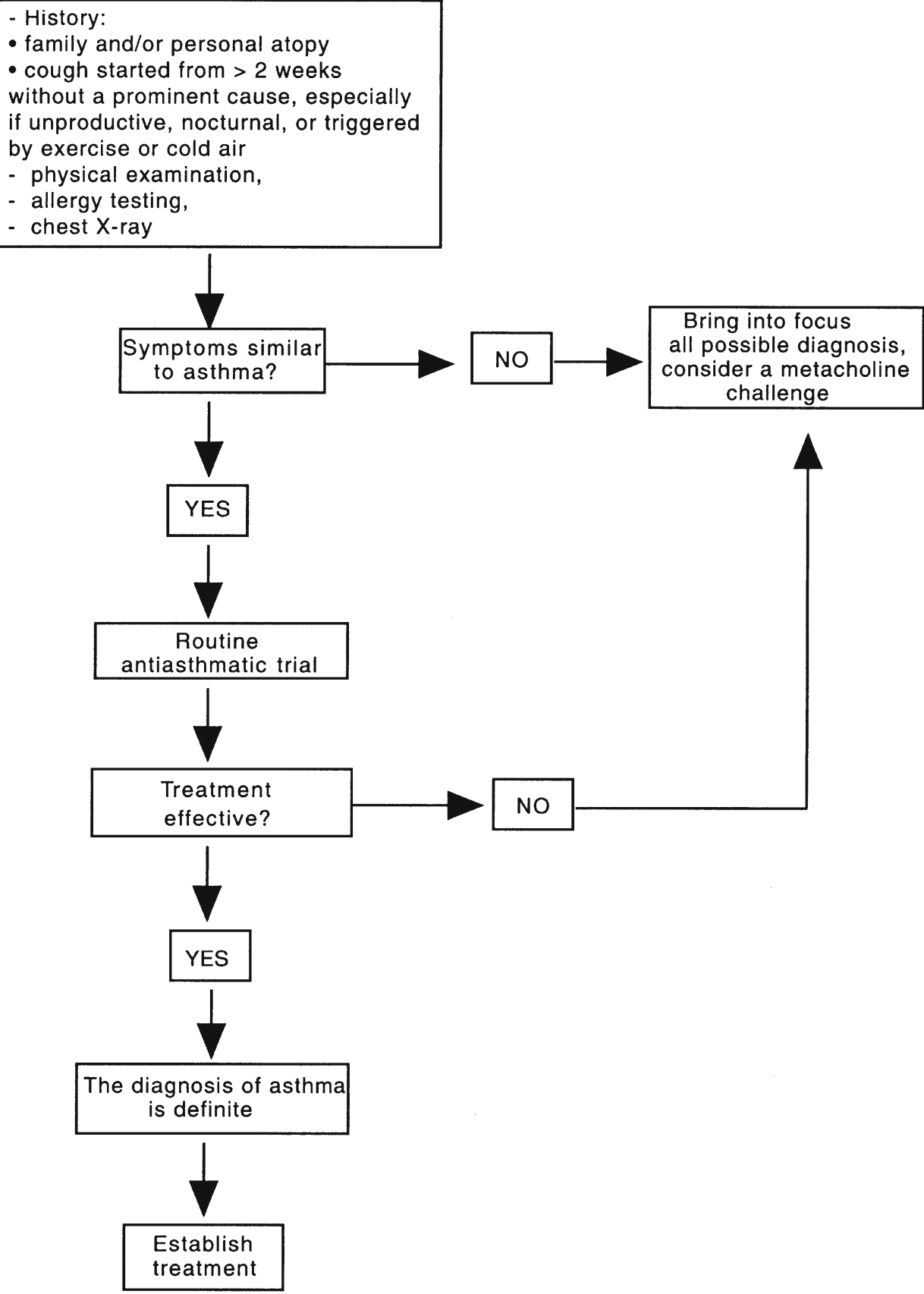

Fig. 11.63. Algorithm for the diagnosis of cough variant asthma. (Data from [279, 472])

dren [624]. On the other hand, children with similar clinical characteristics did not gain any benefits from treatment at a hypoallergenic center [489]. New horizons could be opened by high-dosage IVIg treatment $(2 \mathrm{~g} / \mathrm{kg} / \mathrm{month})$ on steroid-dependent children. After 6 months, doses of oral cortisone for the maintenance and control of asthma exacerbations were reduced 3.2fold, with an equal reduction in PEFR, clinical score and SPT results [388]. The only doubt is that the sample of this study was not very representative: IVIg treat- ment could be appropriate since in severe chronic asthma there is a deficiency of one or more IgG subclasses, which respond favorably to IVIg treatment [468]. The effect could also be due to the correction of steroid-induced hypogammaglobulinemia [253]. A trial with recombinant IFN (rIFN) in a group of children with analogous dependency did not prove effective [46], although rIFN reaches the airway epithelium [276]. In adolescents with unresponsive asthma, only theophylline proved to be effective [707]. Use of methotrex- 
Table 11.52. Apparent failure of therapy: possible causes

\begin{tabular}{l} 
1. Inefficient inhalation technique \\
\hline 2. Lack of confidence in efficacy of the drug \\
\hline 3. Difficulty with the method of administration \\
\hline 4. Poor child compliance \\
\hline 5. Introduction of prophylactic drugs without \\
a clear-cut indication \\
\hline 6. Difficulty with the timing of administration \\
\hline 7. Psychosocial factors \\
\hline 8. Complications (aspirin intolerance, \\
gastroesophageal reflux, etc.) \\
9. Inappropriate therapeutic intervention \\
(no SIT prescription, cough medicines, antibiotics, etc.)
\end{tabular}

Modified from [476].

ate and cyclosporine in the pediatric age raises motivated doubts [365], as they provoke toxicity at the pulmonary and hepatic level and blood alterations as well as nephrotoxicity and hypertension [131].

The collaboration of both children and parents is of primary importance to make a treatment successful. In recent years several educational programs have been proposed. We have mentioned the relevance of psychotherapeutic and psychosocial factors in the whole spectrum of treatment for pediatric asthma. We believe the intervention of a specialist in this area to be particularly appropriate and productive. On the other hand, a group of pediatricians has devised a program aimed at improving the quality of life of children, with particular emphasis on educating the family, who received basic physiopathological information and were followed by a specialized team who tended to the cleaning of the environment, in addition to participating in meetings aimed at ensuring maximum compliance with the prescribed therapy. At the end of the follow-up, an overall improvement was noted in all clinical and psychological parameters [708]. An analogous program should be centered on the following points: an informative meeting, distribution of preprinted forms to assist in the recognition and treatment of acute attacks and follow-up visits every 2 months. At the end of the program, the disappearance of ED visits, the better understanding by parents of the measures to be taken during acute episodes and a greater confidence in their own ability to effectively handle their child's disease are necessary.

\section{Collateral Pathologies}

GER may be one of the possible contributing factors in any child with recurrent and persistent respiratory complaints, so evaluation for GER should be considered in infants with persistent wheezing [170]. Children with
GER-associated cough often show signs of BHR, and therefore the presence of GER should be suspected when faced with persistent wheezing. Symptomatic or functional GER therapy also appears to be necessary for the possible complications related to nocturnal asthma. The basic principles are:

1. Small meals, but more frequently, with food-thickening agents for bottle-fed babies

2. Avoid eating and drinking between meals and $2 \mathrm{~h}$ prior to reclining

3. Avoid excessive intake of lipids

4. Keep the head elevated, in a prone position on a surface inclined $30^{\circ}$ adopting an anti-Trendelenburg position. To this end we suggest placing pillows $(10-15 \mathrm{~cm}$ depending on the child's age) to raise head of the bed and keeping the child upright by lifting the upper part of the body with foam rubber supports or air cushions

Especially in young children, early diagnosis and antireflux therapy in cases with GER-related respiratory complaints can result in significant improvement in symptoms. Drugs heightening lower esophageal sphincter pressure are used, for example domperidone 0.1$0.2 \mathrm{mg} / \mathrm{kg} / 3$ - to 4 -fold a day $20 \mathrm{~min}$ before meals or metoclopramide $0.1 \mathrm{mg} / \mathrm{kg}$ qid by monitoring side effects. If esophagitis is present, antacids, $\mathrm{H}_{2}$-receptors, or in severe cases, omeprazole or lansoprazole are indicated. During follow-up visits to the age of 4.5 years, in $25 \%$ of infants (age, 4-11 months) asthma treatment was necessary [170]. Omeprazole $(0.3-1.0 \mathrm{mg} / \mathrm{kg}$ daily) was effective in 78 children. After 8 weeks of therapy, rebounding was reduced by $66 \%$, heart burn by $56 \%$, vomiting by $33 \%$, abdominal pain by $64 \%$ [132]. The use of $\mathrm{H}_{2}$ antagonists (to impede the production of gastric acid) such as ranitidine $5-10 \mathrm{mg} / \mathrm{kg} /$ day divided into two doses for 8 weeks as an attack therapy, to be subsequently halved during the maintenance phase (in the evenings for 6 months), is justified in the presence of GER-induced disease, documented by endoscopy. We suggest making PFT control before and after antireflux therapy [170]. The outcome over 1-4.5 years of follow-up was excellent; only one patient required further asthma medications [170].

Finally, asthmatic symptoms present in children with FA (Table 9.12), the numerous triggering foods (Tables 9.18, 9.19) and pseudoallergy from additives (Chap. 10) should not be neglected.

\section{Complications}

The clinical situations which can complicate asthma are pulmonary and extrapulmonary [41].

Pulmonary Complications. Atelectasis can be either segmental or subsegmental and often involves the middle lobe. In the absence of immunological deficiencies or of aspiration of foreign bodies and with negative results to the sweat test, even though having undergone regular treatment, hypoxemic children affected with 
middle-lobe syndrome can have frequent relapses, or cough, wheezy breathing or dyspnea persistency, etc. [336]. Using fiberoptic bronchoscopy and a count of BALF cells, a study on 3.3-year-old asthmatic children showed the presence of pathogenic bacteria in $47.6 \%$ of cases, re-evaluating the role of infections [610], often favored by malformations and, in $11.5 \%$ of cases, by GER [336]. In these children, the possibility of underlying asthma must be suspected, especially if faced with a documented parenchymal thickening [481]. In a group of 56 asthmatic children, only 5 of 63 episodes had physical symptoms of middle-lobe syndrome [577]. This was the case of a 7-year-old boy with intubated status asthmaticus complicated by refractory mucus plugging and atelectasis [163]. Atopy is present in 35\% [577] to $38 \%$ of cases [336]. The right middle lobe is most commonly involved due to anatomical factors, which facilitate its obstruction, further aided by the combined action of bronchospasm, edema, mucosal thickening, and mucus plugging, with the possible complication of bacterial infection. It follows that the bronchus tends to twist with hyperinflation, resulting in a partial occlusion with repercussions on the middle lobe [41]. Atelectasis and hypoventilation are also sustained by the particular relationships of this lobe with the others, which are not ideal for ensuring a collateral circulation of alveolar gases [41]. It is not clear if the occlusion of the right middle lobe is more prevalent among females $[41,481,577]$ or males $[163,610]$. The boy was successfully treated with two 10-mg doses of intratracheal rhDNase (see "Treatment of Status Asthmaticus") [163].

Pneumomediastinum presents itself as a sudden complication in $5 \%$ of children with status asthmaticus [554]. The most common cause in children is asthma [93], but it is not a characteristic of severe asthma and rarely is manifest before the age of 2 [336]. The only exception is an 18-month-old girl who also had subcutaneous emphysema [211]. Paroxysmal cough and bronchospasm superimposed on hyperinflation, or related to atelectasis, pneumonia, structural weakness, intermittent positive pressure breathing (IPPB), cause air to rupture alveolar bases and spread along vascular sheaths, consequently causing pulmonary interstitial emphyse$m a$. This may lead to severe cardiovascular insufficiency with reduced venous return, cardiac output and blood pressure [41]. If the air makes its way to the mediastinum and reaches the pericardium, one has auscultatory findings of a crackling sound synchronized with heart beats (Hamman's sign), pathognomonic of pneumopericardium. In other cases, air, through the fascial planes, may escape into the subcutaneous tissue of neck, shoulders and axillae, releasing the mediastinal pressure [41]. Diagnosis is made following tachycardia, retrosternal pains that spread to the arms and neck aggravated by breathing and sometimes by swallowing [336]. However, the association with severe hypoxia, tachycardia, metabolic acidosis, and high ventilation pressures indicates clinically significant tension in the medi- astinum [93]. Pneumomediastinum generally resolves spontaneously within a few days, 6 in the girl or 1-5 days in 5 asthmatic children [211], meaning that ambulatory treatment is usually appropriate. Management consists of treating the underlying cause, rest, analgesics, and simple clinical monitoring [93].

Pneumothorax is the rarest complication of acute infantile asthma, which can, for example, also become present following paroxysmal coughing or as a result of IPPB. It should be suspected in cases when the child suddenly worsens together with signs of hyperphonesis and reduction of the murmur of the affected side [41]. If it is not widespread, is self-limiting, but in case of pneumothorax tension, it provokes respiratory breathing difficulties and continuous aspiration is necessary [313]. Pulmonary infiltrations are frequent radiological findings, but they do not require urgent interventions. Conversely, total pulmonary collapse can be provoked both by the severity of asthma as well as external incidental factors [41].

Extrapulmonary Complications. Generally, extrapulmonary complications are rhinitis, sinusitis and secretory otitis media that interact with asthma, but see Chaps. 12 and 15 for the appropriate treatment. Nonetheless, sinusitis (whether acute or chronic), common among allergic children, can accompany the worsening of asthma or forms of difficult asthma [334], whereas suitable therapy reflects positively on asthma (Chap. 15).

\section{Other Complications}

Other complications are more or less frequent and diverse according to whether the asthma is acute or chronic (Table 11.53) [584]. The most frequent compli-

Table 11.53. Selected complications

\begin{tabular}{l} 
In acute asthma \\
\hline Bronchiectasis \\
Emphysema \\
Pneumothorax \\
Pneumomediastinum ${ }^{a}$ \\
Viral and bacterial infections \\
Bronchitis \\
Acute otitis \\
Pneumonia \\
Sinusitis \\
\hline In chronic asthma \\
\hline Adverse effects of drugs \\
Chest deformity (barrel chest) \\
Clubbing \\
Emphysema \\
Growth retardation \\
Psychological/emotional problems
\end{tabular}

Modified from [584].

a Associated with status asthmaticus. 
cations are bacterial infections, especially in asthmatics $<5$ years, acute and chronic asthma and viral infections, to which asthmatics are particularly susceptible. Another possible complication is vasopressin excessive production (inappropriate $A D H$ secretion): such levels are high in patients with severe asthma, independent of natremia, probably as a result of the effect of severe asthma on pulmonary circulation. Vasopressin levels fall parallel to patient improvement. Since during an asthma attack hyponatremia can occur, in case of hospital admission BE must be carefully monitored as well as electrolyte levels, and the subsequent parenteral fluid administration must be handled accordingly [41].

\section{Death by Asthma}

For a long time asthma mortality was low: only during the first half of the 20th century was it observed that it could be fatal in children [623]. Starting in the 1960s, a few English authors noted a sudden increase in the mortality rate among asthmatics, attributing the cause to the $\beta$-adrenergic isoproterenol, the use of which was discontinued though the doubts had not been clearly proved [479]. The pharmaceutical industry introduced new $\beta_{2}$-adrenergics on the market, but in the $1970 \mathrm{~s}$, other scientists from New Zealand spoke out against the most widely diffused of these: fenoterol. These authors said that the problem was not due to the drug's direct toxic effect, but rather to its abuse because it provided immediate relief [326]. Both isoproterenol and fenoterol are relatively nonselective potent full agonists with both greater long-term and immediate adverse effects than other $\beta$-agonists. Likely the regular use of these agonists led to worsening asthma control and their overuse to treat life-threatening asthma attacks caused an increased risk of death resulting from adverse cardiac effects in the presence of severe hypoxia [569]. In the US, high-dose fenoterol and isoproterenol were not approved for use. In effect, after the ban on the drug, the mortality rate among 5- to 34-year-olds fell from four to $<1$ case $/ 10^{6}$ [480]. Consequently, albuterol was given preference as it was less dangerous according to New Zealand scientists [738] since fenoterol prescription was associated with an increased risk of death compared to albuterol [608]. We have prescribed albuterol without noting adverse effects and a case-control study recognizes that such alarm was unjustified [738]. Recent studies have instead documented that mortality rates decreased, while sales of ICSs increased $[628,630]$, as well as of $\beta_{2}$-adrenergics [331]. Some ecological studies conducted in various countries state that the introduction and sharp increases in the use of ICSs correlate to important reductions in the asthma death rates, whereas other cohort or case-control studies indicate that ICSs might not prevent asthma death [628]. An analysis of 96,258 UK asthmatic patients has confirmed in vivo that regular use of ICSs is associated with a decreased risk of asthma death [331].

\section{Increase or Decrease in the Mortality Rate Due to Asthma}

Despite the progress made in the diagnosis and therapy of asthma, since 1980 the mortality rate is again on the increase at the rate of $6.2 \pm 1.2 \%$ a year, especially among 5- to 14-year-old children [710]. As can be seen in Fig. 5.19, the increase in the world population (19851987) places Israel in the first position, followed by Finland and Denmark, but if the rates in the 5- to 34-yearolds in 1987, compared to the level in 1980, is calculated, Australia ranks first, followed by Singapore and New Zealand, whereas Israel is in ninth place and Finland and Denmark are absent [569] or in the last places (Table 5.17). These rates in patients with severe asthma are markedly greater than mortality rates in asthmatic patients in the general US population, which can be estimated at $0.02 \%$ if the death rate is $1.5 \times 10^{5}$ and asthma prevalence is $3 \%-5 \%$ [595]. After a gradual decline in asthma mortality rates in the 1960s and 1970s, rates have increased progressively in the US during the past 2 decades. In most other Western countries, the rate of asthma mortality decreased during the 1990 s after progressive increase through the $1980 \mathrm{~s}\left(0.36 \times 10^{5}\right.$ in some European countries vs $0.47 \times 10^{5}$ in the US. The underlying reasons, including the role of management, will require further investigation if strategies are to be implemented successfully to reduce asthma mortality rates [29]. In a PICU, a 6-year study resulted in an $8.9 \%$ incidence of acute severe asthma, while all pediatric admission totalled $1.9 \%$ [464]. However, the marked underestimation, varying between $36 \%$ and $127 \%$ for 5 - to 34-year-olds, should be noted, results from a position common to other European countries with the highest levels [375]. Contrary to the statistical findings, beginning from the 5th year, an epidemiological study has established a mortality rate among 11- to 14-yearolds of $0.34 \times 10^{5}$ (Tables $11.54,11.55$ ) [190, 192]. The inclusion of $45.5 \%$ of 13 - to 16-year-olds and of $32 \%$ of 1 - to 4-year-olds is very significant [190] $(p=0.0006)$. In another cohort [192], the death rate among 1- to

Table 11.54. Increase in mortality rate for asthma

\begin{tabular}{lll} 
Age (years) & $\%$ & Female prevalence \% \\
\hline $1-4$ & 32 & \\
\hline $5-12$ & 23 & \\
\hline $13-16$ & 45 & \\
\hline Up to 12 years & & 75 \\
\hline Total & 59
\end{tabular}

Data from [190]. 
Table 11.55. Variations in mortality rate for asthma

\begin{tabular}{lll} 
Age (years) & \multicolumn{2}{l}{ Periods studied } \\
\cline { 2 - 3 } & $1952-72$ & $1973-88$ \\
\hline $1-4$ & $10.4 \%$ & $6.2 \%(p=0.0396)$ \\
\hline $1-19$ & $-34.3 \%$ & \\
\hline $15-24$ & $+50 \%$ & \\
\hline
\end{tabular}

Data from [192].

19-year-olds dropped and among 15- to 24-year-olds doubled, with a male prevalence in the former $(55 \%$ of cases) and of females in the second group (59\%) [190]. Underdiagnosis is more common among females; therefore the cases of death seem to be more frequent precisely in the groups that statistically appear to be least at risk, that is the 15- to 34-year-olds, 5- to 14-year-old females [14] and children 1-4 years of age in general [190].

\section{Examination of Possible Causes}

Tables 11.56 [80, 145] and $11.57[67,403,579,623]$ indicate the possible causes of a worsening of the prognosis, including so-called self-management and possible undertreatment [623]. This assumption is based on the fact that asthma is a very common disease, implies a substantial impairment in children's quality of life and requires challenging medical interventions and treatments often accepted by children reluctantly [375]. Moreover, the deeper understanding of asthma by patients, along with the undeniable positive aspects can, in some cases, give way to a loss of the doctor-patient relationship, which on the contrary is indispensable [67]. Among other frequent disadvantages of self-management is the improper use of some drugs, particularly $\beta_{2}$-adrenergics [569]. Continuous drug dependency occurs, which is reflected negatively on the quality of life and involves a condition of undertreatment, since the patient does not use, or misuses, those drugs that are needed in his or her case [478]. Chronic underuse is also attributable to a false feeling of safety occasioned by masking the effects that systematic use of medications has on symptoms [109]. Thus, on the one hand a masking or an underestimation of the underlying inflammatory process can occur, so that when a sudden emergency materializes, the use of ICSs and/or cromones is delayed $[67,375]$; on the other hand, the rapid beneficial effects on clinical features prevents the patient from realizing that hypoxia worsens [296], dangerously postponing a doctor's visit. Undertreatment increases death cases, since the moderate reduction in mortality is related to the progressive increase of ICS sales [596, 628, 630]. It is recommended to closely follow subjects who have experienced fatal crises or near-fatal attacks [326].
Table 11.56. Features of children at risk for asthma death

Early onset of asthma, especially in the 1st year of life

Severe episodes:

Asthmatic episodes frequently requiring hospitalization

Increasingly severe airway obstruction persistent all day long

Respiratory insufficiency requiring mechanical ventilation

Hypoxic seizures associated with asthma attacks

Nighttime asthma in rapid progression

\section{Attacks precipitated by foods}

Unperceived severity of attacks

Weaning medications, especially oral CSs after exacerbations

Excessive $\beta_{2}$-agonist use neglecting CSs during acute episodes

Steroid dependence with an increase in oral or inhaled CS doses

Inadequate medical and asthma care during hospitalization

Psychological disturbances, overt depression,

self-treatment of asthma

Data from references $[80,145]$.

For example, in Australia, the government has been engaged in distributing guidelines among doctors for treating asthma [598]. A correlation between the increase in both death and asthma prevalence has also been hypothesized; nevertheless, in some countries the mortality rate has diminished, despite the increase in prevalence [598].

\section{Subjects at Risk}

Epidemiological studies have shown that in addition to the age factor, the following categories of pediatric patients are particularly at risk (Table 11.57):

- Children in a home where family interference is dominant. A paradigmatic case of late referral is that of a 5-year-old male whose parents had objected to steroid treatment and had even hesitated 5 days in taking him to hospital [464].

- Adolescents suffering from chronic asthma and from a recent episode of acute asthma, who frequently do not regularly follow treatment for a variety of reasons [478], whose family rarely looks after him or her or on the contrary whose supervision has been rejected [326].

- Adolescents who do not go to their doctor and do not follow their therapy, with periodical relapses of status asthmaticus as a result of undertreatment [595], only occasionally undergoing therapy with insufficient doses 
Table 11.57. Patterns of asthma death in children and adolescents

\begin{tabular}{|c|c|}
\hline Risk factors & Fatal attack \\
\hline $\begin{array}{l}\text { A. Onset of asthma before } 3-4 \text { years of age, } \\
\text { especially in the } 1 \text { st year of life }\end{array}$ & \multirow{4}{*}{$\begin{array}{l}\text { a. Delay in seeking care } \\
\text { b. Failure to recognize severity of deteriora } \\
\text { c. Excessive reliance on bronchodilators } \\
\text { d. Insufficient use of systemic steroids } \\
\text { e. Unclear criteria for initiating treatment } \\
\text { of exacerbations }\end{array}$} \\
\hline B. Age between 10 and 20 years of age & \\
\hline C. Generally severe asthma or near death episodes: & \\
\hline \multirow{2}{*}{$\begin{array}{l}\text { a. Past history of severe asthmatic attacks } \\
\text { b. Frequent admissions to hospital or emergency } \\
\text { wards in the past year or }\end{array}$} & \\
\hline & Negative effects of sedatives during acute asthma \\
\hline $\begin{array}{l}\text { c. One or more emergency department visits } \\
\text { for asthma in the past year with probable } \\
\text { intubation and/or mechanical ventilation }\end{array}$ & \multirow{3}{*}{$\begin{array}{l}\text { 1. Sedatives mask the agitation that is usually a sign of } \\
\text { hypoxemia and delay an adequate clinical evaluation } \\
\text { of progressive bronchial obstruction or of response } \\
\text { to therapy } \\
\text { 2. Sedatives suppress both ventilation and cough }\end{array}$} \\
\hline D. Insufficient patient education from the physician & \\
\hline E. Poor patient collaboration & \\
\hline \multirow[t]{2}{*}{ F. Problems arising from self-treatment: } & Prevention strategy \\
\hline & $\begin{array}{l}\text { A. Physicians should emphasize to parents and/or } \\
\text { children the necessity of initiating medication } \\
\text { to be taken at the onset of symptoms and of } \\
\text { prescribing rapidly effective medications }\end{array}$ \\
\hline G. Poor family support for ongoing and acute care & \multirow{2}{*}{$\begin{array}{l}\text { B. A maintenance therapy should be followed on } \\
\text { a regular basis, with peak flow readings test and PFTs } \\
\text { checked by frequent visits to determine the first } \\
\text { symptoms of airway obstruction }\end{array}$} \\
\hline H. Family history of atopy & \\
\hline I. Increasing use of short-acting $\beta_{2}$-agonists & \multirow{2}{*}{$\begin{array}{l}\text { C. An inadequate response to bronchodilators should } \\
\text { advise to initiate prednisone therapy }\end{array}$} \\
\hline J. Use of three or more antiasthmatic medications & \\
\hline K. Active and passive smoking & \multirow{3}{*}{$\begin{array}{l}\text { D. Parent and physician nonrecognition of asthma } \\
\text { severity is often the cause of delays in care during } \\
\text { the attacks and in planning admission to an ED }\end{array}$} \\
\hline L. Respiratory infections & \\
\hline Contributing factors & \\
\hline 1. Telephone prescriptions & $\begin{array}{l}\text { E. Physicians should ensure patients and/or parents } \\
\text { of his (her) fully availability }\end{array}$ \\
\hline $\begin{array}{l}\text { 2. Poor compliance with long-term treatment } \\
\text { 3. Discontinuity of treatment or medical care }\end{array}$ & $\begin{array}{l}\text { F. Such patients should have home epinephrine } \\
\text { for rapid use and require Medic-Alert bracelets }\end{array}$ \\
\hline 4. Psychosocial factors & \\
\hline $\begin{array}{l}\text { 5. Failure of family (and physician) } \\
\text { to recognize severity of the attack }\end{array}$ & \\
\hline 6. Delays in hospital admission & \\
\hline $\begin{array}{l}\text { 7. Undertreatment during the last attack: } \\
\text { delays in instituting an appropriate treatment, } \\
\text { no use or inappropriate use of CSs }\end{array}$ & \\
\hline
\end{tabular}

Data from $[67,403,579,623]$.

[377], to the point that the prescribed drug serum levels are between 0 and subtherapeutic levels [43]. This is especially alarming when these drugs are ICSs: their regular, uninterrupted use significantly reduces the risk of death from asthma by at least $50 \%$. Instead, treatment interruption is associated with an almost fivefold increase in asthma deaths [630], likely because the CS effect disappears after 2 weeks [593].

- Young patients under proper observation, who are faced with sudden attacks, progressively becoming worse to the point of respiratory insufficiency and death within $20 \mathrm{~min}$ to $3 \mathrm{~h}$ [190], probably because of the effect of a heavy allergenic load or the intervention of other highly negative factors, but who did not avail of help quickly enough [409].

\section{Risk Factors}

It happens, however, that children and/or adolescents do not fall into this classification and die as a result of insufficient management of asthma [623], or for excessive delays in the final moments [190]; $80 \%$ of the cases studied were preventable. They can be divided as follows: 
- A long period of undertreatment, or of scant medical care $(64 \%)$

- Inadequate care in the final stage $(45 \%)$ or delays in asking for help, or insufficient physician knowledge of emergency treatments

Some families hesitate to call a doctor during weekends or at night [190]; In fact $75 \%$ of deaths occur between 7:00 PM and 6:00 AM [375] and life-threatening attacks and deaths show a pattern of occurring on Sunday [527]. Two studies showed that $68 \%$ of Swedish children and $35 \%$ of English children died within $3 \mathrm{~h}$ of the onset of the attack $[190,192]$. Therefore, there is an impact of unpredictability that could also be due to overlooked nonsevere cases [192], whereas in dispatching an ambulance with a doctor on board (the person making the request had indicated asthma to the hospital operator), the arrival of help was shortened by $64 \%$ and the mortality rate decreased sixfold [595]. Survival in the period after the attack is made difficult by mistakes, for example, inadequate monitoring [377] or inadequate actions on the part of patients, especially those at high risk. Some consented to regular check-up requesting a medical visit after $7 \mathrm{~h}$, others refused and died, having requested the medical visit only after 3 days [410].

\section{How Patients Die of Asthma}

The cause of death in cases of severe asthma can vary [76, 578]:

1. Some patients, who died suddenly of a heart attack preceded by tachycardia and extrasystoles, had in all likelihood taken drugs in excessive doses. To this end an abnormal pharmacological mechanism could be regarded as being the cause (possibly prostaglandins), triggered by an exogenous agent [578]; such cases habitually occur at home [190]. However, postmortem examinations carried out on some children have shown equal amounts of specific cardiac lesions among those who had abused $\beta_{2}$-adrenergic drugs and those treated with other drugs [595], although probably the severity of the complications in these patients was due more to hypoxia than to cardiac factors [409].

2 . Often the abuse of inhaled drugs, such as $\beta_{2}$-adrenergics $[375,569,578]$ has been stressed, both because they are very handy to use as they are supplied with dispensers, and because some have a longer and more intense action and an MDI dosed at $200 \mu \mathrm{g} /$ puff [479], whereas, for example, the dosage of albuterol is reduced by half.

3. The theory that the abuse of $\beta_{2}$-adrenergics can lead to a masking of worsened underlying disease, whereas CS treatment could be needed [571], is, however, contradicted by the increase in sales for both classes of these drugs [480].

4. Another probability is that in status asthmaticus the lack of use or underuse of CSs, if taken in a timely manner, prevents the formation of obstructive plugs [76]. In fact, postmortem findings are often marked by highly thickened bronchial walls, with mucus plugs blocking the bronchial lumen and diffused throughout the airways [578].

Death by asthma can be sudden or progressive: in the first instance, the preponderant role of an intense bronchospasm is suspected, as it has been ascertained that recovery from a near-fatal attack occurs more quickly in these forms than in those that evolve more slowly, characterized by respiratory insufficiency following generalized bronchoconstriction and long-term inflammatory phenomena [578] similar to that found in status asthmaticus. Two potentially contributing causes should not be underestimated: drug cardiotoxicity, especially if hypoxemia is present, and the onset of respiratory insufficiency, rapidly aggravated by severe hypoxia [409]. Thus, the underlying mechanism can be represented by a reduced perception of dyspnea and by a very low chemosensitivity to hypoxia [296].

\section{Controversies Surrounding the Risks Linked to Drugs}

Sears et al returned to the risks associated with continuous therapy (in adults) with high doses of fenoterol, including a worsening of asthma and an increase in aspecific BHR [569, 571]. At the end of a retrospective study on 12,301 Canadian asthmatic adults, Spitzer et al [608] declared there was a danger related to the use of bronchodilators in MDI spray cans, maintaining that all $\beta$-adrenergics, including albuterol, but also theophylline and oral steroids, worsen asthma and can be related to fatal cases, without in effect providing clear evidence related to a cause-effect relationship [109]. Cromones as well as ICSs are totally excluded from a careful analysis in this data, as is albuterol if not taken in combination with fenoterol, which was the most common drug used among the deceased [608]. Following the publication of the preliminary results, the North American press underlined the serious dangers deriving from the abuse of $\beta_{2}$-adrenergics and the American College of Allergy and Immunology sent photocopies of the articles to all its associates as well as its Position Statement on the matter [185]. The dissertation by Spitzer et al [608] brought in a large number of controversial letters. The New Zealand authors exclude that in their country the use of albuterol had increased the cases of death between 1969 and 1976 [738], which occurred when the less selective fenoterol was commercialized [571], and therefore it is the prolonged use at high doses that is correlated with the risk of death by asthma or near-fatal asthmatic attacks [331]. The same group [629] subsequently quantified the risk in $10^{5}$ asthmatics/year. The statistics are $42.8 \%$ for nebulized $\beta_{2}$-adrenergics and $19.2 \%$ for oral $\beta_{2}$-adrenergics, $44.6 \%$ for oral CSs; in direct comparison, fenoterol alone vs albuterol alone was $60.2 \%$ vs $7.4 \%$, so the conclusion is that albuterol is safe if inhaled alone 
with MDI, as are inhaled cromones and CSs [629]. Abuse is dangerous because $\beta_{2}$-adrenergics exacerbate BHR. Studies $[110,456]$ have reconfirmed that $\beta_{2}$-adrenergics show tolerance with continued exposure with a loss of clinical effect that can worsen asthma. Abuse and high doses during acute attacks lead to hypoxemia and/or hypokalemia as well as a delay in seeking medical assistance $[409,410,479]$.

Therefore the use of bronchodilators is not intrinsically dangerous, but the substantial irrationality of treatment based only or mainly on $\beta_{2}$-adrenergics should be pointed out; its use should be associated with regular anti-inflammatory and preventive therapy [62, $68]$. The main risk in the use of fenoterol is a dose-effect ratio between mortality and prescription [30], potentially caused by excessive basic dosages, as mentioned above. In line with such reports, on the one hand there is a recourse, as urged by some authors, to halve the dosages [612]; on the other hand the drug has been shown to be effective in moderate forms even in dosages between 10 and $50 \mu \mathrm{g}$, even reduced up to $1 / 20$ of the recommended dosage [196]. However, the adverse effects of fenoterol became known when it was compared to placebo for 6 months [571]. What criteria is used to freely commercialize $\beta$-adrenergics controlled by studies lasting barely 4 weeks [133]? In a large sample of 16,787 patients treated for 16 weeks with salmeterol, the number of cases of death by asthma were 12 [91], among which only one boy 14 years of age $(0.006 \%)$ [197]. There were only two deaths in the group treated with albuterol, one of whom was 20 years old [197]. Therefore we do not agree with the concerns raised by the potential danger of salmeterol in young people, provided that - as with formoterol - it is not used to treat acute asthmatic attacks since it has no effect in a short time, a specification included in illustrated leaflets only in 1994. Therefore it should not be taken too often because of the risk of accumulation causing adverse effects. As a consequence, it is necessary to start preventive maintenance therapy [76] with cromolyn, nedocromil sodium, ICS or long-acting theophylline, resorting to inhaled $\beta_{2}$-adrenergics prn [467], while taking care to eliminate the environmental allergenic load [76]. Theophylline (RR, 1.0), cromolyn and steroids (RR, $<1.0)$ were associated with decreased mortality [331].

It has been highlighted that exposure to high titers of Alternaria alternata spores (Fig. 1.79) can trigger severe asthma exacerbations and is a risk factor for respiratory arrest in asthmatic children and young adults [457], with mortality cases significantly correlated with spore counts $>1,000 / \mathrm{m}^{3}$ [641]. In a total of 6,840 children with respiratory allergy, we have evaluated Alternaria prevalence. Only 89 of 6,840 children (1.3\%) had monosensitization to this allergen, and all were asthmatic. Of these, 29 had perennial manifestations, 18 seasonal prevalence, which was in autumn-winter in 13 of these 18, and in spring-summer in 5 . Thus, this allergy should not underevaluated, due to its protean mani- festations with possible life-threatening reactions [84]. It is therefore necessary for infants and children allergic to this aeroallergen to wear an identification bracelet so that they can be quickly identified in case of need, as well as asthmatic adolescents or children also suffering from $F A$. Of children who died or suffered from severe shock (see Chap. 20), 92.3\% were in fact asthmatic.

It is assumed that some fatal episodes have occurred in countries lacking a widely available National Health Service, or that such fatalities should be related to an absence of specialized medical assistance as well as to a lack of proper facilities to deal with such emergencies in schools [596].

\section{Prevention}

Prevention comprises a series of articulated measures aimed at reducing the occurrences or severity of reexacerbations, to possibly enable children to enjoy a normal life similar to that of their healthy peers. In 1881, it was clear that "to prevent a return of new attacks one must advise the patient to avoid all harmful influences... to all asthmatics one must recommend to live in pure, dry air, to avoid places exposed to dust, smoke and wind..." [82]. As will be seen in Chap. 24, numerous factors have contributed to the increased prevalence of allergic disease. Proof of the negative influences of these factors in children with $\mathrm{FH}+$ is provided by the double reactivity in $74 \%$ of children to Der $\mathrm{p} 1$ and to pets and in $84 \%$ to pollens and molds [483]. Given the severity of infantile asthma, it can be understood how important it is that interventions for allergen avoidance are scrupulously carried out, which, we note incidentally, are overlooked in several guidelines where emphasis is placed above all on drug therapy [272, 435, 504, 545, 698-700]. Some environmental measures are truly effective. It is known that asthmatics improve markedly in aseptic environments, residence in dust-free places can have positive effects on clinical features, children improve when transferring to houses so meticulously cleaned of allergenic factors as to be comparable to a hospital room, with 50-fold reduced Der p levels [624].

\section{Preventive Therapy}

Preventive therapy is required once allergic asthma has been recorded to adopt appropriate measures to prevent recurrences. It is carried out using cromolyn, nedocromil sodium (Figs. 11.64, 11.65), ketotifen and other preventive medications [105]. The indications for prophylactic therapy, especially for a long-term therapy, are summarized in Table 11.58 [476]. These medications have no particular role in international [272] and US guidelines [434-437]. It was proposed to remove cromolyn from international guidelines recommending it as a first choice in prophylactic asthma treatment [642]; however, 

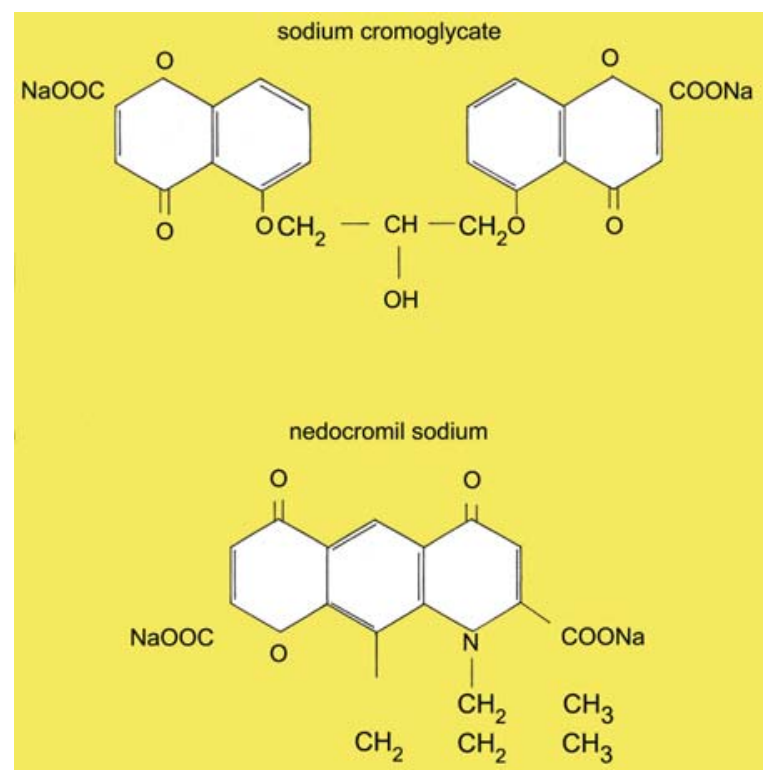

Fig. 11.64. Structural formula of sodium cromoglycate and nedocromil sodium

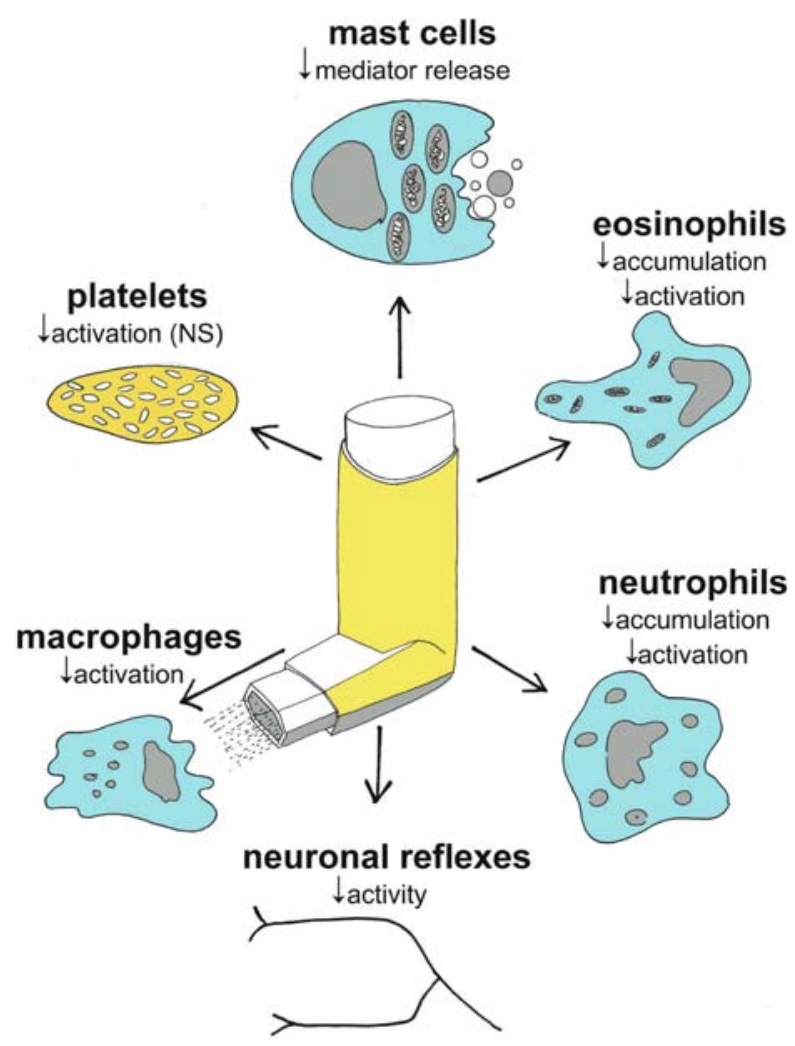

Fig. 11.65. Target cells and tissues for sodium cromoglycate and nedocromil sodium. Cromolyn sodium cromoglycate, NS nedocromil sodium
Table 11.58. Targets for prophylaxis in childhood asthma

Cough and wheezing more than 1-2 times a week or more than 1-2 nights a week

Necessity of bronchodilator use more than 1-2 times a day

Persistent PEFR alterations, an index of airway obstruction

Frequent exacerbations ( $>1$ every 4- 6 weeks) and of $>24$ h duration

Frequent hospital admissions

Modified from [476].

the Expert Panel's opinion is that cromolyn for children of all ages and nedocromil for children $>5$ years of age could be considered in the treatment of persistent asthma, but they are not preferred therapies [434].

Cromolyn is a widely used preventive drug that, by blocking mast cells mediator release, inhibits both early and late bronchospastic reactions induced by allergen inhalation, cold or nebulized air, fog and various irritating chemical substances. Thanks to these characteristics, it is currently used in the prevention of asthma and EIA symptoms in childhood [134,419,458,476], starting from 13 months of age [107], having the advantage of inhibiting the response to BPT with allergens both in the early and late phases, and that of reducing aspecific BHR [251]. The numerous and extensive long-term studies that have been referred to so far in this book have confirmed both its clinical safety and its therapeutic efficacy. A recent meta-analysis on 24 trials out of 251 selected and mostly on hospital-based populations of children [642] demonstrates no improvement in the outcome of 1,000 children aged 1-4 years with mild asthma given inhaled cromolyn at a dose of $10 \mathrm{mg}$ tid. However, cromolyn had a statistically significant effect on cough and wheeze compared to placebo $(95 \% \mathrm{CI}$ ) and an additional decrease in cough score compared to placebo (0.13 to 0.27$)$ and in wheeze score $(0.11$ to 0.26$)$ [ 166 , $642]$. Usually, the relatively low dose of cromolyn used and the inclusion of only children with milder symptoms makes it difficult to draw conclusions about the efficacy of cromolyn in children who are given higher doses for moderate asthma [282]. Several letters commented negatively on the paper, one very well documented [166]. A meta-analysis followed reaching the same conclusions [643]. In our clinic and personally, it has always been prescribed because of its preventive capacity, which has been recognized by two International Consensus papers on the treatment of pediatric asthma $[698,700]$, which places it in the pole position in the prophylaxis of asthma.

Its effects can be summarized as follows $[86,105,251$, 258, 545]:

- Stabilization of mast cell membrane, which can be explained by: 
- Closure of Ca channels, with a subsequent inhibition of mediator release

- Inhibition of phosphodiesterase, with an increase of cAMP intra-mast cell levels

- Blockage of oxidative phosphorylation that prevents mediator release

- Inhibition of the activation of leukocytes, neutrophils, eosinophils and blood platelets

- Inhibition of PAF action

- Attenuation of contractile response to acetylcholine, histamine, serotonin, bradykinin and $\mathrm{PGF}_{2 \alpha}$

- Up-regulation of $\mathrm{PGE}_{2}$ and $\beta_{2}$-adrenergic-mediated bronchodilation

- Prevention of mucociliary clearance alterations

- Inhibition of macrophage degranulation via the following mechanisms of action:

- Inhibition of macrophage lysosomal enzymes (for example $\beta$-glycuronidase)

- Inhibition of chemotactic factor release (for instance $\mathrm{LTC}_{4}$ )

- Inhibition of $\mathrm{O}_{2}$ metabolite production

- Additional properties inhibiting CD4, CD8, CD19 (B cells) and PBMCs expressing sCD23, demonstrated at the skin level (Chap. 7), making cromolyn potentially useful for treating asthma

- In vitro inhibition of IgE synthesis by B lymphocytes [297], probably by blocking their $\mathrm{IL}_{4}$-primed isotypic switching [358], suggesting an early prophylactic effect - Enhancement of IgG production by B cells [297], also with tachykinin antagonizing properties (Chap. 7).

Based on what has been stated, cromolyn's use in acute forms is not advisable.

Cromolyn is administered:

- By DPI, in capsules each containing $20 \mathrm{mg}$, with a predosed dispenser (Spinhaler, see "Predosed Pressurized Sprays"), with a dose of 60-80 mg/day, in 3 fractionated doses

- By MDI (20 mg/2 ml of solution),

- By pressurized MDI, with $5 \mathrm{mg}$ release (average dose of 2 puffs qid).

In the US an MDI formulation of $1 \mathrm{mg} /$ puff is available, which is less able to decrease BHR compared to the MDI formulation of $5 \mathrm{mg} /$ puff which is available elsewhere, thus possibly delivering less medication to the lower airways [252]. Compared with the convenience of 1-2 daily doses of BUD inhalation, the dosing of cromolyn bid-qid likely increased caregiver burden [422].

Several weeks of regular use are required before it is able to carry out its effect on LAR. However, the delivery system may by a limiting factor in the efficient cromolyn delivery to young children [549].

Cromolyn is indicated:

- In short-term prophylaxis: 15-30 min preceding the allergen encounter, or before intense physical exercise

- In long-term prophylaxis: from 4-6 weeks preceding the critical period until the end of exposure

- If a small airway obstruction remains after bronchodilator administration
Cromolyn associated with CSs confers a significant protection against asthma exacerbations, asthma drug therapy, inhaled anti-inflammatory agents, hospitalization, and ED visits [5].

All asthmatic children can derive benefit from cromolyn therapy [436], which is virtually devoid of any side effects [86].

\section{Nedocromil Sodium}

Nedocromil, disodium salt of pyrano-quinoline-dycarboxilic acid, is an antiallergic and anti-inflammatory drug endowed with the following properties [397, 407, 424, 507, 578, 659, 704]:

- In vivo:

- It inhibits mediator release by sensitized mast cells, induced by the specific antigen and anti-IgE antibodies. - It reduces the amount of histamine and $P G D_{2}$ released by sensitized mast cells, both spontaneously and following aspecific stimuli.

- It suppresses IL-dependent IgE production.

- It inhibits chemotactic responses by eosinophils (to FMLP and NAP-1/IL $\mathrm{L}_{8}$ ) that are stimulated by cytokines (GM-CSF and $\mathrm{IL}_{3}$ ).

- It inhibits neutrophil chemotactic action.

- It inhibits BHR induced by $I L_{3} R$-stimulated PAF.

- It may inhibit the activity and functionality of T cells.

- In vitro:

- Even in very low doses, it inhibits chemotactic factorinduced activation of neutrophils and eosinophils, probably acting on proteinkinase $\mathrm{C}$.

- It inhibits the PMN-mediated mechanisms that lead to histamine release.

- It inhibits the release of mediators by neutrophils and eosinophils and the release of $\mathrm{LCB}_{4}$ and 5-HETE by alveolar macrophages.

- It prevents the IgE-mediated monocyte and blood platelet activation and, in high doses, activation of complement-induced proteins associated with eosinophil granules.

- It reduces the release by the bronchial epithelial cells exposed to asthmogenic stimuli of arachidonic acid metabolite able to induce mast cell degranulation and mucus hypersecretion.

- It inhibits the variation in density of eosinophils and $\mathrm{LTC}_{4}$ production.

- As opposed to cromolyn, it also acts on MT mast cells and on basophils.

Moreover, nedocromil mitigates or halts MBP-induced harmful mucociliary dysfunction [648].

Several clinical studies have documented its efficacy in adults, also noting that it is less so in children [71, 113, 134]. In children, it significantly reduced urgent case visits and courses of oral prednisone as compared with placebo [645]. In our experimental double-blind placebo-controlled (DBPC) study [71] in children affected by grass-induced bronchial asthma, the results showed an 
overall improvement both in asthmatic symptoms and in PFT compared to controls. The different opinions expressed by both physicians and parents were statistically highly significant.

The dosage is two puffs $(2 \mathrm{mg})$ qid.

Table 11.14 details the cromone's action mechanism.

Ketotifen has a mechanism partially analogous to that of cromolyn and is capable of the following: $[105,462$, 528, 545]:

- It inhibits mast cell mediator release, in particular of $\mathrm{PGD}_{2}[462]$.

- It interferes with the action carried out by some of these mediators.

- It carries out antihistamine activity at the level of $\mathrm{H}_{1}$ receptors.

- It is active on the two phases of the asthmatic response.

- It inhibits blood platelet migration and PAF release, so that anti-PAF activity can explain many positive effects [528].

Additionally, it prevents $\beta_{2}$-adrenergic hyporesponsiveness and restores their responsiveness, thus improving in asthmatics $\beta_{2}$-adrenoceptor function and $\beta_{2^{-}}$ adrenergic bronchodilation [528].

In five DBPC pediatric studies [74, 511, 545, 589, 667], the following effects were documented:

- Reduction of asthmatic attacks and auscultatory findings of long-term wheezing

- Reduction in the number of days of disease

- Statistical reduction in the use of other drugs [511, 545]

- Anti-asthma prevention in $91 \%$ of cases after 3 years of therapy [74]

The effects in other pediatric studies were controversial $[589,667]$. In one DBPC study, ketotifen proved less effective than cromolyn, possibly because one dose of syrup $4 \mathrm{ml} /$ day was used on subjects weighing $14-18 \mathrm{~kg}$; no data regarding compliance was noted [127].

Dosages. For dosages, see Table 7.19. Younger children find ketotifen particularly pleasant since it is also available in syrup form, has a pleasant taste and is easily administered. Drowsiness is its only side effect [406], which, however, we noted disappears after a few days of use. The prescribing physician should inform parents of this effect. In older children, the capsule form can be taken, preferably at night.

\section{Antihistamines}

For some years second-generation antihistamine drugs have been utilized and experimented in pediatric treatment of asthma [259] (Fig. 11.66). It should be noted, however, that experimental studies have yielded no con-

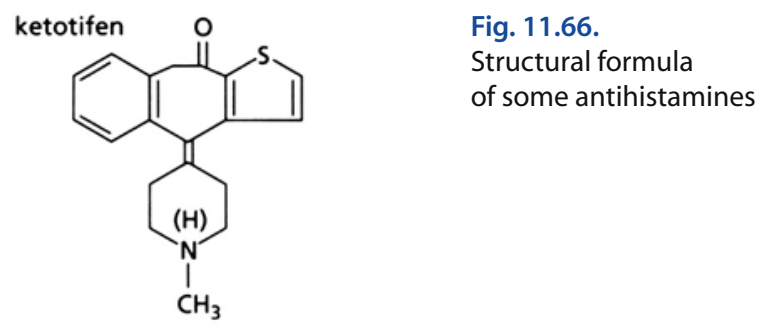<smiles>CN1CCCC(n2nc(Cc3ccc(Cl)cc3)c3ccccc3c2=O)C1</smiles>

loratadine<smiles>CCOC(=O)N1CCC(=C2c3ccc(Cl)cc3Cc3cccnc32)CC1</smiles>

cetirizine

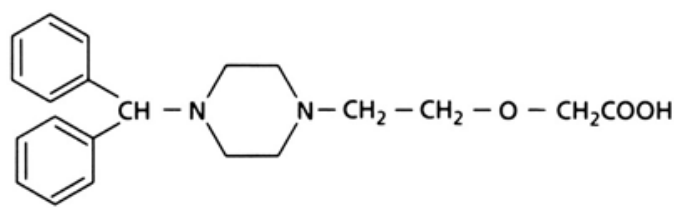

Table 11.59. Possible antiasthmatic effects of second-generation antihistamines

\begin{tabular}{|c|c|c|c|}
\hline \multirow{2}{*}{$\begin{array}{l}\text { Anti- } \\
\text { histamine }\end{array}$} & \multicolumn{3}{|l|}{ Inhibition } \\
\hline & $\begin{array}{l}\text { Histamine } \\
\text { release }\end{array}$ & $\begin{array}{l}\text { Eosinophil } \\
\text { chemotaxis }\end{array}$ & $\begin{array}{l}\text { PGD and LTC } \\
\text { release }\end{array}$ \\
\hline Azelastine & + & + & + \\
\hline Cetirizine & + & + & + \\
\hline Ketotifen & + & & \\
\hline Loratadine & + & + & + \\
\hline Oxatomide & + & & + \\
\hline
\end{tabular}

Data from $[105,139,303,528]$.

sensus since there are noticeable variations regarding the type of molecule or the dosages [105]. While azelastine can reduce IAR, several other drugs seem to express their effect on LAR, an important result for the known correlation between these reactions and symptom severity $[139,303]$. Table $11.59[105,139,303,528]$ summarizes the principal effects that are attributed to these 
drugs; therefore we will simply clarify a few points (see Chap. 12). These drugs fulfill a protective activity related to their half-life with a variable interval of time, ranging from 4 to $12 \mathrm{~h}[105,139]$.

\section{Azelastine}

Azelastine has proved to be a powerful inhibitor of histamine-induced bronchoconstriction. Once optimal serum levels have been reached, however, it is not also able to antagonize $\mathrm{LTC}_{4}$-induced bronchoconstriction, though it is able to significantly reduce SP levels in BALF [439]. A randomized DBPC study has concluded that azelastine is capable of significantly inhibiting IAR, without, however, having any noticeable impact on LAR [662].

\section{Cetirizine}

Various studies on humans have shown that cetirizine can reduce eosinophil, neutrophil and basophil recruitment by $75 \%$ [303], as well as PAF-induced bronchoconstriction, without leading to a similar LAR reduction in the airways, as demonstrated by PFTs, [636]. An antiallergic effect is produced on epithelial cell CD54 expression [81]. Recent evidence suggests that it inhibits eosinophil adhesion to endothelial cells [322]. A randomized DBPC study among adults has shown that cetirizine is able to reduce the number of eosinophils in BALF and inhibit their enrollment [519]; cetirizine's ability to induce consistent reductions of $\mathrm{FEV}_{1}$ and of histamine-induced bronchoconstriction is also known. In 348 children and adolescents with grade II and III asthma, cetirizine reduced the number of days with symptoms as well as the need for other drugs, on a par with cromolyn [303]. The formulation in drops has allowed us to treat very young children (aged 6 months and older), always with very good results.

Long-term Levocetirizine has proved to have a firstrate preventative activity in $>200$ asthmatic children aged 5-6 years and older treated by us (one tablet = $5 \mathrm{mg} /$ day).

Fexofenadine is a new drug that can be given to children. It is more useful in the treatment of AR and has no effects either on the cardiovascular system or the CNS.

\section{Loratadine}

In vitro, loratadine significantly inhibits PAF-induced eosinophil activation, superoxide generation, and nasal secretion of histamine and $\mathrm{PGD}_{2}$ after the challenge [165]. Another long-term antihistamine is desloratadine (a loretadine metabolite), with anti-inflammatory and anti-allergic activities in addition to its antihistaminic activity, it has a 27 -h elimination half-life, which facilitates once-daily dosing. Desloratadine does not cause sedation or prolong the corrected QT interval, can be administered without regard to concurrent intake of food and grapefruit juice (Chap. 12), like the other antihistamines. We have prescribed doses of $2.5 \mathrm{mg}(1.15 \mathrm{ml}$ of syrup)/day to children aged 2-5 years and of $5 \mathrm{ml}$ (2.5 ml of syrup)/day to children aged 6-11.

\section{Oxatomide}

In addition to having an antidegranulation effect on mast cells, inhibiting even the activity of serotonin at the receptor level and, partially, of LTs with spasmogenic activity, oxatomide reduces the bronchoconstriction induced by methacholine and by physical exercise, as has been demonstrated by a DBPC study in asthmatic children [301].

The formulation in drops has allowed us to treat young children, always with effective results.

\section{Nonpharmacological Therapy}

Asthmatic children are strongly encouraged to participate in a sport. As a preventive measure, the family doctor should be consulted on the choice of the most appropriate activity. Though not everyone agrees [359], swimming in a swimming pool is most certainly advisable because the effort is well balanced, warm and humid air is inhaled, it helps to develop respiratory functions and it tones up the body. It is useful to know in advance the percentage of $\mathrm{Cl}$ in the water, because high levels could be an irritant. We have seen how an airway cooling down linked to hyperpnea can provoke EIA. As previously suggested, a preventative measure for this form of asthma - a filtering mask - could protect bicycling or motorbiking asthmatics or those engaged in long-distance skiing in the winter, by covering the nose and mouth and thus avoiding the continued inhalation of asthmogenic cold and dry air [618]. It is possible that the increased prevalence of asthma in long-distance skiing [335] is dependent on the lack of attention given to preventive measures.

\section{Other Interventional Measures}

The disease impact on the asthmatic's everyday life: information on asthma and on its treatment provided to the family (in the manner deemed most appropriate) enables children to better face their disease and follow their medical therapies with greater diligence; moreover, performing their breathing exercises more frequently to help them relax, leads to a possible reduction in the sometimes excessive use of their medications [272].

Greater education also improves the quality of life: by reducing problems such as cough, wheezing, trouble 
Table 11.60. Limitations on the quality of life in children and adolescents

\begin{tabular}{|c|c|c|}
\hline Limitations (\%) & [657] & [213] \\
\hline \multicolumn{3}{|l|}{$\begin{array}{l}\text { Limitations in physical activity, } \\
\text { playing and sports }\end{array}$} \\
\hline Running & 85 & \\
\hline Running up hills & & 78 \\
\hline Playing with pets & 36 & \\
\hline Swimming & 33 & 45 \\
\hline Sleeping & 30 & \\
\hline Playing with friends & 30 & \\
\hline Basketball & 27 & \\
\hline Bicycling & 26 & 55 \\
\hline Playing sport & 20 & 63 \\
\hline Soccer & 29 & \\
\hline Surfing & & 33 \\
\hline \multicolumn{3}{|l|}{ Emotional function } \\
\hline Feeling frustrated & 62 & \\
\hline Feeling uncomfortable & 58 & \\
\hline Feeling different from friends & 54 & \\
\hline Frustrated not having a normal life & 53 & \\
\hline $\begin{array}{l}\text { Feeling concerned/troubled } \\
\text { about asthma }\end{array}$ & 52 & \\
\hline $\begin{array}{l}\text { Feeling frightened by an } \\
\text { asthma attack }\end{array}$ & 49 & \\
\hline Mad or angry because of asthma & 45 & \\
\hline \multicolumn{3}{|l|}{ Problems related to school } \\
\hline $\begin{array}{l}\text { Days missed at school due } \\
\text { to asthma (1-99) }\end{array}$ & & 78 \\
\hline Asthma attacks at school & & 43 \\
\hline
\end{tabular}

breathing, activity limitation, anxiety, loss of sleep or frequently interrupted sleep and worry over the physical symptoms of their disease and the possibility of future attacks, the array of stressors that a child has to face as a consequence of suffering chronic asthma [448], the patient can be ensured a life that is as normal as possible, even regarding school, sports and above all social activities, etc.

The overall situation can be made easier and improved if pediatricians act promptly and effectively not only during the critical attacks, but also in the intercritical times, via focused treatments aimed at preventing future attacks. We are referring to the serious problems faced by a child prone to EIA who will return to play and take part in sporting activities as a direct result of an adequate preventive therapy. A good pediatrician-patient relationship will undoubtedly be beneficial to the psy- chology of asthmatic children and adolescents and will assist them in facing the disease and daily activities.

In adolescents, psychological problems occur more frequently: the doctor should treat these special aspects of the disease, including the refusal to acknowledge symptom presence, widespread emotional problems including lack of cooperation, social aspects, excessive recourse to inhalers, smoking, etc.

Even some aspects related to school can require the doctor's assistance. In schoolboys, schoolgirls, young adolescents, problems related to modifications of behavioral attitudes or learning disabilities caused by medication are often present. It is for this reason that doctors must educate both children and parents about exceptional cases of disturbances possibly occurring, and that such problems often last not less than 2 weeks [711].

To better illustrate the seriousness of the problems relating to pediatric asthma, the results of two studies done on 100 asthmatic children aged 9-13 years [657], and in 4,161 adolescents (958 asthmatics and 3,203 nonasthmatics) and 1,104 of their teachers [213] (Table $11.60[213,657])$ are reported. The trial showed that $42 \%-59 \%$ of the subjects believed that asthmatics can become addicted to their drugs, $70 \%-82 \%$ that there could be fewer problems if the drugs could be taken in class, $36 \%-45 \%$ that asthmatics are embarrassed about using their inhalers and $47 \%-56 \%$ that teachers are worried about taking asthmatics on school outings or to summer camps. Even if comprehension toward asthmatics was greater among students than among school teachers $(p<0.0001)$, the teacher's positive participation in asthmatics' problems is significant [213]. The results of a subsequent trial on 381 students aged 8-18 years are more optimistic: the findings showed that there is only a $30 \%$ restriction on their participation in youth activities [41]. Nevertheless, it is difficult to quantify that many young children will not be able to fulfill their dreams, for example that of being able to run and play football like so many other children with no restrictions whatsoever.

Table 11.61 [284, 435] summarizes the advantages and disadvantages of the main therapeutic methods outlined so far.

\section{Outcome}

We have gathered the relevant data in Table 5.15, defining positive outcome in $41 \%-47 \%$ of cases. In the last few years, a high percentage of children (43\%-76\%) whose asthma persisted as they grew into adulthood has been noted. A large number of children or adolescents who remain asymptomatic can have relapses and/or anomalies in their PFT that do not return to normal, even after 3 years $[219,575]$. It is important that these children are regularly examined by their pediatrician, enabling a timely identification of those subjects who are at risk of suffering from relapses on reaching adult- 
Table 11.61. Advantages and disadvantages of medications for treating pediatric asthma

\begin{tabular}{|c|c|c|}
\hline Medications & Advantages & Disadvantages \\
\hline$\beta$-Agonists & $\begin{array}{l}\text { The most potent and rapid bronchodilator } \\
\text { drugs available at the moment }\end{array}$ & $\begin{array}{l}\text { The regular use may mask the airway } \\
\text { inflammation and disease progression }\end{array}$ \\
\hline Corticosteroids & $\begin{array}{l}\text { The most potent anti-inflammatory drugs } \\
\text { for treating both asthma and airway } \\
\text { hyperreactivity currently available, } \\
\text { topical steroids offer the most secure } \\
\text { therapeutic range }\end{array}$ & $\begin{array}{l}\text { Oral: the side effects suggest their use as } \\
\text { a maintenance therapy in the most severe } \\
\text { forms } \\
\text { Inhaled: unconfirmed growth hypothalamic- } \\
\text { pituitary-adrenal axis suppression. }\end{array}$ \\
\hline Theophylline & $\begin{array}{l}\text { The best available drug to add an } \\
\text { extended bronchodilation and anti- } \\
\text { inflammatory effects }\end{array}$ & $\begin{array}{l}\text { Narrow therapeutic range, variable clearance, } \\
\text { requiring careful monitoring of theophylline } \\
\text { levels, unconfirmed negative effects } \\
\text { on temper, learning, etc. }\end{array}$ \\
\hline Antihistamines & See Table 11.59 & Continual anti-inflammatory therapy \\
\hline Anticholinergic & $\begin{array}{l}\text { Delivered by nebulizers may be added } \\
\text { to } \beta \text {-agonist therapy in acute conditions }\end{array}$ & $\begin{array}{l}\text { Modest bronchodilators less potent than } \\
\beta \text {-agonists }\end{array}$ \\
\hline Cromolyn & $\begin{array}{l}\text { Reduces symptom scores, airway hyper- } \\
\text { reactivity and necessity of other medications }\end{array}$ & $\begin{array}{l}\text { Prophylactic, is less potent than cortico- } \\
\text { steroids, not effective in all children }\end{array}$ \\
\hline Nedocromil sodium & $\begin{array}{l}\text { Reduces symptom scores, airway hyper- } \\
\text { reactivity and necessity of other medications }\end{array}$ & $\begin{array}{l}\text { Prophylactic, is less potent than cortico- } \\
\text { steroids, not effective in all children }\end{array}$ \\
\hline Allergen avoidance & $\begin{array}{l}\text { Allergen-specific, may reduce } \\
\text { and eliminate symptoms }\end{array}$ & $\begin{array}{l}\text { None, major discomfort for children } \\
\text { and families }\end{array}$ \\
\hline $\begin{array}{l}\text { Immunotherapy } \\
\text { (Chap. 13) }\end{array}$ & $\begin{array}{l}\text { Definitive cure of asthma, abatement } \\
\text { of symptoms, airway hyperreactivity } \\
\text { and need of medications }\end{array}$ & $\begin{array}{l}\text { Poor compliance in small children, high cost, } \\
\text { children should be observed } 30 \text { min after } \\
\text { injection should be started early, well before } \\
\text { that asthma agqravates }\end{array}$ \\
\hline
\end{tabular}

Data from $[269,419]$.

hood. On this matter, in the studies cited in Chap. 5, various parameters were predictive of asthma at an adult age. Up to the present time, no study has demonstrated that pharmacotherapy is able to modify the natural history of asthma, whereas SIT is able to do so. On the contrary, as has been noted, the discontinuation of a therapeutic cycle is followed by a reappearance of symptoms [671], which also confirms our experience. The inflammatory process at the basis of asthma persists even after years of CS treatment, even if it can be reduced [652]. Nonetheless, it can resurface displaying all its functional characteristics [671]. As we have seen, there are various antiasthmatic treatments available for use at an infant age and we could additionally recommend ensuring a timely diagnosis and early adoption of preventive measures.

\section{Present and Future Prospects}

In the long term, anti-inflammatory strategies could include nonactivation of $\mathrm{T}$ lymphocytes or of $\mathrm{IL}_{4}$, inhibition of the isotypic conversion after the second signal emitted by the cytokines, and direct elimination of $\mathrm{B}_{\mathrm{IgE}}$ cells through anti-IgE monoclonal antibodies [24,301]. There are two treatments that cure asthma, in addition to SIT.

\section{Anti-lgE}

In a randomized DBPC study, a cohort of 334 entrants aged 6-12 years with moderate or severe allergic asthma received a recombinant humanized monoclonal antibody that binds to free $\operatorname{IgE}$ at the same site as the high-affinity (FcERI) receptor (omalizumab). A 28-week treatment reduced the requirement for ICS while protecting against disease exacerbation, while serum free IgE was reduced in $95 \%-99 \%$ of cases [405]. Children in the omalizumab-treated group reported significant improvements in the activities and symptoms domain scores as well as in the overall asthma-related quality of life compared with placebo [342], but also formoterol [183]. In a pooled analysis of three multicenter, randomized DBPC studies, omalizumab reduced the rate of serious asthma exacerbations and the need for unscheduled outpatient visits, ED treatment, and hospitalization in children with moderate-to-severe allergic asthma [118].

\section{Leukotriene Modifiers}

Anti-LT could have a positive influence on ASA and/or block bronchoconstriction responses in BPT, leading to a decrease in CS use [329]. Figures 11.60 and 11.61 sug- 


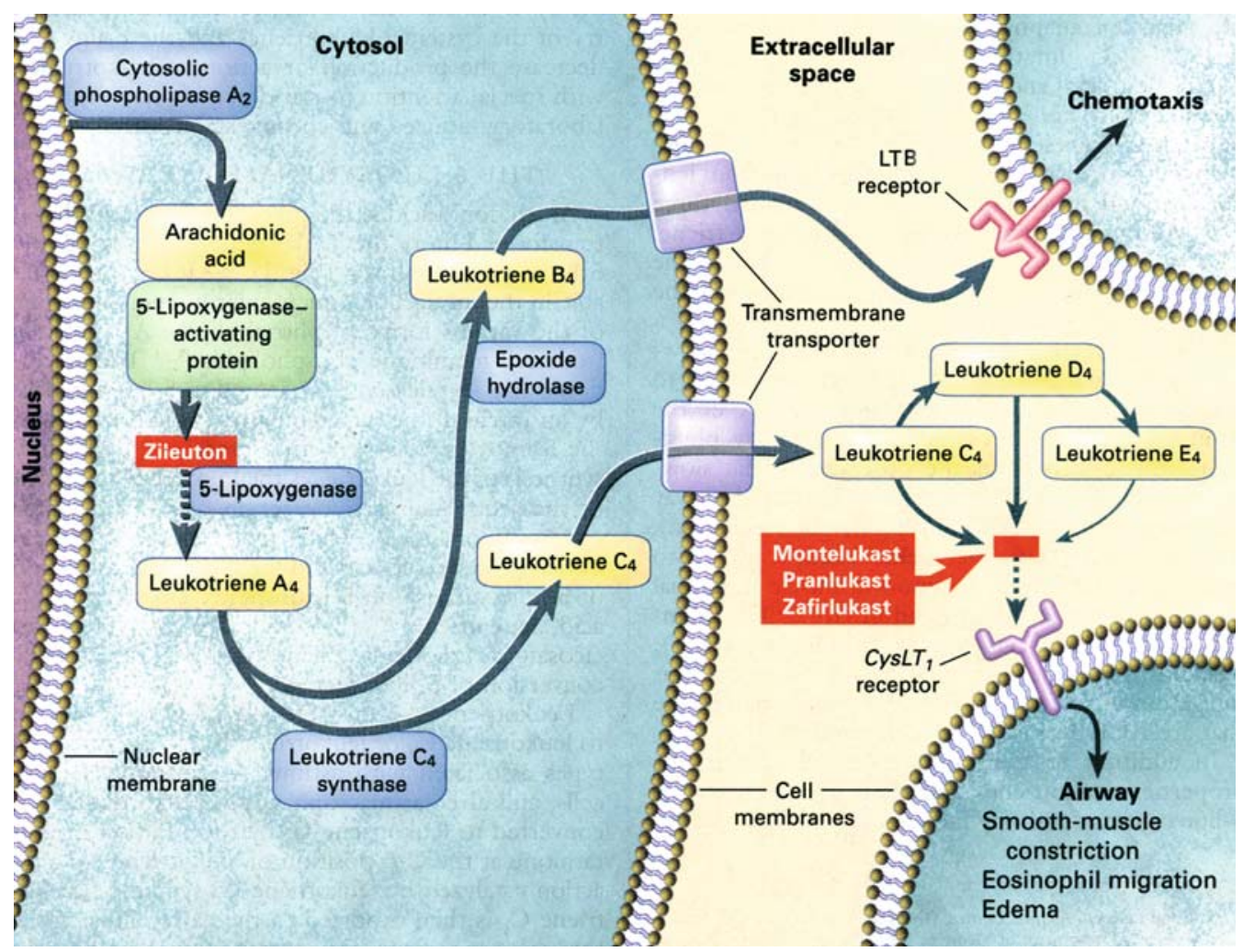

Fig. 11.67. Biochemical pathways of LT formation and action and sites of action of LT-modifying drugs. Enzymes are shown in blue, products in yellow, essential cofactor in green, and drugs in red. Although the synthesis of LTB4 and LTC4 proba- bly takes place in close proximity to the nuclear membrane, for clarity they are shown throughout the cytosol. LTB denotes the LTB receptor. An individual cell may produce the cysteinyl leukotrienes, LTB4, or in rare cases both gest the use of anti-LT drugs, though they have yet to be thoroughly tested: zileuton, an inhibitor of LT synthesis and montelukast, pranlukast and zafirlukast, competitive LT receptor antagonists, as well as pobilukast, tomelukast and verlukast, though the last three are more properly inhibitors of the activation of cysteine-derivatives receptor $\left(\mathrm{LTC}_{4}, \mathrm{LTD}_{4}\right.$ and $\left.\mathrm{LTE}_{4}\right)$. The spirometric data of children of 6-14 years with chronic asthma, who were treated with montelukast (oral monodose of $5 \mathrm{mg}$ ), demonstrated a net improvement [308], added to BUD in 279 children aged $10.4 \pm 2.2$ years, it induced a clinically relevant decrease in the number of asthma exacerbation days [591]. A DB, multicenter, multinational study at 93 centers on 689 children (aged 2-5 years) with persistent asthma given 12 weeks of treatment with $4 \mathrm{mg}$ of oral montelukast has shown significant improvements compared with placebo in multiple parameters of asthma control, including daytime and overnight asthma symptoms, percentage of days with and without asthma symptoms, need for $\beta$-agonists or oral CSs, physicians' global evaluations, and peripheral blood eosinophils [309]. We have treated 40 children aged 3.1-5.1 years (mean, 3.7 years) with montelukast. Compared to 41 controls, there was a significant reduction in the mean incidence of day (74\%) and night (69\%) wheezing in addition to a significant amelioration in EIA compared
Table 11.62. Antileukotriene drugs: route and doses of administration in children

\begin{tabular}{lll} 
Anti-LT & Route & Doses/Ages \\
$\begin{array}{l}\text { Montelukast } \\
\text { (at bedtime) }\end{array}$ & Oral & $\begin{array}{l}4 \mathrm{mg} \text { once } \\
\text { daily/2-6 years }\end{array}$ \\
\hline & & $\begin{array}{l}5 \mathrm{mg} \text { once } \\
\text { daily/6-14 years }\end{array}$ \\
\hline & & $\begin{array}{l}5 \mathrm{mg} \text { once } \\
\text { daily/6-14 years }\end{array}$ \\
\hline $\begin{array}{l}\text { Zafirlukast } \\
\text { or } \text { before } \mathrm{h} \text { after a meal }\end{array}$ & Oral & $\begin{array}{l}10 \mathrm{mg} \text { bid/ } \\
5-12 \text { years }\end{array}$ \\
\hline Zileuton & Oral & $\begin{array}{l}\text { Children aged } \\
12 \text { years and older }\end{array}$ \\
\end{tabular}

Data from [159].

with controls, and in younger children a significant increase in weight. A mean difference between study children and controls was evidenced by spirometric data. We conclude that in pediatric asthma management, chewable tablets of LT modifiers can be used as substitutes of long-acting $\beta$-agonists and ICSs, especially in young children who may have difficulty in using in- 
halers. Montelukast was significantly effective when catsensitive children aged 6-14 years with mild persistent asthma were exposed to high levels of cat allergen [492]. Table 11.62 reports the related doses, and Fig. 11.67 shows the biochemical pathways of LT formation and action. It should be noted that in 32 children 6 to $<24$ years old the $4 \mathrm{mg}$ dose was equally effective and free of untoward adverse effects [402].

Montelukast administration also reduced the increased Th2-like T cell IL mRNA expression in lung tissue and protein in BALF found in OVA-sensitized or challenged mice, and markedly reduced the increased lung mRNA expression of Th2-like T cell ILs [246]. In other animal studies, CD54 modulation during treatment with CSs could represent a new and more selective treatment for the control of the chronic airway inflammation and BHR that characterize asthma. The effect of CD54 could be associated with significant reductions of eosinophilia and consequently, of BHR [230]. Apart from other immune effects, CSs (Figs. 11.43, 11.44) inhibit IL action on eosinophils, and cetirizine and picumast inhibit their activation in vitro, even though not originally introduced for this purpose [528]. The initiative to positively alter the levels of PAF by means of SIT [265] or ketotifen [528] and, finally, that of blocking BHR by inhibiting iNOS by means of CSs [443] is a stimulating prospective. Of special interest would be a method that could monitor NO production in infants and young children, aiming at focusing on the role of the inflammation in early asthma and fostering a strategy for timely intervention [759].

The list of new compounds for the pharmacological control of asthma is long and, in addition to new applications of antihistamines, also include PDE IV inhibitors, anti-TXA $\mathrm{T}_{2}$ and anti-TXA ${ }_{2}$-synthetase, tachykinin antagonists, inhibitors of peptide releases from $\mathrm{C}$-fibers, $\mathrm{K}^{+}, \mathrm{Ca}$ and $\mathrm{Cl}$ channels deactivators, $\mathrm{M}_{3}$-selective antagonists, inhibitors of $\mathrm{IL}_{5}, \mathrm{CD}$ antagonists of adhesion molecules, and inhaled NSAIDs [418]. Also foreseeable are antagonists of PAF, tryptase, quinines and of some chemokines, inhibitors of FLAP and of $\mathrm{PLA}_{2}$, antagonists of $\alpha$-adrenergic receptors, modulators of transcription factors and new antimuscarinic drugs. Finally, the antagonists of tachykinin receptors could eliminate bronchial smooth muscle contractions [426] and cold-induced bronchoconstriction [744].

Most strikingly [243], an anti-IL $\mathrm{IL}_{17} \mathrm{mAb}$ (monoclonal antibodies) treatment regimen has been shown to abate bronchial neutrophilia in parallel with reduction of bone marrow and blood neutrophilia. This treatment also raised eosinophil counts in the bone marrow and bronchial $\mathrm{IL}_{5}$ production, without alteration of allergeninduced BHR.

A crucial role may be played by TARC (thymus and activation-regulated chemokine) CCR4 receptor expressed by Th2 cells in bronchial airway epithelium. Asthmatic patients exposed to a relevant allergen release large amounts of TARC in their BALF; costimula- tion with IFN- $\gamma$, but not with the duo $\mathrm{IL}_{4}-\mathrm{IL}_{13}$, stimulated human bronchial epithelial cells to further increase TARC mRNA and protein expression. TNF- $\alpha$ amplifies IFN- $\gamma$ ability to induce TARC 30 -fold [38]. TARC concentrations are elevated in childhood asthma [345], thus TARC up-regulated in bronchial epithelial cells may play a role in the pathogenesis of allergic asthma [38]. This marker is also linked to plasma total IgE levels and cat allergen sensitization [345]. As a consequence, CCR4 antagonists may have a substantial impact in treating allergic asthma.

In another area of increasing interest, administration of a stable analog of lipoxin A4 (LXA4) blocked both BHR and pulmonary inflammation, as shown by decreased leukocytes and mediators, including $\mathrm{IL}_{5}, \mathrm{IL}_{13}$, eotaxin, prostanoids and cysteinyl LTs [349]. Moreover, blocking $\mathrm{IL}_{13}$ [150], receptors, or the downstream signaling pathway activated by their ligation, could provide one strategy to improve the specificity of asthma treatment [321].

In the animal model, TrkAd5 is able to modify the airway late hyper-responsiveness to histamine, brg sequestering endogenous NGF. Notably, the TrkAd5 administration causes the contractile response to histamine to be lower than control after ovalbumin challenge, thus showing potent effects in allergic asthma [686a].

\section{Other Pediatric Allergic Lung Disease}

These are pulmonary interstitial disorders having an immunological pathogenesis, caused by the inhalation of various antigenical agents active in subjects who are particularly vulnerable; such disorders are rare at the pediatric age.

\section{Extrinsic Allergic Alveolitis}

Also known as hypersensitivity pneumonitis, farmer lung disease (described by Ramazzini in 1713, Chap. 4), and pigeon breeder's, EAA can occur in children aged $3-15$ years [111, 319, 395]. It is rare in infants [168] and $>80$ pediatric cases are known $[111,137,168,223,248$, $319,389,411,748]$. The pathogenesis is of sensitization to inhaled allergens, generally thermophilic actinomycetes or avian antigens $[111,319]$. In addition to the classic cases of actinomycetes that contaminate fodder [111] or of other mycetes that pollute houses [319], there is evidence of a growing number of cases related to contaminated heating and air-conditioning systems [132] and to birds in cages, pigeons, etc., in the barn, house, and the child's bedroom [248,319]. The latter form is the most widely studied in children, with cases caused by free-roaming city pigeons [137], a problem in most countries. There can be type III reactions with formation of antigen-antibody complexes at the alveolar level [411], but by studying BALF a significant lymphocytosis 


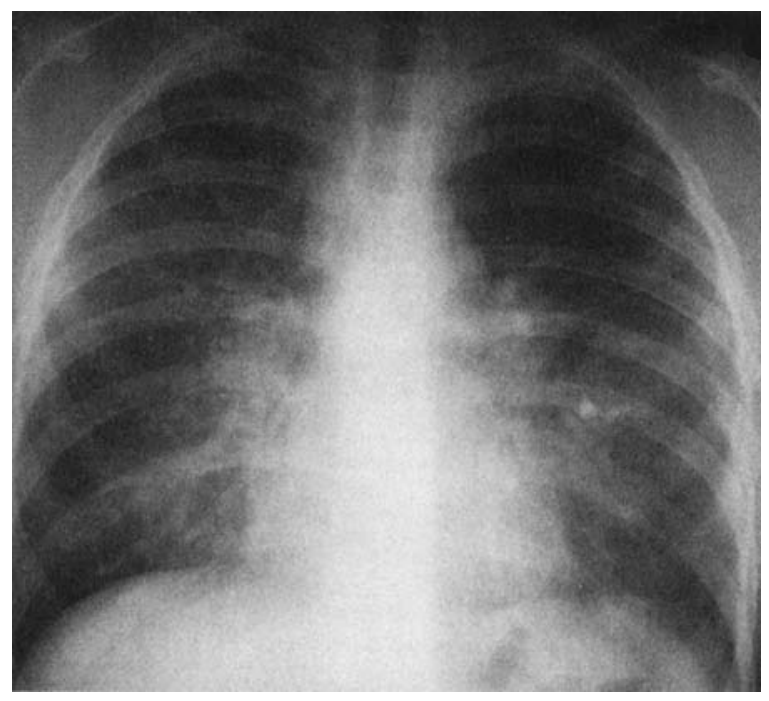

Fig. 11.68. Chest radiograph of an 11-year-old boy with EAA (for details see text). After a short course of oral corticosteroids a complete resolution is seen with fading pulmonary parenchymal shadows

during the acute period, with CD8 predominant intervention, has been demonstrated. These reactions could therefore assume a more significant pathogenetic role [319]. Pulmonary damage is the result of the immune response characterized both by antigen-specific antibodies and cellular hypersensitivity with IL releasing PBMCs. Complement activation in the airways and the irritating effect of thermophilic actinomycetes [95] should also not be overlooked.

The insidious onset of acute forms, secondary to exposure to high concentrations of avian antigens, includes increasing lethargy, weight loss, a febrile bronchopneumopathy with a prevalence of respiratory symptoms such as breathlessness and cough, which can also be spasmodic, and effort-induced dyspnea appearing 4-8 $\mathrm{h}$ after exposure, with auscultatory findings of rales at pulmonary bases $[111,319]$. Complications may occur in the form of sudden respiratory failure [111]. If exposure is prolonged or repeated, the process becomes chronic with aspecific insidious signs such as worsening of the overall general condition, effort-induced dyspnea and even at rest [411], and marked weight loss [319]. More frequently, the picture is subacute with coexisting respiratory and systemic symptoms; among these a general feeling of ill health, anorexia, asthenia, etc. [111].

Diagnosis depends on history of exposure to birds, clinical findings, positive avian precipitins, restrictive defects on PFTs, and a suggestive chest X-ray appearance [223], and in children exposed to birds or playing in barns with a history of breathlessness, or strolling amidst city pigeons [137]. X-ray results reveal a fine miliary pattern, diffused to both lungs with interstitial infiltration in the lower fields and hilar enlargement in acute forms, and diffused interstitial fibrosis with accentuation of the network in chronic forms (Fig. 11.68), but
Table 11.63. Differential diagnosis of EAA

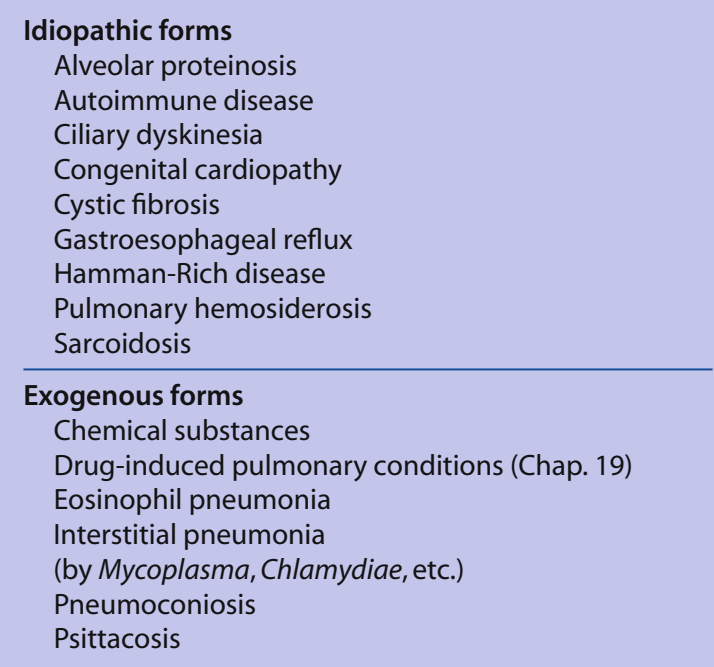

Modified from [319].

may be unremarkable [248]. PFTs show a restrictive ventilatory defect with reduced lung volumes and compliance $[111,319]$. SPTs positive to pet danders provide both immediate and delayed results [411]. Lymphocytosis is found in BALF $(>50 \%)$, a net reduction of both CD4 and CD8 rates and of NK cells, with $87 \%$ sensitivity and $72 \%$ specificity [319], or $85 \%$ of CD8 expressing the activation marker HLA-DR and $32 \%$ of $\mathrm{CD}^{+}$and $16 \%$ of $\mathrm{CD}^{+} 6^{+}$[389]. Follicle-like aggregates of $\mathrm{B}$ cells in the lung interstice may indicate that local antibody synthesis may be involved in an antibody-dependent cellular cytotoxic mechanism [389]. Differential diagnosis is illustrated in Table 11.63 [319] and should be extended to children presenting with unusual respiratory symptoms and signs early [223].

Avoidance of triggering agents entails that clinical findings subside within a few days [425]. CSs are effective and resolutive, especially if accompanied by environmental clearance [75], unless insufficient child compliance or a relapse due to a new exposure to specific allergens occurs [319].

\section{Allergic Bronchopulmonary Aspergillosis}

Though rare in children, ABA is often found in adult studies reporting the onset of symptoms in childhood [75]. Often it is caused by airway colonization by the mold Aspergillus fumigatus (AF) (with 18 different allergens, Table 1.74), which proliferates in soil in great numbers where it can be isolated (Fig. 1.77). In addition to being found in wheat fields and more generally wherever there is vegetation, it is also found in humid houses, trash cans, vegetable substances, rotting wood, freshly cut grass, old hay, fallen leaves, in bedding and 
Table 11.64. Differential diagnosis of allergic bronchopulmonary aspergillosis $(A B A)$

\begin{tabular}{lllll} 
Diagnostic criteria & Comments & ABA & Allergic asthma & Cystic fibrosis \\
\cline { 2 - 5 } Chest X-ray infiltrates & Present in some studies & 100 & 0 & 100 \\
\hline SPT+ to Aspergillus fumigatus & Diagnostic, not specific & 100 & $13-38$ & 30 \\
\hline Raised total serum IgE & Markers of ABA activity & $80-100$ & 50 & 20 \\
\hline Precipitant antibodies to Af & Not specific to ABA & $60-90$ & 25 & 35 \\
\hline Eosinophilia & Absent if treated with steroids & 100 & 40 & 20 \\
\hline High specific lgE/lgG to Af & Essential and specific to diagnosis & 100 & $<5$ & $<5$
\end{tabular}

Data from [95].

in common household floor dust [95]. Since it is very small, with an average diameter of nearly $3 \mu \mathrm{m}$, inhaled spores can reach the peripheral airways where they can proliferate, aided by internal temperature [95]. In ABA other species of AF, Candida albicans, Mucor, Penicillium spp., Cladosporium herbarum, Helminthosporium spp., Stemphylium spp., Torulopsis spp., Curvularia lunata, Rhizopus spp., Drechslera spp., Pseudallescheria spp. [416] are likewise implicated. Mold growth is accompanied by an intense type I, III and IV immune response: antigen release with production of IgE and IgG antibodies. Activation of Th2 T cells orchestrating pulmonary inflammation has also been shown in ABA together with the expression of genes for several ILs present on the $5 q$ chromosome [95]. They are restricted by HLA-DR2 and HLA-DR5 and produce high concentrations of $\mathrm{IL}_{4}$ but few of IFN- $\gamma$ [99].

$A B A$ is characterized by five stages: I acute, II remission, III recurrent exacerbation, IV progressively ingravescent and steroid-dependent, which, if not treated, evolves into stage $\mathrm{V}$, with diffuse pulmonary fibrosis [75, 95]. Some 28 pediatric cases have been reported [ 75,292 , $369,416,425,631,693]$, aged $0.11-18$, including 3 girls aged $0.11-8$ with cystic fibrosis $[292,369,425]$, two girls aged 6-7, with GER of asthmatic origin and multiple sensitizations to mycetes [75] and a 6-year-old boy with cladosporiosis [416]. The symptoms are an asthma-like syndrome with recurring afebrile bronchospastic episodes, coughing, dyspnea, wheezing, and pulmonary infiltration [95].

$A B A$ diagnosis is made on the presence of classic hyphae in the sputum (Fig. 11.69) and on X-rays pulmonary parenchymal wedge-shaped shadows, typical of ABA can be observed depending on the phase of activity (Fig. 11.70a). In the pediatric age, a CAT scan is more appropriate, as it permits a more rapid diagnosis [580]. The presence of marked eosinophilia serves as a guideline. The presence of precipitant antibodies, findings of fungal hyphae in the sputum and fungal isolation in cultures [95] may have diagnostic value. SPTs reveal immediate and/or delayed reactions to the molds. For the diagnosis of the condition, high levels of total $\operatorname{IgE}$ $(>1,000 \mathrm{ng} / \mathrm{ml})$ [369] and IgE antibodies are relevant
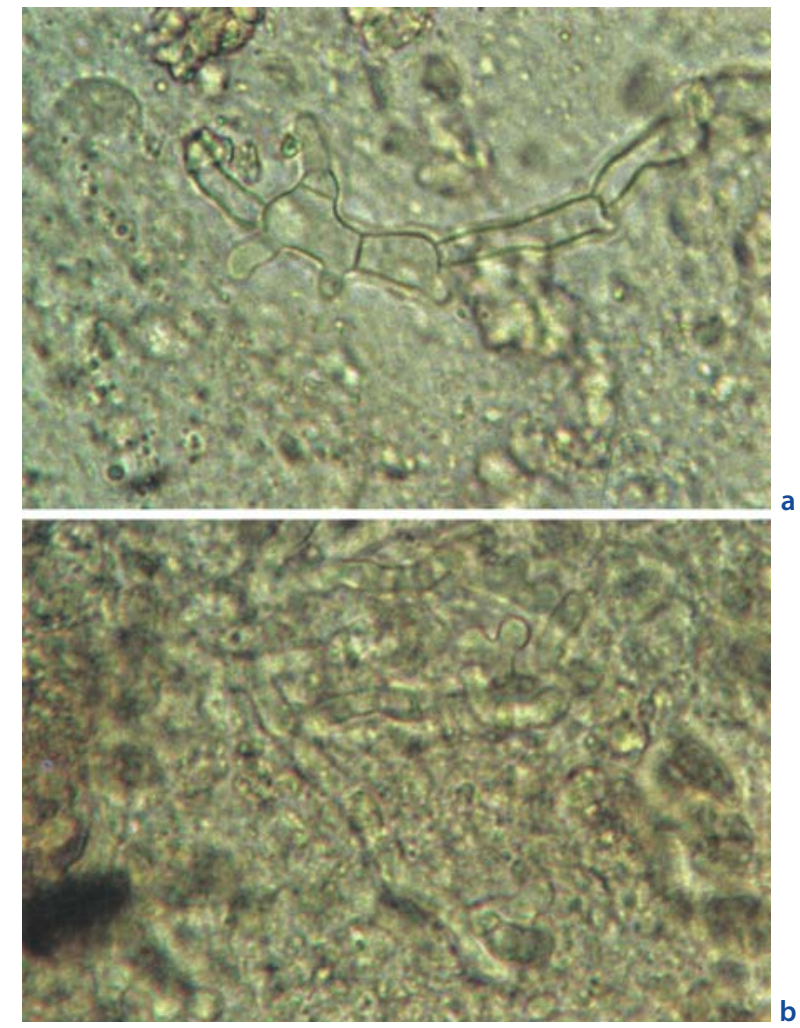

Fig. 11.69 a,b. Allergic pulmonary aspergillosis. Note the characteristic appearance of the hyphae (branching separate structures) in the sputum of a 10-year-old girl

such as the significant association with sIgE and IgGs to AF [75] (Table 11.64) [95]. Children with CF [292, 369] also have anti-AF sIgE [369]; therefore, to obtain a higher specificity, it is safer to take sIgE for CD46 measurements using ELISA [355], but a child with CF and acute symptomatic $\mathrm{ABA}$ with low serum IgE levels has been reported [369].

Steroid therapy for a few months causes remission (stage II), which continues after 10-15 months of followup $[75,416,693]$. Under steroid treatment, clinical findings improve, regression can be seen in the $\mathrm{X}$-rays (Fig. 

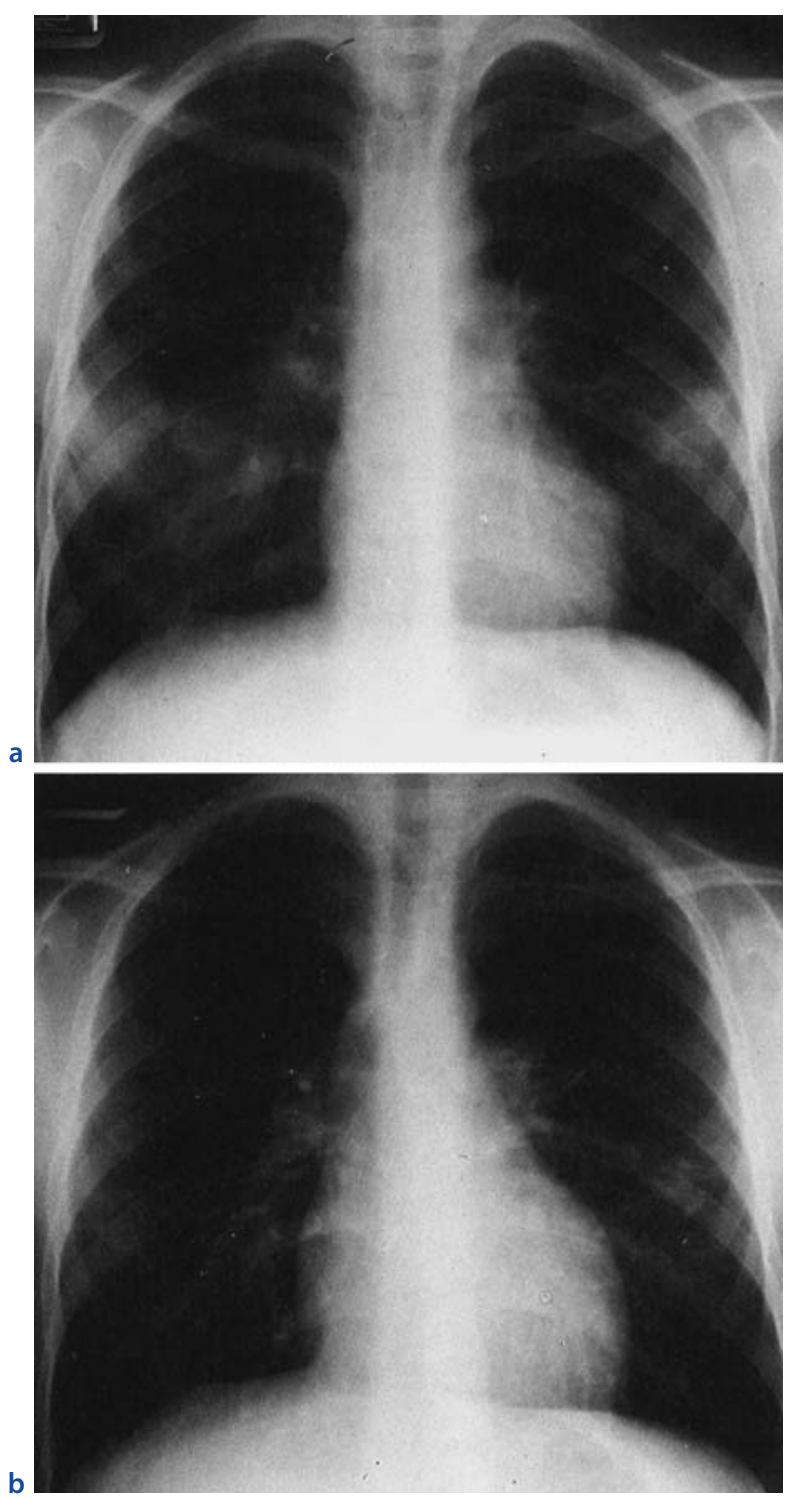

Fig. 11.70. a Chest radiograph of an 8-year-old girl with ABA. b Note the improvement of the girl after 2 weeks of treatment with high dose oral corticosteroids

$11.70 \mathrm{~b})$, peripheral eosinophilia serum IgE titers are reduced, and fungal hyphae are cleared from the airways [75]. Total IgE decrease from 1,596-2,900 kU/1 to $\approx 1,000 \mathrm{ng} / \mathrm{ml}$ after treatment $[75,416]$ and blood eosinophil count from $1,690 \mathrm{~mm}^{3}$ to $980 \mathrm{~mm}^{3}$ [416]. Monitoring total IgE and sIgE/IgGs titers is an excellent index of ABA activity, since their levels are newly increased in case of progression from stage II to III [95]. However very high titers of serum IgE may also be present in several other illnesses, including AD and parasitic disorders.

\section{Pediatricians and Pediatric Asthma}

Apart from pulmonary disease caused by mycetes, the secret of successful management in asthmatic children is to avoid prescribing only symptomatic therapies, but to try to find the casual factors first, specific to each child; therefore every form of treatment should be tailored to the individual child. The negative psychological factors to which we have already referred can trigger asthmatic symptoms, aggravate the attacks and negatively influence compliance with the therapeutic regimen. From our examination of doctor-patient relationships the importance of the pediatrician's role emerges. This doctor must not appear to be simply one who prescribes drugs or tests, but must also fulfill the role of being a friend full of enthusiasm, encouragement and understanding, both to young patients and their families. He must also instill a responsible attitude toward the disease and bring them to an understanding that asthma that is not treated effectively can, more often than not, result in the child or adolescent growing into an invalid adult. Especially in cases such as these, pediatricians should be particularly close to children and their family. Following an asthmatic child for a long time provides an opportunity for acquiring important information on the course of disease and the physical and mental repercussions asthma can have on those involved. Problems related to quality of life and limitations to leading a normal life, or playing and participating in sports are problems with which children must contend. Here we underline the different character of both asthmatic children and adolescents. The real difference is school absenteeism, chronic infirmity, the causes of delayed growth and the effects of HPA-axis depression, which should be considered carefully, especially if alternative treatments are available. The positive aspect, which we have always preferred, is that of giving value to the intercritical periods rather than to side effects, recommending the practice of sports, which can improve quality of life. On the other hand, self-treatment programs have yielded disappointing results, or are based on cycles of hospitalization and do not always achieve positive results. In other cases that we have regularly noted, treatment of children may involve their families in a better understanding of the clinical effects after giving an informative overall picture.

\section{References}

1. Aas K (1981) Heterogeneity of bronchial asthma. Allergy $36: 3-14$

2. Abul-Ainine A, Luyt D (2002) Short term effects of adrenaline in bronchiolitis: a randomised controlled trial, Arch Dis Child 86:276-279

3. Ackerman V, Carpi S, Bellini A, Vassalli G, Marini M, Mattoli $S$ (1995) Constitutive expression of endothelin in bronchial epithelial cells of patients with symptomatic and asymptomatic asthma and modulation by histamine and interleukin-1. J Allergy Clin Immunol 96:618-627 
4. Adachi Y, Murakami G, Matsuno M et al (1992) Longitudinal study of bronchial hyperreactivity in preschool children with bronchial asthma. Ann Allergy 68:261-266

5. Adams RJ, Fuhlbrigge A, Finkelstein JA et al (2001) Impact of inhaled antiinflammatory therapy on hospitalization and emergency department visits for children with asthma. Pediatrics 107:706-711

6. Agertoft L, Pedersen S (2000) Effect of long-term treatment with inhaled budesonide on adult height in children with asthma. N Engl J Med 343:1064-1069

7. Allen DB, Bronsky EA, LaForce CF et al (1998) Growth in asthmatic children treated with fluticasone propionate. Fluticasone Propionate Asthma Study Group. J Pediatr 132: 472-477

8. Allen DB, Mullen ML, Mullen B (1994) A meta-analysis of the effect of oral and inhaled corticosteroids on growth. J Allergy Clin Immunol 93:967-976

9. American Academy of Pediatrics. Committee on Infectious Diseases and Committee on Fetus and Newborn (1997) Respiratory syncytial virus immune globulin intravenous: indications for use. Pediatrics 99:645-650

10. Änggård E (1994) Nitric oxide: mediator, murderer, and medicine. Lancet 343:1199-1206

11. Anhøj J, Bisgaard AM, Bisgaard H (2002) Systemic activity of inhaled steroids in 1- to 3-year-old children with asthma. Pediatrics 109:e40

12. Aoki T, Kojima T, Ono A et al (1994) Circulating endothelin-1 levels in patients with bronchial asthma. Ann Allergy 73:365-369

13. Aretaeus the Cappadocian. (1856) Extant works. Adams F (ed trans) The Sydenham Society, London

14. Arrighi HM (1995) US asthma mortality: 1941-1989. Ann Allergy Asthma Immunol 74:321-326

15. Azzawi M, Bradley R, Jeffery PK et al (1990) Identification of activated $\mathrm{T}$ lymphocytes and eosinophils in bronchial biopsies in stable atopic asthma. Am Rev Respir Dis 142: 1407-1413

16. Backer V, Ulrik CS, Wendelboe D et al (1992) Distribution of serum IgE in children and adolescents aged 7 to 16 years in Copenhagen, in relation to factors of importance. Allergy 47:484-489

17. Baraldi E, Bollini MC, De Marchi A, Zacchello F (1994) Effect of beclomethasone dipropionate on bone mineral content assessed by X-ray densitometry in asthmatic children: a longitudinal evaluation. Eur Respir J 7:710-714

18. Baraniuk JN, Kaliner MA (1990) Neuropeptides in the upper and lower respiratory tract. Immunol Allergy Clin North Am 10:383-407

19. Barbato A, Cracco A, Tormena F, Novello A Jr (1995) The first 20 minutes after a single dose of inhaled salmeterol in asthmatic children. Allergy 50:506-610

20. Bardin PG, Johnston SL, Pattemore PK (1992) Viruses as precipitants of asthma symptoms. II. Physiology and mechanisms. Clin Exp Allergy 22:809-822

21. Barnes PJ (1992) Neural mechanisms in asthma. Br Med Bull 48:149-168

22. Barnes PJ (1993) Pathophysiology of allergic inflammation. In: Middleton E Jr, Reed CE, Ellis EF, Adkinson NF Jr, Yunginger JW, Busse WW (eds) Allergy: principles and practice, 4th edn. CV Mosby, St Louis, pp 243-266

23. Barnes PJ (2002) Corticosteroids. In Barnes J, Drazen S, Rennard S, Thomson N (eds). Asthma and COPD. San Diego: Elsevier Science, pp 2547-2562

24. Barnes PJ (1999) Anti-IgE antibody therapy for asthma. N Engl J Med 341:2006-2008
25. Battistini A (1996) La prevenzione farmacologica della crisi asmatica. Riv Ital Pediatr 22:457-461

26. Battistini A (2000) Medical treatment of respiratory emergencies. Pediatr Med Chir 21:171-179

27. Bauman LJ, Wright E, Leickly FE et al (2002) Relationship of adherence to pediatric asthma morbidity among inner-city children. Pediatrics 110:e6

28. Berger WE, Shapiro GG (2004) The use of inhaled corticosteroids for persistent asthma in infants and young children. Ann Allergy Asthma Immunol 92:387-400

29. Beasley R (2002) The burden of asthma with specific reference to the United States. J Allergy Clin Immunol 109: S482-489

30. Beasley R, Burgess C, Pearce N, Woodman K, Crane J (1994) Confounding by severity does not explain the association between fenoterol and asthma death. Clin Exp Allergy 24:660-668

31. Beasley R, Crane J, Lai CKW, Pearce N (2000) Prevalence and etiology of asthma. J Allergy Clin Immunol 105: S466-S472

32. Bellia V (1991) Diurnal variations of respiratory diseases chronobiology. Res Clin Forums 13:61-67

33. Bellini A, Yoshimura H, Vittori E, Marini M, Mattoli S (1993) Bronchial epithelial cells of patients with asthma release chemoattractant factors for T lymphocytes. J Allergy Clin Immunol 92:412-424

34. Bender B, Milgrom H (1992) Theophylline-induced behavior change in children: an objective evaluation of parent's perceptions. JAMA 267:1621-1624

35. Bensch G, Berger WE, Blokhin BM et al (2002) One-year efficacy and safety of inhaled formoterol dry powder in children with persistent asthma. Ann Allergy Asthma Immunol 89:180-190

36. Bentur L, Canny GJ, Shields MD et al (1992) Controlled trial of nebulized albuterol in children younger than 2 years of age with acute asthma. Pediatrics 89:133-137

37. Berends C, Hoekstra MO, Dijkhuizen B, de Monchy JGR, Gerritsen J, Kauffman HF (1993) Expression of CD35 (CR1) and CD11b (CR3) on circulating neutrophils and eosinophils from allergic asthmatic children. Clin Exp Allergy 23:926-933

38. Berin MC, Eckmann L, Broide DH, Kagnoff MF (2001) Regulated production of the T helper 2-type T-cell chemoattractant TARC by human bronchial epithelial cells in vitro and in human lung xeno grafts. Am J Respir Cell Mol Biol 24:382-389

39. Berman BA, Ross RN (1990) Exercise-induced bronchospasm - is it a unique clinical entity? Ann Allergy 65: 81-83

40. Bienenstock J, McDermott MR, Clancy R (1999) Respiratory tract defenses: role of mucosal lymphoid tissues. In: Ogra PL, Mestecky J, Lamm ME, Strober W, Bienenstock J, McGhee JR (eds) Handbook of mucosal immunology, 2nd edn. Academic Press, San Diego, pp 283-292

41. Bierman CW, Shapiro GG (1996) Evaluation and treatment of the patient with asthma. Pediatric asthma. In: Bierman CW, Pearlman DS, Shapiro GG, Busse WW (eds) Allergy, asthma, and immunology from infancy to adulthood, 3rd edn. WB Saunders, Philadelphia, pp 498-519

42. Birkebæk NH, Esberg G, Andersen K, Wolthers O, Hassager C (1995) Bone and collagen turnover during treatment with inhaled dry powder budesonide and beclomethasone dipropionate. Arch Dis Child 73:524-527 
43. Birkhead G, Attaway MJ, Strunk RC et al (1989) Investigation of a cluster of deaths of adolescents from asthma: evidence implicating inadequate treatment and poor patient adherence with medications. J Allergy Clin Immunol 84: 484-491

44. Bisgaard H (1994) Aerosol treatment of young children. Eur Respir Rev 4:15-20

45. Bisgaard H, Allen D, Milanowski J, Kalev I, Willits L, Davies P (2004) Twelve-month safety and efficacy of inhaled fluticasone propionate in children aged 1 to 3 years with recurrent wheezing. Pediatrics 113:e87-94

46. Boguniewicz M, Schneider LC, Milgrom H et al (1993) Treatment of steroid-dependent asthma with recombinant interferon-gamma. Clin Exp Allergy 23:785-790

47. Bohn D, Kalloghian A, Jenkins J, Edmonsds J, Barker G (1984) Intravenous salbutamol in the treatment of status asthmaticus in children. Crit Care Med 12:892-896

48. Bollinger ME, Wolf B, Schwindt C, Hamilton RG (2004) Contamination of nebulizer equipment with cockroach allergen: there's a bug in the system! Ann Allergy Asthma Immunol 92:475-477

49. Bollinger MB (2002) Efficacy of IV Theophylline in Children with Severe Status Asthmaticus. Pediatrics 110:465466 and Reviewer's Comments

50. Boulet L-P, Turcotte H, Boutet M, Montminy L, Laviolette M (1993) Influence of natural antigenic exposure on expiratory flows, methacholine responsiveness, and airway inflammation in mild allergic asthma. J Allergy Clin Immunol 91:883-893

51. Bousquet J, Chanez P, Lacoste JY et al (1990) Eosinophilic inflammation in asthma. N Engl J Med 323:1033-1039

52. Bousquet J, Chanez P, Michel F-B (1991) Inflammation in chronic asthma. ACI News 3:170-174

53. Bousquet J, Chanez P, Godard B, Michel F-B (1993) Is asthma a disease that remodels the airways? ACI News 5:5-9

54. Brandtzaeg P, Jahnsen FL, Farstad IN (1996) Immune functions and immunopathology of the mucosa of the upper respiratory pathways. Acta Otolaryngol 116:149-159

55. Brightling CE, Bradding P, Symon FA, Holgate ST, Wardlaw AJ, Pavord ID (2002) Mast-cell infiltration of airway smooth muscle in asthma. N Engl J Med 346:1699-1705

56. British guidelines on asthma management 1995: review and position statement. Thorax 52 [Suppl 1]:S1-S21

57. Brofman JD, White SR, Blake JF et al (1989) Eosinophil augmentation of trachealis contraction caused by major basic protein of eosinophils. J Appl Physiol 66:1867-1873

58. Broide DH, Lotz M, Cuomo AJ, Coburn DA, Federman EC, Wasserman SI (1992) Cytokines in symptomatic asthma airways. J Allergy Clin Immunol 89:958-967

59. Broide DH, Payne MM, Firestein GS (1992) Eosinophils express interleukin 5 and granulocyte-colony-stimulating factor mRNA at sites of allergic inflammation in asthmatics. J Clin Invest 90:1414-1424

60. Brunette MG, Lands L, Thibodeau LP (1988) Childhood asthma: prevention of attacks with short-term corticosteroid treatment of upper respiratory tract infection. Pediatrics 81:624-629

61. Brus RHP, Bodenheimer S (1996) High-dose inhaled steroids in asthmatic children. Lancet 348:820-821

62. Burrows B, Lebowitz MD (1992) The $\beta$-agonist dilemma. N Engl J Med 326:560-561

63. Burrows B, Martinez FD, Halonen M, Barbee RA, Cline MG (1989) Association of asthma with serum IgE levels and skin-test reactivity to allergens. N Engl J Med 320:271-277
64. Burrows B, Sears MR, Flannery EM, Herbison GP, Holdaway MD (1992) Relationship of bronchial responsiveness assessed by metacholine to serum IgE, lung function, symptoms, and diagnoses in 11-year-old New Zealand children. J Allergy Clin Immunol 90:376-385

65. Burrows B, Sears MR, Flannery EM, Herbison GP, Holdaway MD (1995) Relationship of bronchial responsiveness to allergy skin test reactivity, lung function, respiratory symptoms, and diagnoses in thirteen-year-old New Zealand children. J Allergy Clin Immunol 95:548-556

66. Bury TB, Corhay JL, Louis R, Radermecker MF (1994) Decrease of T-lymphocyte proliferation in exercise-induced asthma. Allergy 49:605-610

67. Businco L, Cantani A (1988) Prevenzione della morte per asma nel bambino. Atti Congresso Internazionale: aspetti epidemiologici dell'asma bronchiale. Cleup, Padova, pp 131-134

68. Businco L, Cantani A (1990) The prevention of asthma. Interasma News Bull (Jan):3-7

69. Businco L, Ruberto U, Businco E (1968) Contributo allo studio dei rapporti tra bronchiolite ed asma nel bambino. Riv Clin Pediatr 81:1-3

70. Businco L, Frediani T, Lucarelli S, Finocchi M, Puddu M, Businco E (1979) A prospective study of wheezing infants. Clinical and immunological results. Ann Allergy 43:120122

71. Businco L, Cantani A, Di Fazio A, Bernardini L (1990) A double-blind, placebo-controlled study to assess the efficacy of nedocromil sodium in the management of childhood grass-pollen asthma. Clin Exp Allergy 20:683-688

72. Busse WW (1990) Respiratory infections: their role in airway responsiveness and the pathogenesis of asthma. J Allergy Clin Immunol 85:671-683

73. Busse WW, Banks-Schlegel S, Wenzel SE (2000) Pathophysiology of severe asthma. J Allergy Clin Immunol 106: 1033-1042

74. Bustos GJ, Bustos D, Bustos GJ, Romero O (1995) Prevention of asthma with ketotifen in preasthmatic children: a three-year followup study. Clin Exp Allergy 25:568-573

75. Caballero T, Ferrer A, Diaz-Pena JM, Garcia-Ara C, Pascual C, Martin-Esteban M (1995) Childhood allergic bronchopulmonary aspergillosis. J Allergy Clin Immunol 95: 1044-1047

76. Call RS, Ward G, Jackson S, Platts-Mills TAE (1994) Investigating severe and fatal asthma. J Allergy Clin Immunol 94:1065-1072

77. Calverley PMA (1992) Anticholinergics and muscarinic receptors. In: Godard Ph, Bousquet J, Michel FB (eds) Advances in allergology and clinical immunology. Parthenon Publishing, Lancs, pp 543-550

78. Canny GJ, Levison H (1988) Aerosols - therapeutic use and delivery in childhood asthma. Ann Allergy 60:11-19

79. Canny GJ, Levison H (1990) Childhood asthma: a rational approach to treatment. Ann Allergy 64:406-416

80. Canny GJ, Bohn DJ, Levison H (1992) Severe asthma. Rec Adv Pediatr 10:161-171

81. Canonica GW, Pronzato C, Fiorino N, Ciprandi G, Bagnasco M (1993) Adhesion molecules - a review of their clinical roles in allergic inflammation. ACI News 5:80-84

82. Cantani A Sr (1881) Formolario terapeutico ragionato ricavato dalla clinica del Professor Arnaldo Cantani, 3rd edn. Dott Leonardo Vallardi, Naples

83. Cantani A, Businco E, Businco L et al (1983) Evaluation of long-term oral administration of a sustained release theophylline preparation in children with chronic asthma. Allergol Immunopathol 11:11-14 
84. Cantani A, Ciaschi V (2004) Epidemiology of Alternaria alternata allergy in 6840 Italian children. Eur Rev Med Pharmacol Sci 8:289-294

85. Carl JC, Myers TR, Kirchner HL, Kercsmar CM (2003) Comparison of racemic albuterol and levalbuterol for treatment of acute asthma. J. Pediatr 143:731-736

86. Carlsen K-H, Larsson K (1996) The efficacy of inhaled disodium cromoglycate and glucocorticoids. Clin Exp Allergy 26 [Suppl 4]:8-17

87. Carlson M, Håkansson L, Kämpe M, Stålenheim G, Peterson C, Venge P (1992) Degranulation of eosinophils from pollen-atopic patients with asthma is increased during pollen season. J Allergy Clin Immunol 89:131-139

88. Carolan EJ, Casale TB (1993) Effects of neuropeptides on neutrophil migration through noncellular and endothelial barriers. J Allergy Clin Immunol 92:589-598

89. Carter E, Cruz M, Chresrown S, Shieh G, Reilly K, Hendeles L (1993) Efficacy of intravenously administered theophylline in children hospitalized with severe asthma. J Pediatr 122:470-476

90. Casale TB, Baraniuk JN (1998) Neurogenic control of inflammation and airway function. In: Middleton E Jr, Reed CE, Ellis EF, Adkinson NF Jr, Yunginger JW, Busse WW (eds) Allergy: principles \& practice, 5th edn. Mosby, St Louis, pp 183-203

91. Castle W, Fuller R, Hall J, Palmer J (1993) Serevent nationwide surveillance study: comparison of salmeterol with salbutamol in asthmatic patients who require regular bronchodilator treatment. BMJ 306:1034-1037

92. Castro-Rodriguez J, Rodrigo GJ (2004) $\beta$-agonists through metered-dose inhaler with valved holding chamber versus nebulizer for acute exacerbation of wheezing or asthma in children under 5 years of age: a systematic review with meta-analysis. J Pediatr 145:172-177

93. Chalumeau M, Le Clainche L, Sayeg N et al (2001) Spontaneous pneumomediastinum in children. Pediatr Pulmonol 31:67-75

94. Chan KN, Silverman M (1993) Increased airway responsiveness in children of low birth weight at school age: effect of topical corticosteroids. Arch Dis Child 69:120-124

95. Chanarin N, Howarth PH (1995) Special problems: allergic bronchopulmonary aspergillosis. In: O'Byrne P, Thompson NC (eds) Manual of asthma management. WB Saunders, London, pp 607-620

96. Chanez P, Godard Ph, Lacoste JY, Bousquet J, Michel FB (1992) Médiateurs et neuromédiateurs dans l'asthme. Presse Méd 21:259-265

97. Chanez P, Vago P, Demoly P et al (1993) Airway macrophages from patients with asthma do not proliferate. J Allergy Clin Immunol 92:869-877

98. Charpin D, Scheinmann P, Vervloet D (1991) Asthme infantile - fréquence et gravité croissantes. Comment inverser la tendance? Arch Fr Pédiatr 48:523-525

99. Chauhan B, Kutsen AP, Hutcheson PS, Slavin RG, Bellone CJ (1996) T cell subsets, epitope mapping, and HLA-restriction in patients with allergic bronchopulmonary aspergillosis. J Clin Invest 97:2324-2331

100. Chen YZ (2003) National Cooperation Group on Childhood Asthma. A nationalwide survey in China on prevalence of asthma in urban children. Zhonghua Er Ke Za Zhi 41:123-127

101. Chiang AA, Lee J-C (1994) Misunderstanding about inhalers (letter). N Engl J Med 330:1690-1691

102. Chipps BE, Murphy KR (2005) Assessment and treatment of acute asthma in children. J Pediatr 147:288-294
103. Cho Y-J, Hong S-J, Moon H-B (2000) Hydrocortisone enhances allergen-specific IgE production by peripheral blood mononuclear cells from atopic patients with high serum allergen-specific IgE levels, Clin Exp Allergy 30: 1576-1581

104. Chung KF (1996) Theophylline in chronic asthma - evidence for disease modifying properties. Clin Exp Allergy 26 [Suppl 2]:22-27

105. Church MK, Makino S (2001) Drugs for the treatment of allergic disease. In: Holgate ST, Church MK, Lichtenstein LM (eds) Allergy, 2nd edn. Mosby International, St. Louis, pp 353-370

106. Clarke JR, Reese A, Silverman M (1992) Bronchial responsiveness and lung function in infants with lower respiratory tract illness over the first six months of life. Arch Dis Child 67:1454-1458

107. Clough JB, Holgate ST (1994) Episodes of respiratory morbidity in children with cough and wheeze. Am J Respir Crit Care Med 150:48-53

108. Clough JB, Williams JD, Holgate ST (1992) Profile of bronchial responsiveness in children with respiratory symptoms. Arch Dis Child 67:574-579

109. Cockcroft DW (1992) Beta-agonist, airway hyperresponsiveness, and asthma. J Allergy Clin Immunol 90:739-741

110. Cockcroft DW, O’Byrne PM, Swystun VA, Bhagat R (1995) Regular use of inhaled albuterol and the allergen-induced late asthmatic response. J Allergy Clin Immunol 96:44-49

111. Colli C, Haitink O, Vietti F, Quaglia P, Bona C (1992) Alveolite allergica estrinseca: pneumopatia dell'agricoltore. Presentazione di un caso in età pediatrica. Riv Ital Pediatr 18:466-468

112. Collis GG, Cole CH, Le Souëf PN (1990) Dilution of nebulised aerosols by air entrainment in children. Lancet 336:341-343

113. Comis A, Valletta EA, Sette L, Andreoli A, Boner AL (1993) Comparison of nedocromil sodium and sodium cromoglycate administered by pressurized aerosol, with and without a spacer device in exercise-induced asthma in children. Eur Respir J 6:523-526

114. Condino-Neto A, Vilela MM, Cambiucci EC et al (1991) Theophylline therapy inhibits neutrophil and mononuclear cell chemotaxis from chronic asthmatic children. $\mathrm{Br}$ J Clin Pharmacol 32:557-561

115. Connett GJ, Warde C, Wooler E, Lenney W (1993) Audit strategies to reduce hospital admissions for acute asthma. Arch Dis Child 69:202-205

116. Connett G, Warde C, Wooler E, Lenney W (1994) Prednisolone and salbutamol in the hospital treatment of acute asthma. Arch Dis Child 70:170-173

117. Corren J, Berkowitz R, Murray JJ, Prenner B (2003) Comparison of once-daily monometasone furoate versus onedaily budesonide in patients with moderate persistent asthma. Int J Clin Proct 57:567-572

118. Corren J, Casale T, Deniz Y, Ashby M (2003) Omalizumab, a recombinant humanized anti-IgE antibody, reduces asthma-related emergency room visits and hospitalizations in patients with allergic asthma. J Allergy Clin Immunol 111:87-90

119. Corrigan CJ, Kay AB (1990) CD4 T-lymphocyte activation in acute severe asthma: relationship to disease severity and atopic status. Am Rev Respir Dis 141:970-977

120. Covar RA, Leung DYM, McCormick D, Steelman J, Zeitler P, Spahn JD (2000) Risk factors associated with glucocorticoid-induced adverse effects in children with severe asthma. J Allergy Clin Immunol 106:651-659 
121. Crain EF, Weiss KB, Fagan MJ (1995) Pediatric asthma care in US emergency departments. Current practice in the context of the National Institutes of Health Guidelines. Arch Pediatr Adolesc Med 149:893-901

122. Crane J, O’Donnell TV, Prior IA, Waite DA (1989) The relationship between atopy, bronchial hyperresponsiveness, and a family history of asthma: a cross-sectional study of migrant Tokelauan children in New Zealand. J Allergy Clin Immunol 84:768-772

123. Creer TL, Gustafson KE (1989) Psychological problems associated with drug therapy in childhood asthma. J Pediatr115:850-855

124. Crescioli S, Spinazzi A, Plebani M et al (1991) Theophylline inhibits early and late asthmatic reactions induced by allergens in asthmatic subjects. Ann Allergy 66:245-251

125. Crespo M, Antón M, Aguirrezabalaga B (1996) Bronquiolitis aguda. Pediatr Integr 2:126-127

126. Crimi N, Palermo F, Oliveri R, Polosa R, Magrì S, Mistretta A (1994) Inhibition of neutral endopeptides potentiates bronchoconstriction induced by neurokinin A in asthmatic patients. Clin Exp Allergy 24:115-120

127. Croce J, Negreiros EB, Mazzei JAM, Isturiz G (1995) A double-blind, placebo-controlled comparison of sodium cromoglycate and ketotifen in the treatment of childhood asthma. Allergy 50:524-527

128. Crompton GK (1995) Delivery systems. In: O’Byrne P, Thompson NC (eds) Manual of asthma management. WB Saunders, London, pp 341-355

129. Cunningham ME, Huribal M, Bala RJ, McMillen MA (1997) Endothelin-1 and endothelin-4 stimulate monocyte production of cytokines. Crit Care Med 25:958-964

130. Cypcar D, Busse WW (1993) Role of viral infections in asthma. Immunol Allergy Clin North Am 13:745-767

131. Cypcar D, Busse WW (1993) Steroid-resistant asthma. J Allergy Clin Immunol 92:362-372

132. Czerwionka-Szaflarska M, Mierzwa G, Kuczynska R (2004) Effectiveness of omeprazole therapy in children with gastoesophageal reflux (GERD). Pol Merkuriusz Lek $16: 217-219$

133. Dahl R, Earnshaw JS, Palmer JBD (1991) Salmeterol: a four-week study of a long-acting beta-adrenoceptor agonist for the treatment of reversible airway disease. Eur Respir J 4:1178-1184

134. De Benedictis FM, Tuteri G, Bertotto A, Bruni L, Vaccaro R (1994) Comparison of the protective effects of cromolyn sodium and nedocromil sodium in the treatment of exercise-induced asthma in children. J Allergy Clin Immunol 94:684-688

135. De Benedictis FM, Teper A, Green RJ et al, for the International Study Group (2001) Effects of 2 inhaled corticosteroids on growth. Results of a randomized controlled trial. Arch Pediatr Adolesc Med 155:1248-1254

136. De Blic J, Delacourt C, Le Bourgeois M et al (1996) Efficacy of nebulized budesonide in treatment of severe infantile asthma: a double-blind study. J Allergy Clin Immunol $98 ; 14-20$

137. De Marchie Sarvaas GJ, Merkus PJ, de Jongste JC (2000) A family with extrinsic allergic alveolitis caused by wild city pigeons: a case report. Pediatrics 105:E62

138. De Marzo N, Di Blasi P, Boschetto P et al (1989) Airway smooth muscle biochemistry and asthma. Eur Respir J Suppl 6:473s-476s

139. De Weck AL (1990) Does the clinical experience with nonsedating H1-antagonists justify a reassessment of antihistamines in allergy treatment? Clin Exp Allergy 20 [Suppl 2]:51-54
140. Dean T (2002) The Cochrane Collaboration and its contribution towards the management of allergic disease. Clin Exp Allergy 32:1269-1273

141. Del Prete G (1994) Th1 and Th2 cells in atopy. EOS 14:1519

142. Delacourt C, Labbé D, Vassault A, Brunet-Langot D, de Blic J, Scheinmann P (1994) Sensitization to inhalant allergens in wheezing infants is predictive of the development of infantile asthma. Allergy 49:843-847

143. Delgado A, Chou KJ, Silver EJ, Crain EF (2003) Nebulizers vs metered-dose inhalers with spacers for bronchodilator therapy to treat wheezing in children aged 2 to 24 months in a pediatric emergency department. Arch Pediatr Adolesc Med 157:76-80

144. Demoly P, Vachier I, Pène J, Michel FB, Godard P, Damon M (1994) IgE produces monocyte superoxide anion release: correlation with CD23 expression. J Allergy Clin Immunol 93:108-116

145. DeNicola LK, Monem GF, Gayle MO, Kissoon N (1994) Treatment of critical status asthmaticus in children. Pediatr Clin North Am 41:1293-1324

146. Dietrich KA, Conrad SA, Romero MD (1991) Creatine kinase (CK) isoenzymes in pediatric status asthmaticus treated with intravenous terbutaline. Crit Care Med 19: S39

147. Diette GB, Markson L, Skinner EA, Nguyen TTH, AlgattBergstrom P, Wu AW (2000) Nocturnal asthma in children affects school attendance, school performance, and parents' work attendance. Arch Pediatr Adolesc Med 154: 923-928

148. DiGiulio GA, Kercsmar CM, Krug SE, Alpert SE, Marx CM (1993) Hospital treatment of asthma: lack of benefit from theophylline given in addition to nebulized albuterol and intravenously administered corticosteroid. J Pediatr 122: 464-469

149. Devidayal S, Singhi S, Kumar L, Jayshree M (1999) Efficacy of nebulised budesonide compared to oral prednisolone in acute bronchial asthma. Acta Paediatr 88: 835-840

150. Di Lorenzo G, Pacor ML, Pellitteri ME et al (2002) In vitro effects of fluticasone propionate on IL-13 production by mitogen-stimulated lymphocytes. Mediators Inflamm 11:187-190

151. Doan T, Patterson R, Greenberger PA (1992) Cough variant asthma: usefulness of a diagnostic-therapeutic trial with prednisone. Ann Allergy 69:505-509

152. Dodge R, Martinez FD, Cline MG, Lebowitz MD, Burrows B (1996) Early childhood respiratory symptoms and the subsequent diagnosis of asthma. J Allergy Clin Immunol 98:48-54

153. Doi S, Murayama N, Inoue T et al (1996) CD4 T-lymphocyte activation is associated with peak expiratory flow variability in childhood asthma. J Allergy Clin Immunol 97:955-962

154. Dolovich J, O’Byrne P, Hargreave FE (1992) Airway hyperresponsiveness: mechanisms and relevance. Pediatr Allergy Immunol 3:163-170

155. Dooper MWSM, Timmermans A, Weersink EJM, De Monchy JGR, Kaufman HF (1995) Defect in potentiation of adenyl cyclase correlates with bronchoconstriction. J Allergy Clin Immunol 96:628-634

156. Droste JHJ, Wierenga MH, Weiler JJ et al (2000) Does the use of antibiotics in early childhood increases the risk of asthma and allergic disease? Clin Exp Allergy 30:15471553 
157. Doull IJM, Campbell MJ, Holgate ST (1998) Duration of growth suppressive effects of regular inhaled corticosteroids. Arch Dis Child 78:172-173

158. Doull IJM, Freezer NJ, Holgate ST (1995) Growth of prepubertal children with mild asthma treated with inhaled beclomethasone dipropionate. Am J Respir Crit Care Med 151:1715-1719

159. Dubus J-C, Anhoj J (2003) A review of once-daily delivery of anti-asthmatic drugs in children. Pediatr Allergy Immunol 14:4-9

160. Ducharme FM, Chabot G, Polychronakos C, Glorieux F, Mazer B (2003) Safety profile of frequent short courses of oral glucocorticoids in acute pediatric asthma: impact on bone metabolism, bone density, and adrenal function. J Pediatr 111:376-383

161. Duff AL, Pomeranz ES, Gelber LE et al (1993) Risk factors for acute wheezing in infants and children: viruses, passive smoke, and IgE antibodies to inhalant allergens. Pediatrics 92:535-540

162. Durham SR (1991) The significance of late responses in asthma. Clin Exp Allergy 21:3-8

163. Durward A, Forte V, Shemie SD (2000) Resolution of mucus plugging and atelectasis after intratracheal rhDNase therapy in a mechanically ventilated child with refractory status asthmaticus. Crit Care Med 28:560-562

164. Dutau G, Brémont F, Juchet A, Ranché F (1994) La crise d'asthme sévère... et après. Arch Pédiatr 1:350-354

165. Eda R, Sugiyama H, Hopp RJ, Bewtra AK, Townley RG (1993) Effect of loratadine on human eosinophil function in vitro. Ann Allergy 71:373-378

166. Edwards A, Holgate S, Howell J et al (2001) Sodium cromoglycate in childhood asthma (letter). Thorax 56:331

167. Eggleston PA (1988) Upper airway inflammatory diseases and bronchial hyperresponsiveness. J Allergy Clin Immunol 81:1036-1041

168. Eisenberg JD, Montanero A, Lle RG (1992) Hypersensitivity pneumonitis in an infant. Pediatr Pulmonol 12:186190

169. Eid N, Morton R, Olds B, Clark P, Sheikh S, Looney S (2002) Decreased morning serum cortisol levels in children with asthma treated with inhaled fluticasone propionate. Pediatrics109:217-221

170. Eid NS, Sheperd RW, Thomson MA (1994) Persistent wheezing and gastroesophageal reflux in infants. Pediatr Pulmonol 18:39-44

171. Ekins-Daukes S, Simpson CR, Helms PJ, Taylor MW, McLay JS (2002) Burden of corticosteroids in children with asthma in primary care: retrospective observational study. BMJ 324:1374

172. El-Radhi AS, Hogg CL, Bungre JK, Bush A, Corrigan CJ (2000) Effect of oral glucocorticoid treatment on serum inflammatory markers in acute asthma. Arch Dis Child 83:158-162

173. Ellis AG (1906) The pathological anatomy of bronchial asthma. Am J Med Soc 136:407-429

174. Ellis EF (1993) Asthma in infancy and childhood. In: Middleton E Jr, Reed CE, Ellis EF, Adkinson NF Jr, Yunginger JW, Busse WW (eds) Allergy: principles and practice, 4th edn. CV Mosby, St Louis, pp 1225-1262

175. Emre U, Sokolowskaya N, Robling PM et al (1995) Detection of anti-Chlamydia pneumoniae IgE in children with reactive airway disease. J Infect Dis 172:265-267

176. Englund JA, Piedra PA, Ahn Y-M, Gilbert BE, Hiatt P (1994) High-dose, short-duration ribavirin aerosol therapy compared with standard ribavirin therapy in children with suspected respiratory syncytial virus infection. J Pediatr 125:635-641
177. Erjefält J, Persson CGA (1989) Inflammatory leakage of plasma macromolecules into airway tissue and lumen. Pulm Pharmacol 2:93-102

178. Esposito F, Cocco G, Sacerdoti G et al (1992) Valutazione allergologica e funzionale respiratoria di bambini di 12 anni con una storia di grave bronchiolite nella prima infanzia. G Ital Allergol Immunol 2:117-124

179. Evans R III (1991) Origin and progression of childhood asthma. Perspect Asthma 5:17-25

180. Everard ML, Clark AR, Milner AD (1992) Drug delivery from holding chambers with attached facemask. Arch Dis Child 67:580-585

181. Everard ML, Clark AR, Milner AD (1992) Drug delivery from jet nebulisers. Arch Dis Child 67:586-591

182. Everard ML, Swarbrick A, Wrightham M et al (1994) Analysis of cells obtained by bronchial lavage of infants with respiratory syncitial virus infection. Arch Dis Child 71:428-432

183. Everden P, Campbell M, Harnden C et al (2004) Eformoterol Turbohaler compared with salmeterol by dry powder inhaler in asthmatic children not controlled on inhaled corticosteroids. Pediatr Allergy Immunol 15: 40-47

184. Everard ML, Fox G, Walls AF et al (1995) Tryptase and IgE concentrations in the respiratory tract of infants with acute bronchiolitis. Arch Dis Child 72:64-69

185. Executive Committee AAAI (1993) Position statement. Inhaled $\beta_{2}$-adrenergic agonists in asthma. J Allergy Clin Immunol 91:1234-1237

186. Ewerbeck H (1980) Differential diagnosis in pediatrics. Springer-Verlag, New York

187. Ferguson AC, Whitelaw M, Brown H (1992) Correlation of bronchial eosinophil and mast cell activation with bronchial hyperresponsiveness in children with asthma. J Allergy Clin Immunol 90:609-613

188. Finotto S, Neurath MF, Glickman JN et al (2002) Development of spontaneous airway changes consistent with human asthma in mice lacking T-bet. Science 295:336-338

189. Fish JE, Peters SP (1999) Airway remodeling and persistent airway obstruction in asthma. J Allergy Clin Immunol 104:509-516

190. Fletcher HJ, Ibrahim SA, Speight N (1990) Survey of asthma deaths in the Northern region, 1970-85. Arch Dis Child 65:163-167

191. Forbis SG, Aligne CA (2002) Poor readability of written asthma management plans found in national guidelines. Pediatrics 109:e52

192. Foucard F, Graff-Lonnevig V (1994) Asthma mortality rate in Swedish children and young adults 1973-1988. Allergy 49:616-619

193. Fournier M (1992) Intraepithelial lymphocytes in human airways. In: Godard Ph, Bousquet J, Michel FB (eds) Advances in allergology and clinical immunology. Parthenon Publishing, Lancs, pp 245-249

194. Frew AJ, Kay AB (1990) Eosinophils and T-lymphocytes in late-phase allergic reaction. J Allergy Clin Immunol 85: 533-539

195. Frischer T, Kuehr J, Meinert R et al (1992) Maternal smoking in early childhood: a risk factor for bronchial responsiveness to exercise in primary-school children. J Pediatr 121:17-22

196. Fuller RW (1993) Dose equivalence of drugs for asthma. BMJ 306:1006-1007

197. Fuller RW, Castle WM, Hall JR, Palmer JBD (1993) Bronchodilator treatment in asthma. Author's reply. BMJ 306:1611 
198. Furukawa CT (1993) Stepping up the treatment of children with asthma. Pediatrics 92:144-146

199. Gadomski AM, Lichtenstein R, Horton L, King J, Keane V, Permutt T (1994) Efficacy of albuterol in the management of bronchiolitis. Pediatrics 93:907-912

200. Gagro A, Rabatic S, Lokar-Kolbas R, Medar-Lasic M, Zimic L (1995) Effect of an asthmatic attack on CD23 and CD21 expression on lymphocytes from allergic children during the allergen season. Clin Exp Allergy 25:690-697

201. Garofalo R, Kimpen JLL, Welliver RC, Ogra PL (1992) Eosinophil degranulation in the respiratory tract during naturally acquired respiratory syncytial virus. J Pediatr 120:28-32

202. Garofalo R, Dorris A, Ahlstedt S, Welliver RC (1994) Peripheral blood eosinophil counts and eosinophil cationic protein content of respiratory secretions in bronchiolitis: relationship to severity of disease. Pediatr Allergy Immunol 5:111-117

203. Garofalo R, Welliver RC, Ogra PL (1991) Concentrations of LTB4, LTC4, LTD4, and LTE4 in bronchiolitis due to respiratory syncytial virus. Pediatr Allergy Immunol 2:30-37

204. Gaultier C (1992) Development of lung. In: Godard Ph, Bousquet J, Michel FB (eds) Advances in allergology and clinical immunology. Parthenon Publishing, Lancs, pp 625-628

205. Geddes DM (1995) Inhaled budesonide for mild asthma (letter). N Engl J Med 332:683

206. Geller DE, Morgan WJ, Cota KA, Wright AL, Taussig LM (1988) Airway responsiveness to cold, dry air in normal infants. Pediatr Pulmonol 4:90-97

207. Gemou-Engesaeth, Kay AB, Bush A, Corrigan CJ (1994) Activated peripheral blood CD4 and CD8 T-lymphocytes in childhood asthma: correlation with eosinophilia and disease severity. Pediatr Allergy Immunol 5:170-177

208. Gemou-Engesæth V, Fagerhol MK, Toda M et al (2002) Expression of activation markers and cytokine mRNA by peripheral blood CD4 and CD8 T cells in atopic and nonatopic childhood asthma: Effect of inhaled glucocorticoid therapy. Pediatrics 109:e24

209. Gergen PJ, Mullally DI, Evans RE III (1988) National survey of prevalence of asthma among children in the United States, 1976 to 1980 . Pediatrics $88: 1-7$

210. Gerritsen J, Koeter GH, Postma DS et al (1989) Prognosis from asthma from childhood to adulthood. Am Rev Respir Dis 140:1325-1330

211. Gesundheit B, Preminger A, Harito B, Babyn P, Maayan C, Mai-Zahav M (2002) Pneumomediastinum and subcutaneous emphysema in an 18-month-old child. J Pediatr 141: 116-120

212. Gibson PG, Dolovich J, Girgis-Gabardo A et al (1990) The inflammatory response in asthma exacerbation: changes in circulating eosinophils, basophils and their progenitors. Clin Exp Allergy 20:661-668

213. Gibson PG, Henry RL, Vimpani GV, Halliday J (1995) Asthma knowledge, attitudes, and quality of life in adolescents. Arch Dis Child 73:321-328

214. Giffin F, Greenough A, Yüksel B (1994) Relationship between lung function results in the first year of life and respiratory morbidity in early childhood in patients born prematurely. Pediatr Pulmonol 18:290-294

215. Giorgi PL, Oggiano N, Biraghi M, Kantar A (1991) Effects of high doses of inhaled flunisolide on hypothalamic-pituitary-adrenal axis function in children with asthma. Curr Ther Res 49:778-783

216. Gleeson JGA, Price JF (1988) Controlled trial of budesonide given by the nebuhaler in preschool children with asthma. BMJ 297:163-166
217. Gleich GJ (1990) The eosinophils and bronchial asthma: current understanding. J Allergy Clin Immunol 85:422436

218. Godden DJ, Ross S, Abdalla M et al (1994) Symptoms and pulmonary function 25 years alter. J Respir Crit Care Med 149:106-112

219. Gold DR, Wyppij D, Wang X et al (1994) Gender- and racespecific effects of asthma and wheeze on level and growth of lung function in children of six US cities. Am J Respir Crit Care Med 149:1198-1208

220. Goldberg S, Algur N, Levi M et al (1996) Adrenal suppression among asthmatic children receiving chronic therapy with inhaled corticosteroid with and without spaced device. Ann Allergy Asthma Immunol 76:234-238

221. Goldman JM, Bennett JR (1990) Gastro-oesophageal reflux and asthma; a common association, but of what clinical importance? Gut 31:1-3

222. Gozal D, Colin AA, Jaffe M, Hochberg Z (1990) Water, electrolyte, and endocrine homeostasis in infants with bronchiolitis. Pediatr Res 27:204-209

223. Grech V, Vella C, Lenicker H (2000) Pigeon breeder's lung in childhood: varied clinical picture at presentation. Pediatr Pulmonol 30:145-148

224. Gries DM, Moffitt DR, Pulos E, Carter ER (2000) A single dose of intramuscularly administered dexamethasone acetate is as effective as oral prednisone to treat asthma exacerbations in young children. J Pediatr 136:298-303

225. Grimaldi M, Gouyon B, Michaut F, Huet F, Gouyon JB (2004) Burgundy Perinatal Network. Severe respiratory syncytial virus bronchiolitis: epidemiologic variations associated with the initiation of palivizumab in severely premature infants with bronchopulmonary dysplasia. Pediatr Infect Dis J 23:1081-1085

226. Groothuis JR Simoes EAF, Hemming VG et al (1995) Respiratory syncytial virus (RSV) in preterm infants and protective effects of RSV immune globulin (RSVIG). Pediatrics 95:463-467

227. Groothuis JR, Simoes EAF, Levin MJ et al (1993) Prophylactic administration of respiratory syncytial virus immune globulin to high-risk infants and young children. $\mathrm{N}$ Engl J Med 329:1524-1530

228. Guarnaccia S, Pasquali M, Acerbis F et al (1990) Clinicofunctional evaluation of flunisolide + salbutamol combination (VAL 679/A) vs salbutamol in pediatric patients. Minerva Pediatr 42:237-242

229. Gundel RH, Wegner CD, Letts LG (1992) The onset and recovery from airway hyperresponsiveness: relation with inflammatory cell infiltrates and release of cytotoxic granule proteins. Clin Exp Allergy 22:303-308

230. Gundel RH, Wegner CD, Torcellini CA, Letts LG (1992) The role of intercellular adhesion molecule-1 in chronic airway inflammation. Clin Exp Allergy 22:569-575

231. Haahtela T, Järvinen M, Kava T et al (1994) Effects of reducing or discontinuing inhaled budesonide in patients with mild asthma. N Engl J Med 331:700-705

232. Haahtela T, Vidgren M, Nyberg A et al (1994) A novel multiple dose powder inhaler. Salbutamol powder and aerosol give equal bronchodilatation with equal doses. Ann Allergy 72:178-182

233. Håkansson L, Björnsson E, Janson C, Schmekel B (1995) Increased adhesion to vascular cell adhesion molecule-1 and intercellular adhesion molecule-1 of eosinophils from patients with asthma. J Allergy Clin Immunol 96:941-950

234. Hall IP, Wheatley A, Wilding P, Liggett SB (1995) Association of Glu $27 \beta_{2}$-adrenoceptor polymorphism with lower airway reactivity in asthmatic subjects. Lancet 345:12131214 
235. Halsey NA, Chesney PJ, Gerber MA et al (1996) Committee on Infectious Disease of the American Academy of Pediatrics. Reassessment of the indications for ribavirin therapy in respiratory syncytial virus infections. Pediatrics 97:137-140

236. Hamid Q, Azzawi M, Ying S et al (1991) Expression of mRNA for interleukin-5 in mucosal bronchial biopsies from asthma. J Clin Invest 87:1541-1546

237. Hariprakash S, Alexander J, Carroll W, Ramesh P, Randell T, Turnbull F, Lenney W (2003) Randomized controlled trial of nebulized adrenaline in acute bronchiolitis. Pediatr Allergy Immunol 14:134-139

238. Hargreave FE, Pizzichini MM, Pizzichini E (1995) Airway hyper-responsiveness as a diagnostic feature of asthma. Prog Allergy Clin Immunol 3:63-67

239. Hartling L, Wiebe N, Russell K, Patel H, Klassen T (2004) Epinephrine for bronchiolitis. Cochrane Database Syst Rev 1:CD003123

240. Hedlin G, Ahlstedt S, Enander I, Håkansson L, Venge P (1992) Eosinophil cationic protein (ECP), eosinophil chemotactic activity (ECA), neutrophil chemotactic activity (NCA) and tryptase in serum before and during bronchial challenge in cat-allergic children with asthma. Pediatr Allergy Immunol 3:144-149

241. Heino M, Juntunen-Backman K, Leijala M, Rapola J, Laitinen LA (1990) Bronchial epithelial inflammation in children with chronic cough after early lower respiratory tract illness. Am Rev Respir Dis 141:428-432

242. Heinzmann SC, Jerkic A, Haider SP et al (2003) A Clara cell protein 16 (CC16) gene polymorphism influences the degree of airway responsiveness in asthmatic children. J Allergy Clin Immunol 111:515-519

243. Hellings PW, Kasran A, Liu Z et al (2003) Interleukin-17 orchestrates the granulocyte influx into airways after allergen inhalation in a mouse model of allergic asthma. Am J Respir Cell Mol Biol 28:42-50

244. Hendeles L, Weinberger M, Szefler S, Ellis E (1992) Safety and efficacy of theophylline in children with asthma. J Pediatr 120:177-183

245. Henderson FW, Stewart PW, Burchenal MR et al (1992) Respiratory allergy and the relationship between early childhood lower respiratory illness and subsequent lung function. Am Rev Respir Dis 145:283-290

246. Henderson WR, Tang LO, Chu SJ et al (2002) A role for cysteinyl leukotrienes in airway remodeling in a mouse asthma model. Am J Respir Crit Care Med 165:108-116

247. Henriksen JM, Agertoft L, Pedersen S (1992) Protective effect and duration of action of inhaled formoterol and salbutamol on exercise-induced asthma in children. J Allergy Clin Immunol 89:1176-1182

248. Highland KB, Flume PA (2001) A 12-year-old girl with dyspnea and a normal chest radiographic finding. Chest 120:1372-1376

249. Hirai K, Yamaguchi M, Misaki Y et al (1990) Enhancement of human basophil histamine release by interleukin-5. J Exp Med 172:1525-1528

250. Ho L, Collis G, Landaau LI, Le Souef PN (1991) Effect of salbutamol on oxygen saturation in bronchiolitis. Arch Dis Child 66:1061-1064

251. Hoag JE, McFadden ER Jr (1991) Long term effect of cromolyn sodium on nonspecific bronchial hyperresponsiveness: a review. Ann Allergy 66:53-63

252. Hoeger PH, Niggemann B, Ganschow R, Dammann C, Haeuser G (1994) Serum levels of sCD23 and sCD25 in children with asthma and in healthy controls. Allergy 49:217-221
253. Hoeger PH, Niggemann B, Haeuser G (1994) Age related IgG subclass concentrations in asthma. Arch Dis Child 70:179-182

254. Hoekstra MO, Berends C, Dijkhuizen B, Gerritsen J, Kaufman HF (1994) Dextran sedimentation induces a difference in the percentage of hypodense eosinophils in peripheral blood between children with allergic asthma and healthy controls. Clin Exp Allergy 24:969-975

255. Hogg JC (1993) Pathology of asthma. J Allergy Clin Immunol 92:1-5

256. Hogue SL, Phelps SJ (1993) Evaluation of three theophylline dosing equations for use in infants up to one year of age. J Pediatr 123:651-656

257. Holberg CJ, Wright AL, Martinez FD et al (1993) Child day-care, smoking by caregivers, and lower respiratory tract illness in the first three years of life. Pediatrics 91: 885-892

258. Holgate ST (1993) Asthma - pathophysiology. In: Holgate ST, Church MK (eds) Allergy. Gower Medical Publishing, London, pp 13.1-12

259. Holgate ST, Finnerty JP (1989) Antihistamines in asthma. J Allergy Clin Immunol 83:537-547

260. Holgate ST, Lackie PM, Davies DE, Roche WR, Walls AF (1999) The bronchial epithelium as a key regulator of airway inflammation and remodelling in asthma. Clin Exp Allergy 29 [Suppl 2]:90-95

261. Holgate ST, Peters-Golden M, Panettieri RA, Henderson WR Jr (2003) Roles of cysteinyl leukotrienes in airway inflammation, smooth muscle function, and remodeling. J Allergy Clin Immunol 111 [Suppl 1]:S18-S34

262. Holt PG (1995) Environmental factors and primary T-cell sensitisation to inhalant allergens in infancy: reappraisal of the role of infections and air pollution. Pediatr Allergy Immunol 6:1-10

263. Holt PG, McMenamin C (1991) IgE and mucosal immunity: studies on the role of the intraepithelial Ia+ dendritic cells and g/d T-lymphocytes in regulation of T-cell activation in the lung. Clin Exp Allergy 21 [Suppl 1]:148-152

264. Horak E, Roberts M et al (2003) Longitudinal study of childhood wheezybronchitis and asthma: outcome at age 42 BMJ 326:422-423

265. Hsieh K-H, Ng C-K (1993) Increased plasma platelet-activating factor in children with acute asthmatic attacks and decreased in vivo and in vitro production of plasma platelet-activating factor after immunotherapy. J Allergy Clin Immunol 91:650-657

266. Huber AR, Kunkel SL, Todd RF III et al (1991) Regulation of transendothelial neutrophil migration by endogenous interleukin-8. Science 254:99-102

267. Hubert P, Menif K, Jouvet P et al (1994) Les asthmes aigus graves en réanimation pédiatriques. Arch Pédiatr 1:346349

268. Hue V, Deschildre A, Fourier C, Flurin V, Martinot A, Leclerc F (1994) Asthme de l'enfant: qui hospitaliser et où? Arch Pédiatr 1:340-345

269. Hurst SD, Muchamuel T, Gorman DM et al (2002) New IL-17 family members promote Th1 or Th2 responses in the lung: in vivo function of the novel cytokine IL-25. J Immunol 169:443-453

270. IikuraY, Hashimoto K, Akasawa A et al (1996) Serum theophylline concentration levels and preventative effects on exercise-induced asthma. Clin Exp Allergy 26 [Suppl 2]: 38-41

271. Indinnimeo L, De Castro G, Ronchetti R (1993) La bronchiolite. In: Ronchetti R. Malattie dell'apparato respiratorio. Schwarz Tiene Manuale di Terapia, 10th ed. Casa Editrice Ambrosiana, Milan, pp 633-636 
272. International Consensus Report on Diagnosis and Management of Asthma (1992) International Asthma Management Project. Allergy 47 [Suppl 13]:1-61

273. Isles AF, Newth CJL (1995) Management of acute asthma in children. Baillière Clin Pediatr 3:341-378

274. Iwin CG (2000) Interaction between the growing lung and asthma. Role of early intervention. J Allergy Clin Immunol 105:S540-S546

275. Ito S, Noguchi E, Shibasaki M et al (2002) Evidence for an association between plasma platelet-activating factor acetylhydrolase deficiency and increased risk of childhood atopic asthma. J Hum Genet 47:99-101

276. Jaffe HA, Buhl R, Mastrangeli A et al (1991) Organ specific cytokine therapy: local activation of mononuclear phagocytes by delivery of an aerosol of recombinant interferon-g to the human lung. J Clin Invest 88:297-302

277. Jain A, Patwari AK, Bajaj P, Kashyap R, Anand VK (2002) Association of gastroesophageal reflux disease in young children with persistent respiratory symptoms. Trop Pediatr 48:39-42

278. Jarjour NN, Calhoun WJ (1992) Exercise-induced asthma is not associated with mast cell activation or airway inflammation. J Allergy Clin Immunol 89:60-68

279. Johnson D, Osborn LM (1991) Cough variant asthma: a variant of the clinical literature. J Asthma 28:85-90

280. Johnston SL, Bardin PG, Pattemore PK (1993) Viruses as precipitants of asthma symptoms. III. Rhinoviruses: molecular biology and prospects for future interventions. Clin Exp Allergy 23:237-246

281. Johnston SL, Pattemore PK, Sanderson G et al (1995) The relationship between upper respiratory infections and hospital admissions for asthma: a time trend analysis. BMJ 310:1225-1229

282. Kaditis AG, Gourgoulianis K, Winnie G. (2003) Anti-inflamatory treatment for recurrent wheezing in the first five years of life. Pediatr Pulmunol 35:241-252

283. Kaliner MA (1987) Mast cell mediators and asthma. Chest 91 [Suppl]:171-176

284. Kaliner MA (1993) Evolution of asthma treatments. Ann Allergy 71:300-306

285. Kamada AK, Szefler SJ (1995) Glucocorticoids and growth in asthmatic children. Pediatr Allergy Immunol 6:145-154

286. Kaminuma O, Mori A, Kitamura N et al (2005) Role of GATA-3 in IL-5 gene transcription by CDA + T-cells of asthatic patients. Int Arch Allergy Immunol 137(suppl 1):55-59

287. Kay AB (1991) T lymphocytes and their products in atopic allergy and asthma. Int Arch Allergy Appl Immunol 94: 189-193

288. Kelly HW, McWilliams BC, Katz R, Murphy S (1990) Safety of frequent high dose nebulized terbutaline in children with acute severe asthma. Ann Allergy 64:229-233

289. Kelly WJW, Hudson I, Phelan PD, Pain MCF, Olinsky A (1990) Atopy in subjects with asthma followed to the age of 28 years. J Allergy Clin Immunol 85:548-557

290. Kemp JP, Skoner DP, Szefler SJ, Walton-Bowen K, CruzRivera M, Smith JA (1999) Once-daily budesonide inhalation suspension for the treatment of persistent asthma in infants and young children. Ann Allergy Asthma Immunol $83: 231-239$

291. Keogh KA, Macarttur C, Parkin PC et al (2001) Predictors of hospitalization in children with acute asthma. J Pediatr 139:273-277

292. Khan A, Giusti R, Silverman B, Schneider A (2001) An unusual etiology of persistent cough in an 8-year-old girl with cystic fibrosis. Ann Allergy Asthma Immunol 87:191195
293. Kharitonov SA, Yates B, Robbins RA, Logan-Sinclair R, Shinebourne EA, Barnes PJ (1994) Increased nitric oxide in exhaled air of asthmatic patients. Lancet 343:133-135

294. Kikawa Y, Hosoi S, Inoue Y et al (1991) Exercise-induced urinary excretion of leukotriene E4 in children with atopic asthma. Pediatr Res 29:455-459

295. Kikawa Y, Miyamonae T, Inoue Y et al (1992) Urinary leukotriene $\mathrm{E}_{4}$ after exercise challenge in children with asthma. J Allergy Clin Immunol 89:1111-1119

296. Kikuchi Y, Okabe S, Tamura G et al (1994) Chemosensitivity and perception of dyspnea in patients with a history of near-fatal asthma. N Engl J Med 330:1329-1334

297. Kimata H, Yoshida A, Ishioka C, Mikawa H (1991) Disodium cromoglycate (DSCG) selectively inhibits IgE production and enhances IgG4 production by human B cells in vitro. Clin Exp Immunol 84:395-399

298. Kimpen JLL, Garofalo R, Welliver RC, Ogra PL (1992) Activation of human eosinophils in vitro by respiratory syncytial virus. Pediatr Res 32:160-164

299. Kimpen JLL, Garofalo R, Welliver RC, Fujihara K, Ogra PL (1994) An ultrastructural study of the interaction of human eosinophils with respiratory syncytial virus. Pediatr Allergy Immunol 7:48-53

300. Kinberg KA, Hopp RJ, Biven RE, Gallagher JC (1994) Bone mineral density in normal and asthmatic children. J Allergy Clin Immunol 94:490-497

301. Kishida M, Sasamoto A, Saito S, Iikura Y (1992) Oxatomide modifies metacholine- and exercise-induced bronchoconstriction in asthmatic children. Ann Allergy 69:455461

302. Kitchen WH, Olinsky A, Doyle LW et al (1992) Respiratory health and lung function in 8-year-old children of very low birth weight: a cohort study. Pediatrics 9:1151-1158

303. Kjellman N-IM (1993) Is there a place for antihistamines in the treatment of perennial asthma? Pediatr Allergy Immunol 4 [Suppl 4]:38-43

304. Klebl FH, Weber G, Kalden JR, Nüsslein HG (1994) In vitro and in vivo effect of glucocorticoids on IgE and IgG subclass secretion. Clin Exp Allergy 24:1022-1029

305. Klinnert MD, Nelson HS, Price MR, Adinoff AD, Leung DY, Mrazek DA (2001) Onset and persistence of childhood asthma: predictors from infancy. Pediatrics 108:E69

306. Koh YY, Jeong JH, Park Y, Kim CK (1999) Development of wheezing in patients with cough variant asthma during an increase in airway responsiveness. Eur Respir J 14:302-308

307. Koivikko A (1974) Childhood asthma in Finland. Acta Allergol 29:30-72

308. Knorr B, Matz J, Bernstein JA et al (1998) Montelukast for chronic asthma in 6- to 14-year-old children, a randomized, double-blind trial. JAMA 279:1181-1186

309. Knorr B, Franchi LM, Bisgaard H et al (2001) Montelukast, a leukotriene receptor antagonist, for the treatment of persistent asthma in children aged 2 to 5 years. Pediatrics 108:e48

310. Kolski GB, Cunningham AS, Niemec PW, Davignon GF Jr, Freehafer JG (1988) Hypokalemia and respiratory arrest in an infant with status asthmaticus. J Pediatr 12:304-307

311. König P, Shaffer J (1996) The effect of drug therapy on long-term outcome of childhood asthma: a possible preview of the international guidelines. J Allergy Clin Immunol 98:1103-1111

312. König P, Hillman L, Cervantes C et al (1993) Bone mineralization in children with asthma treated with inhaled beclomethasone dipropionate. J Pediatr 122:219-226 
313. Korppi M, Reijonen T, Poysa L, Juntunen-Backman K (1993) A 2- to 3-year outcome after bronchiolitis. Am J Dis Child 147:628-631

314. Korppi M, Kuikka L, Reijonen T, Remes K, Juntunen-Backman K, Launiala K (1994) Bronchial asthma and hyperreactivity after early childhood bronchiolitis or pneumonia. An 8-year follow-up study. Arch Pediatr Adolesc Med 148:1079-1084

315. Koto H, Aizawa H, Takata S, Inoue H, Hara N (1995) An important role of tachykinins in ozone-induced airway hyperresponsiveness. Am J Respir Crit Care Med 151: 1763-1766

316. Kraft M, Torvik JA, Trudeau JB, Wenzel SE, Martin RJ (1996) Theophylline: potential antiinflammatory effects in nocturnal asthma. J Allergy Clin Immunol 97:12421246

317. Krishna MT, Mudway I, Kelly FJ, Frew AJ, Holgate ST (1995) Ozone, airways and allergic airways disease. Clin Exp Allergy 25:1150-1158

318. Kroegel C, Luttman W, Virchow J-C, Matthys H (1995) G-proteins and the lung: nuts and bolts and out of the gutter. Eur Respir J 8:1-4

319. Krüger M, Henschen M, Forster J, Brandis M (1995) Schwierigkeiten bei Diagnose und Therapie der exogenen Alveolitis. Monatsschr Kinderheilkd 143:245-249

320. Kuikka L. Reijonen T, Remes K, Korppi M (1994) Bronchial asthma after early childhood wheezing: a follow-up until 4.5-6 years of age. Acta Pædiatr 83:744-748

321. Kuperman DA, Huang X, Koth LL et al (2002) Direct effects of interleukin-13 on epithelial cells cause airway hyperactivity and mucus over production in asthma. $\mathrm{Na}$ ture Med 8:885-889

322. Kyan-Aung U, Hallsworth M, Haslard D, De Vos C, Lee TH (1992) The effects of cetirizine on the adhesion of human eosinophil and neutrophil to cultured human umbilical vein endothelial cells. J Allergy Clin Immunol 90:270-273

323. La Via WV, Marks MI, Stutman HR (1992) Respiratory syncytial virus puzzle: clinical features, pathophysiology, treatment, and prevention. J Pediatr 121:503-510

324. Laing IA, Eber E, Hayden CM et al (1998) CC16: the A38G polymorphism is associated with airway responsiveness at one month and asthma at 6 years in children unselected for asthma. Am J Respir Crit Care Med 157:A857

325. Laing IA, Hermans C, Bernard A, Burton PR, Goldblatt J, Le Souëf PN (2000) Plasma CC16 levels are associated with the A38G polymorphism and asthma. Am J Respir Crit Care Med 161:124-127

326. Lanier B (1989) Who is dying of asthma and why? In: Lanier B (ed) Priority on pediatric asthma. J Pediatr 115:838-840

327. Laitinen LA, Laitinen A (1996) Remodeling of asthmatic airways by glucocorticosteroids. J Allergy Clin Immunol 97:153-158

328. Laitinen LA, Laitinen A, Haahtela T (1993) Airway mucosal inflammation even in patients with newly diagnosed asthma. Am Rev Respir Dis 147:901-907

329. Laitinen LA, Laitinen A, Haahtela T, Vilkka V, Spur BW, Lee TH (1993) Leukotriene E4 and granulocytic infiltration into asthmatic airways. Lancet 341:989-990

330. Lane SJ, Sousa AR, Lee TH (1994) The role of the macrophage in asthma. Allergy 49:201-209

331. Lanes ST, Garcia Rodriguez LA, Huerta C (2002) Respiratory medications and risk of asthma death. Thorax 57: 683-686
332. Lantner RR, Ros SP (1995) Emergency management of asthma in children: impact of NIH guidelines. Ann Allergy 74:188-191

333. Lantz RC, Dey R (1992) Mechanisms of nonallergic asthma. Immunol Allergy Clin North Am 12:307-327

334. Larché M, Robinson DS, Kay AB (2003) The role of T lymphocytes in the pathogenesis of asthma. J Allergy Clin Immunol 111:450-463

335. Larsen GL (1992) Asthma in children. N Engl J Med 326:1540-1545

336. Le Bourgeois M (1994) Complications. In: Paupe J, Scheinmann P, De Blic J (eds) Allergologie pédiatrique, 2nd edn. Flammarion, Paris, pp 282-293

337. Lei Y, Barnes PJ, Rogers DF (1995) Mechanisms and modulation of airway plasma exudation after direct inhalation of cigarette smoke. Am J Respir Crit Care Med 151:17521757

338. Leickly FE, Wade SL, Crain E et al (1998) Self-reported adherence, management behavior, and barriers to care after an emergency department visit by inner-city children with asthma. Pediatrics 101:1-8

339. Lelong M, Duprey J, Munch ML (1992) Les enfants asthmatiques peuvent-ils rire? (letter). Rev Fr Allergol 32:140

340. Lemanske RF Jr, Busse WW (2003) Asthma. J Allergy Clin Immunol 111:S502-S519

341. Lemanske RF, Dick EC, Swenson CA, Vrtis RF, Busse WW (1989) Rhinovirus upper respiratory infection increases airway hyperreactivity and late asthmatic response. J Clin Invest 83:1-10

342. Lemanske RF Jr, Nayak A, McAlary M, Everhard F, FowlerTaylor A, Gupta N (2002) Omalizumab improves asthmarelated quality of life in children with allergic asthma. Pediatrics 110:e55

343. Lenney W, Pedersen S, Boner AL et al (1995) Efficacy and safety of salmeterol in childhood asthma. Eur J Pediatr 154:983-990

344. Lesouëf PN (1992) Validity of methods used to test airway responsiveness in children. Lancet 339:1282-1284

345. Leung T, Wong C, Ip W, Lam CWK, Wong GWK (2002) Plasma concentration of thymus and activation-regulated chemokine is elevated in childhood asthma. J Allergy Clin Immunol 110:404-409

346. Levison H (1991) The treatment of pediatric asthma: a Canadian consensus. The Medicine Publ Found Symposium Ser 29:97-106

347. Levy J, Zalkinder I, Kuperman O et al (1995) Effect of prolonged use of inhaled steroids on the cellular immunity of children with asthma. J Allergy Clin Immunol 96:806-812

348. Lewis HM (1994) Cough - but is it asthma? (letter) Arch Dis Child 70:554

349. Levy BD, De Sanctis GT, Devchand PR et al (2002) Multipronged inhibition of airway hyper-responsiveness and inflammation by lipoxin A(4). Nat Med 8:1018-1023

350. Li JTC, Gunderson D (1991) Inhalation of the cap of a metered-dose inhaler (letter). N Engl J Med 325:431

351. Lieu TA, Lozano P, Finkelstein JA et al (2002) Racial/ethnic variation in asthma status and management practices among children in managed medicaid. Pediatrics 109: 857-865

352. Lin Y-Z, Hsieh K-H, Chang L-F, Chu C-Y (1996) Terbutaline nebulization and epinephrine injection in treating acute asthmatic children. Pediatr Allergy Immunol 7:9599

353. Lindgren S, Lokshin B, Stromquist A et al (1992) Does asthma or treatment with theophylline limit children's academic performance? N Engl J Med 327:926-930 
354. Little MM, Casale TB (1991) Comparison of platelet-activating factor-induced chemotaxis of normodense and hypodense eosinophils. J Allergy Clin Immunol 88:187-192

355. Little SA, Warner JO (1996) Improved diagnosis of allergic bronchopulmonary aspergillosis with gp66 (formerly antigen 7) of Aspergillus fumigatus for specific IgE detection. J Allergy Clin Immunol 98:55-63

356. Liu M, Wang L, Enhorning G (1995) Surfactant dysfunction develops when the immunized guinea-pig is challenged with ovalbumin aerosol. Clin Exp Allergy 25:10531050

357. Lødrup Carlsen KC, Nikander K, Carlsen K-H (1992) How much nebulised budesonide reaches infants and toddlers? Arch Dis Child 67:1077-1079

358. Loh RKS, Jabara HH, Geha RS (1994) Disodium cromoglycate inhibits $S \mu$ to $S$ deletional switch recombination and IgE synthesis in human B cells. J Exp Med 180:663-671

359. Longo G (1998) I glucocorticoidi per via inalatoria. Med Bambino 17:367-373

360. Lowe L, Murray CL, Custovic A et al (2002) Specific airway resistance in 3-year-old children: a prospective cohort study. Lancet 359:1904-1908

361. Lukacs NW, Tekkanat KK, Berlin A et al (2001) Respiratory syncytial virus predisposes to augmented allergic airway responses via IL-13-mediated mechanisms. J Immunol 167:1060-1065

362. Lundgren JD, Shelhamer JH (1990) Pathogenesis of airway mucus hypersecretion. J Allergy Clin Immunol 85:399417

363. Luparello T, Lyons HA, Bleecker ER, McFadden ER (1968) Influences of suggestion on airway reactivity in asthmatic subjects. Psychosom Med 30:819-825

364. Macdonald JB (1992) Nocturnal asthma. What happens to the airways at night? BMJ 304:998-999

365. MacKenzie CA (1992) Growth of asthmatic children clinical experience with inhaled fluticasone propionate. The management of childhood asthma. Royal Society of Medicine Services, London, pp 20-23

366. Maffei FA, van der Jagt EW, Powers KS, et al (2004) Duration of mechanical ventilation in life-threatening pediatric asthma: Description of an acute asphyxial subgroup. Pediatrics 114:762-767

367. Maggi CA, Patacchini R, Rovero P, Giachetti A (1993) Tachychinins receptors and receptor antagonists. J Auton Pharmacol 13:1-75

368. Mahachoklertwattana P, Sudkronrayudh K, Direkwattanachoi C, Choubtum L, Okascharoen C (2004) Decreased cortisol response to insulin induced hypoglycaemia in asthmatic treated with inhaled fluticasone propionate. Arch Dis Child 89:1055-1058

369. Maiz L, Cuevas M, Quirce S, Pacheco A, Escobar H (1997) Allergic bronchopulmonary aspergillosis with low serum IgE levels in a child with cystic fibrosis. J Allergy Clin Immunol 100:431-432

370. Mallol J, Barueto L, Girardi G et al (1987) Use of nebulized bronchodilators in infants under 1 year of age: analysis of four forms of therapy. Pediatr Pulmonol 3:298-303

371. Mallol J, Barueto L, Girardi G, Toro O (1987) Bronchodilator effect of fenoterol and ipratropium bromide in infants with acute wheezing: use of MDI with a spacer device. Pediatr Pulmonol 3:352-356

372. Mallory MD, Shay DK, Garrett J, Bordley WC (2003) Bronchiolitis management preferences and the influence of pulse oximetry and respiratory rate on the decision to admit. Pediatrics 111:e45
373. Mandelberg A, Tal G, Witzling M et al (2003) Nebulized $3 \%$ hypertonic saline solution treatment in hospitalized infants with viral bronchiolitis Chest123:481-487

374. Marini M, Vittori E, Hollemborg J, Mattoli S (1992) Expression of the potent inflammatory cytokines, granulocyte-macrophage-colony-stimulating factor and interleukin- 6 and interleukin-8, in bronchial epithelial cells of patients with asthma. J Allergy Clin Immunol 89:10011009

375. Marolla F, Mohr P, Borrillo J, Uccella S, Ronchetti R (1992) Fattori epidemiologici associati alla morte per asma in Italia. Pediatra 14:20-24

376. Marone G, Spadaro G, Patella V, Genovese A (1994) The clinical relevance of basophil releasability. J Allergy Clin Immunol 94:1293-1303

377. Marquette CH, Saulnier F, Leroy O et al (1992) Long-term prognosis of near-fatal asthma. Am Rev Respir Dis 146: 76-81

378. Martin RJ (1994) Nocturnal asthma. Ann Allergy 72:5-10

379. Martin RJ, Cicutto LC, Smith HR (1991) Airway inflammation in nocturnal asthma. Am Rev Respir Dis 143:351-357

380. Martin RJ, Cicutto LC, Ackerson LM (1992) Stability of the circadian alteration in lung function in asthma. J Allergy Clin Immunol 89:703-708

381. Martinez FD, Morgan WJ, Wright AL et al (1988) Diminished lung function as a predisposing factor for wheezing respiratory illness in infants. N Engl J Med 319:1112-1117

382. Martinez FD, Morgan WJ, Wright AL, Holberg CJ, Taussig LM (1991) Initial airway function is a predisposing factor for recurrent wheezing respiratory illnesses during the first three years of life. Am Rev Respir Dis 143:312-316

383. Martinez FD, Wright AL, Taussig LM et al (1995) Asthma and wheezing in the first six years of life. N Engl J Med 332:133-138

384. Mascali JJ, Cvietusa P, Negri J, Borish L (1996) Anti-inflammatory effects of theophylline: modulation of cytokine production. Ann Allergy Asthma Immunol 77:3438

385. Massie J, Elfron D, Cerritelli B, et al (2004) Implementation of evidence based guidelines for pediatric asthma management in a teaching hospital. Arch Dis Child 89:660-664

386. Matthews EE, Curtis PD, McLain BI, Morris LS, Turbitt ML (1999) Nebulised budesonide versus oral steroid in severe exacerbations of childhood asthma. Acta Pædiatr 88:841843

387. Mayatepek E, Hoffman GF (1995) Leukotrienes: biosynthesis, metabolism, and pathophysiologic significance. Pediatr Res 37:1-9

388. Mazer BD, Gelfand EW (1991) An open-label study of high-dose intravenous immunoglobulin in severe childhood asthma. J Allergy Clin Immunol 87:976-983

389. McClellan JS, Albers GM, Noyes BE, Sotelo C, Petterchak JA, Knutsen AP (1999) B-lymphocyte aggregates in alveoli from a child with hypersensitivity pneumonitis (bird breeders lung). Ann Allergy Asthma Immunol 83:357-360

390. McConnell R, Berhane K, Gilliland F et al (2002) Asthma in exercising children exposed to ozone: a cohort study. Lancet 359:386-391

391. McConnochie KM, Roghmann KJ (1986) Parental smoking, presence of older siblings, and family history of asthma increase risk of bronchiolitis. Am J Dis Child 140: 806-812

392. McConnochie KM, Russo MJ, McBride JT, Szilagyi PG, Brooks AM, Roghmann KJ (1999) How commonly are children hospitalized for asthma eligible for care in alternative settings? Arch Pediatr Adolesc Med 153:49-55 
393. McFadden ER Jr (1991) Methylxantines in the treatment of asthma: the rise, the fall and the possible rise again. Ann Int Med 115:323-324

394. McFadden ER Jr, Gilbert IA (1994) Exercise-induced asthma. N Engl J Med 330:1362-1367

395. McKenzie SA, Mylonopoulou M, Bridge PD (2001) Bronchodilator responsiveness and atopy in 5-10-year-old coughers. Eur Respir J 18:977-981

396. Meltzer EO, Orgel HA, Ellis EF, Eigen HN, Hemstreet MP (1992) Long-term comparison of three combinations of albuterol, theophylline, and beclomethasone in children with chronic asthma. J Allergy Clin Immunol 90:2-11

397. Mekori JA, Baram D, Goldberg A, Hershkoviz R, Reshef T, Sredni D (1993) Nedocromil sodium inhibits T-cell function in vivo and in vivo. J Allergy Clin Immunol 91:817824

398. Menardo J-L (1995) Assessing nocturnal asthma in children. Pediatr Pulmonol [Suppl 11]:38-39

399. Menon K, Sutcliffe T, Klassen TP (1995) A randomized trial comparing the efficacy of epinephrine with salbutamol in the treatment of acute bronchiolitis. Pediatrics 126: 1004-1007

400. Mertsola J, Ziegler T, Ruuskanen O, Vanto T, Koivikko A, Halonen P (1991) Recurrent wheezy bronchitis and viral respiratory infections. Arch Dis Child 66:124-129

401. Midulla F, Villani F, Panuska JR et al (1993) Respiratory syncytial virus lung infection in infants: immunoregulatory role of infected macrophages. J Infect Dis 168:15151519

402. Migoya E, Kearns GL, Hartford A et al (2004) Pharmacokinetics of montelukast in asthmatic patients 6 to 24 month old. J Clin Pharmacol 44:487-594

403. Miles J, D’Souza W, Burgess C, Beasley R (1995) Management strategies for the "high-risk" asthmatic. Prog Allergy Clin Immunol 3:203-207

404. Milgrom H (1993) Theophylline. Immunol Allergy Clin North Am 13:819-838

405. Milgrom H, Berger W, Nayak A et al (2001) Treatment of childhood asthma with anti-immunoglobulin $\mathrm{E}$ antibody (Omalizumab). Pediatrics 108:e36

406. Miraglia del Giudice M, Decimo F (1990) La terapia farmacologica dell'asma infantile. Riv Ital Pediatr 16:421428

407. Mirone C, Fontana A, Mosca S et al (1994) Effects of nedocromil sodium on bronchospasm and HS-NCA release induced by allergen inhalation in asthmatic patients. Clin Exp Allergy 24:281-287

408. Moens MM, Mertens AV, De Clerck LS, Van Bever HP, Bridts CH, Stevens WJ (1993) Are hypodense eosinophils in children activated or immature? Pediatr Allergy Immunol 4:89-92

409. Molfino NA, Nannini LJ, Martelli AN, Slutsky AS (1991) Respiratory arrest in near-fatal asthma. N Engl J Med 324:285-288

410. Molfino NA, Nannini LJ, Rebuck AS et al (1992) The fatality-prone asthmatic patient. Follow-up study after nearfatal attacks. Chest 101:621-623

411. Molina C, Brun J (1994) Alvéolites allergiques extrinséques. In: Paupe J, Scheinmann P, De Blic J (eds) Allergologie pédiatrique, 2nd edn. Flammarion, Paris, pp 384389

412. Möller GM, Overbreek SE, Van Helden-Meeuwsen CG et al (1996) Increased numbers of dendritic cells in the bronchial mucosa of atopic asthmatic patients: downregulation by inhaled corticosteroids. Clin Exp Allergy 26:517-524
413. Montefort S, Herbert CA, Robinson C, Holgate ST (1992) The bronchial epithelium as a target for inflammatory attack in asthma. Clin Exp Allergy 22:511-520

414. Montgomery GL, Tepper RS (1990) Changes in airway reactivity with age in normal infants and young children. Am Rev Respir Dis 142:1372-1376

415. Morahan G, Huang D, Wu M et al (2002) Association of IL12B promoter polymorphism with severity of atopic and non-atopic asthma in children. Lancet 360:455-459

416. Moreno-Ancillo A, Diaz-Pena J-M, Ferrer A et al (1996) Allergic bronchopulmonary cladosporiosis in a child. J Allergy Clin Immunol 97:714-715

417. Morgan WJ, Martinez FD (1992) Risk factors for developing wheezing and asthma in childhood. Pediatr Clin North Am 39:1185-1203

418. Morley J (1993) Immunopharmacology of asthma. Immunol Today 14:317-322

419. Morton AR, Ogle SL, Fitch KD (1992) Effects of nedocromil sodium, cromolyn sodium, and a placebo in exercise-induced asthma. Ann Allergy 68:143-146

420. Mulligan MS, Polley MJ, Bayer RJ, Nunn MF, Paulson JC, Ward PA (1992) Neutrophil-dependent acute lung injury. Requirement for P-selectin (GMP-140). J Clin Invest 90: 1600-1607

421. Murlas CG, Lang Z, Williams GJ, Chodimella V (1992) Aerosolized neutral endopeptidase reverses ozone-induced airway hyperreactivity to substance P. J Appl Physiol 72:1133-1141

422. Murphy KR, Fitzpatrick S, Cruz-Rivera M, Miller CJ, Parasuraman B (2003) Effects of budesonide inhalation suspension compared with cromolyn sodium nebulizer solution on health status and caregiver quality of life in childhood asthma. Pediatrics 112:e212-219

423. Murray M, Webb MSC, O'Callaghan C, Swarbrick AS, Milner AD (1992) Respiratory status and allergy after bronchiolitis. Arch Dis Child 67:482-487

424. Murray M, Kinnear G, Milner AD (1993) Prevention of fog-induced bronchoconstriction in asthmatic children by nedocromil sodium nebulizer solution. Respir Med 87:65-67

425. Mussaffi H, Greif J, Kornreich L et al (2000) Severe allergic bronchopulmonary aspergillosis in an infant with cystic fibrosis and her asthmatic father. Pediatr Pulmonol 29: 155-159

426. Nadel JA (1994) Neuropeptides in respiratory medicine. In: Kaliner MA (ed) Modulation of neurogenic inflammation by proteases. Marcel Dekker, New York, pp 351-371

427. Nafstad P, Magnus P, Jaakkola JJK (2000) Early respiratory infections and childhood asthma. Pediatrics 106:e38

428. Nagayama Y, Sakurai N (1991) Clinical observations on lower respiratory tract infections with special reference to serum IgE levels. Pediatr Pulmonol 11:44-48

429. Nakai S, Iikura Y, Akimoto K, Shiraki K (1991) Substance P-induced cutaneous and bronchial reactions in children with bronchial asthma. Ann Allergy 66:155-161

430. Nakamura Y, Ghaffar O, Olivenstein R et al (1999) Gene expression of the GATA-3 transcription factor is increased in atopic asthma. J Allergy Clin Immunol 103:215-222

431. Nakano J, Tazikawa H, Ohtoshi T et al (1994) Endotoxin and pro-inflammatory cytokines stimulate endothelin-1 expression and release by airway epithelial cells. Clin Exp Allergy 24:330-336

432. Nall M, Corbett M, McLoughlin J, Petrosko J, Garcia D, Karibo J (1992) Impact of short-term oral steroid use upon children's school achievement and behavior. Ann Allergy 69:218-220 
433. Naspitz CK, Rizzo MC, Arruda LK et al (1995) Environmental control of mite allergy. Progr Allergy Clin Immunol 3:334-339

434. National Asthma Education and Prevention Program Expert Panel (2002) National Asthma Education and Prevention Program Expert Panel Report: guidelines for the diagnosis and management of asthma update on selected topics - 2002. J Allergy Clin Immunol 110:S1-S129

435. National Asthma Education Program (1991) Expert panel report. Guidelines for the diagnosis and management of asthma. NIH Publication No.91-3042A, Bethesda

436. National Heart, Lung and Blood Institute (1995) Global initiative for asthma: global strategy for asthma management and prevention. NIH Publication No. 95-3659, Bethesda

437. National Institutes of Health, National Heart, Lung and Blood Institute. Expert Panel Report 2 (1997) Guidelines for the diagnosis and management of asthma. NIH Publication No. 02-4051, Bethesda, Reprinted 2002

438. Nicolaizik WH, Marchart JL, Pearce MA, Warner JO (1994) Endocrine and lung function in asthmatic children on inhaled corticosteroids. Am J Respir Crit Care Med 150:624628

439. Nieber K, Baumgarten C, Rathsack R et al (1993) Effect of azelastine on substance $P$ content in bronchoalveolar and nasal lavage fluids of patients with allergic asthma. Clin Exp Allergy 23:69-71

440. Nielsen KG, Bisgaard H (2000) The effect of inhaled budesonide on symptoms, lung function, and cold air and methacholine responsiveness in 2- to 5-year-old asthmatic children. Am J Respir Crit Care Med 162(4 Pt 1):15001506

441. Nielsen KG, Bisgaard H (2001) Bronchodilation and bronchoprotection in asthmatic preschool children from formoterol administered by mechanically actuated dry-powder inhaler and spacer. Am J Respir Crit Care Med 164: 256-259

442. Niggemann B, Kleinau I, Schmitt M, Wahn U (1994) Twenty-four-hour time course of eosinophil granule proteins ECP and EPX during bronchial challenges in serum of asthmatic children. Allergy49:74-80

443. Nijkamp FP, Folkerts G (1994) Nitric oxide and bronchial reactivity. Clin Exp Allergy 24:905-914

444. Ninan TK, Russell G (1992) Asthma, inhaled corticosteroid treatment, and growth. Arch Dis Child 67:703-705

445. Ninan TK, Reid IW, Carter PE et al (1993) Effects of high doses of inhaled corticosteroids on adrenal function in children with severe persistent asthma. Thorax 48:599602

446. Nish WA, Schwiez LA (1992) Underdiagnosis of asthma in young adults presenting for USAF basic training. Ann Allergy 69:239-242

447. Noble V, Murray M, Webb MS et al (1997) Respiratory status and allergy nine to 10 years after acute bronchiolitis. Arch Dis Child 76:15-319

448. Noeker M, Petermann E, Walter H-J, Bochmann F, Petermann U, Biberger GA (1993) Asthma bronchiale im Kindes- und Jugendalter. Krankheitsbelastung und Krankheitsbewältigung. Monatsschr Krankheilkd 141:323-329

449. Noguchi E, Yokouchi Y, Shibasaki M et al (2002) Association between TNFA polymorphism and the development of asthma in the Japanese population. Am J Respir Crit Care Med 166:43-46

450. Novembre E, de Martino M, Vierucci A (1988) Foods and respiratory allergy. J Allergy Clin Immunol 81:1059-1065
451. Novembre E, Frongia GF, Veneruso G, Vierucci A (1994) Inhibition of exercise induced asthma by nedocromil sodium and sodium cromoglycate in children. Pediatr Allergy Immunol 5:107-111

452. Nüsslein HG, Weber G, Kalden JR (1994) Synthetic glucocorticoids potentiate IgE synthesis. Allergy 49:365-370

453. O'Brien KP, Fischer TJ (1993) The Dutch hypothesis revisited: recent evidence that children do not outgrow asthma. Pediatr Asthma Allergy Immunol 7:89-97

454. O’Byrne P (1995) Pathogenesis. In: O’Byrne P, Thompson NC (eds) Manual of asthma management. WB Saunders, London, pp 36-50

455. O'Connell EJ, Rojas AR, Sachs MI (1991) Cough-type asthma: a review. Ann Allergy 66:278-285

456. O'Connor BJ, Aikman SL, Bernes PJ (1992) Tolerance to the nonbronchodilator effects of inhaled $\beta_{2}$-agonists in asthma. N Engl J Med 327:1204-1208

457. O'Hollaren MT, Yunginger JW, Offord KP et al (1991) Exposure to an aeroallergen as a possible precipitating factor in respiratory arrest in young patients with asthma. $\mathrm{N}$ Engl J Med 324:359-363

458. Obata T, Matsuda S, Akasawa A, Iikura Y (1993) Preventive effect and duration of action of disodium cromoglycate and procaterol on exercise-induced asthma in asthmatic children. Ann Allergy 70:123-126

459. Oberklaid F, Mellis CM, Le Souëf PN, Geelhoed GC, Maccarrone AL (1993) A comparison of bodyweight dose versus a fixed dose of nebulised salbutamol in acute asthma in children. Med J Aust 158:751-753

460. Ohnishi T, Sur S, Collins DS et al (1993) $\mathrm{IL}_{5}$ is the predominant eosinophil-active cytokine in the antigen-induced pulmonary late-phase reaction. Am Rev Respir Dis 147: 901-907

461. Ohta K, Sawamoto S, Nakajima M et al (1996) The prolonged survival of human eosinophil with interleukin-5 and its inhibition by theophylline via apoptosis. Clin Exp Allergy 26 [Suppl 2]:10-15

462. Okayama Y, Benyon RC, Lowman MA, Church MK (1994) In vitro effects of $\mathrm{H}_{1}$-antihistamines on histamine and $\mathrm{PGD}_{2}$ release from mast cells of human lung, tonsil, and skin. Allergy 49:246-253

463. Ortega AN, Gergen PJ, Paltiel AD, Bauchner H, Belanger KD, Leaderer BP (2002) Impact of site of care, race, and Hispanic ethnicity on medication use for childhood asthma. Pediatrics 109:e1

464. Osundwa VM, Dawod S (1992) Four-year experience with bronchial asthma in a pediatric intensive care unit. Ann Allergy 69:518-520

465. Oswald H, Phelan PD, Lanigan A et al (1997) Childhood asthma and lung function in mid-adult life. Pediatr Pulmonol 23:14-20

466. Owen WF (1991) Cytokine regulation of eosinophil inflammatory disease. ACI News 3:85-89

467. Page CP (1991) One explanation of the asthma paradox: inhibition of natural anti-inflammatory mechanisms by beta2-agonists. Lancet 337:717-720

468. Page R, Friday G, Stillwagon P, Skoner D, Caligiuri L, Fireman P (1988) Asthma and selective immunoglobulin subclass deficiency: improvement of asthma after immunoglobulin replacement therapy. J Pediatr 112:127-131

469. Pajarón M, Sánchez-Solís M, Gutiérrez-Macías A, Pajarón MJ (1996) Bronconeumopatías crónicas: una aproximación inicial a su diagnóstico y tratamiento. Pediatr Integ 2:147-159 
470. Pala P, Bjarnason R, Sigurbergsson F, Metcalfe C, Sigurs N, Openshaw PJ (2002) Enhanced IL-4 responses in children with a history of respiratory syncytial virus bronchiolitis in infancy. Eur Respir J 20:376-382

471. Park ES, Golding J, Carswell F, Stewart-Brown S (1986) Preschool wheezing and prognosis at 10. Arch Dis Child 61:642-646

472. Parks DP, Ahrens RC, Humphries CT, Weinberger MM (1989) Chronic cough in childhood: approach to diagnosis and treatment. In: Lanier B (ed) Priority on pediatric asthma. J Pediatr 115:856-862

473. Patel A, Harrison E, Durward A, Murdoch IA (2000) Intratracheal recombinant human deoxyribonuclease in acute life-threatening asthma refractory to conventional treatment. Br J Anaesth 84:505-507

474. Patel H, Platt RW, Pekeles GS, Ducharme FM (2002) A randomized, controlled trial of the effectiveness of nebulized therapy with epinephrine compared with albuterol and saline in infants hospitalized for acute viral bronchiolitis. J Pediatr 141:818-824

475. Patel L, Wales JK, Kiribige MS, et al (2001) Symptomatic adrenal insufficiency during inhaled corticosteroid treatment. Arch Dis Child 85:330-334

476. Paton JY (1995) Management of chronic asthma in children. In: O'Byrne P, Thompson NC (eds) Manual of asthma management. WB Saunders, London, pp 432-510

477. Pattemore PK, Johnston SL, Bardin PG (1992) Viruses as precipitants of asthma symptoms. I. Epidemiology. Clin Exp Allergy 22:325-336

478. Patterson R, Greenberger PA, Patterson DR (1991) Potentially fatal asthma: the problem of noncompliance. Ann Allergy 67:138-142

479. Pearce N, Crane J, Burgess C, Jackson R, Beasley R (1991) Beta agonists and asthma mortality: déjà vu. Clin Exp Allergy 21:401-410

480. Pearce N, Beasley R, Crane J, Burgess C, Jackson R (1995) End of the New Zealand asthma mortality epidemic. Lancet 345:41-44

481. Pearlman DS, Lemanske RF Jr (1996) Asthma (bronchial asthma): principles of diagnosis and treatment. In: Bierman CW, Pearlman DS, Shapiro GG, Busse WW (eds) Allergy, asthma, and immunology from infancy to adulthood, 3rd edn. WB Saunders, Philadelphia, pp 484-497

482. Pearlman DS, Ostrom NK, Bronsky EA, Bonuccelli CM, Hanby LA (1999) The leukotriene D4-receptor antagonist zafirlukast attenuates exercise-induced bronchoconstriction in children. J Pediatr 134:273-279

483. Peat JK, Britton WJ, Salome CM, Woolcock AJ (1987) Bronchial hyperresponsiveness in two populations of Australian schoolchildren. III. Effect of exposure to environmental allergens. Clin Allergy 17:291-300

484. Peden DB, Berger WE, Noonan MJ et al (1998) Inhaled fluticasone propionate delivered by means of two different multidose powder inhalers is effective and safe in a large pediatric population with persistent asthma. J Allergy Clin Immunol 102:32-38

485. Pedersen S (1995) Management of acute asthma in children. In: O’Byrne P, Thompson NC (eds) Manual of asthma management. WB Saunders, London, pp 511-542

486. Pedersen S, Fost L, Arnfred T (1986) Errors in inhalation technique and efficiency in inhaler use in asthmatic children. Allergy 41:118-124

487. Pedersen S, Ramsgaard Hansen O (1995) Budesonide treatment of moderate and severe asthma in children: a dose-response study. J Allergy Clin Immunol 95:29-33
488. Penna AC, Dawson KP, Manglick P, Tam J (1993) Systemic absorption of salbutamol following nebulizer delivery in acute asthma. Acta Pædiatr 82:963-966

489. Perin PV, Weldon D, McGeady SJ (1994) Objective indicators of severity of asthma. J Allergy Clin Immunol 94: 517-522

490. Persson CGA, Andersson M, Greiff L et al (1995) Airway permeability. Clin Exp Allergy 23:807-814

491. Persson CGA, Erjefält JS, Andersson M et al (1997) Epithelium, microcirculation, and eosinophils - new aspects of the allergic airway in vivo. Allergy 52:241-255

492. Phipatanakul W, Nowak-Wegrzyn A, Eggleston PA (2002) The efficacy of montelukast in the treatment of cat allergen-induced asthma in children. J Allergy Clin Immunol 109:794-799

493. Phillip M, Aviram M, Leiberman E et al (1992) Integrated plasma cortisol concentration in children with asthma receiving long-term inhaled corticosteroids. Pediatr Pulmonol 12:84-89

494. Piacentini GL, Sette L, Peroni DG, Bonizzato C, Bonetti S, Boner AL (1990) Double-blind evaluation of effectiveness and safety of flunisolide aerosol for treatment of bronchial asthma in children. Allergy 45:612-616

495. Piippo-Savolainen E, Remes S, Kannisto S, Korhonen K, Korppi M (2004) Asthma and lung function 20 years after wheezing in infancy: results from a prospective follow-up study. Arch Pediatr Adolesc Med 158:1070-1076

496. Pin I, Radford S, Kolendowicz R et al (1993) Airway inflammation in symptomatic and asymptomatic children with metacholine hyperresponsiveness. Eur Respir J 6:1249-1256

497. Pincus DJ, Szefler SJ, Ackerson LM, Martin RJ (1995) Chronotherapy of asthma with inhaled steroids: the effect of dosage timing on drug efficacy. J Allergy Clin Immunol 95:1173-1178

498. Platts-Mills TAE, Sporik RB, Ward GW, Heymann PW, Chapman MD (1995) Dose-response relationships between asthma and exposure to indoor allergens. Progr Allergy Clin Immunol 3:90-96

499. Plint AC, Johnson DW, Wiebe N et al (2004) Practice varition among pediatric emergency departments in the treatment of bronchiolitis. Acad Emerg Med 11:353-360

500. Pohunek P, Matulka M, Rybnicek O, Kopriva F, Honomichlova H, Svobodova T (2004) Dose-related efficacy and safety of formoterol (Oxis) Turbuhaler compared with salmeterol Diskhaler in children with asthma. Pediatr Allergy Immunol 15:32-39

501. Pohunek P, Warner JO, Turziková J, Kudrmann J, Roche WR (2005) Markers of eosinophilic inflammation and tissue re-modelling in children before clinically diagnosed bronchial asthma. Pediatr Allergy Immunol 16:43-51

502. Potter PC (1995) Genetic abnormalities of the human $\beta 2$ adrenergic receptor. Clin Exp Allergy 25:596-598

503. Poulter LW, Janossy G, Power C, Sreenan S, Burke C (1994) Immunological/physiological relationships in asthma: potential regulation by lung macrophages. Immunol Today 15:258-261

504. Practice parameters for the diagnosis and treatment of asthma. Asthma in children. Spector SL, Nicklas RA (eds) J Allergy Clin Immunol 1995; 96:858-870

505. Prahl P (1991) Adrenocortical suppression following treatment with beclomethasone and budesonide. Clin Exp Allergy 21:145-146

506. Prais D, Danino D, Schonfeld T, Amir J (2005) Impact of Palivizumab on admission to the ICU for respiratory syncytial virus bronchiolitis. A national survey. Chest 128:2765-2771 
507. Pretolani M, Lefort J, Vargaftig BB (1993) Inhibition by nedocromil sodium of recombinant human interleukin-5induced lung hyperresponsiveness to platelet-activating factor in actively sensitized guinea pigs. J Allergy Clin Immunol 91:809-816

508. Price JF (1992) The future management of childhood asthma. The management of childhood asthma. Royal Society of Medicine Services, London, pp 24-29

500. Provisional Committee on Quality Improvement (1994) Practice parameter: the office management of acute exacerbations of asthma in children. Pediatrics 93:119-126

510. Qureshi F Zaritsky A, Poirier MP (2001) Comparative efficacy of oral dexamethasone versus oral prednisone in acute pediatric asthma. J Pediatr 139:20-26

511. Rackham A, Brown CA, Chandra RK et al (1989) A Canadian multicenter study with Zaditen (ketotifen) in the treatment of bronchial asthma in children aged 5-17 years. J Allergy Clin Immunol 84:286-296

512. Rakshi K, Couriel JM (1994) Management of acute bronchiolitis. Arch Dis Child 71:463-469

513. Rao KR, Warner JO (1995) Airway inflammation: is it relevant to childhood asthma? Baillière Clin Pediatr 3:277296

514. Ray GG, Minnich LL, Holberg CJ et al (1993) Respiratory syncytial virus-associated lower respiratory illnesses: possible influence of other agents. Pediatr Infect Dis 12:15-19

515. Ream RS, Loftis LL, Albers GM, Becker BA, Lynch RE, Mink RB (2001) Efficacy of IV theophylline in children with severe status asthmaticus. Chest 119:1480-8

516. Reed CE (1992) Mechanisms of airway obstruction in the asthmatic patient: from past to present. Ann Allergy 69: 245-250

517. Reed CE (1993) Glucocorticoids in asthma. Immunol Allergy Clin North Am 13:903-913

518. Rédier H, Chanez P, De Vos C et al (1992) Inhibitory effect of cetirizine on the bronchial eosinophil recruitment induced by allergen inhalation challenge in allergic patients with asthma. J Allergy Clin Immunol 90:215-224

519. Reese AC, James IR, Landau LI, Lesouëf PN (1993) Relationship between urinary cotinine level and diagnosis in children admitted to hospital. Am Rev Respir Dis 146: 66-70

520. Redington AE, Jones DB, Holgate ST (1994) Mucosal immune function in asthma. In: Ogra PL, Strober W, Mestecky J, McGhee JR, Lamm ME, Bienenstock J (eds) Handbook of mucosal immunology. Academic Press, San Diego, pp 539-549

521. Reigart JR, Etzel RA, Goldman LR, Hendrick JG, Mofenson HC, Simon PR (1993) Ambient air pollution: respiratory hazards to children. Pediatrics 91:1210-1213

522. Reijonen TM, Korppi M, Kuikka L et al (1997) Serum eosinophil cationic protein as a predictor of wheezing after bronchiolitis. Pediatr Pulmonol 23:397-403

523. Renzi PM, Turgeon JP, Yang JP et al (1997) Cellular immunity is activated and a Th2 response is associated with early wheezing in infants after bronchiolitis. J Pediatr 130: 584-593

524. Ricci M, Rossi O (1990) Dysregulation of IgE responses and airway allergic inflammation in atopic individuals. Clin Exp Allergy 20:601-610

525. Ricci M, Romagnani S (1991) Pathogenetic aspects of allergic respiratory syndromes. Ann Ital Med Int 6:183-191

526. Ricci M, Rossi O, Bertoni M, Matucci A (1993) The importance of Th2-like cells in the pathogenesis of airway allergic inflammation. Clin Exp Allergy 23:360-369
527. Richards GN, Kolbe J, Fenwick J, Rea HH (1993) Demographic characteristics of patients with severe life threatening asthma: comparison with asthma deaths. Thorax 48:1105-1109

528. Rimmer SJ, Church MK (1990) The pharmacology and mechanism of action of histamine H1-antagonists. Clin Exp Allergy 20 [Suppl 2]:3-17

529. Ring J (1985) Erstbeschreibung einer "atopischen Familienanamnese" im Julisch-Claudischen Kaiserhaus: Augustus, Claudius, Britannicus. Hautarzt 36:470-474

530. Robinson DS, Hamid Q, Ying S et al (1992) Predominant Th2-like bronchoalveolar T-lymphocyte population in atopic asthma. N Engl J Med 326:298-304

531. Robinson DS, Hamid Q, Bentley A, Ying S, Kay AB, Durham SR (1993) Activation of CD4+ T cells, increased Th2-type cytokine mRNA expression, and eosinophil recruitment in bronchoalveolar lavage after allergen inhalation challenge in patients with atopic asthma. J Allergy Clin Immunol 92:313-324

532. Robinson DS, Ying S, Bentley AM et al (1993) Relationships among numbers of bronchoalveolar lavage cells expressing messenger ribonucleic acid for cytokines, asthma symptoms, and airway methacholine responsiveness in atopic asthma. J Allergy Clin Immunol 92:397-403

533. Robinson GR, Canto RG, Reynolds HY (1993) Host defense mechanisms in respiratory infections. Immunol Clin North Am 13:1-25

534. Roche WR (1991) Fibroblasts and asthma. Clin Exp Allergy $21: 545-548$

535. Romagnani S (2001) T-cell responses in allergy and asthma. Curr Opin Allergy Clin Immunol 1:73-78

536. Ros SP (1991) Emergency management of childhood bronchial asthma: a multicenter survey. Ann Allergy 66: 231-234

537. Rosenwasser LJ (1992) Immunopathophysiology of asthma: mechanism and relation to food allergy. Pediatr Allergy Immunol 3:158-162

538. Rossi GA, Crimi E, Lentero S et al (1991) Late-phase asthmatic reaction to inhaled allergen is associated with early recruitment of eosinophils in the airways. Am Rev Respir Dis 144:379-383

539. Roth M, Johnson PRA, Borges P et al (2004) Dysfunctional interaction of $\mathrm{C} / \mathrm{EBP} \alpha$ and the glucocorticoid receptor in asthmatic bronchial smooth-muscle cells. N Engl J Med 351:560-574

540. Rubilar L, Castro-Rodriguez J, Girardi G (2000) Randomized trial of salbutamol via metered-dose inhaler with spacer versus nebulizer for acutewheezing in children less than 2 years of age. Pediatr Pulmonol 29:264-269

541. Ruiz RG, Price JF (1994) Growth and adrenal responsiveness with budesonide in young asthmatics. Respir Med 88:17-20

542. Rupp NT, Brudno DS, Guill M (1993) The value of screening for risk of exercise-induced asthma in high school athletes. Ann Allergy 70:339-342

543. Rusconi F, Sideri S (1996) Efficacy of epinephrine with salbutamol in treatment of acute bronchiolitis. J Pediatr 128:441-443

544. Rusconi F, Castagneto M, Gagliardi L et al (1994) Reference values for respiratory rate in the first 3 years of life. Pediatrics 94:350-355

545. Rylander E, Eriksson M, Freyschuss U (1988) Risk factors for occasional and recurrent wheezing after RSV infection in infancy. Acta Paediatr Scand 77:711-715 
546. Rylander E, Persson U, Eriksson M (1994) Lymphocyte subsets in infants with wheezy bronchitis. Acta Pædiatr 83:744-748

547. Rylander E, Eriksson M, Pershagen G, Nordvall L, Ernst A, Ziegler T (1996) Wheezing bronchitis in children. Incidence, viral infection, and other risk factors in a defined population. Pediatr Allergy Immunol 7:6-11

548. Saglani S, Malström K, Pelkonen AS et al (2005) Airway remodeling and inflammation in symptomatic infants with reversible airflow obstruction. Am J Respir Crit Care Med 171:722-727. Epub 2005 Jan 18

549. Salmon B, Wilson NM, Silverman M (1990) How much aerosol reaches the lungs of wheezy infants or toddlers? Arch Dis Child 65:401-403

550. Salvatoni A, Nosetti L, Broggini M, Nespoli L (2000) Body composition and growth in asthmatic children treated with inhaled steroids. Ann Allergy Asthma Immunol 85:221-226

551. Sanchez I, De Koster J, Powell RE, Wolstein R, Chernick V (1993) Effect of racemic epinephrine and salbutamol on clinical score and pulmonary mechanics in infants with bronchiolitis. J Pediatr 122:145-151

552. Sanchez-Guerrero I, Albaladejo MD, García-Alonso AM, Muro M, Hernández J, Alvarez MR (1994) Soluble CD23 (sCD23) serum levels and lymphocyte subpopulations in peripheral blood in rhinitis and extrinsic and intrinsic asthma. Allergy 49:587-592

553. Sarkozy F, Boule M, Just J et al (1992) Asthme du nourrisson. Aspects cliniques et fonctionnels. Arch Fr Pédiatr 49:425-428

554. Sarnaik AP, Daphtary KM, Meert KL, Lieh-Lai MW, Heidemann SM (2004) Pressure-controlled ventilation in children with severe status asthmaticus. Pediatr Crit Care Med 5:133-138

555. Sasai K, Furukawa S (1995) Development of bronchial asthma after wheezing associated with respiratory infection in infancy. Acta Pædiatr 84:659-660

556. Scarfone RJ, Loiselle JM, Wiley JF 2nd, Decker JM, Henretig FM, Joffe MD (1995) Nebulized dexamethasone versus oral prednisone in the emergency treatment of asthmatic children. Ann Emerg Med 26:480-486

557. Scarfone RJ, Zorc JJ, Capraro GA (2001) Patient self-management of acute asthma: adherence to national guidelines a decade later. Pediatrics 108:1332-1338

558. Schauer U, Koch B, Michl U, Jäger R, Rieger CHL (1992) Enhanced production of platelet activating factor by peripheral granulocytes from children with asthma. Allergy 47:143-149

559. Scheinmann P, de Blic J, Delacourt C et al (1992) L'enfant asthmatique. Rev Prat 19:2437-2447

560. Schlaghecke R, Kornely E, Santen RT, Ridderskamp P (1992) The effect of long-term glucocorticoid therapy on pituitary-adrenal responses to exogenous corticotropinreleasing hormone. N Engl J Med 326:226-230

561. Schleimer RP, Sterbinsky SA, Kaiser J et al (1992) Interleukin-4 induces adherence of human eosinophils and basophils but not neutrophils to endothelium: association with expression of ICAM-1. J Immunol 148:1086-1092

562. Schlieper A, Alcock D, Beaudry P, Feldman W, Leikin L (1991) Effect of therapeutic plasma concentrations of theophylline, cognitive processing, and affect in children with asthma. J Pediatr 118:449-455

563. Schlosberg M, Liu MC, Bochner BS (1993) Pathophysiology of asthma. Immunol Allergy Clin North Am 13:721-743
564. Schuh S, Parkin P, Rajan A et al (1989) High- versus lowdose, frequently administered, nebulized albuterol in children with severe, acute asthma. Pediatrics 83:513-518

565. Schuh S, Johnson D, Canny G et al (1992) Efficacy of adding nebulized ipratropium bromide to nebulized albuterol therapy in acute bronchiolitis. Pediatrics 90: 920-923

566. Schuh S, Reisman J, Alshehri M et al (2000) A comparison of inhaled fluticasone and oral prednisone for children with severe acute asthma. N Engl J Med 343:689-694

567. Scott MB, Skoner DP (1999) Short-term and long-term safety of budesonide inhalation suspension in infants and young children with persistent asthma. J Allergy Clin Immunol 104:S200-S209

568. Schwiebert LA, Beck LA, Stellato C, Bickel CA, Bochner BS, Schleimer RP (1996) Glucocorticosteroid inhibition of cytokine production: relevance to antiallergic actions. J Allergy Clin Immunol 97:143-152

569. Sears MR (1991) Worldwide trends in asthma mortality. Bull Int Union Tuberc Lung Dis 66:79-83

570. Sears MR (2002) Adverse effects of $\beta$-agonists. J Allergy Clin Immunol 110:S322-S338

571. Sears MR, Taylor DR, Print CG et al (1990) Regular inhaled beta-agonist treatment in bronchial asthma. Lancet 336: 1391-1396

572. Sears MR, Burrows B, Flannery EM, Herbison GB, Hewitt CJ, Holdaway MD (1991) Relation between airway responsiveness and serum IgE in children with asthma and in apparently normal children. N Engl J Med 325:1067-1071

573. Sears MR, Burrows B, Flannery EM, Herbison GB, Holdaway MD (1993) Atopy in childhood. I. Gender and allergen related risks for development of hay fever and asthma. Clin Exp Allergy 23:941-948

574. Sears MR, Burrows B, Herbison GB, Holdaway MD, Flannery EM (1993) Atopy in childhood. II. Relationship to airway responsiveness, and asthma. Clin Exp Allergy 23:949956

575. Sears MR, Greene GM, Willan AR et al (2003) Alongiludinol, population-based, cohort study of childhood asthma followed to adulthood. N Engl J Med 349:1414-1422

576. Sedgwick JB, Bjornsdottir U, Geiger KM, Busse WW (1992) Inhibition of eosinophil density change and leukotriene $\mathrm{C}_{4}$ generation by nedocromil sodium. J Allergy Clin Immunol 90:202-209

577. Sekerel BE, Nakipoglu F (2004) Middle lobe syndrome in children with asthma: review of 56 cases. J Asthma 41: 411-417

578. Serafini U (1992) Can fatal asthma be prevented? A personal view. Clin Exp Allergy 22:576-588

579. Shah A, Pant CS, Bhagat R, Panchal N (1992) CT in childhood allergic bronchopulmonary aspergillosis. Pediatr Radiol 22:227-228

580. Shakib F, Smith SJ (1994) In vitro basophil histamine-releasing activity of circulating IgG1 and IgG4 antibodies from asthma patients and the demonstration that anti-IgE modulates allergen-induced basophil activation. Clin Exp Allergy 24:270-275

581. Shapiro GG (1998) Management of pediatric asthma. Immunol Allergy Clin North Am 18:1-23

582. Shapiro SD, Owen CA (2002) ADAM-33 surfaces as an asthma gene. N Engl J Med 347:936-938

583. Sharek PJ, Bergman DA (2000) The effect of inhaled steroids on the linear growth of children with asthma: a meta-analysis. Pediatrics 106:e8

584. Siegel SC, Rachelefsky GS (1985) Asthma in infants and children: Part I. J Allergy Clin Immunol 76:1-14 
585. Sigurs N, Bjarnason R, Sigurbergsson F (1994) Eosinophil cationic protein in nasal secretion and in serum and myeloperoxidase in serum in respiratory syncytial virus bronchiolitis relation to asthma and atopy. Acta Pædiatr 83:1151-1155

586. Sigurs N, Bjarnason R, Sigurbergsson F, Kjellman B (2000) Respiratory syncitial virus bronchiolitis in infancy is a important risk for asthma and allergy at age 7. Am J Respir Crit Core Med 161:1501-1507

587. Sigurs N, Bjarnason R, Sigurbergsson F, Kjellman B, Björkstén B (1995) Asthma and immunoglobulin E antibodies after respiratory syncytial virus bronchiolitis: a prospective cohort study with matched controls. Pediatrics 95:500-505

588. Silverman ES, Breault DT, Vallone J, et al (2004) Corticoprinreleasing hormone deficiency increases allergen-induced airway inflammation in a mouse model of asthma. J Allergy Clin Immunol 114:747-750

589. Silverman M, Taussig LM (1995) Early childhood asthma: what are the questions? Am J Respir Crit Care Med 151:S1-S44

590. Simon H-U, Grotzer M, Nikolaizik WH, Blaser K, Schöni MH (1994) High altitude climate therapy reduces peripheral blood $\mathrm{T}$ lymphocyte activation, eosinophilia, and bronchial obstruction in children with house-dust mite allergic asthma. Pediatr Pulmonol 17:304-311

591. Simons FE, Villa JR, Lee BW et al (2001) Montelukast added to budesonide in children with persistent asthma: a randomized, double-blind, crossover study. J Pediatr 138: 694-698

592. Simons FER, Persaud MP, Gillespie CA, Cheang M, Shuckett EP (1994) Absence of posterior subcapsular cataracts in young patients treated with inhaled glucocorticoids. Lancet 342:776-778

593. Simons FER, Dolovich J, Moore DW et al (1997) A comparison of beclomethasone, salmeterol, and placebo in children with asthma. N Engl J Med 337:1659-1665

594. Skoner D, Caliguiri L (1988) The wheezing infant. Pediatr Clin North Am 35:1011-1030

595. Sly RM (1989) Mortality from asthma. J Allergy Clin Immunology 84:421-434

596. Sly RM (1994) Changing asthma mortality and sales of inhaled bronchodilators and anti-asthmatic drugs. Ann Allergy 73:439-443

597. Sly PD, Hibbert ME (1989) Childhood asthma following hospitalization with acute viral bronchiolitis in infancy. Pediatr Pulmonol 7:153-158

598. Sly RM, O’Donnell R (1997) Stabilization of asthma mortality. Ann Allergy Asthma Immunol 78:347-354

599. Smith DW, Frankel LR, Mathers LH, Tang ATS, Ariagno RL, Prober CG (1991) A controlled trial of aerosolized ribavirin in infants receiving mechanical ventilation for severe respiratory syncitial virus infection. $\mathrm{N}$ Engl J Med 325:24-29

600. Smith H (1992) Asthma, inflammation, eosinophils and bronchial hyperresponsiveness. Clin Exp Allergy 22: 187-197

601. Soferman R, Bar-Zohar D, Jurgenson U, Fireman E (2004) Soluble CD14 as a predictor of subsequent development of recurrent wheezing in hospitalized young children with respiratory syncytial virus-induced bronchiolitis. Ann Allergy Asthma Immunol 92:545-548

602. Sorva R, Turpeinen M, Juntunen-Backman K, Karonen S-L, Sorva A (1992) Effects of inhaled budesonide on serum markers of bone metabolism in children with asthma. J Allergy Clin Immunol 90:808-815
603. Søyseth V, Kongerud J, Haarr D, Strand O, Bolle R, Boe J (1995) Relation of exposure to airway irritants in infancy to prevalence of bronchial hyper-responsiveness in schoolchildren. Lancet 345:217-220

604. Spahn JD, Szefler SJ (2002) Childhood asthma: new insights into management. J Allergy Clin Immunol 109:3-13

605. Spallarossa D, Sacco O, Girosi D, Rossi GA (1995) Blood eosinophil counts and arterial oxygen tension in acute asthma. Arch Dis Child 73:333-337

606. Spector SL (1993) Update on exercise-induced asthma. Ann Allergy 71:571-577

607. Spencer DA (1992) An update on PAF. Clin Exp Allergy 22:521-524

608. Spitzer WO, Suissa S, Ernst P et al (1992) The use of $\beta$-agonists and the risk of death and near death from asthma. N Engl J Med 326:501-506

609. Sporik R, Holgate ST, Cogswell JJ (1991) Natural history of asthma in childhood - a birth cohort study. Arch Dis Child 66:1050-1053

610. Springer C, Avital A, Maayan C et al (1992) Role of infection in the middle lobe syndrome in asthma. Arch Dis Child 67:592-594

611. Stark JM, Busse WW (1991) Respiratory virus infection and airway hyperreactivity in children. Pediatr Allergy Immunol 2:95-110

612. Staudinger HW, Haas JF (1992) $\beta$-agonists and death from asthma (letter). N Engl J Med 327:355

613. Stein AM, Lerner CA (1993) Behavioral and cognitive effect of theophylline: a dose-response study. Ann Allergy 70:135-140

614. Stein MA, Krasowski M, Leventhal BL, Phillips W, Bender BG (1996) Behavioural and cognitive effects of methylanthine. Arch Pediatr Adolesc Med 50:284-288

615. Stein R, Canny GJ, Bohn DJ, Reisman JJ, Levison H (1989) Severe acute asthma in a pediatric intensive care unit: six years' experience. Pediatrics 83:1023-1028

616. Stein RT, Sherrill D, Morgan WJ et al (1999) Respiratory syncytial virus in early life and risk of wheeze and allergy by age 13 years. Lancet 354:541-545

617. Stempel DA (2003) The pharmacologic management of childhood asthma. Pediatr Clin North Am 50:609-629

618. Stewart EJ, Cinnamond MJ, Siddiqui R, Nicholls DP, Stanford CF (1992) Effect of a heat and moisture retaining mask on exercise induced asthma. BMJ 304:479-480

619. Stewart GA, Thompson PJ, McWilliams AS (1993) Biochemical properties of aeroallergens: contributory factors in allergic sensitization? Pediatr Allergy Immunol 4:163172

620. Stick SM, Burton PR, Clough JB, Cox M, LeSouëf PN, Sly PD (1995) The effects of inhaled beclomethasone dipropionate on lung function and histamine responsiveness in recurrently wheezy infants. Arch Dis Child 73:327-332

621. Strachan DP, Jarvis MJ, Feyerabend C (1990) The relationship of salivary cotinine to respiratory symptoms, spirometry, and exercise-induced bronchospasm in seven-yearold children. Am Rev Respir Dis 142:147-151

622. Strauss RE, Wertheim DL, Bonagura VR, Valacer DJ (1994) Aminophylline therapy does not improve outcome and increases adverse effects in children hospitalized with acute asthmatic exacerbations. Pediatrics 93:205-210

623. Strunk RC (1999) The fatality-prone asthmatic child and adolescent. Immunol Allergy Clin North Am 18:85-97

624. Strunk RC, Fukuhara JT, LaBrecque JF, Mrazek DA (1989) Outcome of long-term hospitalization for asthma in children. J Allergy Clin Immunol 83:17-25 
625. Strunk RC, Bender B, Young DA et al (2002) Predictors of protocol adherence in a pediatric asthma clinical trial. J Allergy Clin Immunol 110:596-602

626. Strunk RC, Sternberg AL, Bacharier LB, Szefler SJ for CAMP (2002) Nocturnal awakening caused by asthma in children with mild-to-moderate asthma in the childhood asthma management program. J Allergy Clin Immunol 110:395-403

627. Sugai T, Sakiyama Y, Matumoto S (1992) Eosinophil cationic protein in peripheral blood of pediatric patients with allergic diseases. Clin Exp Allergy 22:275-281

628. Suissa S, Ernst P (2001) Inhaled corticosteroids: impact on asthma morbidity and mortality. J Allergy Clin Immunol 107:937-944

629. Suissa S, Ernst P, Boivin J-F et al (1994) A cohort analysis of excess mortality in asthma and the use of inhaled $\beta$-agonists. Am J Respir Crit Care Med 149:604-610

630. Suissa S, Ernst P, Benayoun S, Baltzan M, Cai B (2000) Lowdose inhaled corticosteroids and the prevention of death from asthma. N Engl J Med 343:332-336

631. Suzuki K, Iwata S, Iwata H (2002) Allergic bronchopulmonary aspergillosis in a 9-year-old boy. Eur J Pediatr 2002; 161:408-409

632. Sward-Comunelli SL, Mabry SM, Truog WE, Thibeault DW (1997) Airway muscle in preterm infants: changes during development. J Pediatr 130:570-576

633. Szefler SJ (1991) Glucocorticoid therapy for asthma: clinical pharmacology. J Allergy Clin Immunol 88:147-165

634. Szefler SJ (2001) Challenges in assessing outcomes for pediatric asthma. J Allergy Clin Immunol 107:S456-S464

635. Szefler SJ, Bender BG, Jusko WI et al (1995) Evolving role of theophylline for treatment of chronic childhood asthma. J Pediatr 127:176-185

636. Szentivanyi A (1968) The beta adrenergic theory of the atopic abnormality in bronchial asthma. J Allergy 42:203232

637. Tabachnik E, Zadik Z (1991) Diurnal cortisol secretion during therapy with inhaled beclomethasone dipropionate in children with asthma. J Pediatr 118:294-297

638. Tager JB, Hanrahan JP, Tosteson TD et al (1993) Lung function, pre- and post-natal smoke exposure, and wheezing in the first year of life. Am Rev Respir Dis 147:811-817

639. Takeda K, Shibasaki M, Imoto N, Shimakura Y, Takita H (1996) Comparison of basophil histamine release, eosinophil cationic protein and non-specific airway responsiveness between mite-sensitive asthmatics and nonasthmatic children and non-allergic controls. Clin Exp Allergy 26:918-925

640. Tal A, Aviram M, Gorodischer R (1990) Variations in theophylline concentrations detected by 24 -hour saliva concentration profiles in ambulatory children with asthma. J Allergy Clin Immunol 86:238-243

641. Targonski PV, Persky VW, Ramekrishnan V (1995) Effect of environmental molds on risk of death from asthma during the pollen season. J Allergy Clin Immunol 95: 955-961

642. Tasche MJA, van der Wouden JC, Uijen JHJM et al (1997) Randomised placebo-controlled trial of inhaled sodium cromoglycate in 1-4 year old children with moderate asthma. Lancet 350:1060-1064

643. Tasche MJ, Uijen JH, Bernsen RM, de Jongste JC, van der Wouden JC (2000) Inhaled disodium cromoglycate (DSCG) as maintenance therapy in children with asthma: a systematic review. Thorax 55:913-920
644. Tepper RS, Rosenberg D, Eigen H (1992) Airway responsiveness in infants following bronchiolitis. Pediatr Pulmonol 13:6-10

645. The Childhood Asthma Management Program Research Group (2000) Long-term effects of budesonide or nedocromil in children with asthma. N Engl J Med 343:10541063

646. Thomas BC, Stanhope R, Grant R (1994) Impaired growth in children with asthma during treatment with conventional doses of corticosteroids. Acta Pædiatr 83:196-199

647. Thomas EJ, Kumar R, Dasan JB et al (2003) Gastroesophageal reflux in asthmatic children not responding to asthma medication: a scintigraphic study in 126 patients with correlation between scintigraphic and clinical findings of reflux. Clin Imaging 27:333-336

648. Thompson AB, Robbins RA, Romberger DJ et al (1995) Immunological functions of the pulmonary epithelium. Eur Respir J 8:127-149

649. Tinkelman DG, Reed CE, Nelson HS, Offord KP (1993) Aerosol beclometasone dipropionate compared with theophylline in primary treatment of chronic, mild to moderately severe asthma in children. Pediatrics 92:64-77

650. Todd G, Dunlop K, McNaboe J, Ryan MF, Carson D, Shields MD (1996) Growth and adrenal suppression in asthmatic children treated with high-dose fluticasone propionate. Lancet 348:27-29

651. Todd GRG, Acerini CL, Buck JJ et al (2002) Acute adrenal crisis in asthmatics treated with high-dose fluticasone propionate. Eur Respir J 19:1-3

652. Todd GRG, Acerini CL, Ross-Russell R, Zahra S, Warner JT, McCance D (2002) Survey of adrenal crisis associated with inhaled corticosteroids in the United Kingdom. Arch Dis Child 87:457-461

653. Todokoro M, Mochizuki H, Tokuyama K, Morikawa A (2003) Childhood cough variant asthma and its relation to classic asthma. Ann Allergy Asthma Immunol 90:652-659

654. Tonnel A-B, Lassalle Ph, Delneste Y, Wallaert B (1992) Endothelial cells and bronchial asthma. In: Godard P, Bousquet J, Michel FB (eds) Advances in allergology and clinical immunology. Parthenon Publishing, Lancs, pp 153160

655. Toogood JH, Jennings B, Hodsman AB, Baskerville J, Fraher LJ (1991) Effect of dose and dosing schedule of inhaled budesonide on bone turnover. J Allergy Clin Immunol 88:573-580

656. Townley RG (1992) Antiallergic properties of the secondgeneration $\mathrm{H} 1$ antihistamines during the early and late reactions to antigen. J Allergy Clin Immunol 90:720-725

657. Townsend M, Feeny DH, Guyatt GH, Furlong WJ, Seip AE, Dolovich J (1991) Evaluation of the burden of illness for pediatric asthmatic patients and their parents. Ann Allergy 67:403-408

658. Toyoshima K, Doi S, Murayama N (1996) Effect of longterm administration of theophylline on serum immunoglobulin E level in asthmatic children - preliminary study. Clin Exp Allergy 26 [Suppl 2]:28-31

659. Tsai Y-G, Lee M-Y, Yang KD et al (2001) A single dose of nebulized budesonide decreases exhaled nitric oxide in children with acute asthma. J Pediatr 139:433-437

660. Tunon-de-Lara JM, Redington AE, Bradding P et al (1996) Dendritic cells in normal and asthmatic airways: expression of the a subunit of the high affinity immunoglobulin E receptor (FceRI-a). Clin Exp Allergy 26:648-655 
661. Turki J, Pak J, Green SA, Martin RJ, Liggett SB (1995) Genetic polymorphisms of the $\beta_{2}$-adrenergic receptor in nocturnal and nonnocturnal asthma: evidence that Gly16 correlates with the nocturnal phenotype. J Clin Invest 95:1635-1641

662. Twentyman OP, Ollier S, Holgate ST (1993) The effect of H1-receptor blockade on the development of early- and late-phase bronchoconstriction and increased bronchial responsiveness in allergen-induced asthma. J Allergy Clin Immunol 91:1169-1178

663. Ulrik CS, Backer V, Bach-Mortensen N (1992) Bronchodilating effect of ipratropium bromide inhalation powder and aerosol in children and adolescents with stable bronchial asthma. Allergy 47:133-137

664. Urquhart AD (1993) Asthma management guidelines. BMJ 306:1542

665. Van Aalderen WMC, Postma DS, Koeter G, Knol K (1989) Circadian change in bronchial responsiveness and airflow obstruction in asthmatic children. Thorax 44:803-807

666. Van Asperen PP, Kemp AS, Mukhi A (1990) Atopy in infancy predicts the severity of bronchial hyperresponsiveness in later childhood. J Allergy Clin Immunol 85:790-795

667. Van Asperen PP, McKay KO, Mellis CM, Loh RK, Harth SC, Thang YA (1992) A multicentre randomised placebo controlled double blind study on the efficacy of ketotifen in infants with chronic cough or wheeze. J Paediatr Child Health 28:442-446

668. Van Bever JP, Schuddinck L, Wojciechowski M, Steven WJ (1990) Aerosolized budesonide in asthmatic infants. Pediatr Pulmonol 9:177-180

669. Van de Graal E, Out TA, Ross CM et al (1991) Respiratory membrane permeability and bronchial hyperreactivity in patients with stable asthma. Am Rev Respir Dis 143:362368

670. Van der Sande MAB, Kidd IM, Goetghebuer T et al (2002) Severe respiratory syncytial virus infection in early life is associated with increased type 2 cytokine production in Gambian children. Clin Exp Allergy 32:1430-1435

671. Van Essen-Zandvliet EEM (1995) Long-term intervention in childhood asthma: the Dutch study results. Monaldi Arch Chest Dis 50:201-207

672. Van Essen-Zandvliet EEM, Hugues MD, Waalkens HJ et al (1992) Effects of 22 months of treatment with inhaled corticosteroids and/or beta 2 agonists on lung function, airway responsiveness, and symptoms in children with asthma. Am Rev Respir Dis 146:547-554

673. Wang JL, Patterson R, Mintzer R, Roberts M, Rosenberg M (1979) Allergic bronchopulmonary aspergillosis in pediatric practice. J Pediatr 94:376-381

674. VanWoensel JBM, van Aalderen WMC (2002) Treatment for bronchiolitis: the story continues. Lancet 360:101-102

675. Vanhoutte PM (1989) Epithelium-derived relaxing factor(s) and bronchial reactivity. J Allergy Clin Immunol 83:855-861

676. Vells-Karp M (2001) IL-12/IL-13 axis in allergic asthma. J Allergy Clin Immunol 107:9-18

677. Venaille TJ, Mendis AHW, Phillips MJ, Thompson PJ, Robinson BWS (1995) Role of neutrophils in mediating human epithelial cell detachment from native basement membrane. J Allergy Clin Immunol 95:597-606

678. Venge P (1993) Serum measurements of eosinophil cationic protein in bronchial asthma. Clin Exp Allergy 23 [Suppl 2]:3-7

679. Venge P, Håkansson L (1991) Current understanding of the role of the eosinophil granulocyte in asthma. Clin Exp Allergy 21 [Suppl 3]:31-38
680. Verberne AAPH, Hop WCJ, Creyghton FBM et al (1996) Airway responsiveness after a single dose of salmeterol and during four months of treatment in children with asthma. J Allergy Clin Immunol 97:938-946

681. Vignola AM, Kips J, Bousquet J (2000) Tissue remodeling as a feature of persistent asthma. J Allergy Clin Immunol 105:1041-1053

682. Virchow J-C Jr, Oehling A, Boer L et al (1994) Pulmonary function, activated $\mathrm{T}$ cells, peripheral blood eosinophilia, and serum activity for eosinophil survival in vitro: a longitudinal study in bronchial asthma. J Allergy Clin Immunol 94:240-249

683. Viviano GG, Conte M, Benedetto CP, Viviano MT, Tancredi G (1995) Lo stato asmatico. Pediatr Med Chir 17:29-32

684. Volovitz B, Amir J, Malik H, Kauschansky A, Varsano I (1993) Growth and pituitary-adrenal function in children with severe asthma treated with inhaled budesonide. N Engl J Med 329:1703-1708

685. Volovitz B, Kauschansky A, Nussinovitch M, Harel L, Varsano I (1995) Normal diurnal variation in serum cortisol concentration in asthmatic children treated with inhaled budesonide. J Allergy Clin Immunol 96:874-878

686. Volovitz B, Welliver RC, De Castro G, Krystofic DA, Ogra PL (1988) The release of leukotrienes in the respiratory tract during infection with respiratory syncytial virus: role in obstructive airways disease. Pediatr Res 24:504507

687. Von Klot S, Wolke G, Tuch T et al (2002) Increased asthma medication use in association with ambient fine and ultrafine particles. Eur Respir J 20:691-702

688. Waalkens HJ, Van Essen-Zandvliet EE, Hughes MD et al (1993) Cessation of long-term treatment with inhaled corticosteroid (budesonide) in children with asthma results in deterioration. Am Rev Respir Dis 148:1252-1257

689. Wainwright C, Altamirano L, Cheney M et al (2003) A multicenter, randomized, double-blind, controlled trial of nebulized epinephrine in infants with acute bronchiolitis. N Engl J Med 349:27-35

690. Wales JKH, Barnes ND, Swift PGF (1991) Growth retardation in children on steroids for asthma (letter). Lancet 338:1535

691. Walker C, Kaegi MK, Braun P, Blaser K (1991) Activated $\mathrm{T}$ cells and eosinophilia in bronchoalveolar lavages from subjects with asthma correlated with disease severity. J Allergy Clin Immunol 88:935-942

692. Wang J, Homer RJ, Hong L, Cohn L, Lee CG, Jung S et al (2000) IL-11 selectively inhibits aeroallergen-induced pulmonary eosinophilia and Th2 cytokine production. J Immunol 165:2222-2231

693. Wang JL, Patterson R, Mintzer R, Roberts M, Rosenberg M (1979) Allergic bronchopulmonary aspergillosis in pediatric practice. J Pediatr 94:376-381

694. Wardlaw AJ (1995) Eosinophil density: what does it mean? Clin Exp Allergy 25:1145-1149

695. Wardlaw AJ (1993) The role of air pollution in asthma. Clin Exp Allergy 23:81-96

696. Warman KL, Silver EJ, McCourt MP, Stein REK (1999) How does home management of asthma exacerbations by parents of inner-city children differ from NHLBI guideline recommendations? Pediatrics 103:422-427

697. Warner JO (1994) The $\beta$-agonist controversy and its relevance in the treatment of children. Eur Respir Rev 4:21-26

698. Warner JO, Götz M, Landau LI et al (1989) Management of asthma: a consensus statement. Arch Dis Child 64: 1065-1079 
699. Warner JO, Neijens HJ, Landau LI et al (1992) Asthma: a follow-up statement from an international asthma paediatric consensus group. Arch Dis Child 67:240-248

700. Warner JO, Naspitz CK, Cropp GJA (1998) Third international pediatric consensus statement on the management of childhood asthma. Pediatr Pumonol 25:1-17

701. Warringa RAJ, Mengelers HJJ, Maikoe T, Bruijnzel PLB, Koenderman L (1993) Inhibition of cytokine-primed eosinophil chemotaxis by nedocromil sodium. J Allergy Clin Immunol 91:802-809

702. Wasowska-Krolikowska K, Toporowska-Kowalska E, Krogulska A (2002) Asthma and gastroesophageal reflux in children. Med Sci Monit 8:RA64-R71

703. Wasserfallen J-B, Baraniuk JN (1996) Clinical use of inhaled corticosteroids in asthma. J Allergy Clin Immunol 97:177-182

704. Wasserman SI (ed) (1993) Nedocromil sodium: a pyranoquinoline antiinflammatory agent for the treatment of asthma. Proceedings of a symposium. J Allergy Clin Immunol 92:143-216

705. Wegner CD, Gundel RH, Reilly P, Haynes N, Letts LG, Rothlein R (1990) Intercellular adhesion molecule-1 (ICAM-1) in the pathogenesis of asthma. Science 247:456-459

706. Weinberger M (1993) Theophylline: when should it be used? J Pediatr 122:403-405

707. Weinberger M, Hendeles L (1996) Theophylline in asthma. N Engl J Med 334:1380-1388

708. Weinstein AG, Faust DS, McKee L, Padman R (1992) Outcome of short-term hospitalization for children with severe asthma. J Allergy Clin Immunol 90:66-75

709. Weisman Z, Fink A, Alon A et al (1990) Leucotriene C4 decreases the activity of respiratory cilia in vitro. Clin Exp Allergy 20:389-393

710. Weiss KB, Wagener DK (1990) Changing patterns of asthma mortality. JAMA 264:1683-1687

711. Welch MJ, Kemp JP, Meltzer EO, Orgel HA, Ostrom NK (1991) Asthma in the schools. Immunol Allergy Clin North Am 11:173-181

712. Welliver RC. (1997) Therapy for bronchiolitis: help wanted. J Pediatr 130:170-172

713. Welliver RC, Duffy L (1993) The relationship of RSV-specific immunoglobulin $\mathrm{E}$ antibody responses in infancy, recurrent wheezing, and pulmonary function at age 7-8 years. Pediatr Pulmonol 15:19-27

714. Welliver RC, Wong DT, Sun M, Middleton E Jr, Vaughan RS, Ogra PL (1981) The development of respiratory syncytial virus specific IgE and release of histamine in nasopharyngeal secretions after infection. N Engl J Med 306:841-846

715. Welliver RC, Sun M, Rinaldo D, Ogra PL (1986) Predictive value of RSV-specific IgE responses for recurrent wheezing following bronchiolitis. J Pediatr 109:776-780

716. Welliver RC, Wong DT, Sun M, McCarthy N (1986) Parainfluenza virus bronchiolitis. Arch Dis Child 140:34-40

717. Werner HA (2001) Status asthmaticus in children. Chest 119:1913-1929

718. Whitaker K, Webb J, Barnes J, Barnes ND (1996) Effect of fluticasone on growth in children with asthma. Lancet 348:63-64

719. White MV (1995) Muscarinic receptors in human airways. J Allergy Clin Immunol 95:1065-1068

720. Wiens L, Sabath R. Ewing L, Gowdamarjan R, Portnoy J, Scagliotti D (1992) Chest pain in otherwise healthy children and adolescents is frequently caused by exercise-induced asthma. Pediatrics 90:350-353
721. Wild JS, Sigounas A, Sur N et al (2000) IFN-gamma-inducing factor (IL-18) increases allergic sensitization, serum IgE, Th2 cytokines, and airway eosinophilia in a mouse model of allergic asthma. J Immunol 164:2701-2710

722. Williams PV, Shapiro GG (1990) Bronchial hyperresponsiveness in children. Immunol Allergy Clin North Am 10:423-437

723. Wilson JW, Djukanovic R, Howarth PH, Holgate ST (1994) Inhaled beclomethasone dipropionate downregulates airway lymphocyte activation in atopic asthma. Am J Respir Crit Care Med 149:86-90

724. Wilson NM (1994) The significance of early wheezing. Clin Exp Allergy 24:522-529

725. Wilson NM, Phagoo SB, Silverman M (1992) Atopy, bronchial responsiveness, and symptoms in wheezy 3 year olds. Arch Dis Child 67:491-495

726. Wjst M, Dold S, Roell G et al (1994) Bronchial hyperreactivity and history of wheezing in children. Eur J Pediatr 153:682-686

727. Wohl MEB, Majzoub JA (2000) Asthma, steroids, and growth. N Engl J Med 343:1113-1114

728. Wolthers OD (1996) Long-, intermediate- and short-term growth studies in asthmatic children treated with inhaled glucocorticoids, Eur Respir J 9:821-827

729. Wolthers OD, Petersen S (1991) Growth of asthmatic children during treatment with budesonide: a double blind trial. BMJ 303:163-165

730. Wolthers OD, Petersen S (1992) Controlled study of linear growth in asthmatic children during treatment with inhaled glucocorticoids. Pediatrics 89:839-842

731. Wolthers OD, Petersen S (1993) Short-term growth during treatment with inhaled fluticasone propionate and beclomethasone dipropionate. Arch Dis Child 68:673-676

732. Wolthers OD, Allen DB (2002) Inhaled corticosteroids, growth, and compliance. N Engl J Med 347:1210-1211

733. Wolthers OD, Riis BJ, Petersen S (1993) Bone turnover in asthmatic children treated with oral prednisolone or inhaled budesonide. Pediatr Pulmonol 16:341-346

734. Wolthers OD, Juul A, Hansen M, Müller J, Petersen S (1995) The insulin-like growth factor axis and collagen turnover in asthmatic children treated with inhaled budesonide. Acta Pædiatr 84:393-397

735. Wolthers OD, Hansen M, Juul A, Kaspersen Nielsen H, Petersen S (1997) Knemometry, urine cortisol excretion, and measures of the insulin-like growth factor axis and collagen turnover in children treated with inhaled glucocorticosteroids. Pediatr Res 41:44-50

736. Wong JYW, Zacharin MR, Hocking N et al (2002) Growth and adrenal suppression in asthmatic children on moderate to high doses of fluticasone propionate. J Paediatr Child Health 38:59-62

737. Wood RE (1985) Clinical application of ultrathin flexible bronchoscopes. Pediatr Pulmonol 1:244-248

738. Woodman K, Pearce N, Beasly R et al (1992) Albuterol and deaths from asthma in New Zealand from 1969 to 1976: a case control study. Clin Pharmacol Ther 51:566-571

739. Woolcock AJ, Peat JK (1995) Definition, classification, epidemiology and risk factors for asthma. In: O’Byrne P, Thompson NC (eds) Manual of asthma management. WB Saunders, London, pp 3-27

740. Wrenn K, Slovis CM, Murphy F, Greenberg RS (1991) Aminophylline therapy for acute bronchospastic disease in the emergency room. Ann Int Med 115:241-247 
741. Wright AL, Holberg CJ, Morgan WJ, Taussig LM, Halonen M, Martinez FD (1996) Recurrent cough in childhood and its relation to asthma. Am J Respir Crit Care Med 153: 1259-1265

742. Yamaguchi M, Hirai K, Shoji S et al (1992) Haemopoietic growth-factors induce human basophil migration in vitro. Clin Exp Allergy 22:379-384

743. Yamamoto H, Nagata M, Kazuaki T et al (1993) The evidence of platelet activation in asthma. J Allergy Clin Immunol 91:79-87

744. Yoshihara S, Geppetti P, Linden A, Hara M, Chan B, Nadel JA (1996) Tachykinins mediate the potentiation by cold air in guinea pigs. J Allergy Clin Immunol 97:756-760

745. Young S, Le Souëf PN, Geelhoed GC et al (1991) The influence of a family history of asthma and parental smoking on airway responsiveness in early infancy. $\mathrm{N}$ Engl J Med 324:1168-1173

746. Young S, Arnott J, Le Souëf PN, Landau LI (1994) Flow limitation during tidal expiration in symptom-free infants and the subsequent development of asthma. J Pediatr 124:681-688

747. Young S, O’Keeffe PT, Arnott J, Landau LI (1995) Lung function, airway responsiveness, and respiratory symptoms before and after bronchiolitis. Arch Dis Child 72:16-24

748. Zacharisen MC, Schlueter DP, Kurup VP, Fink JN (2002) The long-term outcome in acute, subacute, and chronic forms of pigeon breeder's disease hypersensitivity pneumonitis. Ann Allergy Asthma Immunol 88:175-182

749. Zeck-Kapp G, Kroegel C, Riede UN, Kapp G (1995) Mechanism of human eosinophil activation by complement protein $\mathrm{C} 5 \mathrm{a}$ and platelet activating factor: similar functional responses are accompanied by different morphologic alterations. Allergy 50:34-47

750. Zeiger RS, Heller S, Mellon MH, Wald J, Falkoff R, Schatz M (1991) Facilitated referral to asthma specialist reduces relapses in asthma emergency visits. J Allergy Clin Immunol 87:1160-1168
751. Zelaya CEA, Örvell C, Strannegård Ö (1994) Eosinophil cationic protein in nasopharyngeal secretions and serum of infants infected with respiratory syncytial virus. Pediatr Allergy Immunol 5:100-106

752. Zheng T, Zhu Z, Wang J, Homer RJ, Elias, JA (2001) IL-11: insights in asthma from overexpression transgenic modeling. J Allergy Clin Immunol 108:489-496

753. Zhong NS, Cheng RC, Yang MO, Wu ZY, Zheng JP, Li YF (1992) Is asymptomatic bronchial hyperresponsiveness an indication of potential asthma? A two-year follow-up of young students with bronchial hyperresponsiveness. Chest 102:1104-1109

754. Zieg G, Lack G, Harbeck RJ, Gelfand EW, Leung DYM (1994) In vivo effect of glucocorticoids on IgE production. J Allergy Clin Immunol 94:222-230

755. Zimmerman B, Lanner I, Enander I, Zimmerman RS, Peterson CGB, Ahlstedt S (1993) Total blood eosinophils, serum eosinophil cationic proteins and eosinophilic protein $\mathrm{X}$ in childhood asthma: relation to disease status and therapy. Clin Exp Allergy 23:564-570

756. Zimmerman B, Enander I, Zimmerman R, Ahlstedt S (1994) Asthma in children less than 5 years of age: eosinophils and serum levels of the eosinophil proteins ECP and EPX in relation to atopy and symptoms. Clin Exp Allergy 24:149-155

757. Zimmerman B, Gold M, Wherrett D, Hanna AK (1998) Adrenal suppression in two patients with asthma treated with low doses of the inhaled steroid fluticasone propionate. J Allergy Clin Immunol 101:425-426

758. Zoratti EM, Sedgwick JB, Vrtis RR, Busse WW (1991) The effect of platelet-activating factor on the generation of superoxide anion in human eosinophils and neutrophils. J Allergy Clin Immunol 88:749-758

759. Zoritch B (1995) Nitric oxide and asthma. Arch Dis Child 72:259-262

760. Zwillich CW, Neagley SR, Cicutto L et al (1989) Nocturnal asthma therapy: inhaled bitolterol versus sustained release theophylline. Am Rev Respir Dis 139:470-474 